2018

\title{
Autonomous decentralised M2M Application Service Provision
}

\author{
Steinheimer, Michael
}

http://hdl.handle.net/10026.1/11957

http://dx.doi.org/10.24382/1243

University of Plymouth

All content in PEARL is protected by copyright law. Author manuscripts are made available in accordance with publisher policies. Please cite only the published version using the details provided on the item record or document. In the absence of an open licence (e.g. Creative Commons), permissions for further reuse of content should be sought from the publisher or author. 


\section{Copyright Statement}

This copy of the thesis has been supplied on condition that anyone who consults it is understood to recognise that its copyright rests with its author and that no quotation from the thesis and no information derived from it may be published without the author's prior consent. 



\title{
Autonomous decentralised M2M Application Service Provision
}

\author{
by \\ Michael Steinheimer
}

A thesis submitted to University of Plymouth

in partial fulfilment for the degree of

\section{DOCTOR OF PHILOSOPHY}

School of Computing and Mathematics

Darmstadt Node of the Centre for Security, Communications and Network Research (CSCAN) 



\section{Acknowledgements}

First and foremost, I would like to thank my supervisors Prof. Woldemar Fuhrmann and Dr. Bogdan Ghita for their comprehensive support and guidance during this research work.

My special thanks go to my supervisor Prof. Ulrich Trick, who has always been an excellent mentor for me. Thank you very much for your support during my entire time as a member of the Research Group for Telecommunication Networks at the Frankfurt University of Applied Sciences. The research on which this work is based was only possible through your guidance, support, and motivation.

Many thanks go to current and especially former fellow researchers of the Research Group for Telecommunication Networks. Thank you for your friendship, support, and great inspiration during the past years.

Warm thanks to members of both the graduate school, especially Carole Watson for her kind and comprehensive support in administrative things, as well as the CSCAN Network at University of Plymouth. I would also like to thank the members of the CSCAN Darmstadt Node for their support and helpful comments in academic aspects especially during the Ph.D. seminars.

I would like to thank my family and friends for their appreciation of the fact that my priorities in recent years have been to do this research.

Above all, I would like to thank my loving wife Melanie and our wonderful son Paul for their understanding, great support, and the sacrifices they made on my behalf. This Ph.D. thesis is dedicated to you. 



\section{Author's declaration}

At no time during the registration for the degree of Doctor of Philosophy has the author been registered for any other University award without prior agreement of the Doctoral College Quality Sub-Committee.

Work submitted for this research degree at the University of Plymouth has not formed part of any other degree either at the University of Plymouth or at another establishment.

Relevant scientific seminars and conferences were regularly attended at which work was often presented, and several papers prepared for publication.

Publications:

- Steinheimer, M.; Trick, U.; Ruhrig, P. (2012a), "New approaches for energy optimisation in Smart Homes and Smart Grids by automated service generation and user integration", Proceedings of the Collaborative European Research Conference (CERC), pp. 111-119, April 2012, Darmstadt, Germany

- Steinheimer, M.; Trick, U.; Ruhrig, P. (2012b), "Energy communities in Smart Markets for optimisation of peer-to-peer interconnected Smart Homes", Proceedings of the 2012 $8^{\text {th }}$ International Symposium on Communication Systems, Networks \& Digital Signal Processing (CSNDSP), pp. 1-6, July 2012, Poznan, Poland, IEEE

- Steinheimer, M.; Trick, U.; Ruhrig, P.; Wacht, P.; Tönjes, R.; Fischer, M.; Hölker, D. (2013a), "SIP-basierte P2P-Vernetzung in einer Energie-Community" (translated title: "SIP-based P2P Networking inside an Energy-Community"), Proceedings of the Eighteenth VDE/ITG Mobilfunktagung, pp. 64-70, Mai 2013, Osnabrück, Germany, VDE

- Steinheimer, M.; Trick, U.; Ruhrig, P.; Fuhrmann, W.; Ghita, B. (2013b), "Load Reduction in Distribution Networks through P2P networked Energy-Community", Proceedings of the Fifth International Conference on Internet Technologies and Applications (ITA 13), pp. 90-97, September 2013, Wrexham, UK

- Steinheimer, M.; Trick, U.; Wacht, P.; Ruhrig, P. (2013c), "Decentralised optimisation solution for Smart Grids using Smart Market aspects and P2P internetworked EnergyCommunity“, VDE/IEC World Smart Grid Forum 2013, September 2013, Berlin, Germany, VDE

- Steinheimer, M.; Trick, U.; Wacht, P.; Ruhrig, P.; Fuhrmann, W.; Ghita, B. (2013d), "Decentralised optimisation solution for provision of value added services and optimisation possibilities in Smart Grids", 2013 Collaborative European Research Conference (CERC), October 2013, Cork, Ireland

- Steinheimer, M.; Trick, U.; Fuhrmann, W.; Ghita, B. (2013e), "P2P-based community concept for M2M Applications", Proceedings of the Second International Conference on Future Generation Communication Technologies (FGCT 2013), pp. 114-119, November 2013, London, UK, IEEE

- Steinheimer, M.; Trick, U.; Fuhrmann, W.; Ghita, B. (2015a), "P2P based service provisioning in M2M networks", Proceedings of the Sixth International Conference on Internet Technologies and Applications (ITA 15), pp. 132-137, September 2015, Wrexham, UK, IEEE 
- Steinheimer, M.; Trick, U.; Fuhrmann, W.; Ghita, B. (2015b), "P2P based service provisioning in M2M networks", Second Spanish-Geman Symposium on Applied Computer Science (SGSOACS), Invited Talk, December 2015, Frankfurt, Germany

- Steinheimer, M.; Trick, U.; Fuhrmann, W.; Ghita, B. (2016), "P2P-based M2M Community Applications", Proceedings of the Eleventh International Network Conference (INC 2016), pp. 115-120, July 2016, Frankfurt, Germany

- Steinheimer, M.; Trick, U.; Fuhrmann, W.; Ghita, B.; Frick, G. (2017a), "M2M Application Service Provision: An autonomous and decentralised Approach", Journal of Communications, Vol. 12, no. 9, pp. 489-498, 2017

- Steinheimer, M.; Trick, U.; Fuhrmann, W.; Ghita, B. (2017b), "Decentralised System Architecture for autonomous and cooperative M2M Application Service Provision", Proceedings of the 2017 IEEE International Conference on Smart Grid and Smart Cities (ICSGSC 2017), pp. 312-317, July 2017, Singapore, IEEE

- Steinheimer, M.; Trick, U.; Fuhrmann, W.; Ghita, B. (2017c), "Autonomous decentralised M2M Application Service Provision", Proceedings of the Seventh International Conference on Internet Technologies and Applications (ITA 17), pp. 18-23, September 2017, Wrexham, UK, IEEE

Presentations and Conferences attended:

- Collaborative European Research Conference (CERC), Darmstadt, Germany, April 2012

- $8^{\text {th }}$ International Symposium on Communication Systems, Networks \& Digital Signal Processing (CSNDSP), Poznan, Poland, July 2012

- $18^{\text {th }}$ VDE/ITG Mobilfunktagung, Osnabrück, Germany, Mai 2013

- $5^{\text {th }}$ International Conference on Internet Technologies and Applications (ITA 13), Wrexham, UK, September 2013

- VDE/IEC World Smart Grid Forum 2013, Berlin, Germany, September 2013

- Collaborative European Research Conference (CERC), Cork, Ireland, October 2013

- $\quad 2^{\text {nd }}$ International Conference on Future Generation Communication Technologies (FGCT 2013), London, UK, November 2013

- $6^{\text {th }}$ International Conference on Internet Technologies and Applications (ITA 15), Wrexham, UK, September 2015

- $2^{\text {nd }}$ Spanish-Geman Symposium on Applied Computer Science (SGSOACS), Frankfurt, Germany, December 2015

- $\quad 11^{\text {th }}$ International Network Conference (INC 2016), Frankfurt, Germany, July 2016

- IEEE International Conference on Smart Grid and Smart Cities (ICSGSC 2017), Singapore, July 2017

- $7^{\text {th }}$ International Conference on Internet Technologies and Applications (ITA 17), Wrexham, UK, September 2017

Word count of main body of thesis: 79.804

Signed

Date 


\title{
Autonomous decentralised M2M Application Service Provision
}

\author{
Michael Steinheimer
}

\begin{abstract}
Machine-to-Machine Communication (M2M) service platforms integrate M2M devices and enable realisation of applications using the M2M devices to support processes, mostly in the business domain. Many application-specific vertical implementations of M2M service platforms exist as well as efforts to define horizontal M2M service platforms. Both approaches usually have central components or stakeholders of which the entire M2M system or the user depends. With regards to the end-user, more and more M2M devices provide resources, such as environmental information (e.g. energy consumption data) or control options (e.g. switching energy consumer). These resources offer great potential for supporting smart environments and it would be advantageous if these resources could be used by endusers to create individual smart environments or be accessible for other users to integrate these resources into their processes. Furthermore, it would be advantageous to avoid centralised or domain-specific solutions in order to realise flexible and independent M2M service platforms.
\end{abstract}

This thesis proposes a novel framework for autonomous and decentralised M2M application service provision based on native end-user integration and a distributed M2M system architecture. In order to actively involve end-users in M2M application development, an intuitive methodology for graphical application design through state machine-based application modelling is proposed. To achieve independence from the execution environments, a formal language for modelling M2M applications is introduced enabling a graphically designed M2M application to be represented by a formally described application model, which can be processed automatically and platform-independently. The design of a generalised interface definition enables local M2M applications to be provided as a service to other users. Based on this, an approach is introduced allowing end-users to combine the resources available in their personal environments in order to realise cooperative M2M applications and act as service providers.

The M2M service platform architecture presented does not contain any central components or stakeholders. The distributive nature of central entities and stakeholders is realised by a decentralised system architecture being implemented in the end-user domain. The various M2M service providers and consumers link via a Peer-to-Peer (P2P) network on both the communication level (using communication protocols Constrained Application Protocol, CoAP or Session Initiation Protocol, SIP) and on the data storage level (using structured or unstructured P2P overlay networks). An M2M Community concept complements the P2P network to enable a social network between different M2M service providers and consumers. The thesis also presents a prototypical proof-of-concept implementation used to verify the proposed framework components. 



\section{Contents}

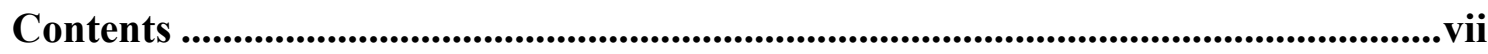

List of Figures...............................................................................................................................

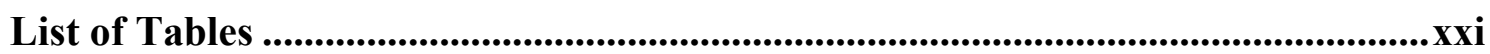

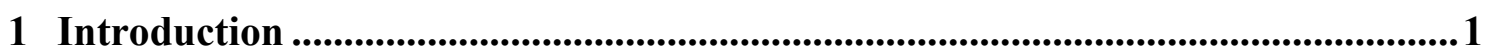

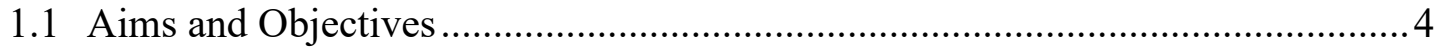

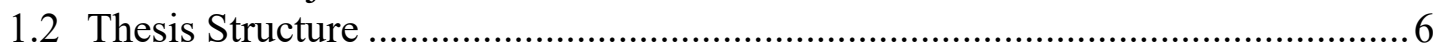

2 Peer-to-Peer, Machine-to-Machine Communication Systems, Use Cases .............9

2.1 Application Execution Environments ............................................................. 10

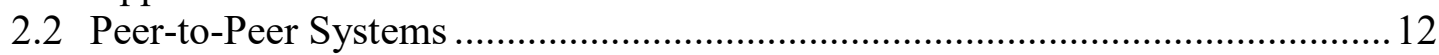

2.2.1 Classification of Peer-to-Peer Systems ……………………………..... 12

2.2.2 Classification of P2P System Architectures ............................................. 15

2.3 Machine-to-Machine Communication Systems.................................................. 19

2.3.1 Classification of Machine-to-Machine Communication ........................... 20

2.3.2 M2M System Architecture ………………………............................... 25

2.3.3 Roles and Stakeholder in M2M Ecosystems …………........................... 31

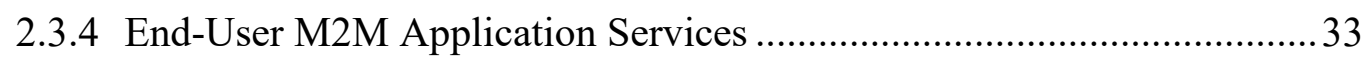

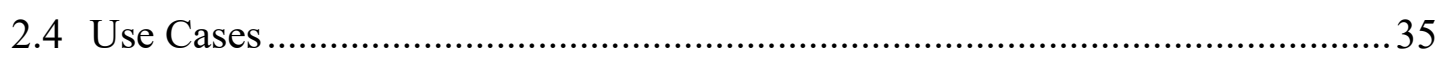

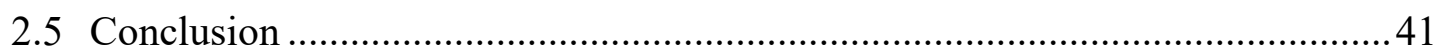

3 Challenges, Requirements, and Use Cases of M2M Application Service Provision................................................................................................................. 43

3.1 Related Work on M2M Service Platforms …………………………………........ 44

3.1.1 OneM2M Specification for M2M Systems …………………………........ 44

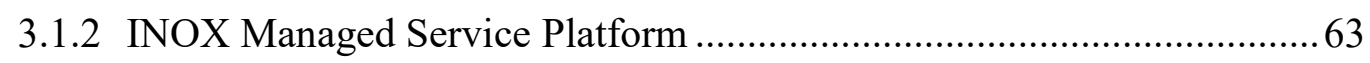

3.1.3 M2M Platform Project based on SOA (M2M on SOA) ............................69

3.1.4 BOSP Business Operation Support Platform …………………………..... 73

3.1.5 IMS enabled M2M Service Platform (IMS M2M SP I) ………………..... 77

3.1.6 M2M horizontal Services Platform Implementation over IP Multimedia Subsystem (IMS M2M SP II) ............................................... 81

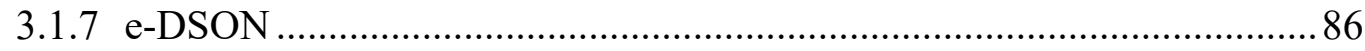

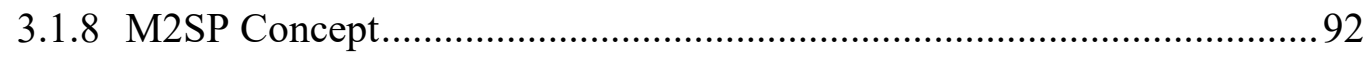

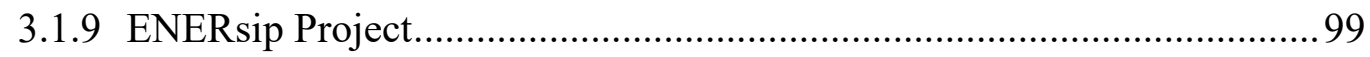


3.1.10Compose Framework

3.1.11Distributed Cooperative M2M System for Flood Monitoring (DistribFloodMon)

3.2 Requirements for a new Framework for Autonomous decentralised M2M Application Service Provision

4 Proposed Framework for autonomous decentralised Application Provision in M2M Systems

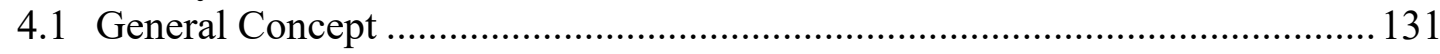

4.2 Framework Architecture and Components ..................................................... 141

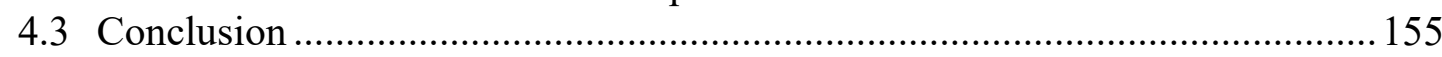

5 Autonomous M2M Application Provision ...................................................... 159

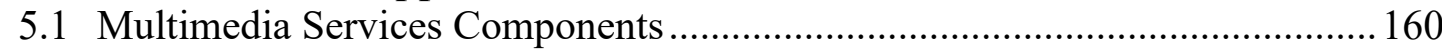

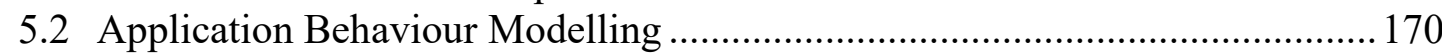

5.3 Graphical Application Behaviour Design.................................................... 177

5.4 M2M Device Management and Communication ........................................... 183

5.5 Formal M2M Application Notation ............................................................... 199

5.5.1 Selection of State Machine-based Modelling Language ........................ 199

5.5.2 Principles of Statechart Modelling …................................................ 212

5.5.3 M2M Application Modelling using Statecharts ...................................2219

5.6 Service Runtime Environment...................................................................... 229

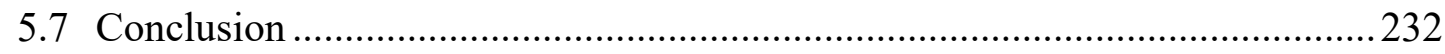

6 Cooperative M2M Application Service Provision................................................. 237

6.1 Provision and Integration of M2M Application Services ...............................2238

6.1.1 M2M Application Service Interface Description .................................239

6.1.2 Performing remote M2M Application Service Requests ...................... 243

6.1.3 Integration of remote M2M Application Services ................................ 246

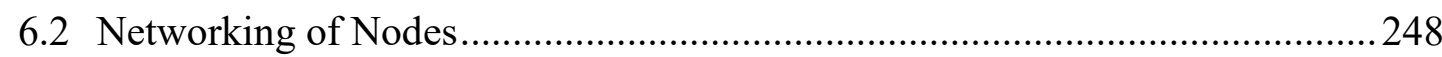

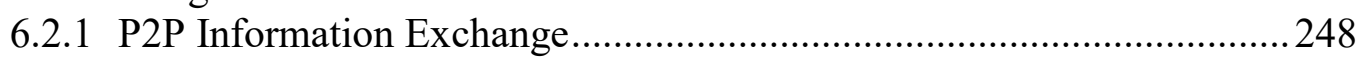

6.2.2 Information Exchange Pattern.......................................................... 256

6.2.3 Selection of appropriate P2P Communication Protocols ...................... 259

6.3 Service Registry and Distributed Data Storage ........................................... 282

6.3.1 M2M Service Registration and Storage of M2M Service IFD .............. 282

6.3.2 Principles of distributed Data Storage ................................................. 284

6.3.3 Analysis of structured and unstructured Overlay Architectures ........... 291

6.4 Cooperative M2M Application Service Provision ............................................ 302

6.4.1 Principles of cooperative M2M Application Service Provision.............. 303

6.4.2 Cooperative M2M Application Configuration and Execution Phase..... 313

6.4.3 Cooperative M2M Application Validation Algorithm .......................... 319 


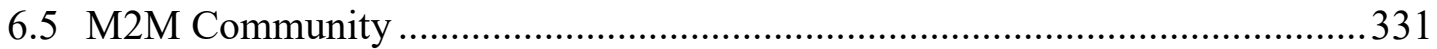

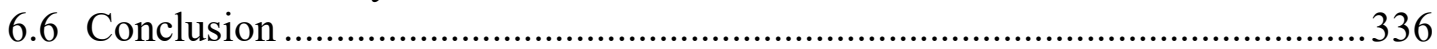

7 Research Prototype and Framework Evaluation ..................................................343

7.1 Evaluation of Framework Requirements .......................................................... 343

7.2 Research Prototype Architecture and Implementation .....................................349

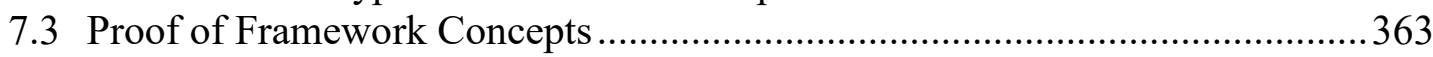

7.3.1 Local M2M Application Execution with remote M2M Service

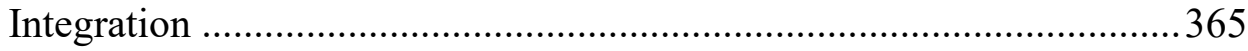

7.3.2 Cooperative M2M Application Service Provision ................................ 371

7.3.3 Communication Scenarios for M2M Service Requests...........................380

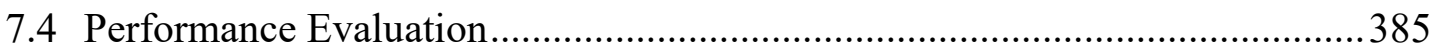

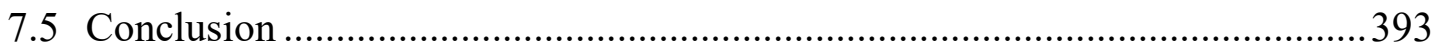

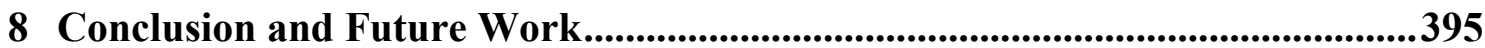

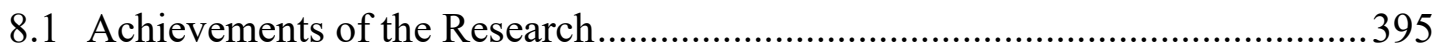

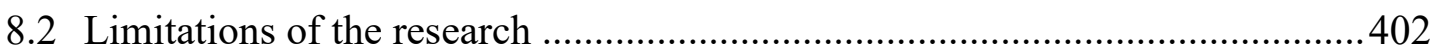

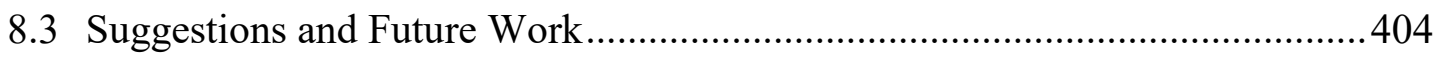

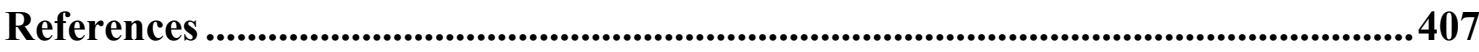

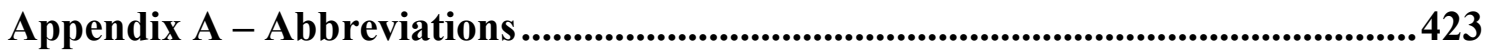

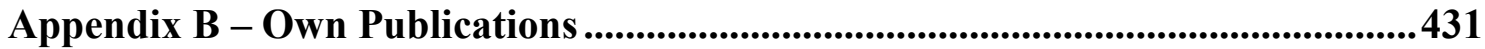

Appendix C - Low Level Descriptions ............................................................507 



\section{List of Figures}

Figure 1.1: End-user's Personal Environment

Figure 2.1: Classification of Execution Environments for Services and Applications acc. (Steinheimer et al., 2016)

Figure 2.2: Classification of P2P Systems based on Degree of (de)centralisation acc. (De Boever, 2007).

Figure 2.3: Correlation among WSNs, M2M, CPS, IoT acc. (Wan et al., 2013; Mehmood et al., 2015).

Figure 2.4: General Perspective of M2M System Architecture acc. (Wan et al., 2013; Mehmood et al., 2015; Bahga and Madisetti, 2014; Boswarthick et al., 2012; Holler et al., 2014)

Figure 2.5: Functional Architecture of M2M System acc. (ETSI TS 102690 V2.1.1, 2013; Boswarthick et al., 2012).

Figure 2.6: Integration of MSP in M2M system architecture acc. (oneM2M TR-0001V2.4.1, 2016; Elloumi, 2014).

Figure 2.7: Functional Roles in the M2M Ecosystem acc. (oneM2M TS-0002-V1.0.1, 2015)

Figure 2.8: Service Orchestration acc. (Terpak et al., 2016) 34

Figure 2.9: Service Choreography acc. (Terpak et al., 2016) 34

Figure 2.10: Use Case local Window Monitoring .36

Figure 2.11: Use Case Neighbourhood Weather Station 37

Figure 2.12: Use Case Building Surveillance 38

Figure 2.13: Use Case Cooperative Energy Optimisation (Load Reduction) 40

Figure 3.1: M2M Common Service Layer acc. (Damour, 2014). 45

Figure 3.2: Vertical Pipes of M2M Business Applications acc. (Arndt and Koss, 2014) 46

Figure 3.3: oneM2M Horizontal Platform Principle acc. (Arndt and Koss, 2014)......... 46 
Figure 3.4: oneM2M Layered Model and Functional Architecture acc. (oneM2M TS0001-V1.13.1, 2016) 48

Figure 3.5: Configurations supported by oneM2M Architecture acc. (oneM2M TS-0001V1.13.1, 2016) 50

Figure 3.6: oneM2M functional Architecture of M2M Application acc. (oneM2M TR0025-V1.0.0, 2016)

Figure 3.7: Possibilities for Node Interconnections acc. (oneM2M TS-0001-V2.10.0, 2016) 55

Figure 3.8: Scope of addressable M2M Common Services by registered CSEs ............56

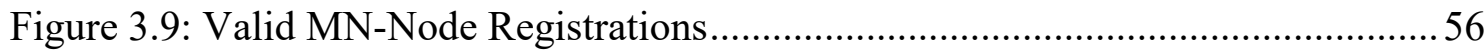

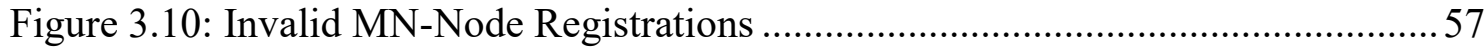

Figure 3.11: Principle of Information Exchange between Entities acc. (oneM2M TS0001-V2.10.0, 2016) 59

Figure 3.12: Primitive Overview acc. (oneM2M TS-0004-V2.7.1, 2016) .....................61

Figure 3.13: INOX Managed Service Platform acc. (Clayman and Galis, 2011)...........64

Figure 3.14: M2M Network Topologies (traditional and proposed) acc. (Zhang et al.,

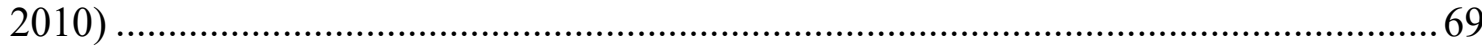

Figure 3.15: M2M Platform Architecture acc. (Zhang et al., 2010) ...............................70

Figure 3.16: M2M System Architecture acc. (Xiaocong and Jidong, 2010) ................. 74

Figure 3.17: IMS-enabled M2M System Architecture acc. (Foschini et al., 2011)........ 78

Figure 3.18: M2M horizontal Service Platform acc. (Padilla et al., 2013) ..................... 81

Figure 3.19: M2M Device Registration Process (Padilla et al., 2013) ........................... 83

Figure 3.20: DSON Service Scenario: Home Multimedia Streaming Service acc. (Kim et

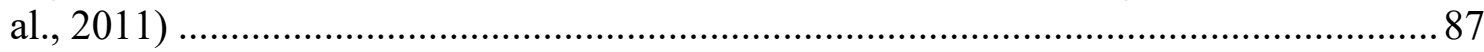

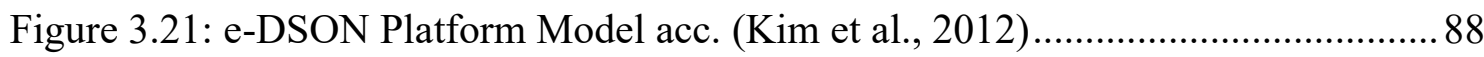

Figure 3.22: e-DSON Platform Architecture acc. (Kim et al., 2012) .............................90

Figure 3.23: M2SP Model acc. (Kim et al., 2014) ..................................................... 93

Figure 3.24: M2SP Architecture acc. (Kim et al., 2014) .............................................. 94

Figure 3.25: M2SP Use Case Scenario acc. (Kim et al., 2014) .................................... 96 
Figure 3.26: ENERsip Architecture acc. (Lopez et al., 2011a; Lopez et al., 2013) 99

Figure 3.27: Logical Architecture of COMPOSE Framework acc. (Mandler et al., 2013)

Figure 3.28: Components of COMPOSE framework acc. (Doukas and Antonelli, 2014)

Figure 3.29: Distributed M2M System acc. (Kitagami et al., 2014) 112

Figure 4.1: Traditional Approach for M2M Application Service Provision 132

Figure 4.2: M2M Application Service Creation and Provision Process with End-User Integration 132

Figure 4.3: ADSP Model: Decentralised M2M Application Design and Execution .... 134 Figure 4.4: HSPU Model: Horizontal M2M Resource Provision and Utilisation 136

Figure 4.5: DCASP Model: Decentralised cooperative M2M Application Service Provision 138

Figure 4.6: BSPU Model: Bottom-Up M2M Service Provision and Utilisation 140

Figure 4.7: M2M Application Service Provision Framework Architecture 142

Figure 4.8: Framework Architecture: Service Design Unit (SDU)............................. 144

Figure 4.9: Framework Architecture: Service Creation Unit (SCU) ........................... 145

Figure 4.10: Framework Architecture: Communication Unit (CU)............................ 146

Figure 4.11: Framework Architecture: Service Runtime Environment (SRE) ............. 149

Figure 4.12: Layer Model of Decentralised Networking Aspect ............................... 151

Figure 4.13: P2P connected M2M Environments within the M2M Community.......... 154

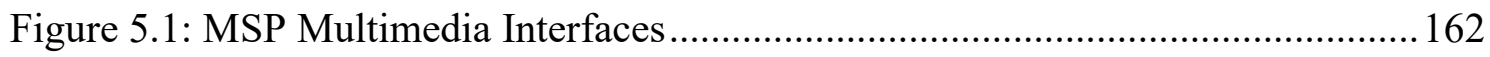

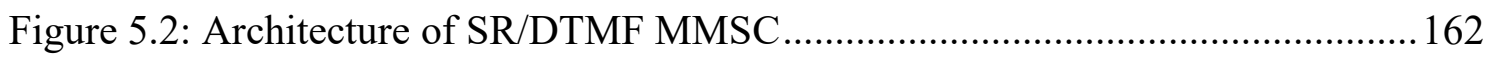

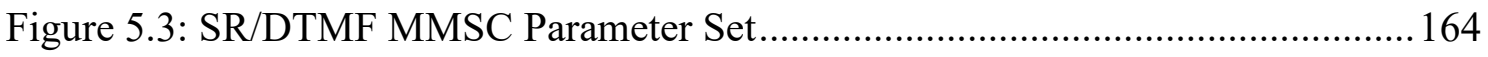

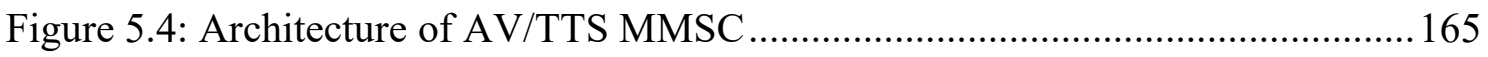

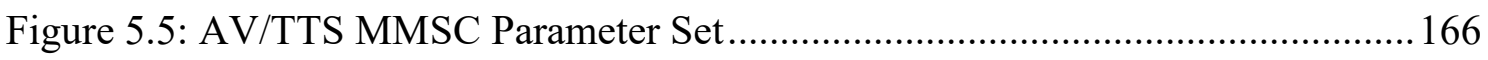

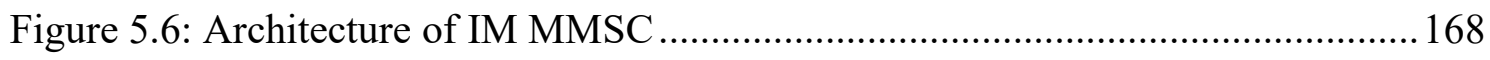

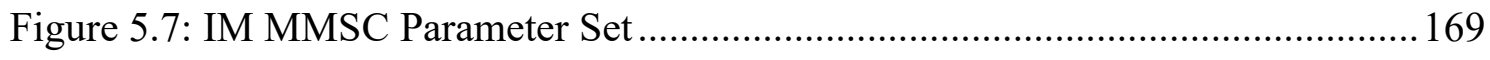




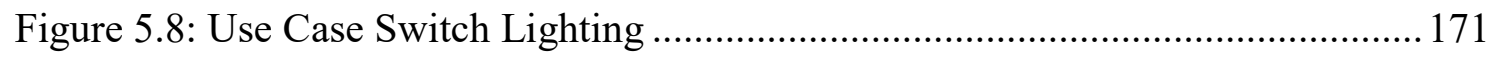

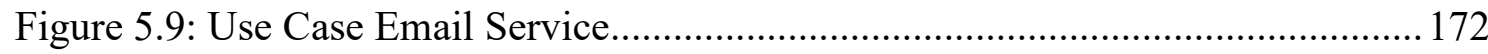

Figure 5.10: General Structure of M2M Device and MMSC ….................................. 173

Figure 5.11: General Representation of M2M Device and MMSC Connections ......... 173

Figure 5.12: FSM derived from (Wagenknecht and Hielscher, 2015)........................ 174

Figure 5.13: M2M Application Use Case 1 represented as FSM ............................... 175

Figure 5.14: Structure of Service Design GUI acc. (Steinheimer et al., 2013c; Steinheimer

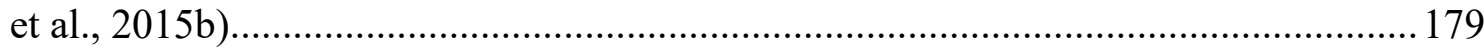

Figure 5.15: M2M Application Design Process...................................................... 181

Figure 5.16: Principles of oneM2M Device Abstraction acc. (oneM2M TR-0007-V1.0.0,

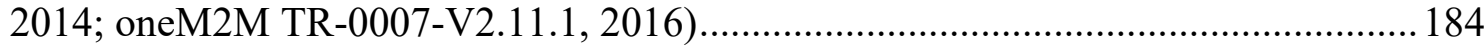

Figure 5.17: Structure of Request Primitive ........................................................... 187

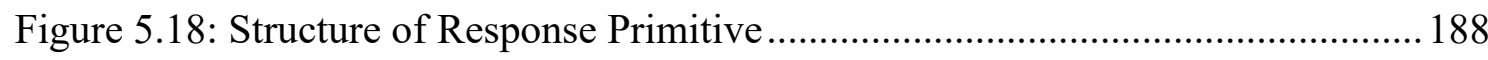

Figure 5.19: M2M Communication Unit (CU) with Abstraction Layer (AL) .............. 188

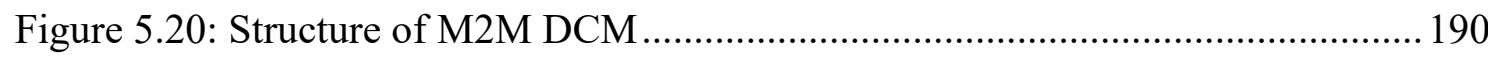

Figure 5.21: M2M CU/AL Register Process ........................................................... 192

Figure 5.22: M2M Application/CU/AL Message Exchange ........................................ 193

Figure 5.23: Mapping M2M DCM to Request Primitive Parameters........................... 195

Figure 5.24: Installation of M2M Device Capability Models.................................... 197

Figure 5.25: Statecharts Basic State and Default Transitions, derived from (Harel and

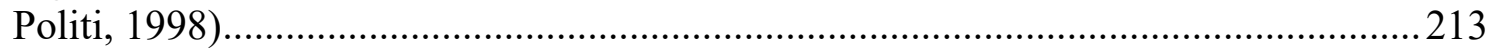

Figure 5.26: Statecharts AND-State, derived from (Harel and Politi, 1998)................ 214

Figure 5.27: Statecharts OR-State, derived from (Harel and Politi, 1998)...................215

Figure 5.28: SCXML Representation of Basic State and Default Transitions ............. 216

Figure 5.29: SCXML Representation of AND-State ............................................. 218

Figure 5.30: Statechart Representation of Use Case 1 ...............................................220

Figure 5.31: Principles of State Parameter Definition and Assign in Statecharts......... 222 
Figure 5.32: Graphical Representation OR-/AND M2M Device Combination 223

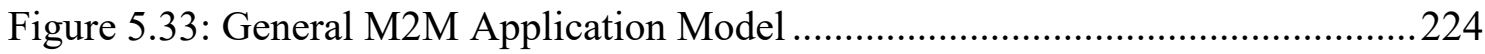

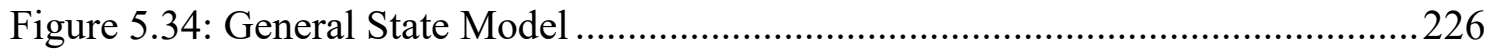

Figure 5.35: Formal M2M Application Description Generation Process .....................2228

Figure 5.36: Assignment graphical Notation Elements to M2M Application Model...229

Figure 5.37: Service Runtime Environment (SRE)..............................................230

Figure 5.38: M2M Application Execution Process...................................................231

Figure 6.1: M2M Application Service Principles ...................................................239

Figure 6.2: Principles of remoteRainSensor Application Service ............................... 242

Figure 6.3: IFD for remoteRainSensor M2M Application Service..............................2242

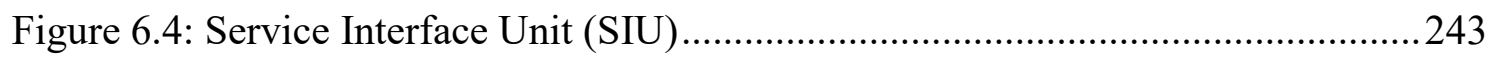

Figure 6.5: SIU: M2M Application Service Request Processing ...............................244

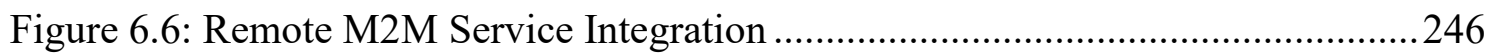

Figure 6.7: Extract Formal Application Description Use Case 2..............................247

Figure 6.8: RELOAD Architecture acc. (Samaniego et al., 2013; IETF RFC 6940, 2014)

Figure 6.9: RELOAD Message Exchange for Service Request acc. (IETF RFC 7904,

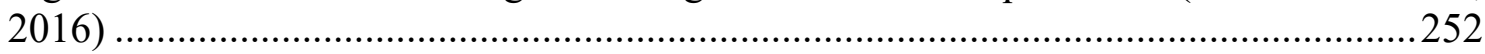

Figure 6.10: Comparison RELOAD and proposed Networking Topology .................255

Figure 6.11: IxPs acc. (Höller, 2014) adopted from (Carrez et al., 2013) ....................256

Figure 6.12: General Service Subscription/Notification/Termination Process .............262

Figure 6.13: CoAP Reliable Message Transmission acc. (IETF RFC 7252, 2014) .....265

Figure 6.14: CoAP Unreliable Message Transmission acc. (IETF RFC 7252, 2014)..265

Figure 6.15: CoAP Message Format acc. (IETF RFC 7252, 2014) .............................266

Figure 6.16: CoAP Messaging for Service/Information Subscription .........................270

Figure 6.17: CoAP Messaging for Service/Information Notification ..........................2 271

Figure 6.18: CoAP Messaging for Service/Information Unsubscription.....................221 
Figure 6.19: SIP Request Message Format acc. (IETF RFC 3261, 2002) ................... 273

Figure 6.20: SIP Response Message Format acc. (IETF RFC 3261, 2002) ................ 273

Figure 6.21: SIP Messaging for Service/Information Subscription ............................2277

Figure 6.22: SIP Messaging for Service/Information Notification ............................... 278

Figure 6.23: SIP Messaging for Service/Information Unsubscription.........................278

Figure 6.24: M2M Services Registration and Request .............................................283

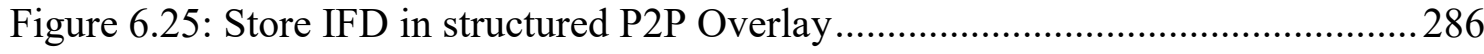

Figure 6.26: Request IFD from structured P2P Overlay .........................................28

Figure 6.27: Store IFD in unstructured P2P Overlay ...............................................28

Figure 6.28: Request IFD from unstructured P2P Overlay........................................ 290

Figure 6.29: Comparison Lookup Costs structured P2P Overlays ...............................2295

Figure 6.30: Comparison Lookup Costs structured P2P Overlays (excl. CAN)...........296

Figure 6.31: Comparison Lookup Costs unstructured P2P Overlays ...........................296

Figure 6.32: Comparison Churn Costs structured P2P Overlays .................................2297

Figure 6.33: Comparison Churn Costs unstructured P2P Overlays .............................298

Figure 6.34: Comparison Lookup Costs structured and unstructured P2P Overlays....299

Figure 6.35: Comparison Churn Costs structured and unstructured P2P Overlays ....... 300

Figure 6.36: Architecture of remote M2M Service Integration ................................... 304

Figure 6.37: Architecture of remote M2M Service Combinations ............................... 304

Figure 6.38: Variants of cooperative M2M Service Combinations .............................. 305

Figure 6.39: M2M Service Composition Message Exchange........................................306

Figure 6.40: M2M Service Aggregation Message Exchange .......................................306

Figure 6.41: Representation of Use Case 3 (Building Surveillance) ............................ 307

Figure 6.42: Statechart Representation AD of cooperative M2M Application Service for

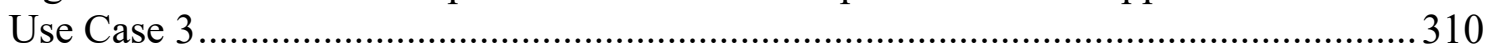

Figure 6.43: Representation of Use Case 4 (Energy Optimisation)............................... 311 
Figure 6.44: Statechart Representation AD of cooperative M2M Application Service for Use Case 4.

Figure 6.45: M2M Application Configuration Process

Figure 6.46: M2M Application Execution Process

Figure 6.47: M2M Service Connections to demonstrate redundant Application Configurations

Figure 6.48: Distributed M2M Application with redundant Service Instances 320

Figure 6.49: M2M Application Configuration and Execution Phase incl. multiple Service Instances

Figure 6.50: M2M Application Configuration with partly redundant Service Instance Connections. 322

Figure 6.51: Algorithm for Documentation of Connection Establishments 325

Figure 6.52: Transitive Closure of Peer 1 and Peer 4 based on Figure 6.50 327

Figure 6.53: Algorithm for Determination of invalid Application Service Configuration 329

Figure 6.54: IFD Element AccessControlPolicy for Definition of (Sub-) Community Assignment. 333

Figure 6.55: M2M application service IFD for Social Service 335

Figure 7.1: Research Prototype Application Architecture 350

Figure 7.2: Research Prototype Emulation System Architecture. 352

Figure 7.3: Research Prototype Emulation System Architecture. 353

Figure 7.4: Research Prototype Architecture Components (illustrated as Packages) ... 354

Figure 7.5: Screenshot of Service Design Unit GUI Web Application 355

Figure 7.6: Screenshot of SDU GUI showing M2M Device/Service Configuration Section .356

Figure 7.7: Screenshot of SDU GUI showing IFD Specification Form .357

Figure 7.8: Screenshot of Instant Message Client. .362

Figure 7.9: Screenshot of SDU GUI Web Application with SM Model Use Case 2 ...365

Figure 7.10: Use Case 2 M2M Application Description 367 
Figure 7.11: Screenshot of Terminal Output ADP Use Case 2 ...................................368

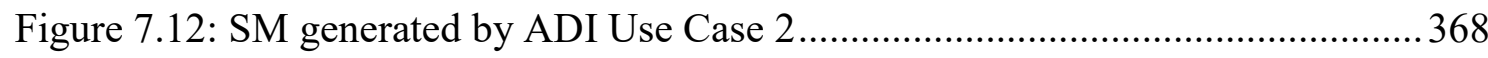

Figure 7.13: Screenshot of Terminal Output ADI Use Case 2 ....................................369

Figure 7.14: SM generated by ADI Use Case 2 .................................................. 370

Figure 7.15: Screenshot of IM Client Use Case 2 .................................................... 371

Figure 7.16: Screenshot of SDU GUI Web Application with SM Model Use Case 3.372

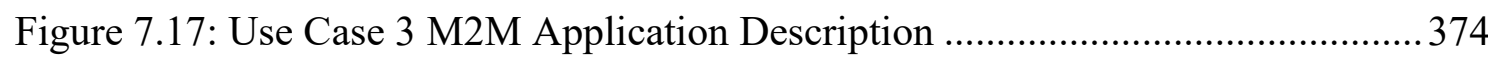

Figure 7.18: Use Case 3 corporateBuildingMonitoringApp IFD ...............................375

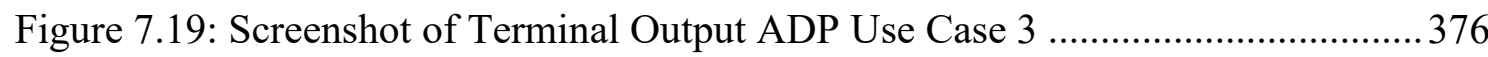

Figure 7.20: Screenshot of Terminal Output ADI Use Case 3 remoteSS1 ................... 377

Figure 7.21: Screenshot of Terminal Output ADI Use Case 3 remoteBMS .................. 377

Figure 7.22: Screenshot of Terminal Output ADI Use Case 3 remoteAS .................... 377

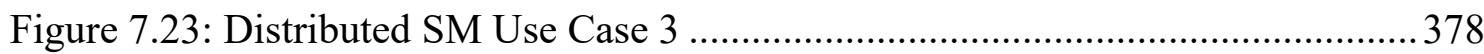

Figure 7.24: M2M Application Configuration Phase Use Case 3 ............................... 378

Figure 7.25: Service Request Process Supporter at remoteBMS Use Case 3 ............... 379

Figure 7.26: M2M Application Execution Process Use Case 3 ..................................379

Figure 7.27: Screenshot of Terminal Output Supporter receiving Alarm Message Use

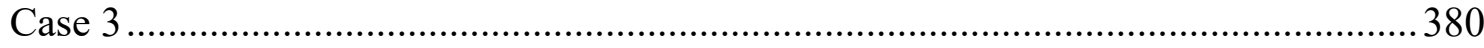

Figure 7.28: Screenshot of Wireshark Trace Request Message via CoAP one-time

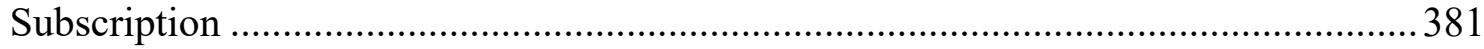

Figure 7.29: Screenshot of Terminal Output received Request Primitive via CoAP ...382

Figure 7.30: Screenshot of Wireshark Trace Response Message via CoAP ................ 382

Figure 7.31: Screenshot of Terminal Output received Response Primitive via CoAP.383

Figure 7.32: Screenshot of Wireshark Trace Request Message via SIP continuous

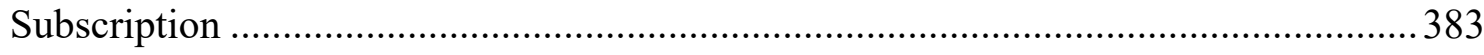

Figure 7.33: Screenshot of Wireshark Trace Terminate Request Message via SIP .....384

Figure 7.34: Screenshot of Wireshark Trace Request/Response Messages via SIP onetime Subscription 386 
Figure 7.35: Signalling Effort using centralised M2M Service Platform (MSP) 387

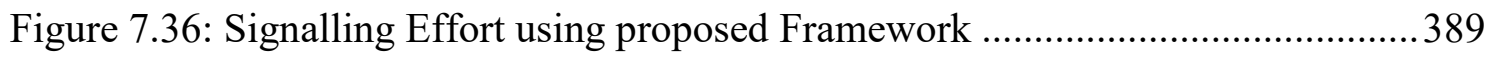

Figure 7.37: Comparison Signalling Effort (\# Messages) ...........................................391

Figure 7.38: Comparison Signalling Effort (Data Volume)..........................................392 



\section{List of Tables}

Table 2.1: Examples of P2P on different levels according to (Hauswirth and Dustdar, 2005)

Table 3.1: Evaluation of related Projects reg. derived Requirements 128

Table 5.1: M2M Application Component FSM Element Mapping 175

Table 5.2: M2M Application Component FSM State Mapping Use Case 1 176

Table 5.3: Relational Operators for logical M2M Device/MMSC Connections 178

Table 5.4: Configuration of TTS MMSC Use Case 1

Table 5.5: Parameter Set of Request Resource Message acc. (oneM2M TS-0001-V2.10.0, 2016) 186

Table 5.6: Parameter Set of Resource Response Message acc. (oneM2M TS-0001V2.10.0, 2016)

Table 5.7: Evaluation of SM-based Modelling Languages acc. (Steinheimer et al., 2017a)

Table 6.1: M2M Application Service Interface Description Parameter 241

Table 6.2: CoAP Message Format acc. (IETF RFC 7252, 2014) 267

Table 6.3: OneM2M Primitive CoAP Message mapping acc. (oneM2M TS-0008-V1.0.1, 2015) 268

Table 6.4: SIP Message Format acc. (IETF RFC 3261, 2002; IETF RFC 3265, 2002) 274

Table 6.5: OneM2M Primitive SIP Message mapping. .275

Table 6.6: Comparison of CoAP and SIP as Communication Protocol..... 281

Table 6.7: Evaluation P2P Algorithms acc. (Lua et al., 2005; Steinmetz and Wehrle, 2005; Malatras, 2015)

Table 6.8: Lookup Costs P2P Overlay Algorithms acc. (Lua et al., 2005; Steinmetz and Wehrle, 2005; Malatras, 2015). .294

Table 6.9: Churn Costs of P2P Overlay Algorithms acc. (Lua et al., 2005; Steinmetz and Wehrle, 2005; Malatras, 2015)..... 295 
Table 6.10: Summary Evaluation of structured and unstructured P2P Overlay Algorithms

Table 6.11: Adjacency Matrix of Graph illustrated in Figure 6.50 .325

Table 6.12: Transitive Closure based on Figure 6.50 328

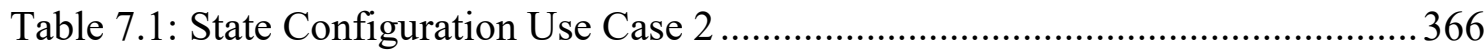

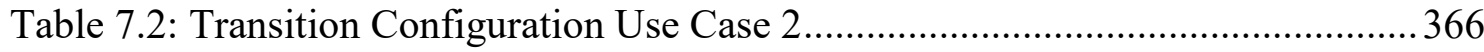

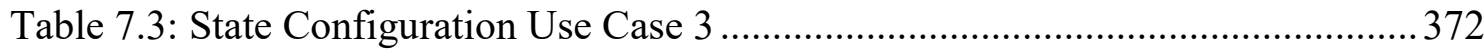

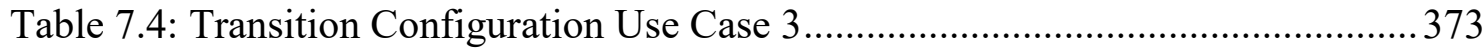

Table 7.5: Signalling Effort for Service Request and Service Response .......................386

Table 7.6: Performance Analysis central M2M Service Platform for Service Requests 388

Table 7.7: Performance Analysis distributed M2M Service Platform Concept for Service Requests 390 


\section{Introduction}

End-user devices, such as domestic appliances (e.g. refrigerator or washing machine), lights, heating or electric vehicles are becoming more and more intelligent. The devices include complex functionality for monitoring and control. Many devices are equipped with the functionality for communication, which enables remote monitoring and control. The increasing number of intelligent, so-called Machine-to-Machine Communication (M2M) devices support the formation of intelligent environments and many new application fields can be established. (Mackenzie, 2014) predicts 3.4 Billion M2M device connections up to 2024. (Danila et al., 2015) mentions application fields such as Transport and Logistic for real time management of vehicles providing vehicle specific data (e.g. fuel consumption or speed), Intelligent Cities for coordination of traffic or street lights, and Healthcare for e.g. remote monitoring of health status.

M2M systems realise the integration of such intelligent devices and provision of specific M2M applications addressing the above mentioned application fields. According to (Danila et al., 2015) many M2M applications already exist in the business domain using the control and monitoring functionality of M2M devices to integrate them in their business processes.

As (Danila et al., 2015) states, the applications for controlling M2M devices and processing the data generated by the $\mathrm{M} 2 \mathrm{M}$ devices are realised via specific vertical application architectures, which means individual solutions have been developed for each specific purpose and are executed on central or distributed application servers located in 
the Cloud. Because the many different solutions existing for different application fields, there are efforts, e.g. from oneM2M (oneM2M, 2015) to develop a horizontal M2M service platform (MSP) that serves as a common basis for M2M applications.

Most of the existing vertical and horizontal MSPs focus on the business domain. They do not integrate the end-user domain, which is an application field with powerful potential because the increasing number o7f controllable devices and Smart Home technologies residing in end-user's environment (refer to Figure 1.1). The personal environment of the end-user, illustrated in Figure 1.1, is difficult to access by external entities (other endusers or service providers), since the activities (controlling and monitoring of M2M devices) carried out in this area would severely affect the end-user or challenge data security.

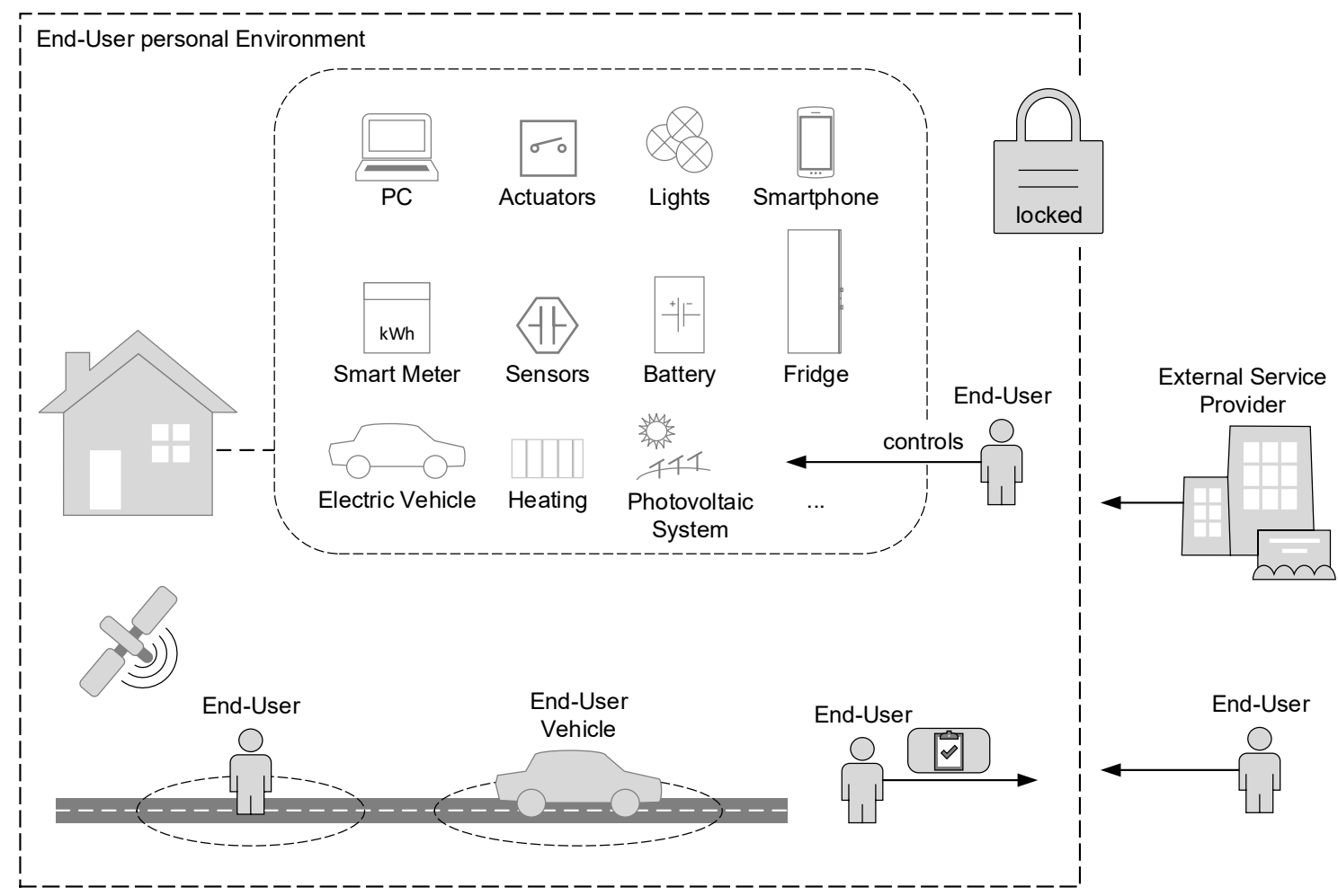

Figure 1.1: End-user's Personal Environment 
It would be advantageous if the potential, respectively the resources available in the enduser domain, could be made accessible for external entities as a service, so that they can be integrated into external applications or processes. For this, it is necessary to integrate end-users actively into the service provision process, by having the option to define M2M applications for their personal environment and additionally having the possibility to make their applications and M2M device resources available to external entities.

The existing horizontal and vertical MSPs have one or more of the following disadvantages:

1. The development of applications or utilisation of MSPs requires expert knowledge. This prevents the end-user from creating applications that integrate the resources in their personal environment or providing the implemented functionalities as a service for external entities.

2. The operation of the MSP requires central platform providers or centralised infrastructures. Both of them are disadvantageous since the users of the M2M system are dependent of this platform provider or the entire system depends on a single centralised component in the M2M system architecture.

Often, central MSP providers only provide platforms that are oriented to a specific application area and thus are restricted in their functionality. Central elements in the M2M system infrastructure are also disadvantageous since they are very resource-intensive and can result in bottlenecks or high costs for the operation and implementation of platform availability.

This research will introduce a more flexible methodology for realisation of M2M systems with the focus on dissolving the bindings to centralised entities (e.g. MSP providers), 
integration of end-users for realisation of M2M applications satisfying their individual requirements, and realisation without specialised and dedicated M2M infrastructures.

\subsection{Aims and Objectives}

The aim of this research is to propose a framework for autonomous, decentralised and cooperative M2M application service provision. The framework should permit M2M systems to become more flexible and provide the ability to integrate end-users in the M2M application provision to support their individual requirements. Furthermore, the framework should dissolve the static binding from M2M application consumer to M2M application provider as well as avoid the requirement of specialised M2M infrastructures for M2M application provision.

The main objectives of this research are subsequently outlined:

1. To analyse existing approaches for service provision in $\mathrm{M} 2 \mathrm{M}$ systems and to define the requirements for decentralised M2M application provision with enduser integration.

2. To specify the architecture and methodologies of the proposed framework for “Autonomous decentralised M2M Application Service Provision”.

3. To design an approach for graphic-oriented modelling of M2M applications by combination of building blocks that bases on modelling the behaviour of an M2M application. 
4. To analyse existing formal description languages and methodologies resulting in a novel formal M2M application description language for automated generation and execution of the graphically designed M2M application semantics.

5. To design a concept for provision and integration of $\mathrm{M} 2 \mathrm{M}$ applications as a service to other end-users and analysis of methodologies for decentralised information exchange between M2M application service provider and consumer based on existing telecommunication infrastructure.

6. To specify a concept for management of M2M application services using decentralised system architecture components.

7. To design an approach for an M2M application architecture based on the combination of distributed $\mathrm{M} 2 \mathrm{M}$ application services for cooperative and composed M2M application provision and to specify an algorithm for automated configuration of distributed cooperative $\mathrm{M} 2 \mathrm{M}$ applications based on formal description language with avoiding of central entities.

8. To produce a proof-of-concept implementation in order to verify and evaluate the proposed framework for “Autonomous decentralised M2M Application Service Provision" based on defined use cases.

The order of objectives declared above corresponds to the general structure of this thesis as presented within the following sections.

Important aspects for research on a framework for autonomous and decentralised M2M application service provision based on native end-user integration and a distributed M2M system architecture are the discussion of security and implementation of associated policies. These aspects are not discussed within this thesis since the research work is part 
of the research project P2P4M2M (P2P4M2M, 2016) performed by multiple team members. In order to avoid overlap with fellow researcher the security aspect is not considered within this research. Publications of fellow researcher, such as (Shala et al., 2017a) and (Shala et al., 2017b) provide an introduction of security concept for autonomous and distributed provision of M2M applications.

\subsection{Thesis Structure}

Chapter 2 describes the theoretical background of the research field in the focus of this research by introducing the principles of Peer-to-Peer (P2P) and Machine-to-Machine Communication (M2M) systems. For this purpose, the principle of $\mathrm{P} 2 \mathrm{P}$ systems is first explained and afterwards the different architectural approaches for implementing a $\mathrm{P} 2 \mathrm{P}$ system are presented. Furthermore, the principle of M2M is introduced and the M2M system architecture is explained. Chapter 2 also presents the various roles and stakeholders in an M2M ecosystem. Finally, use cases are described that serve for illustration and evaluation of the presented concepts in the course of this research.

Chapter 0 introduces existing concepts for application and service provision in the M2M application field. For this purpose, the MSP from the oneM2M standard specification as well as several MSPs from research field are described and analysed. The main result of this chapter is the definition of requirements for a novel framework for providing M2M applications based on the limitations and advantages of the MSPs presented.

Based on the requirements defined in chapter 0 , chapter 4 proposes a novel framework for "Autonomous decentralised M2M Application Service Provision". Additionally, 
chapter 4 briefly introduces the architecture and components of the proposed framework that enables end-users to design and provide individual and cooperative $\mathrm{M} 2 \mathrm{M}$ applications.

Chapter 5 presents the parts of the proposed framework responsible for local M2M application design and execution. It presents a concept that enables end-users to design $\mathrm{M} 2 \mathrm{M}$ applications intuitively and describes how end-users can interact with the M2M platform. Furthermore, chapter 5 describes the integration of M2M devices into M2M platforms as well as the execution of designed M2M applications.

Chapter 6 introduces the framework components responsible for provision of local M2M application functionality as a service to other end-users. Additionally, chapter 6 introduces methodologies for combination and management of distributed M2M application services to provide a cooperative M2M application service.

Chapter 7 focuses on the proof-of-concept of the proposed framework by describing the prototypical implementation and the evaluation of the proposed framework.

Chapter 8 concludes this research by giving a summary of the achievements and limitations as well as aspects for future research and extensions of the proposed framework concept. 



\section{Peer-to-Peer, Machine-to-Machine}

\section{Communication Systems, Use Cases}

This chapter provides the theoretical background for understanding the concepts used in this thesis. Section 2.1 introduces different execution environments for applications and services. Section 2.2 introduces the principles of Peer-to-Peer (P2P) systems, which provide end-to-end communication between participants and decentralised utilisation and management of resources. A decentralised, distributed Machine-to-Machine Communication (M2M) service platform would be beneficial for both providers and consumers of services because its inclusivity and inherent resilience. Section 2.3 introduces the principles of M2M systems, which enable realisation of applications integrating M2M device functionality. Section 2.3 also deals with the separation from $\mathrm{M} 2 \mathrm{M}$ to IoT, as both terms are often used simultaneously in literature. Therefore, first, a general introduction of M2M systems is given (section 2.3.1) and afterwards section 2.3.2 introduces the components and services related to an M2M system architecture. Section 2.3.3 introduces the different roles and stakeholders in a traditional $\mathrm{M} 2 \mathrm{M}$ ecosystem to point out their relationships to each other, which will change in a $p 2 p$-based service provisioning architecture. Finally, section 2.3.4 introduces the service composition approach followed in this project. This chapter closes with a description of the use cases in section 2.4, which will be used to illustrate and evaluate the novel framework proposed in this thesis. 


\subsection{Application Execution Environments}

The realisation of an M2M application or M2M service platform (MSP) requires that a technical environment exists executing the software modules implementing the application logic (hosting of applications). This is called the application execution environment (AEE). The AEE of an application or MSP is to be considered as independent of the application field of the application/MSP. Therefore, the different AEEs are introduced at the beginning and in the further course of this project it is referred to them. Figure 2.1 classifies the different approaches of AEEs based on (Grandison et al., 2010), (Bonomi et al., 2012), (ETSI, 2014), (LeClair, 2014), and (Vaquero and Rodero-Merino, 2014), already related to an M2M environment (through integration of M2M Area Networks).

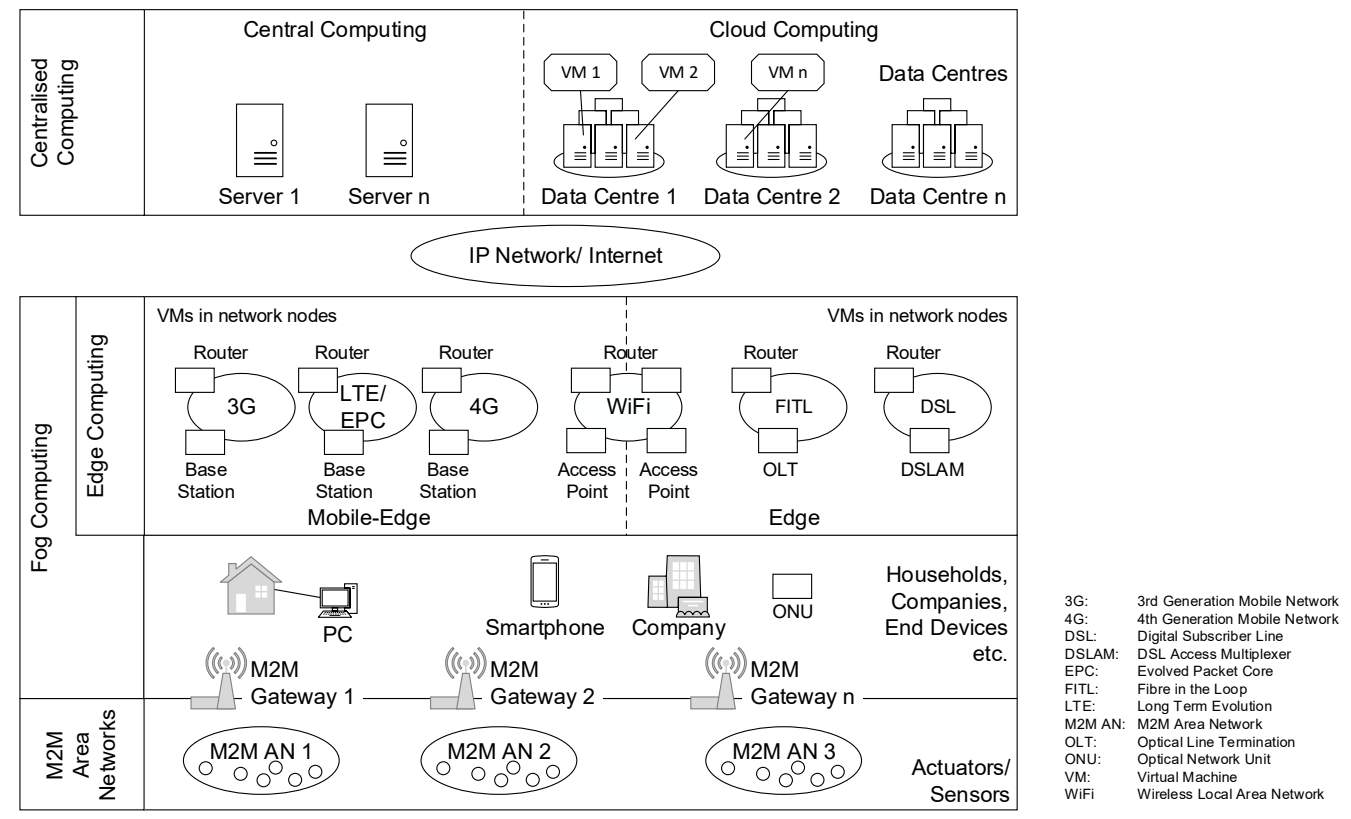

Figure 2.1: Classification of Execution Environments for Services and Applications acc. (Steinheimer et al., 2016) 
Figure 2.1 shows the well-known approaches of AEEs, central server approach (using servers that host the applications on central locations in the network) and Cloud Computing approach. AEEs hosted in the Cloud can be physically distributed across multiple datacentres (Grandison et al., 2010). Despite the decentralised AEE, these approaches are considered to be logically centralised, since they are managed centrally by a single stakeholder. Next to these approaches two new architectural approaches of AEEs came up aiming to reduce the load in the core network: Edge and Fog Computing.

Edge computing is an architectural approach in which data-intensive, isolated applications are moved from central data centres (cloud) to the edge of the network where the data are generated and processed to avoid sending large amount of data through the core network (LeClair, 2014).

Fog Computing is a virtualised platform architecture providing storage, networking and compute services in end-user devices at network edges (Bonomi et al., 2012). End-user devices form the virtualisation platform of Fog Computing architecture (Vaquero and Rodero-Merino, 2014).

Both approaches are realised in the infrastructure provided by the network operator (i.e. access networks or devices located close to the customers, e.g. services executed in base stations), while Fog computing additionally uses devices closer to the customer (e.g. Integrated Access Devices, IADs).

(Osanaiye et al., 2017) gives an overview of specific applications that use the Fog Computing architecture principles, such as a Smart Traffic Light System detecting pedestrians and sending warning signals to driving cars. This shows that the Fog 
Computing architecture is of great relevance for M2M applications. However, (Osanaiye et al., 2017) shows that Fog Computing, same as Edge Computing contains centralised elements in their system architecture. The presented applications are either realised by providers that use the Fog Computing architecture to provide specific, isolated applications or contain central servers that in turn are localised in the Cloud.

The shifting of computing and data capturing tasks to the edge of the network is a promising approach and also a major object in this research. This research additionally goes a step further by presenting a completely decentralised MSP architecture in which the end-user has the possibility to generate and provide applications and services independently.

\subsection{Peer-to-Peer Systems}

P2P systems offer distributed communication and resource management/utilisation. The following section 2.2.1 describes the principles of P2P systems and different areas in which P2P systems can be used. P2P systems can be implemented using different architectural approaches, characterised by their degree of decentralization. Section 2.2.2 introduces the different architectural approaches and describes how resources can be localised in them.

\subsubsection{Classification of Peer-to-Peer Systems}

$\mathrm{P} 2 \mathrm{P}$ is according to (Steinmetz and Wehrle, 2005) a paradigm for communication on the Internet, i.e. for Internet Protocol (IP)-based communication systems (IETF RFC 791, 
1981), following the end-to-end communication principle. P2P communication mechanisms can be applied to access globally distributed resources located closely to peers at network edges.

Commonly instead of the term "P2P system", also the term "overlay network", "P2P overlay" or "P2P overlay network" is used. This illustrates that a $\mathrm{P} 2 \mathrm{P}$ system is a higherlevel network with independent topology, built on top of existing IP networks (Steinmetz and Wehrle, 2005; IETF RFC 7890, 2016).

According to (De Boever, 2007) following characteristics specify a P2P system:

- Decentralisation - The participating entities in $\mathrm{P} 2 \mathrm{P}$ systems are decentralised.

- Cost efficiency - P2P systems utilises resources of peers that are currently unused.

- Self-organisation - P2P systems are self-organised.

- Sharing of resources - Peers share their resources in P2P systems.

- Autonomy - No dependency exist on single entities required for operating a P2P system.

- Scalability - Because sharing resources and distributing content among participating peers a P2P system is highly scalable and avoids bottlenecks.

P2P systems and applications perform a function in a decentralised way by employing distributed resources (e.g. processing power, data storage/content or humans and other resources) provided by participating nodes each adding value to the overall $\mathrm{P} 2 \mathrm{P}$ system (Milojicic et al., 2002; De Boever, 2007). The resources in a P2P system are provided by the participants in the P2P system instead of by few servers as in traditional client/-server architectures (IETF RFC 7890, 2016). 
According to (De Boever, 2007), the P2P system approach was developed because the classical approach of a client/-server model, where resources are managed/deployed by a central server and queried by clients, is very resource intensive. In this context, the resources are content generated by the end-user and consumed by other end-users. In client/-server systems, each user of the system generates additional costs because of the necessary data transmissions to the central server. Because the increasing amount of data to be transferred and increasing size of individual data sets, the traditional client/-server approach is inefficient and its scalability is limited e.g. when network connectivity to external networks and processing/storage are scarce. With an increasing number of users, a classical client/-server system can hardly cover the demand for resources for data transmission (increasing bandwidth requirements) and also for the execution of computation tasks, so that additionally to the single point of failure (in case of breakdown of the central server) bottlenecks can also arise. P2P systems meet these disadvantages because they are naturally very cost-effective and scalable.

According to (Steinmetz and Wehrle, 2005), based on (Oram, 2001) and refined in (Steinmetz and Wehrle, 2004) a P2P system is a self-organised system in which all entities (peers) are equal and act autonomously and the overall system does not involve central entities to organise/coordinate/control utilisation of the resources.

According to (Hauswirth and Dustdar, 2005) P2P architectures can be applied on different level of abstraction, specified as follows:

- Network level - Routing of requests in application-independent way via physical network. 
- Data access level - Search/modify resources using application-specific access structures.

- Service level - Combination and expansion of functionalities of the data access layer to provide higher quality services. The range of these services can vary from simple file sharing to complex business processes.

- User level - Grouping of users ("Communities") and support of user interactions using the service level for community management and information exchange.

According to (Hauswirth and Dustdar, 2005) Table 2.1 shows typical examples of P2P architectures on different levels. Combination of this levels and the utilised P2P concept on individual levels characterise a specific P2P system.

Table 2.1: Examples of P2P on different levels according to (Hauswirth and Dustdar, 2005)

\begin{tabular}{|l|l|l|l|}
\hline Level & Application Domain & Service Class & Example \\
\hline Network & Internet & Routing & TCP/ IP, DNS \\
\hline Data Access & Overlay network & Resource location, file sharing & Gnutella, Freenet \\
\hline Service & P2P applications & Information services, distributed computing & $\begin{array}{l}\text { SETI@Home, Napster, } \\
\text { Skype }\end{array}$ \\
\hline User & User communities & Collaboration & eBay, Ciao \\
\hline
\end{tabular}

Thus, it can be stated that a P2P system is always advantageous when 1. decentralised resources are in the focus of an application, 2. a high scalability is required, 3. a resource is to be provided directly end-to-end and 4. no dependencies on central entities (stakeholders or system components) should exist.

\subsubsection{Classification of P2P System Architectures}

P2P systems can be generally classified according to (De Boever, 2007) based on the degree of decentralisation and "the presence of a structure in object location and routing". 
Although a P2P system is a distributed architecture in which the distribution of resources occurs end-to-end, it can contain more or less central entities to create the topology of the P2P system (illustrated in Figure 2.2) or to locate the resources.

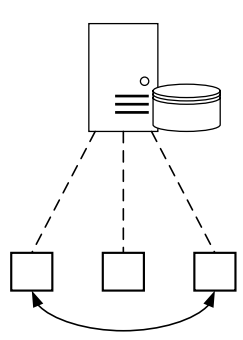

a) Centralised

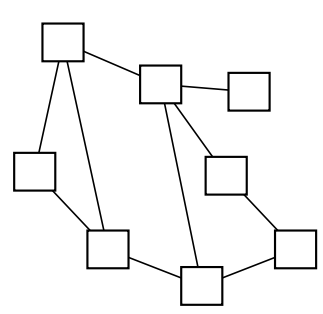

b) Decentralised

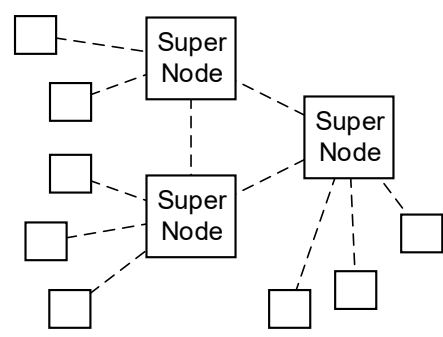

c) Hybrid

Figure 2.2: Classification of P2P Systems based on Degree of (de)centralisation acc. (De Boever, 2007)

(De Boever, 2007) classifies P2P topologies into hybrid, centralised and pure decentralised with the characteristics described subsequently.

- Centralised P2P architectures - Centralised P2P architectures (Figure 2.2-a) contain a central directory server storing a summary of resources and nodes. To locate a resource in the $\mathrm{P} 2 \mathrm{P}$ network, peers contact the directory server and exchange the information for resource sharing directly with the providing peers.

- Decentralised P2P architectures - A decentralised P2P architectures (Figure 2.2b) is completely self-organised and autonomous and involves no centralised components to locate resources in the P2P network.

- Hybrid P2P architectures - Hybrid P2P architectures (Figure 2.2-c) are a mix of both, centralised and decentralised P2P architectures. They contain several socalled "super nodes" having more capabilities and responsibilities than other peers to support resource location. 
Because avoidance of central entities is in focus of this research project, decentralised P2P systems are considered in the further course of the project, since centralised and hybrid P2P system architectures contain central entities.

For implementing a P2P overlay several different approaches exist. A P2P overlay can have a structured topology (structured P2P overlay) or an unstructured topology (unstructured P2P overlay). The P2P overlays are generated by algorithms determining how the structure of an overlay (arrangement of the nodes) is organised. The topology and algorithms for searching and inserting data sets determine which node in the overlay stores a specific data set and how the data sets can be located (without centralised control/coordination) (Steinmetz and Wehrle, 2005).

Structured and unstructured overlays have fundamentally different characteristics in their topology, search algorithms and data management. The main characteristics of both approaches are presented subsequently according to (De Boever, 2007).

- Structured P2P Overlays - In structured P2P overlays the nodes and the resources are assigned to specific locations in the topology. "Distributed Hash Tables (DHT)" enable to identify the location of a specific resource.

- Unstructured P2P Overlays - In unstructured P2P overlays resources and nodes are located in an unstructured way with no relationship between location of the resource and the topology. Identification of resource locations is performed using the so-called "query flooding model".

Structured P2P systems ensure that resources can be localised with limited hops (De Boever, 2007). The global view of the data distributed over several nodes is provided by 
DHTs without dependence on the actual location (Steinmetz and Wehrle, 2005). According to (De Boever, 2007) in a DHT every resource is assigned with a distinct key serving as an ID for the resource. The ID is generated by using hash functions (e.g. SHA1, IETF RFC 6234, 2011). Each peer is responsible for a sequence of that IDs. To be able to locate a resource, the requesting node must know the ID of the resource. When searching for a resource, the search query is continuously forwarded from one node to another until the node responsible for the resource is reached. To be able to forward the search query, each node has a routing table with information about various other nodes possibly providing the requested resource.

A structured P2P network offers according to (Steinmetz and Wehrle, 2005) and (De Boever, 2007) two ways to store data:

- Direct Storage - The data is stored directly in the overlay at the node responsible for this data set. The advantage of this variant is that nodes which inserted the data can leave the overlay after the insertion without losing the inserted data.

- Indirect Storage - The data is not stored directly in the overlay, but only a reference to the data via which the data can be obtained (e.g. link). The data itself remains on the node that inserted the reference. The advantage of this variant is that the load in the overlay is reduced because the reference contains less data volume the data set itself.

In unstructured P2P systems peers only contain an index of data sets stored at their own location and do not have information about data sets stored at other peers. A search request for a data set is forwarded to all peers known to a single peer. Therefore, it is important that one peer knows as many other peers as possible also present in the $\mathrm{P} 2 \mathrm{P}$ 
overlay. To search a data set a peers forward the search query to all known peers. These in turn forward the query to all known peers. This floods the network with the search request. For each search query, a "Time-To-Life" (TTL) value is defined that limits the number of forwardings of a query. Each peer that passes on the search query decrements the TTL value. If the TTL value is zero, the search query is no longer forwarded. Because this process, unstructured $\mathrm{P} 2 \mathrm{P}$ systems cannot guarantee to locate specific data sets because the data set might be stored on a peer that does not receive the search query since it is outside the scope of query forwarding (De Boever, 2007).

Because of the different mechanisms used to generate a P2P overlay and locate resources, it is important to note that choosing an appropriate approach depends on the intended purpose and should be based on criteria such as network load and guarantee to locate a data set.

\subsection{Machine-to-Machine Communication Systems}

In order to create a unified terminology and explain how various terms are used in context of the research project, the following section 2.3.1 describes principles of Machine-toMachine Communication (M2M) and how M2M correlates with the Internet of Things (IoT) since both are used simultaneously in literature. Section 2.3.2 introduces the M2M system architecture by describing the components of an M2M system and points out how M2M application services are realised applying an M2M system. Section 2.3.3 introduces different roles and stakeholders in traditional M2M ecosystem. The different roles will be introduced to show how these are related to each other, as the role assignment will change in a $\mathrm{p} 2 \mathrm{p}$-based service provisioning architecture enabling end-users to take on the role of 
service provider additionally to the role of service consumer. Finally, section 2.3.4 introduces the approach of service choreography followed in this research projects for service provision and shows the difference of it to the approach of service orchestration used in traditional MSPs.

\subsubsection{Classification of Machine-to-Machine Communication}

$\mathrm{M} 2 \mathrm{M}$ describes a communication paradigm that enables devices (machines) to communicate with other devices without or with minimal human intervention during configuration/deployment/operation phase (Dawaliby et al., 2016; Sarigiannidis et al., 2017; Mehmood et al., 2015). M2M enables autonomous measurement, delivery, and processing of information using Information and Communication Technologies (ICT) (Sarigiannidis et al., 2017). Often in publications, such as (Dawy et al., 2017) or (Dawaliby et al., 2016) the terms M2M and Machine-type communications (MTC) are equated. In this research project M2M is used to specify the communication between devices. According to (Dawaliby et al., 2016) M2M can be realised through a P2P or centralised communication model and according to (Holler et al., 2014) an M2M solution focuses on supporting a particular task of one stakeholder and does not "take a broad perspective on solving a larger set of issues or ones that could involve several stakeholders".

Through M2M many applications (e.g. monitoring, infrastructure management, eHealth, smart cities, home automation) are supported or enabled (Dawaliby et al., 2016; Mehmood et al., 2015). M2M is according to (Mehmood et al., 2015) the fundamental 
enabler for the IoT "by providing ubiquitous connectivity between numerous intelligent devices".

Publications such as (Kim et al., 2012) mention that M2M and IoT contain the same aspects. Other publications, such as (Bahga and Madisetti, 2014) or (Holler et al., 2014) try to separate the aspects of M2M and IoT. According to (Mehmood et al., 2015) M2M and IoT domain, but also Wireless Sensor Networks (WSNs) and Cyber-Physical Systems (CPS) domain represent the same key ideas. As stated by (Mehmood et al., 2015) authors of (Wan et al., 2013) made a correlation of M2M, IoT, WSN and CPS. Since (Wan et al., 2013) and (Mehmood et al., 2015) precisely describe the aspects of M2M and IoT and classify the different terms as well as provide the most suitable definitions for the context of this project, these are presented below. The specifications of (Wan et al., 2013) and (Mehmood et al., 2015) are used to classify the focus of this project in the described fields.

To illustrate the correlation of M2M, IoT, WSN and CPS, first IoT is introduced since M2M, WSN, and CPS according to (Wan et al., 2013), from the architectural perspective, belong to IoT.

\section{Internet of Things (IoT)}

According to (Mehmood et al., 2015) the term IoT describes the connection of “objects/machines" (often defined as "things") using an Internet-based infrastructure. According to (Mehmood et al., 2015) and (Wan et al., 2013) the IoT contains the following fundamental components:

- Data Sensing - Sensing and acquiring data of distributed sensors that should be processed in services and applications. 
- Heterogeneous Connectivity - The objects and devices implement different (incompatible) communication technologies.

- Information Processing - The information data acquired needs to be processed to interpret the captured information.

- Services and Applications - Services and applications use the captured information data to generate a benefit out of them and additionally control devices/machines.

Fundamentally, according to (Wan et al., 2013) M2M, WSNs, and CPS "belong to IoT" because they have the same components as described above. The difference between them is the proportion among these components in system design. Figure 2.3 shows the correlation among WSNs, M2M, CPS, as well as IoT according to (Wan et al., 2013) and (Mehmood et al., 2015), while IoT represents the area created by WSNs, M2M, CPS.

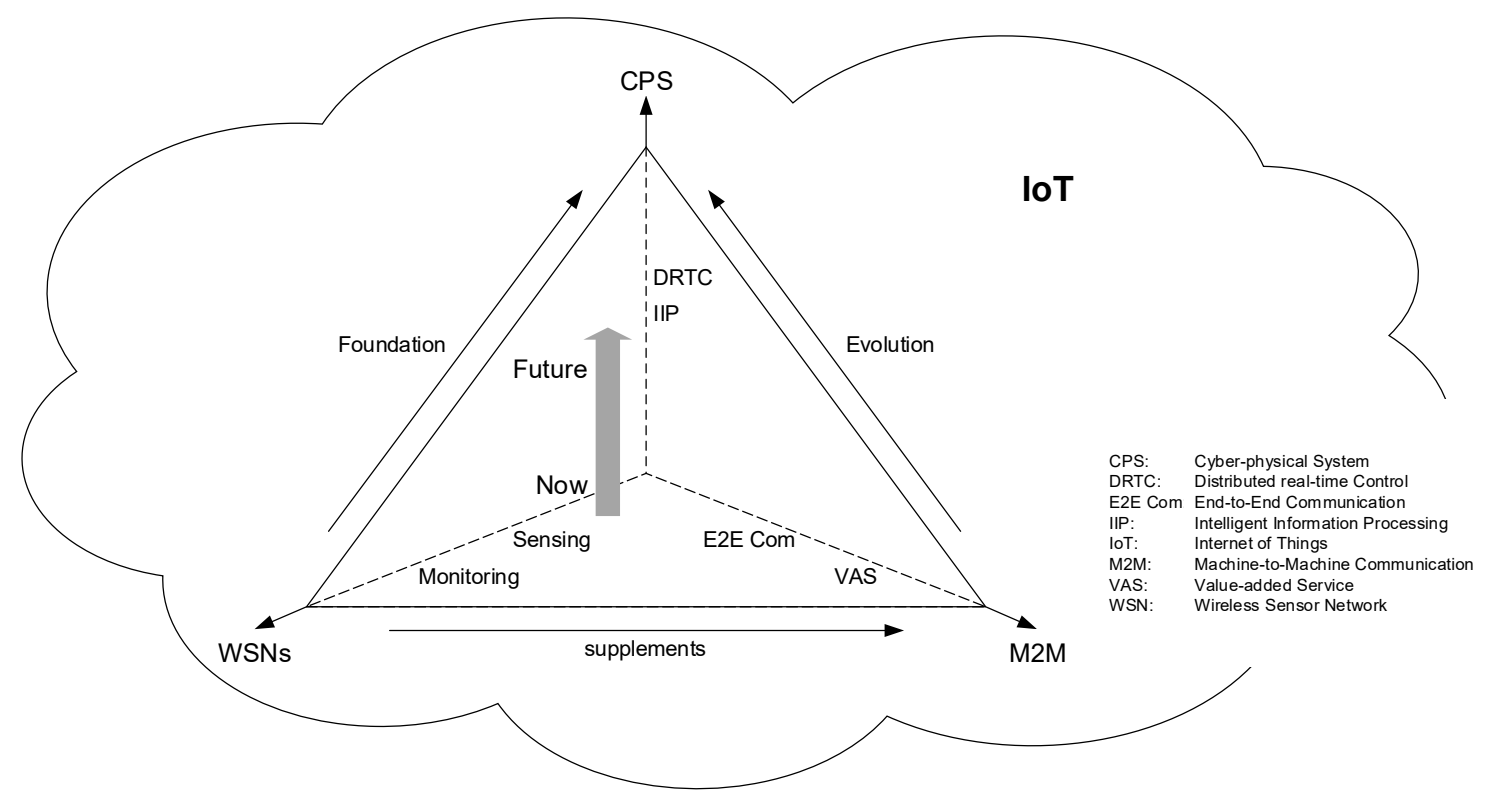

Figure 2.3: Correlation among WSNs, M2M, CPS, IoT acc. (Wan et al., 2013; Mehmood et al., 2015) 
Despite the similarity of WSNs, M2M, CPS in networking aspects they have "major differences from architecture and design philosophy" (Wan et al., 2013). Subsequently the main characteristics of WSNs, M2M, CPS are described according to (Wan et al., 2013), starting with the aspects of M2M.

\section{Machine-to-Machine Communication (M2M)}

- Refers to communication between computers, smart sensors/actuators without or with only minimal human intervention.

- M2M systems have the functionality for making decisions and autonomous control operations to provide value-added services (VAS).

- End-to-end communication between devices and focus on "practical applications (e.g., smart home and smart grid)".

- Through the integration of M2M with WSNs, M2M systems can use the information provided by the WSNs as a basis for performed actions (i.e. WSNs support M2M systems).

\section{Wireless Sensor Networks (WSNs)}

- WSNs, such as Wireless Body Area Networks (WBANs) consist of distributed sensors monitoring environmental conditions. The autonomous sensors cooperate with each other to deliver the collected data to a central location.

- WSNs and Information generation from all sensors is basic scenario of IoT.

- WSNs are the basis of CPS and support M2M.

\section{Cyber-physical System (CPS)}


- CPS is according to (Shi et al., 2011) the closely integration of physical processes into software. Classical application of CPS according to (Shi et al., 2011) are e.g. an operating room where all devices (health sensors or medicine indication systems) are fully networked and accessible for monitoring/control. Another classical CPS application is an "intelligent road with unmanned vehicle". The CPS e.g. monitors and controls the actions of the vehicles, such as stopping or turning left/right.

- CPS introduces interactive and more intelligent operations and represents an enhancement of M2M.

- Characteristics of CPS are: "cyber capability in every physical component", close integration of all devices, high complexity of system architecture, dynamically reconfiguration, "high degrees of automation", dependable and distributed real time operations.

According to (Wan et al., 2013) M2M and CPS address similar domains. While M2M has the perspective of autonomous communication and provision of value-added services supported by WSNs, CPS has the perspective of not only communication but focuses on applications based on WSNs as well as distributed and real-time control.

Since the aspects of this project focus on providing services via MSPs, the scope of the project has to be classified in the M2M field.

The following section introduces the architecture of M2M systems starting from a general perspective of M2M system architecture and refining it afterwards by description of detailed M2M System architecture as defined by European Telecommunications Standards Institute (ETSI). 


\subsubsection{M2M System Architecture}

Implementation of VAS in $\mathrm{M} 2 \mathrm{M}$ requires an $\mathrm{M} 2 \mathrm{M}$ system architecture establishing connection of devices among each other and with a (usually business) application. These M2M applications according to (Holler et al., 2014) can be integrated into business processes of a company to support them.

Figure 2.4 shows the general structure of an M2M system architecture. M2M Devices (providing capabilities for sensing and actuation) communicate via an IP-based Communication Network, such as the Internet or Next Generation Networks (NGNs), with each other and with a single specific application to exchange information (Holler et al., 2014; Boswarthick et al., 2012). Permission to reproduce Figure 2.4 has been granted by the publisher John Wiley and Sons.

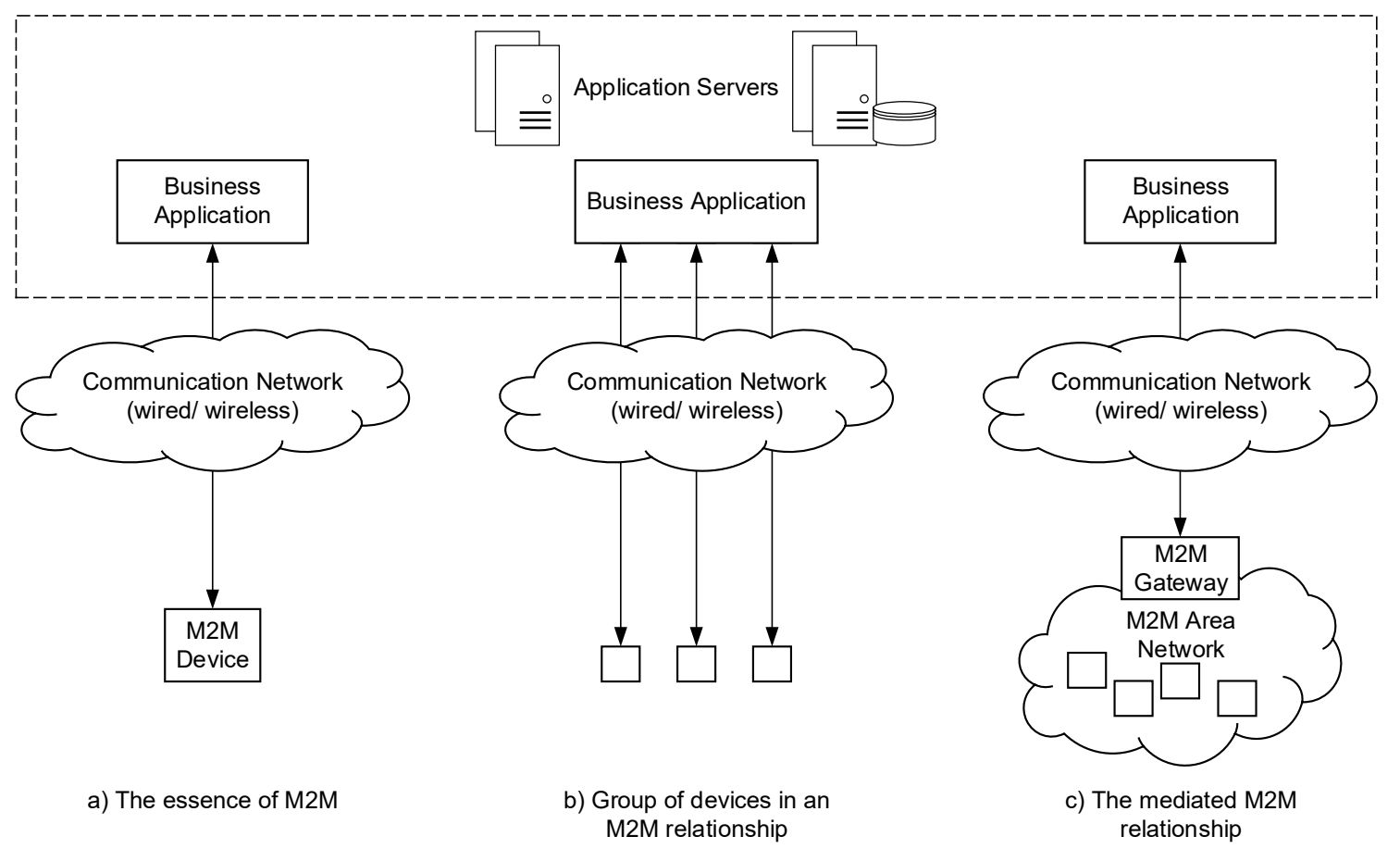

Figure 2.4: General Perspective of M2M System Architecture acc. (Wan et al., 2013; Mehmood et al., 2015; Bahga and Madisetti, 2014; Boswarthick et al., 2012; Holler et al., 2014) 
M2M applications created by "very specialized developers" realise the main logic to achieve the requirements of the M2M system. The M2M applications (e.g. "remote car diagnostics") usually are deployed on Application Servers inside enterprises (Holler et al., 2014). An M2M application can address single M2M Devices (Figure 2.4-a) or a group of M2M Devices (Figure 2.4-b), which can be directly connected to the Communication Network or via a Gateway (GW) (Figure 2.4-b) forming the mediating interface between the M2M Area Network and the Communication Network (Boswarthick et al., 2012). For communication inside an M2M Area Network, several communication technologies and protocols are used (e.g. Bluetooth, ZigBee, Wi-Fi) (Bahga and Madisetti, 2014).

The following section introduces the functional architecture of an M2M system as described by ETSI based on (ETSI TS 102690 V2.1.1, 2013). Figure 2.5 illustrates this functional architecture according to (ETSI TS 102690 V2.1.1, 2013) and refined with information given by (Boswarthick et al., 2012).

The M2M system architecture illustrated in Figure 2.5 is based on so-called Service Capability Layers. Service Capability Layers provide Service Capabilities, which are according to (ETSI TR 102725 V1.1.1, 2013) a set of functions usable commonly by various applications. In the M2M system architecture it is distinguished between different kinds of Service Capability Layers referring to the type/location of equipment implementing these Service Capability Layers. Exemplarily the M2M Device Service Capability Layer resides on M2M Devices and provides functions usable by the applications contained in the M2M Devices. Permission to reproduce Figure 2.5 has been granted by ETSI and the publisher John Wiley and Sons. 


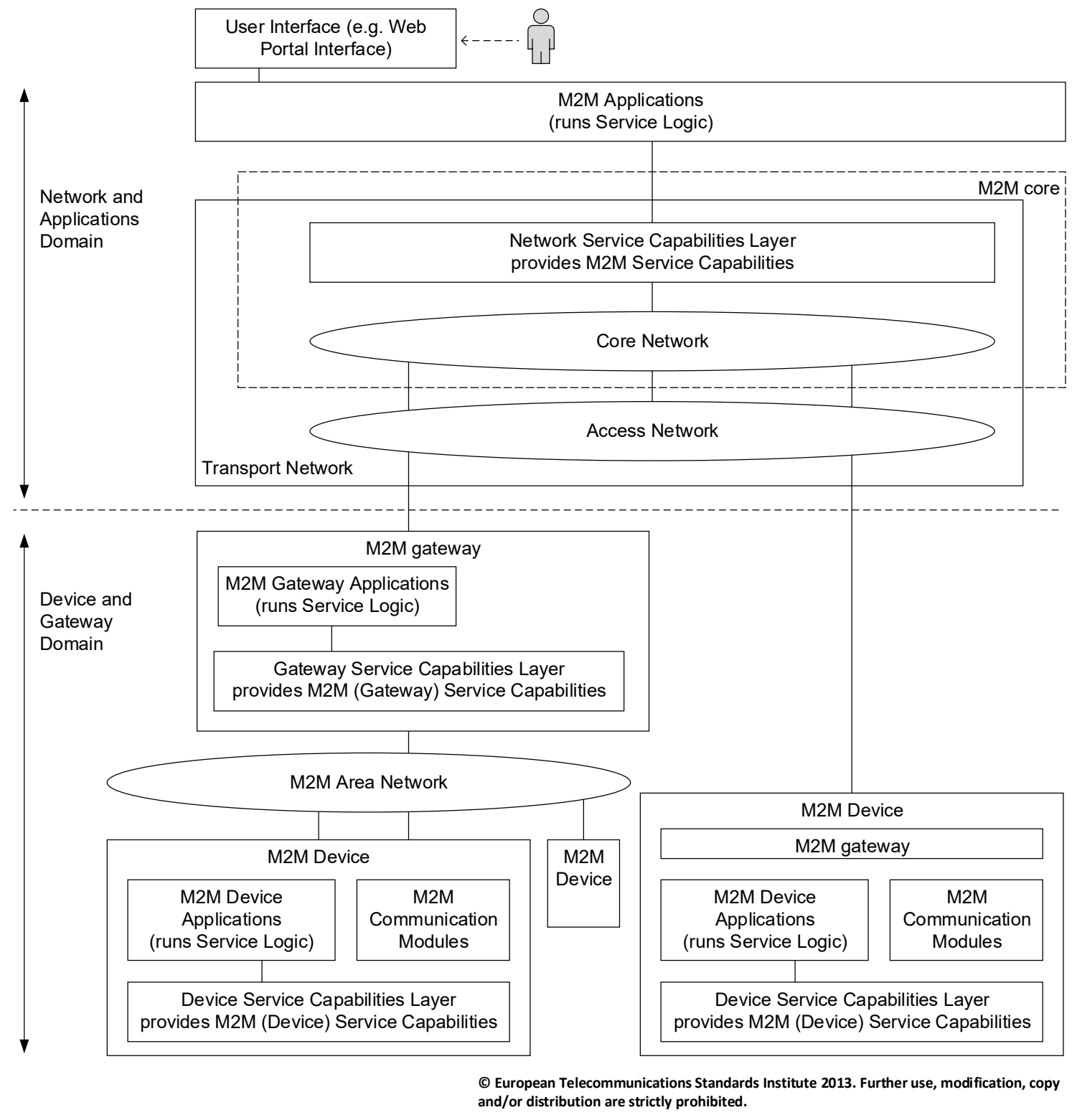

Figure 2.5: Functional Architecture of M2M System acc. (ETSI TS 102690 V2.1.1, 2013; Boswarthick et al., 2012)

According to (ETSI TS 102690 V2.1.1, 2013) and (Boswarthick et al., 2012) the M2M system architecture is separated in the two areas Device and Gateway Domain and Network and Applications Domain. The Access Network, such as xDSL, HFC, Wi-Fi or 3/4G, enables the Device and Gateway Domain to connect with the (IP-) Core Network (ETSI TS 102690 V2.1.1, 2013). Through the Core Network, entities residing in the Device and Gateway Domain connect to M2M Applications that use the data information 
and capabilities of the M2M Devices to realise VAS for supporting various business processes. The separated areas indicate where the architectural elements of an M2M system are located.

An M2M system contains in the Devices and Gateway Domain entities such as M2M Gateways, M2M Area Networks and M2M Devices. These entities are described subsequently according to (oneM2M TS-0011-V2.4.1, 2016), (ETSI TR 102725 V1.1.1, 2013), and (Boswarthick et al., 2012):

- M2M Device - An M2M Device is physical equipment that contains capabilities for communication/computing/sensing/actuation. M2M Devices contain at least one M2M Device Application (executing the application logic and using M2M Device Service Capabilities) as well as one M2M Communication Module (implementing the communication capability).

- M2M Area Network - The M2M Area Network provides functionalities, such as communication technologies/protocols enabling M2M devices to be connected with an M2M Gateway.

- M2M Gateway - A M2M Gateway is equipment implementing connection functionality between M2M Devices and Network and Applications Domain and can be implemented as a stand-alone entity or integrated within M2M Devices.

The Network and Applications Domain contains the Transport Network consisting of various Access Networks and the (IP-based) Core Network forming the communication infrastructure between the Devices and Gateway Domain and the M2M Applications. The Network and Applications Domain additionally contains the Network Service Capability 
Layer providing functionalities to $\mathrm{M} 2 \mathrm{M}$ application service provider (ASP) such as application registration, data storage or support for NAT traversal.

Since M2M Applications and M2M Services are in the focus of this research these and corresponding terms should be specified.

(ETSI TR 102725 V1.1.1, 2013) defines an application as a software entity performing a specific task to support a user operating on a specific goal. Physical devices, such as M2M devices or application servers execute software applications, which according to (ITIL V3.1.24, 2007) realise services. As specified in (ITIL V3.1.24, 2007) software applications provide functions required by services and can be part of multiple services.

A M2M application consists of one or more underlying services that provide capabilities to support the M2M applications (ITU-T Y.101, 2000). Service providers provide these services (ITU-T Y.2301, 2013; OASIS SOA-RM-V1.0, 2006). For using the functionalities of a service, an interface is required to access these functionalities (OASIS SOA-RM-V1.0, 2006). Services consist of service components, which form the building blocks of a service and are combined with other service components to build the whole service (ISO IEC 20000-1:2011, 2013).

According to (oneM2M TS-0011-V2.4.1, 2016) the logic of an M2M application realises the M2M application service and is accessible via a specific interface (M2M application interface). As specified by (UC Santa Cruz, 2017) "applications, themselves, are not services". Services are enabled by applications and "may be provided by someone else". I.e. an M2M application only is an M2M application service if a stakeholder exists which operates the $\mathrm{M} 2 \mathrm{M}$ application and additionally provides an interface to the $\mathrm{M} 2 \mathrm{M}$ 
application that enables an end-user to consume the application logic. The M2M application service is consumed according to (oneM2M TS-0011-V2.4.1, 2016) by the end-user that can be as specified in (ITU-T Y.110, 1998) either a company or a private individual.

Now that the architecture of an M2M system has been described, the following section describes how M2M applications use the functionality of an M2M system to provide specific VAS.

An MSP is used to provide M2M applications in an M2M system. This MSP provides the connectivity between M2M devices and enables the management of participating nodes as well as the exchange of information between the application and M2M devices. The standardisation committee oneM2M published several specifications, such as (oneM2M TS-0007-V2.0.0, 2016) or (oneM2M TS-0001-V2.10.0, 2016) defining the functionality of an MSP and the interworking of it with other components of an M2M system architecture. Figure 2.6 illustrates the principle integration of an MSP in the M2M system architecture according to (oneM2M TR-0001-V2.4.1, 2016; Elloumi, 2014). As Figure 2.6 shows, the $M S P$ is connected to the $M 2 M G W$ via the Transport Network, which in turn connects various M2M Devices via an M2M Area Network. The M2M Application is executed on a central M2M Application Server (e.g. as a Web application) and is provided via an interface to one or more end-users as an M2M application service. An MSP and M2M Application Server are usually operated within data centres. It can be derived that the MSP and M2M Application Server are placed within the Network and Applications Domain, whereas the M2M Application Servers implementing the application logic are 
not part of the MSP. The components of the Device and Gateway Domain are also not part of the MSP.

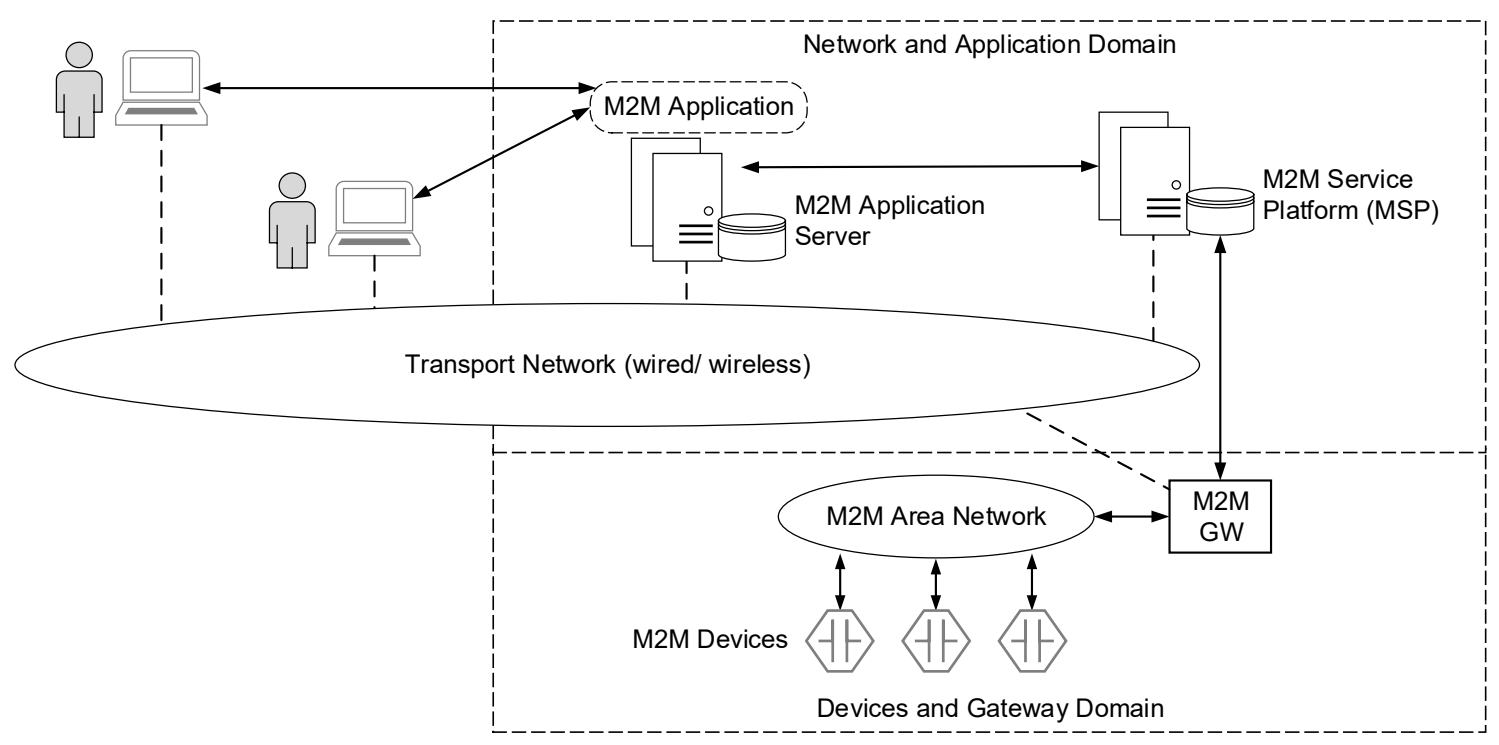

Figure 2.6: Integration of MSP in M2M system architecture acc. (oneM2M TR-0001-V2.4.1, 2016; Elloumi, 2014)

\subsubsection{Roles and Stakeholder in M2M Ecosystems}

In the M2M ecosystem, several separated roles and stakeholders exist providing, operating and consuming M2M applications and M2M services. Figure 2.7 illustrates them as defined by (oneM2M TS-0002-V1.0.1, 2015). Permission to reproduce Figure 2.7 has been granted by ETSI.

As defined by (oneM2M TS-0002-V1.0.1, 2015) and (oneM2M TS-0011-V2.4.1, 2016), the user (end-user) consumes an M2M solution. End-users represent private individuals or companies requiring "information-based services" (ITU-T Y.110, 1998; ISO IEC 20000-1:2011, 2013). 


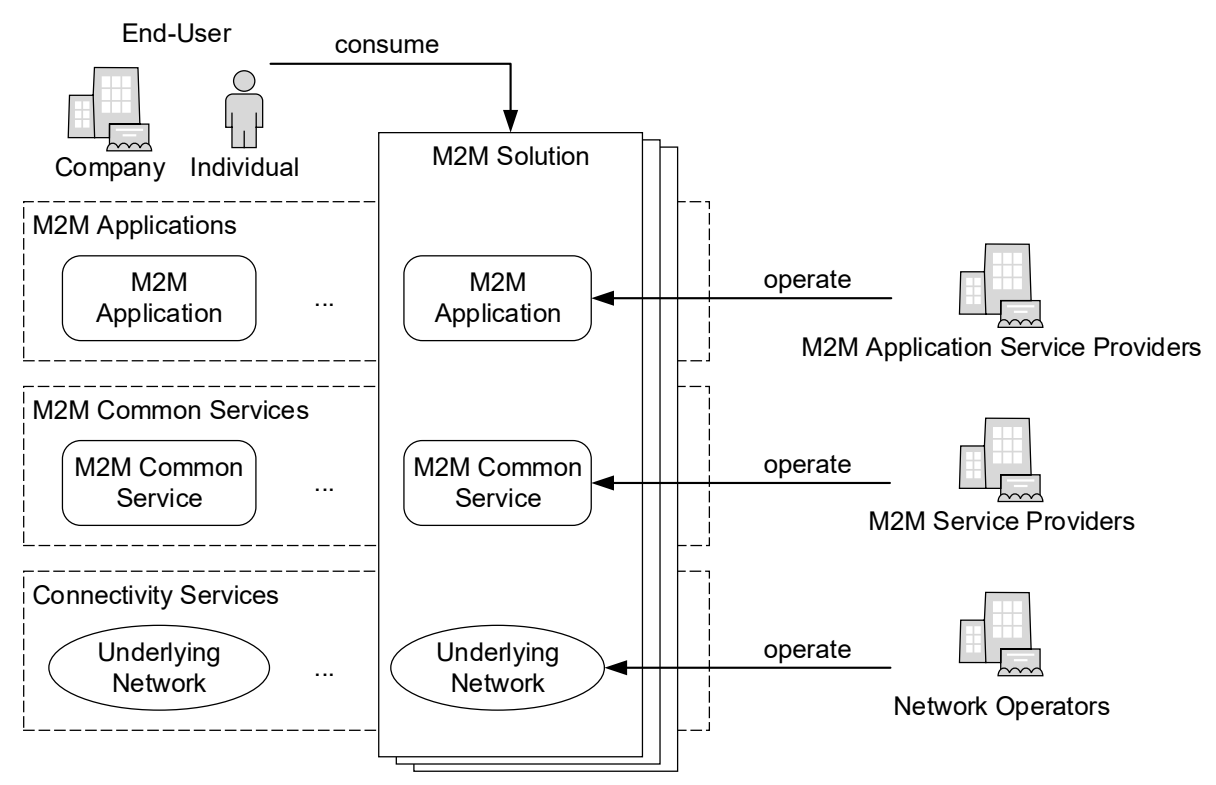

(C) 2015. oneM2M TSs and TRs are the property of ARIB, ATIS, CCSA, ETSI, TIA, TSDSI, TTA and TTC who jointly own the copyright in them. They are subject to further modifications and are therefore provided to you "as is" for information purposes only. Further use is strictly prohibited.

Figure 2.7: Functional Roles in the M2M Ecosystem acc. (oneM2M TS-0002-V1.0.1, 2015)

The M2M application service provider (ASP) is the entity that provides and operates the M2M application service consumed by the end-user (oneM2M TS-0011-V2.4.1, 2016; oneM2M TS-0002-V1.0.1, 2015; ETSI TR 102725 V1.1.1, 2013).

According to (ETSI TR 102725 V1.1.1, 2013) and (oneM2M TS-0011-V2.4.1, 2016) the $\mathrm{M} 2 \mathrm{M}$ (common) service provider provides (and operates) the M2M services to the M2M ASP, which use the services to create the M2M application, or directly to the end-user. As specified by (oneM2M TS-0011-V2.4.1, 2016) every stakeholder subscribing to M2M Services is specified as a service subscriber.

According to (ITU-T E.4110 (2010) service providers generally (i.e. M2M ASP and M2M service provider in particular) offer their services using network resources. The network operator provides and operates according to (oneM2M TS-0002-V1.0.1, 2015) these communication networks used to transmit service information. 
The presentation of the different roles shows that these are different stakeholders. Especially end-users and service providers have separate roles in the M2M ecosystem presented. The concept presented in the course of this research project aims on the dissolution of the static link to a central service provider and enabling end-users taking on the role of a service provider.

\subsubsection{End-User M2M Application Services}

The aim of the research project is to enable end-users to act as service providers in order to make the resources available in their personal environments available to other endusers. Additionally, it should be possible for different end-users to combine their services and provide them as a distributed application. Since this is a P2P-based service provisioning, the service definition from (IETF RFC 7890, 2016) is derived to define an $\mathrm{M} 2 \mathrm{M}$ application service (provided by end-users) in context of this thesis as follows:

- M2M Application Service - An M2M application service is a capability provided by a peer to other peers of the P2P network, while peers can provide different or same M2M application services.

Combination of service is also referred to as service composition. Service compositions using traditional MSPs are realised by service orchestration. Service orchestrations (illustrated in Figure 2.8) according to (Terpak et al., 2016) constitutes a centralised approach based on "a single executable process" that describe the relationship of the participating services. 


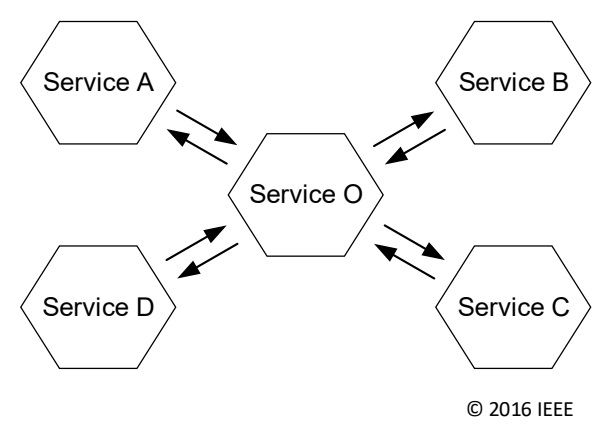

Figure 2.8: Service Orchestration acc. (Terpak et al., 2016)

Service orchestrations include a central coordinator (service orchestrator) for coordination of interactions between the participating services. The service orchestrator actively invokes and combines the services and controls the information exchange between the services. (Terpak et al., 2016)

The service composition followed in this research project is performed by service choreography in order to avoid centralised service coordinators. Service choreographies (illustrated in Figure 2.9) according to (Terpak et al., 2016) constitutes a decentralised approach.

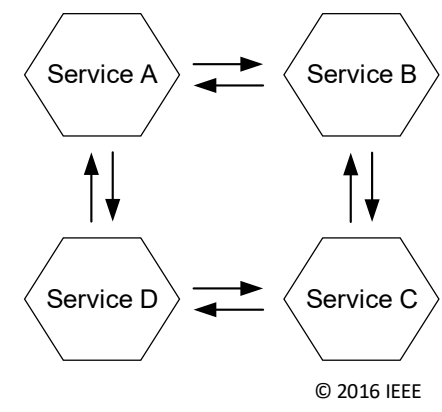

Figure 2.9: Service Choreography acc. (Terpak et al., 2016)

In service choreography, the combination of the participating services is described globally. The global description defines the message exchange between the services as well as the interactions between the service endpoints. In service choreography, each 
service is responsible for information exchange with its corresponding service. The execution of the service composition during runtime takes place without central controlling of the information exchange between the services. (Terpak et al., 2016)

\subsection{Use Cases}

The following section presents use cases for M2M services and M2M application services. The use cases are introduced in this section to increase the overall understanding of the framework presented in the following chapters. The use cases represent the different aspects of the framework and will be used later in chapter 7 to evaluate the framework based on these aspects. Use cases have been defined that address the Smart Home/Smart Building sector, since this area is also a popular field of application for M2M solutions and includes the personal environment of the end-user. These use cases or parts of it are used inside this research to exemplary illustrate specific technologies, functional approaches, system architectures, and methodologies after they have been generally introduced. The use cases presented in this section are close-coupled in its functionality to demonstrate the possibility for realisation of different applications integrating few similar use cases.

\section{Use Case 1: Local Window Monitoring}

The use case "local window monitoring", illustrated in Figure 2.10, demonstrates a service realised in a Smart Home of an individual end-user. The use case combines different M2M device technologies and multimedia communication. 


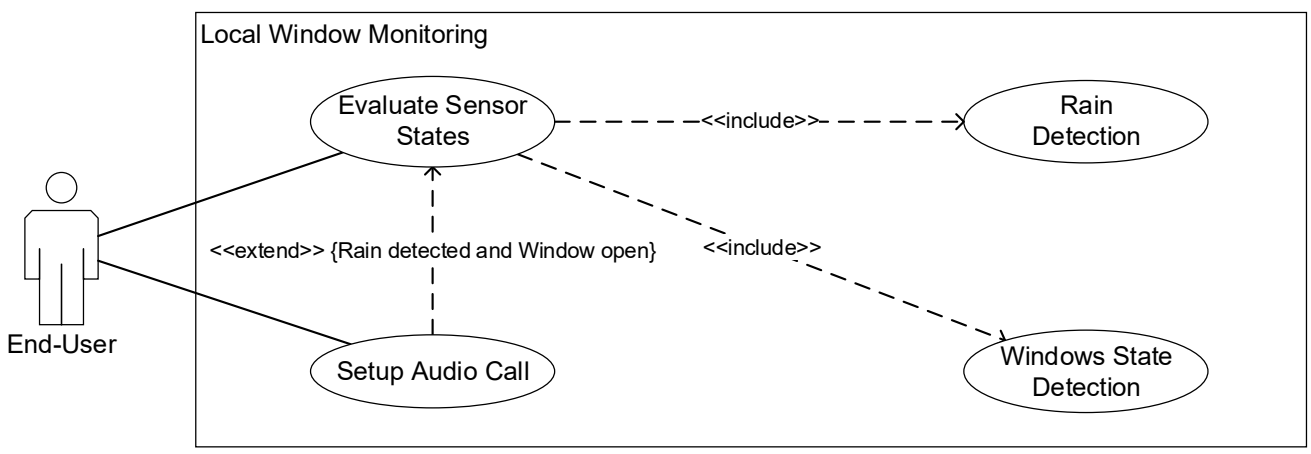

Figure 2.10: Use Case local Window Monitoring

The use case represents an M2M service designed by the end-user and executed in the personal environment of the end-user, without integration of other external entities. The service is realised using a single M2M platform located in end-users environment. It involves a single end-user as actor. The end-user designs the M2M service represented by the use case illustrated in Figure 2.10. The local M2M service detects two different sensor states (window open/closed and raining/not raining) on behalf of sensors using different M2M communication technologies (e.g. ZigBee, Z-Wave) for transmitting their sensor states. Depending on the evaluation result of these sensor states, the M2M service initiates an audio call to inform the end-user in case of raining and window opened at the same time (by playback an announcement).

\section{Use Case 2: Neighbourhood Weather Station}

The use case "neighbourhood weather station", illustrated in Figure 2.11, demonstrates the combination of a service provided remotely with the combination of a local service. The use case represents an M2M application service including the provision of an M2M service by end-user and the utilisation of that M2M service by another end-user. 


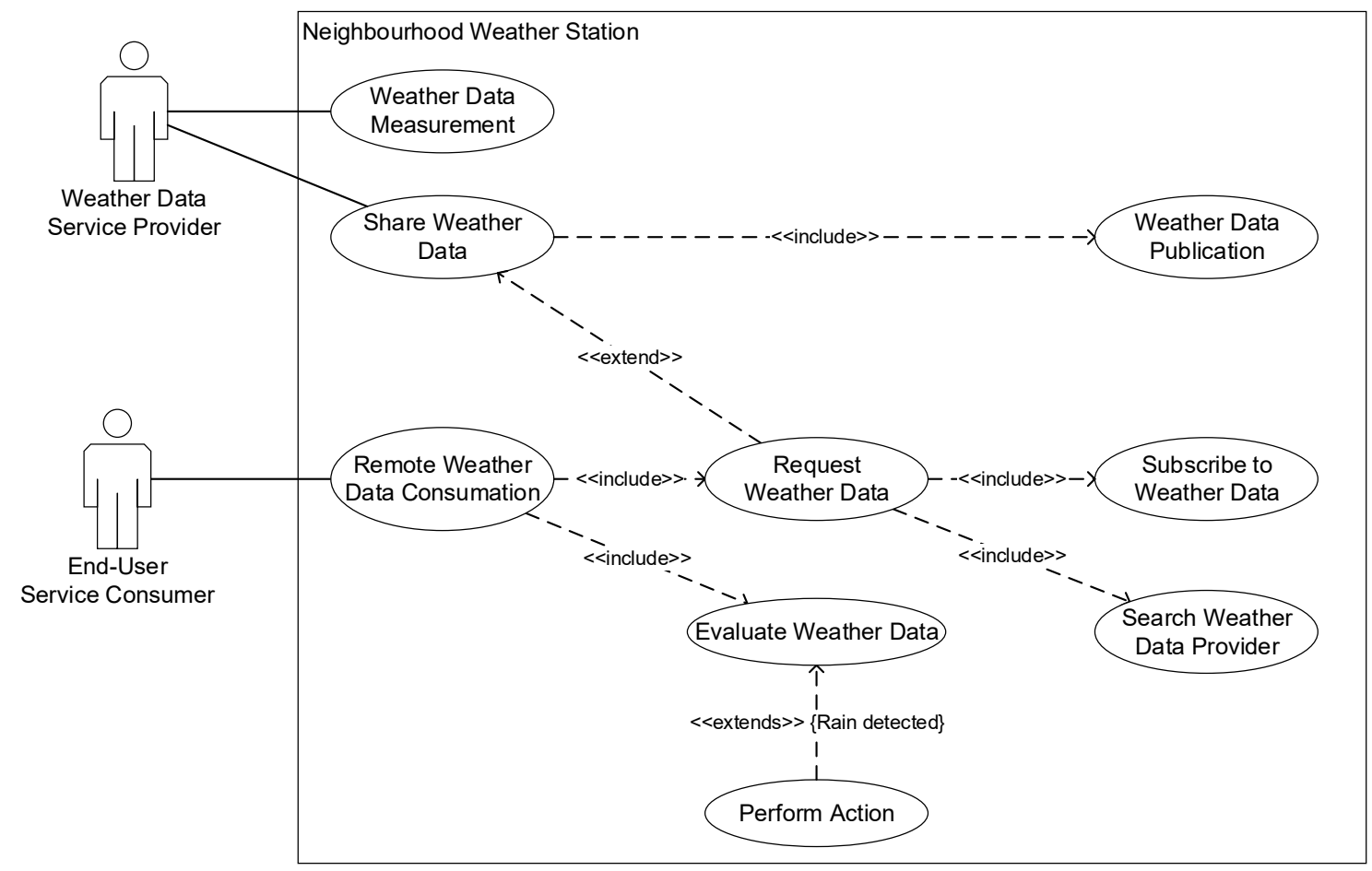

Figure 2.11: Use Case Neighbourhood Weather Station

The use case involves two actors, the SP and the SC. Both actors are end-users. The SP designs an M2M service for measurement of weather data using its local Smart Home equipment and provides, if requested, that weather data to other end-users. The SC designs its individual M2M service with functionality to request and evaluate weather data provided remotely. For this, the M2M service determines automatically a SP providing weather data in the neighbourhood and requests the weather data from the remote service. Depending on the evaluation of the received weather data (e.g. if it is raining), the $\mathrm{M} 2 \mathrm{M}$ service triggers some action (e.g. setup phone call as in previous use case or trigger an actuator for closing the window automatically).

\section{Use Case 3: Building Surveillance}

The use case "building surveillance", illustrated in Figure 2.12, demonstrates the concatenation of multiple services provided by multiple SPs. The use case involves five 
actors, which all are end-users. Four actors provide different M2M services. The different $\mathrm{M} 2 \mathrm{M}$ services are combined to form an M2M application service with the task to monitor specific buildings and react on alerts detected inside that building. The SC consumes the M2M application service.

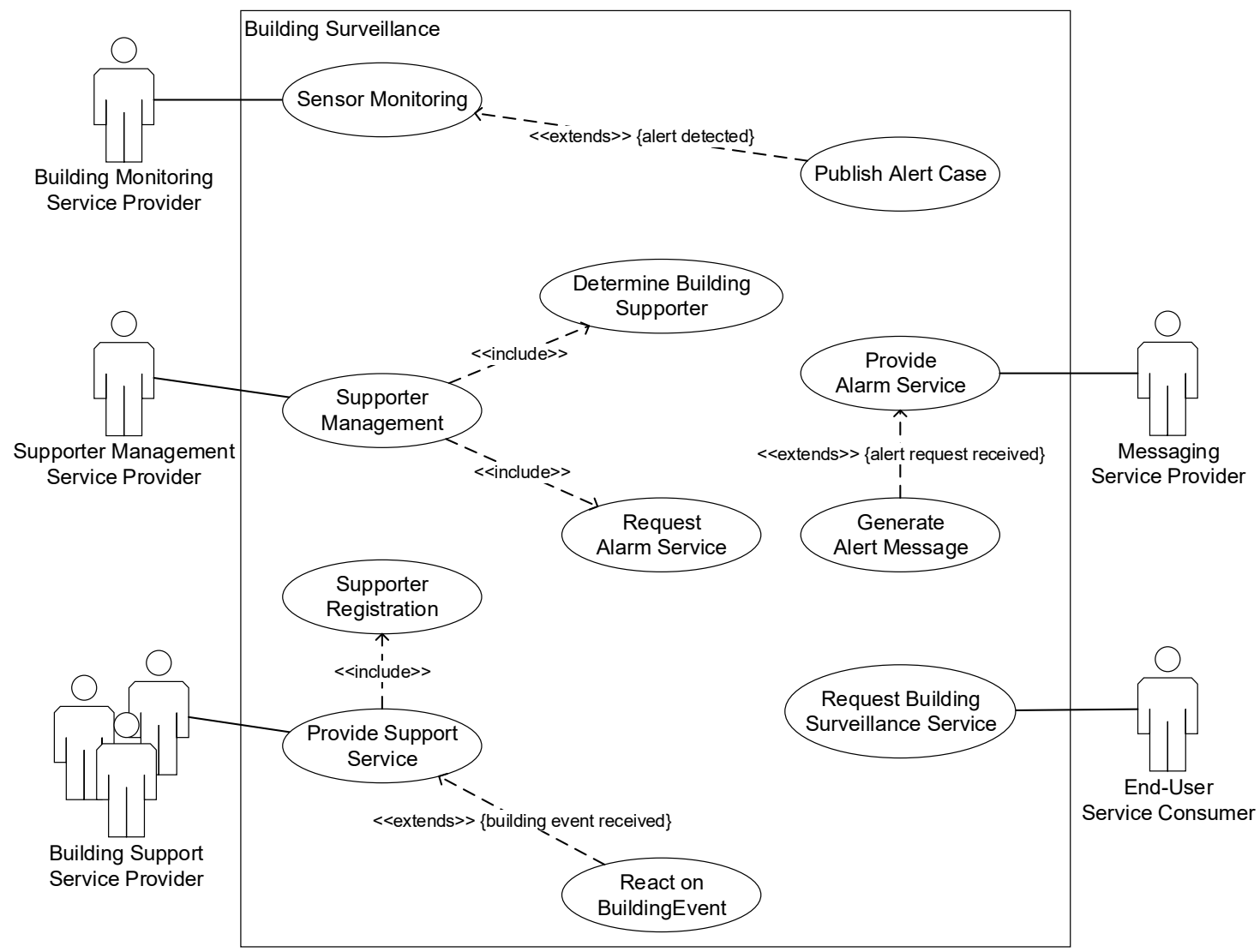

Figure 2.12: Use Case Building Surveillance

The building management SP designs and provides an M2M service for monitoring a sensor in a specific building. If the M2M service evaluates a specific sensor state (e.g. water intrusion or fire alarm), it publishes that alert case to another M2M SP, the supporter management SP. The supporter management SP provides an M2M service for management of supporters. Multiple individual supporters register at that service and offer (as a service) reacting on an alert appearing in a specific building. The task of the 
provided service is to manage the supporters and corresponding buildings. The service identifies the responsible supporter for the received alarm event and requests a service to send an alarm notification. Because the supporter management service cannot provide the functionality for multimedia communication via Instant Message (IM), it requests this functionality as a service provided by the messaging SP. The messaging SP generates an IM and sends the IM to the recipients. Recipients and message text is defined by the supporter management SP and specified at the service request. The building support SP is a group of actors each providing the service to react on an alert appearing in a specific building. All participants of that group register at supporter management service and specify their contact information and specific buildings they support. If an alert appears in a building, the corresponding supporter receives an IM and afterwards reacts on the alert.

The use case "building surveillance" illustrates the combination of services provided by individual SPs, all providing different, encapsulated, and independent services. Each of these services can be integrated in $\mathrm{M} 2 \mathrm{M}$ application services with entirely different contexts.

\section{Use Case 4: Energy Optimisation}

The use case "energy optimisation", illustrated in Figure 2.13, demonstrates an M2M application service realised by multiple end-users each providing individual and independent services in parallel. The functionality of the M2M application service is to reduce energy load by sharing information regarding the total local energy consumption between all participating end-users. 


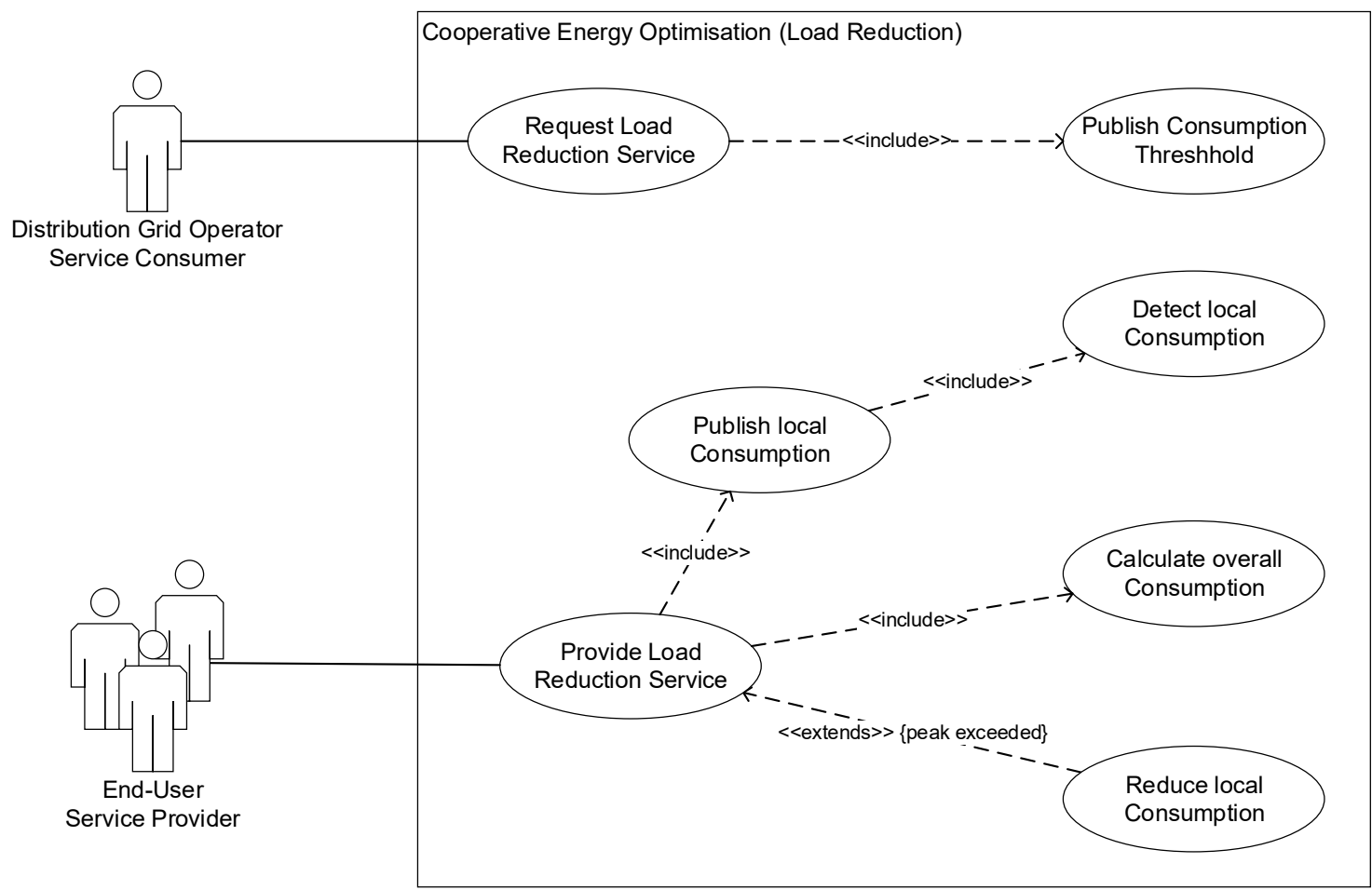

Figure 2.13: Use Case Cooperative Energy Optimisation (Load Reduction)

The use case involves a group of end-users as M2M SPs as well as the distribution grid operator as M2M application service consumer. Each end-user designs and runs a service locally with the functionality to determine the current local energy consumption and ability to reduce the local energy consumption. Each end-user publishes its locally determined total energy consumption to all other involved M2M SPs. The distribution grid operator as $\mathrm{M} 2 \mathrm{M}$ application service consumer specifies at application service request a threshold for total energy consumption. Each individual M2M SP calculates the overall consumption after receiving the consumption information of the other participants. Depending on the local evaluation of the total consumption, the individual end-user service triggers local reduction of energy consumption by e.g. turning of M2M devices or start consuming energy from a local energy storage (battery). 


\subsection{Conclusion}

This chapter introduced the main principles of the research field by discussing AEEs as well as P2P and M2M systems.

Section 2.1 introduced the different AEEs for M2M applications or MSPs. Fog Computing aspect to shift computing and data capturing tasks to the edge of the network is a promising approach also included in the framework concepts proposed in the following chapters.

Section 2.2 presented the $\mathrm{P} 2 \mathrm{P}$ communication paradigm that enables to address resources located at the edge of the network. Using P2P systems can address the disadvantages of traditional client/-server systems, such as single point of failure or bottlenecks, when large amount of data needs to be transmitted and processed. Transferring P2P system approaches to MSPs would be beneficial because a decentralised, distributed MSP could reduce the load in both system and transport networks. The characteristics of P2P system architectures have been described that enables autonomous end-to-end communication between nodes. These principles will be derived to develop a concept for a decentralised MSP with end-user environment integration. Since avoiding central entities in the research project is an important topic, the architecture of decentralised P2P systems rather than centralised or hybrid ones will be applied to the concept of MSP presented in the following chapters.

The M2M system architecture, as main object of this research, has been introduced in section 2.3. The principles of M2M have been described as well as its correlation to IoT. It has been shown that M2M focuses on the provision of value-added services, which is 
the subject area addressed in this research. The several components of an M2M system architecture have been presented and shown how these components are related to each other. It has been shown that an MSP is located as a separate and centralised element in traditional M2M system architectures, which will be changed in the concepts developed in this research to a decentralised and distributed M2M system architecture. The different roles and stakeholder existing in an M2M ecosystem have been introduced. It has been highlighted that M2M ASPs and consumer are separated stakeholder. This relationship will be changed during this research enabling end-users to take the role of both, M2M ASP as well as consumer. Since M2M services and composition of them are in the focus of this research, finally the differences of service choreography (common in traditional MSPs) and service orchestration have been illustrated. Service choreography will be the approach followed in the course of this research since it does not contain a central coordinator for service interactions.

The use cases specified in section 2.4 represent exemplary options to link M2M devices in a Smart Home and to provide the functionality as a service as well as the possibility to combine several services to realise distributed M2M applications. These use cases will be applied later in this research to illustrate architectural approaches and methodologies as well as to evaluate the proposed framework.

The following chapter 0 presents the architecture of MSP defined by standardisation committees ETSI and oneM2M as well as research projects of MSPs. Advantages and disadvantages of these approaches will be determined to define the requirements for optimised architecture and principles for decentralised $\mathrm{M} 2 \mathrm{M}$ application service provision. 


\section{Challenges, Requirements, and Use}

\section{Cases of M2M Application Service}

\section{Provision}

This chapter starts by introducing and analysing in section 3.1 existing concepts for service/application provision in M2M application field, which partly or completely address similar topics as addressed in this research, focusing on their benefits and limitations. The approaches are intended to illustrate how currently M2M service platforms (MSPs) are implemented. OneM2M's approaches for the specification of an MSP will be introduced in detail since it is considered as a reference architecture and because the framework presented in this research should use standardised approaches for the realisation of an optimised MSP. Furthermore, various research projects on MSPs will be presented to consider their advantages and disadvantages in the concept of an optimised MSP. The chapter continues in section 3.2 with an overview of the previously introduced projects, which forms the basis for establishing the requirements for decentralised application provision in M2M networks. In order to define the requirements, the positive aspects of the presented projects are taken into account and new requirements are defined based on the negative aspects. 


\subsection{Related Work on M2M Service Platforms}

This section introduces the related projects on MSPs. They can be categorised e.g. according to (Kim et al., 2014) into "commercial M2M platforms" and "research projects on M2M platforms". According to (Kim et al., 2014) most of the commercial MSPs are realised based on the oneM2M standard specification for M2M systems. The oneM2M standard specification will therefore be introduced as representative of commercial M2M platforms in section 3.1.1, with the remaining subsections covering related projects from the research field.

\subsubsection{OneM2M Specification for M2M Systems}

The standardisation committee oneM2M published several specifications for definition of an architecture of an MSP, which according to (oneM2M TS-0011-V2.4.1, 2016) enable to deploy M2M solutions. The purpose of the developed specifications is to describe a Common Service Layer that enables the connection of myriad devices with M2M application servers (oneM2M TS-0011-V1.2.1, 2015). This section examines relevant specifications with focus on the architecture of an MSP according to oneM2M as well as the mechanisms to realise $\mathrm{M} 2 \mathrm{M}$ application services applying the architecture of an M2M system as defined by oneM2M.

It is specified in (oneM2M, 2015) that the objectives of oneM2M is to define interfaces that enable "individual industries or businesses" for interoperation, but their objective is not to standardise the environment across applications. OneM2M defines an MSP 
(consisting of M2M common services) based on a horizontal service layer (illustrated in Figure 3.1) that is usable across different application fields. (oneM2M TS-0007-V2.0.0, 2016), which describes the specific M2M common services and their interworking in an M2M architecture, defines as its scope the "use of the M2M Services within the context of complex business services". This indicates that the scope of an oneM2M defined M2M system does not designate to end-user domain, respectively M2M application provision by end-users, but describes an M2M system for business applications with the goal to support business processes. Additionally, (oneM2M TS-0011-V2.4.1, 2016) specifies the $\mathrm{M} 2 \mathrm{M}$ service provider (SP), M2M application service provider (ASP) and the M2M service consumer (SC) as different stakeholders in an M2M system. Although the scope of M2M systems according to the architecture defined by oneM2M does not focus on end-user domain and end-user integration in M2M application provision, in the following the architecture is introduced in detail to differentiate the concept defined in this thesis and explain approaches integrated in the proposed framework. Permission to reproduce Figure 3.1 has been granted by author of the referenced publication.

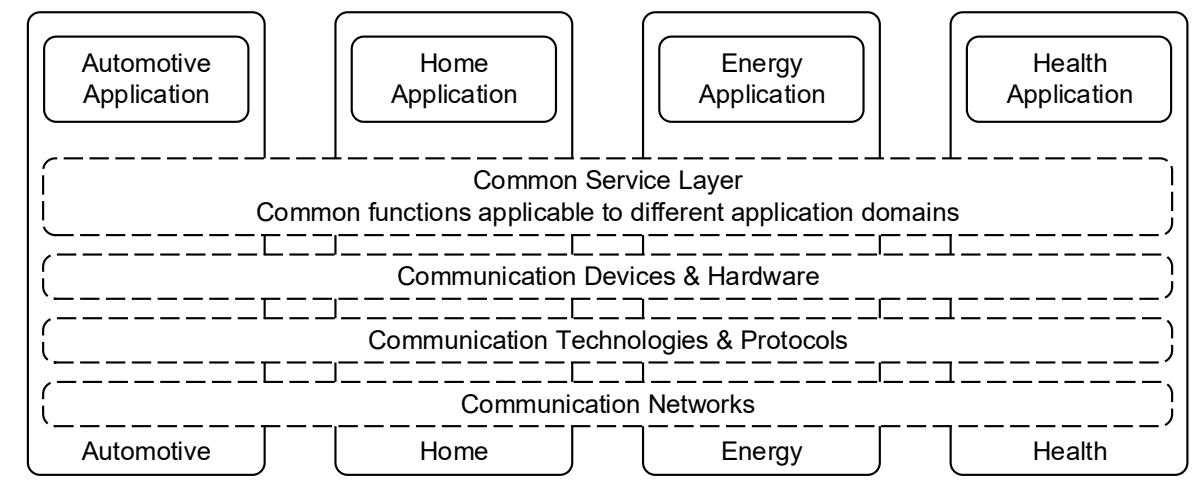

Figure 3.1: M2M Common Service Layer acc. (Damour, 2014)

The purpose of the specified M2M Common Service Layer of the service layer architecture defined by oneM2M is to avoid multiple coexistent solutions for $\mathrm{M} 2 \mathrm{M}$ 
business applications, realised by different centralised infrastructures of M2M SPs as illustrated in Figure 3.2.
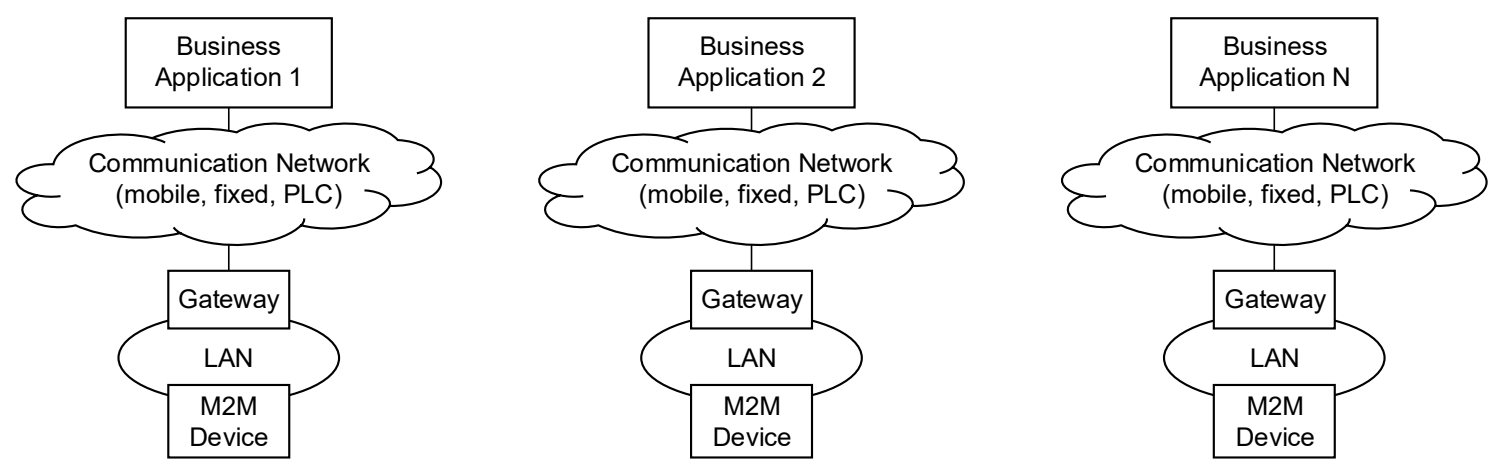

Figure 3.2: Vertical Pipes of M2M Business Applications acc. (Arndt and Koss, 2014)

OneM2M specifies an architecture for realisation of applications that "share common infrastructure, environments and network elements" by definition of a single centralised horizontal MSP architecture as illustrated in Figure 3.3.

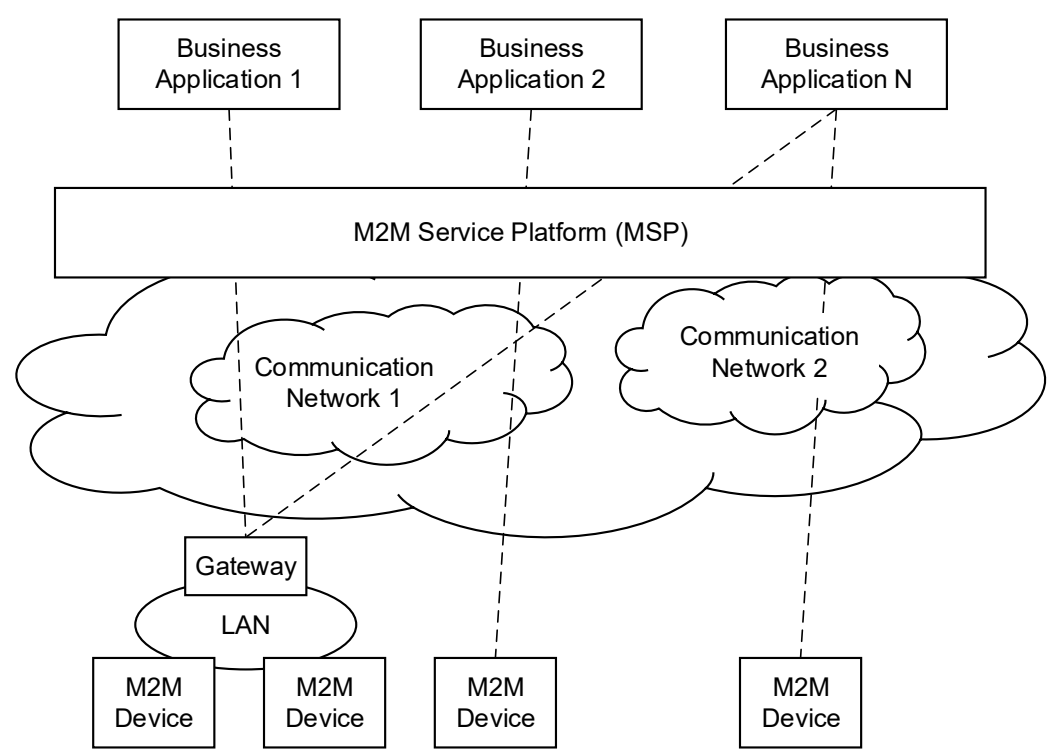

Figure 3.3: oneM2M Horizontal Platform Principle acc. (Arndt and Koss, 2014)

The purpose of the defined horizontal infrastructure is to realise central business applications by central M2M ASPs, but with application of a shared MSP acting as a 
middleware that supports the end-to-end data exchange between customer applications and M2M devices (Arndt and Koss, 2014).

(oneM2M TS-0001-V1.13.1, 2016) defines the following layers (illustrated in Figure 3.4) comprised in an M2M system to support end-to-end M2M Services. Every Layer can use the functionalities of the underlying layer.

- Application Layer - M2M application services are realised by implementation of Application Entities (AE) located in the Application Layer. The AEs can use the functionalities of the Common Services Layer.

- Common Services Layer - The Common Services Layer comprises functionalities that are common for $\mathrm{M} 2 \mathrm{M}$ applications of several $\mathrm{M} 2 \mathrm{M}$ application fields and across different M2M platforms (e.g. device or location management).

- Network Services Layer - The network services layer provides networking functionality to the common services layer, which uses the functionalities provided by the network to connect devices or other entities, using network communication.

oneM2M defines the functional architecture of an M2M system by specifying several AEs and service entities (CSEs and NSEs) located in the defined layers as well as various reference points to connect them for data exchange or to service requests (illustrated in Figure 3.4). Permission to reproduce Figure 3.4 has been granted by ETSI. 


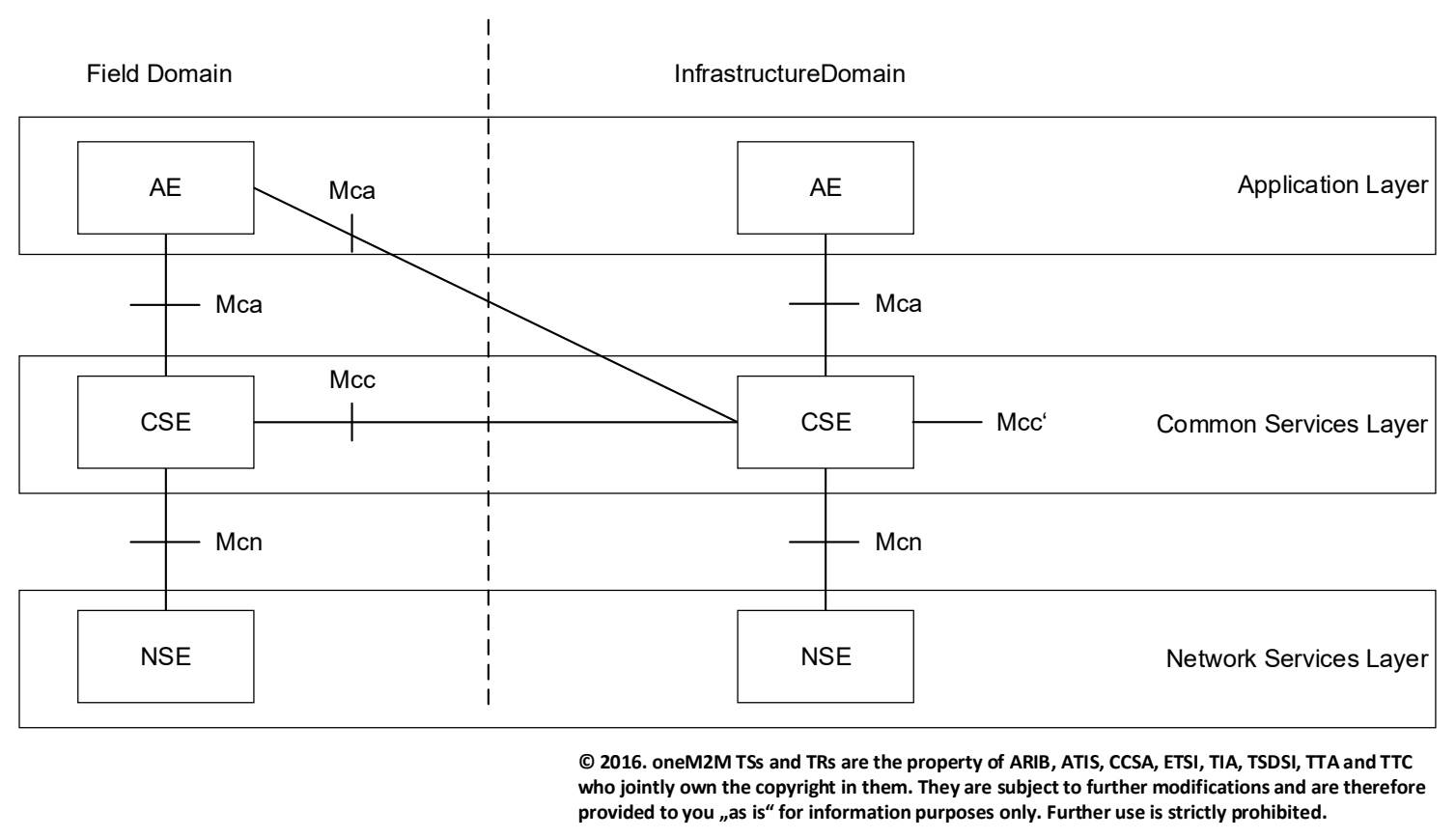

Figure 3.4: oneM2M Layered Model and Functional Architecture acc. (oneM2M TS-0001-V1.13.1, 2016)

Subsequently AE, CSE, NSE of the oneM2M are described as specified in (oneM2M TS0001-V1.13.1, 2016).

- Application Entity (AE) - AEs are entities implementing application logic (e.g. applications for remote blood sugar monitoring or a control applications). Each AE can reside multiple times in various M2M nodes. AEs and CSEs communicate via the Mca reference point to enable the AE to utilise the services provided by the CSE. AE and CSE can but need not be located on the same device.

- Common Services Entity (CSE) - A CSE is an instance "of a set of common service functions" such as services for device management (management of device capabilities) or location service. Other entities of the M2M system can utilise these common service functions (CSF) through the Mca and Mcc reference points illustrated in Figure 3.4. CSEs and NSEs connect via the Mcn reference point to enable the CSE to utilise the services provided by the NSE. Connections 
between CSEs are established via the Mcc reference point to utilise services provided by the other CSEs.

- Network Services Entity (NSE) - A NSE provides underlying network services to the CSEs. Such network services are according to (oneM2M TS-0004-V2.7.1, 2016) e.g. device triggering (3GPP TS 23.682, 2016) or location request (geographical location information).

An oneM2M system consists of several nodes that are whether located in the field domain or in the infrastructure domain. The Field Domain contains M2M devices, gateways and M2M Area Networks. The Infrastructure Domain contains the application infrastructure and the M2M service infrastructure (oneM2M TS-0011-V2.4.1, 2016). According to (Rayes and Salam, 2017) the Infrastructure Domain contains the communication networks (e.g. routers) and servers (e.g. data centres) of the M2M SP. The Infrastructure Domain (i.e. the M2M Service Infrastructure) is according to (oneM2M TS-0011-V1.2.1, 2015) "physical equipment (e.g. a set of physical servers) that provides management of data and coordination capabilities for the M2M SP and communicates with M2M Devices". Therefore the Infrastructure Domain is the place where the main components of an MSP resides. This is intensified by (oneM2M TS-0007-V2.0.0, 2016) describing the M2M services that an oneM2M service platform provides, which are "primarily suitable for the Infrastructure Domain". As specified in (oneM2M TS-0001-V1.13.1, 2016) a node is considered as a logical entity that is contained in a physical entity such as the M2M device or M2M application server. Every node provides specific services that can be consumed by other nodes (CSE of the nodes) in the M2M environment. The AEs, which are also located in nodes, consume the functionalities (services) provided by the 
own node or the functionalities provided by other nodes for implementation of the logic of an M2M application service.

To consume the functionalities by other nodes, the nodes need to be interconnected via the defined reference points. Figure 3.5 illustrates the various node types of an M2M system and shows in which domain they are located. Figure 3.5 also illustrates connection possibilities of various entities according to (oneM2M TS-0001-V1.13.1, 2016). Permission to reproduce Figure 3.5 has been granted by ETSI.

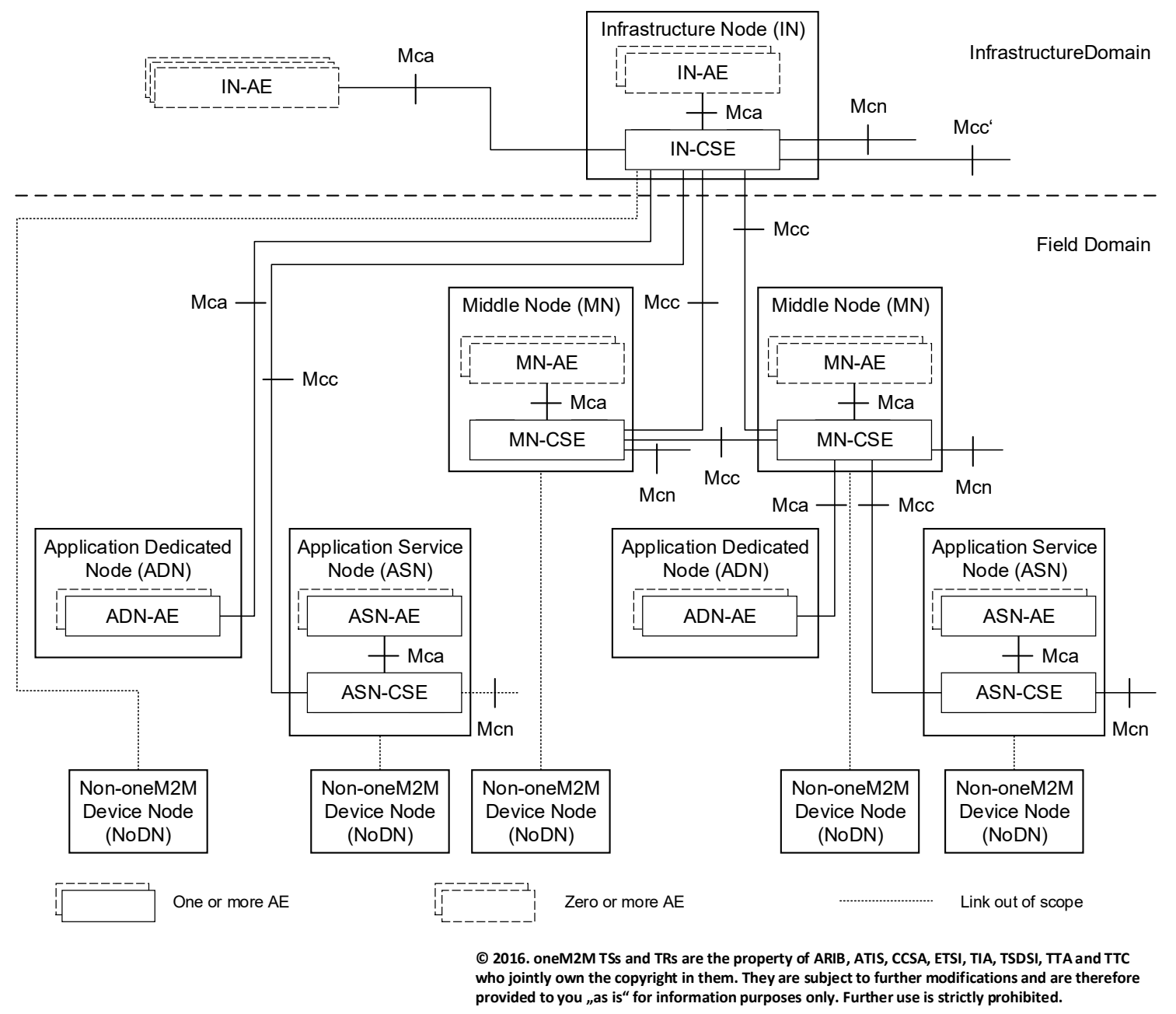

Figure 3.5: Configurations supported by oneM2M Architecture acc. (oneM2M TS-0001-V1.13.1, 2016) 
According to (oneM2M TS-0001-V1.13.1, 2016) the "Infrastructure Domain of any particular M2M SP contains exactly one Infrastructure Node” and the Field Domain can contain multiple Application Service/ Application Dedicated/ Middle/ and Non-oneM2M Nodes. These node types are described subsequently as specified in (oneM2M TS-0001V1.13.1, 2016).

- Application Service Node (ASN) - ASNs (e.g. M2M devices) contain one CSE and at least one AE.

- Application Dedicated Node (ADN) - ADNs (e.g. constrained M2M devices) contain no CSE and at least one AE.

- Middle Node (MN) - MNs (e.g. M2M Gateways) contain one CSE and zero or more AEs.

- Infrastructure Node (IN) - The IN is a single centralised element in the infrastructure of an M2M SP containing one CSE and zero or more AEs.

- Non-oneM2M Device Node (NoDN) - NoDNs not contain AEs or CSEs.

To connect between two Infrastructure Nodes (INs) residing in different M2M SP domains, respectively the CSEs located in the INs, the connection is established using their Mcc' reference points. This connection of the INs enable the CSEs of the INs "to communicate with a CSE of another IN residing in the Infrastructure Domain of another M2M SP to use its supported services and vice versa”.

After the main architectural components of an oneM2M service platform has been introduced, subsequently an exemplary M2M application (illustrated in Figure 3.6) is presented as defined by (oneM2M TR-0025-V1.0.0, 2016). The task of the exemplary $\mathrm{M} 2 \mathrm{M}$ application is to provide functionality for remote monitoring and control of lights 
in a Smart Home using a remote light controller application installed on a Smartphone. The lights (ADNs) are connected to an M2M Gateway (MN) that is connected to the oneM2M service platform. The "oneM2M service platform is modelled as an IN-CSE" and located in the Cloud. The Smartphone application (IN-AE) is connected to the MSP and is controlled by the end-user. Permission to reproduce Figure 3.6 has been granted by ETSI.

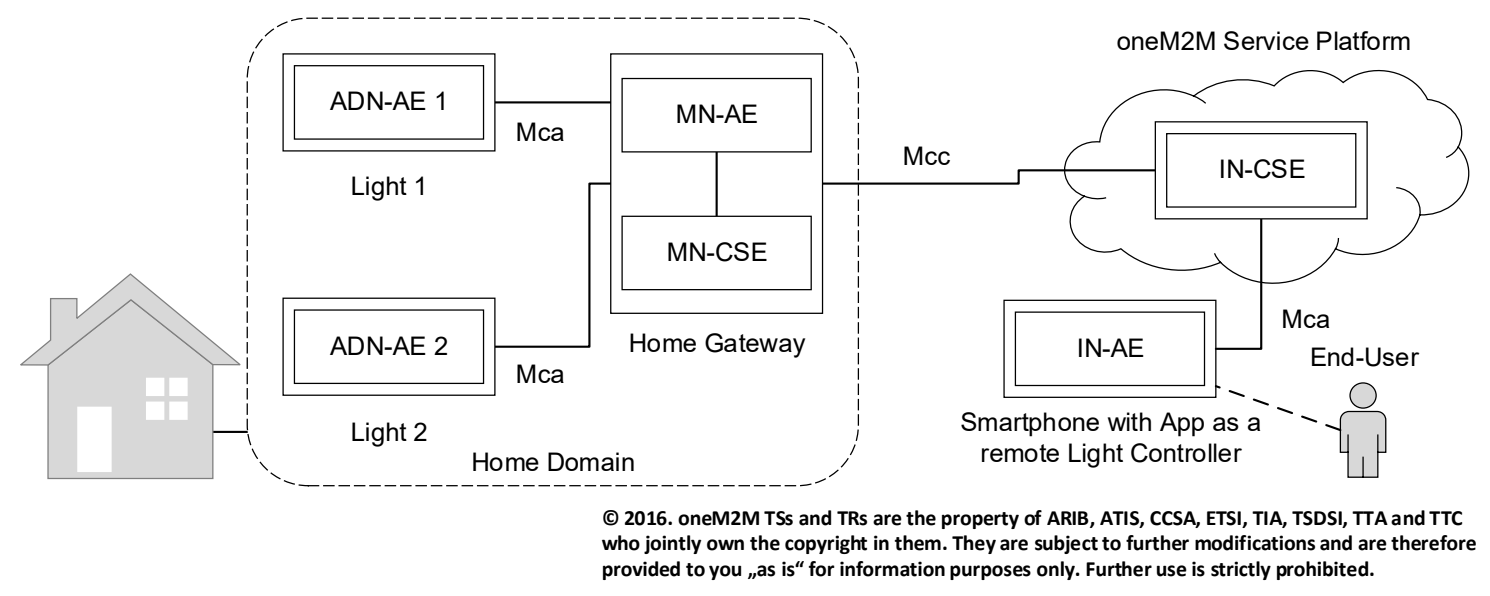

Figure 3.6: oneM2M functional Architecture of M2M Application acc. (oneM2M TR-0025-V1.0.0, 2016)

The Infrastructure Domain illustrated in Figure 3.5 "consists of Application Infrastructure and M2M Service Infrastructure" (oneM2M TS-0011-V2.4.1, 2016). The M2M application infrastructure is "equipment (e.g. a set of physical servers of the M2M ASP) that manages data and executes coordination functions of M2M Application Services" (oneM2M TS-0011-V2.4.1, 2016). The "Application Infrastructure hosts one or more M2M Applications" but "specification of Application Infrastructure is not subject of the current oneM2M specifications" (oneM2M TS-0011-V1.2.1, 2015).

While oneM2M does not specify the Application Infrastructure, they explicitly define the M2M Service Infrastructure. OneM2M specifies which functionality an MSP should 
provide by specifying so-called M2M common services. According to (oneM2M TS0011-V2.4.1, 2016) a common service is a "set of oneM2M specified functionalities that are widely applicable to different application domains made available through the set of oneM2M specified interfaces“. M2M common services (also referred to as Common Service Functions, CSFs) are realised by the CSE of a node that contains a set of M2M common services. The M2M common services have so-called service capabilities, which are the functions of an M2M common service usable by AEs or CSEs.

OneM2M separates between definition of functionalities (M2M common services) specific for an MSP (CSE in IN) and the definition of M2M common services that are applicable to all nodes in an M2M system. In the following first the M2M common services, i.e. the functionality of an MSP, is described as specified by (oneM2M TS-0007V2.0.0, 2016). After description of the MSP functionality, the M2M common services for general M2M nodes are described. Because not all M2M common services specified by oneM2M need to be integrated in an MSP or in M2M nodes, the description of the M2M common services is limited to the functionalities that are relevant in the scope of this research.

- Service Subscription - Invoke of AEs and associated M2M service capabilities, i.e. invoke of functionalities provided by a specific $\mathrm{AE}$.

- Data Exchange - Possibility for data (information) exchange between AEs supporting "Subscribe-Publish-Notify" and "Request-Response" data exchange patterns.

- Registration - Functionality to register AEs at the MSP and to refresh or terminate AE registrations. 
The following M2M common services applicable to general nodes are specified by (oneM2M TS-0001-V2.10.0, 2016).

- Communication Management and Delivery Handling (CMDH) - Functionality for communication with other entities (CSEs, AEs or NSEs). The CMDH controls (i.e. decides) which communication connection (communication protocol, network interface) should be used for message delivery. The transmission of the message is done without consideration of the message content (i.e. the $\mathrm{CMDH}$ "is not aware of the specific operation requested at the target entity").

- Data Management and Repository - Functionality for data storage (of e.g. application data, subscriber information or location information).

- Discovery - Functionality to search for applications and services.

- Registration - Registration functionality to enable registered entities (other AEs or CSEs) to utilise offered services.

- Subscription and Notification - Functionality for notification to track events occurred at a resource. AEs or CSEs have to subscribe for a resource they want to track. While the resource subscription is active, the hosting CSE sends notification events to the subscriber.

For realisation of an $\mathrm{M} 2 \mathrm{M}$ application service it is necessary to interconnect the nodes, respectively the CSEs of the nodes to use their provided M2M common services. These interconnections are done by registration of the nodes with each other, respectively forming a uni-directional connection of nodes. Figure 3.7 illustrates the possible node connections and the corresponding cardinalities that can exist in an oneM2M system as 
specified in (oneM2M TS-0001-V2.10.0, 2016). Permission to reproduce Figure 3.7 has been granted by ETSI.

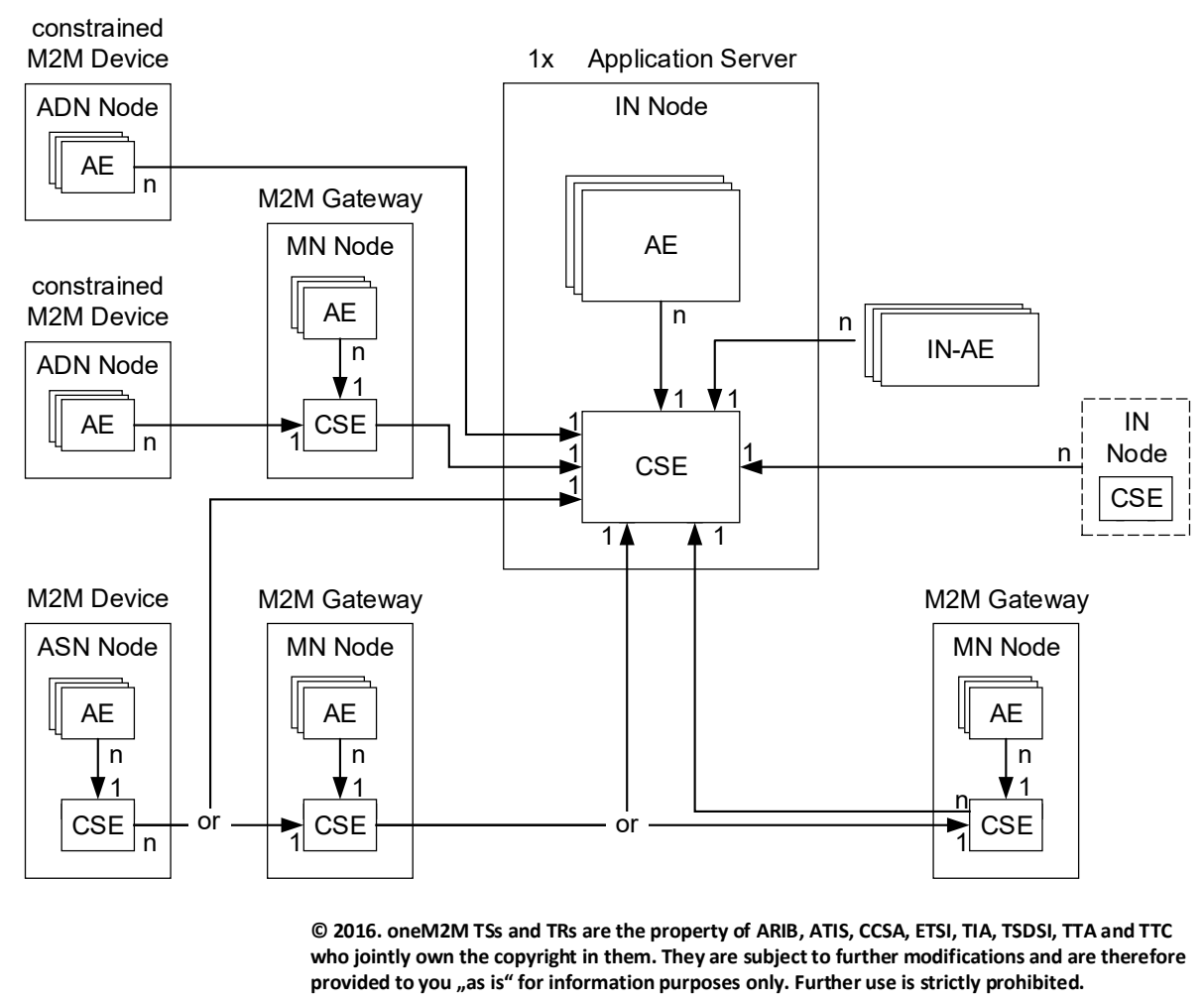

Figure 3.7: Possibilities for Node Interconnections acc. (oneM2M TS-0001-V2.10.0, 2016)

ADN-Nodes can register either to an IN-Node or to a MN-Node. An ADN-Node can exclusively register to a single IN-Node or MN-Node. ASN-Nodes can also directly register to an IN-Node or to a MN-Node. A single ASN-Node can exclusively register to a single MN-Node or IN-Node. MN-Nodes can register with other MN-Nodes or with IN-Nodes. The registration also can be exclusively to a single MN-Node or an IN-Node. The IN-Node can register to IN-Nodes of other M2M SPs.

The registration of a node (registree) at another node (registrar) enables the registrar node using the M2M common services provided by the registree node and vice versa. Additionally, the registration of an $\mathrm{AE}$ at a CSE (on same or remote node) enables the 
AE using the M2M common services provided by that CSE and vice versa. The connections of two CSEs enable both CSEs to consume the M2M common services reachable by both CSEs (oneM2M TS-0001-V2.10.0, 2016). Figure 3.8 illustrates exemplarily the set of addressable M2M common services by two registered nodes (MNCSE C and MN-CSE D).

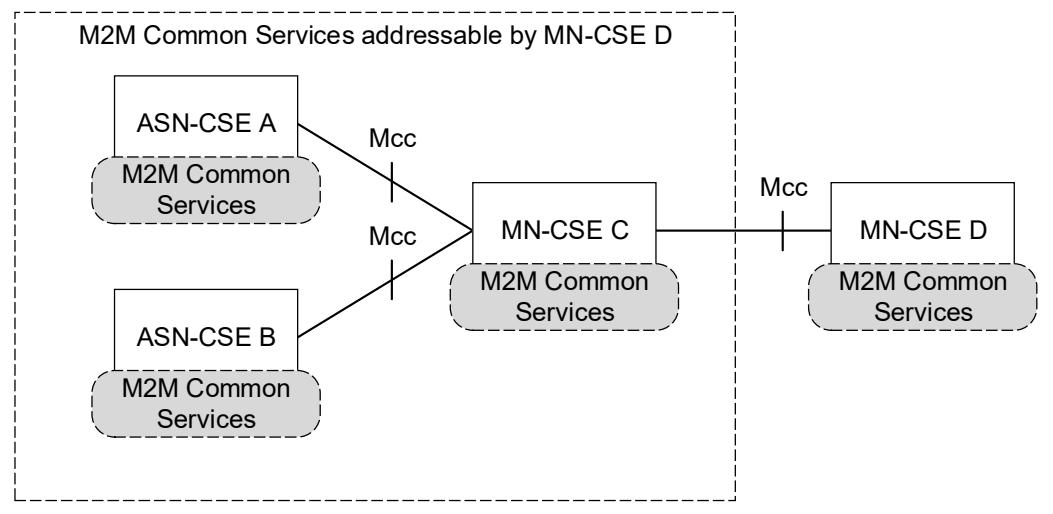

Figure 3.8: Scope of addressable M2M Common Services by registered CSEs

(oneM2M TS-0001-V2.10.0, 2016) specifies several restrictions for the possibilities to connect MN-Nodes (i.e. M2M gateways). It specifies that a MN-Node can only support one uni-directional registration. I.e. a MN-Node can only register at one other MN-Node. Furthermore, at an MN-Node only one other MN-Node can register. Figure 3.9 illustrates some valid connection of MN-Nodes.

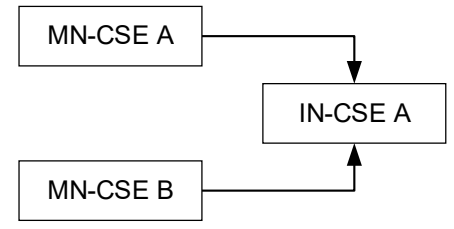

a)

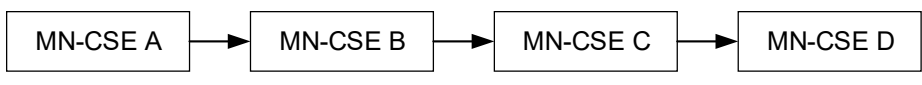

b)

Figure 3.9: Valid MN-Node Registrations

Multiple MN-CSEs can register at a single IN-CSE (Figure 3.9a). This enables the MN-

Nodes A and B to consume the M2M Common Services provided by IN-Node A and vice 
versa. Figure $3.9 \mathrm{~b}$ illustrates "a concatenation (registration chain) of multiple unidirectional registrations" (oneM2M TS-0001-V2.10.0, 2016). Here each MN-Node has maximum registered at one other $\mathrm{MN}-\mathrm{Node}$ and has maximum one other $\mathrm{MN}-\mathrm{Node}$ that is registered to the MN-Node itself. According Figure 3.8 e.g. MN-CSE D can use the M2M Common Services provided by MN-CSE A, MN-CSE B or MN-CSE C. Figure 3.10 illustrates some invalid connection of MN-Nodes as specified in (oneM2M TS-0001V2.10.0, 2016).

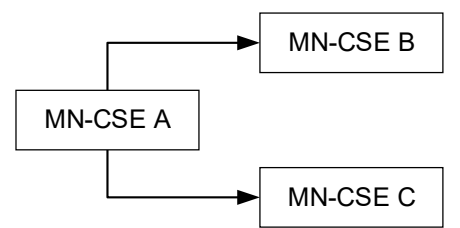

a)

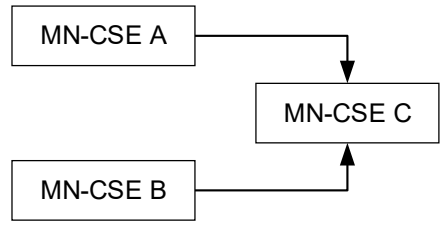

c)

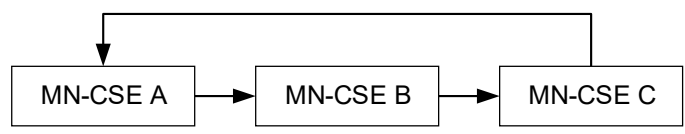

b)

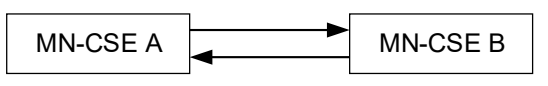

d)

Figure 3.10: Invalid MN-Node Registrations

According to (oneM2M TS-0001-V2.10.0, 2016) a MN-CSE can only register at one other MN-CSE. MN-CSE A therefore cannot register at two other MN-Nodes (Figure 3.10a). The connection of MN-Nodes by e.g. a registration chain (Figure 3.10b and d) are not allowed to form a loop. Additionally, it is not possible to register more than one MNCSE at the same other MN-CSE (Figure 3.10c).

Especially the restrictions for connection of MNs to other entities in an M2M system highlight why it is necessary to have an IN available in the architecture of an M2M system specified by oneM2M. 
For realisation of an $\mathrm{M} 2 \mathrm{M}$ application service, integrating several $\mathrm{M} 2 \mathrm{M}$ devices for controlling and monitoring, following two approaches exist:

1. All integrated $\mathrm{M} 2 \mathrm{M}$ devices are registered at a central $\mathrm{IN}$ inside the infrastructure domain of the M2M SP (directly or using an M2M gateway as proxy, as illustrated in Figure 3.6) and a centralised AE located at the IN or outside the IN coordinates the application process (i.e. message exchange between M2M devices) or

2. the M2M application service logic is distributed to the MNs inside the field domain, which are connected uni-directional by a registration chain as illustrated in Figure 3.9b.

Realisation according the first approach requires the existence of a central network element (IN), which is located in the infrastructure domain. This results in several disadvantages, such as this central entity is a single point of failure, a complex centralised infrastructure domain is required, and system user are dependent of a single M2M SP.

Realisation according the second approach in principle seems possible without necessity of a central IN, but limits the flexibility of the M2M system because MNs are only allowed to connect to one other MN. As a consequence other MNs cannot be connected to the $\mathrm{MN}$ and therefore not use the functionality provided by a specific M2M Gateway or devices connected to the M2M Gateway.

The following section describes the utilisation of M2M common services by different nodes after successful registration of the nodes. According (oneM2M TS-0001-V2.10.0, 2016) the utilisation of $\mathrm{M} 2 \mathrm{M}$ common services is based upon information exchange between the provider and the consumer, respectively CSEs and AEs, via the defined 
reference points. The information exchange is realised by request and response messages as illustrated in Figure 3.11. Permission to reproduce Figure 3.11 has been granted by ETSI.

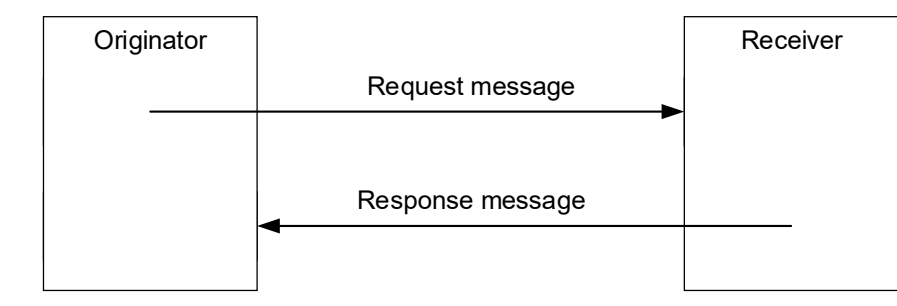

(c) 2016. oneM2M TSs and TRs are the property of ARIB, ATIS, CCSA, ETSI, TIA, TSDSI, TTA and TTC who jointly own the copyright in them. They are subject to further modifications and are therefore provided to you ",as is" for information purposes only. Further use is strictly prohibited.

Figure 3.11: Principle of Information Exchange between Entities acc. (oneM2M TS-0001-V2.10.0, 2016)

According to (oneM2M TS-0001-V2.10.0, 2016) and (oneM2M TR-0025-V1.0.0, 2016) entities that are interested in a specific information provided by another entity, request that information via a request message. The requested entity responses the requested information via a response message to the requesting entity. This kind of communication occurs between AEs and CSEs as well as between CSEs. Inside an M2M system, everything is considered as a resource, identifiable uniquely by a URI and consumed or provided by an entity. Controlling of a resource is done in the same way as requesting information using CRUD (Create Retrieve Update Delete) operations.

The above-specified registrations of nodes are related to a single M2M SP domain and the intra-domain interconnection of the nodes. This kind of interconnection therefore is only realisable with existence of a central M2M SP that obtains an infrastructure domain. Without a central M2M SP the realisation could only be possible if end-users appear in the role of an M2M ASP hosting an individual IN. For addressing a resource located in the domain of another M2M SP, it is required to interconnect the domains of both M2M 
SPs via their INs. The interconnection of the M2M SPs and the routing of messages is realised including the public domains of the M2M SPs. Precondition is that the domains of the SPs are reachable via a public DNS server and the IN registers the "public domain names of the CSEs" at the DNS (oneM2M TS-0001-V2.10.0, 2016).

The disadvantage of this addressing mechanism is that the interconnection of domains is only possible if INs and additionally registered public domains exist. Both are usually not common in the application environment of end-users and demonstrate again that the scope of the defined architecture for M2M systems is limited to the business application field and not to that of end-user environments.

According to (oneM2M TS-0004-V-2014-08, 2014) the approach for request and control resources contains an abstraction mechanism for information exchange between entities and follows the RESTful architecture principles (Fielding, 2000; Bayer, 2002). The message exchange occurs via Primitives specified in (oneM2M TS-0004-V-2014-08, 2014) that are messages exchanged in the service layer. Figure 3.12 illustrates this kind of information transmission. Permission to reproduce Figure 3.12 has been granted by ETSI.

For transport of the messages from one entity to another entity, the primitive is mapped to application layer communication protocol such as HTTP (IETF RFC 2068, 1997), CoAP (IETF RFC 7252, 2014), WebSocket Protocol (IETF RFC 6455, 2011) or MQTT (OASIS mqtt-v3.1.1, 2014). Transport layer protocol (UDP/TCP) with underlying IP network is used to transmit the messages between the entities. In case of information exchange between a CSE and a NSE across the Mcn reference point appropriate Mcn protocols are used. Through the abstraction of the communication inside the 
application/service layer from communication in transport layer, the Primitives are transport protocol independent.

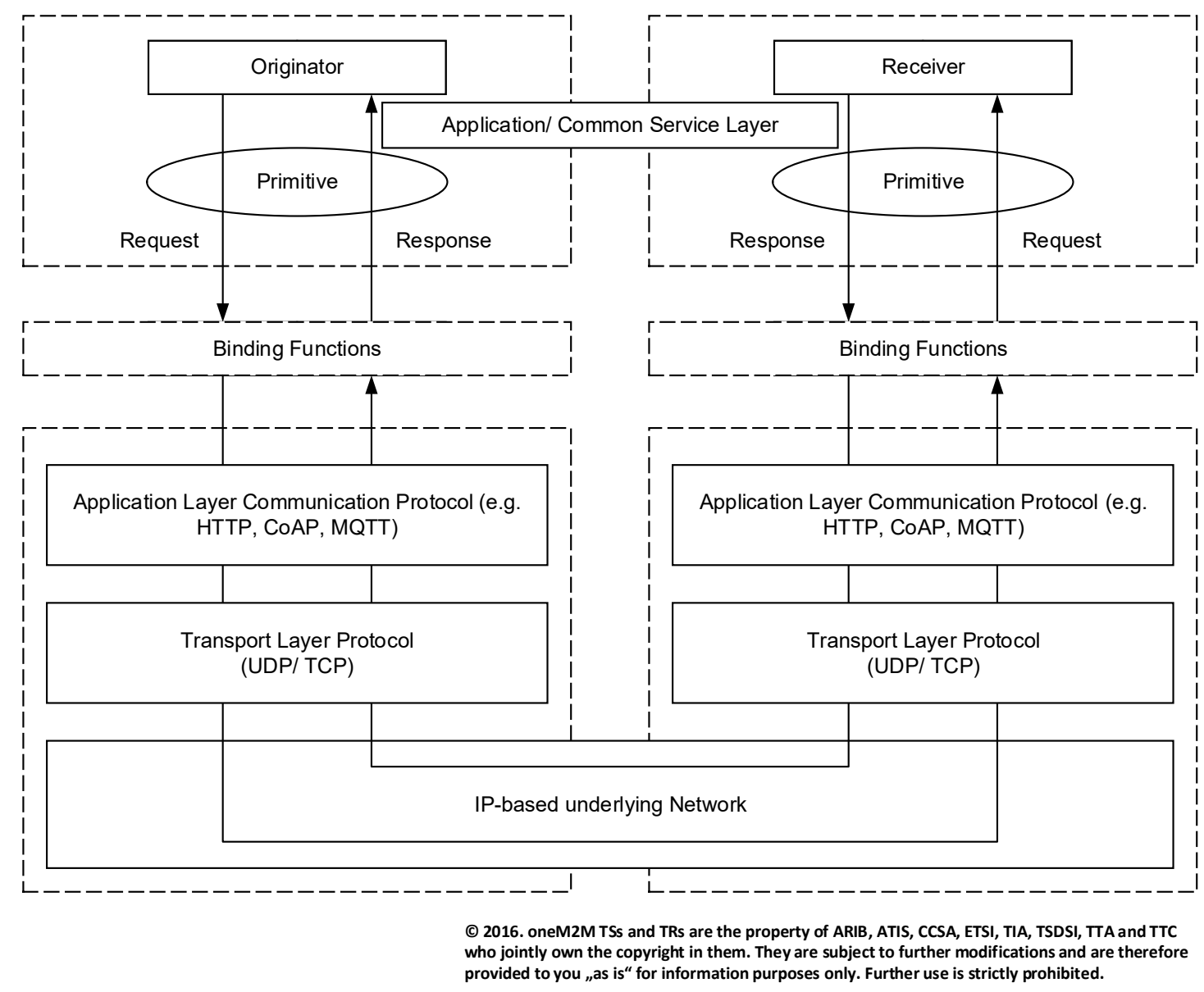

Figure 3.12: Primitive Overview acc. (oneM2M TS-0004-V2.7.1, 2016)

Benefit of the oneM2M systems architecture is that the purpose of oneM2M is to share infrastructure and network elements across multiple M2M applications. This is beneficial with regards to the MSP because sharing infrastructure elements has various advantages, such as reducing the costs for platform provision (e.g. for maintenance or operation) or enable the application provider to focus on the business aspects of their applications without regard of basic functionalities such as $\mathrm{M} 2 \mathrm{M}$ device communication or accounting. The concept of a central MSP has the advantage that communication among 
applications components, M2M devices as well as the structure of M2M application services is unified. This enables the interoperability of different M2M application services and M2M devices. Especially the protocol translation functionality of nodes to interoperate between different M2M protocols, support the integration of multiple M2M devices in different M2M applications.

Next to the advantages presented above, the MSP architecture of oneM2M contains several disadvantages presented in the following. First disadvantage is that oneM2M focuses on the interoperation of industries and businesses and the support of complex business processes but not the integration of end-user environment or application provision by end-users. OneM2M does not specify the M2M Application Architecture and limits its specifications to the MSP architecture. Despite the advantages of a common service layer regarding the shared functionalities, this platform needs a complex setup of the environment with additional network elements (physical or virtual servers) and endto-end communication technologies. It does not reuse commonly existing communication technologies and network elements for realisation of the MSP. The M2M ASP are dependent of a single MSP provider. Furthermore, the M2M application service itself is designed and realised by a central M2M ASP. Through this, the consumer of an M2M application service is dependent of the M2M ASP. The M2M system as defined by oneM2M includes the $\mathrm{IN}$ as a single centralised element in an oneM2M system architecture. The IN is e.g. required for $\mathrm{M} 2 \mathrm{M}$ application provision and interconnection of different M2M SP domains. Required central elements in an architecture usually have negative aspects because realisation of continuous availability and maintenance result in large costs for the provider. Furthermore, all other participants in the M2M system are dependent of the central IN. By considering the OM2M project (Monteil and Alaya, 2014; 
OM2M, 2016), a specific implementation of the oneM2M architecture, it becomes clear that not only the design and operation of an oneM2M system requires expert knowledge, but also creating an application. Because the required expert knowledge development of oneM2M compliant applications and the provision in a corresponding oneM2M architecture is not feasible by end-users.

\subsubsection{INOX Managed Service Platform}

The INOX Managed Service Platform (Clayman and Galis, 2011) specifies "a M2M service architecture" (Kim et al., 2014). The INOX platform has been designed to avoid silo applications that coexist next to each other providing monitoring/control functionality using sensors and smart objects. According to (Amaral et al., 2015) INOX is a platform that provides a "M2M service architecture for Io $\mathrm{T}$ " including functionality to register and discover things as well as object virtualisation and capabilities for orchestration to control and manage services.

The purpose of the INOX architecture and infrastructure is to integrate the smart objects/things with the "common services and management architectural model" (Clayman and Galis, 2011). INOX provides a framework to combine the smart objects "for higher-level processing" (Clayman and Galis, 2011). The INOX platform focusses intensively on the service approach and combination of different sensor networks inside the platform (Savaglio and Fortino, 2015).

According to (Clayman and Galis, 2011) and (Savaglio and Fortino, 2015) the INOX Platform contains the following three layers. Figure 3.13 displays these layers including the platform components contained in them. 
- Service Layer - The Service Layer contains the Services and Applications connecting through Service APIs to elements in Platform Layer.

- Platform Layer - The Platform Layer provides the functionality for management and orchestration to deploy Services and Applications as well as virtualises the Servers, Smart Objects and Things located in the Hardware Layer.

- Hardware Layer - The physical devices (e.g. sensors and servers) and devices (routers) providing networking functionality over the internet via IP-based communication protocols are located in the Hardware Layer. The functionality for hosting services and virtual machines is also provided by the servers in the Hardware Layer.

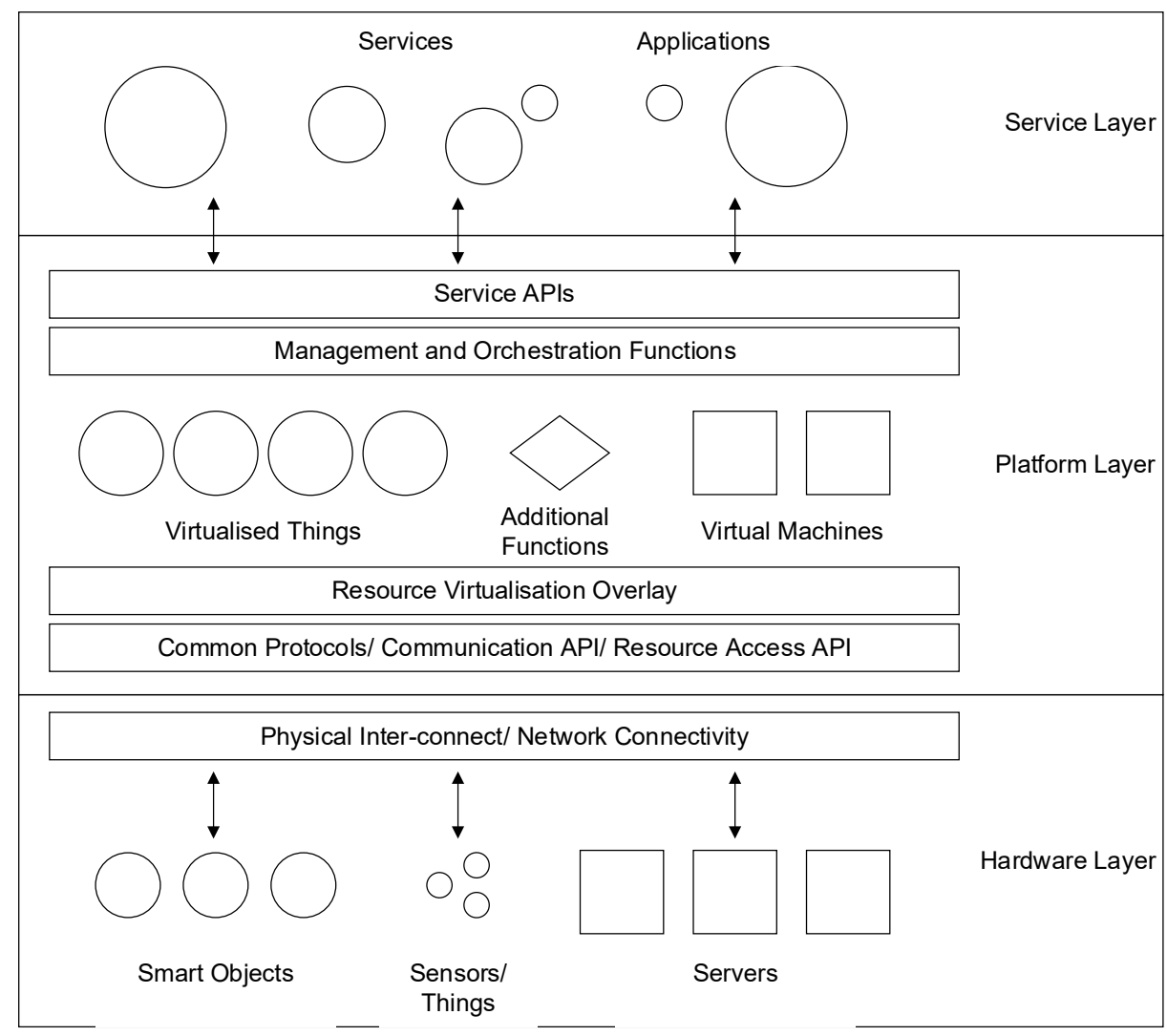

Figure 3.13: INOX Managed Service Platform acc. (Clayman and Galis, 2011) 
The physical devices are located in the same local environments or distributed across different locations. An application provided by the INOX platform should be able to use every device or service provided by the devices. According to (Clayman and Galis, 2011) it does not matter if the devices that are used and managed by services are located in different domains because INOX platform provides capabilities to connect them.

Derived from (Clayman and Galis, 2011) the Platform Layer forms the service platform middleware and contains the main functional elements of the INOX platform. According to (Clayman and Galis, 2011) this middleware contains a Resource Virtualization Overlay representing all resources of the Hardware Layer as virtual resources and links both, virtual and physical resources. The virtualisation overlay provides APIs for manipulation of resources abstracted from the real resources (virtual resources) or physical resources (without virtualisation). The abstraction of the devices isolates the devices in the Hardware Layer and enables upper layers (services, end-user application) using devices without considering implementation specific device interfaces.

The Management and Orchestration Functions element in the Platform Layer can be specified as a kind of resource scheduler which is according to (Clayman and Galis, 2011) responsible to allocate portions of virtual resources to given tasks. It provides capabilities for controlling and management of services located in various domains.

The Common Protocols/Communication API/Resource Access API element in the INOX platform provides the physical interfaces to the devices in Hardware Layer. This element contains the communication protocol stacks to interact with the specific devices (Clayman and Galis, 2011). 
The Service APIs element provides according to (Clayman and Galis, 2011) the interfaces to the INOX service platform that are used by the user services. The Service APIs form interfaces to integrate functionality of smart objects into user services. These interfaces enables smart objects to interact via the internet and to query/update information or behaviour of objects.

In addition to the functionality provided by the platform elements above, (Clayman and Galis, 2011) specifies additional functions of the INOX platform like function for registration and discovery of smart objects by utilisation of e.g. device specific identifiers or location. The INOX platform supports a large amount of devices and services because scalability is a requirement provided by the INOX platform.

(Clayman and Galis, 2011) does not describe how to build applications or the architecture of applications provided by the INOX platform but defines that the platform provides service applications automatically by processing a manifest specifying the service elements. This leads to the assumption that applications are defined in a declarative way.

(Clayman and Galis, 2011) does not specify the execution environment of the INOX platform but mentions that the platform "has the functionality of a service cloud $[\ldots]$ and the ability to run shared applications" as well as the INOX project is "working towards an IoT cloud environment". This leads to the assumption that the presented platform is provided as a (logically) centralised cloud-based solution or is executed in a physically centralised server environment.

The benefits of the INOX service platform for M2M applications is that it provides mechanisms to integrate distributed things and smart objects as well as existing services 
into complex applications. The presented approach of the architecture enables avoiding previous existing implementations of applications that are statically bound to the corresponding smart objects (silo-applications). According to (Clayman and Galis, 2011) previously applications have been realised that were statically connected to the sensors or smart objects. The approach presented in the INOX project defines a dynamic architecture that enables sharing of resources between different applications (Clayman and Galis, 2011). The mechanism integrated in the INOX platform for device lookup enables the applications to address (search and discover) the devices without regard of the specific device location or contact address. Providing a service-based infrastructure enables a unified mechanism for integration and combination of M2M devices as well as other resources located in the Internet into applications based on common service principles. The introduced virtualisation layer enables the communication between devices and applications independent of the applied communication protocol and realises the inter-networking of different M2M protocols and networks (Clayman and Galis, 2011). The seamless integration of different M2M device technologies enlarges the flexibility of services and applications because the higher number of usable devices. The abstraction of the devices from the implementing M2M technology enables the use of the devices without considering implementation specific technologies and permit focussing on core application aspects instead of technology aspects for realisation. Device abstraction additionally enables the portability of services and application regardless of the M2M technology of the integrated devices. The declarative way to describe applications instead of statically and execution system specific implementation of application logic supports the independence of application logic from the execution 
environment and enables to move applications from one execution system to a different one without adapting the application logic description.

Besides the advantages of INOX platform, the presented approach also contains several disadvantages. As the INOX architecture forms a cloud-based centralised integration platform of smart objects and because the functionality provided by the platform layer for orchestration to deploy services and application, the user of the platform (SC) is dependent of both, the central platform provider (for application development and platform operation) and the central platform components (for service and application execution). Centralised platform infrastructures require many resources for service provision (operating costs for hardware and availability as well as service maintenance or costs for service development). The large costs for operating the platform are transferred to the consumer, which in turn enlarges the costs for service utilisation. Additionally, central infrastructures for storing and calculating data by a single entity always is critical regarding data safety and end-users privacy. (Clayman and Galis, 2011) mentions end-user applications which can be considered as applications that are consumed by end-users and not provided by end-users. It can therefore be concluded that the end-user is no active stakeholder in the context of application creation and also not able to provide service based on end-users individual personal environment. Therefore end-users also cannot cooperate with each other to combine their local M2M resources and provide their functionality as a cooperative M2M service. 


\subsubsection{M2M Platform Project based on SOA (M2M on SOA)}

(Zhang et al., 2010) presents an M2M platform for bridging business applications and M2M devices using Java 2 Enterprise Edition (J2EE) framework and service oriented architecture (SOA) concept (Kim et al., 2014). The proposed M2M platform aims to integrate business applications and M2M devices using Web Service (WS) technology, which is able to reduce dependencies of system parts and can react on changes in short terms (Kim et al., 2014; Zhang et al., 2010).

(Zhang et al., 2010) identifies as deficit of traditional M2M solutions (illustrated in Figure 3.14a) that several M2M devices are connected to "different business applications" and the necessary interfaces are implemented multiple times providing the same functionality. According to (Zhang et al., 2010) in that architectural approach, all devices are connected to the applications that integrate the devices. The interfaces to the devices are created newly for each connection and are rarely reused.

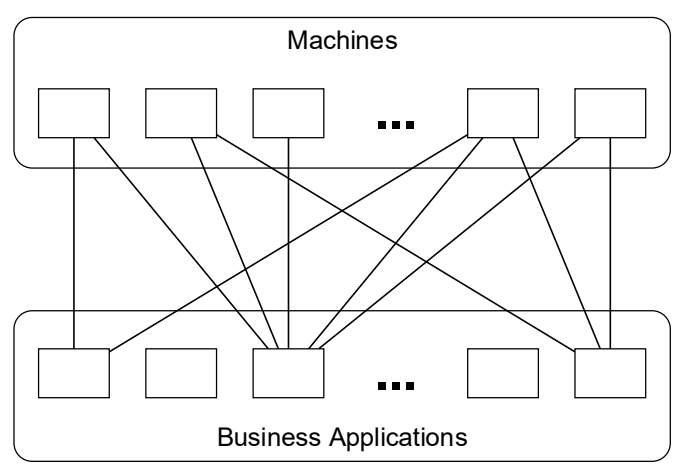

a) Traditional M2M Network Topology

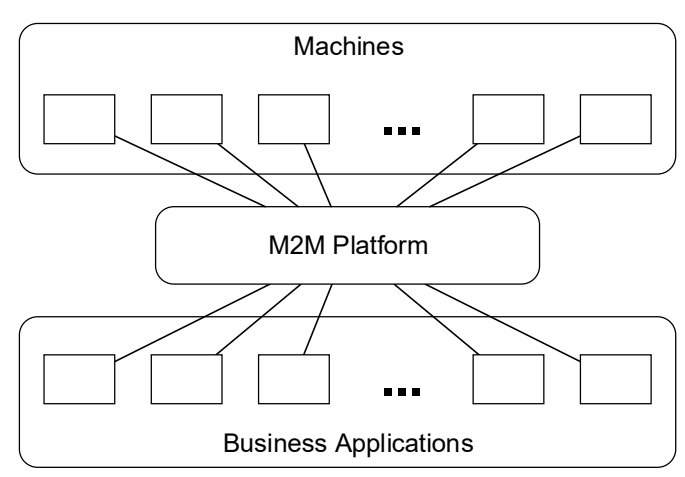

b) Proposed M2M Network Topology

Figure 3.14: M2M Network Topologies (traditional and proposed) acc. (Zhang et al., 2010)

Therefore (Zhang et al., 2010) proposes a platform architecture that has a larger reusability of interfaces. For this they proposes that a "bridge platform" is used as M2M 
platform (illustrated in Figure 3.14b) that connects applications and devices in "startopology”. (Zhang et al., 2010) proposes an M2M platform as service-oriented and distributed system that has a loosely-coupled and multi-layer architecture.

For integration of the M2M Platform with the devices and business applications (Zhang et al., 2010) proposes a SOA (OASIS SOA-RM-V1.0, 2006) implementation based on WS and a Message Broker because it enables realisation of applications as encapsulated services that are accessible via the internet. The participants in such a system structure includes SP deploying and maintaining services, service broker responsible for registration and location of services, and service requestor consuming services (Zhang et al., 2010).

(Zhang et al., 2010) separates an M2M system in four parts: M2M platform, devices, business applications, and access devices. Figure 3.15 illustrates the parts of the platform as well as the internal components.

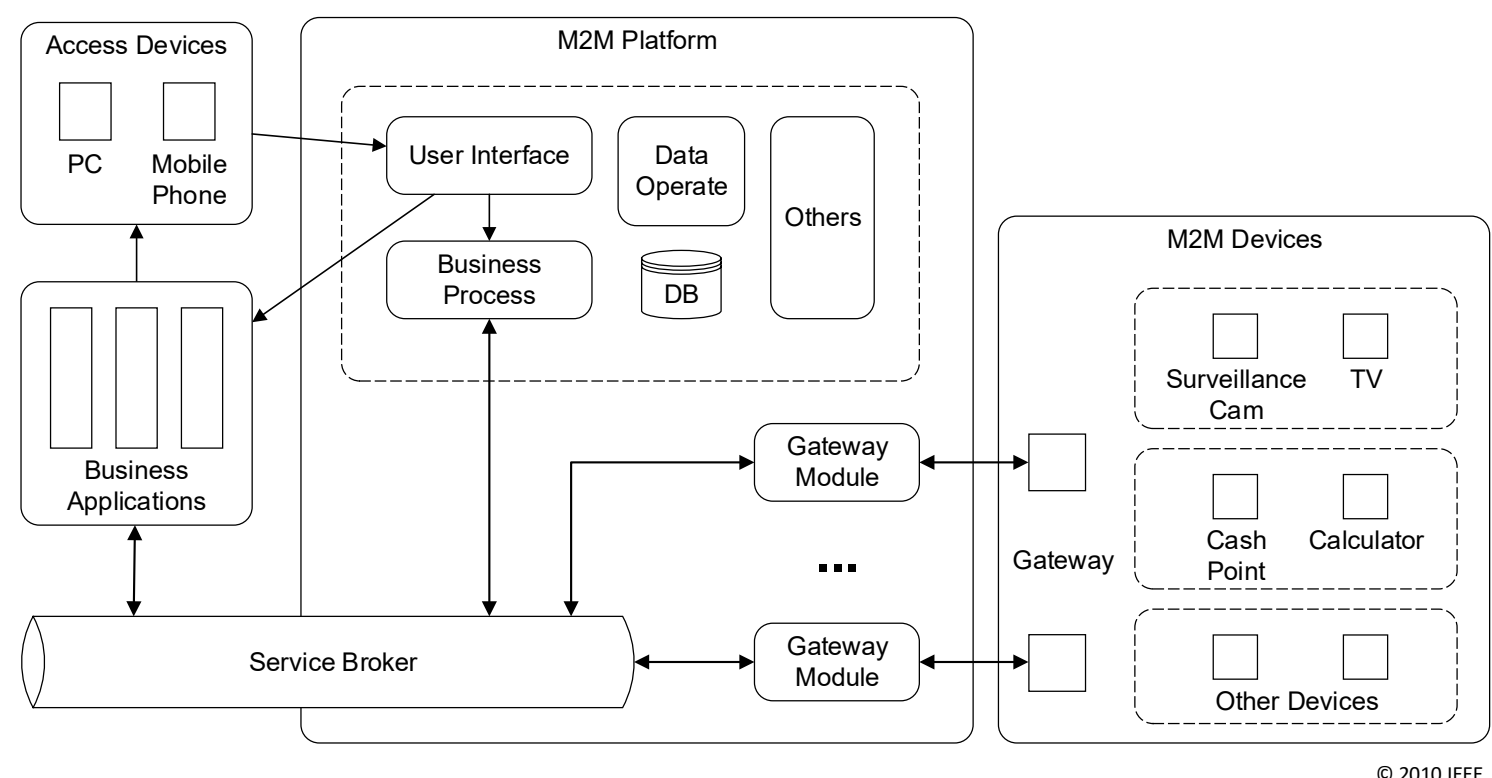

Figure 3.15: M2M Platform Architecture acc. (Zhang et al., 2010) 
In the proposed architecture all parts of the M2M system are separated from each other (i.e. are executed on separated locations). The M2M devices (e.g. surveillance cameras or TVs) are connected to the M2M platform via M2M gateways. The gateways are located at the place where the M2M devices reside. The M2M gateways connect to the M2M platform via gateway module components of the M2M platform. Business applications and user interface hardware are also located outside of the M2M platform.

According to (Zhang et al., 2010) the user utilises an end-device (e.g. PC or mobile phone) to access the business application by sending a request to the M2M platform (or directly to the business application). The operation request is analysed by the application system, which connects to the Webservice provided by M2M platform. The M2M platform afterwards requests the WS that the M2M gateway provides to trigger operation at the device or request information from it.

As mentioned above, the M2M system proposed by (Zhang et al., 2010) is focused on services, especially WS. All parts of the M2M system are connected via WS. The service broker element of the $\mathrm{M} 2 \mathrm{M}$ platform provides message routing functionality as well as functionality for transformation of message format to exchange information between the WS provided by the system elements (Zhang et al., 2010).

The M2M platform provides the core functionality of the M2M system including a User Interface for interaction with users (e.g. to display users' information data). It also includes internal elements for management of e.g. users, application or M2M devices as well as functionality for sms/email notification and provides functionality for several business needs (e.g. payment functionality). For internal data storage and operation, the M2M platform includes a database system (Zhang et al., 2010). 
The architecture of an M2M system as presented by (Zhang et al., 2010) has the following advantages. The bridge functionality of the platform reduces the dependence of business applications and M2M devices. Additionally, the SOA-based approach provides the benefit that the (physical) dependence of applications, application users, the M2M platform as well as the M2M devices is reduced. The SOA principles as well as the included asynchronous service/message broker makes the applications and the other components of the M2M system loosely coupled which enables the M2M system to quickly response to change request in the runtime system (Zhang et al., 2010). The presented approach includes the abstraction of communication between device specific technologies and the platform internal system and application components. This enables to realise applications without consideration of the specific details of different M2M device technologies. The M2M system of (Zhang et al., 2010) provides a graphical interface for the end-user, which enables the user to interact with the M2M system using commonly available hardware. Additionally, provision of commonly available communication functionality using email and SMS notification functionality support the information exchange with the user.

Besides the advantages presented above, the approach of (Zhang et al., 2010) contains several drawbacks. It focuses exclusively on business applications, which are according to (ITIL V3.1.24, 2007) and (ISO IEC 20000-1:2011, 2013) provided by single centralised providers. (Zhang et al., 2010) proposes specifically the realisation of an M2M system with a single encapsulated M2M platform in the focus that is located in the public network. This creates a dependency of the platform provider as well as limits the extension of platform functionality, which requires detailed knowledge about and access to the M2M platform. The approach considers the end-user as a simple user of the M2M 
system but does not integrate the end-user in the application design. Implementation of business applications using the frameworks Struts (Apache Struts, 2017), Spring (Spring, 2017), Hibernate (Hibernate, 2017) as proposed by (Zhang et al., 2010) as well as design principles of SOA requires expert knowledge to design and implement M2M services or applications. This especially would make the integration of end-users into application development impossible.

\subsubsection{BOSP Business Operation Support Platform}

(Xiaocong and Jidong, 2010) presents an architecture of "a business operation support platform for M2M" (Kim et al., 2014). The presented business operation support platform (BOSP) focuses on the support of carriers (Kim et al., 2014; Xiaocong and Jidong, 2010).

(Xiaocong and Jidong, 2010) proposes that carriers operate a BOSP according to architecture presented subsequently. Smart devices provide functionalities (e.g. video, phone or sound recording) that can be used by carriers to provide "precise services". Additionally (Xiaocong and Jidong, 2010) proposes that carriers provide their abilities like voice and data communication for external usage. Furthermore, the carriers have large potential for creating "new abilities by processing the data" that they store, analyse and integrate from various industries.

The proposed M2M architecture of (Xiaocong and Jidong, 2010), illustrated in Figure 3.16 contains four layer. The Perception Layer contains the various sensor networks, M2M terminals or other equipment like mobile phones, and provides the functionality for collecting object information. These devices are connected via gateways to the access networks and the internet, which both reside in Transportation Layer. M2M applications 
reside in the Application Layer and are provided by external ASP that use the functionalities/data information provided by the carrier. The defined BOSP resides inside the operation supporting layer (Xiaocong and Jidong, 2010).

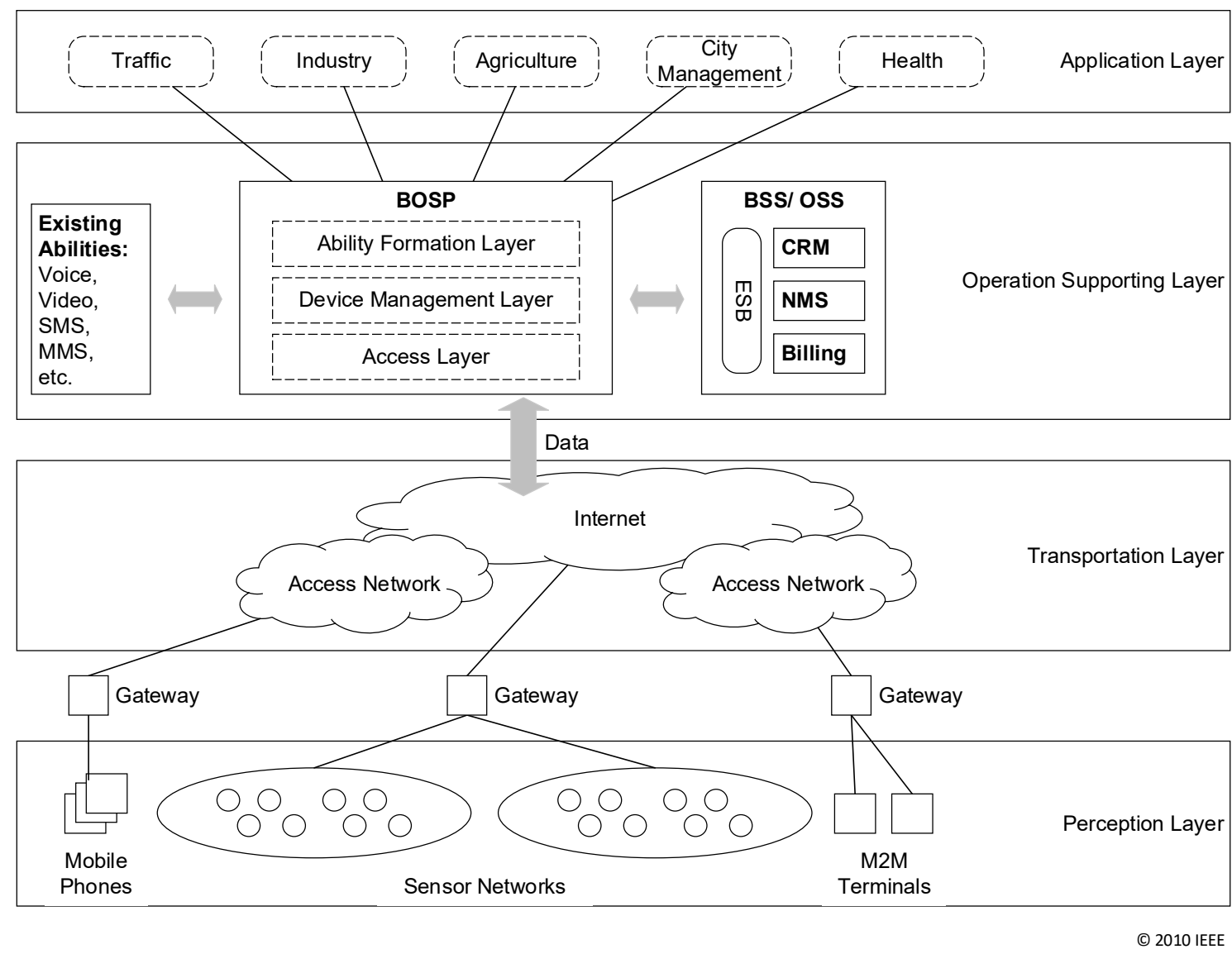

Figure 3.16: M2M System Architecture acc. (Xiaocong and Jidong, 2010)

The BOSP according to (Xiaocong and Jidong, 2010) is separated in three layers:

- Access Layer - The Access Layer provides protocol adaptation and access control functions. It translates messages, which are sent to the BOSP in proprietary protocols into standard protocols for internal processing and vice versa.

- Device Management Layer - The Device Management Layer manages the devices deployed by the carrier and offers a management portal for monitor/control/check 
devices or performing software updates. The Management portal forms the interface to device vendor for device status feedback, carrier staff for status monitoring and control of "operating services and associated devices", and administrator for device monitoring and account supervision.

- Ability Formation Layer - The Ability Formation Layer provides communication functionalities like voice/video or data transportation and messaging (SMS, MMS). Additionally, it provides information of devices like geographical position, temperature, movement, energy consumption.

The BOSP according to (Xiaocong and Jidong, 2010) contains four interfaces for access. The Data Interface receiving IP packets via heterogeneous networks (e.g. NGN), Application Interface forwarding the data to the applications, Existing Abilities Interface used for communication with the BOSP to provide the abilities of carriers' network to the applications using Application Interface. The Existing BSS/OSS Interface can connect various systems already operated by the carrier like customer relationship management system (CRM), billing system, network management system (NMS) via the Enterprise Service Bus (ESB) of the carrier (Xiaocong and Jidong, 2010).

The architecture of an M2M system according to (Xiaocong and Jidong, 2010) with a dedicated BOSP provided and operated by the carrier has various advantages. Especially the provision of carriers' abilities like Voice over IP (VoIP) communication and messaging functionality via mobile networks is advantageous to form an interface to M2M system users. Integration of VoIP communication and messaging functionality in M2M systems offers the benefit that it can be applied for communication with end-user using commonly existing hardware like PC, Smart Phone or fixed telephone that all 
usually exist in end-users environment. Additionally, the functionality of device/protocol abstraction integrated in the BOSP according to (Xiaocong and Jidong, 2010) enlarges the flexibility for connecting various M2M communication technologies to the platform without consideration of technology specific aspects in application provision.

Next to the advantages presented above, (Xiaocong and Jidong, 2010) contains several disadvantages. First disadvantage is that it focusses only on carriers. It does not consider the integration of the end-user in system or application design. (Xiaocong and Jidong, 2010) announces benefits by using its proposed BOSP for industries like "government, agriculture, military, manufacturing business, construction business" to make them more intelligent, but does not promise advantages for the end-user. The end-user interfaces of the applications have to be provided by the M2M ASP, which can use the application interfaces of BOSP to integrate the abilities of the M2M devices and communication functionality. According to (Xiaocong and Jidong, 2010) "carriers are abundant with information of things because they monopolize perception devices and communication tunnels". This in turn is a big disadvantage because the ASP as well as the end-user are dependent of a single monopolist. Additionally storing and calculating data by a single entity always is critical regarding data safety and end-users privacy. The threatening "BigBrother effect" becomes clear by looking at the provider's goals presented in (Xiaocong and Jidong, 2010). According to (Xiaocong and Jidong, 2010) the smart devices and the travellers can be identified. For example by the "abilities offered by carriers, readers in the doors of the trains and busses will enable an accurate tracking of every connection and route of every traveller". Applying standard protocols for M2M technology abstraction is advantageous because it supports the interoperability and independence of 
applications, but (Xiaocong and Jidong, 2010) does not give hints regarding the applied/standardised protocols of BOSP.

\subsubsection{IMS enabled M2M Service Platform (IMS M2M SP I)}

(Foschini et al., 2011) presents the architecture of an M2M system based on IP Multimedia Subsystem (IMS) technologies (Magedanz and de Gouveia, 2006; Poikselkä and Mayer, 2009). The authors present its work with the help of a case study for realisation of a retractable bollard management system (Kim et al., 2014; Foschini et al., 2011). The presentation of the case study as well as the system elements explanation has been used to derive the architecture of an M2M system according to the principles of (Foschini et al., 2011).

The M2M system introduced by (Foschini et al., 2011) is based on seamless integration of M2M devices (i.e. M2M area network) into the IMS of a specific provider. In particular, (Foschini et al., 2011) proposes to reuse existing network technologies and the services provided by the access network provider or other possibly existing wireless network infrastructures (e.g. Wi-Fi) for the development and provision of M2M application services (Foschini et al., 2011).

The M2M system specified by (Foschini et al., 2011), illustrated in Figure 3.17, is a distributed architecture and separated into three domains: M2M Device Domain, Network Domain, and Application Domain. The M2M devices reside in the M2M Device Domain. The Network Domain represents the access network infrastructure and consists of IMS components as specified in (Trick and Weber, 2015). These components are used to 
realise the communication of $\mathrm{M} 2 \mathrm{M}$ devices with the M2M servers that are located in the Application Domain (Foschini et al., 2011).

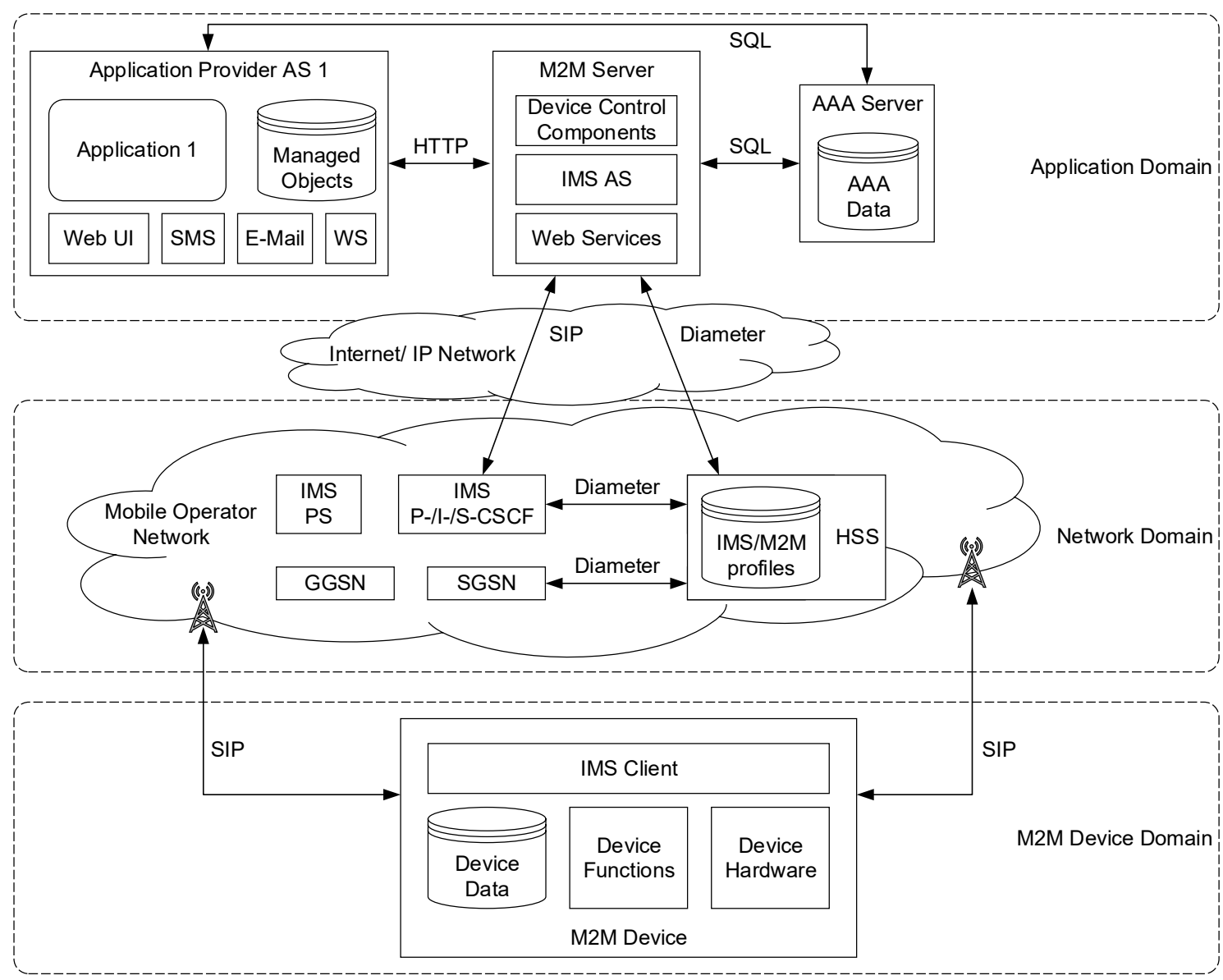

(C) 2011 IEEE

Figure 3.17: IMS-enabled M2M System Architecture acc. (Foschini et al., 2011)

The core component of the M2M system is according to (Foschini et al., 2011) the M2M server, residing in the Application Domain. The M2M server interacts with M2M devices and other elements/systems required for application service provision (authorisation, authentication, accounting server storing information required for system usage or application server for M2M application execution) via the IMS. A dedicated application server of the M2M SP executes the M2M application. 
The application server element communicates with the M2M server via "RESTful interactions" that are controlled by the WS element in the AS. Additionally, the application server element provides possibilities for communication (notification of application user) via SMS or email adopting the communication services of the IMS (Foschini et al., 2011).

The coordination of information exchange between M2M server and M2M devices via IMS using SIP messages as well as adopting IMS services (e.g. for sending SMS) is coordinated by the IMS AS element of the M2M server. The information exchange between M2M server and M2M devices is based on the Publish/Notify Principle (IETF RFC 3903, 2004). For realisation of information exchange both, M2M server and M2M devices subscribe for the presence status of each other at the IMS presence server (IMS PS). For sending a specific information from $\mathrm{M} 2 \mathrm{M}$ device to $\mathrm{M} 2 \mathrm{M}$ server and vice versa, they publish this information to the IMS PS. The IMS PS notifies M2M server or M2M device about that information by sending a notify message (Foschini et al., 2011).

For realisation of device specific functionality in the M2M devices, the M2M devices contain corresponding function elements. To persist information in the M2M device, it contains an element for data storage. Next to the device specific functions an M2M device requires a component to realise the communication functionality via IMS. For this the M2M devices host an IMS client (Foschini et al., 2011).

The M2M application user (end-user) can interact with the M2M application service via "a web-based user interface" (Web UI element), provided by the AS of the application provider, to monitor the status of the M2M application or M2M devices (Foschini et al., 
2011). The presented case study does not consider controlling the devices but it can be assumed, that the same user interface is used for device controlling.

The architecture of an M2M system as presented by (Foschini et al., 2011) has the following advantages. The presented approach utilises already deployed communication technologies and networks (IMS) for realisation of an M2M system (Foschini et al., 2011). Reusing existing network technologies and communication services benefits in that no extra communication networks need to be established. Reusing existing communication system avoids additional costs for establishing a communication network. Additionally the M2M system provider can focus on its competence related to M2M and application service provision without providing and maintaining the communication network it requires.

Besides the advantages presented above, the approach of (Foschini et al., 2011) contains the following drawbacks. A single central provider provides M2M server and other system components required for realisation of an M2M application (application provider AS and AAA server, see Figure 3.17) as well as M2M application itself. According the presented approach, the end-user not only is dependent of the M2M system provider, but also is dependent of the IMS provider whose network elements are seamless integrated in the M2M system. Particularly the presented approach requires that the M2M ASP have technical access to the network elements of IMS provider (e.g. HSS). This is a rather unrealistic scenario, unless the IMS provider itself is the M2M ASP. Furthermore, the presented approach envisages that network elements of the IMS provider can be used (IMS PS for Information exchange). Although the utilisation of the IMS is beneficial for message transport, it can be assumed that functionality of the network elements itself is 
unavailable for external SP. The presented approach again does not consider the end-user as M2M ASP.

\subsubsection{M2M horizontal Services Platform Implementation over IP Multimedia Subsystem (IMS M2M SP II)}

(Padilla et al., 2013) introduces a horizontal MSP (illustrated in Figure 3.18) applying IP Multimedia Subsystem (IMS) network elements (Magedanz and de Gouveia, 2006; Poikselkä and Mayer, 2009). The aim of the presented research project according to (Padilla et al., 2013) is to connect M2M devices and M2M application server (AS) through the IMS core network to develop a horizontal MSP. The reason of (Padilla et al., 2013) to propose using the IMS is because its independency from the access network.

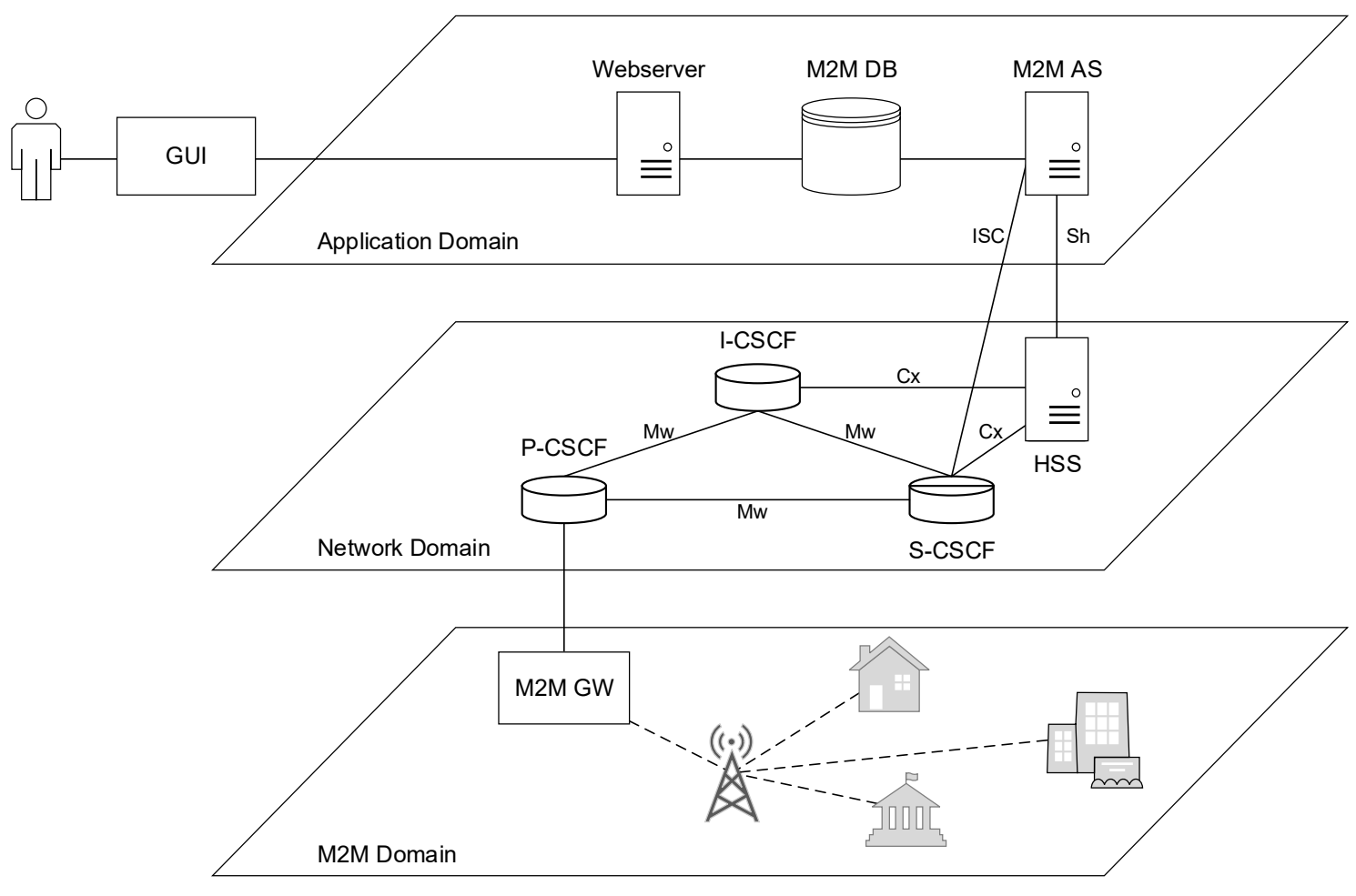

(c) 2013 IEEE

Figure 3.18: M2M horizontal Service Platform acc. (Padilla et al., 2013) 
(Padilla et al., 2013) separates the platform architecture into the layers described subsequently:

- M2M Domain - The M2M devices are located in the M2M Domain, which enables the M2M devices to communicate with each other for exchanging information and connect to public network.

- Network Domain - The Network Domain represents the IMS and is connected with the M2M Domain via an M2M gateway (M2M GW). The functionality of the M2M GW is to provide protocol translation functionality from protocols used in the M2M Domain to protocols used in the IMS, which is usually Session Initiation Protocol (SIP) (IETF RFC 3261, 2002).

- Application Domain - In the Application Domain the M2M application server (AS) resides providing the functionality to store M2M GW related data and characteristics of devices connected to it. Additionally M2M AS receives the data sent by the M2M devices. The Application Domain also contains a webserver providing the data stored in the database (M2M DB) by the M2M AS, to the customer via a webpage. The webserver processes the data stored in the M2M DB according the business model of the customers.

According the concept defined by (Padilla et al., 2013) the M2M devices send the data generated by them to the M2M AS. For this the M2M GW registers the M2M devices to the M2M AS. Figure 3.19 shows the process for registration of M2M devices at the M2M AS. For clarification of the registration process, subsequently the network elements P-/I/S-CSCF and HSS of the IMS are introduced briefly according to (Magedanz and de Gouveia, 2006; 3GPP TS 23.228 v5.15.0, 2006). 
- Proxy-Call Session Control Function (P-CSCF) - The P-CSCF forms the gateway to the IMS. All signalling messages send from or to the IMS passes the P-CSCF.

- Interrogating-Call Session Control Function (I-CSCF) - The I-CSCF provides functionality to determine the S-CSCF for a specific user and creates charging records.

- Serving-Call Session Control Function (S-CSCF) - The S-CSCF is responsible for session control and user registration. Every user of the IMS have to register to the S-CSCF for using the IMS. Additionally, S-CSCF detects and integrates Application Server (AS), such as the M2M AS to provide a requested service.

- Home Subscriber Server (HSS) - The HSS is a database storing user related information, such as user location and user policy information.

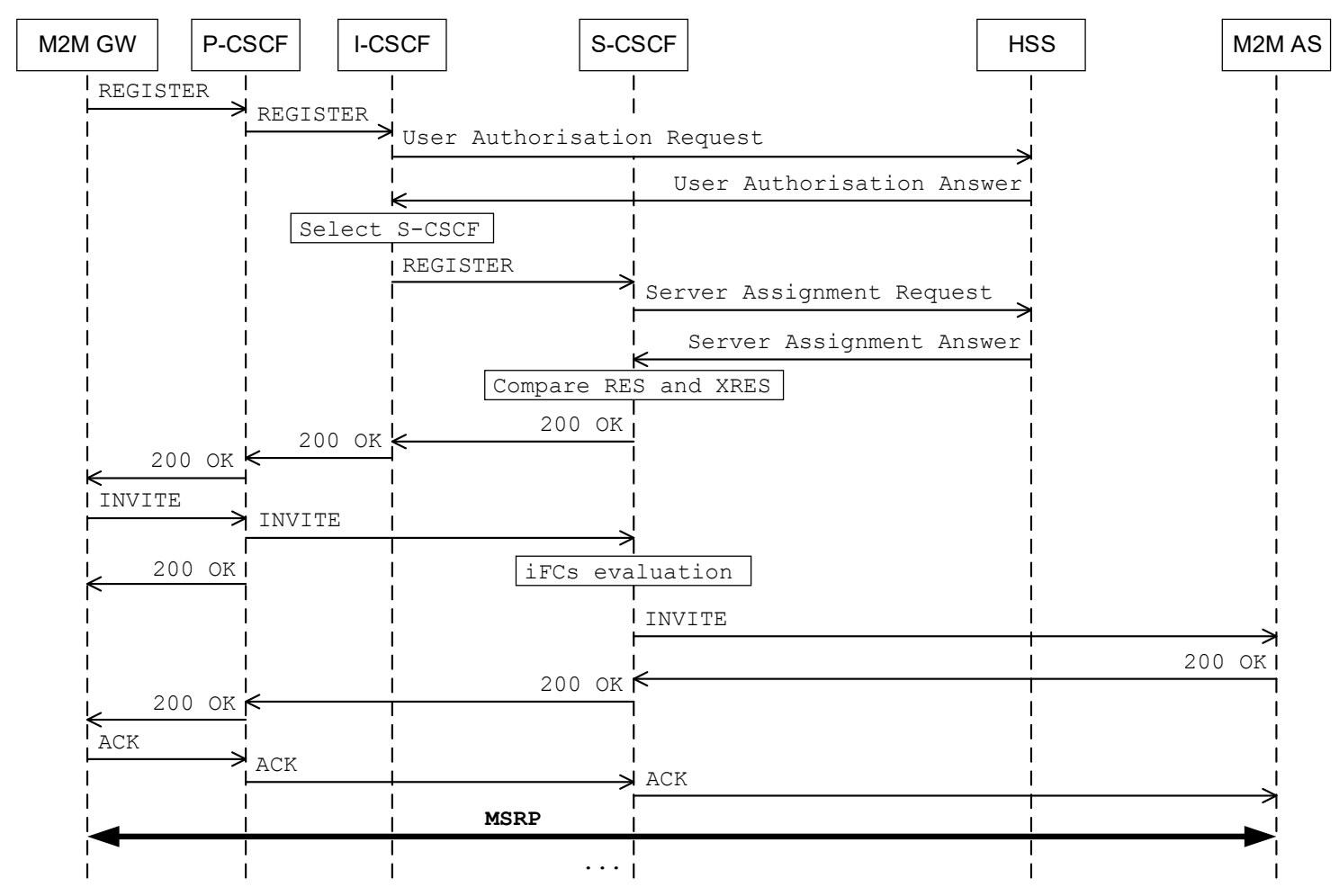

Figure 3.19: M2M Device Registration Process (Padilla et al., 2013) 
Initially the M2M GW registers itself at the network domain. For doing this the M2M GW sends a register request to the P-CSCF using SIP REGISTER method (IETF RFC 3261, 2002). The P-CSCF forwards the request to the I-CSCF. The I-CSCF requests the corresponding S-CSCF for the specific M2M GW at the HSS. After receiving the response of the HSS, the I-CSCF forwards the register request to the S-CSCF specified in the response of the HSS After successful registration of the M2M GW at the Network Domain the M2M GW establishes a session with the M2M AS. The session establishment is done by sending a SIP INVITE (IETF RFC 3261, 2002) message to the M2M AS. This SIP INVITE message contains a Session Description Protocol (SDP) message (IETF RFC 4566, 2006) that specifies to use Message Session Relay Protocol (MSRP) (IETF RFC 4975, 2007) for information exchange between M2M AS and M2M GW, respectively the M2M devices (Padilla et al., 2013).

It can be derived from (Padilla et al., 2013) that after initial session establishment the $\mathrm{M} 2 \mathrm{M}$ GW requests a list of M2M devices from the M2M AS that are connected to the M2M GW and which should send its information data to the M2M AS. M2M GW and M2M AS exchange this information via MSRP messages. After receiving the list of M2M devices, the M2M GW establishes a MSRP session between each of the M2M devices and the AS. For this, the M2M GW sends a SIP re-INVITE request (IETF RFC 3261, 2002) and (IETF RFC 6141, 2011) to the M2M AS including SDP with specification of the MSRP URIs of the specific M2M devices. This MSRP URIs include the devicespecific session-IDs which enables both, M2M GW and M2M AS to assign the MSRP messages to the M2M devices within the same SIP session. After linking the M2M devices to the M2M AS, the M2M devices start to transmit their information data to the 
M2M AS which can be monitored by the user via the GUI provided by the webserver (Padilla et al., 2013).

The architecture of an M2M system as presented by (Padilla et al., 2013) has the following advantages. The presented approach enables the integration of the M2M devices located in end-user environments and is based on the IMS. This is advantageous because existing communication network infrastructure and protocols are utilised to exchange information between the M2M devices and the MSP. This enables the IMS provider to seamless integrate M2M systems in its infrastructure and provide the data generated by the M2M devices as a service to the user. Reusing existing communication network technologies benefits in that no extra communication networks need to be established which results in avoiding additional costs for operation and maintenance.

Besides the advantages, the approach of (Padilla et al., 2013) has several drawbacks as described in the following. The approach only specifies that the M2M GW translates between protocols in M2M Domain and those in Network Domain but does not specify the protocol translation between protocols inside the M2M Domain. Interoperability of M2M device technologies is required to integrate those devices in the M2M platform and to use their functionality in the M2M services. The architecture of (Padilla et al., 2013) contains several centralised system entities in the Application Domain and in the Network Domain (e.g. the M2M AS). The MSP is dependent of these central entities, which limits the scalability as well as availability of the MSP. Additionally because a central IMS provider is integrated, the user of the MSP is dependent of this provider. Additionally, the data storage and processing according customers' business model is done by the web server as a centralised element in platform architecture, which might by critical for the 
end-user data security and privacy aspects. The functionality of the MSP as introduced by (Padilla et al., 2013) is limited to monitoring the data, produced by the M2M devices. The authors do not describe additional functionalities, such as control of the M2M devices or realisation of control applications.

\subsection{7 e-DSON}

(Kim et al., 2012) introduced the enhanced Dynamic Service Overlay Network (e-DSON) platform project that has its focus on distributed service provision. (Kim et al., 2014) identified the e-DSON platform as "a distributed M2M service platform architecture using a service overlay network".

According to (Kim et al., 2012) service overlay networks are used to create "a logical topology on a physical network" to provide adaptable services and applications. In a service overlay network, the features of a service are programmable because the service overlay network is located at application level. The overlay consist of distributed nodes, each providing controllable services and data delivery. A SP can link the nodes to compose an individual service.

According to (Kim et al., 2012) the e-DSON platform is based on the DSON platform introduced in (Kim et al., 2009), (Kim et al., 2010), and (Kim et al., 2011). The DSON platform is according to (Kim et al., 2012) a "service overlay network framework" that is based on management of service information and the control of service entities, to realise the desired application logic. The DSON platform provides the functionality to compose user-centric distributed multimedia services. Figure 3.21 shows an exemplary scenario of 
the DSON platform in which clients in one home network B use media content (e.g. movie files) provided by a server in another home network A.

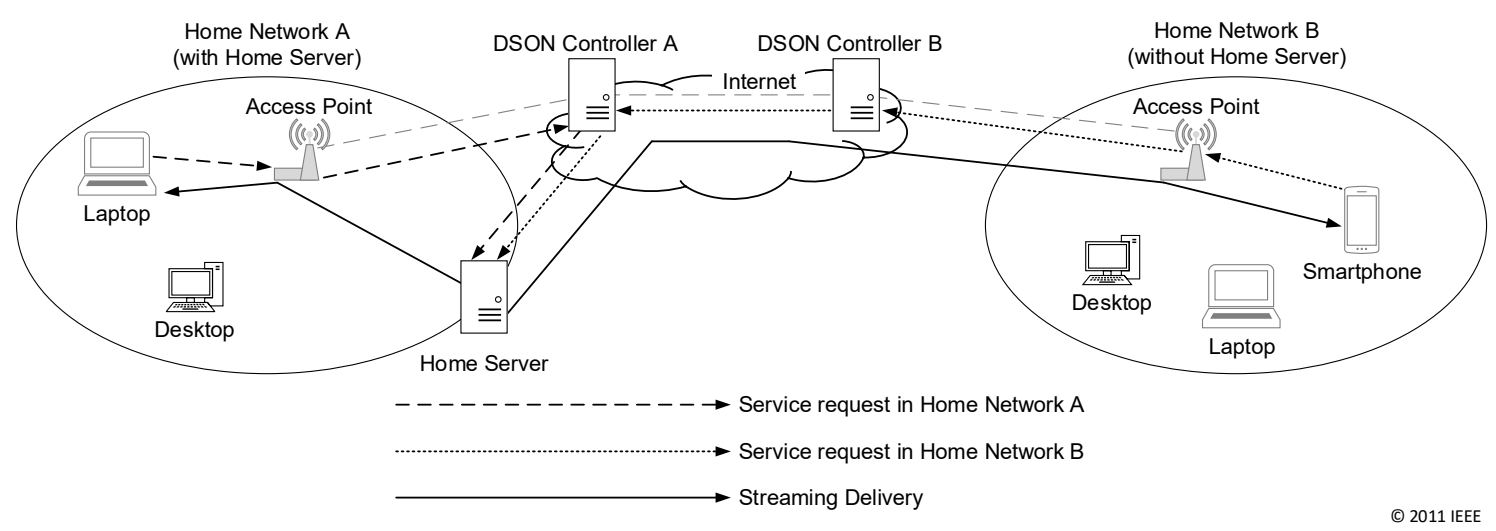

Figure 3.20: DSON Service Scenario: Home Multimedia Streaming Service acc. (Kim et al., 2011)

According to (Kim et al., 2011) the DSON platform is realised by several servers located in the Internet and operated by the DSON platform provider. The media content of the home server located in home network A is registered at DSON controller A. DSON controller A shares its content list with other DSON controller. Clients in other home networks can search for desired content by contacting an appropriate DSON controller (e.g. DSON controller B). DSON controller B is aware about the content provided by the home server located in home network A and forwards the media request to DSON controller A that again forwards the request to the home server providing the media content. Through this dynamic forwarding, the DSON platform creates a dynamic service overlay network to share content between clients located in distributed networks. According to (Kim et al., 2011), ,with DSON, all the service information is stored in DSON" and "all content and data are managed and controlled by DSON".

According to (Kim et al., 2012) the DSON platform is restricted to multimedia service and does not support sensor environments. Because this aspect, (Kim et al., 2012) 
developed the e-DSON platform with the aim to apply the DSON platform concept to M2M environments. (Kim et al., 2012) identifies that the user is primary stakeholder consuming M2M services and it is important that the M2M platform used for service provision satisfies the requirements of the end-user. (Kim et al., 2012) defines several requirements, such as interoperability or communication efficiency for an MSP to realise M2M application services based on information exchange between services, M2M devices and the user. Additionally, (Kim et al., 2012) defines that MSPs have to provide functions, such as composition and service management or location management and M2M services must support many different technologies to exchange service messages between M2M devices. Figure 3.21 shows the platform model of the proposed e-DSON platform.

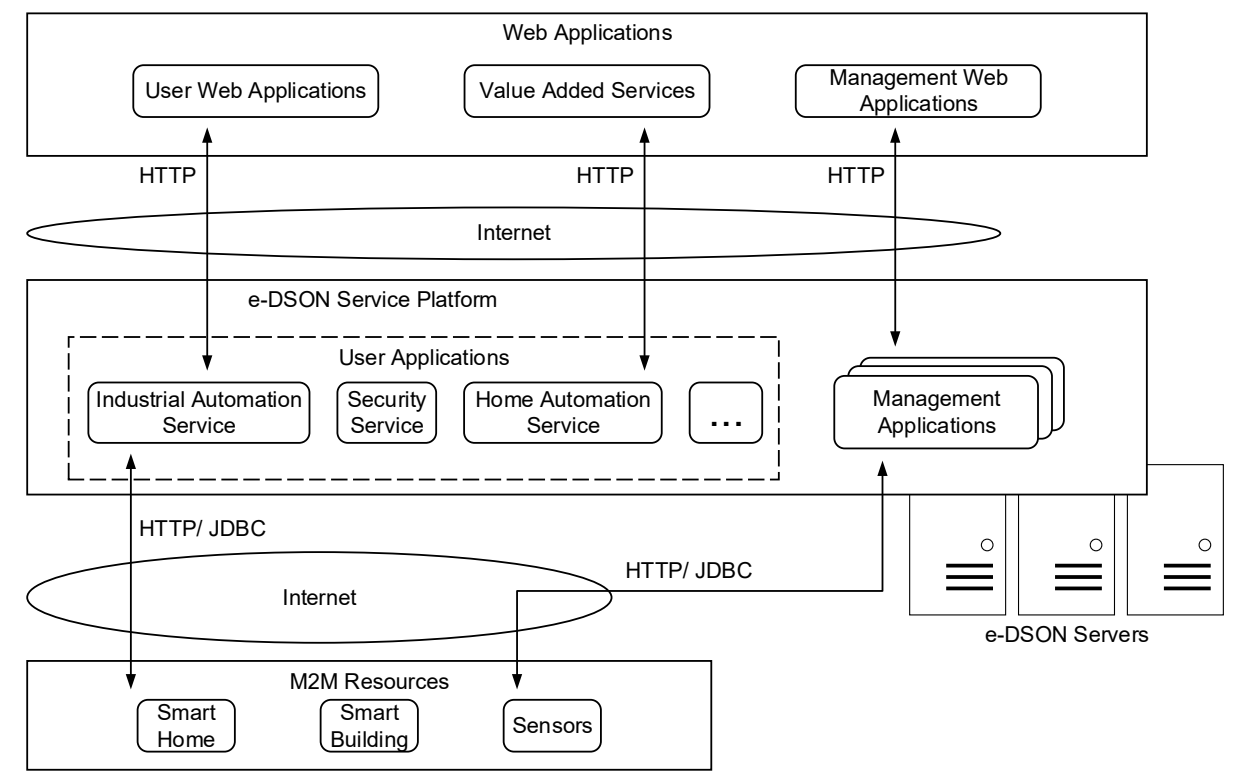

Figure 3.21: e-DSON Platform Model acc. (Kim et al., 2012)

As described above, the e-DSON platform is based on the DSON platform. The DSON platform has been extended by (Kim et al., 2012) by providing not only the information about media streaming content in the platform (i.e. registering this information in several 
DSON controllers), but also the information about M2M devices or local services (e.g. residing in smart buildings). The registered $\mathrm{M} 2 \mathrm{M}$ devices and services can now be combined by linking them via a service overlay network. This makes it possible to offer applications that are specific to the different requirements of the user (Kim et al., 2012).

The user interface is realised via a web application. The user can access the M2M services provided by the platform via the user interface or the user can register devices and services. The e-DSON platform uses HTTP to exchange information from the MSP and web application because HTTP is a common protocol and available in common user environments (Kim et al., 2012).

The e-DSON platform contains services of two types: user service and management service (for things and services). Service and device providers use the management services for uploading service/device specification. A user service is the user-specific application provided by the e-DSON platform. A user-specific application is e.g., when devices trigger events, another device can be controlled by the e-DSON platform (Kim et al., 2012). Figure 3.22 illustrated details of the e-DSON platform architecture.

According to (Kim et al., 2012) the architecture of the e-DSON platform is separated in three layers: presentation layer, business layer and data access layer. The presentation layer is used to provide an interface for the user via a web-based GUI. Using this GUI the user can interact with the user application. The data access layer provides the interfaces to the M2M devices and external data sources as well as the smart servers (M2M gateway in M2M environments) to be connected to the e-DSON platform. The business layer contains the core functionality of the e-DSON platform and provides the functionality to realise user-specific applications. 


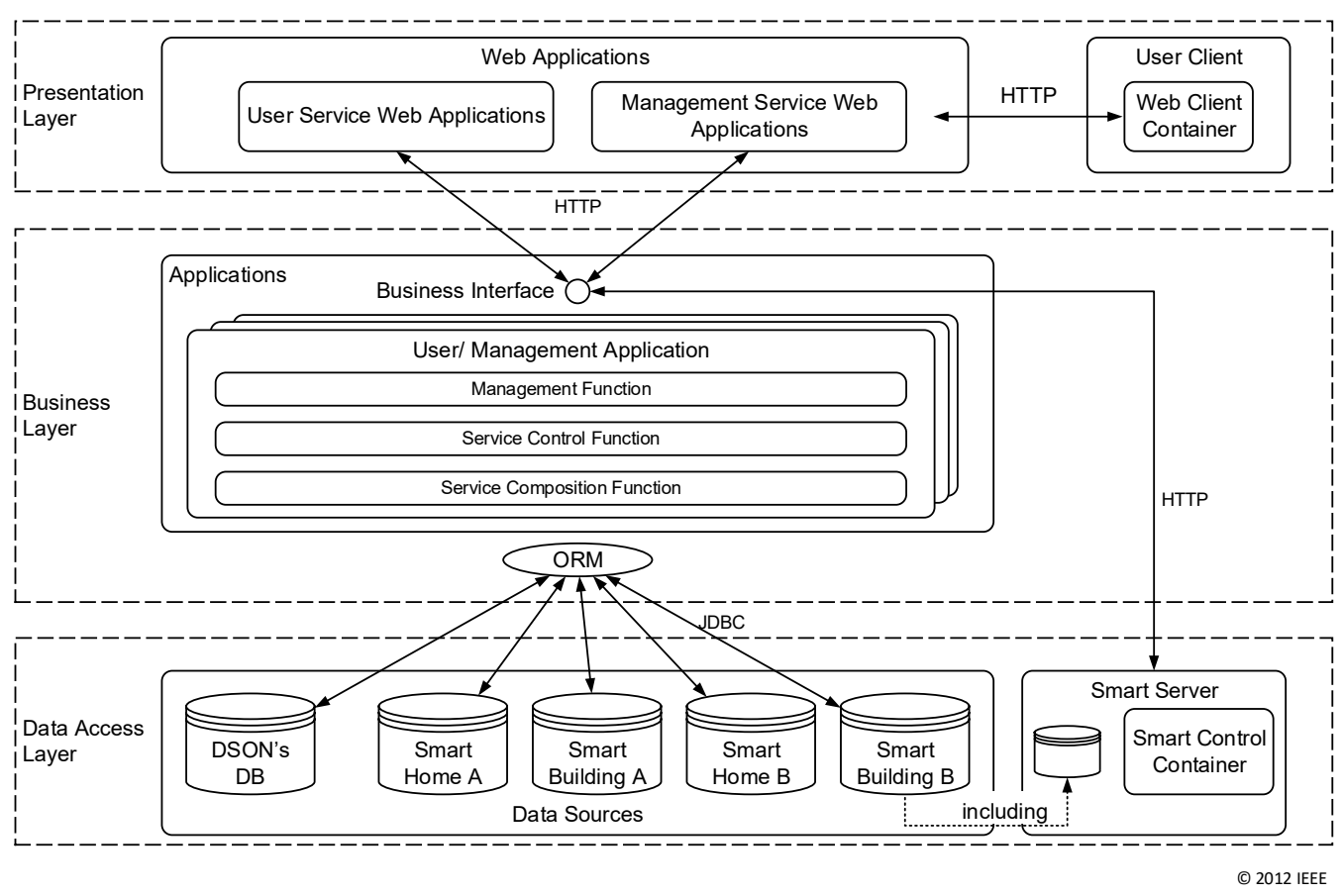

Figure 3.22: e-DSON Platform Architecture acc. (Kim et al., 2012)

The application contains as illustrated in Figure 3.22 service composition function, service control function, and management function. The service composition function creates the service overlay network based on the specified service topology, i.e. connects the distributed service endpoint with each other. The service control function provides the functionality for e.g. registration and release of services or request and control services. The management function is responsible for storage and management of device information, user profiles, location information, and service topology (i.e. specification about connection of services). Using these functions, the e-DSON platform composes the user-specific application out of several devices and services registered to the platform. The e-DSON platform supports user-centric M2M services because it can provide a specific service for each user by dynamic composition of services (Kim et al., 2012).

The e-DSON MSP as presented by (Kim et al., 2012) has various advantages as presented subsequently. The presented approach focuses on addressing the requirements of the end- 
user by providing user-centric, i.e. user individual services and integrates end-users' environment. The possibility for dynamic composition of services enlarges the flexibility of the provided M2M applications as well as the re-use of devices and services in multiple M2M applications. Especially realisation of M2M applications by service composition using a service overlay network that links several nodes, each providing resources or services, enables the flexible combination of device resources and services on service layer. Using common available technologies and interfaces to connect to the service platform and access the user applications, such as HTTP protocol, XML as data format, and web interfaces simplifies the integration in external systems and the access of the users to the M2M application services. According to (Kim et al., 2012) using overlay networks for service provision reduces the costs for system operation.

Besides the advantages of the e-DSON platform, the presented concept has several drawbacks as the following section specifies. As illustrated in Figure 3.20 and Figure 3.21 , the e-DSON platform architecture is realised by distributed, but centralised operated servers, located in the Internet. If one or more servers fail, the platform is limited or no longer available. Furthermore, the user is dependent on a central platform operator that operates the e-DSON platform. According to (Kim et al., 2012) the e-DSON platform does the service composition, but specifies that the user "is not involved in the composition". That means a separate stakeholder have to be involved defining the service composition structure which the e-DSON platform interprets and dynamically allocates. Although the e-DSON platform can be connected to external systems via a HTTP interface and the user can use the applications via a web interface, it is not described whether different M2M technologies in the smart environment can be integrated into the platform. Although the services and resources can be linked dynamically to each other 
via a service overlay network, the authors do not give any indication of how this link is made (whether formally defined or programmed). Because the application is composed by the e-DSON platform and as specified by (Kim et al., 2011) "all the service information is stored in $\mathrm{DSON}^{\text {" }}$ as well as "all content and data are managed and controlled by DSON" it can be derived that the application itself is defined statically and stored in the e-DSON platform. The defined applications are stored in the business layer of the e-DSON platform and are therefore centrally defined and coordinated. Therefore, no dynamic applications are generated, but rather statically defined applications are linked with resources (e.g. an application for streaming multimedia content is statically defined in its functionality, the source and the destination of the contents is generated dynamically). If, on the other hand, a new application is desired, a developer must first define the service topology and store this configuration in the central e-DSON platform. The e-DSON platform offers the possibility to provide specific user-based applications, but such applications as described in (Kim et al., 2014) more target to the business layer instead of targeted to private individuals.

\subsubsection{M2SP Concept}

(Kim et al., 2014) classifies and evaluates currently existing concepts for MSPs from industry and research area. Based on that research (Kim et al., 2014) proposes its optimised M2M service platform (M2SP) model.

According to (Kim et al., 2014) their proposed M2SP model, illustrated in Figure 3.23, includes all functionalities of currently existing MSPs from industry and research area. Additionally (Kim et al., 2014) extends these functionalities by P2P communication from 
SCs and M2M devices, mediated by the M2SP, to avoid the issue of other MSPs which require that all the traffic have "to go through the platform between user devices and objects".

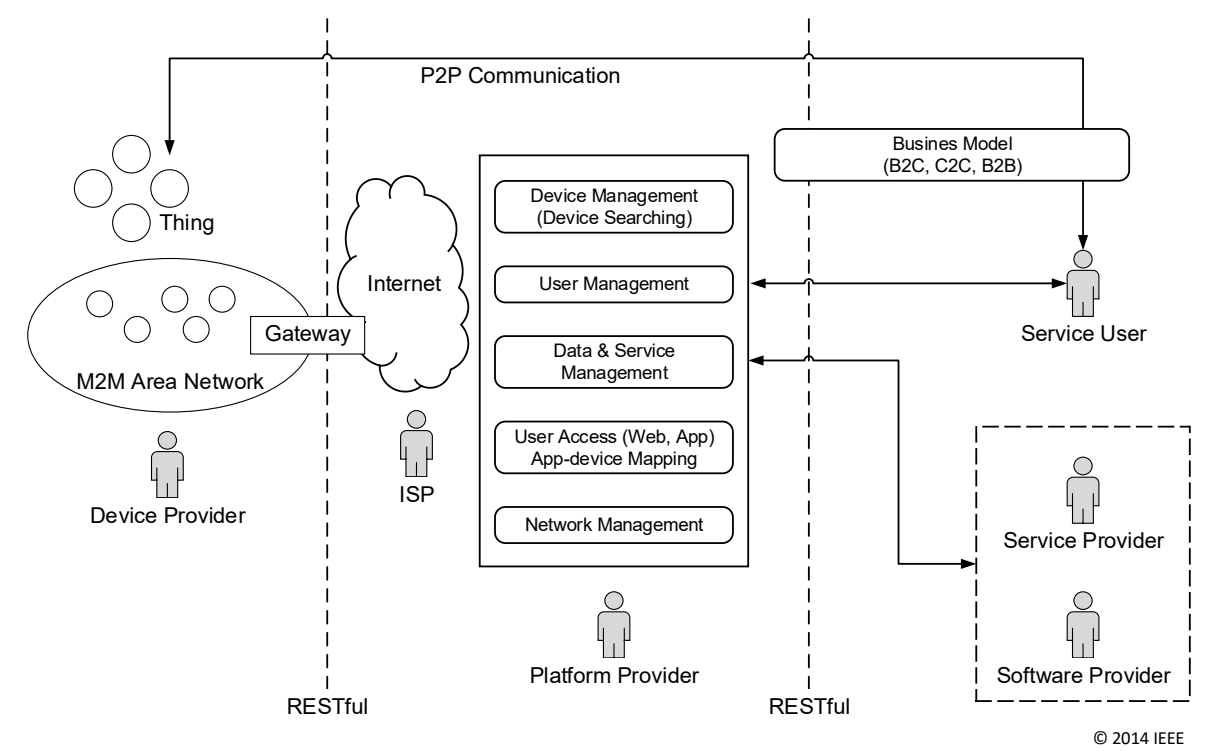

Figure 3.23: M2SP Model acc. (Kim et al., 2014)

According to (Kim et al., 2014) their M2SP model consists of M2M devices connected either directly to the Internet or are located in an M2M area network and connected via a gateway to the Internet. The Internet, respectively any IP-based network, realises the communication network to connect the M2M devices to the central M2SP. The proposed platform model includes the stakeholders: device provider, Internet Service Provider (ISP), platform provider, service users, service provider (SP), and software provider. Device providers represent the manufacturers of the M2M devices. They equip the M2M devices with the required functionality and provide them to the SP. The SP places the $\mathrm{M} 2 \mathrm{M}$ devices received by the device provider in physical areas to use them for realising specific services. The ISP provides the communication network (Internet) to link M2M devices with the M2SP as well as provide access to the M2SP for users and 
service/software providers. Software providers develop apps and web applications in cooperation with the SP to provide specific services that use the devices. The platform provider provides and operates the M2SP. Service user, SP, and software provider connect to the M2SP also via the Internet. Additionally, the service user can connect directly ( $\mathrm{P} 2 \mathrm{P})$ to the M2M devices, after mediation by the M2SP, to reduce the load of the M2SP by continuously forwarding the M2M device data (Kim et al., 2014).

Figure 3.24 shows the architecture of the M2SP and the M2M network which consists according to (Kim et al., 2014) out of M2M area/access networks and a core network. M2M area networks are heterogeneous networks implemented by different M2M technologies. M2M nodes located in the M2M area networks connect either direct or via an M2M gateway "through various access networks" to the core network. M2M devices and users "connect through the core network" and the M2SP manages M2M devices and users as well as provides M2M services.

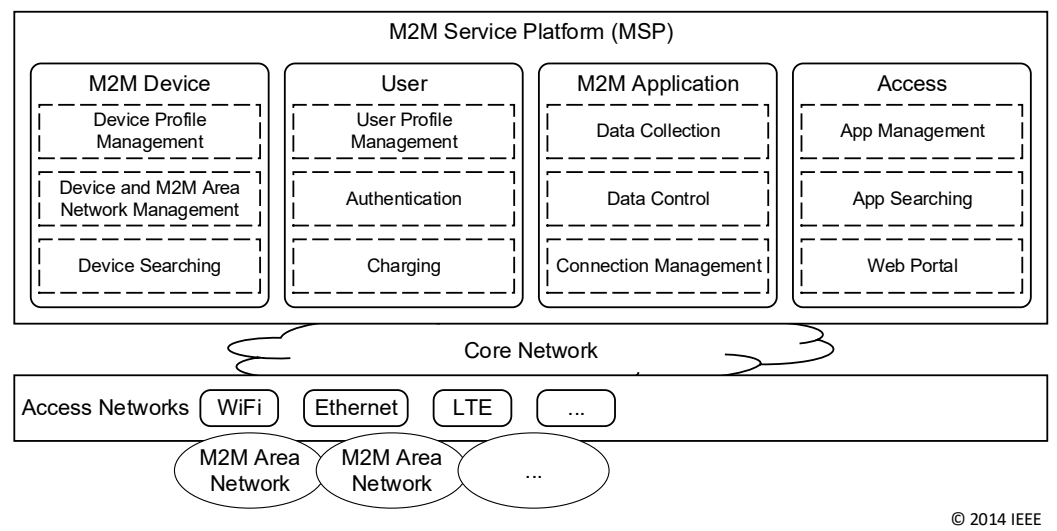

Figure 3.24: M2SP Architecture acc. (Kim et al., 2014)

The M2SP according to (Kim et al., 2014) consists of four separate platforms described subsequently that can be executed on different locations but are integrated by a single M2SP provider. 
The M2M Device platform holds a database where all M2M devices register. Services, managers, and users utilise the M2M device platform. The Device Platform provides functionality for management of device profiles (e.g. location, type, address), query devices, monitoring status of devices and controlling of devices (Kim et al., 2014).

The M2M User platform provides functionality for managing user profiles or registration of M2M service users. The M2M User platform interoperates with the M2M Device platform and manages access restriction of users to devices and services (Kim et al., 2014).

The M2M Application platform interoperates with the M2M Device platform and provides the M2M application consisting of multiple services using data collected from M2M devices (Kim et al., 2014).

The M2M Access platform provides the access for the users. Users connect with the M2M Access platform to receive the app or access the web page that both form the interface to the M2M application that is provided by the M2M Application platform or M2M devices. App developers use the app management functionality of the M2M Access platform to register their apps (Kim et al., 2014).

Figure 3.25 illustrates a use case scenario and interaction of platform components and stakeholders as presented by (Kim et al., 2014). 


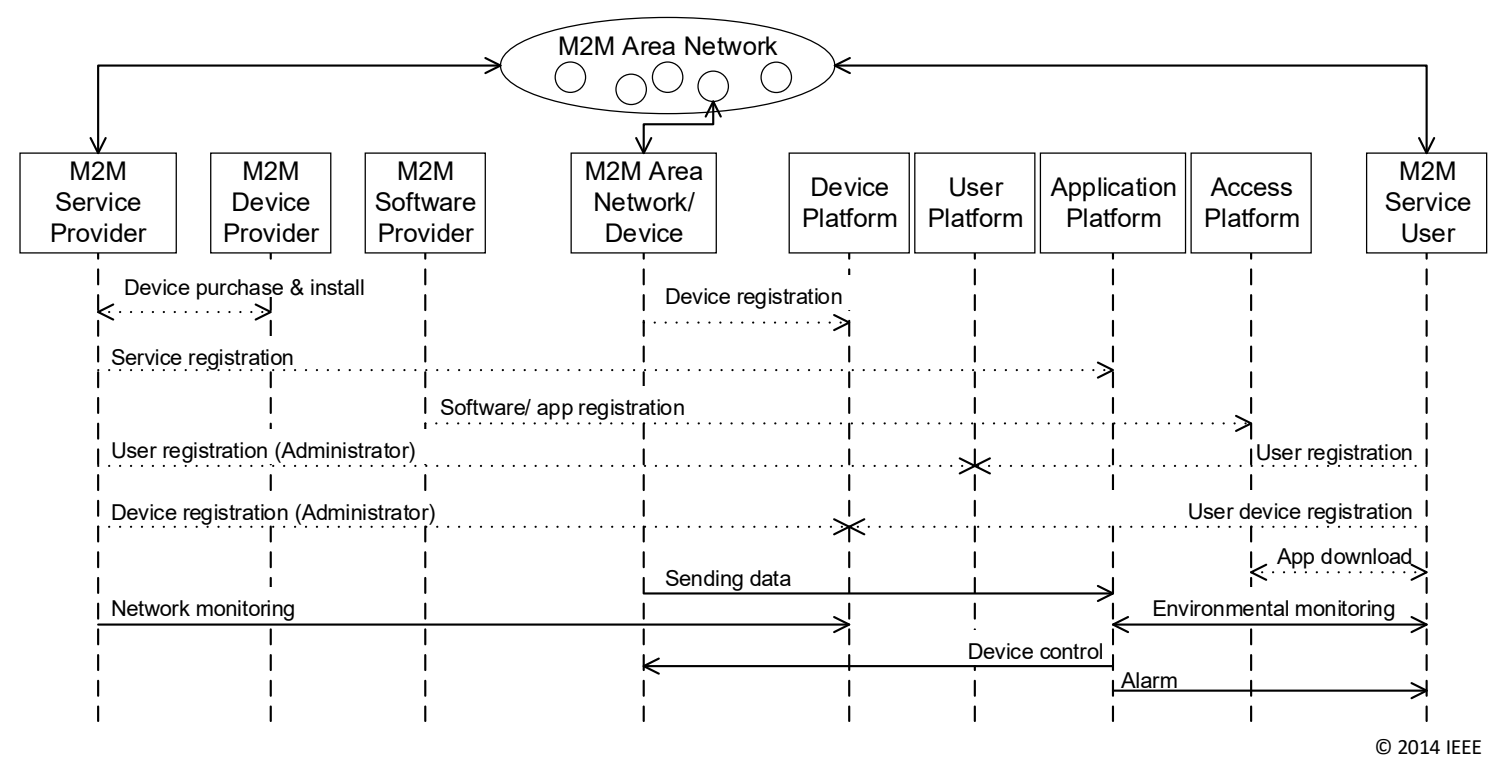

Figure 3.25: M2SP Use Case Scenario acc. (Kim et al., 2014)

The overall task of the use case is to realise an environmental monitoring application service. Various $\mathrm{M} 2 \mathrm{M}$ devices are placed in an $\mathrm{M} 2 \mathrm{M}$ area network monitoring environmental conditions. User of the application service monitor the M2M devices and "the M2M service provider manages the M2M area network for service maintenance" (Kim et al., 2014).

An M2M SP receives several sensor devices from the device provider and places them in the area of interest as well as register them to the M2M Device Platform. Services which the devices provide, such as actuation services, are registered additionally at the M2M Application Platform. A software provider develops an app and registers it to the M2M Access Platform. M2M service user and administrator register themselves to the M2M User Platform and their mobile devices to the M2M Device Platform. After registration "they download the appropriate app for the environmental monitoring services by searching from the Access-platform". The M2M Application Platform periodically receives measurement data from the M2M devices. The M2M service user uses the 
environmental monitoring app to access the information of sensor devices, provided from the M2M application Platform to the app. Additionally, the M2M application (executed by the M2M Application Platform) contains the functionality to control other M2M devices (e.g. actuators) and send alarm messages to the M2M service user, depending on pre-defined conditions (Kim et al., 2014).

The M2SP concept as presented by (Kim et al., 2014) has various advantages presented subsequently. The presented concept targets not only to the business domain. As (Kim et al., 2014) specifies its concept for MSPs can address multiple business models, especially the Customer-to-Customer (C2C) business model, it can be derived that also end-user are able to register their devices to the platform and therefore their individual environments can be addressed. The presented concept proposes to use the Internet, respectively an IP network as communication network between M2M devices, M2SP and other stakeholders (e.g. service users). This has the advantage that common available communication network technology is used to realise the networking of the participants. As the M2M devices can connect directly or via a gateway to the core network it can be derived that the gateway does protocol translation for connecting M2M devices located in an $\mathrm{M} 2 \mathrm{M}$ area network with the Internet. This offers the possibility to connect multiple M2M devices, implementing different M2M technologies, in the same way to the M2SP. Because the central registration of the M2M devices and services as well as the possibility of the M2SP to combine them, flexible applications can be realised using a single platform. The M2SP concept proposes to use apps or web interfaces to access the MSP or the $\mathrm{M} 2 \mathrm{M}$ application services. Both are common available technologies that can be used with common hardware (e.g. smartphone), which enables the platform and SPs to provide interfaces to the platform in a comfortable way. The P2P communication from 
SCs and M2M devices is especially advantageous because it reduces the load of the MSP and the communication network.

Besides the positive aspects of the M2SP presented above, the MSP concept proposed by (Kim et al., 2014) contains the following disadvantages. The M2SP represents a centralised platform architecture with central elements included that request device information or execute application logic. P2P communication with devices that provide applications is only possible after mediation by M2SP. The user of the M2SP is dependent of the central stakeholders, such as SPs, software providers and the central M2MSP platform provider. The M2SP holds a central database as well as manages the M2M devices, users, and provides the M2M services. These aspects on the one side create a dependence of the M2SP provider and SC as well as on the other side risks data safety and end-user privacy because a single entity stores the data and is able to process the data. As another central element, the M2SP concept includes the M2M Access Platform for provision of the web-based GUI as well as the apps. Both, app development and webpage generation needs to be done by expert developers and especially the apps provide an interface that is highly dependent of the executing device. It can be derived from (Kim et al., 2014) that individual apps for different M2M application services are required. This enlarges the effort for development, provision, and maintaining the apps by different expert developers and creates a dependence of the end-user access device to the manufacturer of the device operating system, because individual app stores needs to be involved for installation of the apps. The authors of (Kim et al., 2014) do not mention how the application logic is realised (i.e. implemented). They only specify that software developer provide the application software. Although end-user can register their devices to the M2SP to be used in M2M applications, the end-user are not involved in the M2M 
application creation process. As (Kim et al., 2014) identifies in order to "support the P2P communication between devices or users and devices in an M2M network, a signalling process is required for resource allocation and authorization between end devices". This is according to (Kim et al., 2014) one of the "main research issues of P2P communication in an M2M network". Because this aspect, it can be derived that both is not implemented in the approach as presented by (Kim et al., 2014).

\subsubsection{ENERsip Project}

(Lopez et al., 2011a), (Lopez et al., 2011b), (Lopez et al., 2012), (Lopez et al., 2013), and (Carreiro et al., 2011) present the ENERsip project proposing a service-oriented monitoring and control concept for energy grids. The aim of the project is to reduce consumption by increasing the user's awareness of consumption and coordinating their needs with energy generation facilities in the neighbourhood (Lopez et al., 2011a).

Figure 3.26 illustrates the architecture of the proposed ENERsip system according to (Lopez et al., 2011a; Lopez et al., 2013).

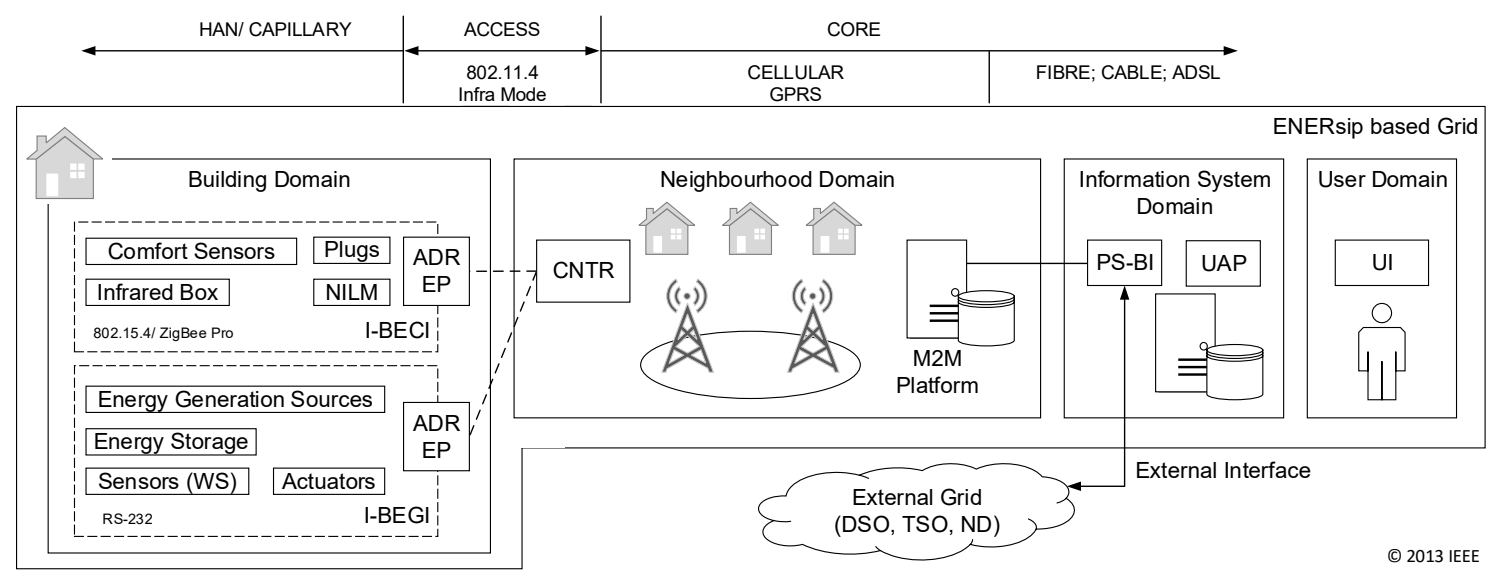

Figure 3.26: ENERsip Architecture acc. (Lopez et al., 2011a; Lopez et al., 2013) 
The Building Domain represents the smart buildings with energy generating/consuming devices and is separated in In-Building Energy Consumption (I-BECI) and In-Building Energy Generation (I-BEGI) infrastructure. Both parts contain different types of devices: I-BECI devices are sensors for measuring environment values, plugs switching and measuring energy consumption of non-intelligent devices, infrared box for controlling more intelligent devices (e.g. TVs), and the Non-invasive Load Monitoring (NILM) module determining consumption of devices "based on their electrical signature". I-BEGI devices are energy generation/storage devices and actuators controlling these devices or determining weather conditions. Both, I-BECI and I-BEGI represent the Home Area Network (HAN), which is connected via an Automated Demand Response End Point (ADR EP), i.e. an M2M gateway to the M2M core infrastructure (Lopez et al., 2011a).

The core M2M infrastructure according to (Lopez et al., 2011a) is represented by the neighbourhood domain, which allows remotely control/manage/monitor a large number of devices producing/consuming energy. The neighbourhood domain provides functionality for transmitting data produced in the building domain to the information system (IS) domain and in turn forwarding control commands initiated by the IS to the building domain. The neighbourhood domain contains a set of concentrators (CNTR) as well as the M2M platform for managing/monitoring CNTR and forwarding data to the IS as well as forwarding control commands generated by the IS to the CNTRs (Lopez et al., 2011a).

According to (Lopez et al., 2011a) the IS domain contains two different components: the Power Saving Business Intelligence (PS-BI) and the User Application Platform (UAP). The PS-BI collect all data information generated by the devices, processes them and 
coordinate efficient usage of available resources. The UAP provides services for monitoring, visualisation and controlling of energy-efficiency and comfort based on user profiles and collected data.

The User Domain according to (Lopez et al., 2011a) provides applications enabling the users to interact with the ENERsip platform through a web-based GUI. The user can consume the services provided by the UAP to monitor and manually control users' appliances remotely using the web-based GUI. External SPs (e.g. Distribution System Operators) could receive information regarding user's consumption "depending of the contract with the prosumer". Additionally the user has the possibility to define rules executing pre-defined actions, such as switch on/off devices depending on pre-defined conditions.

Advantages of the concept presented by the ENERsip project are that the project targets on the end-user domain. It allows integrating several M2M devices of different types, such as intelligent energy producer or consumer as well as non-intelligent M2M devices and therefore provides a large range of devices that the user can monitor and control using the proposed platform. The ENERsip platform provides a web-based interface to the enduser that can be used for remote monitoring and control M2M devices. Additionally, the ENERsip platform provides the functionality that the end-user can define individual rules for automatically control of its devices. This has the positive effect that the end-user can define the control rules according its requirements and is not limited by the control services that the service platform provider offers, which has no information about the requirements of the end-user. According to (Lopez et al., 2013) the ENERsip platform supports the integration of different $\mathrm{M} 2 \mathrm{M}$ technologies via the $\mathrm{M} 2 \mathrm{M}$ gateway and 
additional communication technologies are supported in principle as long as a RS-232 hardware modem exists to connect the communications technologies to the M2M gateway.

Besides the advantages described above, the concept of the ENERsip platform has the following disadvantages. First disadvantage is, that the use cases of the ENERsip platform are limited to the smart grid application field by environmental and device monitoring as well as switching electrical devices. Declarative definition of application logic (in particular defining control rules) makes the application logic independent from the execution environment because the rules should be interpretable on different execution systems by providing an appropriate rules interpreter engine. However, none of the publications mentioned above specify any underlying mechanism for rule definition or mention a standard used to ensure the portability. The ENERsip platform architecture includes several centralised components, such as the M2M platform (server) or the information system providing the business logic and the user interface. This makes the ENERsip platform dependent of those centralised elements. Additionally, the user of the platform is dependent of the ENERsip platform provider as a single stakeholder. As (Carreiro et al., 2011) specifies the ENERsip platform uses the proprietary ENERsip protocol inside the platform for communication between the platform components (ADR EP, CNTR, M2M platform, IS), which limits the compatibility with other M2M systems and requires an individual protocol stack in each system component, next to common protocol stacks existing by default. The end-user has no possibility to offer the realised automation functionality as a service to others instead of e.g. offering the collected information from its devices to Distribution and Transmission System Operators "depending on the contract with the prosumer" (Lopez et al., 2011a). 


\subsubsection{Compose Framework}

(Doukas and Antonelli, 2013), (Mandler et al., 2013), (Doukas and Antonelli, 2014), (Doukas et al., 2015), and (Doukas and Antonelli, 2015) present the COMPOSE (Collaborative Open Market to Place Objects at your Service) framework that provides according to (Doukas and Antonelli, 2013) infrastructure and methodologies to build applications communicating with smart objects (smartphones, sensors, actuators). The COMPOSE framework according to (Doukas and Antonelli, 2015), (Doukas and Antonelli, 2014), and (Doukas and Antonelli, 2013) provides an end-to-end solution for developers and entrepreneurs for designing, implementing, and deploying IoT services and applications. The COMPOSE framework provides tools for development of apps that form the end-user interface, integrate smart objects for environment integration, a processing infrastructure to run the applications and tools for service discovery/composition or application deployment. All components of the COMPOSE framework (development tools) and back-end infrastructure for running the applications according to (Doukas and Antonelli, 2015), (Doukas and Antonelli, 2014), and (Doukas and Antonelli, 2013) run in "a cloud-based infrastructure". The COMPOSE framework according to (Mandler et al., 2013) realises a SOA where all resources are consumed and provided as a service. The COMPOSE framework aims to enable developers to create applications using existing services and M2M devices. 


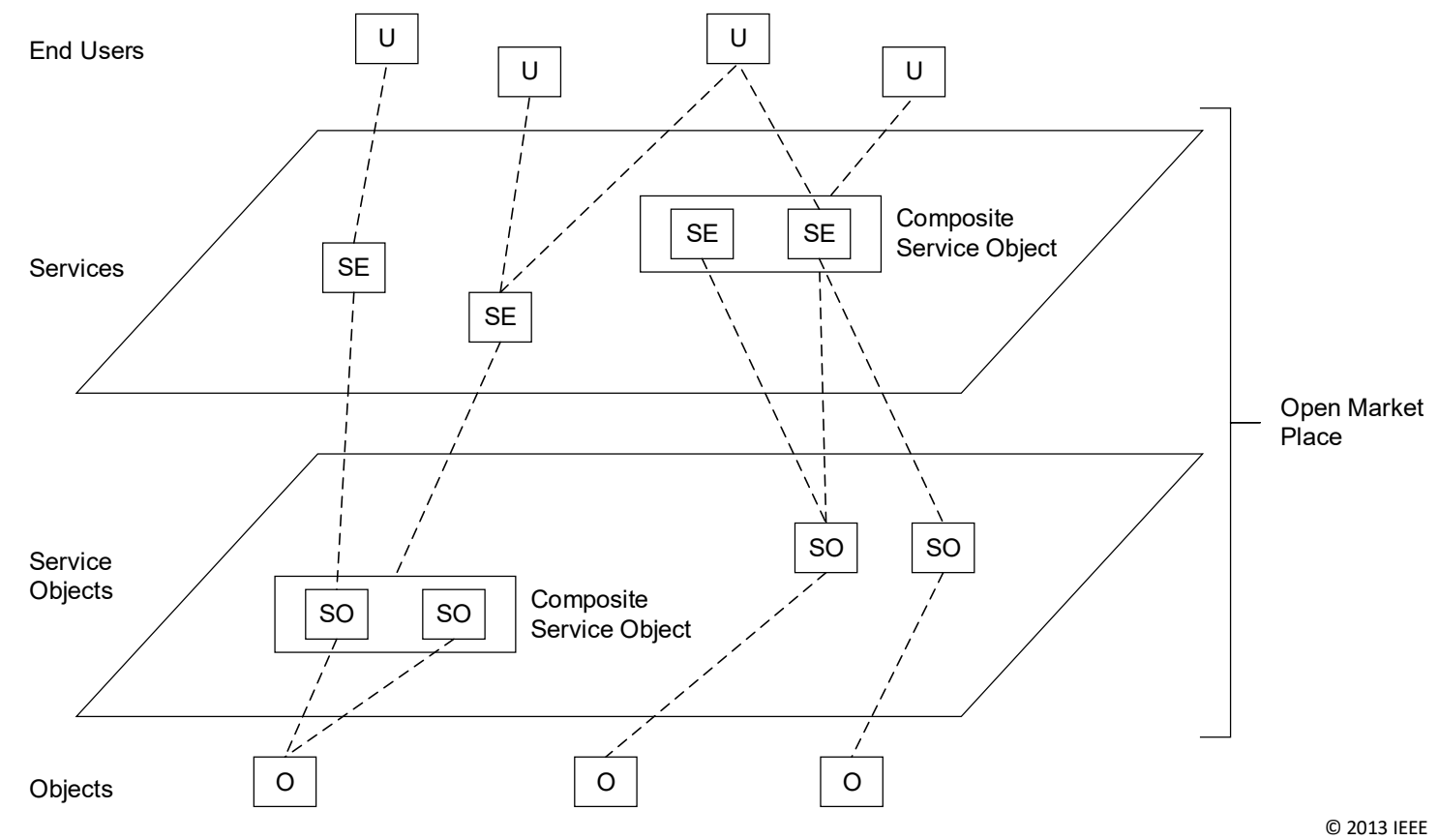

Figure 3.27: Logical Architecture of COMPOSE Framework acc. (Mandler et al., 2013)

The logical architecture of COMPOSE (illustrated in Figure 3.27) is separated according to (Mandler et al., 2013) and (Doukas and Antonelli, 2013) in the four layers described subsequently.

- Object Layer - The object layer contain all smart objects, which are physical devices able to communicate and provide sensing/actuating functionality.

- Service Object Layer - The service object layer contains virtual representations of the objects that can be accessed through the network for obtaining data or control the actuation functionality. Service objects can represent standalone smart objects or composite smart objects and can be used by various services.

- Service Layer - The service layer contains services, which are "ICT abstractions of software systems" providing functionality that can be used for a specific task. Services consume information data from service objects or control them. A 
service can be a single service or a composition of services processing data and implementing the application logic.

- End-User Layer - The end-user layer contain the end-users consuming the services. End-users are persons that access the marketplace using their personal end devices "through the installation of a given application" or a machine to integrate the service into their business processes.

The COMPOSE framework consists of three parts. Figure 3.28 shows these parts and the components of the COMPOSE framework.

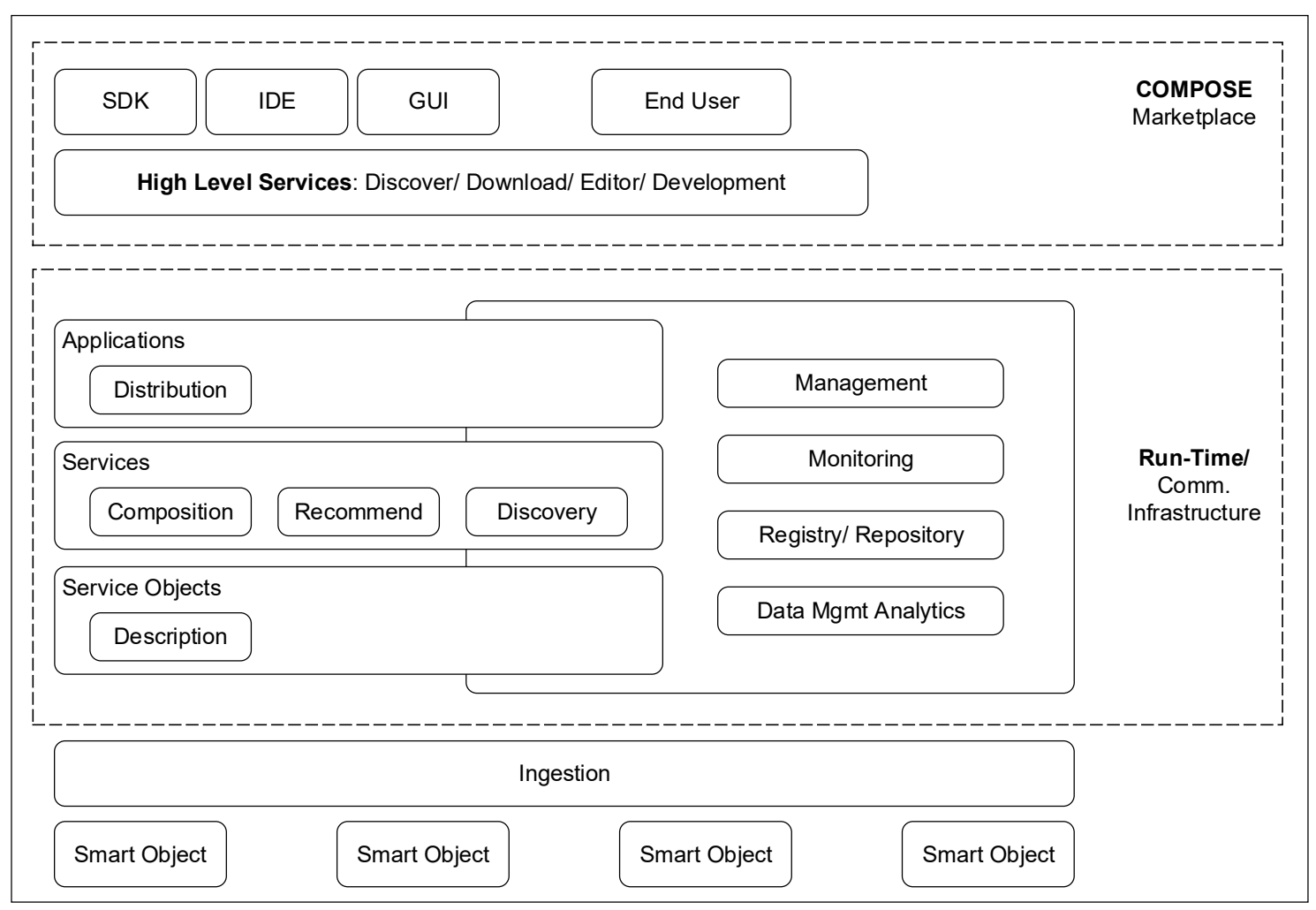

(C) 2014 IEEE

Figure 3.28: Components of COMPOSE framework acc. (Doukas and Antonelli, 2014)

The Ingestion Layer contains according to (Doukas and Antonelli, 2013) the smart objects and devices and forms according to (Doukas and Antonelli, 2014) the interface to these 
devices. According to (Doukas and Antonelli, 2015) and (Doukas and Antonelli, 2014) the devices in the Ingestion Layer communicate with the COMPOSE Runtime Engine via HTTP or different M2M protocols (MQTT, CoAP) to transmit device information (e.g. sensor data) or receive control commands.

According to (Mandler et al., 2013) the Runtime Engine "comprise of a cloud environment" and is responsible for management of services and the platform including the discovery and monitoring of services and devices as well as control of Smart Objects. The Runtime Engine contains according to (Doukas and Antonelli, 2014) the service objects, services, and applications. The services according to (Doukas and Antonelli, 2013) consume the information provided by one or multiple service objects and control the devices via the service objects "to perform a given task".

The service registry component according to (Doukas and Antonelli, 2014) provides the functionality for discovery of services. To implement the service registry component the COMPOSE framework uses iServe (Pedrinaci et al., 2010) as a central service registry. Developers use the service registry to query existing services resulting in "a list of available services and their endpoints that meet the requested criteria".

Applications are a combination of services and implement a user-specific application logic to satisfy the requirements of a user.

The COMPOSE Marketplace according to (Doukas and Antonelli, 2013) is an environment to share services and applications with other developers. According to (Doukas and Antonelli, 2015) and (Doukas and Antonelli, 2014) the COMPOSE Marketplace is the "front-end interface" developers use to publish and access services. 
The COMPOSE Marketplace contains an IDE, libraries and SDKs to create application logic and apps as well as functionality for registration and discovery of services and the deployment of applications.

According to (Mandler et al., 2013) developers use the IDE and the SDK to develop services, applications and apps. In this context applications are the combinations of services to realise the application logic executed in the COMPOSE runtime and apps are the smartphone applications to interact with the end-user. The COMPOSE framework according to (Doukas and Antonelli, 2014) provides the functionality to create applications graphically using a "visual workflow editor". The visual workflow editor is realised according to (Doukas and Antonelli, 2014) using Node-RED (NodeRed, 2017), which is a Node.js-based tool that enables developers to create workflows of services by graphical combination of service nodes. The result of the combination of services is a Node.js application that is executed in the CloudFoundry (CloudFoundry, 2017), which is a cloud-based application platform.

The GUI element of the COMPOSE Marketplace forms the interface to the end-user. According to (Doukas and Antonelli, 2013) the smartphone application is the "main graphical interface the user is using to interact with the application for configuration of user preferences or receiving information data. The apps bi-directionally communicate with the back-end infrastructure using Web 2.0 technologies.

To illustrate the mechanisms for service and application development using the COMPOSE framework, the following describes the development process from the perspective of a developer. According to (Doukas and Antonelli, 2013), (Doukas and 
Antonelli, 2014), and (Doukas and Antonelli, 2015) a developer that is going to create and deploy applications has to perform the following four steps.

1. Create new services or discover existing services using IDE and SDKs. The services can either be "a set of classes or high level scripts" that define the service logic (e.g. request and process specific data information).

2. Defining and implementing the interaction with the smart objects (e.g. request the information data from specific smart object) using existing SDKs and libraries.

3. Deploying the implemented application to COMPOSE infrastructure.

4. Developing the mobile app for accessing the developed application.

While the developer can use the above-mentioned Node-RED workflow editor to design the back-end application logic, for development of the mobile app the developer uses a mobile application framework based on Titanium (Appcelerator, 2017). Titanium is a development SDK that enables the unified development of mobile apps and transformation to specific mobile runtimes (mobile operating systems, e.g. iOS or Android) (Doukas and Antonelli, 2013; Doukas and Antonelli, 2015).

The COMPOSE framework as introduced above contains several advantages presented subsequently. The approach that developers create services and applications as well as deploy and register them in a global accessible infrastructure enables other developers to reuse already existing services and applications. This enlarges the reusability of services and application which in consequence safe time and costs for development of new services. Reusing the existing services and creation of new services by composition of the existing services enlarges the flexibility of the platform because several services can be used to create applications in multiple application fields. Reusing service 
functionalities inside new applications also avoid the implementation of many vertical silo applications as in case of implementing a different M2M application for different use cases. The COMPOSE framework targets to the end-user domain, but it has to be considered that in context of COMPOSE framework the end-user is a business stakeholder that benefits by integration of the M2M application realised with COMPOSE into its business process. Designing back-end application logic graphically by defining workflows that combine service nodes is advantageous because that is an intuitive methodology to create application, even when the developer has less experience in application development. Using smartphone application, as a GUI for interaction with the application is advantageous because it is a common available interface technology. Additionally, communication of apps with the back-end infrastructure using Web 2.0 technologies reuses common existing protocols and prevents to provide an additional protocol stack for communication in the end device of the end-user.

Besides the advantages presented above, the COMPOSE framework has several disadvantages as presented in the following. Most of the elements in the COMPOSE infrastructure, such as central service registry and execution engine for services and application, is provided by a single platform provider and run in a cloud-based infrastructure. This makes the user of the platform dependent of the platform provider and the platform dependent of specific platform components, including the particular solutions proposed for realisation of platform components, such as CloudFoundry as central application platform. Especially according to (Doukas and Antonelli, 2015) the app server providing the user interface apps as well as the COMPOSE server are standalone elements in the COMPOSE architecture. The output of the graphical combination of services is a Node.js application. This makes the solution dependent of that technology 
and complicates the portability and adaptability because Node.js is no standard specification. Additionally, the services the developer can create and reuse are static classes or scripts representing the service logic, which are platform dependent. This also complicates reusing the services in other platforms. Although the smartphone as runtime environment for the GUI app is commonly available, it is required to develop a specific app for each application running in the back-end. Although the COMPOSE framework provides tools that unifies and simplifies the development, still expert knowledge is required. Although the developer has the possibility to create application logic graphically, this is not fully integrated in the development process because particular parts for providing the M2M solution still have to be developed requiring expert knowledge. E.g., the developer has to use existing libraries or SDKs for creating the mobile app as an interface for the application. Mobile application framework is based on Titanium Appcelerator, which is on the one side advantageous with regards to the platform independency of app development, but on the other side has the negative effect that, because using the Titanium SDK as development framework, requires expert knowledge of the developer to develop the app logic. The COMPOSE framework targets to developers and entrepreneurs, but not to private individuals as end-users. The end-users are only provided with the possibility to interact with the applications in the back-end using apps as input/output interface, but are not provided with the functionality to design applications by themselves. The COMPOSE framework has another disadvantage regarding the data safety and end-user privacy aspect. Because according to (Doukas and Antonelli, 2014) "every data transaction between the different entities (users, applications and smart objects) is logged inside COMPOSE" as well as the data storage engine of COMPOSE logs every action of the end-user, the COMPOSE provider has generally 
access to the data created inside the end-user environment. This can represent the behaviour of the end-user and its environmental conditions.

\subsubsection{Distributed Cooperative M2M System for Flood Monitoring (DistribFloodMon)}

(Kitagami et al., 2014) presents a concept of a distributed cooperative M2M system for the use case of flood disaster prevention. The task of the presented use case is to apply multiple water level sensors and rainfall sensors in a specific area to realise a system for flood disaster prevention based on data analysis generated by the sensors. (Kitagami et al., 2014) proposes to realise the M2M system based on a distributed approach, because traditional server-centric approaches of M2M systems for data analysis and device control contain the following disadvantages: M2M devices and the M2M server application continuously exchange data, which generates a large data volume and delays the control messages generated by the M2M server. Additionally (Kitagami et al., 2014) criticises that if communication link between the M2M gateway and the M2M server fails, the whole system will fail.

Based on this aspects (Kitagami et al., 2014) introduces a distributed M2M system for the special use case of a flood prevention application as illustrated in Figure 3.29. 


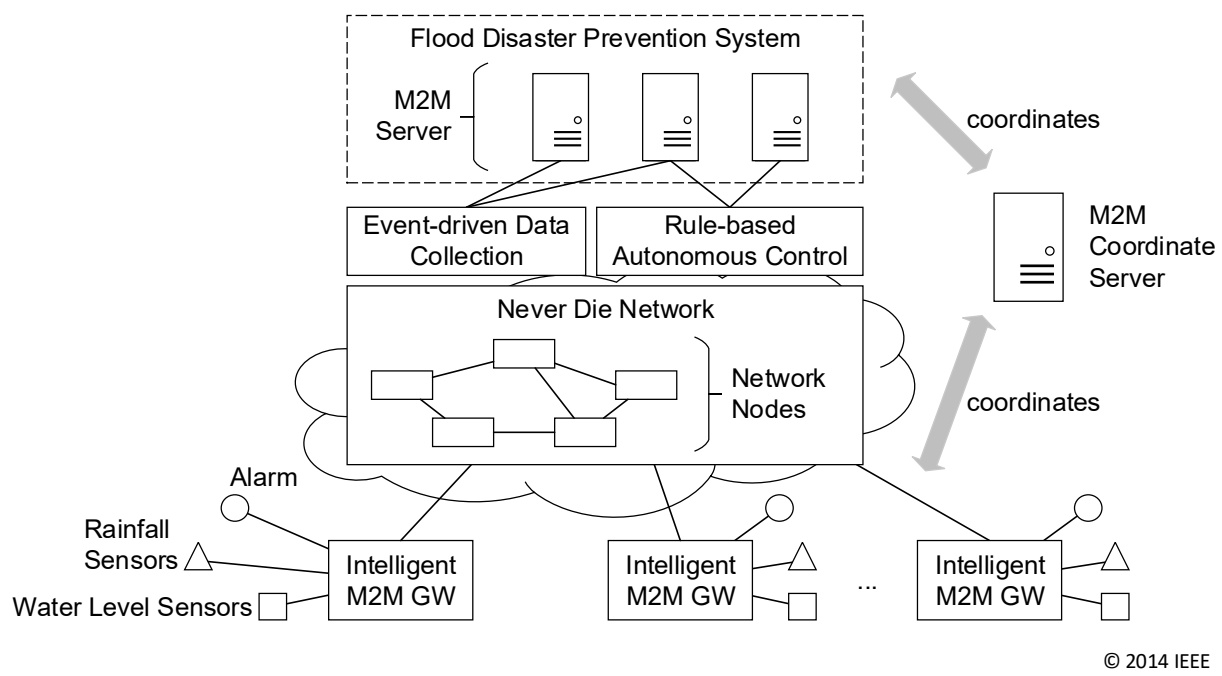

Figure 3.29: Distributed M2M System acc. (Kitagami et al., 2014)

According to (Kitagami et al., 2014) the proposed M2M system consists of central M2M servers located outside of the M2M area networks, respectively outside the domain where the sensors and actuators reside. The task of the M2M server is to monitor and control the sensors and actuators to generate statistics about the sensor values for further analytics, forecasts and in case of an alert, control the actuators. The M2M coordinate server adjusts and coordinates the $\mathrm{M} 2 \mathrm{M}$ gateway and $\mathrm{M} 2 \mathrm{M}$ server for the usage in flood disaster prevention application.

The sensors and actuator are connected to multiple intelligent M2M gateways (M2M GWs). These intelligent M2M GWs not only connect the sensors and actuator to the communication network, but also include "a rule-based autonomous control mechanism". The measurement values of the sensors are aggregated in the M2M GWs, which send the aggregated measurement data continuously to the M2M servers and stores the original measurement values locally for later processing. Additionally, the M2M GWs autonomously monitor the measurement values and transmits the original values (not 
aggregated measurement values) for a specific interval to the M2M server if e.g. predefined variations of measurement values occurs (Kitagami et al., 2014).

To prevent a delayed control of the actuators in the area of interest (e.g. alarm actuators) caused by connection interrupts from M2M server and M2M GWs, the M2M GWs include a local rule engine for controlling the actuators. The M2M coordinate server configures the conditions in the gateways for transmission of the detailed measurement data, i.e. defines the corresponding thresholds by sending the rules in advance to the M2M GWs (Kitagami et al., 2014).

According to (Kitagami et al., 2014) the M2M GWs connect via a Never Die Network (Shiratori et al., 2012) with each other and with the M2M servers. The Never Die Network is a mobile adhoc network including redundancy of links between the nodes.

The presented approach of (Kitagami et al., 2014) includes various advantages specified as follows. The transmission of aggregated measurement data enables detection and storing of measurement values. Through this, it is possible to generate statistics of the measurement values without stressing the communication network because large amounts of data. Additionally, the autonomous execution of the parts of the application logic by local monitoring, evaluation and control of sensors/actuators enlarges the system availability and reduces the disadvantages of traditional server-centric M2M systems.

Besides the advantages presented above, the M2M system introduced by (Kitagami et al., 2014) includes several drawbacks. (Kitagami et al., 2014) presents only one single and very static use case, respectively application field of flood monitoring. They does not define a general concept of distributed M2M applications or providing the application as 
a service for other users. Setup of an underlying Never Die Network for communication of the participating nodes seems necessary in the case of the introduced flood prevention application, especially with regard to the area to deploy the application with disrupted or not existing communication network. However, considering application field of end-user domain, which usually provides existing and working communication networks, additional communication networks are not necessary to implement. The overall use case application is a combination of decentralised control and central management and data collection, including central system elements, which again results in the negative aspects of including central elements or stakeholders in the M2M system architecture, as described in previous sections. The presented approach of does not focus on or include end-user integration in application development or the integration of M2M devices located in end-user environment. The application definition by specification of semantical rules, representing the application logic, enlarges the independence of application logic from execution systems because e.g. change of an execution system only requires exchange of rules interpreter engine. However, (Kitagami et al., 2014) does not specify a standardised language for definition of the rules executed in the intelligent M2M GWs, which in consequence again limits the platform independency.

\subsection{Requirements for a new Framework for Autonomous decentralised M2M Application Service Provision}

The previous section 3.1 introduced current M2M system architectures for M2M application services. The positive aspects and weaknesses have been mentioned at the end of the corresponding sections. This section derives requirements for a new approach for 
application service provision in M2M environments to enable end-users for autonomous and independent M2M application service provision. The definition of requirements is based on the strengths and weaknesses of previously introduced M2M system architectures. For definition of appropriate requirements reasonable aspects of related work projects will be adopted as requirements and additional ones defined based in order to avoid the negative related work project aspects. The requirements defined in this section will be the basis for the proposed framework for "Autonomous decentralised M2M Application Service Provision” presented in chapter 4.

Many of the related projects introduced above, such as oneM2M specification for M2M systems focus on the support of interoperation of individual industries or businesses in professional domains as well as the realisation of complex business applications for support of business processes. Other ones also focus on the end-user domain with endusers' M2M device integration. The personal environment of the end-user has powerful potential for providing applications with the integration of the M2M devices located at the end-user environment. Therefore, the end-user environment should be addressable by the MSP. Based on this aspect, the following requirement can be derived:

- End-user environment integration - The MSP should address end-user environment.

The projects BOSP, ENERsip, COMPOSE, e-DSON, and M2SP satisfy this requirement because they directly address M2M devices located in end-user environment. The other projects are related to the business domain or special use cases without integration of endusers' M2M devices. 
Considering the related projects, end-users mostly have no option to be involved in application development for their personal environments. Because the M2M applications that effect the environment of the end-users have to meet end-users' individual requirements, the end-user should get involved in defining the application semantic individually regarding end-users' personal requirements. Based on this aspect, the following requirement can be derived.

- End-user integration - The end-user has to be integrated in application creation for its personal environment.

None of the above-mentioned related projects fully satisfies this requirement.

The e-DSON framework partially satisfies this requirement because the e-DSON platform has its focus on user-centric M2M applications. The end-user is not explicitly integrated in the application creation process, but the flexible structure of the application provision concept via service overlay networks allow the application developer to simply define individual user applications.

The next set of requirements refer to service provision aspects of an MSP. The approaches described above to define services for the personal environment are limited to personal use of the services by the owner of the environment in which the M2M devices reside, respectively to the end-user who has defined the services. Other end-users or external SPs could benefit from using the services or resources available inside the end-users' personal environment to include them in their own environments or processes. This enlarges the possible functionality of applications defined by others by also including the resources 
and functionalities of other personal environments into own applications. Therefore, the following requirement is derived.

- End-user service provision and utilisation - End-users should be able to provide resources and applications inside their personal environment as a service to other end-users or SPs.

Only the related e-DSON project satisfies this requirement by static development of usercentric applications including distributed resources that other end-users can offer inside a centralised system architecture.

Although making services and resources that are available in end-users environment accessible for other entities is advantageous to enlarge the functionalities of end-user applications, this would be limited to integrate them in applications for single environments. As mentioned above the end-user environment has powerful potential for providing services to others. Combination and coordination of those services could enable applications with higher impact and flexibility regarding realisable functionalities. Therefore, the next requirement is defined as follows.

- Cooperative end-user service provision - Services of end-users should be combinable to provide a cooperative application service that can be utilised by other end-users or SPs.

None of the above projects satisfies this requirement. Only the e-DSON framework partially satisfies this requirement because the e-DSON platform offers the possibility to make services of local device resources and services available to others, but does not 
allow to define the cooperative service logic by the end-users themselves, instead a central expert developer have to define the structure of the service composition.

The next set of requirements refer to the system architecture of the MSP. Almost all of the related projects are based on centralised architectures or contain centralised elements in the system infrastructure. Centralised architecture means that the MSPs or parts of the platforms contain physically central network element (such as a server) or logical central elements or stakeholders (such as cloud environments or platform providers). This research should explore alternatives to centralised and cloud-based M2M service platform architectures. A decentralised system architecture could avoid the following disadvantages of centralised or partly centralised architectures in context of MSP provision with end-user integration. 1) A centralised MSP often requires high performance hardware for providing the platform functionality to be used by many users or to connect many $\mathrm{M} 2 \mathrm{M}$ devices. A single stakeholder has to provide large resources for service development and maintenance as well as for platform setup, management, and maintenance. The platform provider additionally has to spend large effort for ensuring the reliability of the platform. Each of these topics will result in high costs for operating such a centralised MSP and for developing applications running on those platforms. The (as usual) companies providing the platforms need to transfer the required costs to the user of the platform. Since the target group in the focus of this research are the end-users instead of companies that usually utilise M2M solutions, the costs have to be as low as possible because the end-users will not accept the costs. 2) The MSP users (SP and SC) could be dependent on the platform provider as a single stakeholder included in the M2M system architecture. If that stakeholder stops platform provision, the entire M2M system solution fails. If a single M2M SP creates and hosts an M2M application service, the 
consumer of this application service is dependent of that central provider too. 3) Centralised M2M platform solutions often are limited in flexibility. Centralised service provision via service platforms mostly intend specific application fields (e.g. MSPs provides solution for Ambient Assisted Living but is not able to address the requirements for a Smart Grid solution). I.e. centralised solutions might limit the application fields for specific domains. 4) A further negative aspect of centralised elements has to be considered regarding the data safety and end-user privacy. Storing a large amount of data that is produced by $\mathrm{M} 2 \mathrm{M}$ applications at a central location (physically or logically) is always critical. The stakeholders having access to that data storage could misuse the data for e.g. analysing the end-users' behaviour by getting detailed information provided by their M2M devices. Based on disadvantages of centralised architectures for MSPs identified above, the next criterion is derived to ensure the independence of central provider, enlarging the flexibility of the platform, avoiding costs for the end-user and ensures the privacy aspect, which in consequence enlarges the acceptance by the enduser.

- Decentralised system architecture - The approach for MSP has to avoid centralised entities in the system architecture to provide the service platform or the services. Avoidance of centralised entities means that no centralised system components in the MSP architecture (such as e.g. central service repositories) should be integrated as well as no central stakeholder involved required for operation of the MSP and the service provision (development, operation, maintenance, management, controlling) except the end-user itself.

None of the above-mentioned related projects fully satisfies this requirement. 
The oneM2M service platform specification partially satisfies this requirement because the highly distributed nature of the components in field domain, but requires a central IN in the infrastructure domain. The DistribFloodMon project partially satisfies this requirement because the autonomous mechanism for local sensor monitoring and controlling the actuators in case of an alert, but the total application infrastructure includes central M2M servers for data storage and analytics as well as a dedicated M2M coordinate server for configuration of the intelligent M2M gateways and the central M2M servers. The e-DSON framework partially satisfies this requirement because the e-DSON platform allows linking decentralised device resources and service to realise a more complex composed service. However, the service topologies are defined by a central stakeholder as well as the e-DSON platform itself includes centralised system elements.

Considering the related projects, almost all of them are able to integrate different M2M device technologies. The provided applications can request information data from devices realised with different $\mathrm{M} 2 \mathrm{M}$ communication technologies or control them. This is an important aspect because the applications realised with the MSPs should be independent from M2M device technologies to ensure the flexibility of applications. Therefore, the following requirement is derived.

- M2M device technology abstraction - The MSP should abstract the M2M device technology to include different M2M device technologies.

Multimedia communication functionalities such as instant messaging or audio/video calls form a well interface to generate outputs of the MSP and to interact with the end-user as the required end devices (e.g. mobile phone) are already present. Multimedia communication can also be used for realisation of an input interface to the platform using 
the same device. Additionally, multimedia communication forms a flexible interface for communication between SP and SC to organise service provision/utilisation. Especially for social services, often personal communication between SP and SC is required to arrange an appointment (e.g. via email, IM or call). Multimedia communication is required to build up services such as surveillance services (e.g. monitoring buildings via video streams). Therefore, the next requirement is defined as follows.

- Multimedia communication - The MSP should integrate multimedia communication functionality.

Only the related projects BOSP and IMS M2M SP I satisfy this requirement. BOSP integrates carriers' abilities, such as VoIP communication and messaging functionality into its platform concept. IMS M2M SP I utilises the IMS communication technologies for exchange of information data between the M2M system elements. Although IMS M2M SP I uses only messaging functionality of the IMS, because the IMS integration it is possible to include also audio/video communication.

The M2M on SOA project partially satisfies this requirement because it includes email and SMS as notification options of users. The M2M on SOA project restricts multimedia communication to notification functionality and does not use the same kind of communication to control applications or integrates audio/video communication.

Considering the related projects, almost all of them include a mechanism to manage the location of M2M devices or M2M services for addressing them. The related approaches usually realise this functionality by a central management entity in the system architecture. Additionally, different end-users reside on different and variable locations 

(geographical and topological), the devices and provided services have to be continuously addressable. E.g., because end-user environments usually cannot be addressed via static IP addresses, the changing IP addresses must be managed. As specified above, an MSP should avoid centralised components adopting such management tasks. Therefore a decentralised management solution is required, which results in the next requirement.

- Device/service lookup mechanism - The devices used for service provision and the services itself needs to be identifiable and addressable. Therefore a mechanism for device and service lookup is required.

Beside the functional requirements specified above, several non-functional requirements can be derived from the related projects as the following section presents. Because no central entity has to be integrated in M2M system architecture for MSP provision, the user of the platform, i.e. the end-user is responsible for operating the platform. It is assumed that the end-user has common technical knowledge but no expert knowledge in application development or operating a specific service platform. Because the end-user has to operate the service platform and is responsible for application design, both have to be simple and intuitive. Based on these aspects the following requirement is derived.

- Simplicity - Both, application development and operating the MSP have to be simple.

None of the above-mentioned related projects satisfies this requirement.

The COMPOSE framework project partially satisfies this requirement because the developer is enabled to design the back-end application logic graphically by designing workflows with the included nodes. This simplifies the development process of the back- 
end application, but operating the system platform, consisting of several different platforms as well as e.g. development of the GUI apps by using mobile SDKs, complicates both, application development and platform provision. The ENERsip platform partially satisfies this requirement because the end-user has the possibility to define control rules for its devices and can interact with the platform using a web-based GUI. However, operating multiple system elements located in different parts of the network that communicate with each other using the specific ENERsip protocol stack complicates the system architecture.

Most of the related projects contain elements in their platform architecture that require high performance hardware for e.g. operating central architecture components or network entities. To ensure less financial effort for the end-user, the components of the MSP should be executed applying low cost hardware or existing hardware and network facilities. Therefore, the following requirement is derived.

- Minor hardware requirements - The hardware requirements necessary for providing the MSP as well as accessing the provided applications have to be low.

None of the above-mentioned related projects fully satisfies this requirement.

The IMS M2M SP I and II architecture partially satisfies this requirement because it reuses existing network topology of the IMS, but also requires central M2M AS inside the Cloud. Because the IMS M2M SP I and II approach seamless include the network elements of the IMS, such as P-/I-/S-CSCF/HSS in the M2M system architecture, instead using the IMS as plain communication network, these entities can be considered as parts of the MSP. 
Because the increasing amount of M2M devices, as well making the platform usable for large number of end-user the following requirement is derived.

- Scalability - The MSP should meet scalability in both directions (M2M devices and users).

None of the above-mentioned related projects fully satisfies this requirement.

The oneM2M service platform specification partially satisfies this requirement when assuming the MSP is operated as a cloud solution, which naturally provides scalability depending on the allocated resources. However, central elements in system architecture limits the scalability. The INOX service platform project partially satisfies this requirement because the authors define scalability as a requirement that is provided by the INOX platform, but does not specify how the scalability is realised. The INOX service platform runs as a cloud environment, which requires central elements that again limit the scalability. The IMS M2M SP I and II approach partially satisfy this requirement. The presented approaches include the IMS as communication network, which is rather scalable. However, the included central system components, such as the AS or M2M server in turn limits the scalability. The COMPOSE framework project partially satisfies this requirement. (Doukas and Antonelli, 2013) specifies the COMPOSE framework as a scalable cloud-based solution. However, on the opposite side the central framework entities, such as the compose server, naturally limits the scalability of an architecture. The M2SP concept partially satisfies this requirement. Included central elements, such as the app stores required for app installation as well as the $\mathrm{P} 2 \mathrm{P}$ communication possibility with the devices enable scalable systems. However, other central elements in system 
architecture, such as the centralised M2M Application Platform or the M2M User Platform limits the scalability.

Most of the related projects introduced above contain proprietary components in their architecture or service implementations, which make them dependent of it. Independence of platform components and service implementations from specific implementation technologies is necessary to provide best options for adaptability of platform components as well as ensure the portability of the MSP components. Portability means that the services and platform components does not have a static binding to the execution environment (e.g. using proprietary solutions). Using standardised protocols is advantageous for ensuring portability and adaptability. Portability is especially important because of the many existing different devices inside end-user environment that should be used for MSP execution as well as for access the services. Based on these aspects, the following requirement is derived.

- Platform independency - The realisation of $\mathrm{M} 2 \mathrm{M}$ platform architecture components as well as applied methodologies should be platform independent.

Only the INOX service platform project satisfies this requirement due it proposes a loosely-coupled system architecture with high service orientation and additionally, defines a declarative way to describe applications instead of static and execution system specific implementation of application logic.

The oneM2M service platform specification partially satisfies this requirement because the oneM2M specification does not specify implementation details regarding execution environments, but also no concept for independent application definition. Examination of 
OM2M implementation shows the dependence of application definition on the platform because the application logic has to be defined programmatically and is translated in platform dependent software executables. The M2M on SOA project partially satisfies this requirement because the SOA principles considered in that approach as well as the combination of system elements based on a message broker enable realisation of independent application and system elements. In contrast the specific frameworks proposed by the authors such as Struts, Spring, Hibernate make the application and execution environment dependent on these frameworks. The IMS M2M SP I approach partially satisfies this requirement because it applies standardised protocols for communication between M2M devices and MSP as well as between platform components, but (Foschini et al., 2011) does not specify how the application architecture is structured or implemented inside the Application Domain. The ENERsip platform project partially satisfies this requirement because declarative definition of application logic makes the application logic independent of the execution environment. However, the authors did not mention any underlying mechanism or standards for rule definition. The e-DSON framework partially satisfies this requirement because the e-DSON platform uses the common available HTTP protocol for communication between platform, user and home environment as well as XML (Extensible Markup Language) (W3C, 2008) as data format, but does not specify any standard-based methodology to describe the application logic for generation of the underlying service overlay network.

The data generated in end-user environments during application execution or M2M device integration into MSP, such as sensor states or communication data, are highly sensitive because they can represent the behaviour of the end-user and the environmental conditions. If the end-users knows their data are secure, this increases the acceptance of 
an MSP. Therefore it is necessary to keep this data protected, keep the end-user as owner of this data, and avoid the so called "Big Brother Effect". Based on these aspects, the following requirement is derived.

- Data safety, end-user privacy - The MSP has to provide the functionality for data safety and end-user privacy.

Almost none of the related projects satisfy this requirement mainly because the single central architecture elements storing the data or single platform providers that could have access to the data and misuse it e.g. for determination of end-users 'behaviour.

The oneM2M service platform specification partially satisfies this requirement because the standard specification includes security functionality such as access control. However, because the oneM2M architecture includes central elements may monitoring or storing data produced in M2M area networks the requirement is not fully satisfied.

Table 3.1 shows the requirements specified above including the evaluation of the related projects regarding these requirements. Requirements the related project satisfy are marked with "+"; requirements not satisfied by the projects are marked with “_“; requirements the projects partially satisfy are marked with "o". Evaluations that cannot be determined based on the published information about the projects are marked with "/".

Table 3.1: Evaluation of related Projects reg. derived Requirements 
3.2 Requirements for a new Framework for Autonomous decentralised M2M Application Service Provision

\begin{tabular}{|c|c|c|c|c|c|c|c|c|c|c|c|}
\hline \multirow[t]{2}{*}{ Requirements } & \multicolumn{11}{|c|}{ Related M2M Service Platform Projects } \\
\hline & 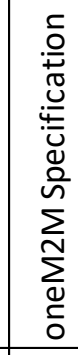 & $\stackrel{x}{\underline{O}}$ & 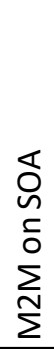 & ڤે & 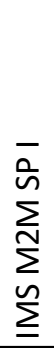 & $\begin{array}{l}= \\
\bar{n} \\
\sum_{n}^{n} \\
\sum_{n}^{n} \\
\underline{n}\end{array}$ & $\begin{array}{l}z \\
\text { ¿ } \\
\text { d } \\
\end{array}$ & $\stackrel{n}{\Sigma}$ & 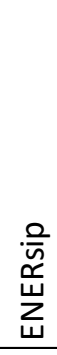 & $\begin{array}{l}\text { Uू } \\
\text { O } \\
\text { ¿ } \\
\text { О }\end{array}$ & $\begin{array}{l}\frac{1}{0} \\
\sum_{0}^{0} \\
0 \\
0 \\
\frac{0}{4} \\
\frac{0}{2} \\
\frac{.0}{0} \\
0\end{array}$ \\
\hline End-user environment integration & - & / & - & + & - & + & + & + & + & + & - \\
\hline End-user integration & - & - & - & - & - & - & 0 & - & + & - & - \\
\hline $\begin{array}{l}\text { End-user service provision and } \\
\text { utilisation }\end{array}$ & - & - & - & - & - & - & + & - & - & - & - \\
\hline $\begin{array}{l}\text { Cooperative end-user service } \\
\text { provision }\end{array}$ & - & - & - & - & - & - & 0 & - & - & - & - \\
\hline Decentralised system architecture & 0 & - & - & - & - & - & 0 & - & - & - & 0 \\
\hline $\begin{array}{l}\text { M2M device technology } \\
\text { abstraction }\end{array}$ & + & + & + & + & / & - & / & + & + & + & / \\
\hline Multimedia communication & / & / & 0 & + & + & + & - & - & - & - & - \\
\hline $\begin{array}{l}\text { Device/ service lookup } \\
\text { mechanism }\end{array}$ & + & + & + & + & + & / & + & + & / & + & - \\
\hline Simplicity & - & / & - & - & - & / & - & - & 0 & 0 & - \\
\hline Minor hardware requirements & - & - & - & - & 0 & 0 & - & - & - & - & 0 \\
\hline Scalability & 0 & 0 & - & - & 0 & 0 & + & 0 & / & 0 & - \\
\hline Platform independency & 0 & + & $\mathrm{o}$ & / & 0 & + & 0 & - & 0 & - & - \\
\hline Data safety, end-user privacy & 0 & / & - & - & - & - & - & - & - & - & - \\
\hline
\end{tabular}

With regard to the above-specified requirements, a novel framework for autonomous decentralised application service provision in M2M networks will proposed in chapter 4. Chapter 5 and chapter 6 will introduce the underlying concepts of the proposed framework. 


\subsection{Conclusion}

This chapter introduced related projects on MSPs. The oneM2M standard for M2M system architectures has been introduced as representative of commercial MSPs in section 3.1 as well as approaches from the research field. Each of the related projects has been evaluated regarding its strengths and weaknesses. It can be noted that most related projects pursue a centralised approach by including central entities or stakeholders in their system architecture. Furthermore, it can be stated that most of the presented approaches do not integrate the end-user into the application creation, but this is important to take account of the requirements of the end-user.

Based on the evaluation of the related projects in section 3.2 requirements have been derived. One of the main requirements is that an MSP not only addresses the end-user application field, but also integrates the end-user into the application creation process. Furthermore, it can be noted that it is important to avoid central entities or stakeholders, such as central platform provider or network element provider, to ensure the independence of such entities. Reuse of existing resources for operation of an MSP and networking of M2M devices and services is important because it reduces the cost of operating the platform and increases the acceptance of an M2M solution. Additionally to these aspects, it is important that an MSP integrates the different M2M device technologies and allows them to be combined with each other. 



\section{Proposed Framework for autonomous}

\section{decentralised Application Provision in}

\section{M2M Systems}

This research proposes a novel framework for autonomous and decentralised application and service provision in M2M systems. The framework addresses the requirements defined in section 3.2 and eliminates the deficits of currently existing approaches for application and service provision in $\mathrm{M} 2 \mathrm{M}$ systems. In this chapter, section 4.1 first introduces the overall concept of the proposed framework to enable end-users to design and provide individual or cooperative M2M applications as a service to other end-users or central service providers (SPs) such as companies or organisations. Afterwards section 4.2 describes the framework architecture and its components realising the proposed framework architecture.

\subsection{General Concept}

The concept described in this research changes the method of M2M application and service design, provision as well as utilisation significantly from previous approaches. Figure 4.1 illustrates the process of service and application development, deployment and utilisation for traditional M2M service platforms. (MSPs) 


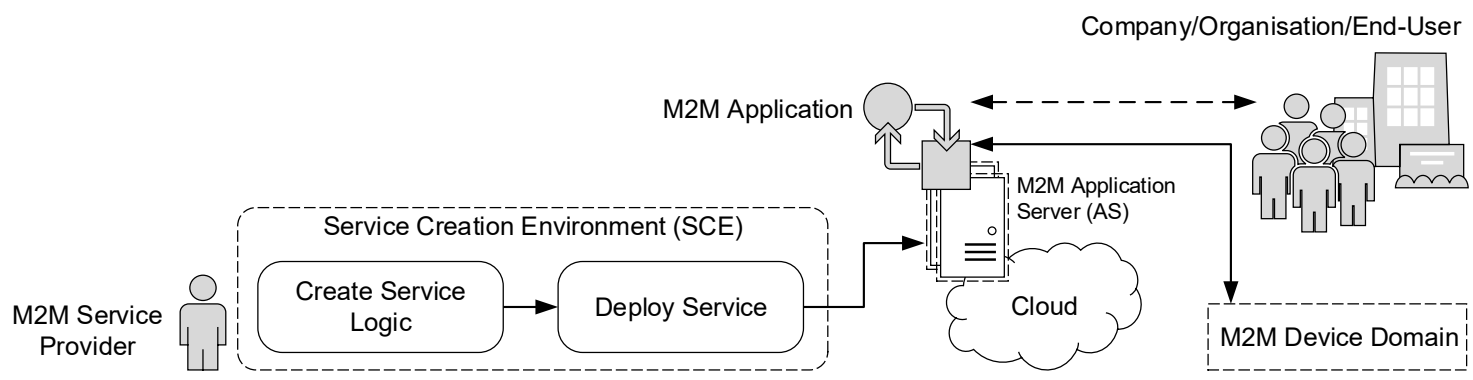

Figure 4.1: Traditional Approach for M2M Application Service Provision

As constituted in section 3.1 in the traditional approaches for provisioning M2M applications, highly specialised software developers design and implement M2M applications supporting specific business process. Such M2M applications are deployed on application servers (AS) running in centralised infrastructures like the Cloud or dedicated servers. Application consumers (companies, organisations, end-user) utilise these applications as a service. The M2M applications control and monitor devices located in the M2M device domains.

This research project proposes, as illustrated in Figure 4.2, that end-users design M2M applications corresponding to their personal requirements (Steinheimer et al., 2012a; Steinheimer et al., 2017a).

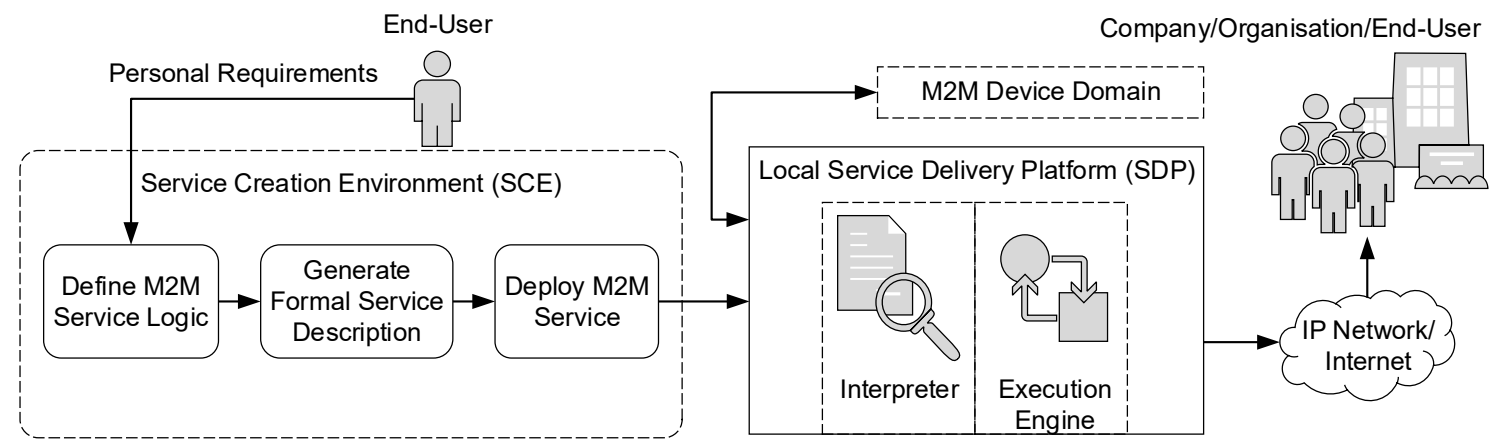

Figure 4.2: M2M Application Service Creation and Provision Process with End-User Integration 
According to (Steinheimer et al., 2013a) and (Steinheimer et al., 2015b) a formal service description language describes the M2M service logic, which is deployed in the local environment of the end-user that is also responsible for service execution (instead a central M2M AS). Here it has to be considered that end-users are no specialised software developers, but users with common technical knowledge. The designed applications can be provided as a service to other end-users or SPs (Steinheimer et al., 2013e).

This research defines conceptual models for M2M system architectures enabling autonomous decentralised M2M service provision as well as horizontal and bottom-up M2M service provision (Steinheimer et al., 2015a). This project additionally defines new conceptual models for M2M system architectures to realise cooperative M2M application service provision consisting of aggregation and composition of $\mathrm{M} 2 \mathrm{M}$ services provided by end-users (Steinheimer et al., 2013e). The conceptual models define novel methods to provide services and applications in $\mathrm{M} 2 \mathrm{M}$ application fields and therefore offer possibilities for new and highly flexible M2M applications. M2M applications, realised according to the defined models, are able to address application ranges not addressable by traditional M2M SPs such as control or monitoring applications including equipment located in end-users personal environment (e.g. reducing demand for energy by turning off or shifting energy-consuming devices or monitoring environmental conditions by accessing devices that include various sensors). The conceptual models are described subsequently and section 4.2 describes the functional architecture of the framework to realise these models. 


\section{Autonomous Decentralised M2M Service Provision (ADSP) Model}

As examined in section 3.2 the provision of an M2M service requires an MSP. Most of the current approaches for $\mathrm{M} 2 \mathrm{M}$ application provision require centralised infrastructures for hosting these service platforms. As examined in section 3.2, such centralised infrastructures result in less flexibility and dependence on the platform provider/operator as well as require many resources. More decentralised approaches including Edge or Fog Computing aspects execute the M2M services closer to end-users, but also do not avoid the dependence of an MSP provider or make it impossible to use the MSPs by end-users to deploy their individual services.

To avoid the disadvantages of centralised approaches for M2M service provision, this research project defines the ADSP model (illustrated in Figure 4.3) as fundamental structure of the proposed M2M system architecture (Steinheimer et al., 2013a; Steinheimer et al., 2015b).

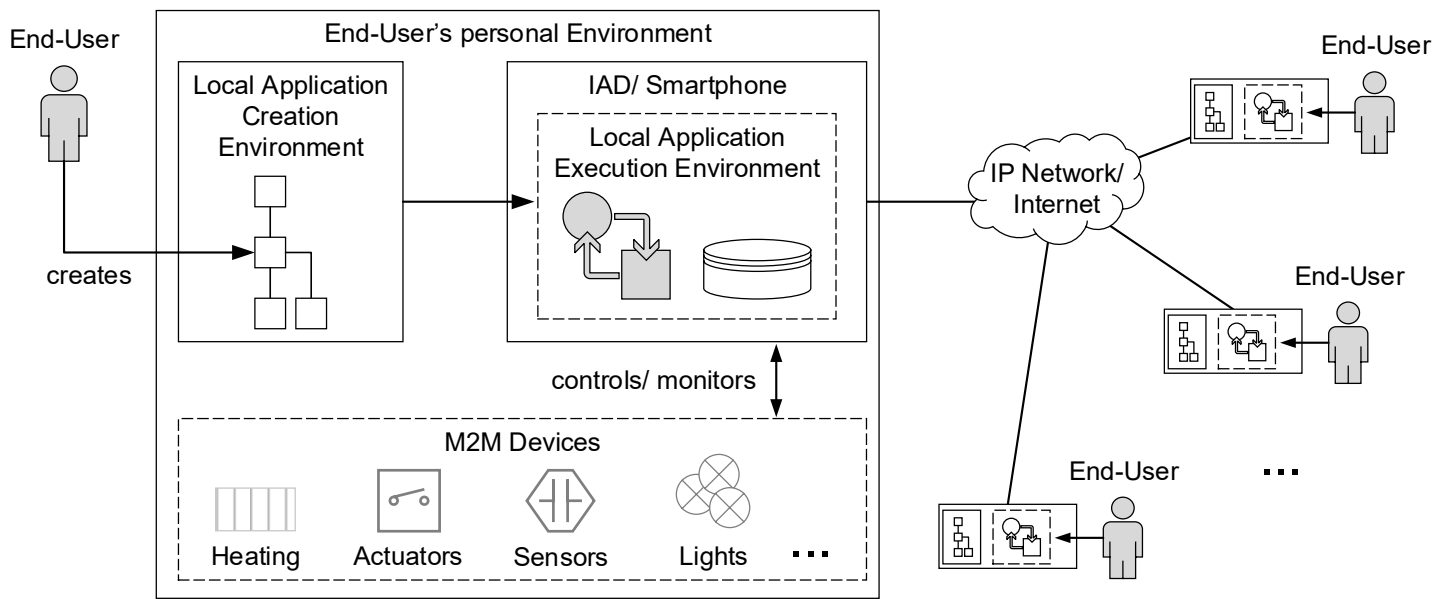

Figure 4.3: ADSP Model: Decentralised M2M Application Design and Execution

This conceptual model defines that end-users use the resources available in their personal environments to design and deploy M2M applications themselves (within their personal 
environments). The end-users provide the M2M applications without involving a central entity (e.g. a central MSP provider) or centralised execution environments for M2M applications. Therefore, the execution environment for M2M applications are arranged in the areas that are accessible and manageable by the end-users, which are their personal environments. The personal environment of end-users are either their permanent place of residence (home/apartment) or their current locations. In the further course of this research, the local execution environments will be linked so that end-users can realise cooperative services.

As section 3.2 defines the requirement that the execution system for the MSP does not require additional hardware, the Integrated Access Device (IAD) already existing at most permanent locations of end-users is used and the end-user's smartphone at the mobile location. The IAD represents the M2M-Gateway in end-users environments, which execute the M2M applications close to the end-user and processes the data where they are collected (Steinheimer et al., 2013a).

The ADSP model satisfies the requirements of the distributed system architecture and the end-user is included in the M2M application creation. Compared to the traditional centralised approaches for MSPs, the above-defined model avoids the resource-intensive provision of M2M applications by means of additional network elements since the execution environments use the locally available and cost-efficient resources. This will make better use of existing resources (network capacity, computing power), avoid bottlenecks, and reduce the cost of service creation, provision and maintenance. This satisfies the requirement that low hardware resources are required for platform provisioning. Furthermore, this model resolves the fixed binding to a central M2M 
application service provider (ASP) or a central execution platform, since the M2M applications are performed locally, which increases the independence of the application users from a central provider, and meets the data security and end-user privacy requirements.

The following section defines the HSPU model, which builds on the previously defined ADSP model.

\section{Horizontal M2M Service Provision and Utilisation (HSPU) Model}

As stated in section 3.2 the common concepts for MSPs allow end-users to consume M2M application services defined for their personal environments. However, end-users are not able to make the functionality of their M2M devices or the applications defined for their M2M device domain available to other end-users.

The HSPU model defined in this section extends the above-described ADSP model by providing the functionality of local $\mathrm{M} 2 \mathrm{M}$ devices and $\mathrm{M} 2 \mathrm{M}$ applications to other endusers as a service (Steinheimer et al., 2013e; Steinheimer et al., 2015a). Figure 4.4 illustrates the principles of HSPU model.

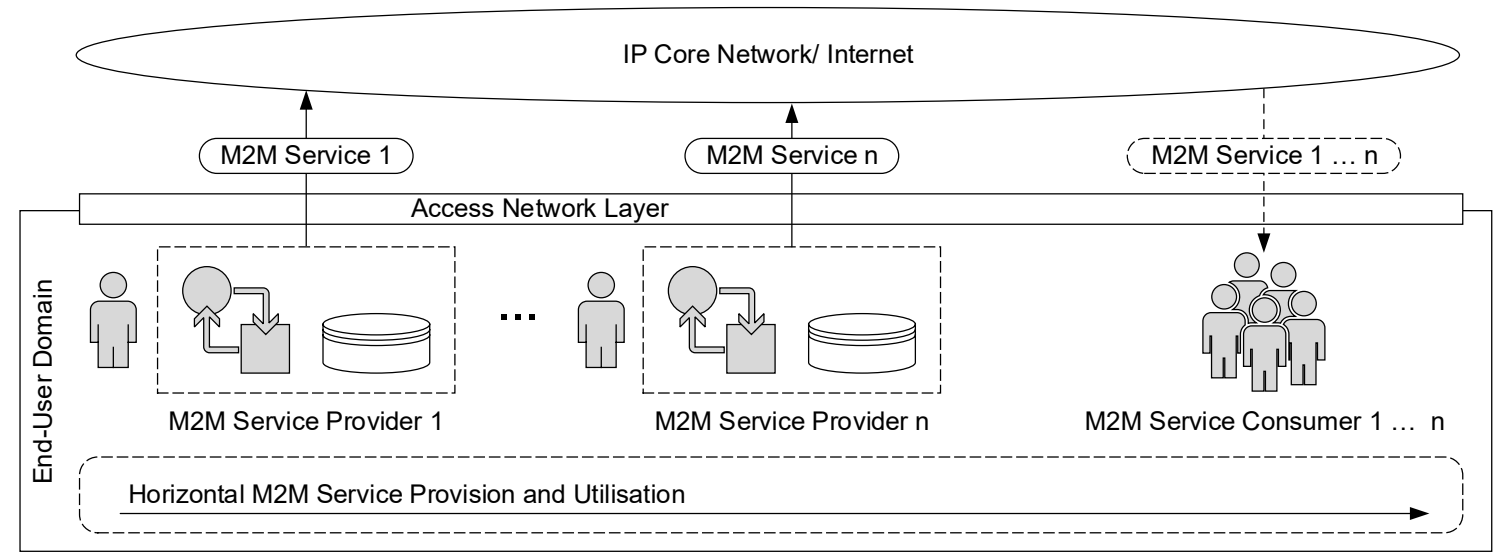

Figure 4.4: HSPU Model: Horizontal M2M Resource Provision and Utilisation 
The service provision and utilisation are performed horizontally at end-user level, i.e. without any central, mediating entity in the M2M system architecture. According to this model, an end-user takes on the role of an M2M SP and provides independent M2M services to other end-users that run in the execution environment of this M2M SP. The connection of M2M SP and M2M service consumer (SC) as well as every required information exchange occurs directly (P2P) between the participants (Steinheimer et al., 2012a).

A MSP based on the architecture implementing the HSPU model has the advantage that both, M2M service provision and utilisation are realised without any central entity and M2M SCs are independent of such entities. This again satisfies the requirement of decentralised system architecture. Additionally, realisation of the HSPU model enlarges the flexibility of an MSP because provided M2M services can address various application fields, not limited by the functionality of traditional MSPs that are often implemented for specific application domains. Because all end-users can publish their individual M2M services a large diversity of available services can exist (because of large number of different SPs). End-users can search for M2M services satisfying their individual requirements. Besides these advantages horizontal M2M service provision and utilisation reduces the costs for service development and provision because end-users provide their own resources and no additional system elements are required. The HSPU model includes a naturally redundancy of M2M services, because multiple M2M SPs can provide identical services.

The following section defines the DCASP model, which extends the above-specified conceptual models. 


\section{Decentralised Cooperative M2M Application Service Provision (DCASP) Model}

As stated in section 3.2 none of the related projects offer the functionality to combine individually provided M2M services to form a complex M2M application service on endusers level. Therefore, this section defines the DCASP model, which is based on the previous defined conceptual models. Figure 4.5 illustrates the principles of DCASP model.

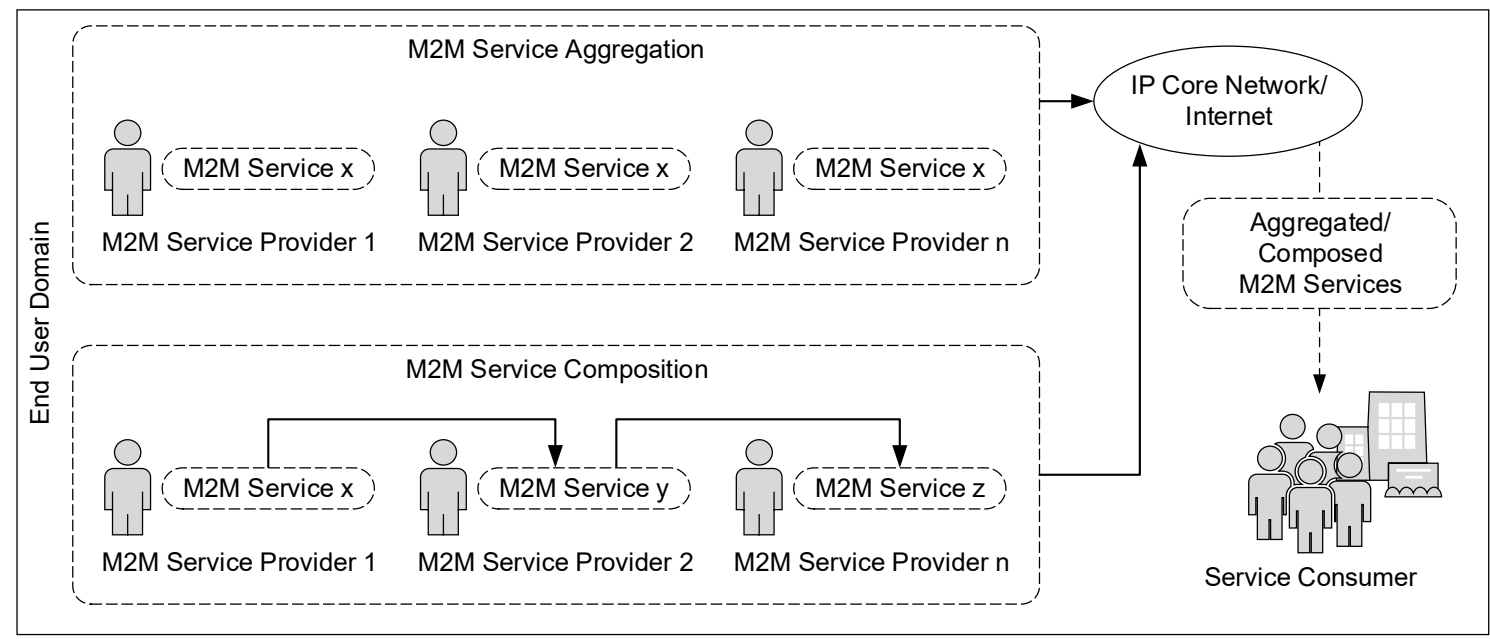

Figure 4.5: DCASP Model: Decentralised cooperative M2M Application Service Provision

In the DCASP model the individually provided M2M services are combined to build a complex M2M application (Steinheimer et al., 2013e). Other end-users or external SPs (companies/organisations) can utilise these complex M2M applications as a service. The DCASP model distinguishes two methods for decentralised M2M service combinations: service aggregation and service composition. In case of service aggregation end-users (peers) provide a cooperative $\mathrm{M} 2 \mathrm{M}$ application service by simultaneously offering identical local M2M services (with optional information exchange). In case of service composition end-users provide a cooperative M2M application service by concatenation (linking) of different individual M2M services. The M2M SPs and SCs are again linked 
via a P2P network without any central entity required to manage the service combination or information exchange.

A M2M system architecture that implements the DCASP model by the distributed provision of M2M application services, avoids the resource-intensive provision of M2M applications that central approaches use. This will make better use of existing resources (network capacity, computing power), avoid bottlenecks, and reduce the cost of M2M service creation, provision and maintenance. Because multiple end-users can deploy the same M2M application using this approach, the fixed relationship to a central M2M service or platform provider is resolved, which in turn increases the independence of the M2M application users.

The DCASP model offers the possibility to provide different M2M applications in a flexible way. Different M2M services can be flexibly combined. Through the cooperation of multiple M2M SPs on end-user level new M2M applications can be realised that could not be realised before because resources located in end-users' environments were not easy accessible. By combining several local M2M applications, various use cases can be realised that are only possible through the cooperation of end-users, such as the reduction of the load in the energy distribution grid. Such jointly provided M2M application services could provide an advantage to the end-users involved, if they receive appropriate incentives from the application SCs.

The following section describes the BSPU model which is based on the ADSP and DCASP model. 


\section{Bottom-Up M2M Service Provision and Utilisation (BSPU) Model}

The above-specified conceptual models offer new possibilities to make resources located in end-users personal environment accessible and enable the combination of them. The BSPU model (see Figure 4.6) extends the previously defined models by specifying that end-users can provide their individual or cooperative M2M services to external SPs. This was not possible before without centralised MSPs or defining certain terms and conditions between end-users and external SPs, which again would be complicated and individual solutions. (Steinheimer et al., 2015a; Steinheimer et al., 2016)

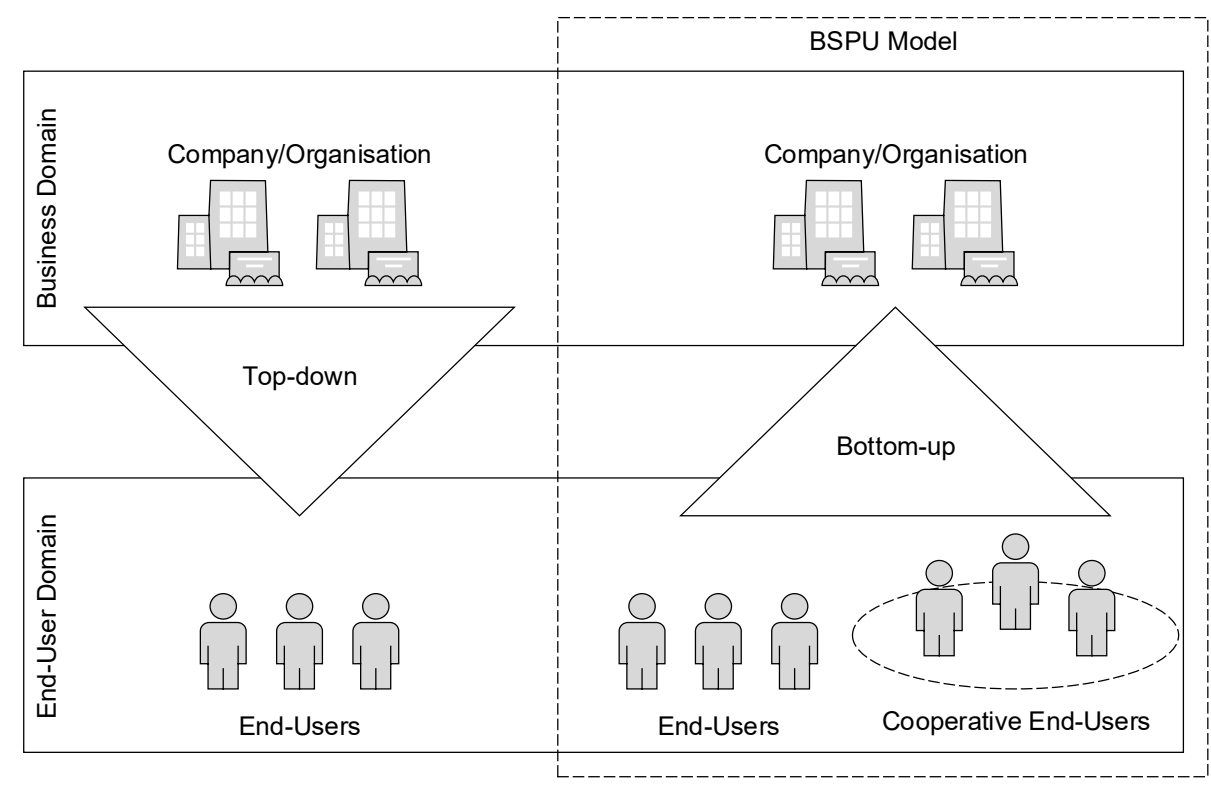

Figure 4.6: BSPU Model: Bottom-Up M2M Service Provision and Utilisation

The BSPU model reverses the direction of service delivery. Central SPs do not provide their services to end-users as before, but distributed end-users provide their M2M services to the central SPs.

External SPs usually are interested in information regarding the personal environment of end-users to use that information to create a benefit for their individual business 
processes. Another interest of external SPs could be to control M2M devices located in the personal environment of the end-user, such as energy consumers. The BSPU model enables central SPs to address the personal environment of the end-user as an application field of their M2M applications. This area was previously not addressable, e.g. because of legal restrictions or data-safety regulatory. It is only possible for central SPs to integrate the end-users' personal environment into their business processes, if the end-users provides their information data or control functionality by themselves to the central SP. Due to the customers' active participation in the provision of M2M services, it is within their discretion to determine the scope of control tasks or data collections in their personal environments.

\subsection{Framework Architecture and Components}

The previous section 4.1 introduced the principles of conceptual models that form the basis of the proposed novel framework for "Autonomous decentralised M2M Application Service Provision". The proposed framework aims to eliminate the gaps and disadvantages of current approaches of MSPs identified in section 3.1 by enabling the end-users to design individual M2M services and make them available to other end-users or central SPs. Additionally, the service providing end-users can cooperate with each other to provide complex M2M application services. Figure 4.7 illustrates the functional basis for the proposed novel framework for autonomous decentralised service and application provision in M2M application field, with focus on end-users domain. 


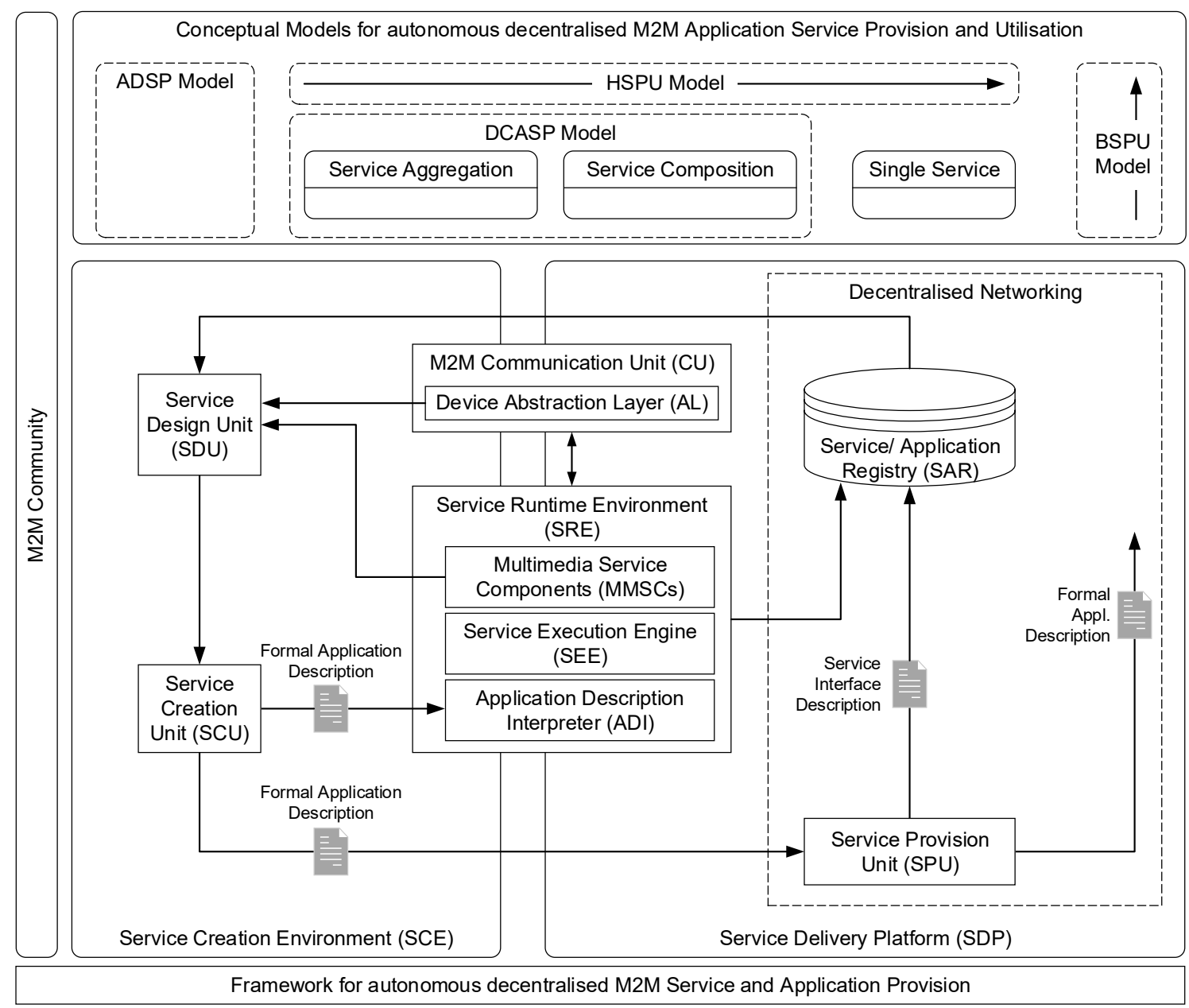

Figure 4.7: M2M Application Service Provision Framework Architecture

The framework consists of four essential parts: Service Creation Environment (SCE), Service Delivery Platform (SDP), Conceptual Models for autonomous decentralised M2M Application Service Provision and Utilisation, and the M2M Community (Steinheimer et al., 2012a; Steinheimer et al., 2013e). SCE and SDP are completely decentralised as they are located in end-users' personal environment. The task of the SCE is to provide functionality for application and service design by end-users. The SDP is responsible for $\mathrm{M} 2 \mathrm{M}$ application/service management and networking of the participating platforms in end-users domain. The M2M Community is a component in the proposed architecture supporting the networking of participants and the management of 
M2M services. The conceptual models are realised by means of the functional components of SCE and SDP. While section 4.1 described the principles of the conceptual models, the subsequently section describes the components of $S C E, S D P$ and $M 2 M$ Community.

\section{Service Design Unit (SDU)}

Integration of end-users implies that the end-users have the possibility to create M2M applications for their personal environment (e.g. their Smart Home). Currently no possibility for comfortable and simple M2M application creation exists as identified in section 3.1.

An intuitive development of M2M applications could be realised by a graphical development process and by modelling the behaviour of an M2M application independently from underlying technologies (Steinheimer et al., 2012b; Steinheimer et al., 2017b). In the e-SCHEMA research Project (e-SCHEMA, 2015) the graphical development of M2M applications was demonstrated. The use of the proposed principle of statemachine-based modelling was evaluated as a practicable approach by probands with different technical backgrounds. The majority (90 percent) of the candidates stated that they found it easy to model simple or complex M2M applications using the proposed approach. Therefore this approach was chosen as methodology to graphically model the semantics of an M2M application. The graphical design of M2M applications by endusers for their individual personal environments realises the integration of end-users. The $S D U$ provides the interface for graphically designing M2M applications via a Graphical User Interface (GUI). The definition of an M2M application is done by the end-users by modelling the behavior of the M2M application through the combination of graphical 
building blocks (refer to Figure 4.8). The graphical building blocks represent the control and monitoring functionalities of $\mathrm{M} 2 \mathrm{M}$ devices (available in their personal environment) as well as Multimedia Service Components (basic communication services). The definition of $\mathrm{M} 2 \mathrm{M}$ application semantic is abstracted from technical realisation meaning the end-user defines the application logic graphically and the designed application is automatically transformed into a formal description representing the application semantic (Steinheimer et al., 2013a; Steinheimer et al., 2017b).

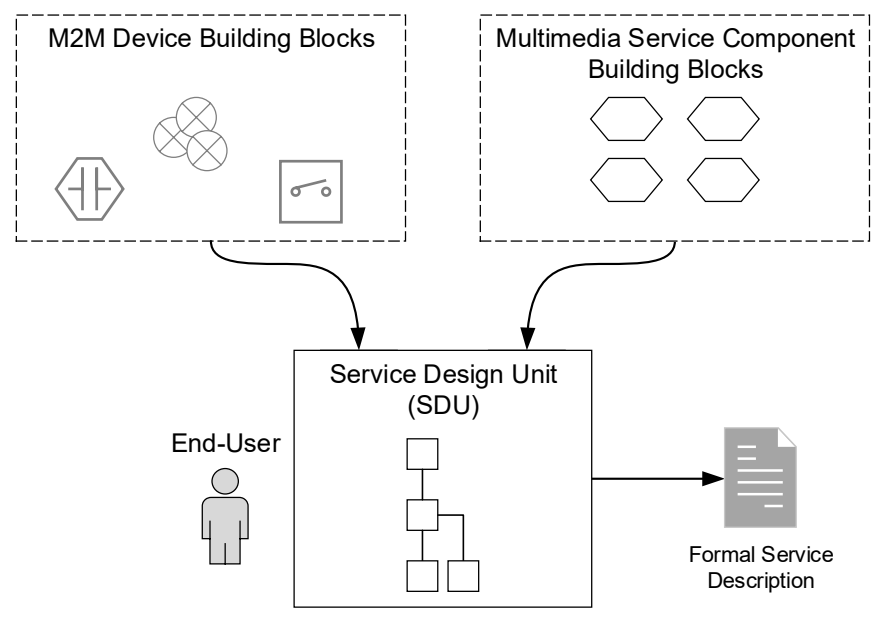

Figure 4.8: Framework Architecture: Service Design Unit (SDU)

The convenient graphical design of M2M applications enables end-users to create M2M applications for their personal environment according their individual requirements and satisfies the requirement of end-user integration into the process of M2M application design. The integration of end-users by offering the possibility to design individual applications expands the diversity of available M2M services enormous.

\section{Service Creation Unit (SCU)}

The approaches for creation and execution of M2M applications, described in section 3.1, often lack in portability because the service executables are dependent on the specific 
platform. I.e. the platform where the service executables are deployed is not simply exchangeable. Thus, the service executables are close-coupled to the platform and other execution environments cannot execute them. Only the INOX Managed Service Platform (Clayman and Galis, 2011) and the ENERsip platform (Lopez et al., 2013) make use of a declarative description of M2M application logic instead of programmatically implementing it, but the authors do not mention any standard used for the declarative description.

This project proposes an abstraction mechanism for service executables. The graphically designed M2M application logic is not transformed to application code that is dependent on the execution environment and therefore dependent on the platform. The $S D U$ (refer to Figure 4.9) automatically transforms the application logic into a formal application description using a unified, standardised and machine-readable formal description language describing the application semantic.

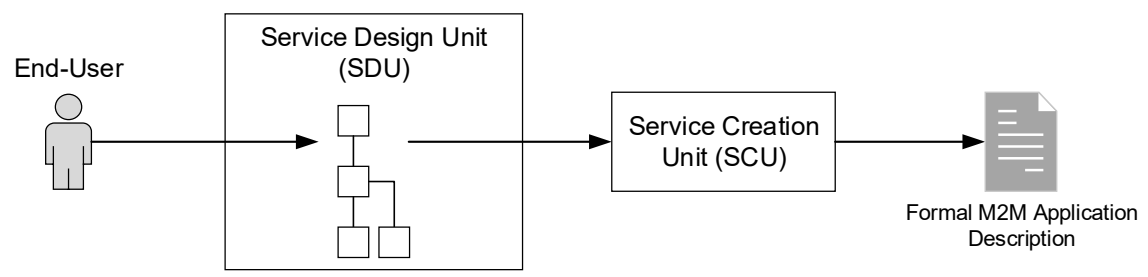

Figure 4.9: Framework Architecture: Service Creation Unit (SCU)

The formal application description can be parsed, interpreted, and the application logic described can be executed independently of the underlying execution environment. This makes the formally defined M2M application independent of the execution environment and can be easily ported to other execution environments, which meets the requirements of platform independence. Each execution environment that contains a parser for the unified and standardised formal description language is able to execute the application. 
The machine readability of the formal description language enables the fully automated application execution. The application of unified mechanisms for M2M application description using a standardised formal description language supports the realisation of an application description parser on different platforms.

\section{M2M Communication Unit (CU) with Device Abstraction Layer}

The intention of the presented concept is not only the integration of end-users, but also the M2M device domain of end-users. The basis of M2M application creation is the integration and the networking of M2M devices. M2M devices have different, mostly incompatible communication abilities (i.e. the M2M devices communicate via different M2M technologies). As a result, M2M devices that use communication technology A will not be able to communicate with other M2M devices that use communication technology B. Therefore, it is impossible to connect M2M devices using different communication technologies with each other for controlling or information processing.

The $M 2 M C U$, as illustrated in Figure 4.10, includes multiple M2M device technology interfaces and integrates different device technologies as most of the approaches introduced in section 3.1 do (Steinheimer et al., 2012a; Steinheimer et al., 2015b).

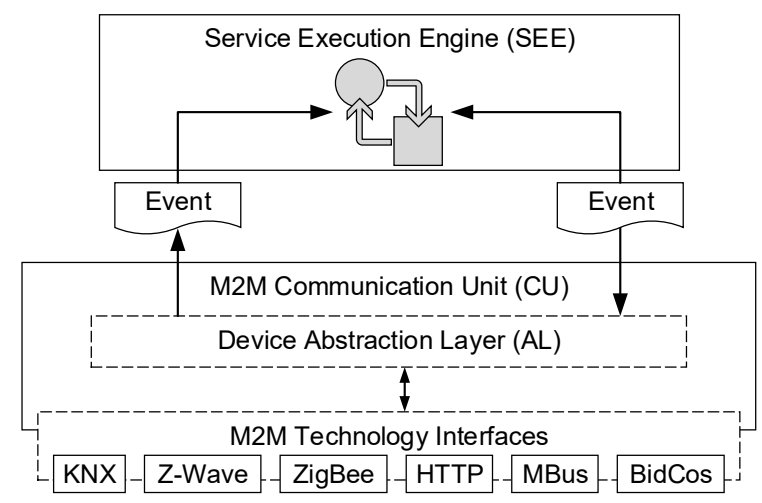

Figure 4.10: Framework Architecture: Communication Unit (CU) 
The unified integration of several M2M device technologies is realised by the Device Abstraction Layer (AL) component of the proposed framework, which is included in the $M 2 M C U$. According to (Steinheimer et al., 2012a) and (Steinheimer et al., 2015b) the Device $A L$ abstracts the communication between the execution environment and the different M2M device technologies. Different M2M devices are represented inside the M2M CU by a unified interface. An M2M application executed by the Service Execution Engine (SEE) integrates M2M devices using the unified interface. The M2M application uses this interface to request data from the M2M device or send control commands to it. The Device $A L$ converts the unified requests into technology specific requests so that they can be transferred to the M2M device via a specific M2M technology interface.

The Device $A L$ of the $M 2 M C U$ accomplishes the compatibility of different M2M device technologies. This part of the proposed concept also enables the integration of M2M device technologies defined in the future, by definition and integration of corresponding $\mathrm{M} 2 \mathrm{M}$ device technology interfaces into the $M 2 M C U$. This realises the separation and independence of service functionality from communication technologies of the M2M devices and satisfies the requirement for M2M device technology abstraction, as defined in section 3.2.

\section{Multimedia Service Components (MMSC)}

As identified in section 3.1 in most MSPs the integration of multimedia communication is not considered or rather not integrated as essential interface. Often in MSPs no interfaces exist that are simple operable and comparably flexible. 
This project proposes to provide reusable MMSCs as part of the SRE, which end-users can utilise to interact with the MSP using common multimedia communication equipment (Steinheimer et al., 2013a). Integration of multimedia communication forms an interface that is usable on several ways. Multimedia interfaces are usable by audio/video communication (e.g. audio/video call), by textual communication (Instant Message) or combined (speech recognition, text-to-speech). Therefore, the framework contains MMSCs the end-user can apply to generate an interface for $\mathrm{M} 2 \mathrm{M}$ applications. The interface can work as input and output interface or as control channel between MSP and end-user.

Central integration of multimedia communication enables the simple realisation of intuitive interfaces to MSPs. Through the integration of MMSCs it is possible to receive and exchange information as well as trigger control instructions, just using multimedia communication devices such as a mobile phone. The precondition to serve a multimedia interface still exists in the environment of most involved entities. Most end-user have a multimedia communication device (e.g. a Smartphone) and are familiar with operating it. Integration of multimedia communication extends the M2M onto the Machine-to-Human Communication via natural and human readable language.

\section{Service Runtime Environment (SRE)}

The SRE (see Figure 4.11) provides the MMSCs and contains the Application Description Interpreter (ADI) and the Service Execution Engine (SEE) for realisation of the M2M application execution. The $S R E$ receives the formal application description from the $S C U$ and executes the defined application logic on behalf of the $A D I$ (parsing and interpreting 
the formal application description) and the SEE (triggering the defined actions) (Steinheimer et al., 2013a; Steinheimer et al., 2015b).

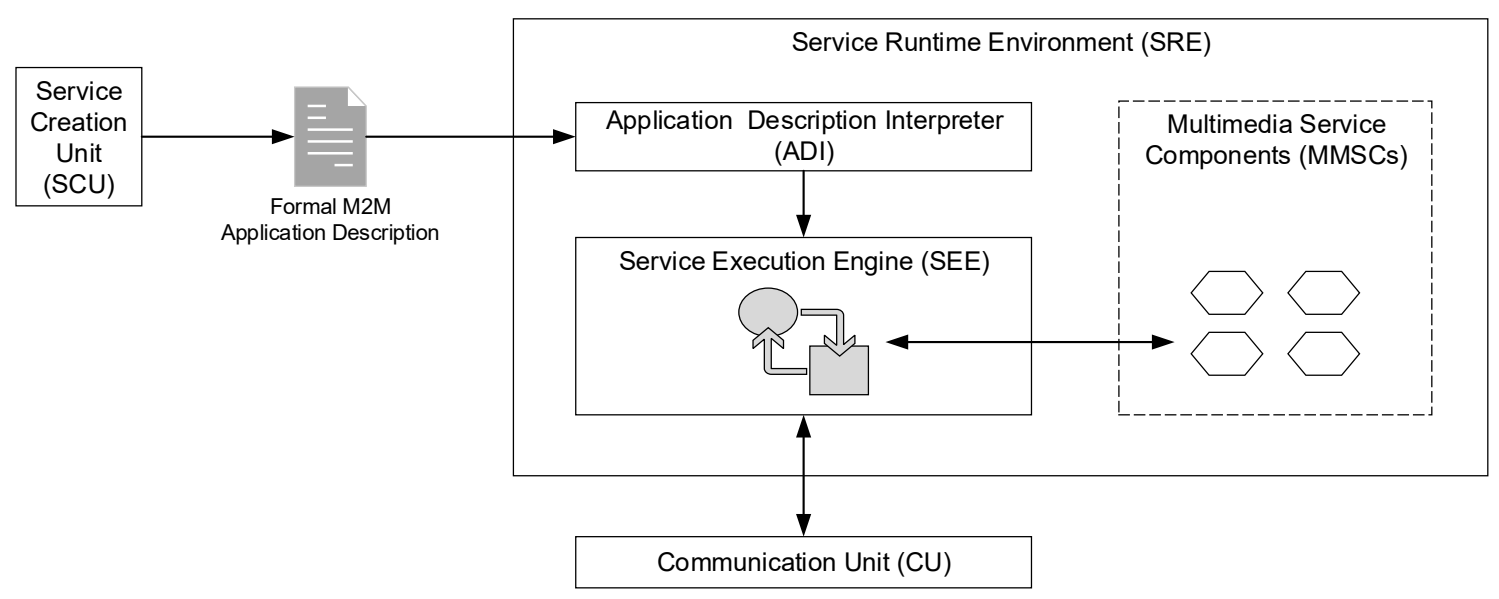

Figure 4.11: Framework Architecture: Service Runtime Environment (SRE)

Most of the projects introduced in section 3.1 realise the M2M application execution using remote execution environments or central components in their system architecture. As mentioned above the $S R E$ is located in the local environments end-users, i.e. the M2M application is also executed locally and the proposed concept does not require any central MSP, which satisfies the requirement of decentralised system architecture and also ensures the data safety and end-user privacy.

The components presented up to this point enable end-users to graphically create a local M2M application in a simple way and execute it locally. These framework components enable the realisation of the ADSP model defined in section 4.1. The framework components described subsequently are proposed to realise the HSPU and BSPU conceptual models. 


\section{Service Provision Unit (SPU) and Service/Application Registry (SAR)}

For realising the HSPU and BSPU model, a possibility is still required to provide the $\mathrm{M} 2 \mathrm{M}$ application as a service to other users. This is realised by the $S P U$ component, which extracts an interface description from the formal application description and registers it at the $S A R$. The interface description contains all necessary information describing the M2M service and its interfaces. Users who intent to use an M2M service offered by another user, request the $S A R$ for available M2M services and integrate the M2M service into their graphical application description. The $S P U$ contacts the M2M SP and requests the desired $\mathrm{M} 2 \mathrm{M}$ service.

The decentralised structure of the M2M system architecture presented in this project is an essential aspect of the designed framework. Figure 4.12 shows the designed approach as a layer model representing the functional architecture for networking of SPs and SCs as well as for realisation of M2M application services (Steinheimer et al., 2017a; Steinheimer et al., 2017b; Steinheimer et al., 2017c). The functionality of the respective layers is described below.

\section{Network Layer}

Since the M2M system architecture, as defined in section 3.2, should not require any additional resources accept the existing at end-users environment, the end-user's M2M gateways are connected with each other via an IP based communication network, respectively the Internet. Therefore, the networking fundamentals are realisable with minimal infrastructural costs. End-users can be located at different geographic locations 
as well as in different access networks (wireless mobile networks or fixed access networks), which are interconnected via the core network.

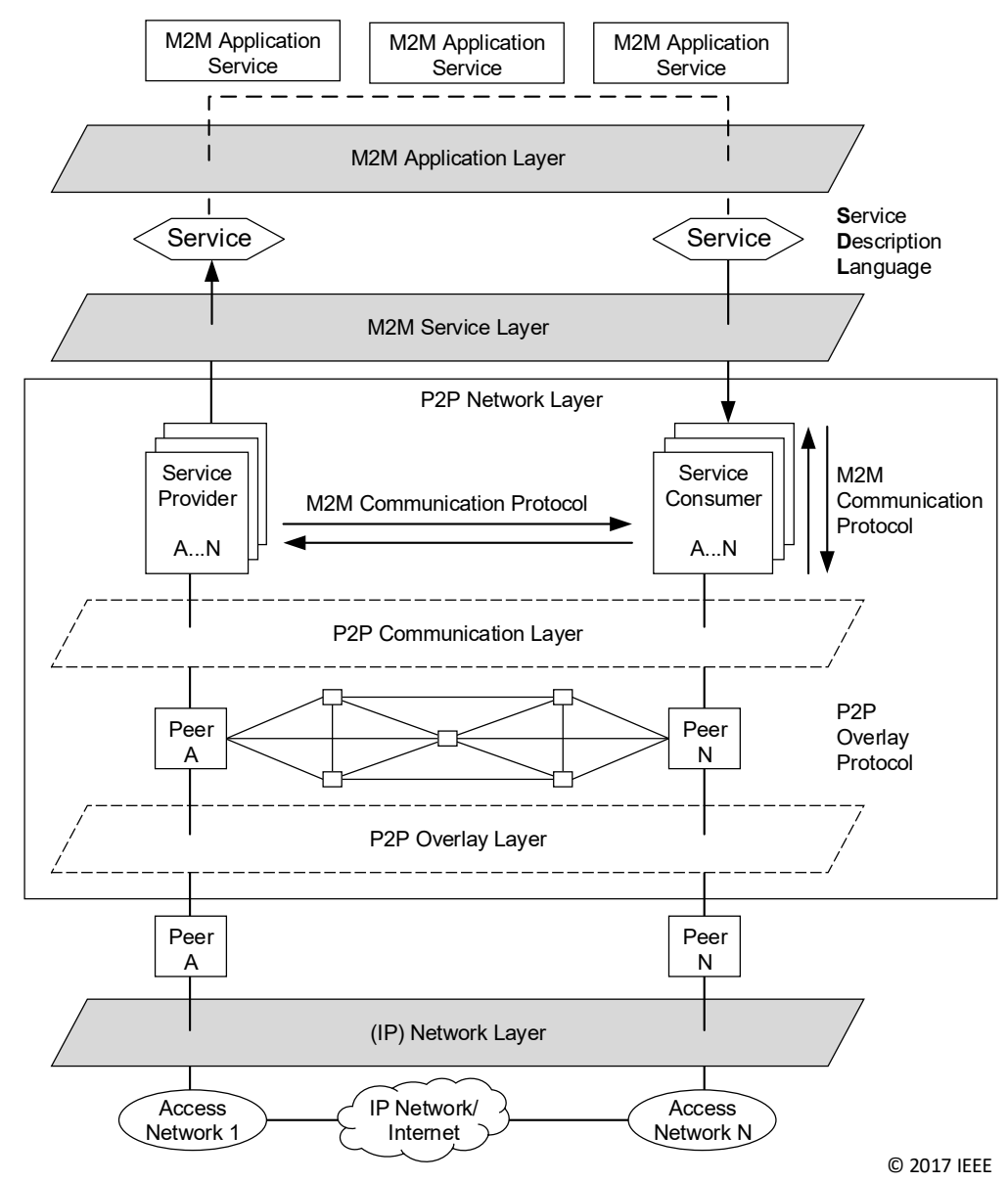

Figure 4.12: Layer Model of Decentralised Networking Aspect

\section{P2P Network Layer}

As defined as a requirement in section 3.2, the entire system architecture must be decentralised and no central stakeholders should be present. Therefore, end-user nodes represent equivalent entities and are connected to each other via a P2P network. Each end-user node in the M2M system architecture represents an individual peer in the P2P network providing or consuming a service or just behave as passive node supporting the P2P network infrastructure. The $P 2 P$ Network Layer includes the sub-layers $P 2 P$ Overlay 
Layer and $P 2 P$ Communication Layer realising a decentralised infrastructure for communication and data storage.

The P2P Communication Layer realises the information exchange between the participants (i.e. the peers). The information exchange between the participants for the service utilisation (service requests, confirmations) as well as the necessary signalling to generate cooperative applications automatically occurs directly between the peers using M2M communication protocols (e.g., CoAP, SIP). The communication in the $P 2 P$ Communication Layer occurs end-to-end between peers providing and consuming correspondent services. Peers that are not involved in a specific M2M application context are not integrated in the communication activities between M2M SP and SC.

In addition to the communication between the participants, the required data management in the M2M system architecture, such as registration of M2M services, must also be decentralised to prevent central components in the M2M system architecture. Distributed data storage can be realised with a P2P overlay network (e.g., Chord, Gnutella). The $P 2 P$ Overlay Layer realises this functionality by forming the P2P overlay network out of all existing end-user nodes. All end-user nodes are member of the P2P overlay network, independent if they are involved in a specific M2M application context or not. Any data storage in the presented M2M system architecture is decentralised via the end-user nodes within the $P 2 P$ Overlay Layer.

\section{M2M Service Layer}

The services defined and provided by the end-users are available via the M2M Service Layer and can be used via their corresponding interfaces. The service interface is 
described by an interface specification and is available in the M2M Service Layer (explained in section 6.1).

\section{M2M Application Layer}

The distributed M2M application services are realised within the M2M Application Layer. For this purpose, the services available via the M2M Service Layer are combined with each other following the same approach as defining local M2M applications (combination of graphical building blocks and application description using formal language). The exchange of information between the M2M services takes place via the $P 2 P$ Network Layer using P2P communication protocols.

The decentralised networking of nodes allows to realise the entire M2M system architecture decentralised without the inclusion of central entities. The application composition on the M2M Application Layer makes it possible to compose flexible applications from fine-grained, independent services. According to the proposed concept, the infrastructure is provided cooperatively by all participants, whereby the applicationspecific communication takes place between the nodes involved in the context of an M2M application. As a result, the effort required for networking is distributed to the nodes and does not stress a single system component that would have to be provided centrally. Because no central component is involved in the networking of nodes, the system architecture is not dependent on this single component. The entire M2M system architecture uses existing resources in the end-user domain such as existing IP/NGN network infrastructure. This makes it possible to realise a communication network for M2M without providing additional networking infrastructure. Using IP-based information exchange guarantees the applicability by everyone that has access to the 
Internet. Therefore, the realisation is possible with minimal costs. The P2P networking structure offers advantages like redundancy and scalability as well as data security and end-user privacy because necessary data storage is realised via the $\mathrm{P} 2 \mathrm{P}$ overlay network and distributed over the participating nodes that are decoupled from each other.

The following section describes the M2M Community component of the proposed framework system architecture.

\section{M2M Community}

Up to this point in the proposed framework concept for "Autonomous decentralised M2M Application Service Provision" it has not been discussed how the participating nodes, i.e. the end-users, can join the P2P network and how the presented framework manages different interests of end-users utilising the distributed MSP. To address these topics the designed concept includes the M2M Community component (see Figure 4.13).

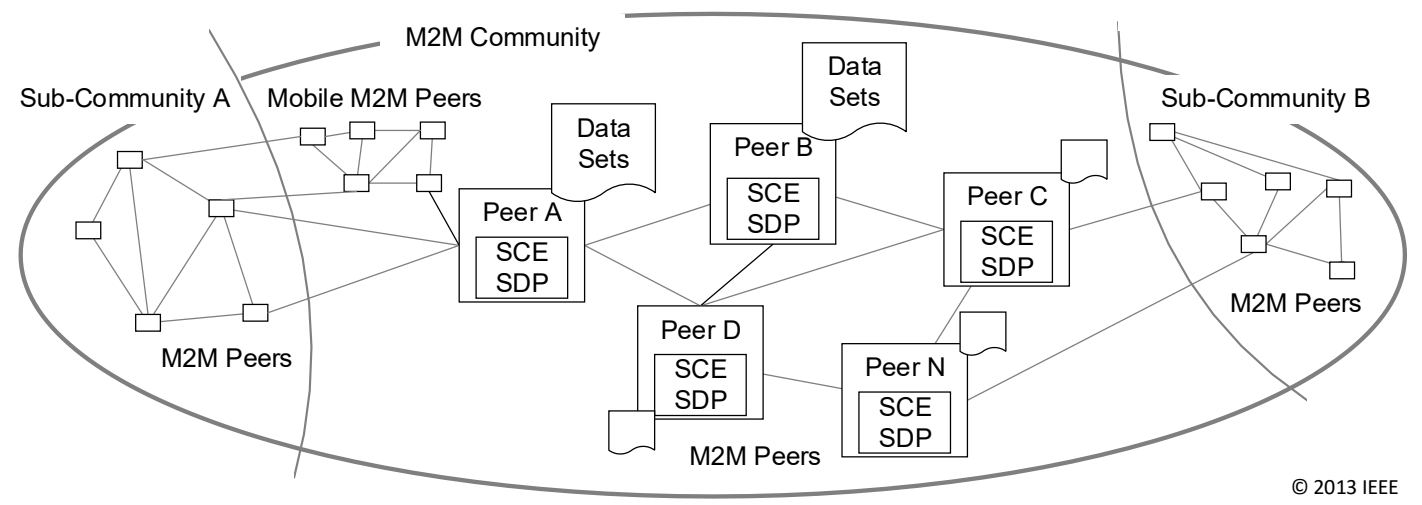

Figure 4.13: P2P connected M2M Environments within the M2M Community

The M2M Community extends the P2P networking approach by adding social networking functionalities to the P2P network (Steinheimer et al., 2012a; Steinheimer et al., 2013e). End-users that want to participate in the distributed M2M platform architecture join the 
4 Proposed Framework for autonomous decentralised Application Provision in M2M Systems

M2M Community and as part of the community can utilise the MSP functionalities. Therefore, the M2M Community forms the basis for networking the peers. The $M 2 M$ Community follows the principles of a social network in which users with the same interests group together. The M2M community has the task to create such groups of interest. The respective groups of interest are represented by sub-communities, which group the M2M services by their characteristics or the user groups of the M2M services. For this purpose, it is e.g. possible to form sub-communities that describe a specific geographic area in which M2M services are available, such as e.g. the neighbourhood of an end-user. Figure 4.13 illustrates the structure of the P2P connected nodes within the M2M Community consisting of various sub-communities. It shows that the nodes are connected P2P with each other inside the M2M Community and some peers form specific sub-communities. The M2M Community and its sub-communities form logical groups of M2M nodes independent of the geographical location of the peers.

\subsection{Conclusion}

This chapter introduced the concept of a novel framework to realise an M2M system architecture for "Autonomous decentralised M2M Application Service Provision". The combination of application and service provision by end-users with Fog Computing aspects of execution environments next to the end-users with possibility of information exchange between end-users individual platforms forms a novel concept for provision of $\mathrm{M} 2 \mathrm{M}$ application services and enables end-users to participate in application provision on end-users domain. 
Section 4.1 introduced the general concept of the proposed framework proposing conceptual models for M2M service design and provision: The ADSP model that enables end-users to design individual M2M applications by modelling the behaviour of $\mathrm{M} 2 \mathrm{M}$ applications using an intuitive GUI. The designed M2M applications are executed in the local SDP of the end-users and therefore does not require any remote or centralised entity involved in the M2M application execution process. The HSPU model enables end-users to provide the designed $\mathrm{M} 2 \mathrm{M}$ application functionality as a service to other end-users. The BSPU model enables the provision of the M2M service to external SPs not located in the end-user domain, such as central distribution grid operators. Both models again use the resources already present in end-users environment and therefore do not require any additional hardware or networking resources. The DCASP model enables the different M2M SPs to combine their M2M services as a service aggregation or a service composition and provide the combined M2M application service to other end-users or external SPs.

Section 4.2 introduced the proposed framework architecture and components consisting of SCE and SDP as well as M2M Community. The SCE provides the functionality for M2M application design and automatically generates a formal application description out of the designed graphical model. The formal application description is processed for application execution in the SDP component of the framework. The SDP additionally provides the functionality for $\mathrm{P} 2 \mathrm{P}$ networking of the participating nodes to realise the decentralised data exchange between M2M SPs and SCs or required decentralised data storage functionality. Finally, the M2M Community concept realises the management functionality of different end-user interests and user groups as well as forms the entry point to the decentralised MSP. 
The introduced novel framework concept enables addressing M2M application fields that traditional concept of MSPs are not able to address, such as monitoring and control functionalities in end-users personal environment. The proposed framework concept has the advantage that the M2M system architecture is realisable with cost efficient resources already existing in end-user's environments and therefore existing resources (e.g. network capacity or computing power) are used more efficient. Decentralised M2M application service provision additionally prevents bottlenecks as well as reduces the costs for M2M application service creation/provision/maintenance and resolves the binding to a specific central MSP provider.

This chapter defined the basis for "Autonomous decentralised M2M Application Service Provision". The following chapter 5 covers aspects of M2M application creation with native end-user integration and local execution of M2M applications. The aspects of cooperative application provision through the composition of distributed M2M services are discussed in chapter 6 . Both chapters introduce related projects that are used to derive the optimal approaches for the design of individual framework components. 



\section{Autonomous M2M Application Provision}

Chapter 4 introduced the principles of the proposed concept for "Autonomous decentralised M2M Application Service Provision". This chapter 5 introduces the parts of the framework responsible for M2M applications design by end-users and their execution in local M2M environments. The next chapter 6 will cover the distributed and cooperative M2M application service provision. Section 5.1 presents the Multimedia Service Components that this project proposes as part of the local M2M platform to provide an interface between end-user and M2M applications using existing multimedia communication equipment. Afterwards section 5.2 focuses on the proposed principles of application design by end-users via graphical application behaviour modelling. It describes an intuitive, platform independent methodology to define M2M application semantics. Section 5.3 presents an approach of a GUI that enables end-users to graphically design the behaviour of an M2M application by combining building blocks representing the M2M devices in their personal environments and previously mentioned Multimedia Service Components. Section 5.4 presents the concept to integrate different M2M device technologies into the local M2M platform and make them available to be integrated in $\mathrm{M} 2 \mathrm{M}$ applications. For this, an abstraction mechanism is specified enabling $\mathrm{M} 2 \mathrm{M}$ application definition without regard of the specific M2M technologies used to communicate with the M2M devices. Section 5.5 goes back to the principles of M2M application definition and presents an approach for describing state machine-based applications using a modelling language. After selecting an appropriate modelling 
language, the discussion introduces a formal description of the designed M2M application behaviour that allows automated execution of the service logic. Finally, section 5.6 defines the principles for processing the formal M2M application description by parsing and interpreting the defined semantics and executing the service logic.

\subsection{Multimedia Services Components}

End-user integration is an important aspect of this project. For this reason it is proposed that an M2M system is not only limited to the communication between M2M devices, but also that end-users themselves can communicate with an M2M application. This extends the functionality of traditional M2M systems with machine-to-human communication capabilities. Thus end-users can be informed about events that occur in an M2M system or can actively control an M2M application. For this purpose, the requirement was defined in section 3.2 that an M2M Service Platform (MSP) should have multimedia communication interfaces via which end-users can communicate with the M2M system, since the required equipment for communication already exists in end-users' environment. In order to realise these communication interfaces, this section introduces the Multimedia Service Components (MMSCs) which, as mentioned in section 4.2, can be integrated by end-users as interfaces into their individual M2M applications.

A typical scenario should assume that end-users have a smartphone or other multimedia over IP capable end device, which is used to establish an interface to the M2M application. In such a scenario, a smartphone may send and receive text messages as well 
as audio and video calls. This offers the following possibilities to interact with the M2M platform or with an M2M application:

- Natural Language - Natural language that is converted to text and processed in the M2M application or natural language that is generated and played back via the audio channel.

- Dual-Tone Multi-Frequency (DTMF) Signalling -DTMF signalling can be used for signalling during a telephone call using key tones of telephone key pad (ITUT Q.23, 1993 and ETSI ES 201 235-1 V1.1.1, 2000).

- Text Message - A text message sent to or generated by the M2M platform to transmit text-based information.

- Video - A video stream can be transmitted, generated by a video source inside the M2M environment during a video call.

In order to enable these communication capabilities, it is proposed that the MMSCs introduced below use the User Agent (UA) (IETF RFC 3261, 2002) integrated in the enduser's Integrated Access Device (IAD) to communicate with the end-user's multimedia devices (refer to Figure 5.1). The MMSCs are designed to make use of the Session Initiation Protocol (SIP) (IETF RFC 3261, 2002) for communication with IAD UA, respectively with the end-user's multimedia device because SIP is the common standard protocol in multimedia communication for controlling communication sessions and most end-user IADs are equipped with a SIP stack to serve for public telephony connections. (Steinheimer et al., 2013a) 


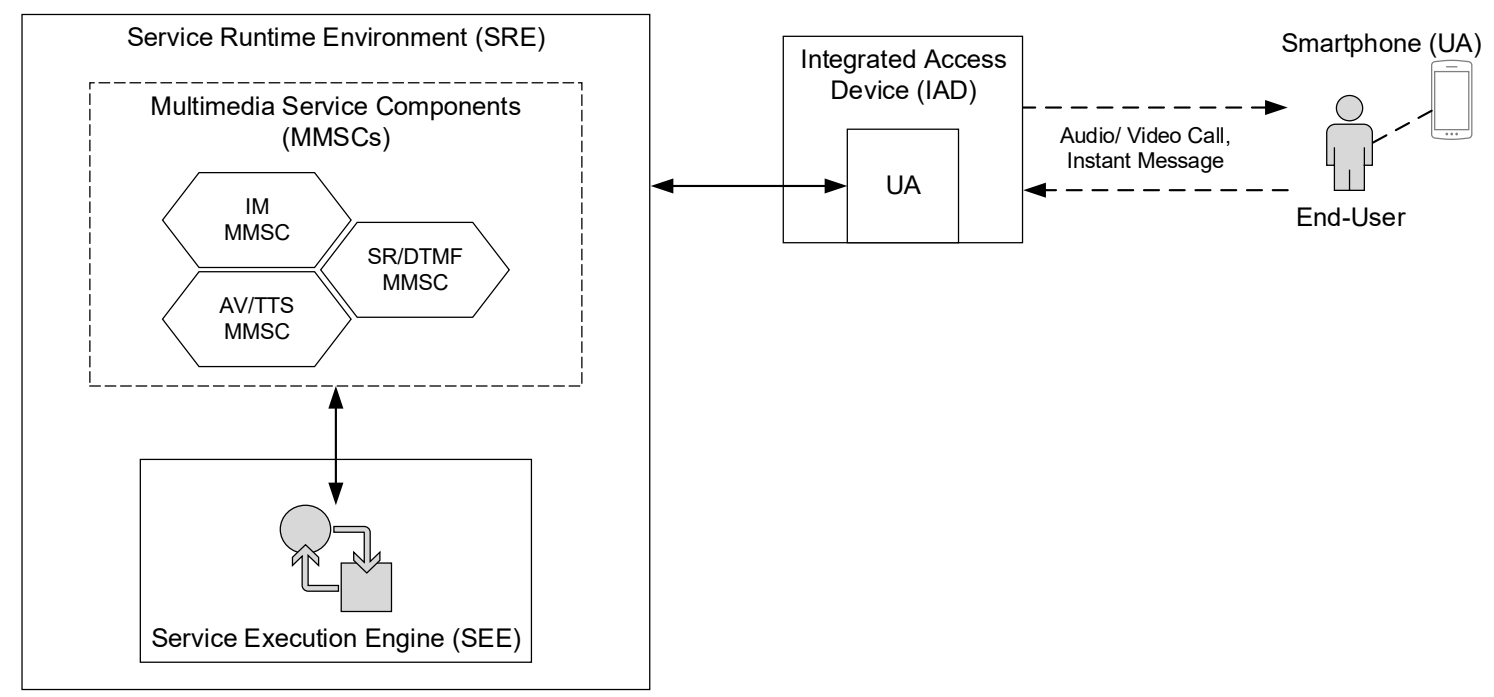

Figure 5.1: MSP Multimedia Interfaces

\section{Speech Recognition/DTMF (SR/DTMF) MMSC:}

First the $S R / D T M F M M S C$ (see Figure 5.2) is introduced offering the functionality to receive audio calls with a SIP UA, which is integrated in the local M2M platform. The designed $S R / D T M F M M S C$ can serve as an easy operable input interface to the local M2M platform in two ways:

- Detect Spoken Text - During an audio session, this $M M S C$ performs speech recognition (SR) to detect spoken text by analysing the received Audio Stream.

- Detect DTMF Signals - During the audio session, end-users have the possibility to generate DTMF signals using the dial pad of their multimedia devices.

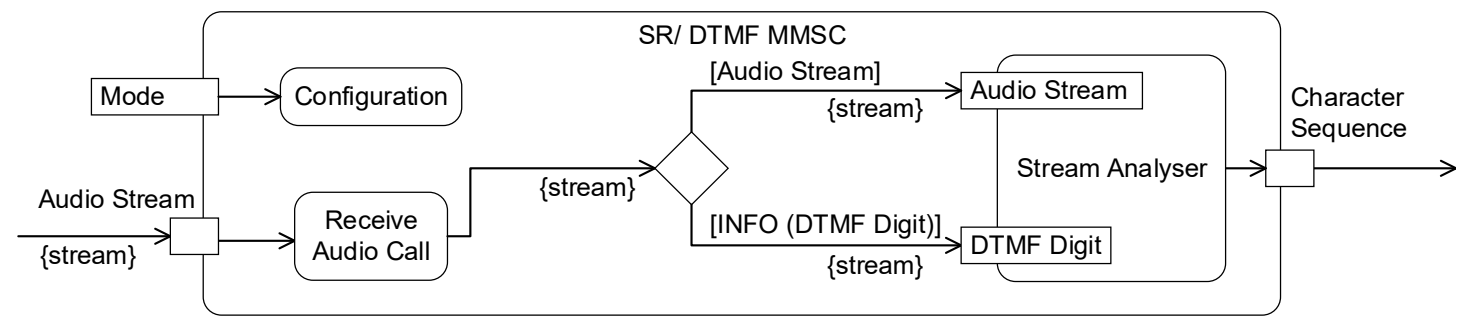

Figure 5.2: Architecture of SR/DTMF MMSC 
The SR/DTMF MMSC provides either the text detected from the Audio Stream or the DTMF Digit sequence as an input value to the local M2M application. It consists of the three parts Receive Audio Call, Stream Analyser, and Configuration. The Receive Audio Call part receives the call initiated by the End-user UA executing a SIP Three Way Handshake (IETF RFC 3261, 2002 and IETF RFC 3665, 2003). The platform UA accepts the call by confirming the session establishment. After the audio session has been established, the End-User UA starts transmitting the Audio Stream. The SR/DTMF MMSC has two options for configuration specifying if it serves detecting DTMF signals or spoken text. The configuration is managed by the Configuration part of this $M M S C$ in dependence on the Mode parameter. When the MMSC receives the Audio Stream, it starts analysing the Audio Stream to detect either the spoken text or the DTMF Digit sequence (depending on the configuration). If the $S R / D T M F M M S C$ is configured for detecting spoken text, the Stream Analyser performs the SR. If it is configured for detecting DTMF signals the Stream Analyser detects the DTMF Digits sent by the End-User UA via one SIP INFO request per DTMF Digit (IETF RFC 6086, 2011). After call termination the SR/DTMF MMSC provides the detected Character Sequence for further processing to the local M2M application.

Figure 5.3 illustrates the parameter set of the SR/DTMF MMSC. The Input parameter Audio Stream is assigned internally by the Receive Audio Call part of this MMSC and represents the Audio Stream that corresponds to the RTP (Realtime Transport Protocol) (IETF RFC 3550, 2003) stream received by the platform UA. The Output parameter Character Sequence is assigned by the Stream Analyser of the SR/DTMF MMSC and represents the character sequence detected by this $M M S C$ during audio stream analysis. This Output parameter is usable for further processing in M2M applications. 


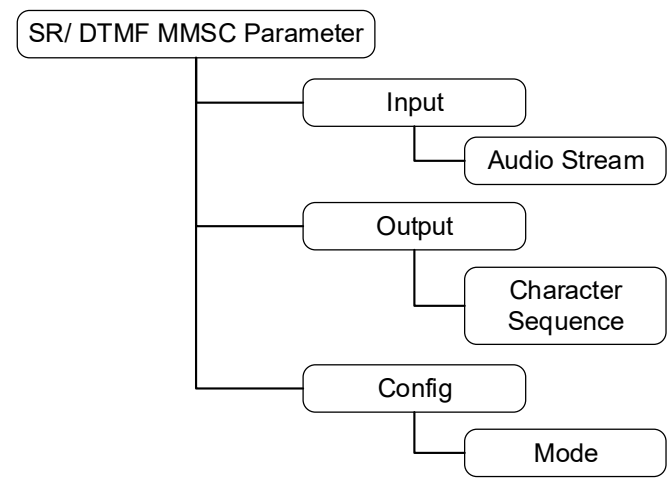

Figure 5.3: SR/DTMF MMSC Parameter Set

The Config parameter Mode specifies the operation mode of the MMSC. This parameter has to be specified by the end-user during application design for configuring this $M M S C$ to be operated for SR (Mode = "SR Call") or for DTMF recognition (Mode = "DTMF Call").

The following MMSC has been designed to be used as output interface for M2M applications.

\section{Audio/Video Text-to-Speech MMSC (AV/TTS MMSC):}

The AV/TTS MMSC (refer to Figure 5.4) offers the functionality to establish an audio/video call using the SIP UA integrated in the local M2M platform. This MMSC, has two options for configuration to serve as an output interface of M2M applications:

- Play back Announcements - Based on predefined text this MMSC can generate an announcement and play back this announcement during the audio session.

- Forward Audio/Video Streams - During the audio or video session, this MMSC can forward Audio and Video Streams generated by multimedia sources connected to the local M2M platform, such as a surveillance cam or door intercommunication system. 


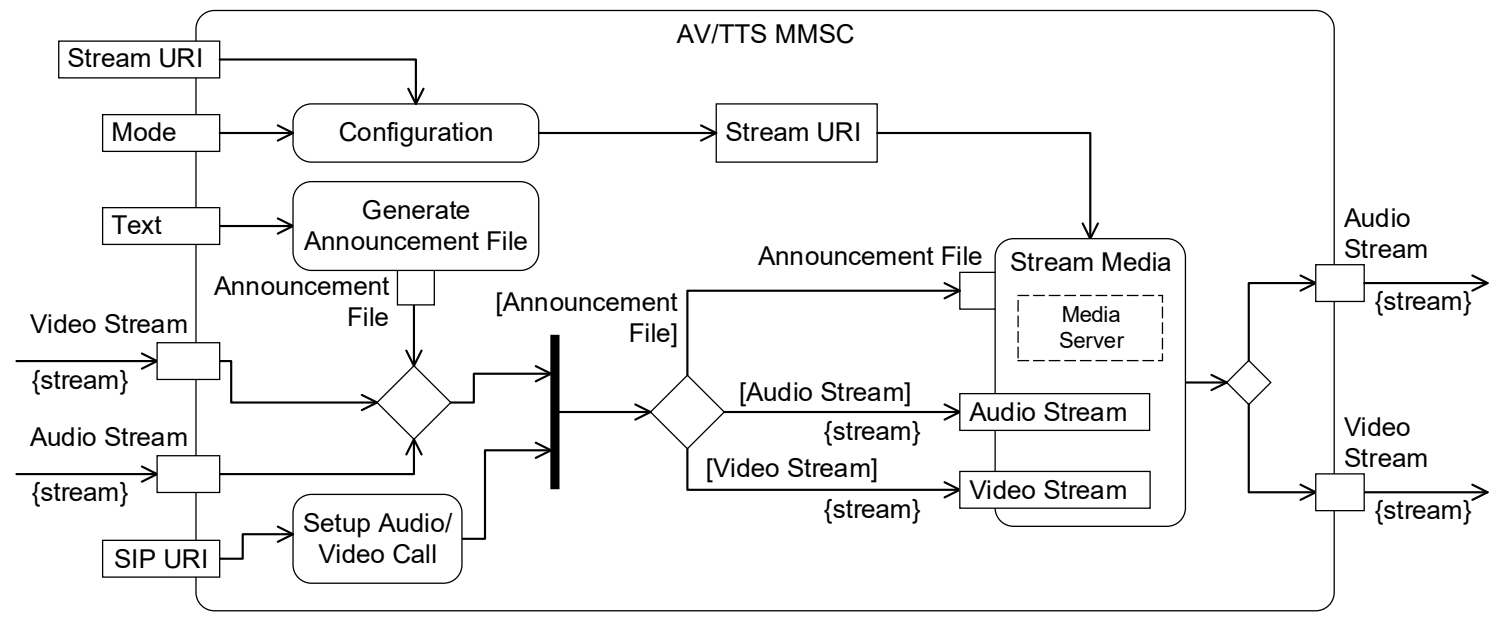

Figure 5.4: Architecture of AV/TTS MMSC

The AV/TTS MMSC consists of the parts Configuration, Generate Announcement File, Setup Audio/Video Call, and Stream Media. The Setup Audio/Video part establishes an audio or video session with the target UA of the end-user. The required address information of receivers are specified via their SIP URIs (IETF RFC 3261, 2002) and defined by the SIP URI input interface parameter of this MMSC. The Configuration part manages, in dependence on the Mode parameter, whether the MMSC serves for generating announcements (TTS) or forwarding an Audio or Video Stream. The Stream Media Part contains a local Media Server element for generating or forwarding audio/video streams. Depending on the configuration, the Stream Media part forwards Audio or Video Streams, which are connected to the Audio or Video Stream input interfaces, to the End-User UA or plays back an announcement file. The Generate Announcement File part, when MMSC is configured for TTS, generates an Announcement File (audio) out of the text sequence defined via the Text input parameter. After the Announcement File has been generated and the call is established with the End-User UA, the local Media Server starts play back of this announcement. 
Figure 5.5 illustrates the parameter set of the AV/TTS MMSC. The Input parameter Text has to be specified by the end-user during application design. It defines the text that the AV/TTS MMSC announces after the call has been established with the End-user UA. The Input parameters Audio and Video Stream is assigned internally by the Stream Media part of this MMSC representing the Audio or Video Stream that corresponds to the stream that the MMSC should forward. The Input parameter SIP URI has to be specified by the enduser during M2M application design and corresponds to the destination address of EndUser $U A$ that should receive the call.

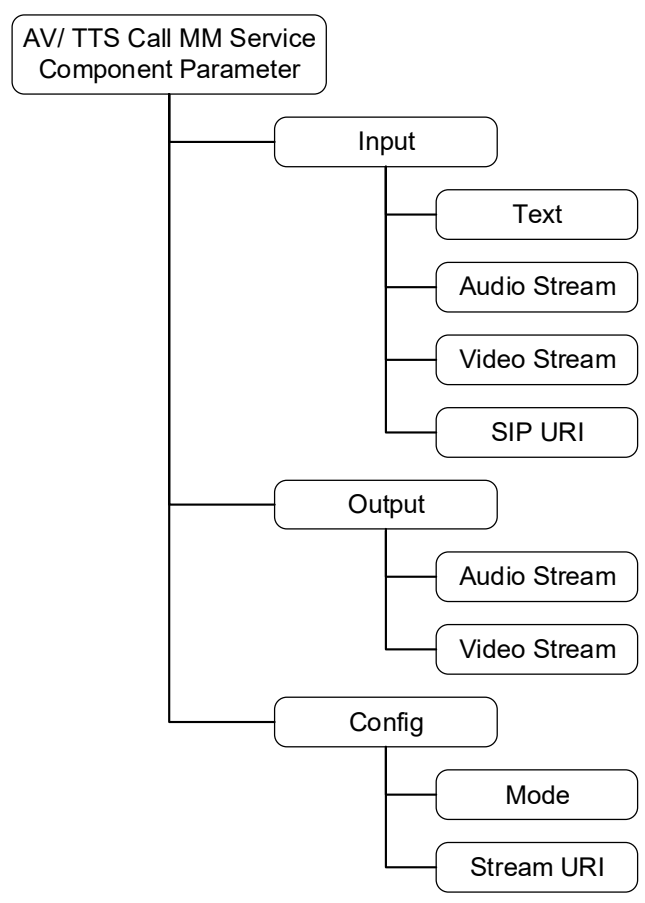

Figure 5.5: AV/TTS MMSC Parameter Set

The Output parameters Audio and Video Stream are assigned by the Stream Media part of the MMSC. They represents the Audio or Video Stream that is send to the End-User $U A$. The Config parameter Mode specifies the operation mode of the MMSC and has to be specified by the end-user during application design for configuring if the $M M S C$ forwards audio stream $($ Mode $=$ "audio"), video stream $($ Mode $=$ "video") or announce a 
predefined text $($ Mode $=$ "TTS"). The Config parameter Stream URI has also to be specified by the end-user during application design and defines the source address of the stream to be forwarded to the End-User UA, such as Real Time Streaming Protocol (RTSP) (IETF RFC 2326, 1998) URI (e.g. rtsp://192.168.0.22/surveillanceCam Indoor.stream1) often used for requesting RTSP media streams of local multimedia devices.

The following MMSC has been designed to be used as both, text-based input and output interface for M2M applications.

\section{Instant Message MMSC (IM MMSC):}

The IM MMSC (refer to Figure 5.6) offers the functionality for text-based communication with M2M applications using the SIP UA integrated in the local M2M platform. This MMSC offers communicating abilities in the following ways depending on its configuration:

- Receiving Instant Message (IM) - The MMSC receives an IM and extracts the message text for further processing in local M2M applications.

- Sending Instant Message - The MMSC generates an IM based on a character sequence for transmitting information generated by the M2M applications.

The IM MMSC has been designed to serve as communication interface for both, input and output interface using the SIP protocol extension for Instant Messaging specified in (IETF RFC 3428, 2002). 


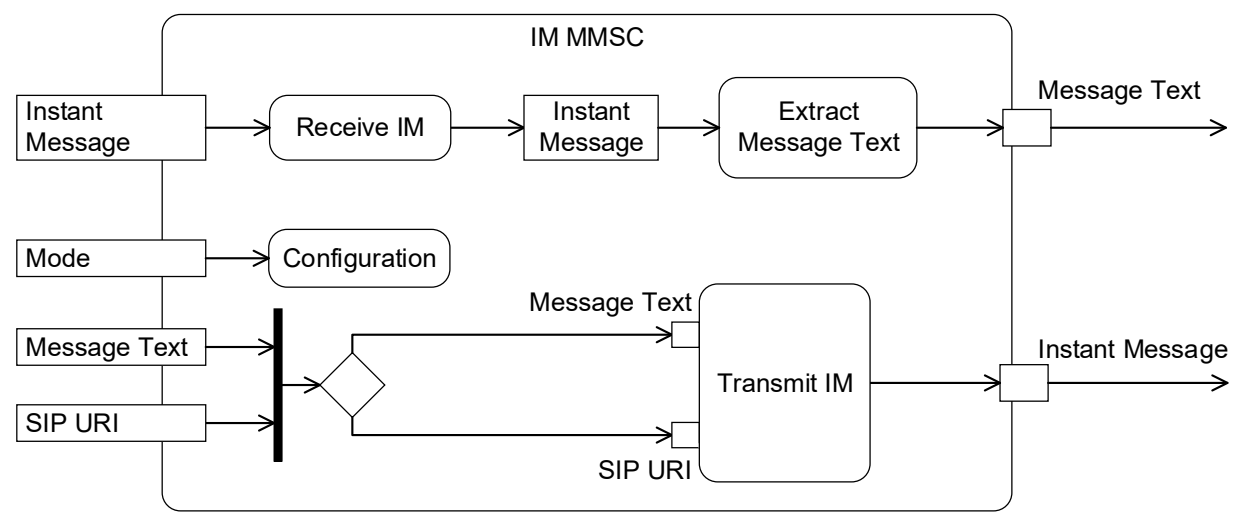

Figure 5.6: Architecture of IM MMSC

The IM MMSC consists of the parts Receive IM, Configuration, Extract Message Text, and Transmit IM. The Configuration part manages the configuration of this MMSC in dependence on the Mode parameter, which specifies whether the MMSC serves for generating IMs or receiving IMs. The Receive IM part realises the ability to receive IMs sent by the End-User UA using SIP protocol. The Extract Message Text part extracts the message text from the received IM and provides it via the output interface Message Text of the IM MMSC for further processing in the M2M application. The Transmit IM part realises sending of an IM by generating a SIP request of type MESSAGE carrying the message text in its message body and sends it to the End-user UA.

Figure 5.7 illustrates the parameter set of the IM MMSC. The Input parameter Instant Message is assigned internally by the Receive IM part of this MMSC and represents the IM received by the Platform UA. The Input parameter Message Text has to be defined by end-users during application design. It specifies the message text that should be transmitted to the End-user UA. The Input parameter SIP URI has to be defined by the end-user during application definition and represents the recipient address (SIP URI) of the IM generated by the IM MMSC carrying the text-based information. The Output parameter Instant Message is assigned internally by the Transmit IM part of that MMSC 
representing the SIP MESSAGE generated by the IM MMSC that is transmitted to the End-user UA. The Output parameter Message Text is assigned by the Extract Message Text part of that MMSC corresponding to the message body of the received IM, respectively the text-based information send from End-user $U A$.

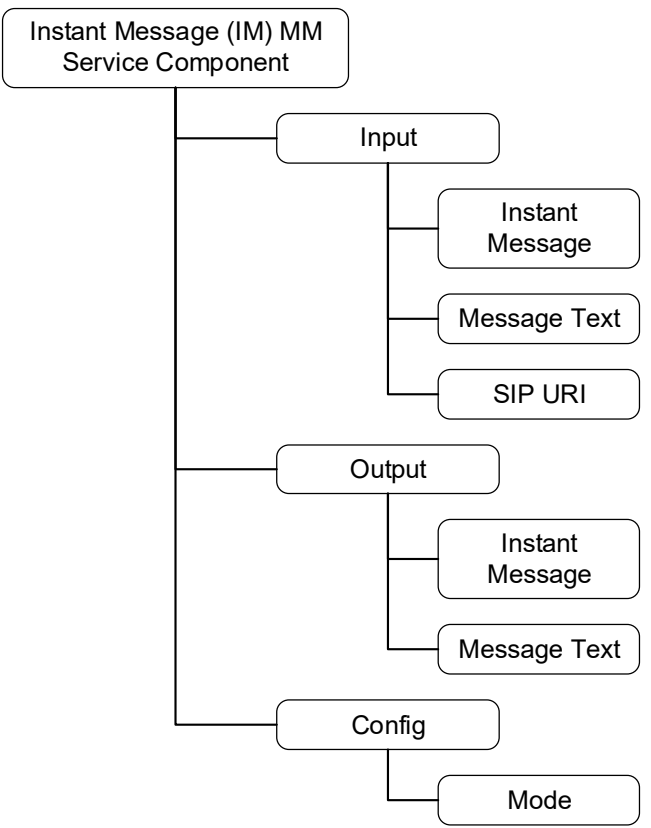

Figure 5.7: IM MMSC Parameter Set

The Config parameter Mode specifies the operation mode of the IM MMSC. The Mode parameter has to be specified by the end-user during application design for configuring that MMSC to be operated as input interface (Mode = "inIM") or as output interface (Mode = "outIM"). It can be stated that the MMSCs proposed in this section extend the interface functionality of the MSPs presented in section 3.1 by the use of multimedia communication. The designed interfaces can be used with existing equipment, so no additional devices need to be purchased.

The following section 5.2 introduces the methodology to create M2M applications by the end-user in a comfortable and intuitive way. 


\subsection{Application Behaviour Modelling}

As identified in section 3.1, in existing platforms for providing $\mathrm{M} 2 \mathrm{M}$ applications, the configuration of automation tasks or the creation of application logic is highly dependent on the implementing systems. Thus, the tools for definition of M2M applications can be indicated as domain specific languages, which are utilised for creation of $\mathrm{M} 2 \mathrm{M}$ applications that are strictly bound to the executing system. A portability of the configured applications to another, different system is not possible without redefining the application (suitable for the new execution platform). Therefore, this research proposes a methodology for application creation following MDA (Model Driven Approach) principles derived from (OMG, 2017a) by defining a platform independent application model (Steinheimer et al., 2017b). Such an abstract application model is expressed by a modelling language describing the behaviour of an application, separated from the technology-specific realisation of it (OMG, 2014; OMG, 2017a). The definition of the abstracted model is independent of the realising platform and can therefore be modelled without specific knowledge about the platform that implements the application (Petrasch and Meimberg, 2006). The executing system has to interpret the abstract description of the application and convert it into a specific structure that is appropriate to the executing system. In the concept designed in this project end-users graphically create a behaviour model of the M2M applications, which allows them to define the M2M application semantic without having specific knowledge of the executing platform (Steinheimer et al., 2017a).

In order to specify an adequate methodology how end-users can intuitively model the behaviour of an M2M application, this project proposes that end-users design state 
machines (SMs) describing the activities of an application by connecting building blocks representing M2M devices and MMSCs (Steinheimer et al., 2017c). According (Rupp et al., 2007) SMs describe the behaviour of a system that is specified using states. A SM describes how a system residing in a specific state acts at specific events. This approach to describe the behaviour of a system has been derived because end-users are able to understand the principles behind (i.e. describing sequence of activities) as described subsequently.

Devices have specific functionalities that are executed when the devices are triggered (e.g. powered on). The functionality of a device is used to perform an activity that corresponds to the functionality of the device. The end-user knows in principle how a device works and is familiar with the use case that devices are connected with each other such as illustrated in Figure 5.8. It shows that if the end-user triggers a device (switch button by pushing), the switch button activates the lighting.

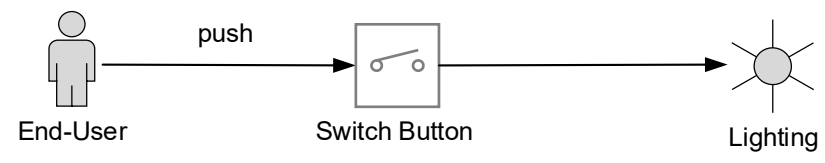

Figure 5.8: Use Case Switch Lighting

End-users are also familiar in utilising services, respectively service components, such as illustrated in Figure 5.9. It shows an end-user that uses an email service utilising an appropriate software, respectively service component on a personal computer (PC) and inputs the text as well as the email address of the receiver. The service component has also a specific functionality, which is to generate the email and sends it to the receiver defined by the end-user as input parameter. 


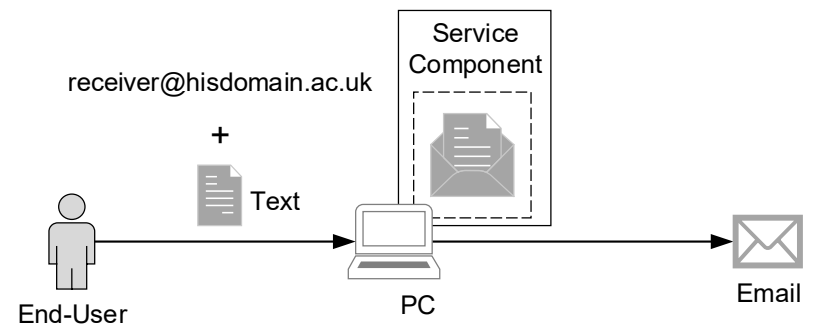

Figure 5.9: Use Case Email Service

Considering both use cases, end-users are able to relate that devices are connected to each other. It is irrelevant whether they exchange data (as in M2M environments) or are interconnected by an electrical connection (as in traditional electrical circuits). More generally speaking, devices and services have inputs from which an activity results which in turn generates outputs. At this point, three classes of devices are specified that can be present in the personal environment of an end-user:

- Actuators - Actuators control actions depending on the inputs and supply output values if necessary.

- Sensors - Sensors are used to acquire data which they supply as output values, possibly triggered by an input.

- Combined - These devices cannot be assigned directly to one of the other groups, since they offer both, possibility to control and supply of sensor values. E.g. a surveillance camera offers abilities to detect movements and to communicate this as an event and can also be triggered to start recordings.

Figure 5.10 illustrates the general structure of $M 2 M$ devices/MMSCs defined to specify a general perspective of them (but also of M2M applications). They can have multiple Input and Output parameters as well as Config (configuration) parameters whereby all of them in general perspective are optional. 


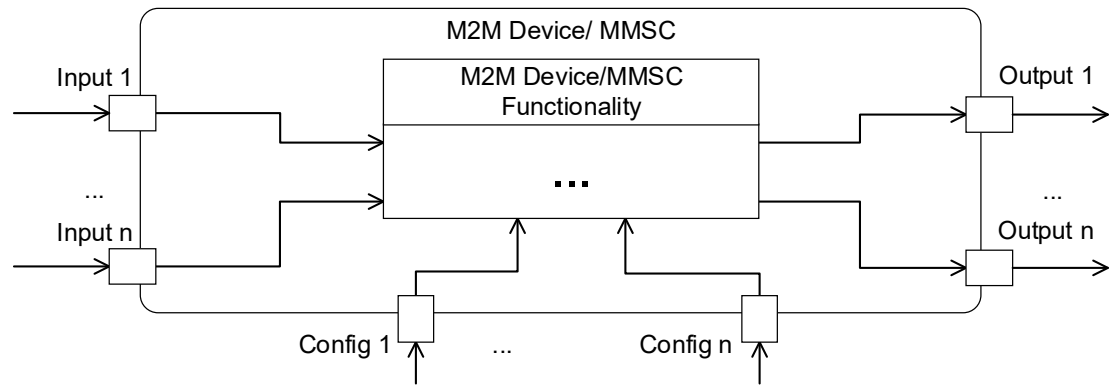

Figure 5.10: General Structure of M2M Device and MMSC

- Input Parameter - Input parameters are processed from an M2M Device/MMSC to perform the M2M Device/Service Functionality. Input parameters can be set statically or connected with an Output parameter of another M2M Device/MMSC.

- Output Parameters - Output parameters are generated by the M2M Device/MMSC as a result of the performed activity. Output parameters can be queried for inspection or directed as an Input parameter to another M2M Device/MMSC.

- Config Parameters - Config parameters are used to (statically) predefine specific configuration options of M2M Devices/MMSCs.

From a general perspective, end-users can define M2M applications by connecting the Output parameter of one M2M Device with the Input parameter of another M2M Device. It is defined at this point that not only M2M Devices can be combined, but also $M 2 M$ devices and MMSCs because they work according to the same principle (input, processing, and output). Figure 5.11 shows the general representation of connections between M2M Devices and MMSCs as described above.

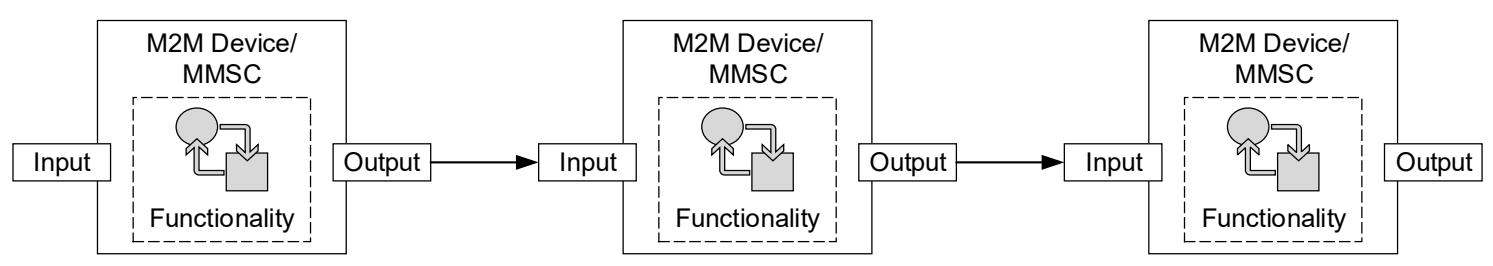

Figure 5.11: General Representation of M2M Device and MMSC Connections 
In general, the behaviour of an application can be defined as devices/services that are in a particular state and depending on the input data they generate an output, which in turn is passed on to another device or service. A SM, in particular a deterministic Finite State Machine (FSM) specifying this behaviour is used in the concept designed in this project. A deterministic FSM $A$ is formally defined according (Vossen and Witt, 2016) as follows.

$$
\mathrm{A}=\left(\sum, \mathrm{S}, \delta, \mathrm{S}_{0}, \mathrm{~F}\right)
$$

Thereby $\sum$ is the input alphabet and $S$ is the state quantity of $A, S_{0} \in S$ is the start state, $F \subseteq S$ is the quantity of final states, and $\delta: S \times \sum \rightarrow S$ is the transition function of $A$ (Vossen and Witt, 2016).

Figure 5.12 exemplarily shows a simple FSM derived from (Wagenknecht and Hielscher, 2015). Figure 5.12-a shows the FSM definition and Figure 5.12-b shows the corresponding transition function that can be illustrated according (Böckenhauer and Hromkovic, 2013) as a table. Figure 5.12-c shows the graphical representation of that FSM. This FSM can be described as follows: The FSM consists of three states $S_{0}, S_{1}, S_{2}$. The FSM starts at state $S_{0}$. If the current state of FSM is $S_{0}$ and the input is " 0 " then the current state moves to state $S_{1}$. If the input is " 1 " the current state moves to $S_{2}$. As both states, $S_{1}$ and $S_{2}$ are end states the SM stops processing after moving to state $S_{1}$ or $S_{2}$.

$$
\begin{aligned}
\Sigma & =\{0,1\} \\
\mathrm{S}_{0} & =\mathrm{S}_{0} \\
\mathrm{~F} & =\left\{\mathrm{S}_{1}, \mathrm{~S}_{2}\right\} \\
\mathrm{S} & =\left\{\mathrm{S}_{0}, \mathrm{~S}_{1}, \mathrm{~S}_{2}\right\}
\end{aligned}
$$

(a) FSM Definition

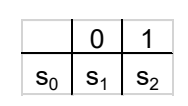

(b) Transition Function

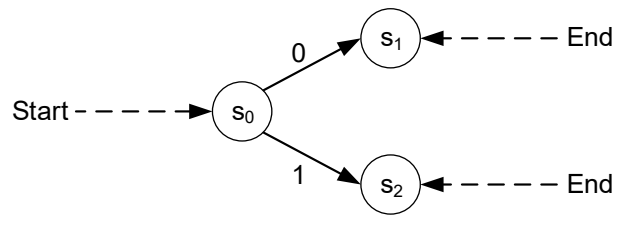

(c) Graphical Representation of FSM

Figure 5.12: FSM derived from (Wagenknecht and Hielscher, 2015) 
To model an application consisting of linked M2M devices and MMSCs with a FSM, the components of M2M applications are mapped to the elements of the FSM as specified in Table 5.1.

Table 5.1: M2M Application Component FSM Element Mapping

\begin{tabular}{|l|l|l|}
\hline \multicolumn{1}{|c|}{$\begin{array}{c}\text { M2M Application } \\
\text { Component }\end{array}$} & FSM Element & \multicolumn{1}{c|}{ Description } \\
\hline $\begin{array}{l}\text { M2M Device/MMSC } \\
\text { Component }\end{array}$ & State & $\begin{array}{l}\text { M2M Device/MMSC components are assigned to the states } \\
\text { of a FSM representing them inside the FSM. }\end{array}$ \\
\hline $\begin{array}{l}\text { M2M Device/MMSC } \\
\text { Component Connection }\end{array}$ & Transition & $\begin{array}{l}\text { Connections between the M2M Devices and MMSCs are } \\
\text { assigned to transitions connecting the states of a FSM and } \\
\text { representing the information flow between M2M Devices } \\
\text { and MMSCs inside the FSM. }\end{array}$ \\
\hline
\end{tabular}

The following Figure 5.13 illustrates exemplarily the M2M application as defined by Use Case 1 (refer to section 2.4) represented as a FSM. The graphical representation of the FSM is shown in Figure 5.13-c.

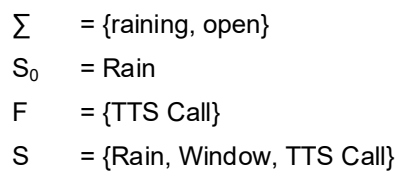

(a) FSM Definition Use Case 1

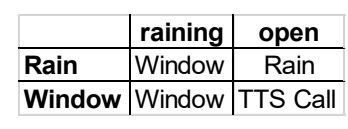

(b) Transition Function Use Case 1

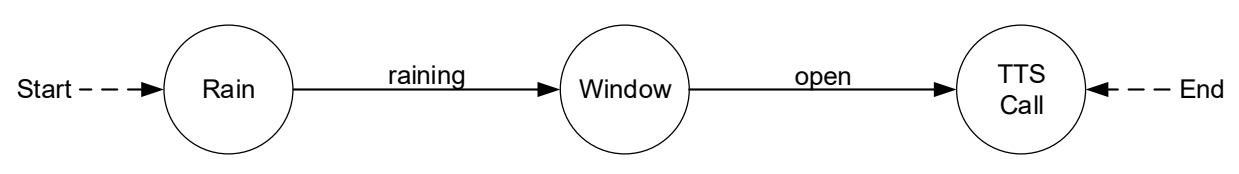

(c) Graphical Representation of FSM

Figure 5.13: M2M Application Use Case 1 represented as FSM

To realise Use Case 1, an application is defined that detects whether it rains with the help of a rain sensor as M2M device. Furthermore, within the application, a window sensor is used as M2M device to check if the window is opened. If it rains and the window is opened at the same time, a corresponding information should be transmitted to the end- 
user by phone call. This is done using the AV/TTS MMSC defined in section 5.1, which is configured to announce a specific text using TTS.

According to the above defined principles the M2M devices and MMSC are mapped with the states as specified in Table 5.2. Thus the state quantity S (refer to Figure 5.13-a) of the FSM is $\{$ Rain, Window, TTS Call $\}$.

Table 5.2: M2M Application Component FSM State Mapping Use Case 1

\begin{tabular}{|l|l|}
\hline M2M Application Component & \multicolumn{1}{|c|}{ FSM Element } \\
\hline M2M Device Rain Sensor & FSM state Rain \\
\hline M2M Device Window Sensor & FSM state TTS Call \\
\hline
\end{tabular}

Since the M2M application should check first if it rains, the start state $S_{0}$ is specified as Rain (Rain Sensor). As the M2M devices and MMSC have been assigned to the states of the FSM, the connections between them need to be specified. This is done by first defining the input alphabet $\sum$ representing the sensor states. Therefore, the input alphabet $\sum$ is $\{$ raining, open $\}$. Additionally, the Transition Function $\delta$ needs to be defined as illustrated in Figure 5.13-b. This Transition Function specifies that if checking the state of rain sensor results in raining, the current state of the FSM moves to Window checking if its state is open. If this is the case, the current state of the FSM moves to TTS Call, which is equivalent to establishing the call with the end-user UA using AV/TTS MMSC. Since the application should terminate after announcing the text to the end-user, the quantity of final states $F$ is $\{$ TTS Call $\}$.

After the principles of behavior-oriented M2M application modelling by means of FSMs have been introduced and exemplified, the following section describes how the graphical modelling is proposed. 


\subsection{Graphical Application Behaviour Design}

As described in section 4.2, the graphical design of an M2M application is realised via a GUI provided by the Service Design Unit (SDU) component of the designed framework. A GUI is proposed as the end-user interface for application design, since most users are familiar with the use of GUIs. An interface could also be implemented, e.g. through a text-based input/output system, but this would not satisfy the requirements for simplicity and usability.

This section deals with the structure of the GUI and the process of modelling an M2M application graphically in the form of a state machine (SM) to define the application semantics, i.e. the application model. The description of the process for the graphical modelling is again illustrated with the help of M2M application represented by Use Case 1 (refer to section 2.4). As introduced in section 4.2 the graphically designed application is transformed by the SCU component of the proposed framework into a formal description, which can be executed by the M2M platform (Steinheimer et al., 2013b). Section 5.5 will specify an adequate modelling concept, respectively a formal modelling language to describe the behaviour of an M2M application abstracted as a SM.

A GUI should contain the following aspects to provide an adequate interface to end-users for M2M application modelling:

- M2M Device/MMSC Indication - The GUI should indicate M2M devices and MMSCs available in the personal environment of end-users (and also remote available M2M services). 
- M2M Device/MMSC Interface Integration - The interface of the M2M devices and MMSCs must be available to specify their Input/Output/Config parameters.

- Connection of M2M Devices/MMSCs - The M2M device and MMSCs, respectively their Input and Output interfaces have to be connectable.

- Display designed M2M Applications - The GUI should provide the possibility to manage or change already designed M2M applications.

- Specification of Conditions - To create a decision-based M2M application logic, the logical connections between the M2M Devices/MMSCs should be equipped with conditions to process the steps in the $\mathrm{M} 2 \mathrm{M}$ application workflow in dependence on defined conditions. Relational operators illustrated in Table 5.3 are proposed for defining conditions that are understandable for end-users.

Table 5.3: Relational Operators for logical M2M Device/MMSC Connections

\begin{tabular}{|l|l|l|}
\hline Relational Operator & Example & Descripion \\
\hline Greater than $(>)$ & $\mathrm{A}>\mathrm{B}$ & Checks if a value $A$ is greater than another value $B$. \\
\hline Less than $(<)$ & $\mathrm{A}<\mathrm{B}$ & Checks if a value $A$ is less than another value $B$. \\
\hline Equal to $(==)$ & $\mathrm{A}==\mathrm{B}$ & Checks if two values $A$ and $B$ are equal. \\
\hline Not equal to $(!=)$ & $\mathrm{A} !=\mathrm{B}$ & Checks if two values $A$ and $B$ are equal. \\
\hline
\end{tabular}

The GUI is presented in this project as a web-based application, but could also be realised as e.g. App on a smartphone or tablet. It is not the kind of application that is important, but a simple interface to the M2M platform making it possible to design a SM for the M2M applications.

In the following, the structure of the GUI and the process for service creation by endusers is described starting with the architecture of the GUI (illustrated in Figure 5.14). 
5 Autonomous M2M Application Provision

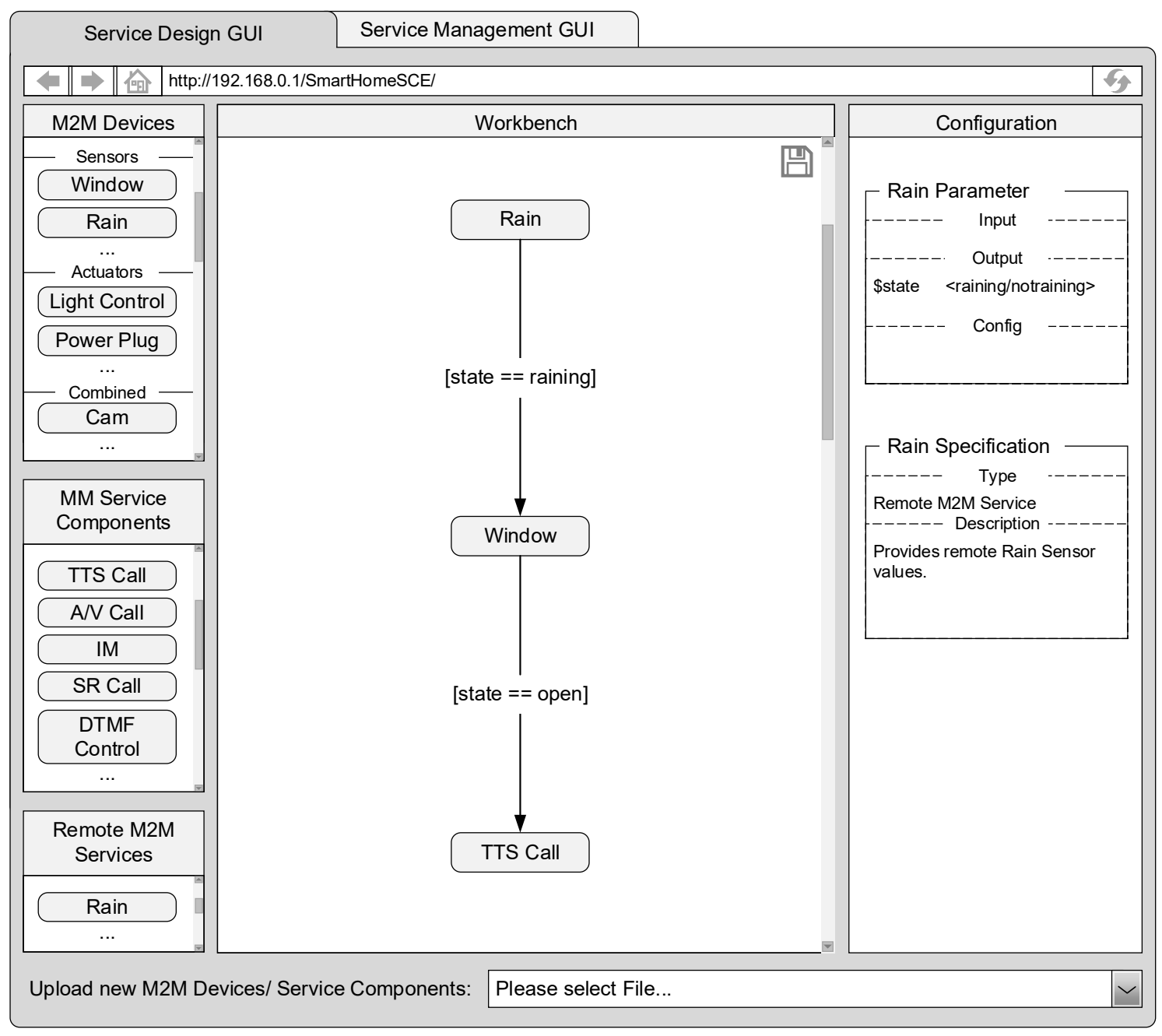

Figure 5.14: Structure of Service Design GUI acc. (Steinheimer et al., 2013c; Steinheimer et al., 2015b)

The GUI consists of the subsequently described sections:

- M2M Devices Section - The M2M Devices section lists M2M devices present in end-users' personal environments (represented by graphical building blocks). These building blocks represent the interfaces to the M2M Devices and makes them available for integration in M2M applications.

- MMSCs Section - The MMSCs section lists the MMSCs provided by the local M2M platform also represented by graphical building blocks representing the interfaces to the MMSCs for integrating them in M2M application workflows. 
- Remote M2M Services Section - The Remote M2M Services section lists building blocks of M2M services provided by other end-user (refer to section 6.1). These remote services can be integrated into local $\mathrm{M} 2 \mathrm{M}$ applications according to the same principles as integration of M2M devices/MMSCs. Additionally, these building blocks are used to model cooperative M2M applications (refer to section $6.4)$.

- Configuration Section - The Configuration section provides configuration details of the individual M2M devices/MMSCs, such as the Config parameters of M2M devices or information regarding the values provided by Input/Output parameters (Parameter area). The Configuration section also provides additional information regarding the specification of M2M devices/MMSCs, such as type specification of the M2M device (e.g. actuator or sensor), a prose description of the functionality (e.g. control functionality of an M2M Device) or annotations regarding the configuration like value ranges parameters (Specification area).

- Workbench Section - The Workbench section is used to create the M2M applications by graphically combining the building blocks of M2M devices and MMSCs. This process is illustrated in Figure 5.15 and described subsequently.

According to (Steinheimer et al., 2015b) End-users starts modelling the SM of the desired M2M application by dragging all the M2M device/MMSC building blocks they want to integrate in their local M2M application to the Workbench section (Select M2M Device/Service Component Building Blocks). For Use Case 1, integrated into the illustration of the GUI structure of Figure 5.14, these are the building blocks of Rain sensor, Window sensor, and TTS Call MMSC. After placing the M2M Device and MMSC building blocks to the Workbench section the end-user connects the building blocks with 
an arrow to specify the semantical workflow of the M2M application (Define Logical Combinations). In the exemplary Use Case 1 the Rain sensor building block is connected to the Window sensor building block that again is connected to the TTS Call MMSC building block.

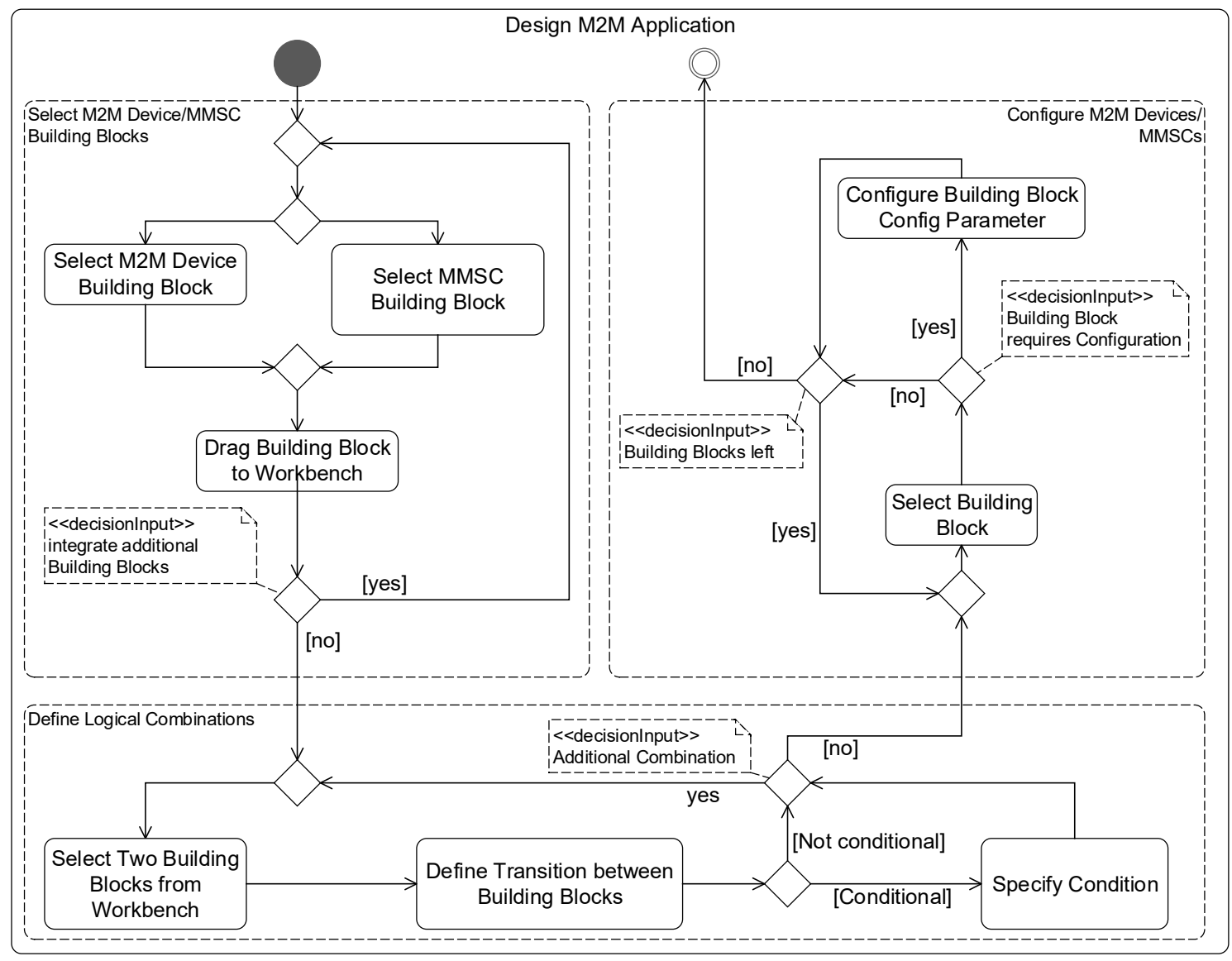

Figure 5.15: M2M Application Design Process

These connections model that first the Rain Sensor is processed (in particular requested), then the Window Sensor is processed (also requested) and finally the TTS Call MMSC is processed (to setup up a call). If the end-users want to design, that a specific step of the application workflow is processed only if a defined condition becomes true, they can add a condition to each connection between the M2M Devices and MMSCs. In the exemplary Use Case 1, illustrated in Figure 5.14 following conditions are defined: 
- $\quad[$ state $==$ raining $]-$ For the connection of Rain sensor and Window sensor it is specified that the step to process requesting state parameter of Window sensor is performed, if the state parameter of Rain sensor holds the value "raining". Otherwise, the application resides at the step requesting the Rain sensor. Transferred to the SM paradigm the SM resides in the state corresponding to the Rain sensor and the transition to the state corresponding to the Window sensor is performed only if the input of the SM is raining (element of FSM input alphabet).

- $\quad[$ state $==$ open $]-$ For the connection of Window sensor and TTS Call MMSC it is specified that the step to setup the announcement call is performed, if the window is open, i.e. if the state parameter of the Window sensor holds the value "open". Transferred to the SM paradigm the SM resides in the state corresponding to the Window sensor until the input of the SM is open (element of FSM input alphabet).

After end-users have defined all logical connections between the M2M devices/MMSCs they configure those (Configure M2M Devices/MMSCs). For this, they select the corresponding building block in the Workbench section and define the Config parameter inside the Configuration section of the GUI. In exemplary Use Case 1 the TTS MMSC is the only building block that requires configuration. Table 5.4 shows its configuration according the specification of $A V / T T S$ MMSC introduced in section 5.1 to initialise it for call setup with end-user's UA and announcement of a pre-defined text.

Table 5.4: Configuration of TTS MMSC Use Case 1

\begin{tabular}{|l|l|l|}
\hline TTS MMSC Parameter & Parameter Value & Descripion \\
\hline config.mode & TTS & Configuration for TTS call. \\
\hline input.text & "warning window open and starts raining" & $\begin{array}{l}\text { Warning message to be } \\
\text { announced. }\end{array}$ \\
\hline input.sipURI & "sip:username@domain" & $\begin{array}{l}\text { Destination SIP URI of end-user } \\
\text { UA specified to receive the call. }\end{array}$ \\
\hline
\end{tabular}


This section introduced the architecture of the GUI that provides an easy usable interface to the end-users for graphical design of $\mathrm{M} 2 \mathrm{M}$ applications for their local environment with integration of M2M devices and MMSCs enabling input/output interfaces of M2M applications. The process for graphical application design has been introduced in detail and the behaviour of $\mathrm{M} 2 \mathrm{M}$ application representing exemplary Use Case 1 was modelled graphically, which has been defined in previous section 5.2 by means of a FSM.

\subsection{M2M Device Management and Communication}

The basis for the creation of an M2M application is the integration of M2M devices into the M2M platform and the networking of M2M devices. As described in section 3.2, the M2M devices are mostly implemented with different mutually incompatible M2M communication technologies. If the necessary details for the connection of different M2M devices were taken into account in an M2M application (e.g. message format, communication protocols, control commands), a separate interface would have to be implemented for each M2M technology within the M2M application. The M2M application would have to consider the devices as well as M2M technology-specific details. (ETSI TR 101584 V2.1.1, 2013) confirms this aspect according to the previously valid standard for M2M (ETSI TR $102966 \mathrm{~V} 1.1 .1,2014$ ). In this way, the M2M application would again be close coupled to the executing platform, or could only be used with the M2M technologies provided inside the M2M application. Porting the application to a different system would not be possible. It is therefore essential that the M2M devices can be connected to the M2M platform and be used in the M2M applications independently of the underlying technology. Therefore, in (Steinheimer et al., 2013a), 
(Steinheimer et al., 2013b), and (ETSI TR 101584 V2.1.1, 2013) the abstraction of M2M devices, respectively $\mathrm{M} 2 \mathrm{M}$ communication technologies from platform-internal utilisation of $\mathrm{M} 2 \mathrm{M}$ devices has been introduced. The abstraction of communication within the M2M platform from the M2M technology-specific communication has been defined as a requirement for an M2M system with (oneM2M TS-0002-V1.0.1, 2015; oneM2M TS-0002-V2.7.1, 2016). Section 4.2 of this thesis introduced the M2M Communication Unit (CU) with Abstraction Layer (AL) as component of the proposed framework. The M2M CU with AL component realises the integration of different M2M device technologies into the local M2M platform. This section describes the principles of unified M2M device integration.

OneM2M introduces in (oneM2M TR-0007-V1.0.0, 2014) and (oneM2M TR-0007V2.11.1, 2016) an approach for M2M device abstraction that allows handling of $\mathrm{M} 2 \mathrm{M}$ devices inside an $\mathrm{M} 2 \mathrm{M}$ application independent of the underlying specific $\mathrm{M} 2 \mathrm{M}$ technology (illustrated in Figure 5.16).

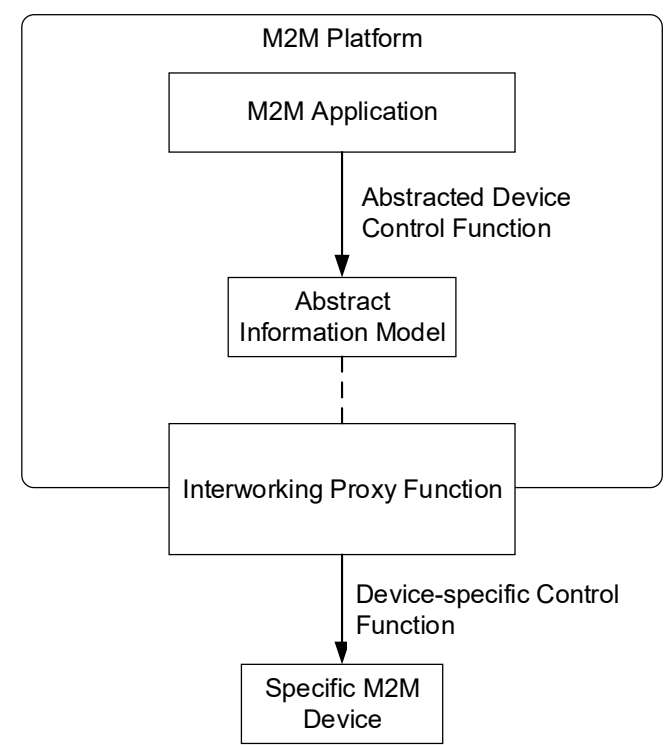

Figure 5.16: Principles of oneM2M Device Abstraction acc. (oneM2M TR-0007-V1.0.0, 2014; oneM2M TR-0007-V2.11.1, 2016) 
The presented approach is based on mapping native device interfaces into an abstracted oneM2M resource addressable by an $\mathrm{M} 2 \mathrm{M}$ application. The M2M application does not communicate with the native device API, but with the abstract representation of the M2M device. A so-called Abstract Information Model specifies the abstract device, which is a formal representation of the device including e.g. its operations or parameters. OneM2M defines the "Interworking Proxy Function" responsible for translating the Abstract Information Model into the Device-specific Control Functions (oneM2M TR-0007V2.11.1, 2016).

As described in section 3.1.1 within an oneM2M architecture according (oneM2M TS0001-V2.10.0, 2016) everything is considered as resources that are provided by entities and are queried or controlled by other entities. Therefore, M2M devices connected to the $\mathrm{M} 2 \mathrm{M}$ platform are considered as resources. Considering now the M2M application as an entity that consumes a resource and the M2M Technology API as an entity which provides a resource and the device-specific communication with the physical M2M equipment, this principle can be transferred to connecting $\mathrm{M} 2 \mathrm{M}$ devices to the $\mathrm{M} 2 \mathrm{M}$ platform.

Query and control of resources is done according (oneM2M TS-0001-V2.10.0, 2016) using specific Create (C), Retrieve (R), Update (U), Delete (D), Notify (N) operations (CRUDN operations). To query/manipulate a resource, consuming and providing entity communicate applying the request/response principle described in section 3.1.1.

Table 5.5 and Table 5.6 describe an extract of the parameter sets according (oneM2M TS0001-V2.10.0, 2016) that request and response messages contain that are generated by resource consuming/providing entities. 
Table 5.5: Parameter Set of Request Resource Message acc. (oneM2M TS-0001-V2.10.0, 2016)

\begin{tabular}{|c|c|c|}
\hline Parameter & & Description \\
\hline To & Address of $\mathrm{tl}$ & e resource or URI of an attribute of a resource to be requested. \\
\hline From & Identifier of & he message originator. \\
\hline \multirow[t]{6}{*}{ Operation } & \multicolumn{2}{|c|}{$\begin{array}{l}\text { Operation the receiving resource should execute. The operation parameter is } \\
\text { separated into the specific operations stated below. }\end{array}$} \\
\hline & Create (C) & $\begin{array}{l}\text { Creates a new resource at the destination address specified in To } \\
\text { parameter. }\end{array}$ \\
\hline & Retrieve (R) & Requests a resource addressed by the To Parameter. \\
\hline & Update (U) & $\begin{array}{l}\text { Sets the content of a resource addressed by the To parameter. The } \\
\text { content to be set in the resource is specified in the Content } \\
\text { parameter }\end{array}$ \\
\hline & Delete (D) & $\begin{array}{l}\text { Deletes a resource at the destination address specified in To } \\
\text { parameter. }\end{array}$ \\
\hline & Notify (N) & Information that is sent to the receiving resource. \\
\hline Content & \multicolumn{2}{|c|}{$\begin{array}{l}\text { Content that should be transferred to the receiving resource and relates to the } \\
\text { operation specified by Operation parameter. Only applicable for following } \\
\text { operations: Create (content of resource to be created), Retrieve (list of attribute } \\
\text { names to be retrieved), Update (content to be updated in the destination } \\
\text { resource), and Notify (information should be notified to the receiver). }\end{array}$} \\
\hline Request Identifier & \multicolumn{2}{|c|}{ Identifies of the specific request message. } \\
\hline
\end{tabular}

According (oneM2M TS-0004-V2.7.1, 2016) oneM2M defines Primitives that are messages exchanged between entities in an M2M system. Originator and receiver can be located on the same M2M Node or on M2M nodes connected via a network. Primitives are modelled as a data structure specifying the operations and parameters processed on the receiver and originator entity. According (oneM2M TS-0004-V2.7.1, 2016) each (CRUDN) operation related to a resource "comprises a pair of primitives: Request and Response". These Primitives become serialised, e.g. as XML or JSON format that can be exchanged between the entities using e.g. HTTP, CoAP, MQTT protocol to transmit the Primitives over an IP network. OneM2M specifies so-called Bindings to define unified 
Table 5.6: Parameter Set of Resource Response Message acc. (oneM2M TS-0001-V2.10.0, 2016)

\begin{tabular}{|c|c|c|}
\hline Parameter & & Description \\
\hline \multirow[t]{3}{*}{$\begin{array}{l}\text { Response Status } \\
\text { Code }\end{array}$} & \multicolumn{2}{|c|}{$\begin{array}{l}\text { Indicates the result of an operation specifies in request message. The result can } \\
\text { describe the status as stated below. Successful status codes are e.g. acc. } \\
\text { (oneM2M TS-0004-V2.7.1, 2016) } 1000 \text { ACCEPTED, 2000 OK, or } 2004 \text { UPDATED. } \\
\text { Exemplary unsuccessful status codes are } 4004 \text { NOT_ALLOWED, } 5000 \\
\text { INTERNAL_SERVER_ERROR, or } 6005 \text { EXTERNAL_OBJECT_NOT_FOUND. }\end{array}$} \\
\hline & $\begin{array}{l}\text { successful } \\
(2 x x x)\end{array}$ & Indicates the requested operation has been successful. \\
\hline & $\begin{array}{l}\text { unsuccessful } \\
(4 x x x, 5 x x x \\
6 x x x)\end{array}$ & Indicates the requested operation has been unsuccessful. \\
\hline Request Identifier & \multicolumn{2}{|c|}{ Request Identifier of the corresponding request message. } \\
\hline Content & \multicolumn{2}{|c|}{$\begin{array}{l}\text { Content of the resource. Only applicable for following requested operations: } \\
\text { Create (address and/or the content of the resource created), Update (content of } \\
\text { the resource that has been updated), Delete (all existing information about the } \\
\text { deleted resource), and Retrieve (content that has been requested by the } \\
\text { originator). }\end{array}$} \\
\hline
\end{tabular}

interfaces for the communication protocols HTTP (oneM2M TS-0009-V2.6.1, 2016), WebSocket Protocol (oneM2M TS-0020-V2.0.0, 2016), CoAP (oneM2M TS-0008V1.0.1, 2015), MQTT (oneM2M TS-0010-V2.4.1, 2016) describing how to convert oneM2M primitives into these specific communication protocol messages. Figure 5.17 and Figure 5.18 illustrate the structure of a Request and Response Primitives that can be exchanged between M2M entities. A corresponding XML representation is illustrated in Listing C.1 and Listing C.2.

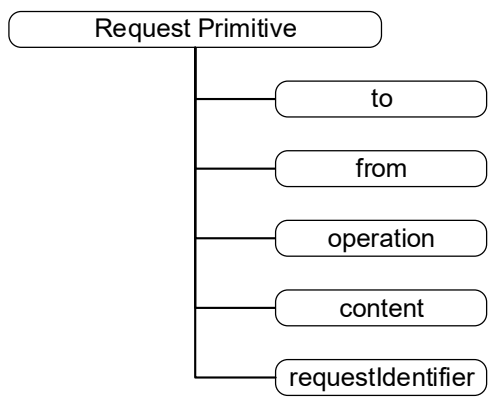

Figure 5.17: Structure of Request Primitive 


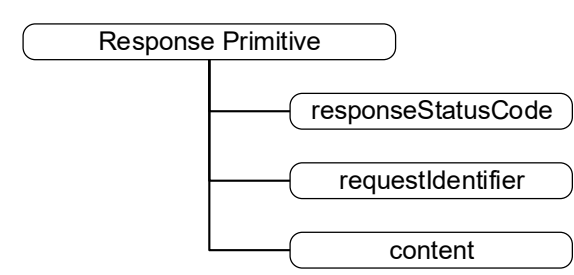

Figure 5.18: Structure of Response Primitive

In order to define an appropriate abstract device representation and abstraction mechanism to integrate $\mathrm{M} 2 \mathrm{M}$ devices of different $\mathrm{M} 2 \mathrm{M}$ technologies into the $\mathrm{M} 2 \mathrm{M}$ platform, the principles of device handling and communication between M2M entities have been derived from oneM2M as described subsequently.

The framework architecture designed in this project contains the $M 2 M C U$ with $A L$ element (illustrated in Figure 5.19). The $M 2 M C U$ realises the unified communication between M2M applications executed inside the M2M platform and the M2M devices of different M2M technologies connected to the M2M platform. (Steinheimer et al., 2012a; Steinheimer et al., 2013a; Steinheimer et al., 2015b)

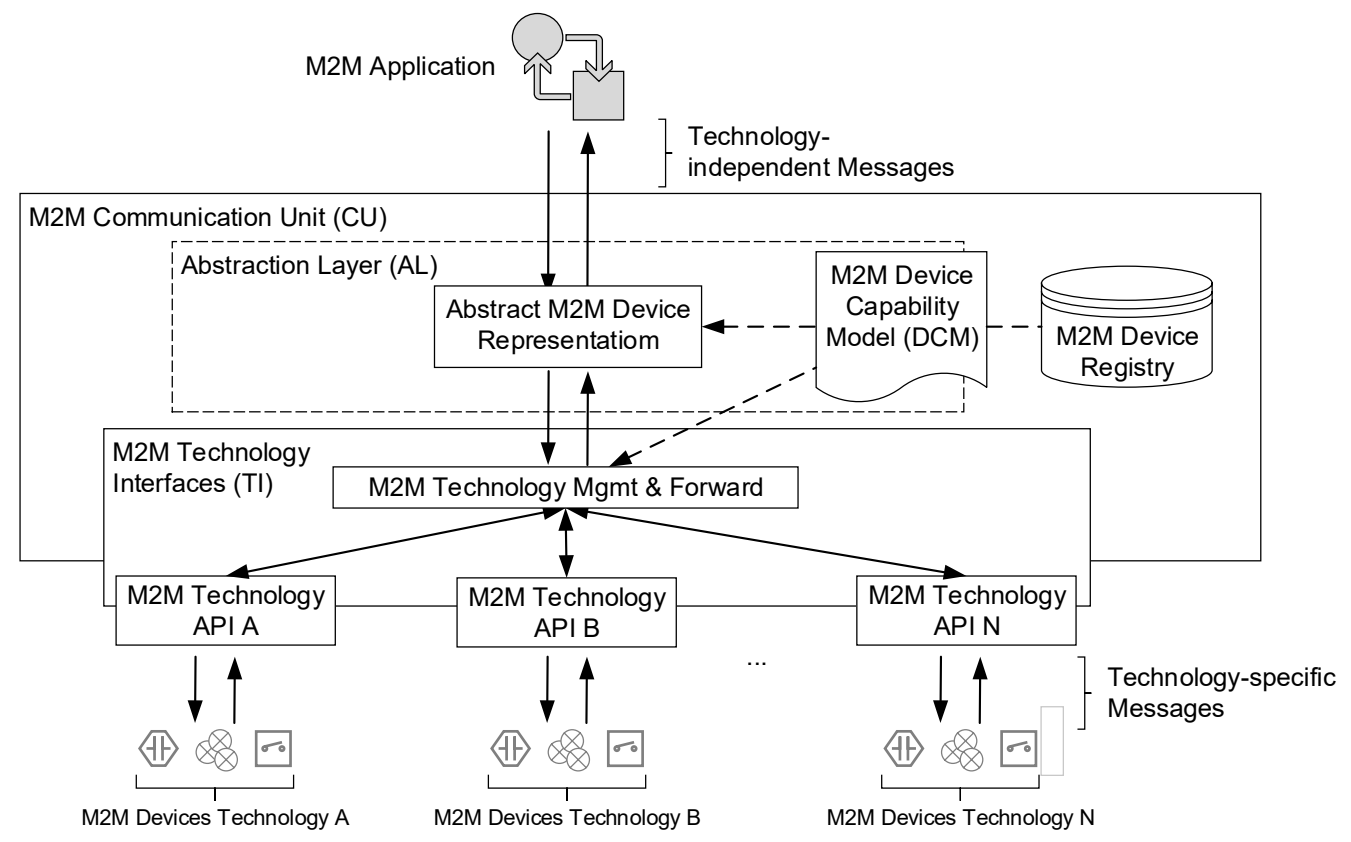

Figure 5.19: M2M Communication Unit (CU) with Abstraction Layer (AL) 
The $A L$ as part of the $M 2 M C U$ abstracts the devices inside the M2M platform from specific communication technologies, such as KNX or ZigBee. To achieve this integration, all devices are considered independent of their specific communication technologies as Abstract M2M Device Representations. To realise the abstraction of specific M2M device technologies the $A L$ contains the following functionality:

- Message Coordination - The $A L$ coordinates controlling of different types of $\mathrm{M} 2 \mathrm{M}$ equipment and thus offers the possibility to define M2M applications without depending on the M2M technologies used.

- Abstract M2M Device Management - Management of abstracted M2M devices by $\mathrm{M} 2 \mathrm{M}$ device registration, assignment of abstract device-IDs and administration of the assignments of abstract and specific device-IDs.

To realise the abstraction, derived from (oneM2M TR-0007-V2.11.1, 2016) an abstract information model has been designed (M2M Device Capability Model, M2M DCM), describing the M2M devices. The M2M applications do not communicate with the specific M2M device, but with the abstract device representation within the platform described by the $M 2 M D C M$.

This $M 2 M D C M$ is also used by the $S D U$ component of the framework to display and specify an M2M device representation within the GUI. Since also MMSCs are usable in applications, they are also described with the $M 2 M D C M$ and processed in the same way.

To specify an appropriate $M 2 M D C M$, following information should be included in it:

- Device-ID - To uniquely identify an M2M device, a device-ID should be included. 
- Input/Output/Config Parameters - In section 5.2 was specified that $M 2 M$ devices/MMSCs each have a set of Input/Output/Config parameters. For unified description of $M 2 M$ device/MMSC, these parameters should be contained in a $M 2 M D C M$.

- Type and Description - In section 5.3 was specified that additional information on M2M devices/MMSCs should be displayed in the GUI (description and type). The $M 2 M D C M$ therefore should also contain these information.

Considering these requirements, a general structure of an $M 2 M D C M$ is designed as shown in Figure 5.20.

M2M Device/MMSC Capability Model

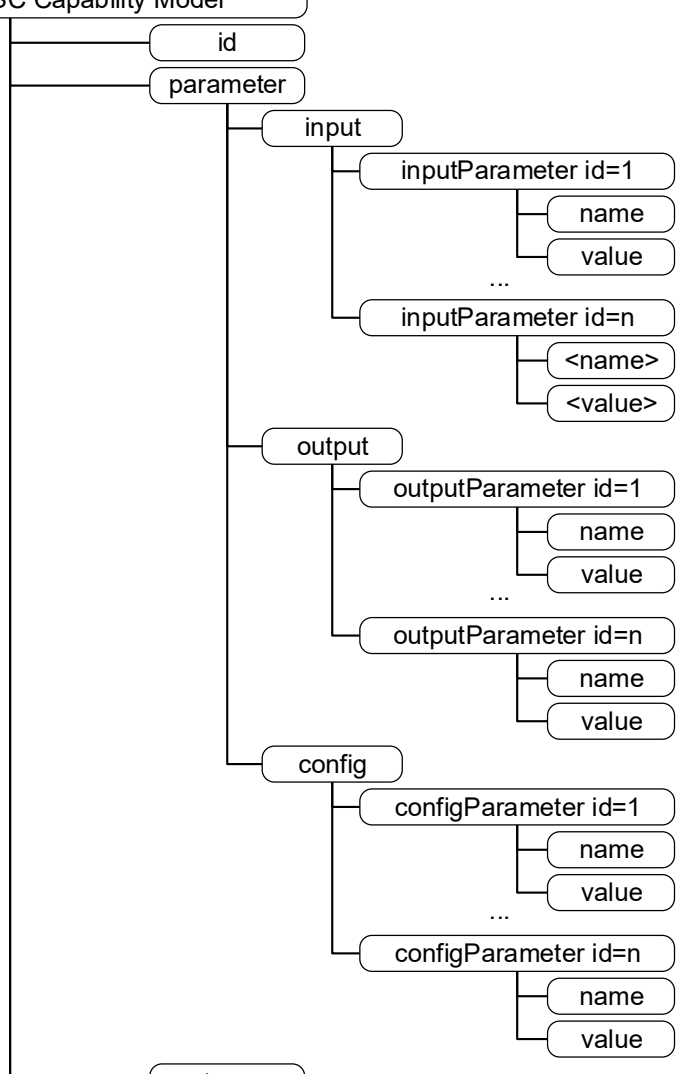

Figure 5.20: Structure of M2M DCM 
To formally describe this structure of an $M 2 M D C M$, the XML format is selected since it is standardised, expandable and machine-readable. Listing C. 3 shows a representation of an uniformly defined $M 2 M D C M$ in XML. If only predefined values are allowed for specification of parameters, this can be defined as enumeration within the parameter specification in a specific instance of an $M 2 M D C M$ (e.g. for the rain sensor of Use Case 1 this could be defined in this way: \{raining| notraining $\}$ ).

The M2M Technology Interfaces (M2M TI) element of the $C U$ (see Figure 5.19) realises management of and communication with M2M devices of various M2M technologies. It is designed to realise abstracted and M2M technology-specific communication, and contains various $\mathrm{M} 2 \mathrm{M}$ technology-specific APIs communicating with the respective M2M devices via a device-specific technology.

To realise this management and communication the $M 2 M T I$ contains the following functionality:

- M2M Device Technology APIs - The M2M TI element contains the M2M Technology APIs for communication with the M2M devices of the specific technology to query or control them.

- Message Transformation - Transforms abstracted request messages in technology specific request messages and transforms technology-specific response and notification messages into abstracted response and notification messages.

- Message distribution - Distribute technology-specific messages to $M 2 M$ Technology APIs implementing the communication with the M2M equipment.

- Device Registration - Registration of technology-specific M2M devices at the $A L$ component of the $M 2 M C U$. 
As described above, the M2M application does not communicate with the physical M2M device but with an abstract representation of it. For this purpose, an application communicates with the $A L$ to query a value of an $\mathrm{M} 2 \mathrm{M}$ device or to control the $\mathrm{M} 2 \mathrm{M}$ device. If the $A L$ receives a request for an abstracted M2M device, it must forward this request to the corresponding $M 2 M T I$. For this purpose, it is necessary for the $A L$ to manage the allocation of abstract to technology-specific M2M devices. To realise this, the $M 2 M T I$ element registers the connected devices at the $A L$, which records this registration and assigns them an abstract device-ID. The $A L$ stores the assignment of abstract and specific device-ID within the M2M Device Registry contained in the $C U$. The process of the M2M device registration is shown in Figure 5.21.

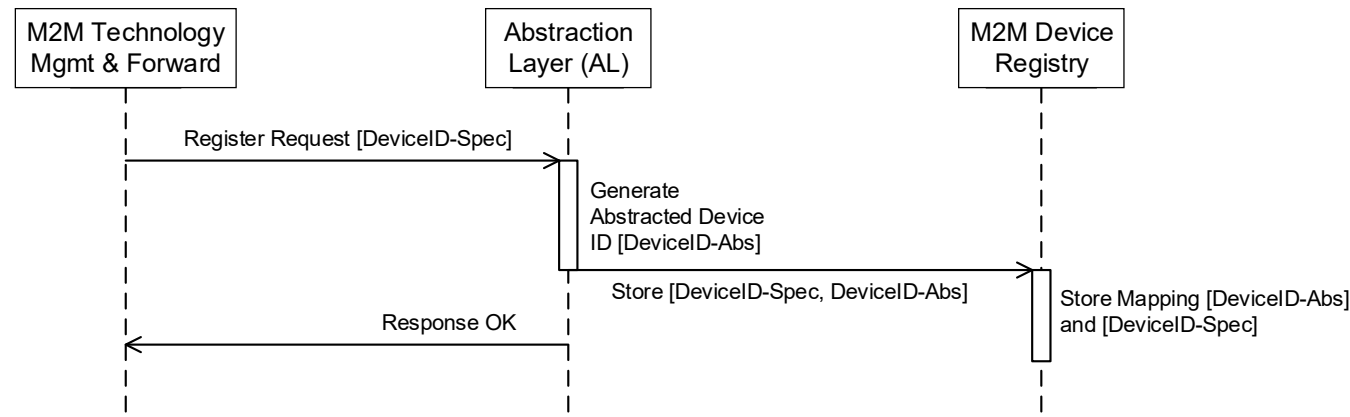

Figure 5.21: M2M CU/AL Register Process

To register an M2M device with the $A L$, the M2M Technology Mgmt \& Forward element sends a Register Request to the $A L$ containing the specific device-ID of the M2M devices. The $A L$ generates an abstract device-ID for the M2M device specified in the Register Request and stores it within the M2M Device Registry component. The abstraction of the $\mathrm{M} 2 \mathrm{M}$ devices offers the advantage that the M2M devices, or the technology of an M2M device can be changed or exchanged, but the M2M application itself is not affected by such changes since it communicates with the abstracted devices. 
If an M2M application queries a value of an abstract M2M device or sends a control command to it, this is realised as shown in Figure 5.22.

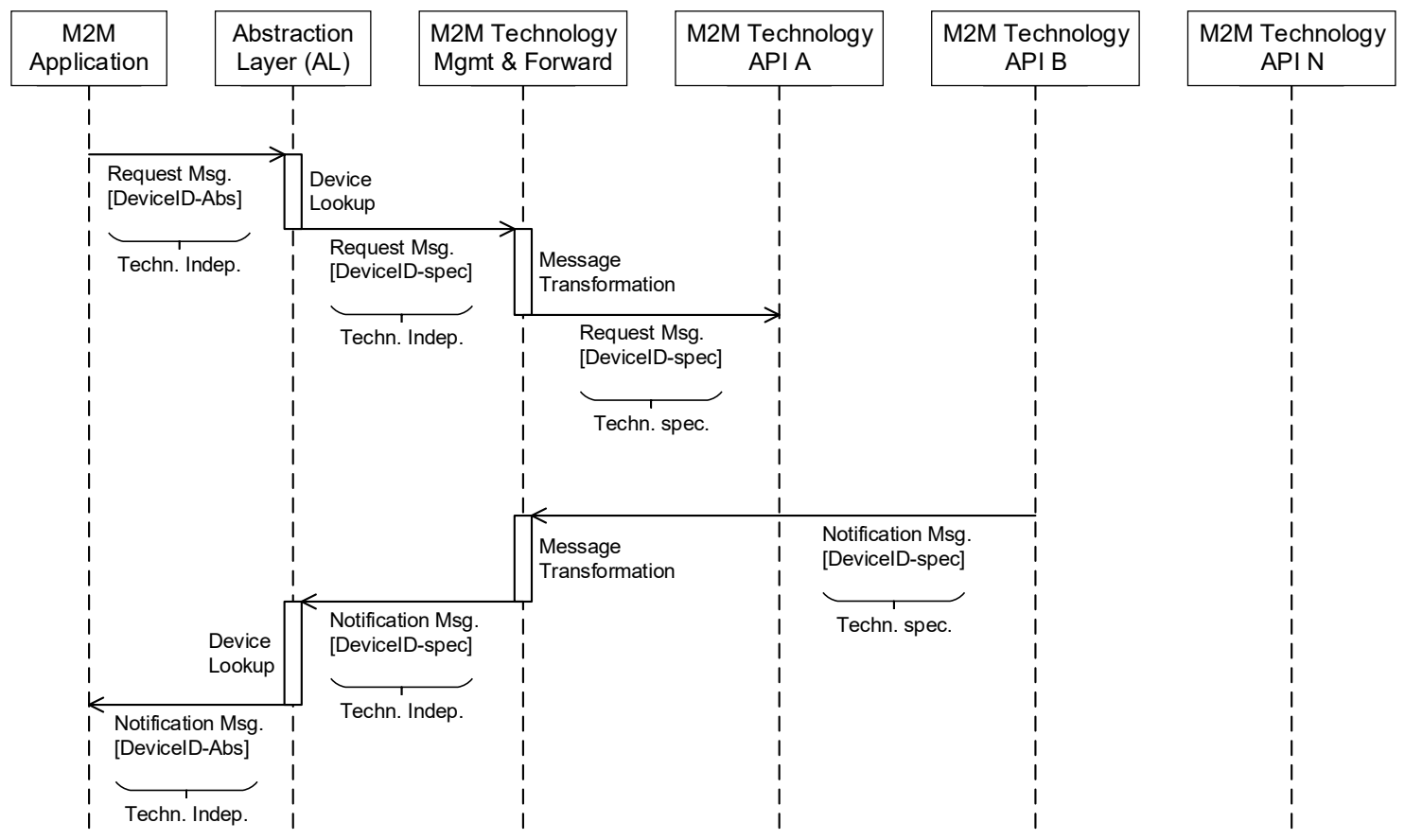

Figure 5.22: M2M Application/CU/AL Message Exchange

The M2M application sends a technology-independent request message to the $A L$ containing the abstracted device-ID of the M2M device. Upon receiving the request, the $A L$ assigns the specific device-ID to the abstracted device-ID (by requesting the $M 2 M$ Device Registry) and replaces the abstracted device-ID with the specific device-ID. The $A L$ now sends this modified request to the M2M Technology Mgmt \& Forward element of the M2M TI. Since the M2M Technology Mgmt \& Forward element has done the registration of the M2M devices with the $A L$, it knows to which specific M2M Technology $A P I$ the request must be forwarded. For this purpose, the M2M Technology Mgmt \& Forward element translates the abstract request message into a technology-specific request message and forwards this request message to the corresponding $M 2 M$ Technology API, which ultimately realises the communication with the device. Figure 
5.22 also contains the representation of the message exchange by means of a Notify message sent from a specific M2M device to an M2M application. In this case, the transformation of a specific request is done analogously to the described method of a request message initiated by an $\mathrm{M} 2 \mathrm{M}$ application.

The $M 2 M D C M$ is stored within the $A L$, but must also be known to the $M 2 M T I$ element because the M2M TI element receives a query specified for an abstracted M2M DCM and has to map this request to a specific M2M device.

Up to now, the communication between the M2M application and the M2M device has been specified via an abstracted communication mechanism. Additionally, it is necessary to define a uniform message format, which is used for the exchange of information between the M2M platform components. For this purpose, the approach defined by oneM2M is used to exchange messages between entities using Primitives. Following this approach, a Request Primitive must be defined containing the necessary information to query or control M2M devices. This Request Primitive is generated by the M2M application or the $M 2 M T I$ element and sent to the $A L$. The $A L$ contains an Abstracted M2M Device Representation (described by the M2M DCM). The M2M TI element of the $M 2 M C U$ also contains the $M 2 M D C M$. The $M 2 M D C M$ is therefore a central component on which the $M 2 M C U$ performs the abstracted message exchange between M2M application and M2M devices. It is therefore necessary to integrate the information into a Request Primitive that is required for querying or controlling M2M devices, as specified in the $M 2 M D C M$. Figure 5.23 illustrates the $M 2 M D C M$ (specified in Figure 5.20) as well as the structure of a Request Primitive (specified in Table 5.5) and assigns the relevant information of an M2M device to the elements of a Request Primitive. Listing 
C.4 illustrates the XML representation of the fully specified Request Primitive template including the $M 2 M D C M$ parameters.

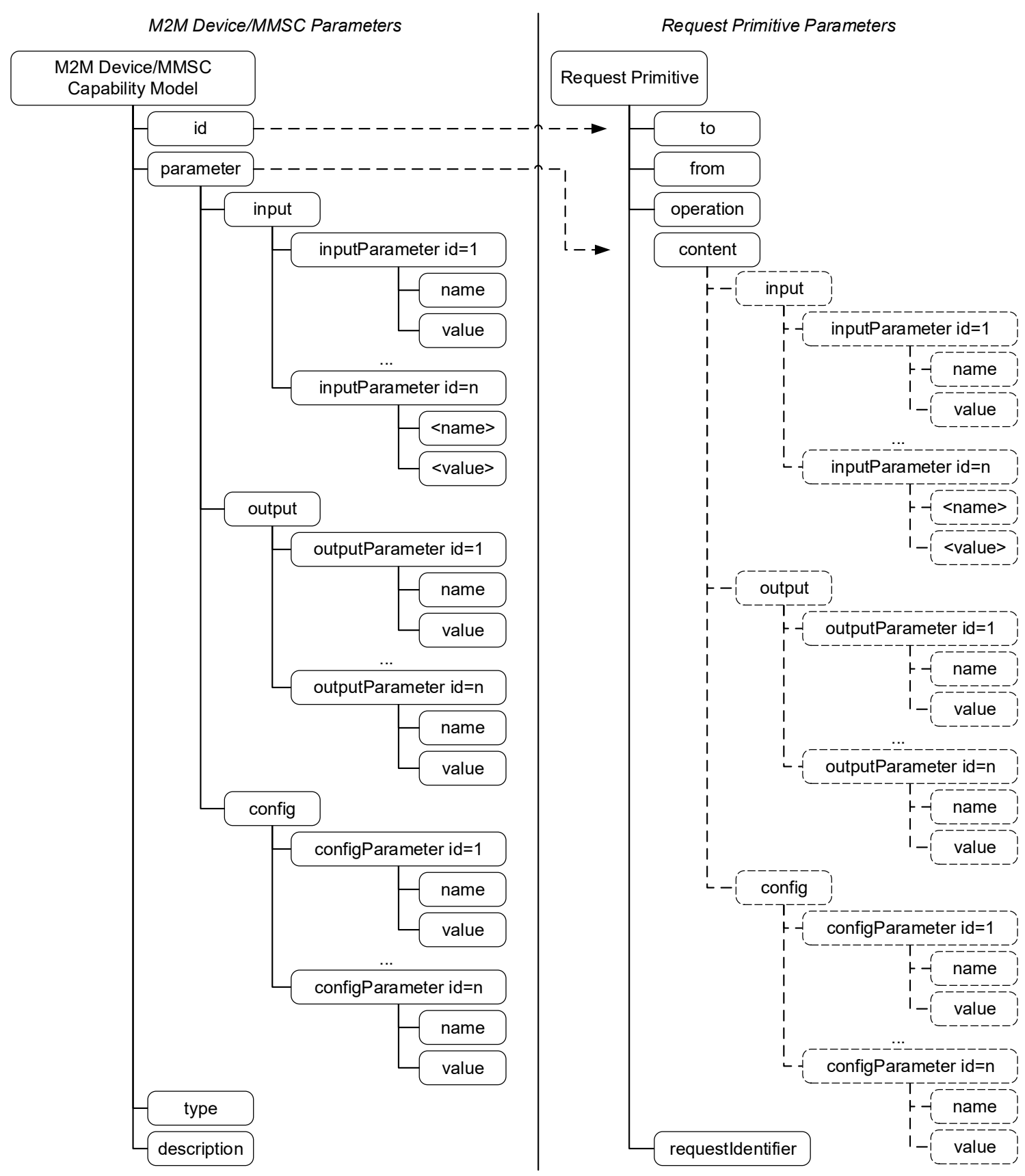

Figure 5.23: Mapping M2M DCM to Request Primitive Parameters

Since an M2M device is uniquely identified via an M2M device-ID, this parameter can be transferred to the To parameter of a Request Primitive. The M2M DCM parameters 
describe the Input/Output/Config parameters of an M2M device. It is therefore necessary information that must be transferred to an M2M device. Such information should be transmitted, as specified in Table 5.5, using the content parameter of a Request Primitive.

Because both, Request Primitive and $M 2 M D C M$ are specified using XML format, the Parameter element of $M 2 M D C M$ as complex datatype can be integrated in the Content parameter of the Request Primitive XML description. Using these two mappings, all information required for request/control $\mathrm{M} 2 \mathrm{M}$ devices are integrated in the Request Primitive. Further information, respectively parameter to be specified are:

- From Parameter - The From parameter specifies the Request Primitive originator. Specification of this parameter depends on the element of the M2M platform originating the request (M2M Application, $A L$ or $M 2 M T I)$.

- Operation Parameter - The operation parameter specifies the operation to be executed at the request receiver (create for M2M device registration, update for control and config operations, retrieve for value requests, notify for notification requests)

Up to this point, it has been specified that the communication of $\mathrm{M} 2 \mathrm{M}$ application and physical M2M equipment occur in an abstracted way and how the communication between the M2M platform components occurs to exchange information for request and control of M2M devices. The following approaches are proposed for automatically or partly automatically integration of machine-readable M2M DCMs into the M2M platform (illustrated in Figure 5.24 including particular steps of installation process). 
- Installation by End-user - End-users installs M2M DCMs as files using the GUI (1.1). The M2M DCMs are stored inside the M2M platform in the M2M Device Registry (1.2) containing all installed M2M DCMs. During invocation process of the GUI, available M2M DCMs are requested from M2M Device Registry and displayed as M2M device/MMSC building blocks in the GUI $(1.3,2.3,3.5)$ to be available for application design (refer to section 5.3).

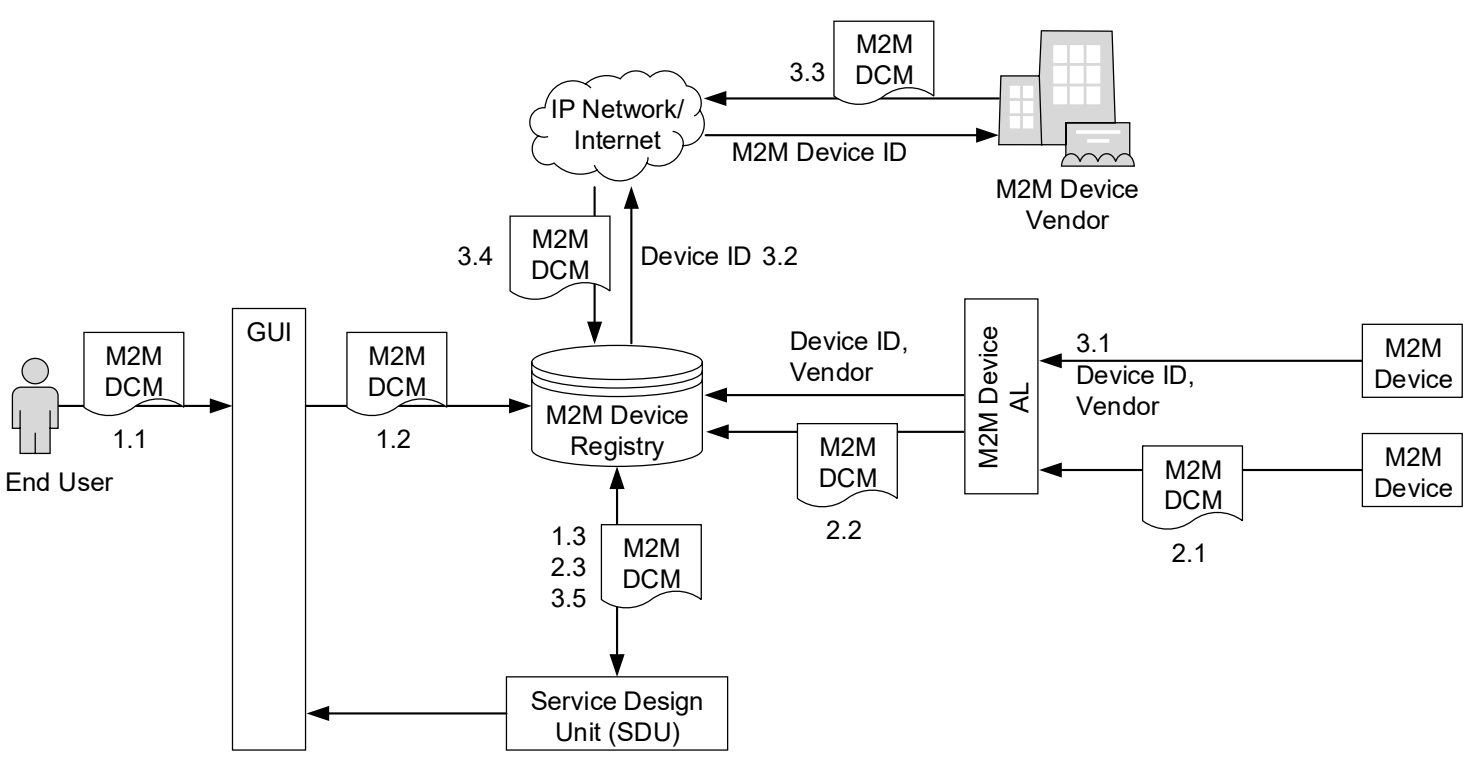

Figure 5.24: Installation of M2M Device Capability Models

- Installation by M2M Device - The M2M DCMs are stored at the M2M device that should connect to the M2M platform. While connecting M2M devices to the M2M platform the M2M DCMs transfer to the M2M platform (2.1) and are stored in M2M Device Registry (2.2).

- Download from M2M Device Vendor - The M2M device to be installed contains a reference (e.g. a link) incl. M2M device-ID to the vendor of the M2M device for download the M2M DCM using a network connection to the vendor. While connecting the M2M device to the M2M platform the reference and M2M device- 
ID transfer to the M2M platform (3.1). The M2M Device Registry contacts the M2M Device Vendor (3.2) using the provided information and requests the M2M DCM stored at vendor-side for download (3.3). After downloading the M2M DCM, the M2M Device Registry stores the M2M DCM in its repository for all available M2M DCMs (3.4).

The lastly presented approach to automatically download the M2M DCMs from M2M device vendor has the advantage that M2M devices do not need to provide storage space for storing M2M DCMs and at the same time enables provision of updated M2M DCMs. Additionally, the fully automated installation of M2M DCMs is realisable according this approach. Drawback of this approach is that an active network connection to the download server of the M2M device vendor must exist. Additional disadvantage is that the download server represents a centralised element in the M2M system architecture, which results in risks for e.g. availability (refer to section 3.2) and violates the principle requirement of the proposed framework to avoid central components. The firstly presented approach to install M2M DCMs by end-users has the disadvantage, that it requires manual installation of the M2M DCMs by end-users and therefore is no fully automated mechanism. Additionally, this approach requires providing the M2M DCM on a separate installation medium usable for installation or downloading the M2M DCM from the M2M Device Vendor download server. Therefore, the second approach is the most practical solution for automated installation of M2M DCMs with the drawback that the additional storage space on the M2M device needs to be provided.

The following section describes the formal description of graphically designed M2M application as introduced in section 4.2. 


\subsection{Formal M2M Application Notation}

The previous sections introduced how end-users can model M2M applications in the form of a SM. This section determines a concept for SM-based modelling (section 5.5.1) and introduces the principles of modelling using Statecharts (section 5.5.2). Section 5.5.3 describes how a specific M2M application can be modelled and formally described using Statecharts according to the approach proposed in this research.

\subsubsection{Selection of State Machine-based Modelling Language}

As introduced in section 5.2, the end-user should describe the M2M application be means of an abstract model that is based on a SM. Therefore, a modelling language or a modelling concept is needed which allows to specify the behaviour of an application in form of a SM.

Describing an M2M application with a FSM allows to map M2M devices/MMSCs to the states of an FSM and to map the connections between them to state transitions. Nevertheless a FSM as introduced in section 5.2 cannot be applied to the description of an $\mathrm{M} 2 \mathrm{M}$ application in particular and generally to the description of combinations of devices and service components since FSMs do not allow state transitions to be equipped with data that is required for inputs or outputs of states. However, this is necessary if the introduced concept should be followed by combining input and output of devices and services. FSMs also do not allow to configure a state because the states do not have parameters that could be used for this. Since M2M devices/MMSCs are usually 
configurable and cannot be controlled or configured exclusively by input parameters, definition of configuration parameters is required.

Therefore, an alternative is needed to define the behavior of an application, similar to an FSM. Since this definition is to be made with a formal language, languages and concepts are presented and compared with each other that enable a behavioural modelling and formal description of M2M applications.

To identify an appropriate modelling language enabling the formal description of the graphically modelled application using SM concept, the following section first defines requirements according to (Steinheimer et al., 2017a) for selecting an optimal modelling language for this purpose. Subsequently, potential candidates are presented and then evaluated with regard to the defined requirements.

- Standardised language - It should be a standardised modelling language to ensure portability.

- M2M Device/MMSC Mapping - The modelling language must provide elements that enable representation of $\mathrm{M} 2 \mathrm{M}$ devices/MMSCs and connection between them. The connections between the M2M devices/MMSCs, i.e. information flow between them or activation should be equipped with a condition.

- Intuitive usability - Since the system is to be used by an average technically experienced end-user after a short training, the complexity of the graphical notation should be low. Complex and non-intuitive forms of modelling reduce or eliminate usability.

- Parallel flows - Within an application, it should be possible to define parallel sequences to realise concurrent tasks. 
- Synchronisation of states - To synchronise parallel flows there must be a synchronisation possibility.

- Machine readability - Since the M2M application should be generated automatically after graphical modelling and automatically processed by the M2M platform, the modelling language should be a formal language which is machinereadable.

- State parametrisation - The elements to be combined should be able to be parameterised (definition of input/output/configuration parameters). Therefore, the states in the SM must also be parameterisable.

- Existing parser/interpreter implementation - To be able to process the formal language automatically, an implementation of a corresponding parser or interpreter should exist.

- Domain independent - The modelling language cannot be a domain specific language to prevent limiting the scope to the specific domain.

An application according (Harel and Politi, 1998) can be modelled with three different views onto the system:

- Functional View - The Functional View specifies the processes, activities, and functions of a system. It includes inputs and outputs of activities, which is the information flow between the internal and external activities. The Functional View of a system is described using Activity Diagrams as Modelling Language (Harel and Politi, 1998). An Activity Diagram describes complex processes, focuses on the task of the system that has to be divided into single steps, and 
answers the question "how a system realises a specific behaviour" (Rupp at al., 2007).

- Behavioural View - The Behavioural View specifies the behaviour of a system. It specifies how a system acts on defined conditions and when the activities defined in the Functional View become active and when the information flow between the activities take place. The Behavioural View of a system is described using State Diagrams as Modelling Language (Harel and Politi, 1998). A State Diagram describes the behaviour of a system using states and transitions between states that are triggered by external or internal events. A state diagram answers the question "how the system behaves when residing in a specific state and a specific event occurs" (Rupp at al., 2007).

- Structural View - The Structural View specifies the modules and subsystems "constituting the real system and the communication between them". The Structural View is considered as the "physical model" of the system describing the specific hardware and software implementations (Harel and Politi, 1998).

Since the end-user should describe the application in an abstract way and do not need to know any hardware- and software-specific details, the application cannot be modelled according to the Structural View. Because modelling the behaviour of an M2M application is in the focus of the concept described in this research, formal descriptions based on the Behavioural View appear to be a suitable approach. According (Rupp et al., 2007), (ISO/IEC 19514, 2017) and (OMG, 2017b) SMs (Behavioural View) and Activity Diagrams (Functional View) are equivalent and can describe both the same behaviour of a system, therefore also modelling languages for process-based modelling are considered in the evaluation for the optimal modelling language. 
According to the above-defined requirements, the following common modelling languages form a group of SM-based modelling languages that potentially satisfy the requirements (Steinheimer et al., 2017a):

- Business Process Model and Notation (BPMN) (OMG, 2011)

- UML Activity Diagram (UML AD) (OMG, 2015)

- UML StateMachine Diagram (UML SMD) (OMG, 2011)

These modelling languages are described below and evaluated according to the defined requirements. The first two modelling languages represent candidates for Functional View (describing process-oriented modelling, workflows). The last modelling language represents a candidate for Behavioural View (describing behaviour-oriented modelling). All of these modelling languages specify a set of elements that can be combined graphically to describe a process or the behaviour of an application. To evaluate the modelling language candidates regarding the defined evaluation criteria, the following section describes if each modelling language can satisfy the specific requirement.

BPMN is a standard, defined by the Object Management Group (OMG) to provide a formal notation of business processes that is easy to understand by all involved users (e.g. business analysts, technical developers or people manging/monitoring the defined processes) (OMG, 2011). BPMN provides a graphical notation that is "widely accepted for modelling business processes" (Geambasu, 2012).

UML AD is a diagram specified by the Unified Modelling Language standard (OMG, 2015) for describing the behaviour of a system using graphs. The graph consists out of nodes that are connected to other nodes using edges. Some of the nodes represent "lower- 
level steps in the overall Activity" and other nodes "hold data that is input to and output from executable nodes". UML AD describes e.g. procedural computations or can be applied for modelling business processes or workflows in organisations (OMG, 2015). The focus of modelling with a UML AD is in coordination "lower-level behaviours" of a system (Geambasu, 2012).

UML SMD is used to model event-driven behaviour of a system with the use of "finite state-machine formalism". The finite state-machine formalism applied in UML SMDs is based on Statecharts as defined by David Harel (Harel, 1987). The UML SMD consists of states that can be connected to other states by transitions. UML SMD distinguishes between Simple States without internal transitions or states and Composite States that contains other states and transitions (OMG, 2011). According (Rupp et al., 2007) UML StateMachines are an extension of FSMs. The behaviour of a system is specified by means of states. A SM describes how a system behaves in a certain state during certain events.

It has been defined as a precondition for selecting appropriate candidates that the modelling language is specified in a common standard. This illustrates the first advantage against the FSM introduced in section 5.2, which can be described mathematically but is not specified by any standardisation committee.

\section{M2M Device/MMSC Mapping and Conditional Transition}

Using BPMN as modelling language for M2M applications the M2M devices/MMSCs can by mapped to Activities representing the performed action. Sequence Flows representing the information flow between M2M devices/MMSCs can connect Activities. BPMN distinguishes between Conditional Flows and Sequence Flows. A Conditional 
Flow can be equipped with a condition specifying whether a Flow is only processed if the condition is true. In UML AD the M2M devices/MMSCs can be mapped to Actions that can be connected using Activity Edges. The Activity Edges in an UML AD represent the control flow of a process and can be used to describe the connection of M2M devices/MMSCs. The UML AD contains so-called Control Nodes. The Decision Node is a specific Control Node that can be used for specifying conditional control flows. To specify a condition the Activity Edge connected to the Decision Node can be equipped with a condition. The control flow then only is performed if this condition is satisfied. Using UML SMD as modelling language the M2M devices/MMSCs can be mapped to States and connected by a Transition. The Transition between two States can be equipped with a so-called "Guard Condition" specifying if a Transition from one State to another State is executed only if the "Guard Condition" is fulfilled.

All three candidates include elements in their modelling language specification that enable the mapping of M2M devices/MMSCs to elements in the modelling language. Also all candidates provide connection functionality of elements to represent the connection of M2M devices/MMSCs, respectively the information flow between them. The connection can be defined with either direct connections or connections based on a condition.

\section{Intuitive Usability}

BPMN offers a wide range of elements that can be used to model processes. BPMN enables modelling in a very high degree of detail. This makes the language versatile but also significantly more complex than e.g. UML SMD. Therefore, when using BPMN, it is important to select the elements describing an M2M application that are understandable 
for the users and to limit the number of elements needed to model an M2M application. UML AD offers the possibility to describe processes in a very detailed way using a large number of available elements for the modelling. Since there are many different elements for the modelling of a process using UML AD, UML AD can only be used if the selection of the available elements is limited. The elements of UML SMD are clear and become combined graphically. The basic structure of the UML SMD corresponds to the structure as M2M applications are modelled using the GUI presented in section 5.3. UML SMDs describes an abstract view of the system. According (Labiak and Miczulski, 2004), Statecharts offer the possibility to describe the complex behaviour of a system in sufficient detail, so that it can be used by non-specialists (i.e. persons who do not have technical training).

BPMN, UML AD, and UML SMD allow the modelling of the behaviour of an application with their elements. BPMN and UML AD offer here a very large number of available elements for modelling. At the same time, this represents a high complexity that restricts the intuitive use of the modelling language. Using BPMN and UML AD, a system is modelled using the bottom-up approach. An abstract description with the top-down approach is not possible because the degree of detail. But this would be better for the enduser to understand, since not the system details but the overall behaviour should be described. Since the elements available for the modelling of UML SMDs are limited in their diversity compared to BPMN and UML AD, but the desired behaviour can be modelled at the same time, intuitive modelling is possible rather than with the other candidates.

\section{Parallel Flows}


BPMN provides a Fork element that can be used to divide a path into multiple paths. Using the Fork element enables parallel processing of activities and therefore parallel processing of M2M devices/MMSCs. The UML AD enables describing parallel flows by providing Fork Nodes for splitting control flows into multiple parallel control flows. This enables the parallel processing of actions (i.e. the parallel information flow between different M2M devices/MMSCs). UML SMD distinguishes between simple States and Composite States. A Simple State does not include other States. A Composite State can include other States connected by Transitions (i.e. another SM). A Composite State can be separated into so-called Regions that specify two or more included SMs executed in parallel.

All three candidates enable modelling of parallel sequences in the application structure. FSM cannot model this kind of behaviour because it specifies only one target state per transition and does not allow multiple target states be reachable from one originating state.

\section{Synchronisation of States}

The counterpart to a Fork element in BPMN is the Join element. Via the Join element, parallel Sequence Flows (i.e. parallel M2M devices/MMSCs) are recombined and synchronised. UML AD provides Join Nodes as counterpart to Fork Nodes to synchronise (i.e. recombine) parallel control flows. UML SMDs have so-called Join and Fork nodes. A Fork node can be used to split a transition and a Join node can merge them again. This makes it possible to generate parallel sequences, which are also recombined again synchronously. Above described Parallel Flows defined by regions in a Composite State 
can also be synchronised. Then the composite state is left by transition only if all including parallel SMs have reached their Final States.

All three candidates support merging and synchronisation of parallel flows. This FSM naturally do not support because missing parallel flow capability.

\section{Machine Readability}

BPMN is a graphical notation and not machine-readable. According (OMG, 2011) the standardised Web Service Business Process Execution Language (WSBPEL) (OASIS, 2007) has been specified as a formal description language for definition of business processes. WSBPEL is a "web service-based XML execution language" for business processes and is machine-readable. The BPMN standard specification includes mapping definitions for a subset of BPMN elements to WSBPEL (Geambasu, 2012 and OMG, 2011). According (Geambasu, 2012) no specification exist that maps the UML AD components "to any business process execution language", which means that UML AD is not machine-readable because no formal description exists. UML SMDs are not machine-readable, since they are a graphical notation. However, with State Chart extensible Markup Language (SCXML) (W3C, 2015), an execution language for SMs specified by World Wide Web Consortium (W3C) exists that is based on Harel Statecharts (same as UML SMDs) and allows formal description of SMs using XML. Since SCXML is a formal language, it is machine-readable and can therefore be processed automatically, which means that through UML SMDs described by SCXML automated processing of the application description is possible. 
None of the three candidates support machine readability out of the box, but for BPMN and UML SMD standardised execution languages exist enabling the formal description of modelling languages and therefore are machine-readable. The scope of UML SMDs is fully implemented by SCXML. WSBPEL only implements a subset of BPMN elements. For UML AD no standard specification exist for formal description of UML AD which prohibits the automated execution of M2M applications described using UML AD.

\section{State parametrisation}

BPMN represents data using Data Objects that are added to Flows between Activities. These Data Objects define the data exchanged between the Activities, respectively the M2M devices/MMSCs. Inside of a BPMN Activity this data need to be associated to Data Input field of the Activity. The Data produced by Activities are represented as Data Output field of an Activity. Both, Data Inputs and Data Outputs need to assign the data contained in the Data Object to the corresponding Input and Output fields of an Activity using InputOutputSpecification, which is an element of the Activity itself. In a UML AD, data is represented by Object Nodes exchanged between the Actions. An Action can generate an Object Node as an output, which in turn is processed as an input in another Action. The Data Objects leave an Action via an interface and are imported into an Action via an interface. These interfaces are referred to as parameters of an Action and can be uniquely identified by name. For the States in UML SMDs, so-called Entry and Exit Activities can be defined. These Activities are executed as soon as a State is entered or exited. These Activities are methods that are called and can be used to define input and output parameters for the State. For input and config parameters the Entry Activity would be used and for Output parameter the Exit Activity. In SCXML, it is also possible to 
explicitly define parameters for a State. They can be used to define the parameters in the XML description of the Statechart.

All three candidates provide the functionality to equip states with parameter and therefore enable the configuration of M2M device/MMSC parameters contained in the corresponding element of the modelling language.

\section{Existing parser/interpreter implementation}

For WSBPEL, there are a number of Execution Engines which can interpret and execute the processes described by WSBPEL, e.g. Apache ODE (Orchestration Director Engine) (ODE, 2017) or Oracle BPEL Process Manager (Oracle, 2017a). Since UML AD are not described with a formal language, there is no parser/interpreter implementation. The Apache Commons Foundation (Commons SCXML, 2016) provides a parser/interpreter for SMs described with SCXML.

The candidates BPMN and UML SMD therefore satisfy the requirement that a parser/interpreter exists for the formal description language.

\section{Domain independent}

BPMN is a domain independent language but requires WSBPEL as formal execution language. WSBPEL is a domain specific language because it is limited to integration of web services. According (Thakar et al., 2016) the WSBPEL standard (OASIS, 2007) is specified for traditional web service architecture to integrate web services as components into business processes using Simple Object Access Protocol (SOAP) (W3C, 2000). The details of the components, such as input and output parameter of services and service endpoints are described using web services Description Language (WSDL) (W3C, 2001). 
The WSDL specification is designed for SOAP-based web service compositions, not usable for RESTful architectures, which is the architectural approach for M2M environments (refer to section 3.1.1). UML AD is a domain independent language because it can describe processes in various application domains. Since SCXML is realised using XML, it is a domain independent language. Since its application area is not limited to a specific field SCXML is a domain independent modelling language for SMs.

The modelling languages UML AD, UML SMD, and FSM are not specified for use in a specific application domain, but can be used for modelling/describing behaviour-based systems in various application domains. BPMN itself is not limited, but WSBPEL as execution language is limited for use in web service application field.

Table 5.7 summarises the evaluation results of the modelling language candidates and compares it with the FSM principle introduced in section 5.2.

Table 5.7: Evaluation of SM-based Modelling Languages acc. (Steinheimer et al., 2017a)

\begin{tabular}{|c|c|c|c|c|}
\hline \multirow[t]{3}{*}{ Requirements } & \multicolumn{4}{|c|}{ Modelling Language } \\
\hline & \multicolumn{2}{|c|}{ Functional View } & \multicolumn{2}{|c|}{ Behavioural View } \\
\hline & $B P M N$ & $U M L A D$ & $U M L S M D$ & FSM \\
\hline Standardised Language & + & + & + & - \\
\hline M2M Device/MMSC Mapping & + & + & + & + \\
\hline Intuitive Usability & 0 & 0 & + & + \\
\hline Parallel Flows & + & + & + & - \\
\hline Synchronisation of States & + & + & + & - \\
\hline Machine Readability & + & - & + & - \\
\hline State Parametrisation & + & + & + & - \\
\hline Existing Parser/ Interpreter & + & - & + & - \\
\hline Domain independent & 0 & + & + & + \\
\hline
\end{tabular}

The result of the evaluation is that UML SMDs fully satisfy the requirements and therefore the standardised description language SCXML as formal service description is proposed for describing M2M application behaviour. BPMN and UML AD represent both 
very extensive modelling languages allowing a fine granular and detailed description of processes, whereby BPMN serves more for the description of business processes. BMPN also fulfils almost all requirements for the formal description language. However, the fact that WSBPEL is limited to Web service environments prevents WSBPEL from being used for the application description in a RESTful M2M environment.

Since UML SMDs, respectively Statecharts as modelling language and SCXML as execution language were selected, these are presented in detail below.

\subsubsection{Principles of Statechart Modelling}

The M2M application semantic is graphically designed by creating an SM-based behaviour model (see section 5.3) that should be described using UML SMDs or Harel Statecharts in the formal execution language SCXML (see section 5.5.1). The following section presents the relevant elements and principles for modelling using Statecharts.

The main elements of Statecharts are states and transitions. States are illustrated as boxes with rounded corners and the name of the state is placed inside the box. Arrows connecting the states represent transitions (Harel and Politi, 1998). States in the diagram can be active representing the current state of the system and by a transition, it can move to another state of the system (Harel and Naamad, 1996). In particular a state represents an active configuration of a system and can move from one state (i.e. configuration) to another state based on specific triggers acting on the system, such as external events or predefined conditions causing the state transition (Harel and Kugler, 2004). 
The system moves from one state to another state following the specified transitions and performs defined actions while residing in states or moving along a transition. Execution of a Statechart starts always at an initial state specifying the entry-point of a system (Harel and Politi, 1998). Via Transitions, it is described according (Harel and Naamad, 1996) from which state it should be moved to another state. Transitions describe the connection between states. The state from which a transition originates is referred to as a Start State and the state to which the transition terminates is referred to as a Target State. Transitions can be provided with conditions that specify whether to perform a transition from an active state.

According (Harel and Kugler, 2004) a Statechart can contain Basic States and Hierarchical States. A Basic State does not contain substates and forms the lowest instance in state hierarchy. Hierarchical States are composed out of other states and can exist in two different configurations: OR-States containing substates that are "related to each other by exclusive OR" and AND-States including substates that contain “orthogonal components that are related by AND".

Figure 5.25 illustrates a Basic State as well as the Initial State specifying the entry point of the Statechart and the Final State specifying the termination of the described behaviour.

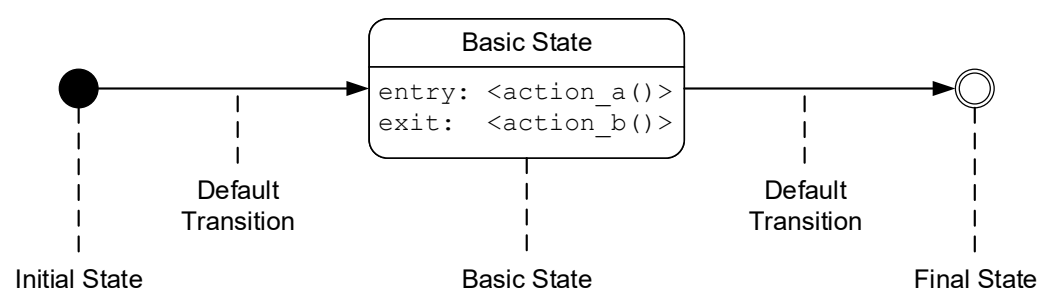

Figure 5.25: Statecharts Basic State and Default Transitions, derived from (Harel and Politi, 1998) 
A Default Transition to the states in a Statechart connects Initial State and Final State (Harel and Politi, 1998). States and transitions can be equipped with Actions that are executed during a transition or while either entering a state (Entry Action) or leaving a state (Exit Action) (Harel and Kugler, 2004). A Default Transition is a special kind of transition that cannot by equipped with a condition or action. The Statechart in Figure 5.25 describes that after entering the Basic State the action_a() is executed and while leaving the Basic State the action_b() is executed. Actions can be static calculations or method calls to trigger any additional functionality to be executed while residing in a state or moving from one state to another.

As mentioned above two kinds of hierarchical states exist describing different behaviour. Figure 5.26 illustrates the hierarchical AND-State A. According (Harel and Politi, 1998) AND-States consist of one or more orthogonal components (sections) executed simultaneously. AND-States can therefore be used to describe parallel sequences, which again are defined by states. For each parallel section it has to be defined which state is the Initial State and therefore should begin with the execution

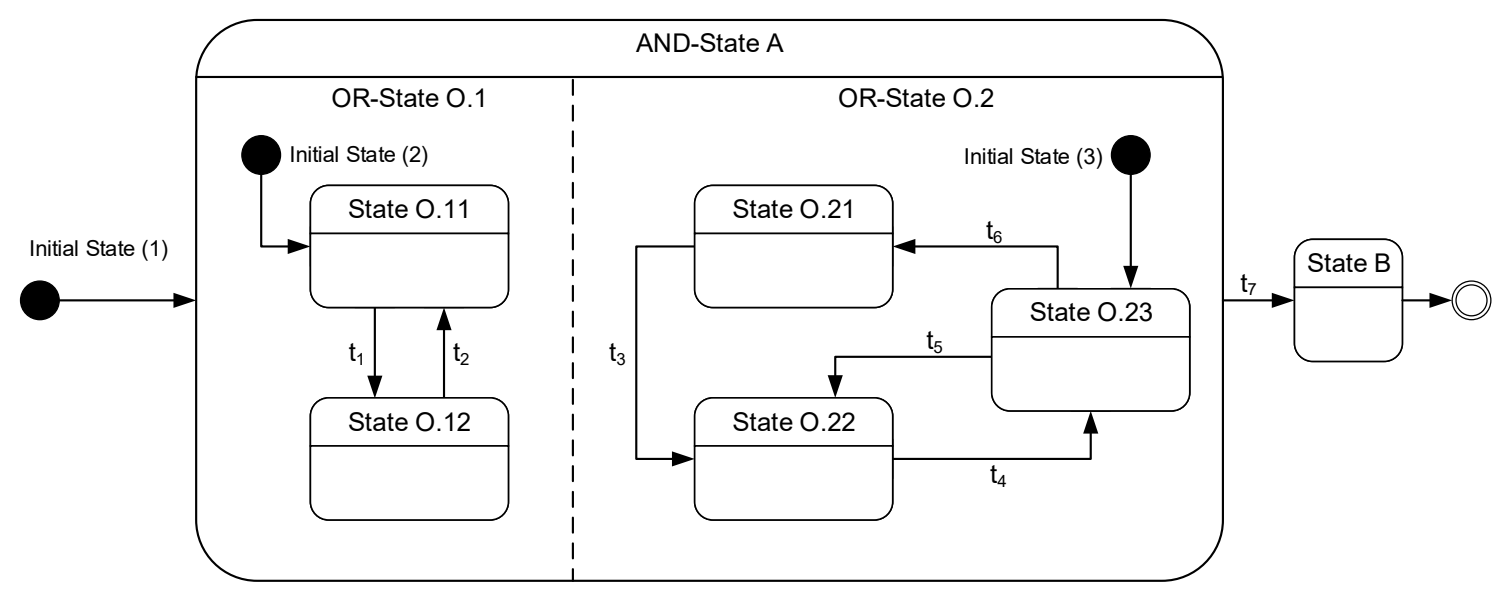

Figure 5.26: Statecharts AND-State, derived from (Harel and Politi, 1998) 
In an AND-State, additionally to the enclosing AND-State itself, the embedded states are always active within a section and execute actions. A Parallel State is left when all sections have reached their final state (which was not defined in the exemplary ANDState $A$, so the $A N D$-State $A$ remains within the contained sections). Another way to leave an AND-State is when a transition is generated from an inner state to a state outside the Hierarchical State, or when a transition of the comprehensive Parallel State is triggered. In the example shown in Figure 5.26, AND-State $A$ is therefore only left when the transition $t_{7}$ has been triggered. The active state then changes to State $B$ without regards of which internal state the $A N D$-State $A$ currently resides.

The second type of Hierarchical State is the OR-State. Figure 5.27 shows an exemplary OR-State $O$. The OR-State also contains states that in turn are connected with transitions.

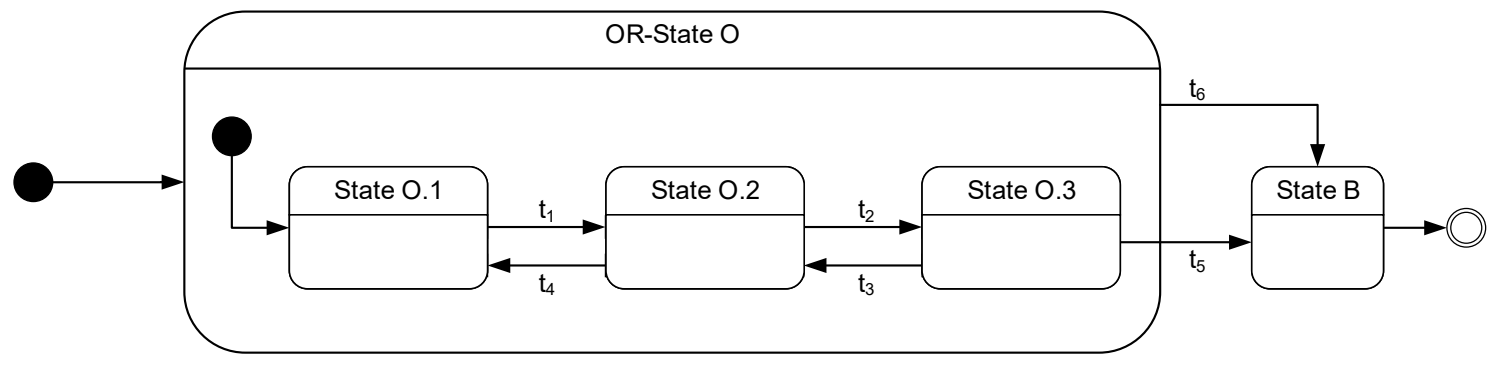

Figure 5.27: Statecharts OR-State, derived from (Harel and Politi, 1998)

An OR-State has no parallel sections, but group's individual states. It is also necessary to define for an OR-State which of the contained states is the initial state, i.e. which state should start processing as soon as the comprehensive OR-State is reached. An OR-State can terminate its processing when a transition is triggered within the Hierarchical State (e.g., $t_{5}$ ) targeting to a state outside the Hierarchical State. A further possibility to leave an OR-State is when a transition connected to the comprehensive OR-State is triggered and leads to an external state (e.g. $\left.t_{6}\right)$. 
After the principles of modelling using Statecharts have been introduced, the following section introduces the formal description of Statecharts using SCXML.

SCXML has been specified by $\mathrm{W} 3 \mathrm{C}$ as recommendation for an executable SM language that combines Harel semantics of Statecharts with XML syntax. It has been defined because UML SMDs only are specified as "graphical specification language" without XML representation for automated execution (W3C, 2015). Subsequently the basic structure of a formal description of Statecharts using SCXML is presented using two examples.

Figure 5.28 shows the formal description by SCXML of the example of a minimal Statechart, shown in Figure 5.25, consisting of a basic state and two default transitions.

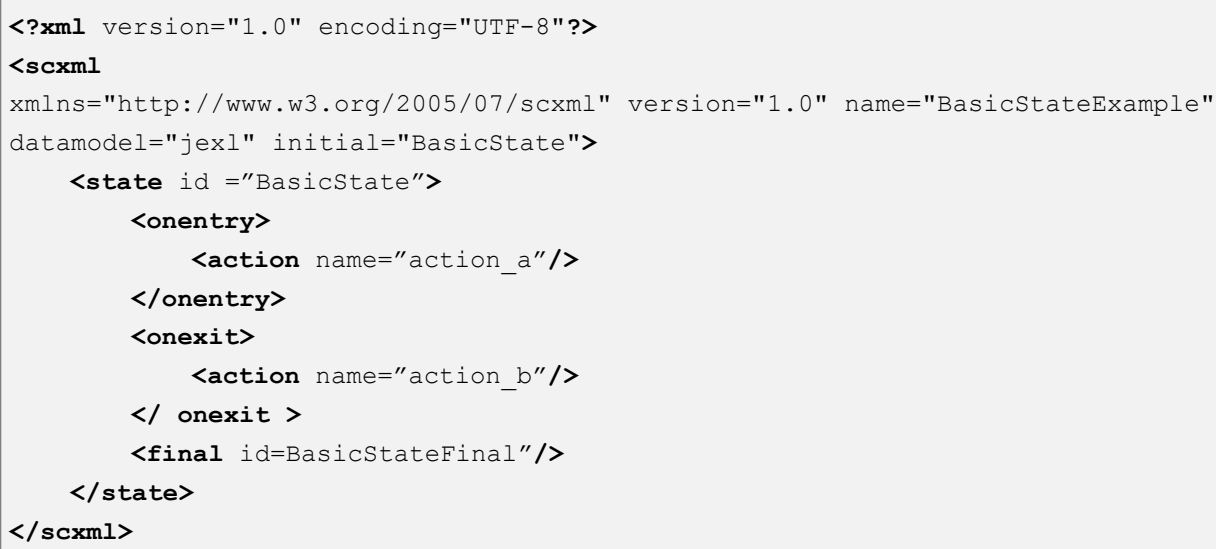

Figure 5.28: SCXML Representation of Basic State and Default Transitions

Since the document format of a SCXML description is XML, every SCXML description starts in the first line with the XML declaration, specifying the XML version and the encoding used. Next the root element has to be specified as $<$ SCXML $>$ which is the element encompassing all components of the Statechart. Inside the root element the namespace and version of SCXML is specified. The root element additionally contains 
the name of the Statechart and the datamodel used in the description. Lastly, the SCXML root element contains the definition of the Initial State, which is in that example the state with ID BasicState. The other elements contained in the SCXML description are states and transitions in different variants. States are defined using the $<$ state $>$ element including the ID of the state and transitions are defined using the <transition> element. In the exemplary Statechart, $<$ BasicState $>$ is the only state defined. The action to be executed when entering a state is defined using the $<$ onentry $>$ element. The actions to be executed when leaving the state is defined using the <onexit> element. Both elements include the $<$ action $>$ element specifying the action by name that the system should execute when entering or leaving the state. If a state should be defined as a Final State, this is defined using the $<$ final $>$ element defining the encompassing state as a Final State. The Final State element includes a specific ID, here BasicStateFinal to uniquely identify the Final State.

Figure 5.29 illustrates a more complex example describing the SCXML representation of the Statechart illustrated in Figure 5.26.

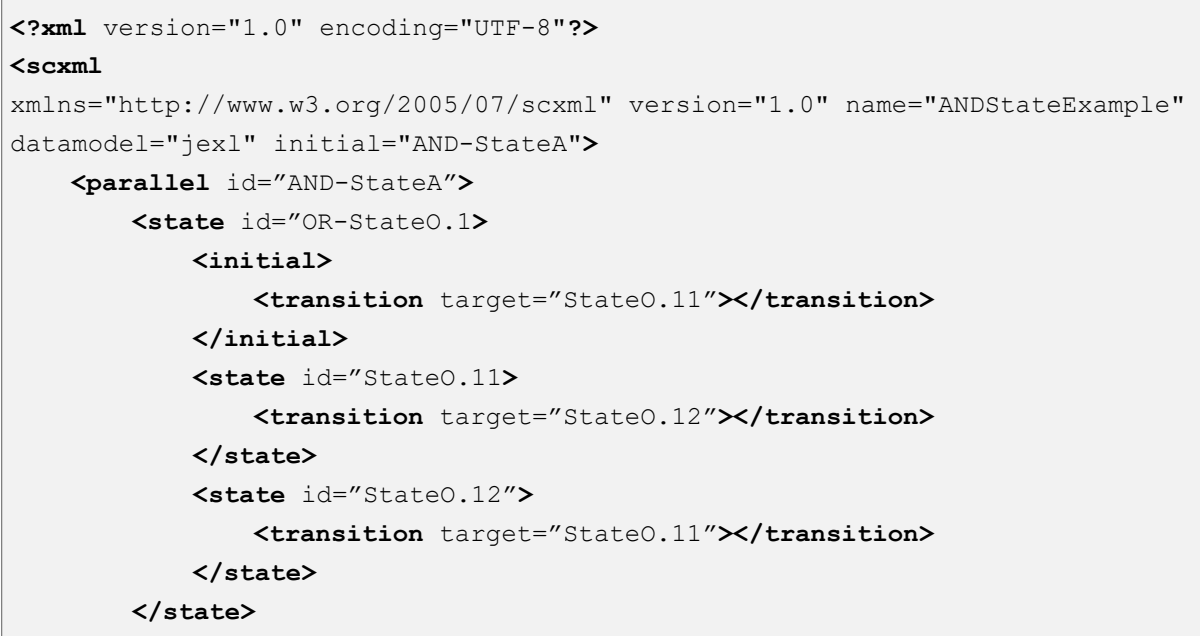




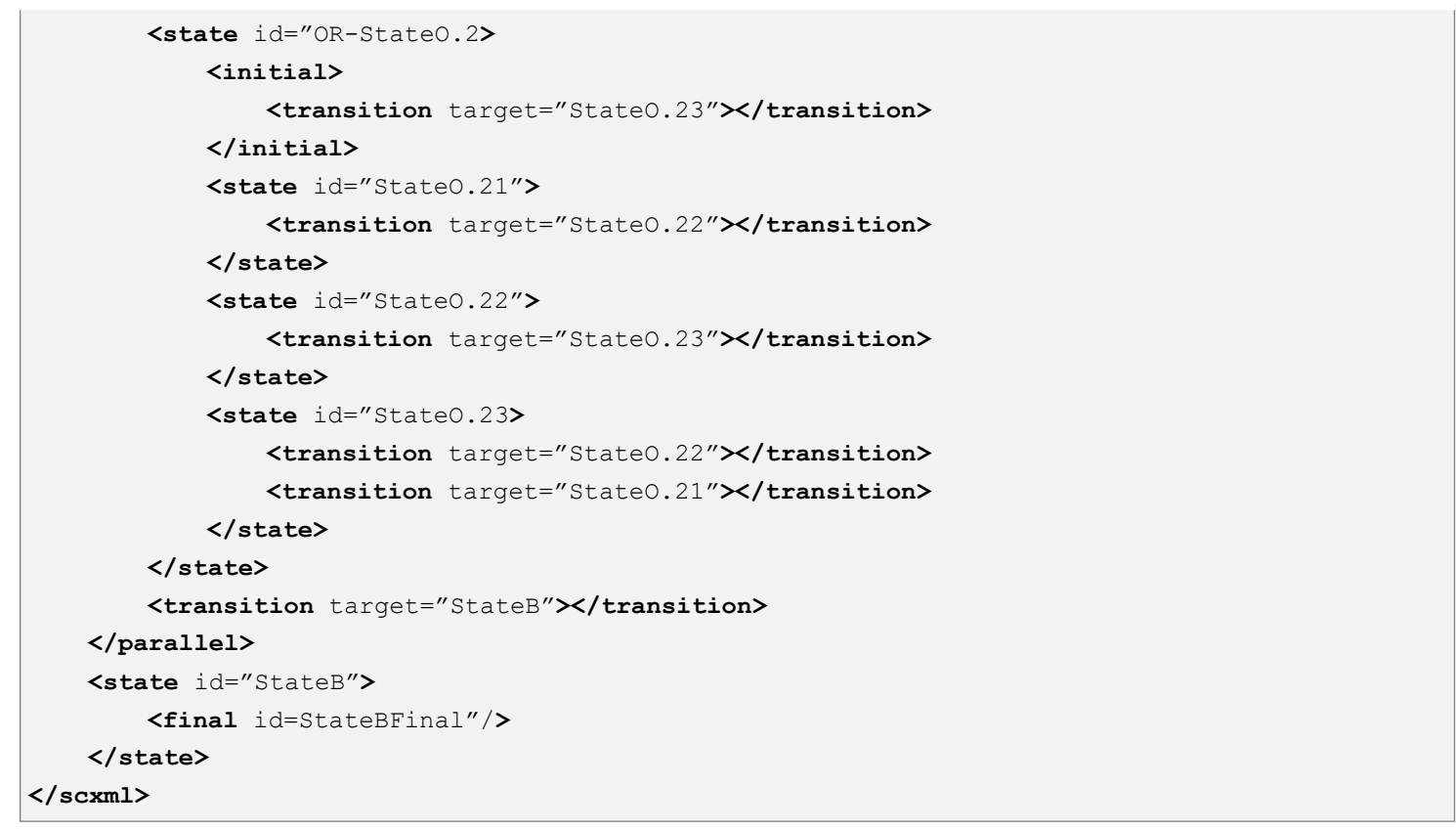

Figure 5.29: SCXML Representation of AND-State

This example contains an AND-State (AND-StateA), which SCXML specifies using the $<$ parallel $>$ element and another simple state $($ StateB $)$. StateB is defined as Final State using the $<$ final $>$ element. The AND-State, respectively Parallel State encompasses the two OR-States OR-StateO.1 and OR-StateO.2. Parallel States and OR-States must specify the Initial State for their containing states. This is done using the <initial $>$ element that specifies the Initial State as a Transition Target with the ID of the Initial State element. In the example Statechart for the hierarchical OR-States StateO.11 and StateO.23 are defined as Initial States. If states are connected to another state by transition, this is defined using the $<$ transition $>$ element. Inside the $<$ transition $>$ element, ID of the Target State defines the Target State of a transition.

This section introduced the principles of behaviour modelling with Statecharts as modelling language and introduced SCXML as standardised SM execution language. Both bring the possibility to model and describe systems flexible and very detailed but 
with reduced complexity. The following section describes how to apply the power of Statecharts and SCXML to formally describe M2M applications.

\subsubsection{M2M Application Modelling using Statecharts}

In the previous sections it was introduced how MMSCs can be integrated into M2M applications to create an interface to them (section 5.1). Furthermore, a unified structure for an M2M Devices/MMSCs was specified, consisting of input/output/config parameters as interfaces to a component performing variable functionality (section 5.2). Moreover, it was worked out that the end-user graphically models the behaviour of an application in the form of a SM and thereby defines the application logic (section 5.2 and section 5.3). The modelling is done using Statecharts that are formally described with SCXML (Steinheimer et al., 2017b). To determine how such a modelled M2M application can be formally described by means of Statecharts, it is first necessary to define a general structure for an M2M application and to transfer this structure to the modelling principles using Statecharts.

It has already been determined that an M2M device/MMSC is represented as a state. It was also defined that the connection between M2M devices/MMSCs is made by defining transitions between the states. This is now to be described using Statecharts. Figure 5.30 exemplarily illustrates the Statechart representation of an M2M application with the help of Use Case 1 (refer to section 2.4), also described as FSM in Figure 5.13 and graphically modelled as illustrated in Figure 5.14. The corresponding SCXML representation of the Statechart is illustrated in Listing C.5. 


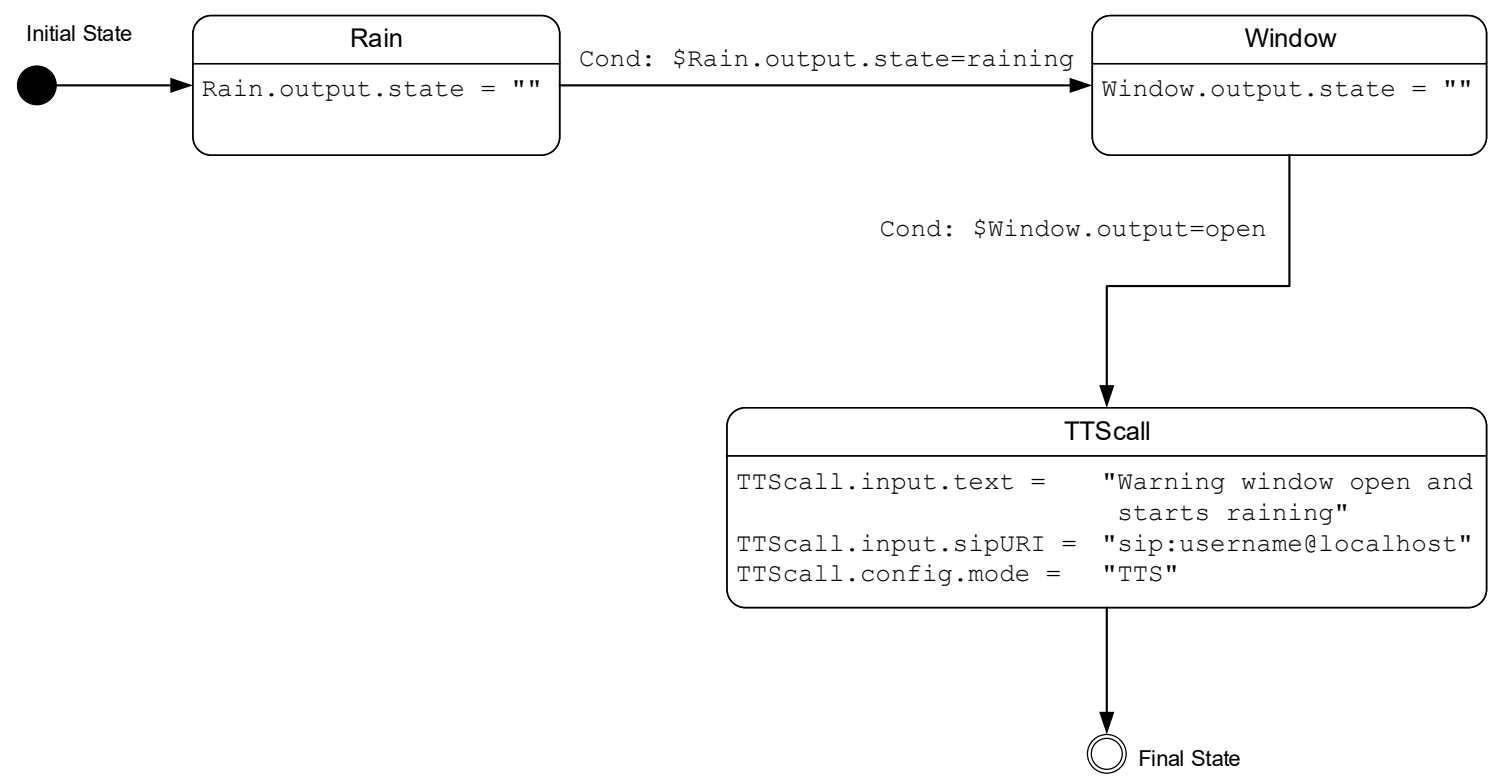

Figure 5.30: Statechart Representation of Use Case 1

M2M devices/MMSCs each are defined as a state (state-id: "Rain", "Window", "TTScall”). The configuration of an M2M device/MMSC and the data exchange between the components is defined by the parameters of a state. This can be mapped to the parameters of a state in Statechart representation, respectively SCXML. To determine which parameters are defined in a state for the specification of an M2M devices/MMSC, first it has to be defined which parameters an M2M device/MMSC can have. This has already been specified by the general structure of an M2M devices/MMSC by having any M2M device/MMSC 0...n Input/Output/Config parameter. The M2M DCM for each M2M device describes the device-specific structure. The parameters specified by the DCM are mapped using the parameters of a state. To obtain the best flexibility in determining the data structure, JEXL (Java Expression Language) (Commons JEXL, 2017) is proposed as the format for the Data Model. By integrating JEXL as a regular expression language in SCXML, it is possible to define the structure of the parameter declaration according to the ANT-style (Apache ANT, 2017). This makes it possible to 
categorise the parameters according to their structure within their naming according to input/output/config. The following structure is defined for the parameters:

- $<$ deviceID $>$.input.<parameter name $>$

- $\quad<$ deviceID $>$.output. $<$ parameter name $>$

- $\quad<$ deviceID $>$.config. $<$ parameter name $>$

Each parameter starts with the ID of an M2M device/MMSC followed by a ".". Attached to this is the category of the parameter. For this purpose, the input/output/config key words define the type of parameters. Finally, the parameter name is added to this category with a previous ".". For assigning the parameters to a state, it is necessary to define a Data Model for the state. This Data Model is an element of the state and contains a separate element for each parameter defining the name of the parameter via the entry data id, and the value of the parameter via the entry expr. In the example for Use Case 1 the output parameters of Rain and Window were created. Since they are output parameters which are assigned by the M2M device itself, these are not initialised within the Statechart or SCXML description, but are defined as "". The MMSC TTScall, respectively the state TTScall, has input and config parameters that must be specified. This is done in the Data Model of the state TTScall by assigning the corresponding configuration values to the input and config parameters via the expr entry.

The connection of the M2M devices/MMSCs is realised by the description of a transition between the M2M device/MMSCs. As described above, the connection between M2M application components should be conditional. This condition is specified within the transition element using the entry cond. In the example of Use Case 1, e.g. the following condition is defined for the state Rain: \$Rain.output.state=raining. This condition defines 
that the transition is only executed if the Rain.output.state parameter is equal to the value "raining".

Expressions can be specified not only in the conditions of a transition, but also in $<$ assign $>$ elements of SCXML, respectively through onentry/ onexit operations or transitions of states. These elements allow values to be assigned to parameters in the Data Model. Figure 5.31 shows, by means of two connected states, an overview of definition, query and assignment of parameters in a Statechart. The corresponding SCXML description in illustrated in Listing C.6. However, not only the static assignment of values to the state parameters is possible, but also the access by the expression to the parameters of the states or the global parameters of the Statechart. The ability to access the parameters of a state within a regular expression gives the possibility to define not only the values for the parameters of the states statically, but to access the contents of the parameters by means of the parameter name with preceding "\$".

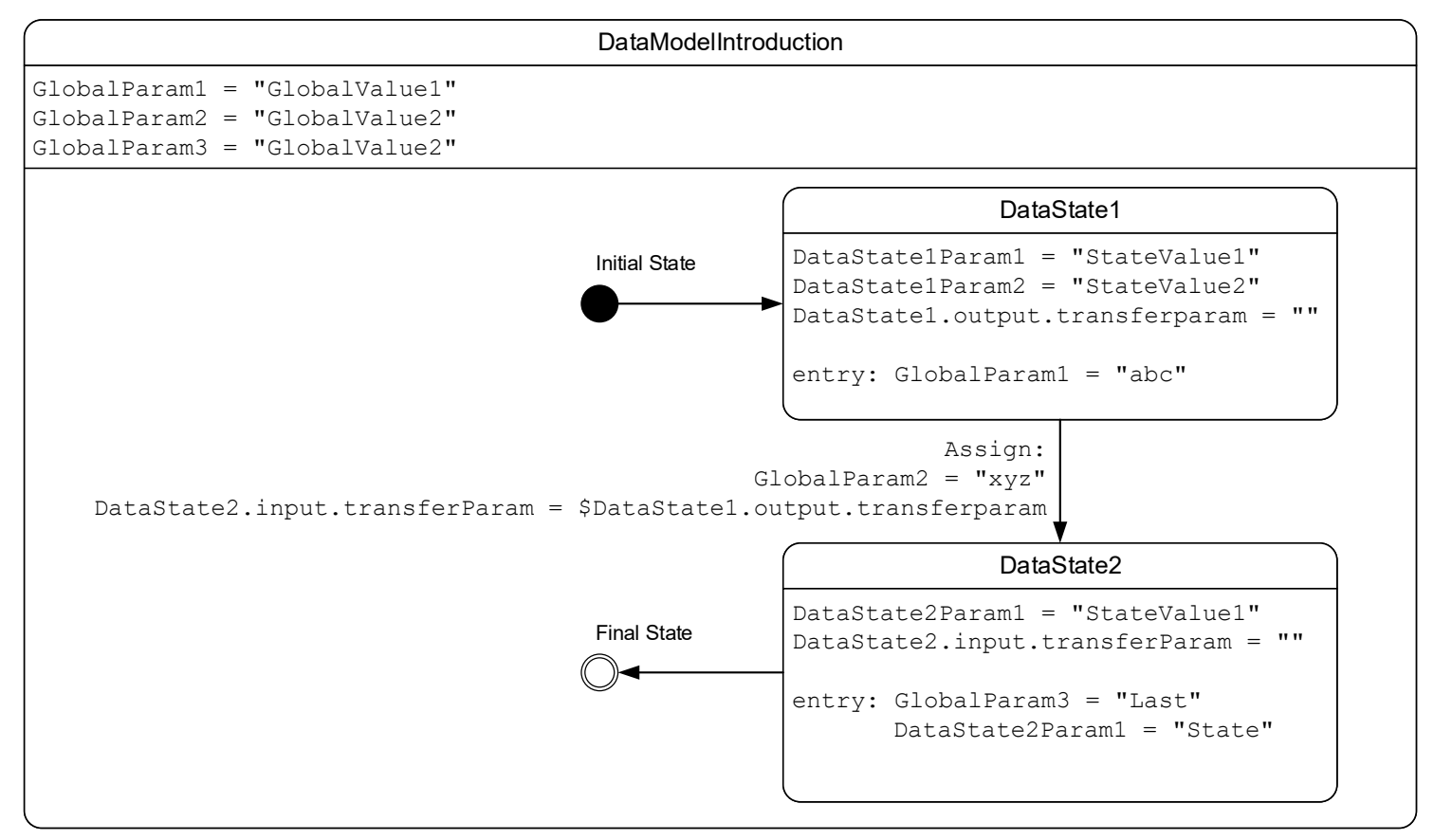

Figure 5.31: Principles of State Parameter Definition and Assign in Statecharts 
This enables that the M2M device/MMSC receives the output parameters of another M2M device/MMSC as an input value and can further process it within its service logic. This extends the original concept by allowing transitions between devices to have parameters and thus to transfer values to another device or to have transitions without parameters.

Up to this point it was specified how M2M devices can be connected with each other and exchange data. It has not been specified yet, how the structure of the application can be described as generalised as possible. For this purpose, the structure of a Statechart was derived and transferred to the structure of an M2M application. Thus, an M2M application can consist of different M2M devices/MMSCs connected sequentially (OR-connections), or in parallel sequences (AND-connections). Figure 5.32 shows the graphical representation of a combined OR-/AND-connection. Listing C.7 illustrates the corresponding SCXML pattern.

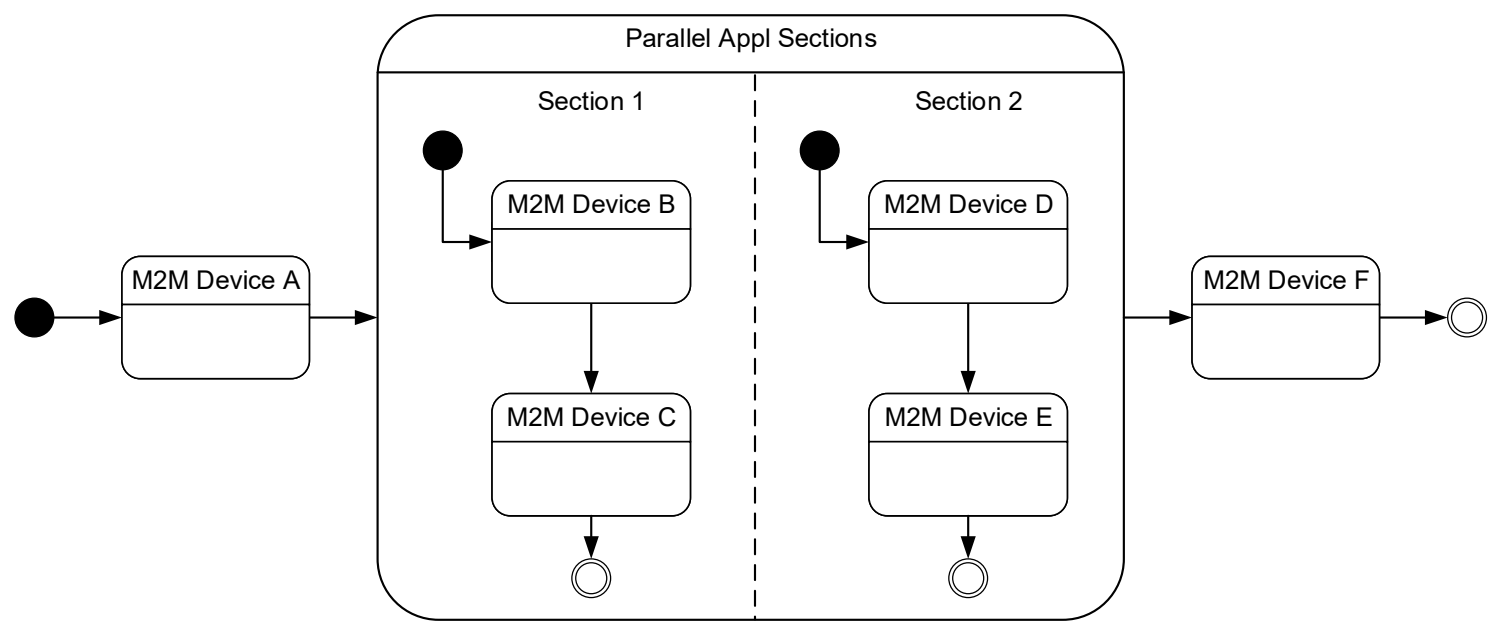

Figure 5.32: Graphical Representation OR-/AND M2M Device Combination

Through the generally defined, reusable structure of an M2M application description by means of SCXML, a possibility is created to formally describe M2M applications 
according to a unified pattern. This generally defined structure serves for the formal M2M application description process (described subsequently) to transform the graphically modelled application into a formal description.

To automate the formal description of the M2M application, the following section presents the process generating a formal description from the graphically modelled application. The Service Creation Unit (SCU) of the proposed framework performs this process. As an input, the process described here receives the graphical notation of the application description and generates the formal notation from it.

To define an adequate algorithm performing the generation of the formal description, a General M2M Application Model (see Figure 5.33) and a General State Model (see Figure 5.34) have been derived from the above-described principles of M2M application modelling by means of Statecharts.

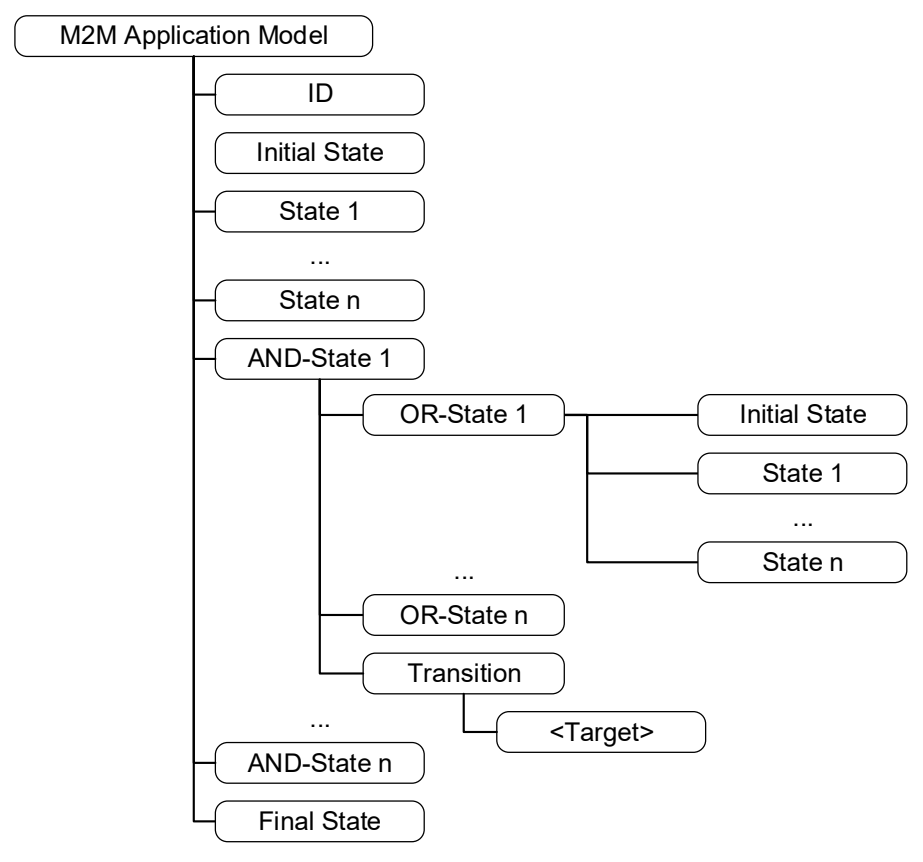

Figure 5.33: General M2M Application Model 
The General M2M Application Model describes the generalised structure of an M2M application. This defines that M2M applications always have an ID uniquely identifying them. Furthermore, the entry point must be defined for each M2M application realised by the Initial State definition. An M2M application also requires a defined exit point marking the end of the application realised by the Final State element. Additionally to these mandatory elements, an M2M applications have states as optional elements. These can be simple states not containing other states or complex states describing parallel sequences in M2M applications. Parallel sequences are modelled using AND-States, which have individual sections executed in parallel. For each of the parallel sections, it is necessary to define an OR-State that includes the states to be executed in parallel. For each OR-State, it must also be defined which of the contained Simple States is the entry point (defined as Initial State). Additionally AND-States have a transition to integrate them into an application flow.

The General State Model describes the generalised structure of a simple state. They have an ID to uniquely identify the state or the M2M device. Via OnEntry and OnExit elements of a state, an action can be defined by name that is executed when the state is entered or exited. Furthermore, a state has a Data Model (input/output/config parameters) that specifies the interfaces to an M2M component. 


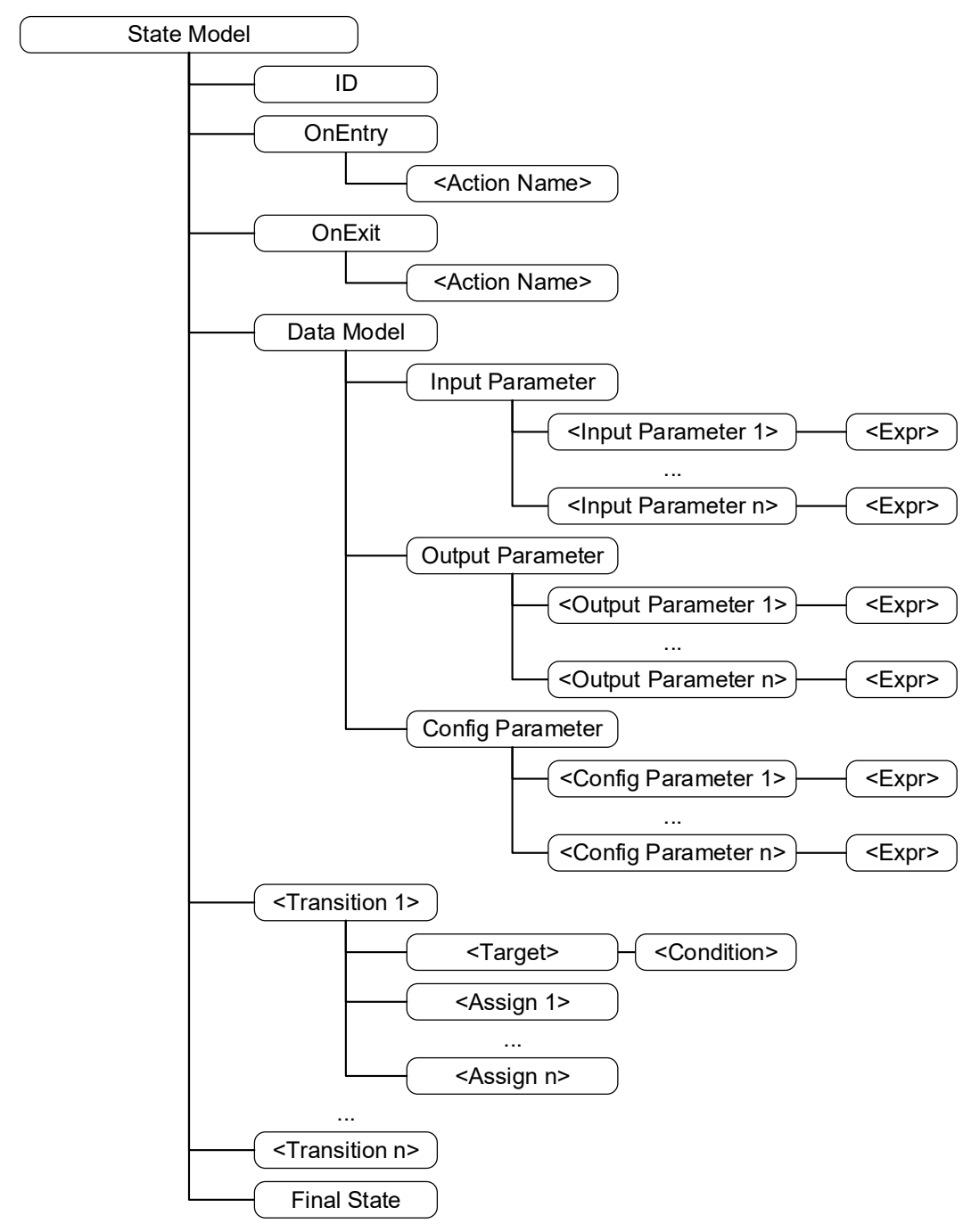

Figure 5.34: General State Model

A value can be assigned to each Input/Output/Config parameter using the $<$ Expr $>$ element of the parameter. Connections between $\mathrm{M} 2 \mathrm{M}$ components are represented by the Transition elements of a state. The Transition elements are used to specify which state is the target of the transition defined by the <target $>$ element. For each Transition, a condition can be defined specifying when the transition is executed. The condition is defined by the $<$ Condition $>$ element of the transition. If value assignments should be made to the M2M Components during a transition, this is specified via the $<$ Assign $>$ 
elements of the Transitions. A state can be declared as a Final State. If so, the state contains a Final State element in the specification.

The SCU now uses the General M2M Application Model, General State Model and the $M 2 M D C M s$ to generate the formal M2M application description applying the algorithm described in Figure 5.35. First, the SCXML frame is generated based on the General M2M Application Model. Where the name of the modelled application is specified as the ApplicationID. Furthermore, the start element is defined as initial state. Afterwards, all graphically modelled states are captured. Each of these states is analysed and inserted into the SCXML frame based on the result of the analysis. It is first checked whether it is a simple state or a complex state (parallel flow). If it is a simple state, a state element is generated based on the General State Model. Figure 5.36 shows the mapping of the elements from graphical notation into the formal description of the state according to (Steinheimer et al., 2017b). If it is a parallel sequence element, a parallel state element is first created in SCXML. All parallel sections are then captured. For each parallel section, an OR-State element is created and all states that are contained within the parallel section are captured. The collected states are simple states that are analysed as described above. After the formal definition of the state is done, the state is inserted into the OR-State element. After all states are captured and added to the OR-State element, the OR-State element is added to the previously generated parallel state element. As soon as all parallel sections have been processed, the parallel state element is added to the SCXML frame. 


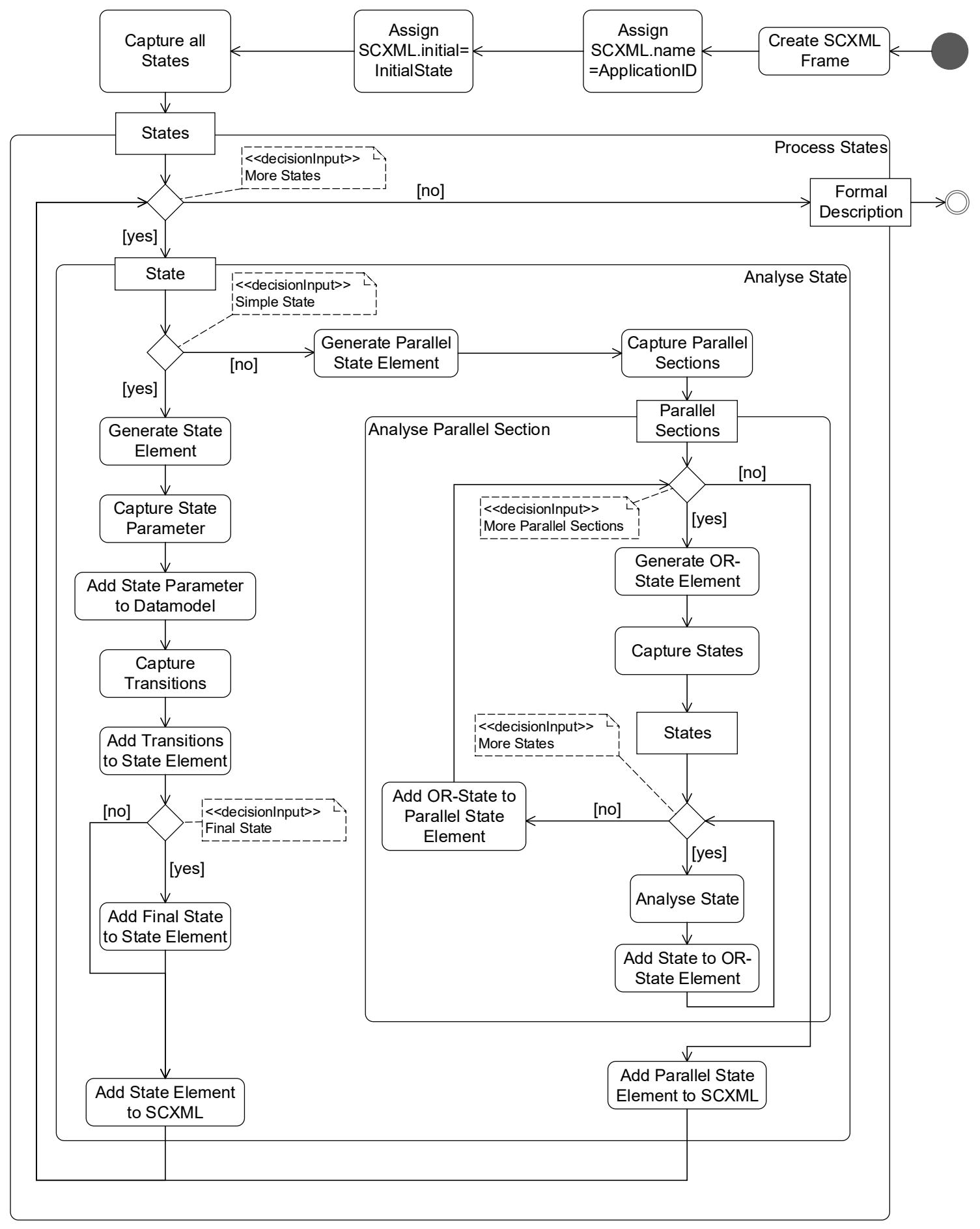

Figure 5.35: Formal M2M Application Description Generation Process 
5 Autonomous M2M Application Provision

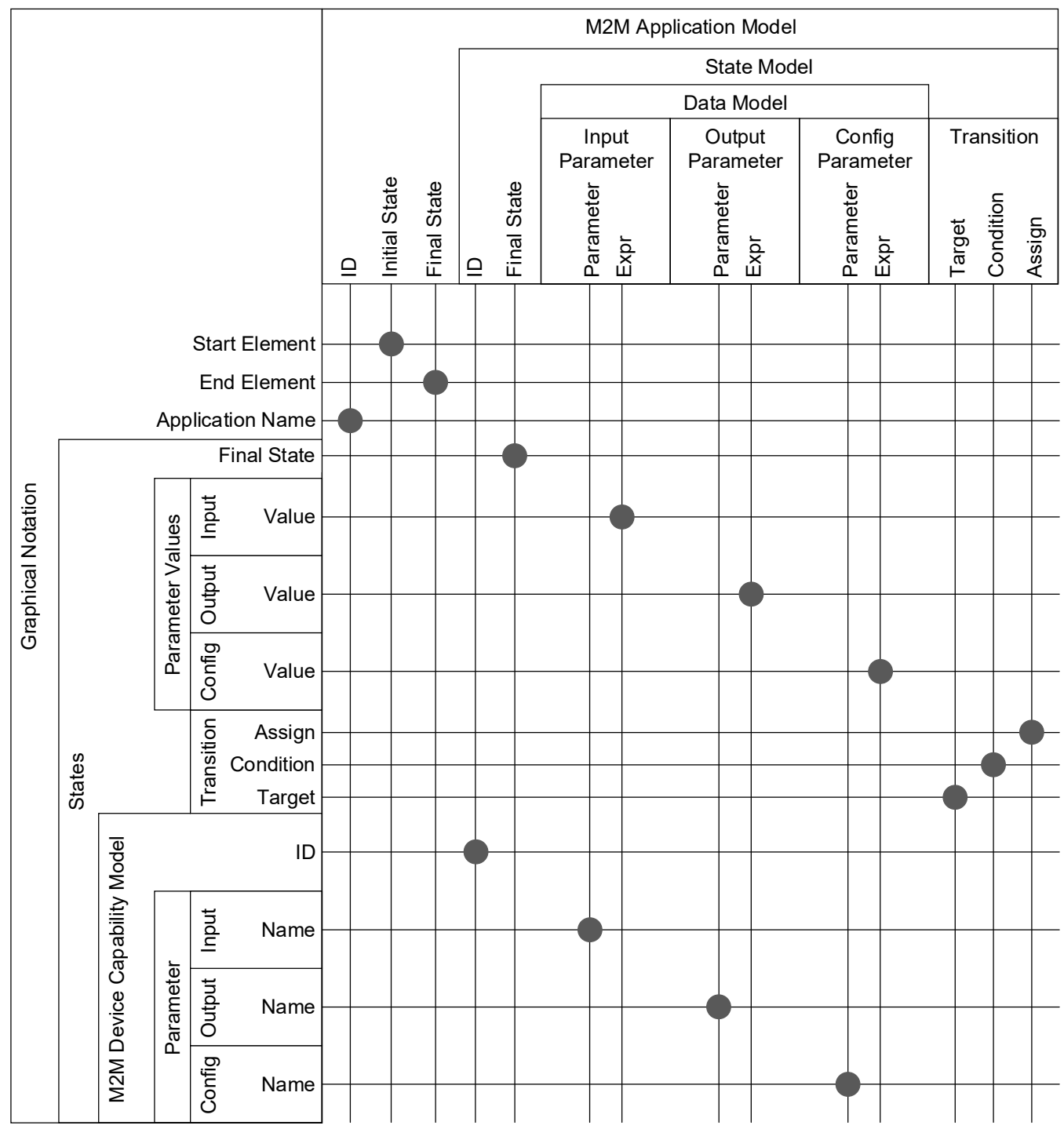

Figure 5.36: Assignment graphical Notation Elements to M2M Application Model

\subsection{Service Runtime Environment}

The previous sections introduced the SM-based modelling and automated formal description of local $\mathrm{M} 2 \mathrm{M}$ applications. As described in section 4.2, the formal M2M application description is passed from the $S C U$ to the $S R E$ for further processing. Figure 5.37 shows the structure of the $S R E$, which is responsible for executing the application. 


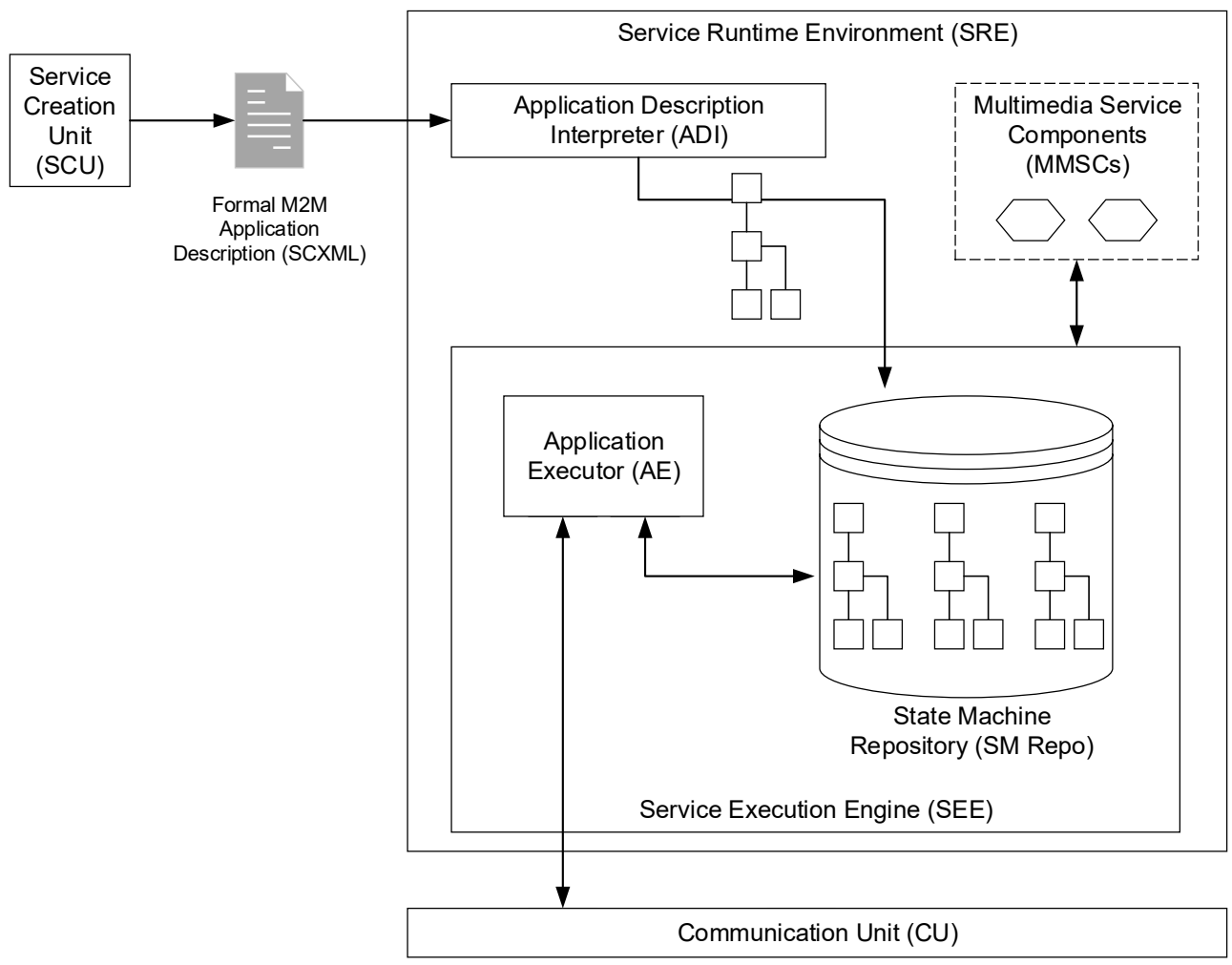

Figure 5.37: Service Runtime Environment (SRE)

The Application Description Interpreter (ADI) first parses the formal M2M application description after receipt and generates a SM from the described semantics defining the application logic. The generated SM is stored in the State Machine Repository (SM Repo) holding all SMs included in the M2M platform. The Application Executor (AE) holds an instance of each SM and receives messages containing Input/Output values from the $C U$ or the MMSCs. By analysing the defined SM behaviour, the $A E$ executes the defined actions for each state, depending on the defined conditions and parameter values. For determining the defined behaviour of the M2M application, the SEE holds the current state of the SM (starting with the initial state). Figure 5.38 illustrates the AE's analysis process for receiving messages from the CU and the corresponding SM evaluation. 


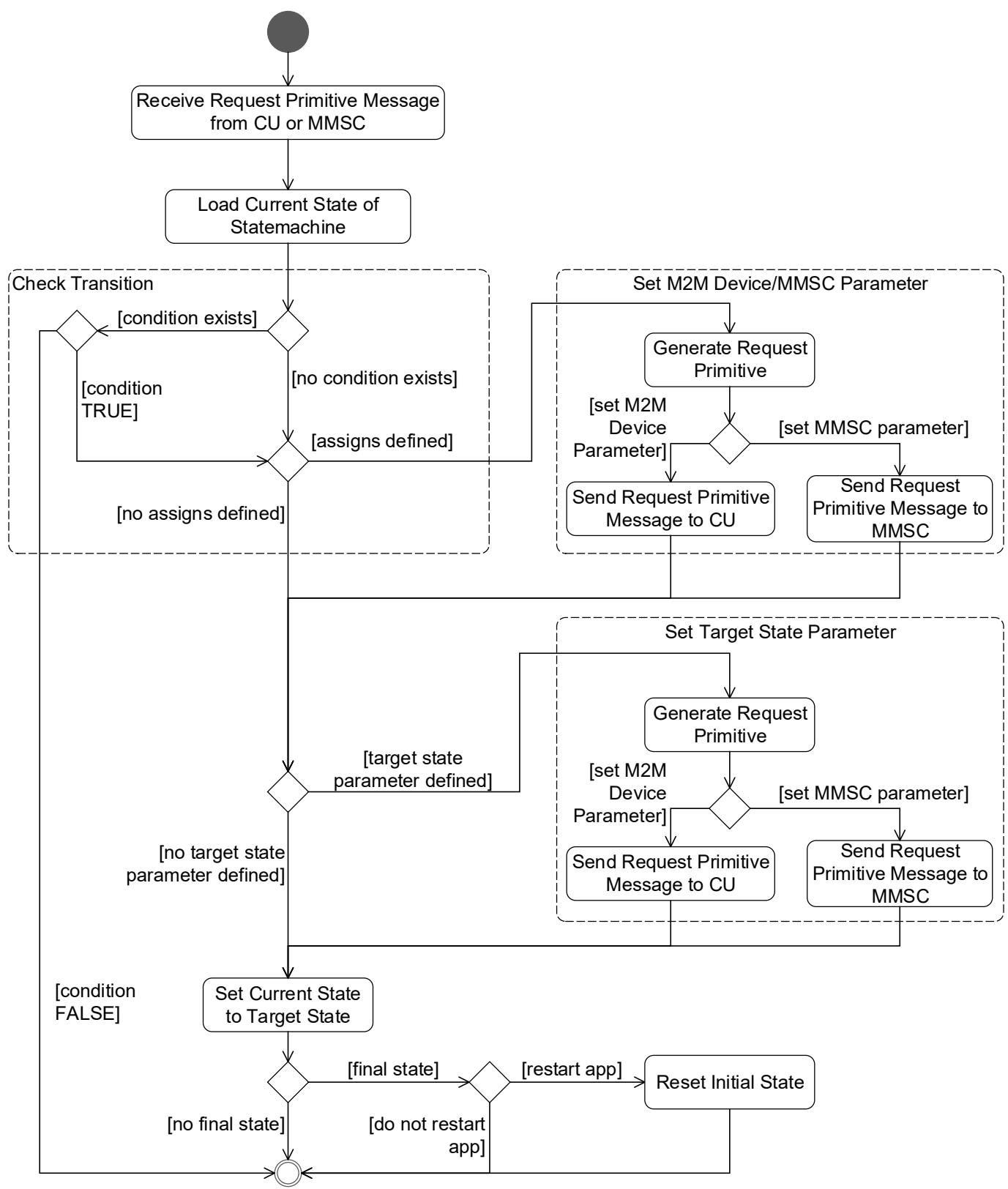

Figure 5.38: M2M Application Execution Process

After receiving a Request Primitive message from the $C U$ or $M M S C$, the $A E$ loads the current state of the SM, analyses it and executes the defined operations. First, the $A E$ checks the transitions. If condition are fulfilled or do not exist the $A E$ checks whether assigns have been defined to set $M 2 M$ device/MMSC parameters. If the condition of the transition is FALSE the SM resides in the current state. If assigns have been defined, then the $A E$ generates a Request Primitive message with the content of the parameter to be set 
and sends it to either $C U$ or $M M S C$, which set the values of the parameter as specified in the Primitive content. Afterwards the $A E$ checks whether target state parameter have been defined. If yes, the $A E$ triggers setting the specific parameter by sending Request Primitive messages. After executing the control operation by setting the M2M device/MMSC parameter the $A E$ sets the defined target state as the new current state of the SM and checks if it is a final state. If current state is final state, then the $A E$ checks if application should restart. If this is the case, the initial state is reset and the application starts from beginning.

\subsection{Conclusion}

This chapter 5 introduced MMSCs (section 5.1) that form a comfortable input/output interface to the M2M platform using existing multimedia communication equipment. Multimedia communication can serve e.g. for controlling M2M applications or to realise a communication from M2M platform and end-users to e.g. inform them about events occurred in their local M2M environment.

The principles of M2M application definition by means of modelling the behaviour of the M2M application has been introduced in section 5.2. It has been illustrated that the behaviour of an M2M application can be modelled by defining SM-based application flows. To achieve this a general structure of M2M device/MMSCs containing input/output/config parameters has been defined and mapped to the states of a SM. This enables the representation of an M2M device/MMSC inside the application behaviour model. The connections of M2M devices/MMSCs have been mapped to the transitions of the SM and representing the connection between them. The defined concept of 
behavioural modelling by means of SMs forms a generalised, intuitive and platform independent methodology to define the semantic of M2M applications.

Furthermore, it has been presented a structure of a GUI that can be applied for graphical design of the application model (section 5.3). The GUI forms an end-user interface for application generation by exposing M2M/MMSCs present in the personal environment of the end-user. The GUI can be used to graphically design the application model by combining M2M devices/MMSCs that are represented as building blocks. Conditions can be defined to make the application flow (connection between M2M devices/MMSCs) dependent on predefined conditions.

Then, section 5.4 described the principles for integration of different M2M technologies into the local M2M platform. To achieve this, a concept has been defined to abstract the communication between the specific M2M technologies and the communication inside the M2M platform, respectively the communication between M2M devices and defined M2M application using the AL component of the defined M2M platform architecture. The communication between M2M devices and M2M platform components takes place using RESTful communication principles and exchange of Primitive messages. A M2M DCM has been defined describing the abstract structure of M2M devices/MMSCs and forms as an interface description of them. It has been specified how to map the M2M DCM to Primitive messages exchanged between the M2M platform components to realise a generalised communication principle. Section 5.4 also presented different approaches for automatically or partly automatically integration of M2M DCMs into the M2M platform. Presented possibilities were the installation by the end-user, installation by the $\mathrm{M} 2 \mathrm{M}$ device, and the download from M2M device vendor. Installation by the M2M 
device has been identified as the most practical way because it prevents manual steps to be performed by the end-user and does not violate the general requirement of the presented concept to avoid central entities in M2M platform infrastructure.

Section 5.5 introduced the modelling languages BPMN, UML ADs, and UML SMDs for SM-based modelling. These modelling languages have been evaluated regarding defined requirements. The result of the evaluation was that UML SMDs satisfy the defined requirements and therefore has been selected as graphical modelling language for M2M applications. Since UML SMDs are based on Harel Statecharts, the principles of Statechart modelling has been introduced in detail as well as the standardised formal description language SCXML as machine-readable execution language for Statecharts. After introduction of Statecharts modelling and SCXML, the semantics have been defined to model and formally describe M2M applications using Statecharts. For this a general M2M Application Model and a General State Model were defined enabling the formal description of M2M applications using SCXML. For automatically generating the formal $\mathrm{M} 2 \mathrm{M}$ application description, a process has been defined that uses the General M2M Application Model and General State Model to create the SCXML description out of the graphical notation of the M2M application.

Finally section 5.6 introduced the Service Runtime Environment (SRE) of the proposed M2M platform architecture concept. The SRE processes the formal M2M application description by parsing and interpreting the SCXML description and generates a SM from the described semantics. The SEE of the SRE holds and executes the SMs and therefore controls the M2M application. To achieve this a process for M2M application execution 
has been defined that controls M2M devices/MMSCs as defined by the M2M application semantics.

This chapter 5 presented a continuous concept of autonomous M2M application provision starting with the M2M application design by the end-user followed by automated formal description and execution of the application logic. The M2M device abstraction offers the possibility to control different M2M devices and enables independence of the used M2M technology from M2M application execution. The concept includes a loose coupling of application description and application execution. It is platform independent and adaptable because M2M application logic is defined at a higher level than realisation by means of specific programming languages and compilation of application executables. By using a modelling and description of applications independent of the target language, the approach is independent of the technical realisation/programming. This makes it portable to other M2M platforms and does not require reimplementation or recompilation of application/service logic. The advantage of using a formal description that is based on an official standard is that there is a loose coupling between the application generation tool and the executing environment. Therefore, another GUI or approach for application definition, such as plain text-based application design, could replace the GUI. The presented concept uses standardised notation for application modelling (UML SMDs/Statecharts) and SCXML as standardised formal description language for application description. The presented concept also uses a standardised approach for communication between the platform components. This makes the presented M2M platform architecture concept both, adaptable and portable. Because the defined general structure of an M2M application and the definition of M2M device capabilities, which describe the query and control of M2M devices and MMSCs, a generic language has been 
defined that allows to model various M2M applications. The communication between the platform components is realised consistently with the RESTful communication principle. According (Bayer, 2002) the generic interfaces defined in this way make it possible to implement and offer generic services.

In this chapter it has been illustrated how to model and execute individual M2M applications. The concept up to this point forms a local M2M platform realisable without any central entity. It forms the basis for the second part of the proposed framework (presented in the following chapter 6) that realises a global, distributed MSP and enables end-users to cooperate and provide fully distributed M2M application services. 


\section{Cooperative M2M Application Service}

\section{Provision}

The previous chapter 5 introduced the principles of the proposed concept for individual M2M application design and local execution. Multimedia Service Components (MMSCs) have been introduced as interface to $\mathrm{M} 2 \mathrm{M}$ applications usable with existing multimedia communication equipment. The end-user integration in M2M application design has been enabled through modelling the behaviour of application semantic by graphical design. A concept has been defined for integrating different M2M device technologies by abstraction of M2M technology-specific communication. Additionally chapter 5 defined a Statechart-based formal description language for M2M applications describing the graphically designed M2M applications as machine-readable format for automated processing and execution.

This chapter 6 introduces the parts of the proposed framework responsible for provision of local M2M application functionality as a service to other end-users. Additionally this chapter introduces the principles for combining distributed M2M application services to provide cooperative M2M application services. Section 6.1 introduces the concept to make local M2M devices and M2M applications available as a service to other end-users and integrate them into local M2M applications. For this purpose, an interface description of the M2M application service is specified and it is described how service requests are generated and processed inside the M2M platform architecture. 
Afterwards section 6.2 presents the networking principles of the participating nodes by introducing the P2P communication between nodes and identifying an appropriate information exchange pattern for data exchange. Additionally, section 6.2 identifies an appropriate communication protocol for data transmission between distributed $\mathrm{M} 2 \mathrm{M}$ platforms. Section 6.3 introduces the management and distribution of M2M application service interface descriptions (IFDs) without central entities for data storage applying the principles of distributed data storage using a P2P overlay network. The common P2P overlay algorithms are analysed and evaluated to select an optimal methodology for distributed data storage in context of the proposed framework. Section 6.4 introduces the concept for cooperative M2M application service provision by defining mechanisms to enable distributed M2M application service composition and aggregation. Finally, section 6.5 presents details about the M2M community approach enabling networking of endusers through social networking principles.

\subsection{Provision and Integration of M2M Application Services}

In section 4.1 it was shown that the functionality of M2M devices/applications should also be available to other end-users. For this purpose, it is necessary to define an interface enabling to access those. Following steps need to be performed to provide the functionality of M2M devices/applications as a service:

1. An interface must be specified to access the M2M devices/applications. 
2. A component must be integrated into the local M2M platform, which accepts requests for services, passes them on to the $\mathrm{M} 2 \mathrm{M}$ devices/applications and returns the application's response value to the requestor.

3. It must be specified how to integrate the remotely provided M2M application services into local M2M applications.

4. It must be specified how to register the M2M application services to make them available to other end-users and where to store the IFDs.

The following sections 6.1.1 - 6.1.3 address these topics. Section 6.1.1 introduces the IFD specifying a unified interface to provide M2M application services. Section 6.1.2 shows how requests for an $\mathrm{M} 2 \mathrm{M}$ application service are performed in local $\mathrm{M} 2 \mathrm{M}$ platforms of the M2M application service provider (ASP) and finally, section 6.1.3 introduces how to integrate remote $\mathrm{M} 2 \mathrm{M}$ application services in local $\mathrm{M} 2 \mathrm{M}$ applications.

\subsubsection{M2M Application Service Interface Description}

Figure 6.1 shows the characteristics of applications that are behind an M2M service.

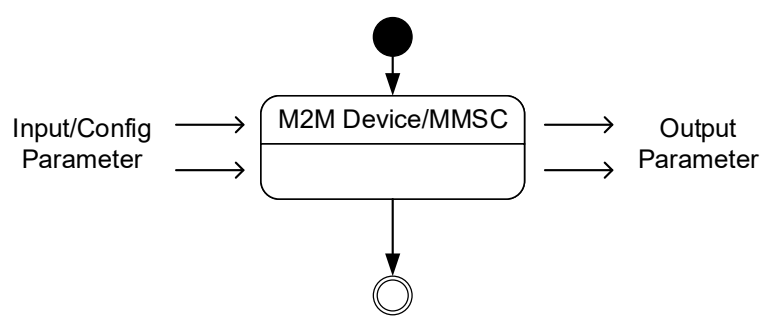

a) Single Component Service

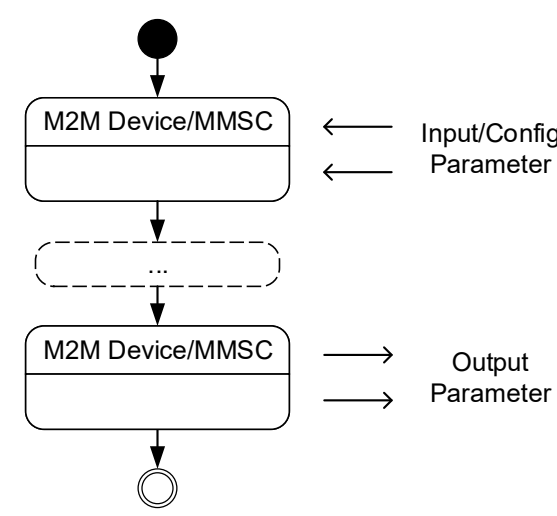

b) Multi Component Service

Figure 6.1: M2M Application Service Principles 
If the functionality of an M2M device should be made available to others, an application can be defined having only one component whose functionality is to be provided (Figure 6.1-a). A more complex M2M application consists of several connected components (Figure 6.1-b). The principle of both applications is the same: an application has an input (these are the Input/Config parameters of the initial state), and an application has an output (these are the Output parameters of the final state). Therefore, an IFD must contain information about these parameters (Steinheimer et al., 2017c). Since according to (oneM2M TS-0001-V1.13.1,2016) M2M applications are considered as resources (refer to section 3.1.1), the resource type specifications of oneM2M for Application Entity (AE) resource and Common Resource (CR) (oneM2M TS-0001-V1.13.1, 2016) has been used as a basis to define an appropriate IFD in the context of this project.

Table 6.1 shows the designed IFD which should be described in machine-readable XML format to enable automated processing.

Parameters appName, App-ID, pointOfAccess, requestReachability, and contentSerialisation have been adopted from the resource type specification of AEs as well as parameters creationTime, lastModifiedTime, and accessControlPolicy from resource type specification of CRs. Additionally parameters have been added to the IFD representing the parameters applications expect as input or generate as output. These parameters could be determined automatically using the formal M2M application description (AD). For this purpose, it is not necessary to analyse the entire M2M AD, but only the parts describing initial and final state, since these are start and end points of the application, respectively represent the input and output interface. These two elements are already marked as initial and final state in the M2M AD so that they are easily identifiable. 
The parameters of these states can now be transferred to the IFD. It is sufficient if only the Input/Config parameters of the initial state are transferred and from the final state only the Output parameters.

Table 6.1: M2M Application Service Interface Description Parameter

\begin{tabular}{|c|c|c|c|}
\hline Parameter & \multicolumn{3}{|c|}{ Description } \\
\hline appName & \multicolumn{3}{|c|}{ Application name specified by developer of application. Type: String. } \\
\hline App-ID & \multicolumn{3}{|c|}{ Application identifier. ID of the application itself. Type: String. } \\
\hline pointOfAccess & \multicolumn{3}{|c|}{$\begin{array}{l}\text { Contact address as list of URIs to communicate with the resource. Type: } \\
\text { String. }\end{array}$} \\
\hline requestReachability & \multicolumn{3}{|c|}{$\begin{array}{l}\text { Specifies if the AE resource can receive requests, i.e. defines if the service is } \\
\text { currently available. Type: Boolean. }\end{array}$} \\
\hline creationTime & \multicolumn{3}{|c|}{$\begin{array}{l}\text { Specifies when the application has been created. Specified by the system that } \\
\text { has created the resource. Type: Unix Timestamp. }\end{array}$} \\
\hline lastModifiedTime & \multicolumn{3}{|c|}{$\begin{array}{l}\text { Specifies when the application has been modified to show if it is a current } \\
\text { development or outdated. Type: Unix Timestamp. }\end{array}$} \\
\hline contentSerialisation & \multicolumn{3}{|c|}{$\begin{array}{l}\text { Specifies the supported serialisation formats of primitive content parameter, } \\
\text { such as XML, JSON. Type: String. }\end{array}$} \\
\hline \multirow[t]{4}{*}{ accessControlPolicy } & \multirow{3}{*}{ privileges } & accessControlOriginators & $\begin{array}{l}\text { Defines which originator is } \\
\text { allowed to use the application. } \\
\text { Comma-separated list of } \\
\text { "all" } \mid<\text { OriginatorURIs }>\mid<\text { Groups }>\mid \\
\text { <SP-Domains or Subdomains }>\text {. }\end{array}$ \\
\hline & & accessControlContexts & $\begin{array}{l}\text { Defines when and where the } \\
\text { application can be used. Comma- } \\
\text { separated list of }<\text { Time-Window } \\
\text { as Unix TimeStamp }>\mid<\text { Location as } \\
\text { GPS Coordination with Radius }>\text {. }\end{array}$ \\
\hline & & accessControlOperations & $\begin{array}{l}\text { Specifies which of the CRUD } \\
\text { operations can be used for } \\
\text { requesting the application. }\end{array}$ \\
\hline & expirationTime & \multicolumn{2}{|c|}{$\begin{array}{l}\text { Specifies how long the resource is valid/ exists. Type: Unix } \\
\text { Timestamp. }\end{array}$} \\
\hline \multirow[t]{3}{*}{ content } & input & \multicolumn{2}{|c|}{$\begin{array}{l}\text { Specifies the Input parameter as a list of parameter names. } \\
\text { Type: String. }\end{array}$} \\
\hline & output & \multicolumn{2}{|c|}{$\begin{array}{l}\text { Specifies the Output parameter as a list of parameter names. } \\
\text { Type: String. }\end{array}$} \\
\hline & config & \multicolumn{2}{|c|}{$\begin{array}{l}\text { Specifies the Config parameter as a list of parameter names. } \\
\text { Type: String. }\end{array}$} \\
\hline description & \multicolumn{3}{|c|}{ Gives a prose description of the application. Type: String. } \\
\hline
\end{tabular}


Figure 6.2 illustrates exemplarily as part of Use Case 2 (Neighbourhood Weather Station) an $\mathrm{M} 2 \mathrm{M}$ application service providing the status of a local rain sensor. Other end-users that want to integrate the sensor data in their local M2M application can request for that service as described in subsequent section 6.1.3. Figure 6.3 shows the corresponding IFD based on the interface parameter specified in Table 6.1. Listing C.8 illustrates the corresponding XML representation of the IFD.

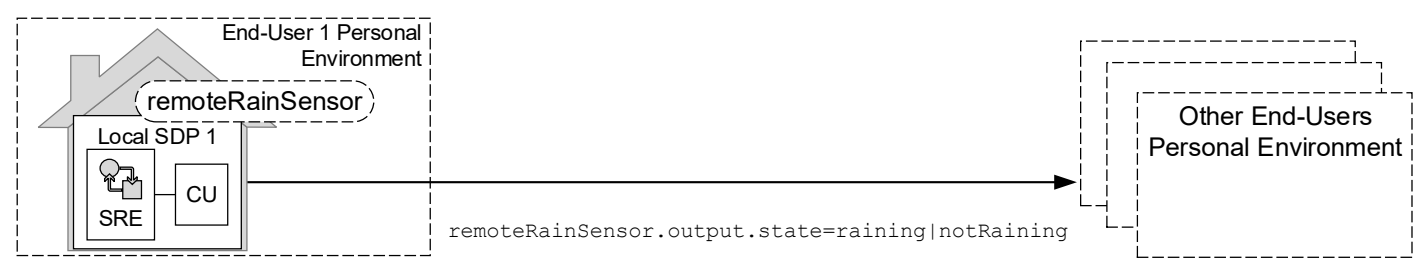

Figure 6.2: Principles of remoteRainSensor Application Service

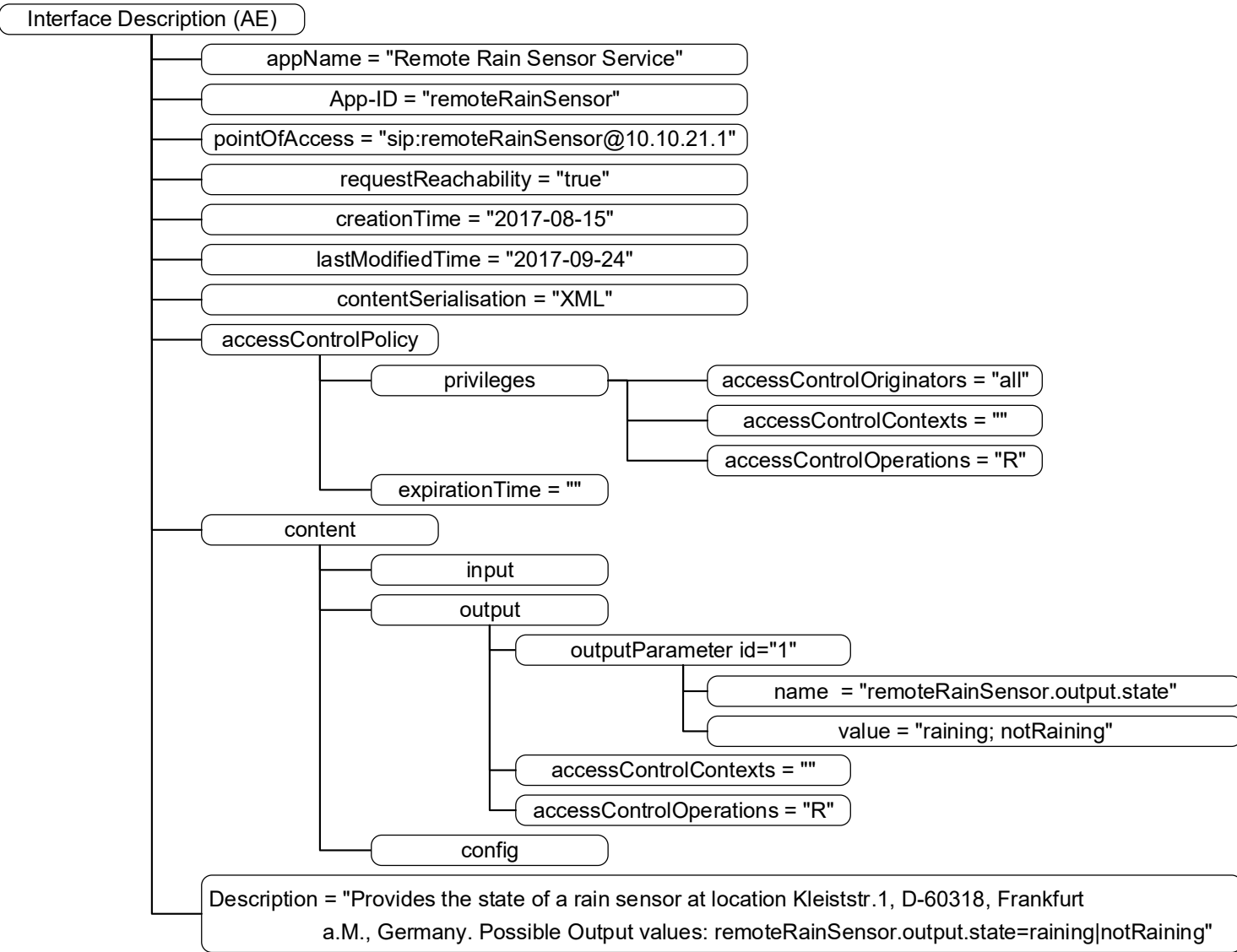

Figure 6.3: IFD for remoteRainSensor M2M Application Service 


\subsubsection{Performing remote M2M Application Service Requests}

An application provided as a service can be used by sending a request message to the service provider's platform. As indicated in section 5.4, the exchange of messages between M2M entities, which are also the applications, is realised using Primitives. For requesting a service, the requestor must therefore generate a Request Primitive based on the IFD (containing the required Input/Output/Config parameter) and send it to the service provider (SP). This then responds with a Response Primitive (containing, if intended, the Output parameter). The content and structure of the Primitive messages have already been described in section 5.4 .

As mentioned above, a component in the local M2M platform needs to be defined that accepts and handles the requests. The M2M Service Interface Unit (SIU) as part of the Communication Unit (CU) has been designed for this purpose (see Figure 6.4).

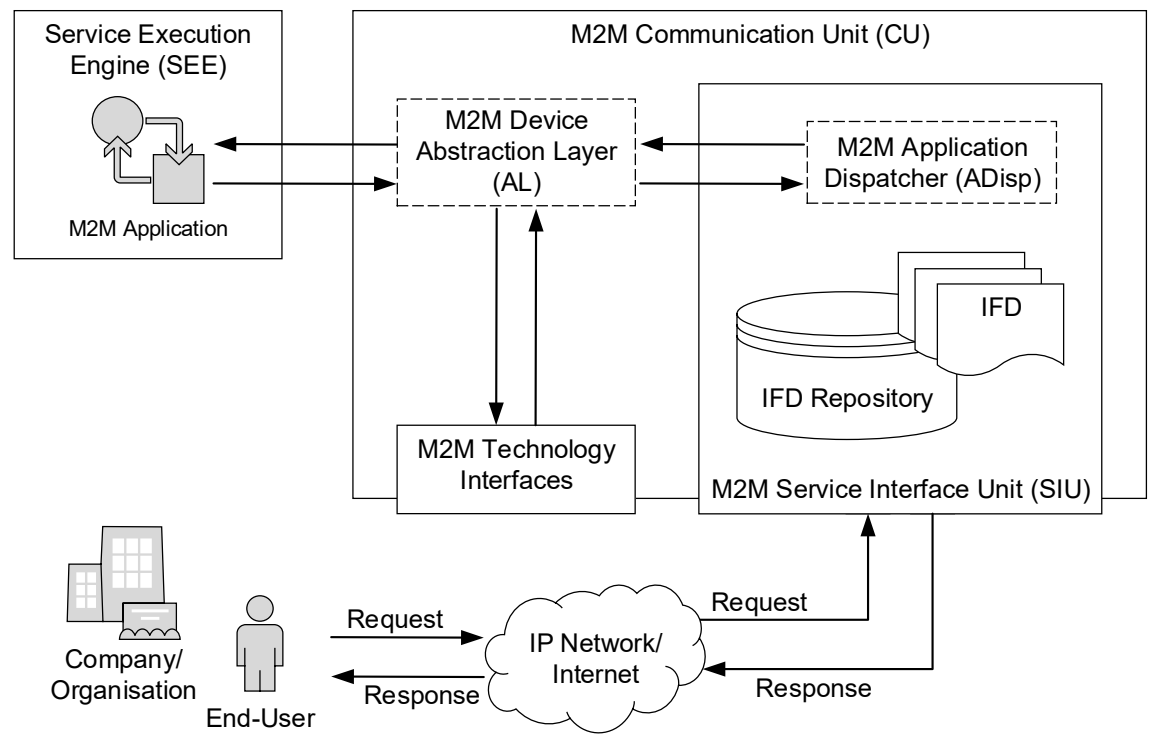

Figure 6.4: Service Interface Unit (SIU) 
The SIU includes an IFD Repository storing the IFDs and an M2M Application Dispatcher (M2M ADisp) forwarding the service requests to the corresponding M2M application. Figure 6.5 shows the process of request processing by the $S I U$.

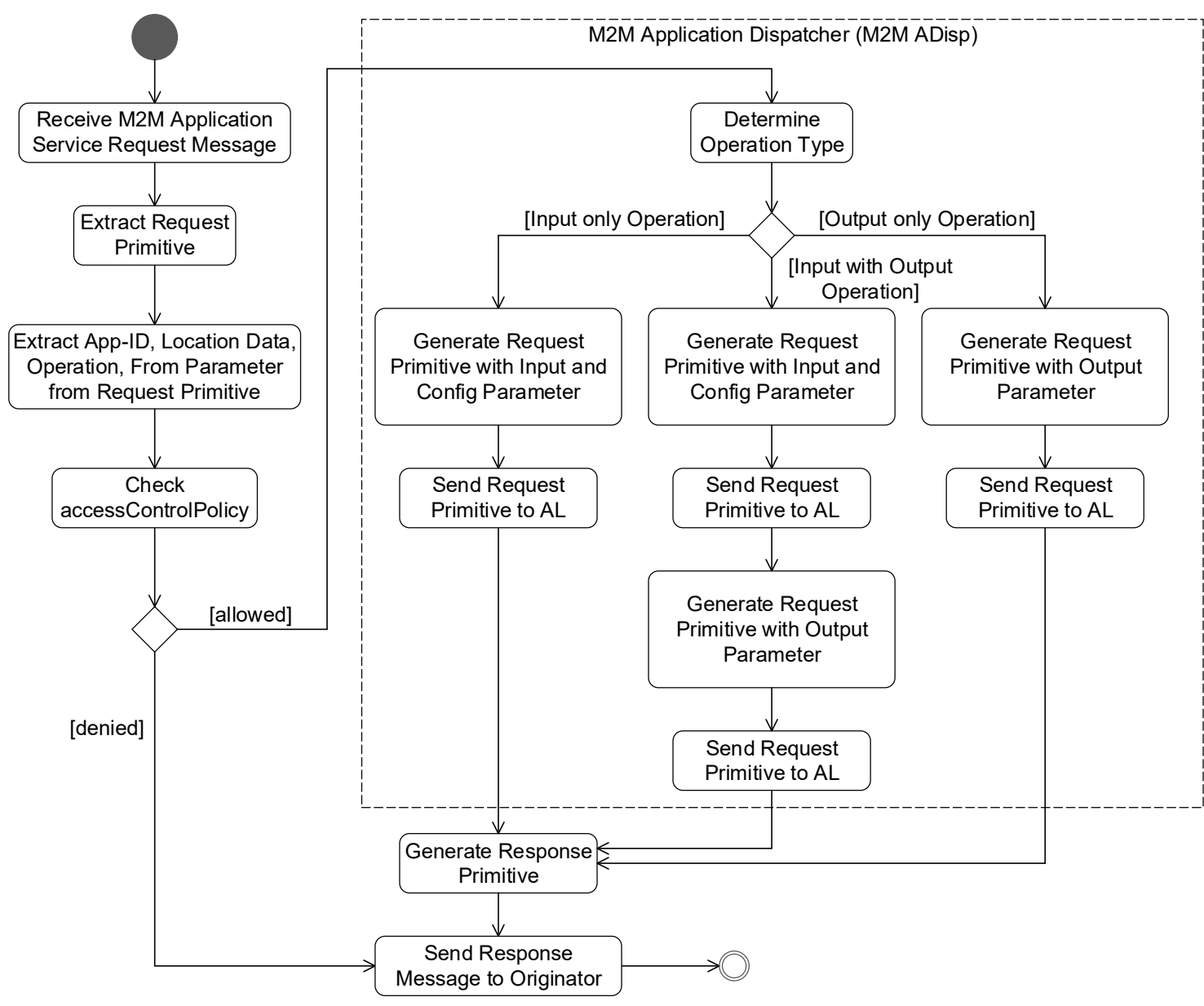

Figure 6.5: SIU: M2M Application Service Request Processing

The $S I U$ receives the request for a service and extracts the Request Primitive from the request message. The structure of the Request Primitive essentially corresponds to the Request Primitive shown in Figure 5.23 containing the Input/Output/Config parameter in the Content section. If the authorisation should be validated, the Request Primitive message shall contain the corresponding information for checking the access ControlPolicies. 
The SIU extracts App-ID, location data, operation, and from parameters from the Primitive and checks the permissions for application usage based on the IFDs stored in the IFD Repository. After successful authorisation the M2M ADisp processes the Request Primitive. The processing steps depend on which operations the M2M application should perform and can be classified as follows:

- Input only Operation - The application is intended to trigger only one action in the platform (e.g., switch a device). The application has only Input/Config parameters and no Output parameters.

- Input with Output Operation - An operation should be triggered that requires input values and returns output values (e.g., calculation tasks). The application has both Input and Output parameters.

- Output only Operation - The application has no Input parameters but provides an Output (e.g., request of a single sensor value).

For operations by which input/config parameters should be set, the M2M ADisp generates a Request Primitive containing these parameters and the corresponding values and sends it to the Abstraction Layer (AL) (refer to section 5.4). For operations that request output parameters, the M2M ADisp generates another Request Primitive containing the output parameters and sends it to the $A L$ to query the output parameters. The $S I U$ then generates a Response Primitive (which may contain the output values) and returns it to the requestor. 


\subsubsection{Integration of remote M2M Application Services}

After having introduced how M2M applications can be made available as a service via an interface, this section describes how to integrate these remote $\mathrm{M} 2 \mathrm{M}$ services into $\mathrm{M} 2 \mathrm{M}$ applications that are executed locally (see Figure 6.6).

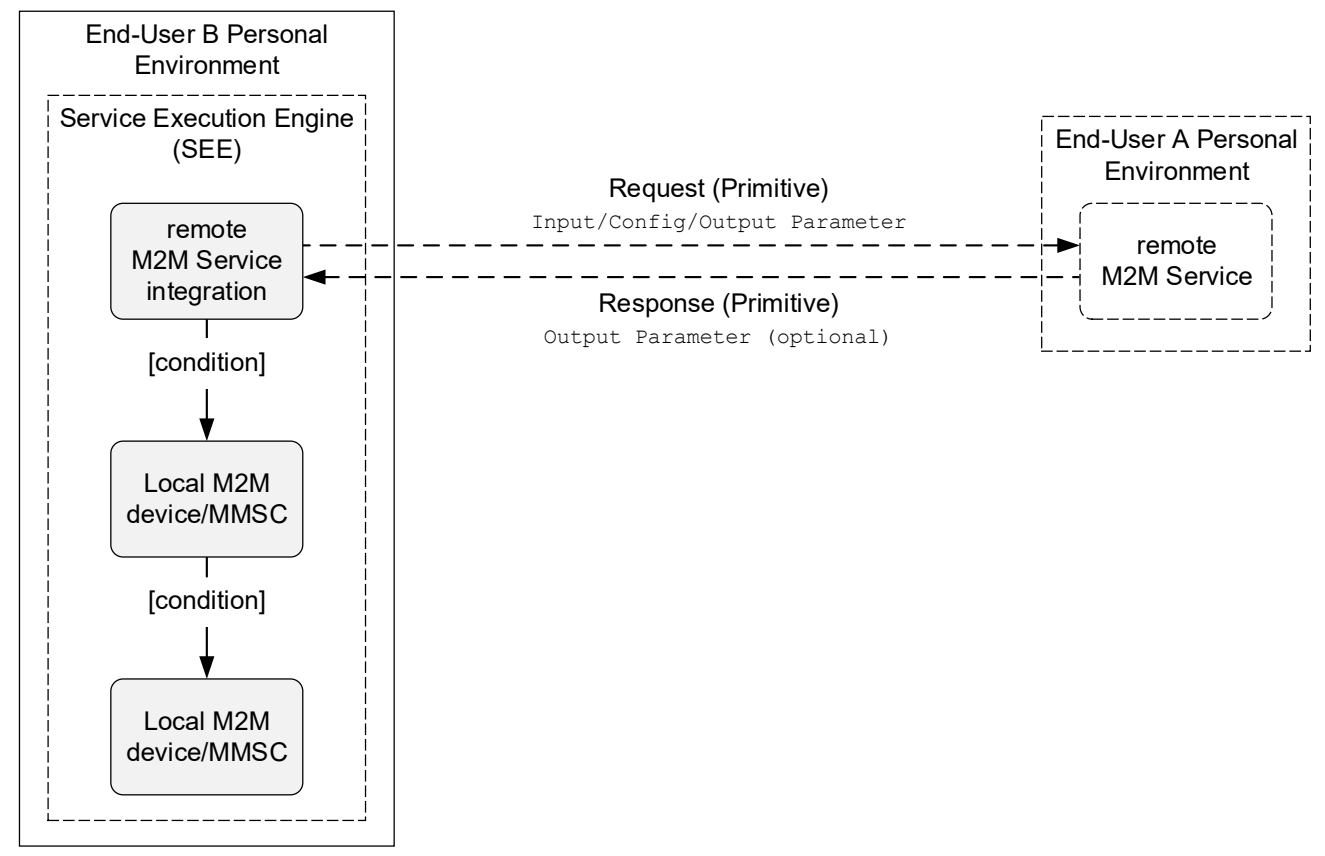

Figure 6.6: Remote M2M Service Integration

The graphical design of an M2M application integrating a remote M2M service is done in the same way as modelling an application containing only M2M devices/MMSCs that are locally available (see section 5.3). The remotely offered M2M services are displayed in the GUI in the section Remote M2M Services as building blocks (see Figure 5.14) and can be integrated into the information flow of the application (as state in the application model and by connecting to other M2M devices/MMSCs by using transitions) (Steinheimer et al., 2017c). This makes it possible to integrate the functionality of remote M2M applications into local M2M applications. 
The formal application description is created in the same way as described in section 5.5. While in the formal description of a local application, the device/MMSC-IDs are specified as identifiers for a state (see Figure 5.36), the identifier for the integration of remote services corresponds to the service-ID (field $A p p-I D$ in IFD). Figure 6.7 gives an exemplary section of a formal application description (as part of a Statechart) in which a remote M2M service is integrated (see Use Case 2). Here it can be seen that there is no difference to the format when locally available $M 2 M$ devices/MMSCs are described. The corresponding SCXML representation is illustrated in Listing C.9.

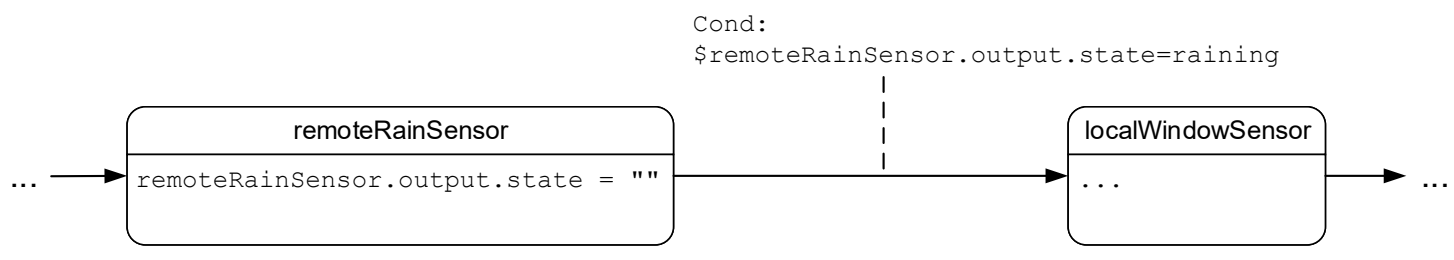

Figure 6.7: Extract Formal Application Description Use Case 2

The state machine (SM) representing the application semantic is processed as described in section 5.6 by the Service Execution Engine (SEE) by generating an instance of the SM and executing it. To utilise a remote M2M service, the Service Execution Engine (SEE) must identify (e.g., by means of the state-ID) whether the application contains a remote service that must be requested. Remote services are identifiable because they are defined by a state whose corresponding device/service logic is locally not available. If such elements are identified, the service is requested by sending a Request Primitive to the SP via the $C U$. The Request Primitive message contains the parameters (Input/Config/Output parameters) that serve as input for the remote service and are delivered by it as output. If the requested remote service has a response parameter, it is returned by the service 
providing platform in the Response Primitive message and can be processed further within the local M2M application.

It should be noted that the formal application description does not contain information about the specific SP (i.e., no contact data). SPs offering a service register this via the IFD. This describes the service and contains the information about the addressing options by specifying the URI of the service endpoint (field pointOfAccess). The administration of the IFD and how service consumers can request the IFD to obtain this information is described in section 6.3.1.

\subsection{Networking of Nodes}

Chapter 4 has proposed that service providers (SPs) and service consumers (SCs) communicate P2P (i.e. without central entities involved for communication) to exchange information required for service utilisation. This section introduces how the networking of SP and SC is proposed in this project. First, the kind of P2P networking between the nodes is introduced (section 6.2.1). Subsequently, in section 6.2.2, an adequate information exchange pattern is selected for information exchange between the nodes. Finally, section 6.2.3 describes which communication protocols can be used to exchange information between the nodes.

\subsubsection{P2P Information Exchange}

The P2P Communication Layer (refer to Figure 4.12) realises the communication between the participating nodes in the proposed framework. $\mathrm{P} 2 \mathrm{P}$ communication is a 
common methodology to avoid central entities in system infrastructures. As avoiding central entities in the described framework is a mandatory requirement, the communication between the involved nodes also follows the $\mathrm{P} 2 \mathrm{P}$ communication principle (Steinheimer et al., 2012a; Steinheimer et al., 2017a).

The Resource Location and Discovery Protocol (RELOAD) project (IETF RFC 6940, 2014) specifies a fully decentralised P2P signalling protocol usable over the Internet. Figure 6.8 shows the architecture of RELOAD adopted from (Samaniego et al., 2013) and (IETF RFC 6940, 2014). RELOAD enables nodes to route signalling messages to other nodes with the help of additional peers in the overlay. In the RELOAD project an overlay topology is created using a specific overlay algorithm, which also defines how to route the messages in the overlay. The overlay algorithm that is used to generate the topology is generic, i.e. exchangeable with other overlay algorithms (IETF RFC 6940, 2014). The nodes in a RELOAD overlay request services from other peers or provide services to them, such as a Traversal Using Relays around NAT (TURN) service (IETF RFC 7374, 2014). Permission to reproduce Figure 6.8 has been granted by authors of the referenced publication and IETF.

\begin{tabular}{|c|c|c|c|}
\hline Internet Model & $\begin{array}{c}\text { Internet Model } \\
\text { Equivalent in Overlay }\end{array}$ & \multicolumn{2}{|c|}{ RELOAD Architecture } \\
\hline \multirow{4}{*}{ Application } & Application & $\begin{array}{l}\text { SIP } \\
\text { Usage }\end{array}$ & $\begin{array}{l}\text { CoAP } \\
\text { Usage }\end{array}$ \\
\hline & Transport & \multicolumn{2}{|c|}{$\begin{array}{l}\text { Message } \\
\text { Transport }\end{array}$} \\
\hline & (Routing) & \multicolumn{2}{|c|}{ Topology Plugin } \\
\hline & Internet & \multicolumn{2}{|c|}{$\begin{array}{l}\text { Forwarding and Link } \\
\text { Management }\end{array}$} \\
\hline Transport & Link & TLS & DTLS \\
\hline
\end{tabular}

Figure 6.8: RELOAD Architecture acc. (Samaniego et al., 2013; IETF RFC 6940, 2014) 
RELOAD does not only provide the functionality for P2P message routing, but also provides the functionality for distributed storage of data items inside the overlay using the selected overlay algorithm (IETF RFC 7374, 2014; IETF RFC 6940, 2014). According to (IETF RFC 7374, 2014) through the definitions of so-called "Usages" (which are again protocol definitions) it is specified how the RELOAD overlay is used to support a specific application. I.e. Usages define how the data items are structured (ID, data structure) that should be stored in the RELOAD overlay for specific applications and define how the RELOAD messaging functionality should be applied.

The components of the RELOAD framework are specified according to (IETF RFC 6940, 2014) as follows:

- Usage Layer - Implements application specific functionality by using the “overlay services provided by RELOAD”. The Usage Layer defines how applications map their application specific data into data items that can be stored in and retrieved from the RELOAD overlay.

- Message Transport - Provides the message routing functionality of RELOAD. The applications defined by Usages use the Message Transport service of RELOAD to exchange messages between peers. Applications that want to store data items also use the Message Transport component by sending a "Store Request" to the RELOAD overlay.

- Storage - Implements "one of the major functions of RELOAD" that is storing data in the overlay. The Storage component uses the Topology Plug-in to store the data items using the specified overlay algorithm. 
- Topology Plug-in - Provides the implementation of the overlay algorithm and maintains "the overlay algorithm Routing Table" which the Forwarding and Link Management Layer contacts for routing of messages.

- Forwarding and Link Management Layer - Establishes the network connections and transports the messages between peers "as determined by the Topology Plugin".

As described above, so-called Usages are defined specifying how a RELOAD overlay is used to support a specific application. The SIP Usage for RELOAD (IETF RFC 7904, 2016) is presented below and demonstrates the way of operating a RELOAD-based application, but also indicates the disadvantages of RELOAD.

The SIP Usage for RELOAD provides the functionality for a distributed VoIP system without the requirement of Registrar or Proxy Server (IETF RFC 7904, 2016). It is defined for application in "server-less, peer-to-peer SIP deployments" (IETF RFC 7890, 2016). The SIP Usage defines the following functionality according to (IETF RFC 7904, 2016):

- Registration - Storage functionality for mapping of SIP User Agent (UA) URIs to RELOAD Node-IDs and functionality to request the Node-ID of other UAs.

- Rendezvous - Functionality for message routing to establish "a direct connection for exchanging SIP messages".

Figure 6.9 shows the information flow defined for a service request in a RELOAD overlay system. It illustrates the principles of RELOAD overlay functionality with the help of specific SIP Usage according to (IETF RFC 7904, 2016) enabling to establish a SIP 
session between two UAs (Alice and $B o b$ ). Permission to reproduce Figure 6.9 has been granted by IETF.

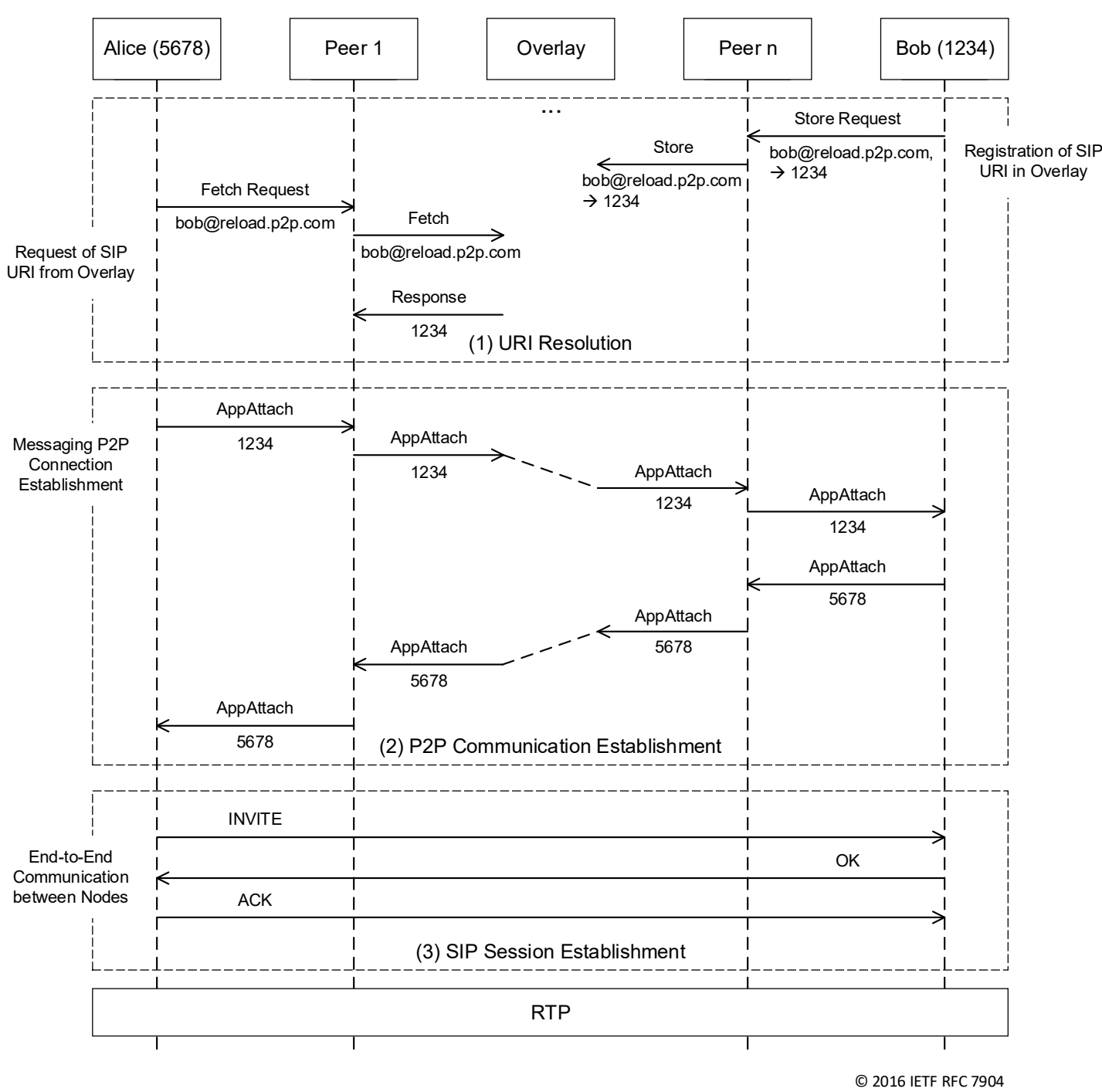

Figure 6.9: RELOAD Message Exchange for Service Request acc. (IETF RFC 7904, 2016)

The preconditions for communication are performed in the URI Resolution phase. For addressing nodes in a RELOAD overlay specific RELOAD Node-IDs are used (e.g., Alice has the Node-ID "5678" and Bob "1234"). For being addressable the nodes store a mapping of their (SIP) URIs to their Node-IDs in the overlay. For contacting a node to establish a SIP session, the nodes request the Node-ID of the peer to contact at the overlay. 
The overlay searches and responses the corresponding data item applying the specified overlay algorithm.

Afterwards (P2P Communication Establishment phase) the peer that wants to establish the connection generates a RELOAD protocol-specific AppAttach message directed to the destination Node-ID and sends it to the overlay network, which routes it to the destination host (applying the specified routing algorithm). The destination host responses also with an AppAttach message to the requestor (routed through the overlay). After the originator has received the AppAttach message from destination host, both nodes are connected and ready for using the provided services.

For final session establishment (SIP Session Establishment phase) the originator initiates a SIP Three-Way-Handshake directly with the destination host using plain SIP signalling messages.

Another Usage for RELOAD that has been defined is the Constrained Application Protocol (CoAP) Usage for RELOAD (IETF RFC 7650, 2015). This describes according to (Rodrigues et al., 2016) a lookup service for resources and how to cache sensor data in a RELOAD overlay. This Usage again describes the connection establishment between the end nodes via routing through the P2P network.

The above-described approach of RELOAD to connect nodes P2P is advantageous for avoiding central entities in a system architecture. The use of P2P overlay algorithms to generate a shared, distributed database is also an advantage. At the same time, however, the RELOAD approach has the following disadvantages: 
- RELOAD Stack required - RELOAD is a complex system defined by the RELOAD protocol which requires a corresponding protocol stack to exist at the runtime environment of the end-user. While most end-users have a SIP stack integrated in their IAD, this is not the case with a RELOAD stack. Thus end-users could not use a RELOAD-based system with the available resources and network access (provided by their Internet Service Provider, ISP).

- Networking of Nodes - RELOAD is building its own routing infrastructure on the underlying Internet/IP network, which already has an infrastructure for addressing nodes (refer to Figure 6.10-a). To connect two nodes in a RELOAD overlay, they must first establish a connection with each other using special RELOAD messages before they can exchange the actual messages that are associated with a service. The exchange of the service-specific messages is then end-to-end, but beforehand, other nodes for the routing of the messages are unnecessarily involved.

In order to eliminate these disadvantages, this research proposes an $\mathrm{P} 2 \mathrm{P}$ networking approach for linking SPs and SCs as illustrated in Figure 6.10-b, which is optimised in the following aspects:

- Direct Communication of M2M SP and SC - The communication between the SP and SC takes place on the application layer exclusively end-to-end between the nodes involved in a service utilisation, meaning for connecting SP and SC the messages are exchanged directly between these two nodes without intermediary entities.

- Application of Internet Communication Technology - The involved peers are all connected to the Internet or another continuous IP network. As a result, the 
corresponding routing mechanisms can be reused which are provided in the underlying network. Addressing is done directly via the URI, which the end-user platform has been assigned by the ISP. This makes an additional routing mechanisms unnecessary.

- Storage of Service URI in IFD - The URI of the SP is directly stored in the IFD in the designated field pointOfAccess (refer to Table 6.1) since the IFD should be available to the $\mathrm{SC}$ when requesting a $\mathrm{M} 2 \mathrm{M}$ service.

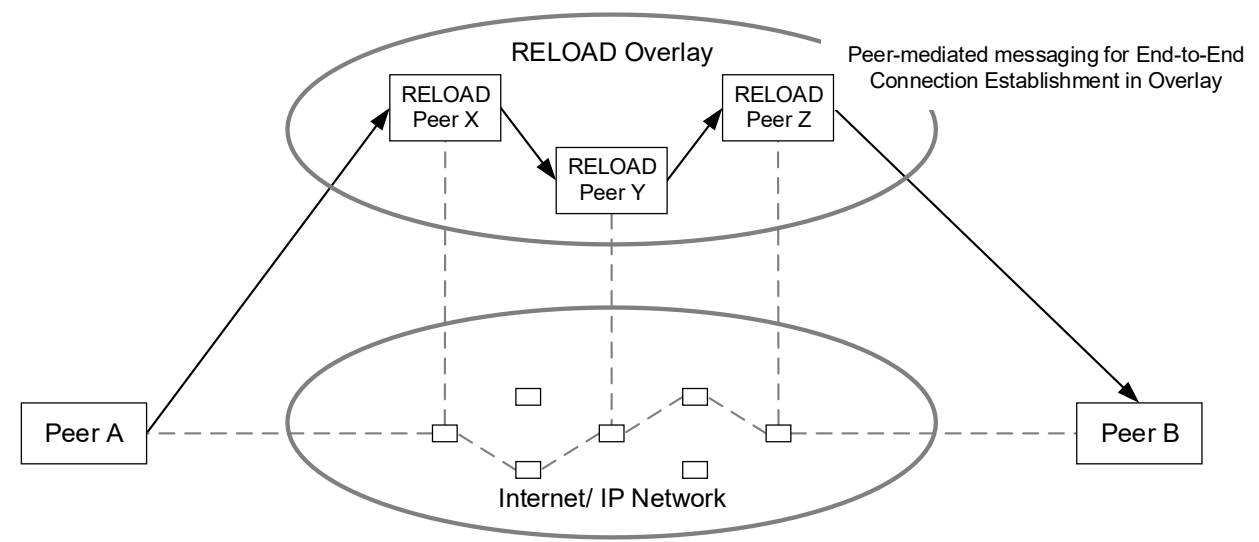

a) RELOAD Networking Topology

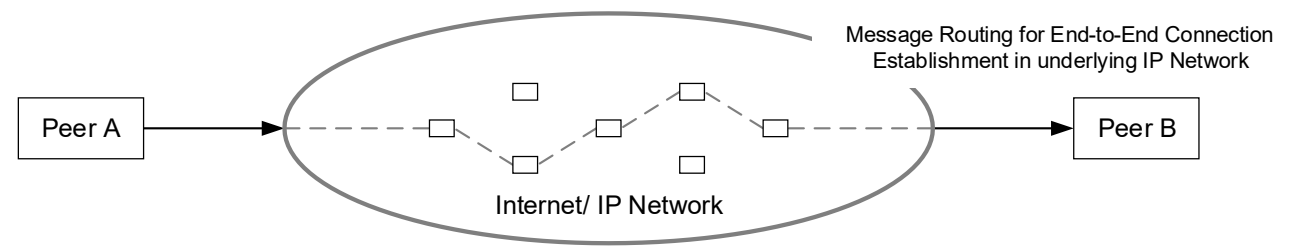

b) Proposed Networking Topology

Figure 6.10: Comparison RELOAD and proposed Networking Topology

The following improvements compared to a RELOAD-based architecture result from the proposed optimisation approaches.

1. Since the IFD is already available to SCs when they plan to request a remote M2M service, they can use the URI stored in the IFD without the need for an additional address resolution (no URI Resolution phase). 
2. No additional nodes on the application layer are required for routing messages, since the service request is sent directly to the SP (no P2P Communication Establishment phase). This is possible because the SP's URI is contained in the IFD.

3. A RELOAD stack in the execution environment is not required since the IP-based connection functionality of the ISP is used. The SIP stack contained in the enduser's IAD could also be used for communication between SP and SC. This means that the resources available to the end-user environment can be used without having to provide additional communication protocol stacks.

\subsubsection{Information Exchange Pattern}

For exchanging information between M2M entities several information exchange patterns (IxPs) exist as specified by (Holler et al., 2014) adopted from (Carrez et al, 2013). This section describes these IxPs (see Figure 6.11) and evaluates their application for information exchange in the context of the concept designed in this research. Permission to reproduce Figure 6.11 has been granted by authors of the referenced publication and publisher Elsevier.
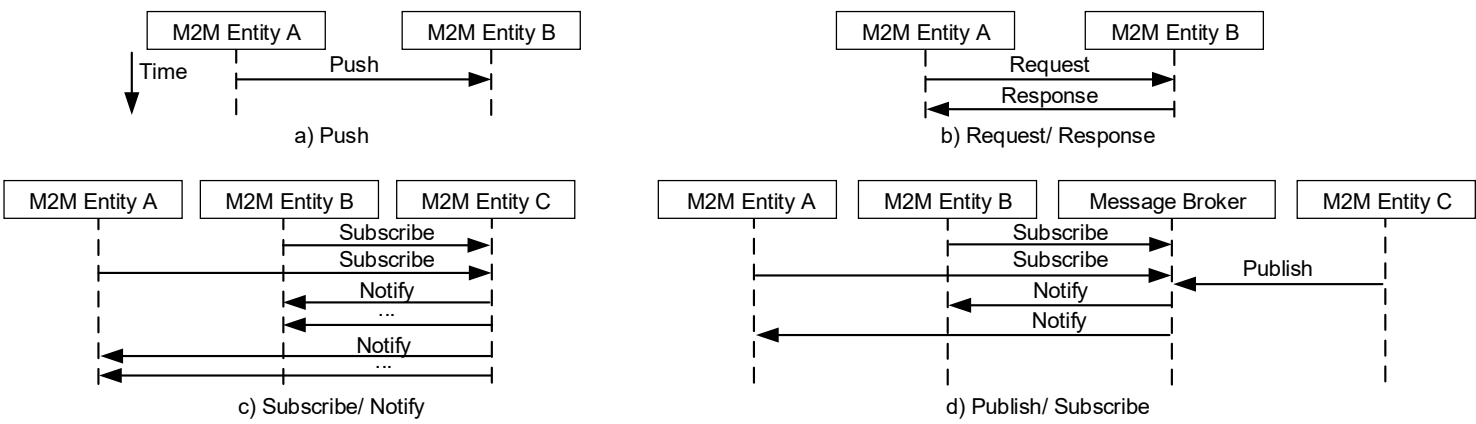

Figure 6.11: IxPs acc. (Höller, 2014) adopted from (Carrez et al., 2013) 


\section{Push IxP}

In push IxP (Figure 6.11-a) information are pushed from an M2M entity to another M2M entity. This IxP requires that the contact information of $M 2 M$ Entity $B$ is already configured in $M 2 M$ Entity $A$ and that $M 2 M$ Entity $B$ listens for the information might be pushed (Holler et al., 2014).

\section{Request/Response IxP}

In request/response IxP (Figure 6.11-b) an M2M Entity $A$ requests an information at another $M 2 M$ Entity $B$. M2M Entity $B$ responses the information after receiving the request. The interaction in this $\mathrm{IxP}$ is synchronous, i.e. $M 2 M$ Entity $A$ has to wait for the response until it continues further processing. In practice, this limitation is addressed by performing multi-threading capabilities in M2M Entity $A$. Additionally, M2M Entity $B$ has to perform capabilities "to handle concurrent requests and responses from multiple components" (Holler et al., 2014).

\section{Subscribe/Notify IxP}

In subscribe/notify IxP (Figure 6.11-c) multiple $M 2 M$ Entities $A$ and $B$ subscribe for an information provided by an M2M Entity $C$. M2M Entity $C$ sends a notify message to all subscribers of that information once the information is ready for transmission. The interaction in this IxP is asynchronous. M2M Entity $C$ needs to perform the capability for storing and managing the contact data and corresponding request topics. The subscribe/notify IxP is applicable when a single M2M entity provides information of interest for multiple other M2M entities (Holler et al., 2014). 


\section{Publish/Subscribe IxP}

In publish/subscribe IxP (Figure 6.11-d) a centralised Message Broker is involved mediating publications and subscriptions between information publisher and consumer. M2M entities interested in an information subscribe at the Message Broker for a specific information. $\mathrm{M} 2 \mathrm{M}$ entities that provide that information publish the information to the Message Broker which send a notify message to the entities interested in that information. As subscribe/notify IxP, publish/subscribe IxP is also an asynchronous pattern. Different from the subscribe/notify IxP, here the information producer does not need to manage information about the information subscriber (Holler et al., 2014).

The evaluation of the IxPs specified by (Holler et al., 2014) leads to the result, that subscribe/notify IxP is the best fitting pattern to exchange information between $\mathrm{M} 2 \mathrm{M}$ entities in the approach presented in this research with regards to the specified requirements of section 3.2. The push IxP is not applicable because the contact information of the receiving $\mathrm{M} 2 \mathrm{M}$ entity needs to be configured statically in the information producing entity. Because the dynamic nature of the services forming an application in the presented concept, specific service instances cannot be known by configuration time and furthermore are highly volatile. The request/response IxP is partly usable for information requests of single services during runtime and receiving information instantly. However, it is not the best choice for general information exchange due to the synchronous principle of that pattern and parallel response management that needs to be performed by the receiving entity. Also for receiving information by multiple $\mathrm{M} 2 \mathrm{M}$ entities during runtime, every $\mathrm{M} 2 \mathrm{M}$ entity interested in an information has to request every information always it wants to consume/process the information. 
Configuring an application before running the application and validating the correct application configuration as proposed in this project is not realisable according to the principle of synchronous information exchange, since service links are not yet available at configuration time (see section 6.4.3). The publish/subscribe IxP contains a central Message Broker. This violates the decentralised principle and requirements of the approach presented in this research. This leads to select the subscribe/notify IxP as the best choice for information exchange between distributed M2M entities (Steinheimer et al., 2013e). Benefit of this pattern is that it is based on asynchronous principles and additionally entities that want to receive an information have to register a single time for an information at an information producer and keep informed always the information is ready for transmission.

\subsubsection{Selection of appropriate P2P Communication Protocols}

After the principles of proposed P2P communication between SP and SC has been introduced in section 6.2.1 and 6.2.2, a standardised application layer protocol for exchanging messages via an IP network is required. As transport protocol usually TCP (IETF RFC 793, 1981) or UDP (IETF RFC 768, 1980) is used. Since UDP avoids overhead for connection-oriented communication at transport level, this should be selected as transport protocol. The Primitive messages are then exchanged with an application layer protocol. OneM2M defines so-called Protocol Bindings (refer to section 5.4) specifying how the Primitive messages between M2M entities (i.e. SP and SC) are embedded in an application layer communication protocol. OneM2M defined Protocol Bindings for HTTP (oneM2M TS-0009-V2.6.1, 2016), MQTT (oneM2M TS-0010- 
V2.4.1, 2016), CoAP (oneM2M TS-0008-V1.0.1, 2015) and WebSocket protocol (oneM2M TS-0020-V2.0.0, 2016).

HTTP follows the Request/Response IxP and MQTT uses the Publish/Subscribe IxP with a central message broker. Both approaches do not meet the requirements of the framework introduced in this project (avoidance of central components) or are inconvenient for implementation of application logic (blocking at invocation). WebSocket Protocol enables bi-directional end-to-end communication, but has the disadvantages that it relies on the additional protocols HTTP and TCP and therefore WebSocket communication results in large overhead for connection establishment and termination. WebSocket connections using UDP are not possible. Each WebSocket connection requires a separate TCP connection establishment (Three-Way-Handshake) and HTTP Opening Handshake as well as for closing connection HTTP Closing Handshake and TCP Connection Teardown. While the connection exists it is required to exchange keep alive messages continuously keeping the connection open. Beside the unnecessary overhead for communication with the WebSocket protocol, at least one HTTP, TCP, and WebSocket stack is required on the platform of both communication partners, which enlarges the complexity of the system itself and is not commonly existing on end-users equipment.

Therefore, CoAP remains the only protocol suggested by oneM2M for exchanging messages between M2M entities in this context. Since multimedia communication is a central component of the introduced framework, Session Initiation Protocol (SIP), which is the standard protocol for signalling in multimedia communication networks, is proposed as an alternative to CoAP (Steinheimer et al., 2013e). 
The following illustrates how Subscribe/Notify IxP can be realised using CoAP and SIP. For this purpose, a general mechanism is first described to specify how a service can be requested and how the service providing peer manages and processes the requested information. Afterwards, CoAP is introduced and the message exchange for the service request is depicted according to the CoAP Protocol Binding. Subsequently, SIP will be introduced. Since no Protocol Binding has been defined for SIP so far, this Protocol Binding is designed to realise the exchange of Primitive messages via SIP.

\section{Principle of Service Subscription, Notification, and Termination}

A peer requests a service from other peers by sending a Request Primitive message to them. Mapping this to the Subscribe/Notify IxP, this means that peers subscribe services (i.e. send subscribe message to service providing peers). Peers providing services then execute the individual service logic and, if necessary, delivers requested information to the requesting peer using a Response Primitive message. Mapping this to the Subscribe/Notify IxP, this means that service providing peers send a notify message to service requesting peers.

When requesting services according to Subscribe/Notify IxP, the following cases should be considered:

- Request Once - Requesting peers should be able to request a service once.

- Continuous Request - Requesting peers should be enabled to permanently request information provided by a service and receive their outputs continuously. 
- Terminate Request - Service requesting peers should be able to terminate a continuous service request (i.e. inform service providing peers they do not continue using a service).

Figure 6.12 shows these cases and thus the general principles of service requests/terminations.

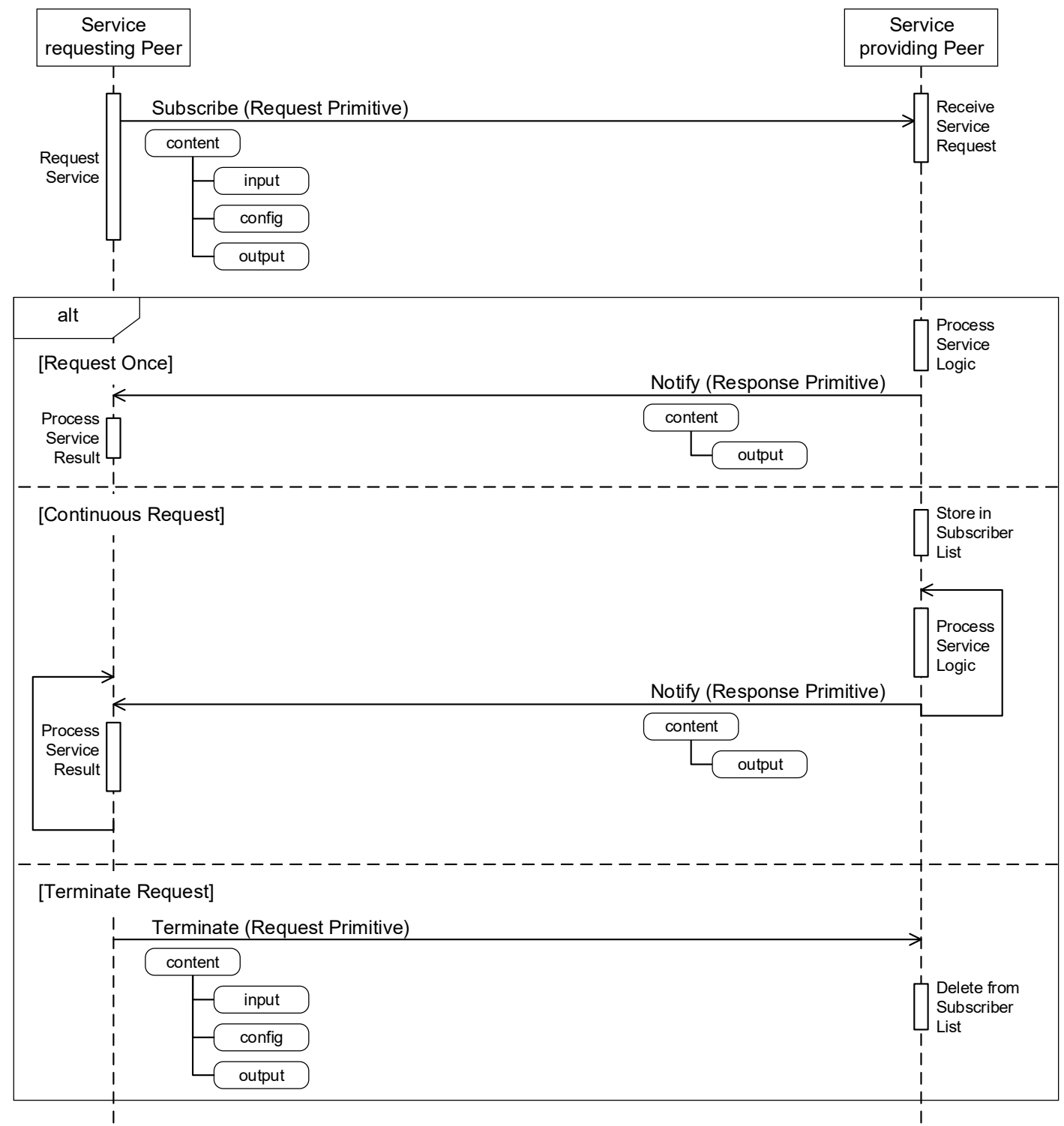

Figure 6.12: General Service Subscription/Notification/Termination Process 
A service is requested by sending a Subscribe Request which contains the parameters to be queried and, if necessary, the parameters to be set. If request are one-time requests of a service (Request Once), the service providing peers respond directly and return the output parameters of the service (if defined). These are then processed by the requesting peer. If the output values of a service are to be transmitted and processed continuously (Continuous Request), the service providing peers store the contact information (of requesting peers) in a local subscriber list. To terminate a service subscription (Terminate Request), the peers that requested the service before send a Terminate Request message to the service providing peers. The subscriber will then be deleted from the local subscriber list.

\section{Realisation of Subscribe/Notify IxP using CoAP}

CoAP is a protocol for communication in "Constrained RESTful Environments" with the goal of realising a "REST architecture". A REST architecture is according to (Bayer, 2002) an architectural approach for distributed applications in which all entities or components are considered as resources. These resources can be addressed directly via a unique URI. Communication with the resources is done by exchanging representations of the resources between the client (using the resource) and the server (hosting the resource). Constrained environments contain nodes that are limited in CPU power, RAM and ROM capacity. Additionally to the nodes, the networks for transmission of information are also limited in their transmission capacity (low bandwidth, low power for transmission of messages) so that message packets to be transmitted should be as small as possible (IETF RFC 7252, 2014). 
According to (IETF RFC 7252, 2014) CoAP offers the following essential features for message exchange:

- UDP binding with (optional) reliability support - Use of UDP for connectionless transmission and realisation of reliability on CoAP protocol level.

- Asynchronous message exchange - Messages can be transmitted asynchronously, so that senders do not block until they have received a response.

- Low Header Overhead and parsing Complexity - CoAP has low header overhead and because the low complexity of CoAP messages less effort is required to parse the messages.

Communication between nodes takes place by exchanging messages between so-called endpoints installed on the nodes. An endpoint is an "entity participating in the CoAP protocol". Endpoint correspond to a socket on a node (IP address + port) and the associated additional addressing information of an application that should process the CoAP messages (URI-Path). CoAP distinguishes between client and server endpoints. Client endpoints initiate messages and server endpoints receive messages. Server endpoints process the Request messages and reply with a Response message, which in turn is processed by the client. The nodes in a CoAP implementation can take on both of these roles, i.e. in contrast to HTTP implementation, they can act both as clients and servers (IETF RFC 7252, 2014).

CoAP distinguishes between Confirmable, Non-confirmable, and Acknowledgement messages to realise the message exchange between endpoints. CoAP enables reliable and unreliable message transmission as described subsequently. 
Figure 6.13 shows the "Reliable Message Transmission". Reliability is implemented by marking the Request message as "Confirmable (CON)". A confirmable message must be answered by the recipient with an "Acknowledgement message (ACK)". The ACK message contains the same Message-ID as the corresponding Request message. If no acknowledgement message is sent, the client repeats the sending of the Request message (IETF RFC 7252, 2014). Permission to reproduce Figure 6.13 has been granted by IETF.

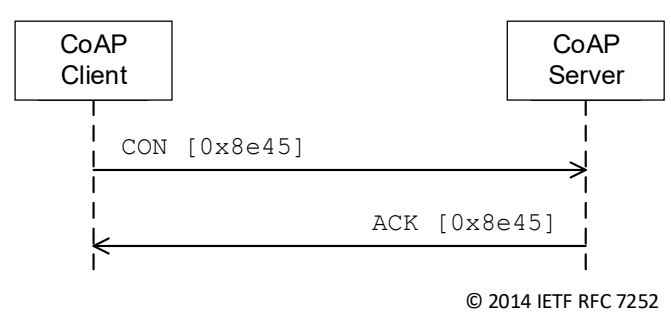

Figure 6.13: CoAP Reliable Message Transmission acc. (IETF RFC 7252, 2014)

Figure 6.14 shows the "Unreliable Message Transmission". Unreliable message transmissions does not require acknowledgement messages. Such messages are marked as "Non-confirmable message (NON)". NON messages also have a Message-ID to enable duplicate message detection (IETF RFC 7252, 2014). Permission to reproduce Figure 6.14 has been granted by IETF.

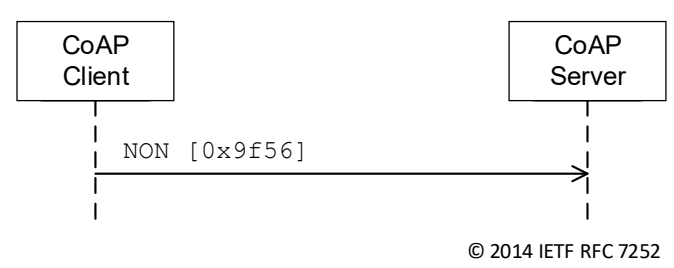

Figure 6.14: CoAP Unreliable Message Transmission acc. (IETF RFC 7252, 2014)

CoAP uses, similar as HTTP, the methods GET, PUT, POST, DELETE to request resources by a client. Subsequently the semantics of these methods are introduced as described in (IETF RFC 7252, 2014). 
- GET - Retrieves representations of information corresponding to resources identified by request URIs.

- $\quad$ POST - Requests to process representations included in the request message.

- PUT - Requests to update/create resources with representations included in the request message.

- DELETE - Deletes resources identified by request URIs.

The resource hosting servers response to a request with Response messages indicating they have received, understood and performed the request (or indicate errors occurred, such as requested resource not found). The individual response code, given in the Response message, indicates the result of the request (IETF RFC 7252, 2014).

Figure 6.15 shows the message format of a CoAP message. Table 6.2 describes the meaning of the sections in the CoAP message format. The contents of the sections Ver, $T, T K L$, Code, Message ID are specified in each case encoded in the Unsigned Integer format and have the number of bits as shown in Figure 6.15 (IETF RFC 7252, 2014). Permission to reproduce Figure 6.15 has been granted by IETF.

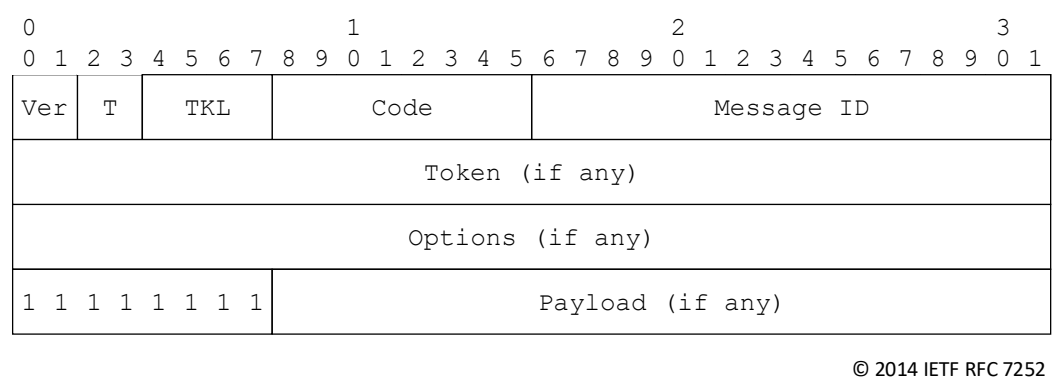

Figure 6.15: CoAP Message Format acc. (IETF RFC 7252, 2014)

A resource is addressed with the CoAP protocol using a URI that is structured as follows: coap: $/ /<$ host address $>:[<$ port $>] /<$ Path $>[?<$ query $>]$. The port is optional and if not 
specified "default port 5683 is assumed". The path specifies the resource located at the server. The query is also optional and specifies arguments that serve "to further parameterize the resource". Arguments are often specified as "key=value" pairs (IETF RFC 7252, 2014).

Table 6.2: CoAP Message Format acc. (IETF RFC 7252, 2014)

\begin{tabular}{|c|c|}
\hline Section & Description \\
\hline Version (Ver) & Defines the CoAP version. \\
\hline Type (T) & $\begin{array}{l}\text { Defines the type of the CoAP message: } 0 \text { (Confirmable), } 1 \text { (Non-confirmable), } 2 \\
\text { (Acknowledgement). }\end{array}$ \\
\hline Tokel Length (TKL) & Defines the length of the token specified in the Token field. \\
\hline \multirow[t]{3}{*}{ Code } & $\begin{array}{l}\text { Indicates type of message (request or response) and gives details about the } \\
\text { request methods or response status. }\end{array}$ \\
\hline & $\begin{array}{l}\text { Following request method codes are specified: } 0.01 \text { (GET), } 0.02 \text { (POST), } 0.03 \\
\text { (PUT), } 0.04 \text { (DELETE). }\end{array}$ \\
\hline & $\begin{array}{l}\text { Exemplary response codes are: } 2.01 \text { (Created), } 2.02 \text { (Deleted), } 4.04 \text { (Not Found), } \\
5.00 \text { (Internal Server Error). }\end{array}$ \\
\hline Message ID & $\begin{array}{l}\text { Indicates a message ID in order to detect corresponding acknowledgement } \\
\text { message (for reliable message transmission) or detect duplicate messages (for } \\
\text { unreliable message transmission). }\end{array}$ \\
\hline Token & $\begin{array}{l}\text { Clients can set the token value that can be used "to correlate requests and } \\
\text { responses". }\end{array}$ \\
\hline Options & $\begin{array}{l}\text { The CoAP message can include individual options (indicated by numbers) } \\
\text { specifying details corresponding to the CoAP message, such as conditions when } \\
\text { to perform a request or content format. }\end{array}$ \\
\hline Payload & $\begin{array}{l}\text { The CoAP message can carry optional payload that carries application specific } \\
\text { data. }\end{array}$ \\
\hline
\end{tabular}

The size of a CoAP message should be small enough to be transported in a single IP packet to avoid IP packet fragmentation. I.e. the CoAP message may only be so large that it can be encapsulated in the payload of a single UDP message. This depends on the MTU size of the IP network. If the MTU size is not known, an MTU size of 1280 Byte should be assumed. Minus the UDP header (IETF RFC 7252, 2014) recommends a maximum 
message size of 1152 Byte, whereby 1024 Byte are proposed for the payload size (IETF RFC 7252, 2014).

OneM2M defines in (oneM2M TS-0008-V1.0.1, 2015) a so-called "CoAP Protocol Binding" specifying how (Request/Response) Primitive messages are mapped to CoAP messages. This Protocol Binding defines the mapping of parameters of an oneM2M Primitive to corresponding message fields of a CoAP message. The following introduces how to realise the Subscribe/Notify IxP with CoAP messaging.

Table 6.3 shows the mapping of a Primitive message to a corresponding CoAP message according to (IETF RFC 7252,2014) required in the context of this project.

Table 6.3: OneM2M Primitive CoAP Message mapping acc. (oneM2M TS-0008-V1.0.1, 2015)

\begin{tabular}{|c|c|c|}
\hline $\begin{array}{l}\text { OneM2M Primitive } \\
\text { Parameter }\end{array}$ & \multicolumn{2}{|c|}{ CoAP Message Parameter } \\
\hline To & \multicolumn{2}{|c|}{$\begin{array}{l}\text { URI (in Request message). The To parameter is mapped to the URI-Path of } \\
\text { the CoAP message. }\end{array}$} \\
\hline From & \multicolumn{2}{|c|}{$\begin{array}{l}\text { Field: Option. Option no } 256 \text { (oneM2M-FR). The From parameter (in } \\
\text { Request message) is mapped to the oneM2M-FR option. }\end{array}$} \\
\hline \multirow[t]{6}{*}{ Operation } & \multicolumn{2}{|c|}{$\begin{array}{l}\text { Field: Code. Only in case of a request. Operation parameter is mapped to } \\
\text { the CoAP method as follows. }\end{array}$} \\
\hline & oneM2M operation & CoAP Method \\
\hline & CREATE & POST \\
\hline & DELETE & DELETE \\
\hline & NOTIFY & POST \\
\hline & \multicolumn{2}{|c|}{$\begin{array}{l}\text { In case of Subscribe message (oneM2M operation CREATE) additionally } \\
\text { Resource Type parameter in URI query ty=23. }\end{array}$} \\
\hline Request Identifier & \multicolumn{2}{|c|}{$\begin{array}{l}\text { Field: Option. Option no } 257 \text { (oneM2M-RQI). The Request Identifier } \\
\text { parameter is mapped to the oneM2M-RQI option. }\end{array}$} \\
\hline Response Status Code & \multicolumn{2}{|c|}{$\begin{array}{l}\text { Response Status Code is mapped to the Code field of a Response message. } \\
\text { oneM2M } 2000 \text { (OK) corresponds to CoAP status code } 2.05 \text { (Content). }\end{array}$} \\
\hline Content & \multicolumn{2}{|c|}{$\begin{array}{l}\text { Field: Payload. Contains the Service Specific Input/ Config/ Output } \\
\text { Parameter. }\end{array}$} \\
\hline
\end{tabular}


6 Cooperative M2M Application Service Provision

\begin{tabular}{|l|l|}
\hline$n / a$ & Field: Version (Ver). The Version field should be set to 1. \\
\hline$n / a$ & $\begin{array}{l}\text { Field: Type (T). O (Confirmable) in case of reliable message transmission, } 1 \\
\text { (Non-confirmable) in case of unreliable message transmission, } 2 \\
\text { (Acknowledgement) indicates an acknowledgement message. }\end{array}$ \\
\hline $\mathrm{n} / \mathrm{a}$ & Field Token Length (TKL). Length of the Token field. \\
\hline $\mathrm{n} / \mathrm{a}$ & Field: Message ID. Unique message ID. \\
\hline $\mathrm{n} / \mathrm{a}$ & Field: Token. Unique ID set by the client. \\
\hline & $\begin{array}{l}\text { Field: Option. Option no 259 (oneM2M-OT) = 0 specifies retrieve of } \\
\text { service/ information once. Otherwise continuous information delivery. }\end{array}$ \\
\hline
\end{tabular}

It should be noted that according to (oneM2M TS-0008-V1.0.1, 2015) both, oneM2M CREATE requests and oneM2M NOTIFY requests are transmitted with a CoAP POST (method) message. The distinction between CREATE and NOTIFY requests is realised using the Resource Type parameter in the URI-query. If this parameter is set, the request is handled as a CREATE request, i.e. a SUBSCRIBE request. (oneM2M TS-0004-V2.7.1, 2016) defines several Resource Types, such as $t y=23$ (Subscription). If the parameter has not been set, the request is handled as a NOTIFY request.

Furthermore, a distinction must be defined as to whether information (i.e., a service) should be requested once, or whether the requested information should be continuously sent to the requesting peer. For this purpose, it has been specified that this information is delivered in the Request message. The option parameter no 259 (oneM2M-OT) is used, which specifies an originating timestamp as defined in (oneM2M TS-0008-V1.0.1, 2015). If this is set to " 0 ", the request is handled as a one-time request otherwise it is handled as a continuous subscription. If the information should be continuously transmitted to the peer, the information-providing peer stores the contact data locally and sends the 
information continuously to the peer. If this is a one-time request, the peer returns the information directly and does not store any further information about the requesting peer.

The following Figure 6.16 - Figure 6.18 illustrate the comprehensive message exchange using the CoAP protocol including the specific protocol message fields to realise the Subscribe/Notify IxP. Figure 6.16 shows the subscription of an information/service by sending a CREATE request. The receiving server replies with an Acknowledgement message. Figure 6.17 shows the notification messages generated by the server delivering the requested information (once or continuous) to the requesting peer. Figure 6.18 illustrates the message exchange for terminating a subscription. In that case clients generate a DELETE message indicating that they are not interested in the previously requested information/service anymore. Message Sequence Charts C. 1 - C. 3 show the overall message exchange including message content for subscription, notification, and termination via CoAP (refer to Appendix C).

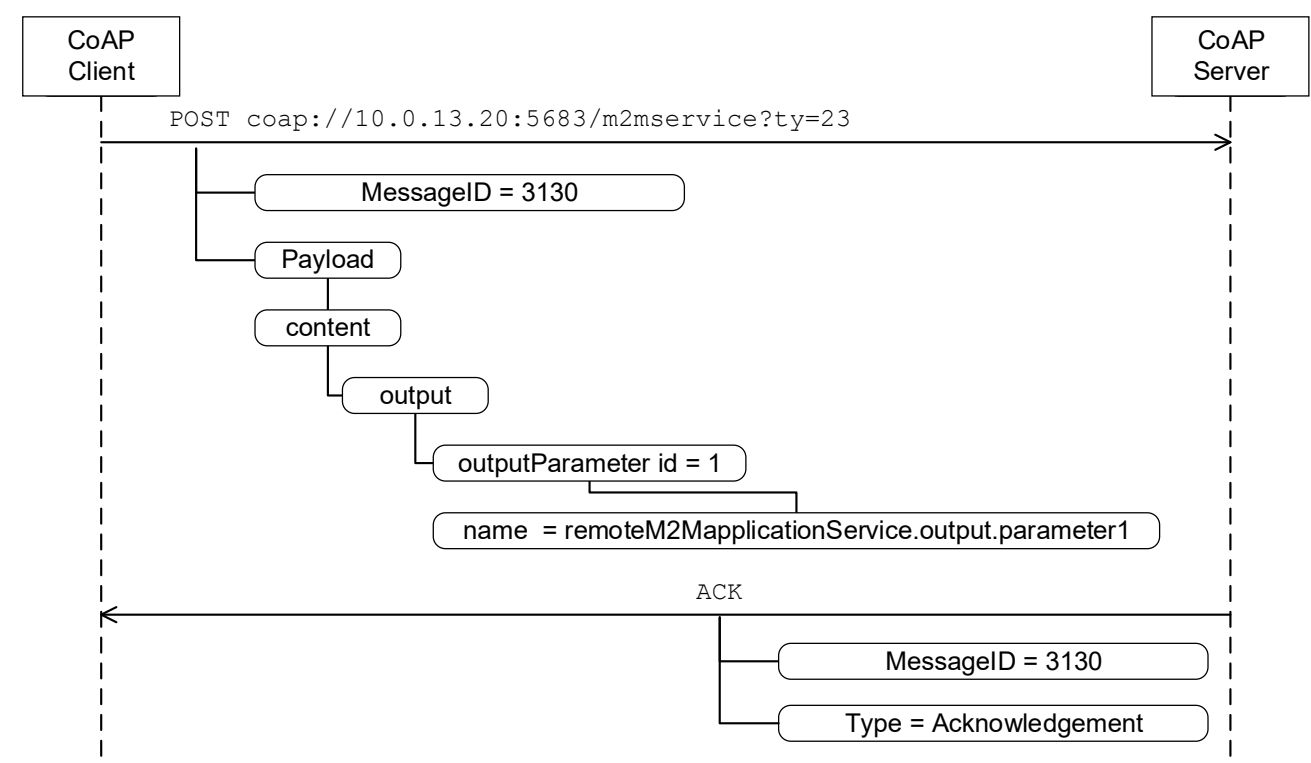

Figure 6.16: CoAP Messaging for Service/Information Subscription 


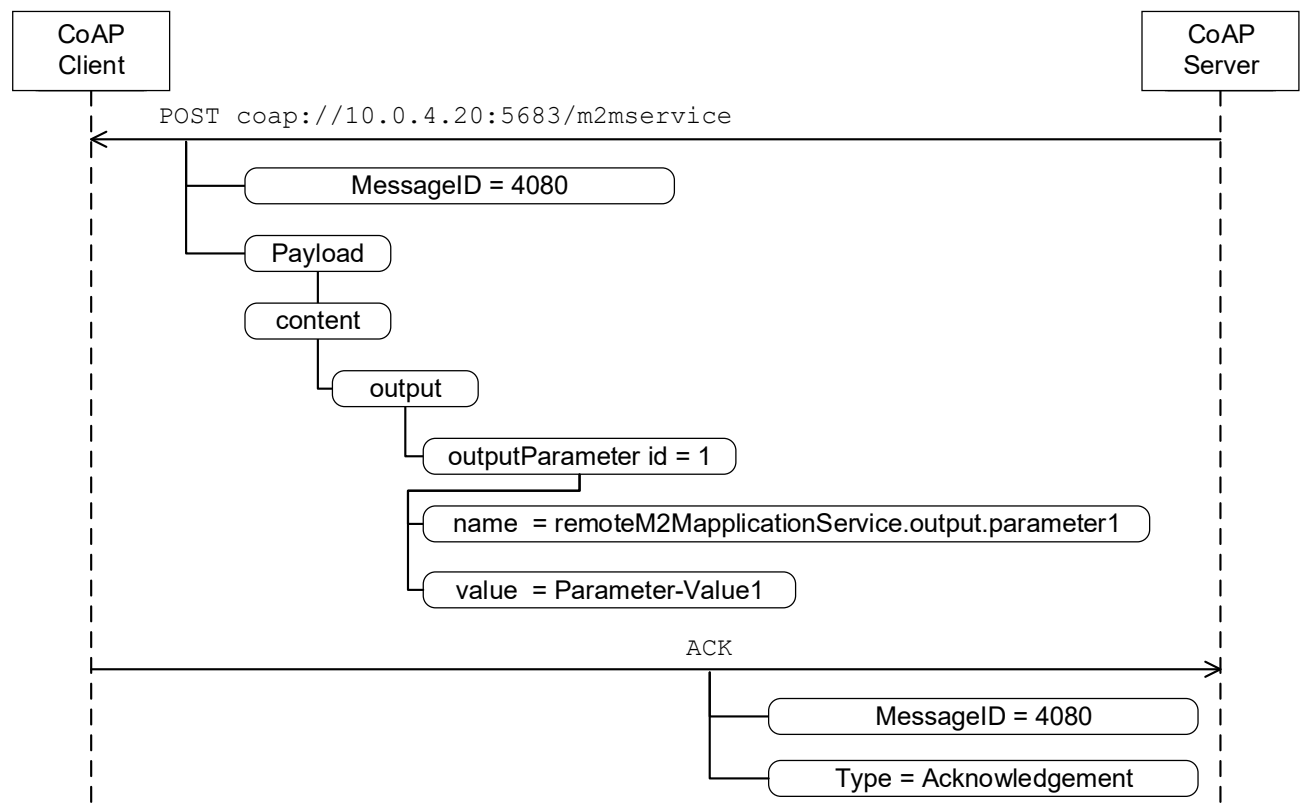

Figure 6.17: CoAP Messaging for Service/Information Notification

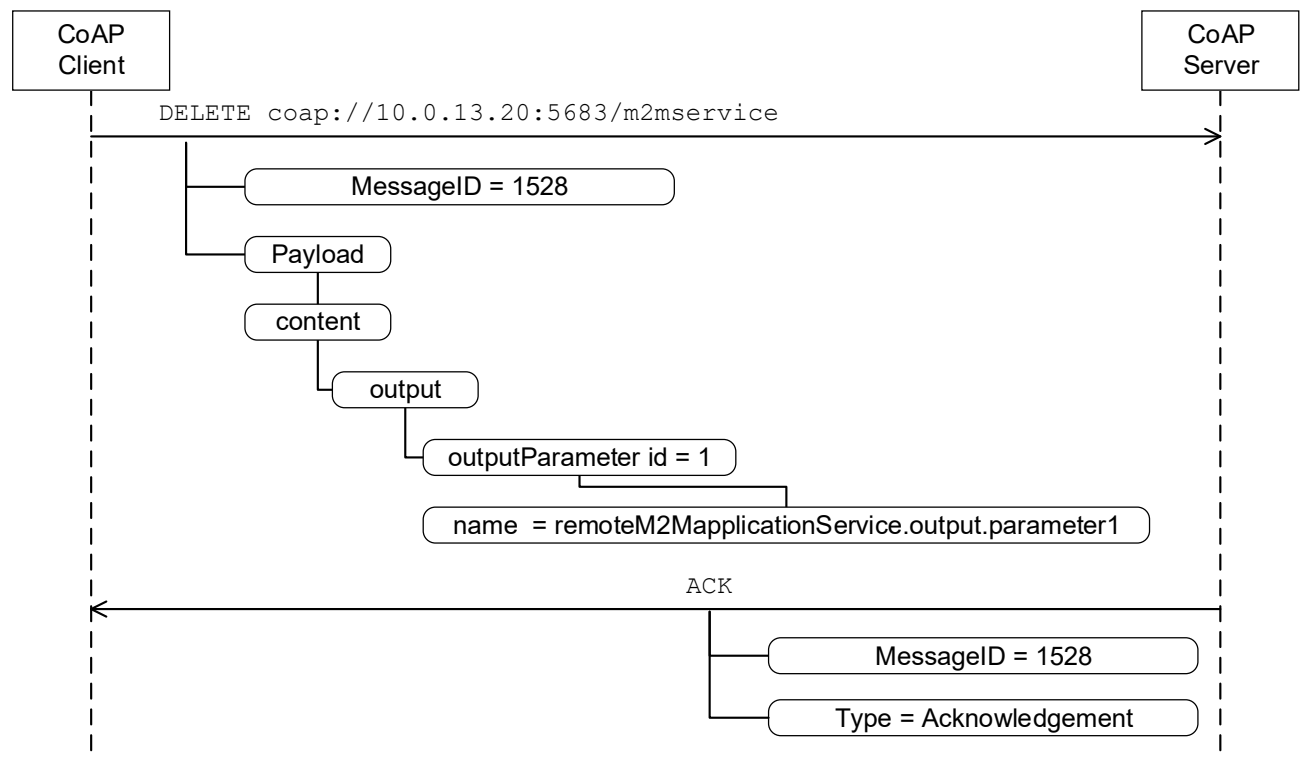

Figure 6.18: CoAP Messaging for Service/Information Unsubscription

As described above no Protocol Binding for SIP exist. Therefore, a SIP Protocol Binding is introduced below to also enable the exchange of Primitive messages using SIP. 


\section{Implementation of Subscribe/Notify IxP using SIP}

SIP is a text-based "application-layer control protocol” enabling ,internet endpoints“, socalled user agents (UAs) to establish a session with each other (shared thread in context of an application) and exchange information data. SIP is independent of underlying protocols, such as transport protocol TCP or UDP. SIP is the standard protocol for signalling (controlling communication sessions) in internet-based telephony environments and therefore its protocol stack is usually available on common IADs in end-users environment. Same as CoAP, SIP implements an "HTTP-like request/response transaction model" and integrates a reliability mechanisms. I.e. a transaction consists of a request invoking a specific method on the server which is answered by a Response message. An UA can take the role of both, client (User Agent Client, UAC) initiating SIP requests and server (User Agent Server, UAS) responding to SIP requests (IETF RFC 3261, 2002).

Additionally to the basic SIP standard RFC 3261 extensions of this standard were defined, such as extension for "SIP-Specific Event Notification" (IETF RFC 3265, 2002) enabling Subscribe/Notify IxP or „Extension for Instant Messaging“ (IETF RFC 3428, 2002) enabling Instant Messaging via SIP.

Figure 6.19 illustrates the structure of a SIP Request message and Figure 6.20 shows the structure of a SIP Response message. 
6 Cooperative M2M Application Service Provision

\begin{tabular}{|c|c|c|c|c|}
\hline Start Line & Method & Request URI & SIP Version & CRLF \\
\hline \multirow{4}{*}{ Header } & Contact & Via & From & To \\
\hline & Call-ID & CSeq & Content-Type & Content-Length \\
\hline & Event & Max-Forwards & Expires & $\ldots$ \\
\hline & \multicolumn{4}{|c|}{ Blank Line } \\
\hline $\begin{array}{r}\text { Message } \\
\text { Body }\end{array}$ & \multicolumn{4}{|c|}{ Message Body } \\
\hline
\end{tabular}

Figure 6.19: SIP Request Message Format acc. (IETF RFC 3261, 2002)

\begin{tabular}{|c|c|c|c|c|}
\hline Start Line - & SIP Version & Status Code & Reason Phrase & CRLF \\
\hline \multirow{3}{*}{ Header - } & CSeq & Call-ID & From & To \\
\hline & Via & CSeq & Content-Length & Max-Forwards \\
\hline & Expires & $\begin{array}{c}\text { Subscription- } \\
\text { State }\end{array}$ & \multicolumn{2}{|c|}{$\ldots$} \\
\hline
\end{tabular}

Figure 6.20: SIP Response Message Format acc. (IETF RFC 3261, 2002)

A SIP Request message always starts with a Start Line. This specifies the method to be executed on the UAS containing the address information (SIP URI) of the target SIP UA. A SIP URI has the following structure: sip: $<$ username $>@<$ host address $>:[<$ port $>]$. The port is optional and if not specified default port 5060 is assumed. A SIP Response message also starts with a Start Line containing information about the corresponding Request message (e.g. Status Code of message transmission). Beside the Start Line, a SIP message contains various Header Fields giving more details of the session.

Table 6.4 describes an extract of SIP Header Fields as contained in Request/Response messages. SIP messages can additionally include a Message Body containing required information in the application context, such as Session Description Protocol information (describing details of an audio/video call) (IETF RFC 3261, 2002). 
Table 6.4: SIP Message Format acc. (IETF RFC 3261, 2002; IETF RFC 3265, 2002)

\begin{tabular}{|c|c|}
\hline Section & Description \\
\hline $\begin{array}{l}\text { Start Line in } \\
\text { Request } \\
\text { Messages }\end{array}$ & $\begin{array}{l}\text { The Start Line has the format }\langle\text { Method name }\rangle\langle\text { Request-URI }\rangle\langle\text { SIP-Version }\rangle \text {. } \\
\text { Method Name specifies the method to be executed on the UAS. Request-URI } \\
\text { specifies the addressing information (SIP URI) of the UAS. SIP-Version specifies } \\
\text { the Version of the SIP Protocol used (usually "SIP } / 2.0^{\prime \prime} \text { ) }\end{array}$ \\
\hline $\begin{array}{l}\text { Start Line in } \\
\text { Response } \\
\text { Messages }\end{array}$ & $\begin{array}{l}\text { The Start Line has the format }\langle\text { SIP Version }>\langle\text { Status Code }\rangle\langle\text { Reason Phrase }>\text {. SIP- } \\
\text { Version specifies the Version of the SIP Protocol used (usually "SIP } / 2.0 \text { "). Status } \\
\text { Code indicates the response status of a request (e.g. } 200 \text { for successful request). } \\
\text { Reason Phrase is a textual description of the Status Code (e.g. "OK" for Status } \\
\text { Code 200). }\end{array}$ \\
\hline Contact & $\begin{array}{l}\text { Defines the SIP URI of the message originating UA. Indicates where to send } \\
\text { future requests. }\end{array}$ \\
\hline Via & $\begin{array}{l}\text { Contains information about the routing of Response messages (IP }+ \text { port }+ \text { branch } \\
\text { parameter to identify a specific transaction). Indicates where the request } \\
\text { initiating UA expects to receive the Response message and possibly other } \\
\text { network elements included in the routing path. }\end{array}$ \\
\hline From & $\begin{array}{l}\text { Contains the SIP URI as well as a display name of the originator of a request. } \\
\text { From header field of Response messages equals to the From header value of the } \\
\text { original Request message. }\end{array}$ \\
\hline To & $\begin{array}{l}\text { Contains the SIP URI as well as a display name of the receiver of a request. To } \\
\text { header field of Response messages equals to the To header value of the original } \\
\text { Request message. }\end{array}$ \\
\hline Call-ID & $\begin{array}{l}\text { Globally unique identifier to correlate messages send in a specific context } \\
\text { (dialog). }\end{array}$ \\
\hline CSeq & $\begin{array}{l}\text { Command Sequence containing an Integer number as well as a method name. } \\
\text { Implements a sequents number that is incremented for every new Request } \\
\text { message inside the same dialog. }\end{array}$ \\
\hline Content-Type & Specifies the type of content in the Message Body (e.g. application/xml). \\
\hline Content-Length & Describes the length of the Message Body. \\
\hline Event & $\begin{array}{l}\text { Indicates the type of event a subscriber registers for. Can contain additional } \\
\text { parameter specifying details about the type of event. }\end{array}$ \\
\hline Max-Forwards & $\begin{array}{l}\text { Integer value indicating the maximum number of hops on the routing path and is } \\
\text { decremented by each forwarding network element. }\end{array}$ \\
\hline Expires & $\begin{array}{l}\text { Defines the time in seconds a avent subscription is active. Value set to " } 0 \text { " } \\
\text { defines terminating a subscription. }\end{array}$ \\
\hline Message Body & The SIP message can carry optional payload that carries application specific data. \\
\hline Subscription-State & $\begin{array}{l}\text { Indicates the status of the subscription. "Active" indicates that the subscription is } \\
\text { active and will be processed by the notifier and "terminated" means that the } \\
\text { subscription is terminated. }\end{array}$ \\
\hline
\end{tabular}


To realise the Subscribe/Notify IxP, an "event notification mechanism" is defined in (IETF RFC 3265, 2002). Here UAs (subscriber) who are interested in information (i.e. in specific events) register themselves at the event producing UA (notifier). The notifier then sends information about this to the subscriber at regular intervals or if a corresponding event has occurred (IETF RFC 3265, 2002).

To implement the event notification mechanism, (IETF RFC 3265, 2002) defines the following methods:

- $\quad$ SUBSCRIBE - The SUBSCRIBE method is used in a SIP Request message to register for an event at another UA.

- NOTIFY - The NOTIFY method is used by the notifier to send an event to a subscriber.

To specify a SIP Protocol Binding analogous to the CoAP Protocol Binding, it must be defined how the elements of the Primitive messages are mapped to the elements of a SIP message. Table 6.5 shows this mapping.

Table 6.5: OneM2M Primitive SIP Message mapping

\begin{tabular}{|c|c|c|}
\hline $\begin{array}{l}\text { OneM2M Primitive } \\
\text { Parameter }\end{array}$ & \multicolumn{2}{|c|}{ SIP Message Parameter } \\
\hline To & \multicolumn{2}{|c|}{$\begin{array}{l}\text { Request Line SIP URI and To header field. The To parameter is mapped to the } \\
\text { SIP URI in the Request Line as well as the To header field of the SIP message. }\end{array}$} \\
\hline From & \multicolumn{2}{|c|}{$\begin{array}{l}\text { From header field and Contact header field. The From parameter is mapped to } \\
\text { the From header field as well as the Contact header field of the SIP message. }\end{array}$} \\
\hline \multirow[t]{6}{*}{ Operation } & \multicolumn{2}{|c|}{$\begin{array}{l}\text { The Operation parameter is mapped to the Method Name in Request Line. } \\
\text { Operation parameter is mapped to the SIP method as follows. }\end{array}$} \\
\hline & oneM2M operation & SIP Method \\
\hline & CREATE & SUBSCRIBE \\
\hline & DELETE & SUBSCRIBE \\
\hline & NOTIFY & NOTIFY \\
\hline & \multicolumn{2}{|c|}{ In case of Subscribe message for unsubscription the Expires header is set to " 0 " } \\
\hline
\end{tabular}




\begin{tabular}{|c|c|}
\hline Request Identifier & Event header parameter reqld has been defining the Request Identifier. \\
\hline $\begin{array}{l}\text { Response Status } \\
\text { Code }\end{array}$ & $\begin{array}{l}\text { Response Status Code is mapped to the Response Line Status Code of a } \\
\text { Response message. oneM2M } 2000 \text { (OK) corresponds to SIP status code } 200 \\
\text { (OK). }\end{array}$ \\
\hline Content & Message Body. Contains the Service Specific Input/ Config/ Output Parameter. \\
\hline $\mathrm{n} / \mathrm{a}$ & Content-Length header field. Indicates the size of the Message Body \\
\hline $\mathrm{n} / \mathrm{a}$ & Field Token Length (TKL). Length of the Token field. \\
\hline $\mathrm{n} / \mathrm{a}$ & CSeq header field: Command Sequence Number set by the UAC. \\
\hline $\mathrm{n} / \mathrm{a}$ & Call-ID header field: Unique ID set by the UAC. \\
\hline $\mathrm{n} / \mathrm{a}$ & $\begin{array}{l}\text { Event header parameter once has been specified indicating retrieve of service/ } \\
\text { information once (once=true). Otherwise (once=false ) continuous information } \\
\text { delivery. }\end{array}$ \\
\hline$n / a$ & Via header field: SIP URI of the client, set by the UAC. \\
\hline $\mathrm{n} / \mathrm{a}$ & Max-Forwards header field. Indicates the maximum number of hops. \\
\hline
\end{tabular}

The designed SIP Protocol Binding enables transmission of Primitive messages with SIP and makes it possible to use the existing communication infrastructure, in particular the existing communication equipment (SIP stack in IAD) in end-users' environments for communication between SPs and SCs.

It should be noted that Primitive operations CREATE and DELETE are both sent via a SIP Request message with the method SUBSCRIBE. The distinction between CREATE and DELETE is made based on the Expires header field. If this field has the value " $O$ ", the message is handled as an unsubscription message.

It has also been designed to specify whether a service should be used once or whether the associated information should be transmitted continuously. For this purpose, the additional parameter "once" has been defined in the Event header field. The parameter reqId has also been specified as an additional parameter in the Event header field defining the Request Identifier of a Primitive. 
The following Figure 6.26 - Figure 6.28 illustrate the comprehensive message exchange using SIP including the specific protocol message fields to realise the Subscribe/Notify IxP.

Figure 6.26 shows the subscription of an information/service by sending a SUBSCRIBE request. The receiving UAS replies with an acceptance message. Figure 6.27 shows the notification message generated by the UAS that delivers the requested information (once or continuous) to the requesting peer. Figure 6.28 shows the message exchange for terminating a subscription. In that case the UAC generates a SUBSCRIBE message with Expires header field set to " 0 " indicating that it is not interested in the previously requested information/service anymore. Message Sequence Charts C.4 - C.6 show the overall message exchange including message content for subscription, notification, and termination via SIP (refer to Appendix C).

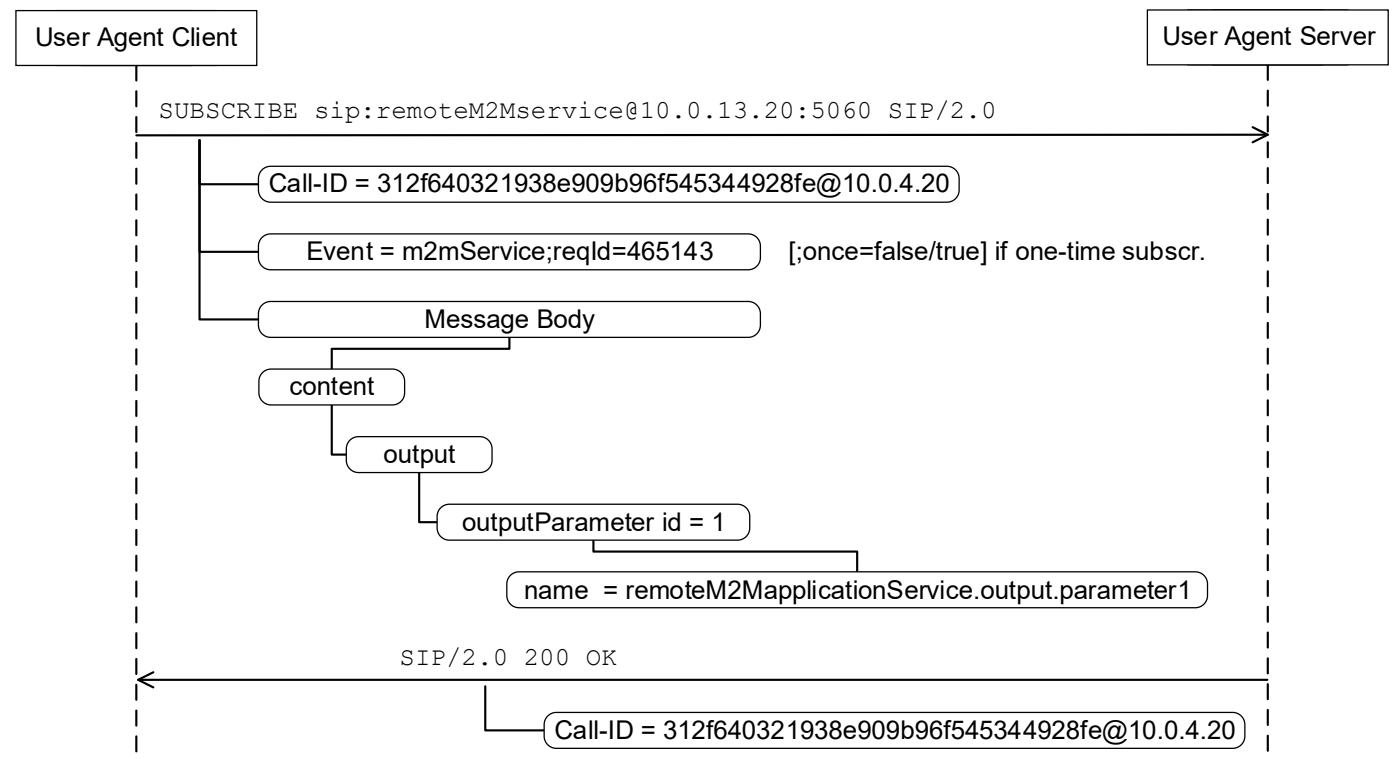

Figure 6.21: SIP Messaging for Service/Information Subscription 


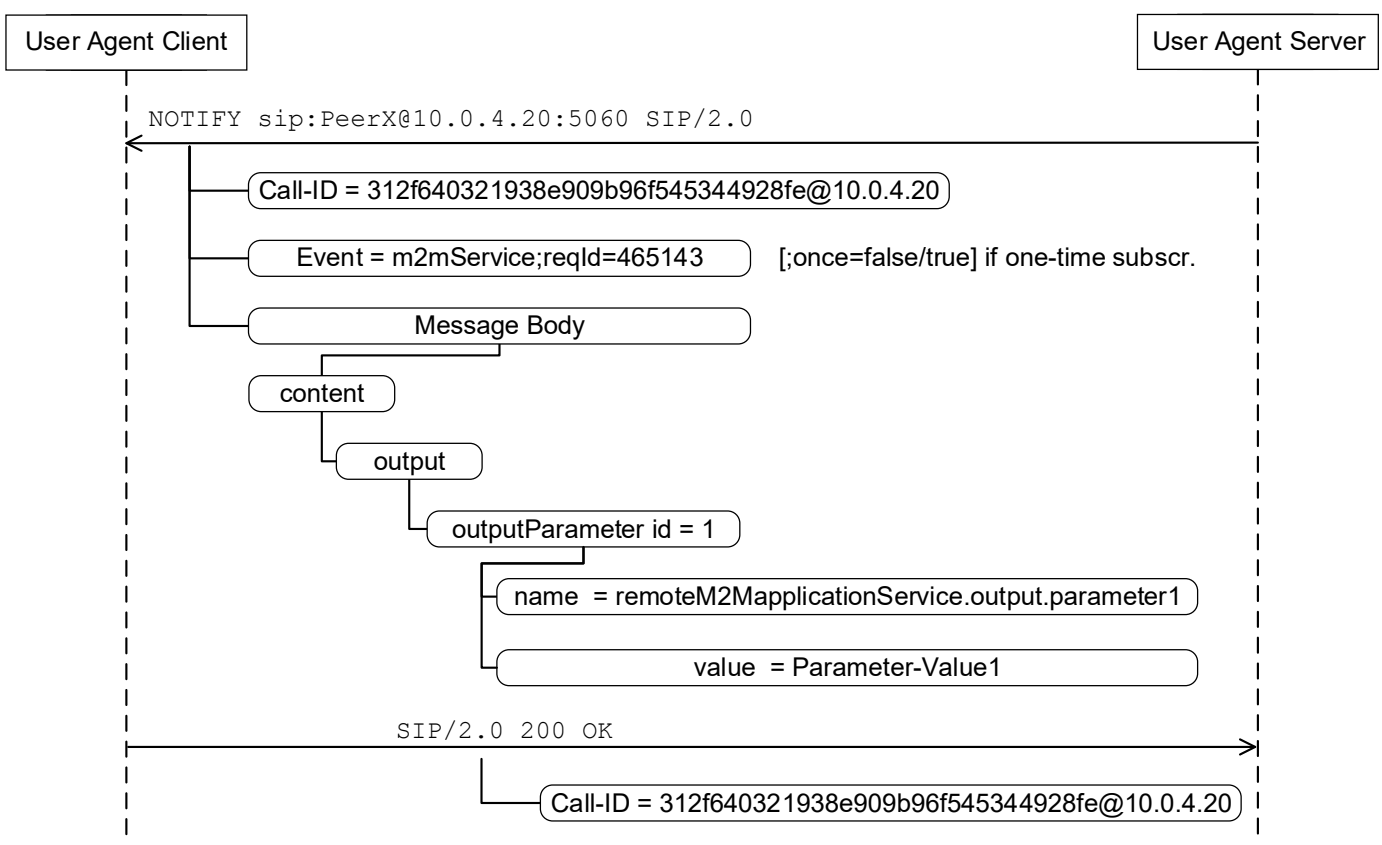

Figure 6.22: SIP Messaging for Service/Information Notification

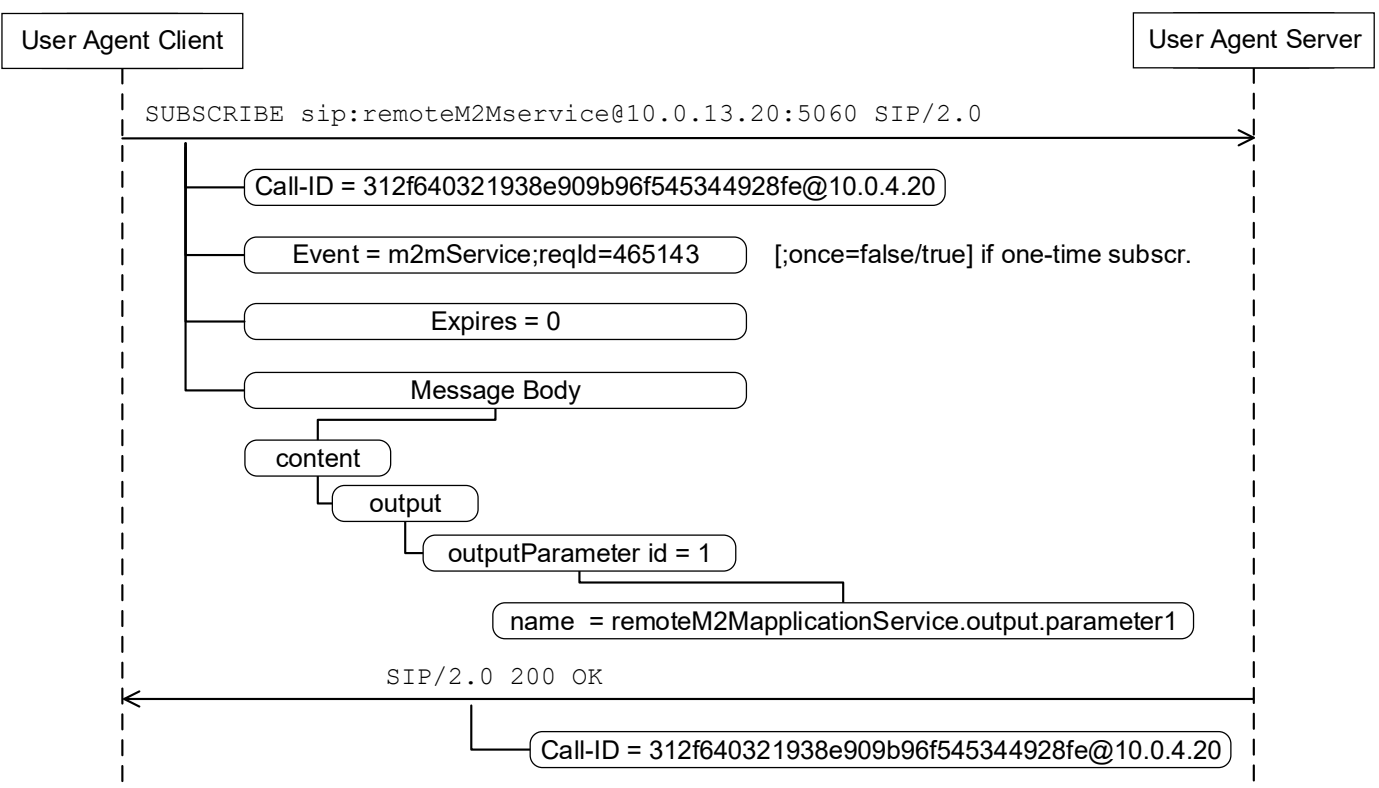

Figure 6.23: SIP Messaging for Service/Information Unsubscription

It should be emphasized once again that all communication is $\mathrm{P} 2 \mathrm{P}$, meaning that no additional mediating network elements on the application layer are involved in the communication between SP and SC. Thus, there are no dependencies on additional network elements or on the operators of the network elements. 
The entities contained in the architecture of the M2M service platform (MSP) presented in this research have the possibility to use both, CoAP and SIP as communication protocols between the peers by using the CoAP Protocol Binding or introduced SIP Protocol Binding.

In the following, an evaluation of CoAP and SIP as communication protocol in the context of the presented architectural approach is performed.

Both CoAP and SIP have functionality transporting XML-data and mechanisms for reliable communication enabling reliable exchange of Primitive messages. CoAP and SIP are realised in realised in Application Layer (OSI) that offers the functionality to act in heterogeneous networks. Both protocols can be used for end-to-end communication between end-users' platforms. Furthermore, CoAP and SIP offer the functionality for location-independent availability via unique identifiers (globally defined SIP/CoAP URIs).

CoAP has an advantage when considering the message size. The header fields of a CoAP message are smaller than the header fields of a SIP message resulting in more overhead for a SIP message, because the header fields are not encoded and compressed like in CoAP, but in text format. The content of the header fields and the payload is the same for both protocols. However, the overhead of a SIP message according to (IETF RFC 3261, 2002) can be reduced by providing the SIP header fields in a compact form. In this case, the header fields are abbreviated by a single letter (e.g. compact form of the "From:" header is "f:"). This reduces the length of the header field name to 4 Byte, since UTF-8 (IETF RFC 3629, 2003) is used for character encoding. 
The implementation of the MMSCs within the local M2M platform requires a SIP stack. The use of SIP as compared to CoAP for message exchange between the distributed M2M platforms has the advantage that an additional stack is avoided, which in turn reduces the complexity of the platform architecture. End-users also usually have an end-device (e.g. smartphone) in which a UA is already included that may be used for communication with the local M2M platform via SIP. In addition to the implementation of the MMSCs, SIP can also be used to realise a permanent VoIP communication or messaging (IM) between SP and SC using additional protocols (e.g., SDP, RTP) whose stacks are also already included in the common end-user multimedia communication equipment (RTP stack). This enables communication independent of a public VoIP provider using P2P VoIP communication. In particular, cellular mobile networks with LTE radio access networks (3GPP TS 23.002 v8.7.0, 2010) require packet-switched message transport and therefore SIP is used for implementation of multimedia over IP communication services. SIP provides in comparison to CoAP integrated presence functionality to request information regarding the availability of users as well as Instant Messaging functionality for exchange of text messages between the M2M entities. In contrast to CoAP, a SIP-based platform architecture could easily be integrated into Next Generation Networks (NGNs) (ITU-T Y. 2001, 2004; ITU-T Y. 2012, 2006) or IP Multimedia Subsystems (IMS) (ETSI TS 123 $228 \mathrm{~V} 11.10 .0,2013)$ if the provider agrees to do so, since these are based on network elements, end devices and gateways that communicate with SIP. For example, the NGN/IMS provider could also act as a provider for the communication platform and, if necessary, take over some of the platform functionality defined in this research (e.g. registration of services). Additionally, a standard for NAT traversal practices has already 
been defined for SIP in (IETF RFC 6314, 2011), which in turn simplifies the integration of the M2M services in existing end-user equipment.

Table 6.6 summarises the comparison of the protocols CoAP and SIP in the context of the presented framework.

Table 6.6: Comparison of CoAP and SIP as Communication Protocol

\begin{tabular}{|l|c|c|}
\hline & CoAP & SIP \\
\hline Application Layer Protocol & + & + \\
\hline Reliable Communication & + & + \\
\hline Transport of XML Application Data & + & + \\
\hline Possibility for end-to-end Communication & + & + \\
\hline Location-independent availability & - & + \\
\hline Low Overhead & - & + \\
\hline No additional Protocol Stacks in Platform required & - & + \\
\hline Existing (cliend-side) User Interface & - & + \\
\hline Possibility for VolP Communication & - & + \\
\hline Integrated Presence Functionality & + & + \\
\hline Possibility for NGN/ IMS integration & + & + \\
\hline NAT traversal functionality & + & + \\
\hline$+:$ advantageous; o: moderate; -: disadvantageous & + & + \\
\hline
\end{tabular}

As result of the evaluation it can be stated that both protocols have advantageous functionality enabling the communication between M2M SP and SC and therefore are integrated in the proposed MSP architecture. CoAP has the advantage of low header size and therefore less overhead in message transmission, which is advantageous in constrained environments. SIP has the advantage that it offers additional (standardised) functionalities that prevent integration of additional protocol stacks and client interface 
software. Because the extended functionality of SIP, it is therefore suggested to use SIP as the communication protocol.

\subsection{Service Registry and Distributed Data Storage}

After explaining how peers can use services offered by other peers, and how networking is realised between peers, the following section describes the management and publication of services. For this, section 6.3.1 defines a common data base for the administration of the IFDs. Section 6.3.2 introduces the concepts to realise the common database using structured and unstructured P2P overlays. Finally, section 6.3.3 presents an evaluation of structured and unstructured overlay algorithms in the context of this project.

\subsubsection{M2M Service Registration and Storage of M2M Service IFD}

To enable the use of services offered by end-users, it is necessary to register the services at an accessible location and to make the IFD available to other end-users. In this project, a distinction is made between a service (service functionality) that can be uniquely identified by a service-ID and service instances (specific implementation of the service). A service provides a particular functionality, whereas a service instance is an actual implementation of a service by an end-user that can be addressed via a service endpoint. The IFD combines this information by describing the functionality of a service, specifying the interface and including the service endpoints (pointOfAccess). 
The Service/Application Registry (SAR) has been designed for the registration of the services and has the task to manage which services exist, how the IFDs are defined for these services, and under which URI services are reachable (Steinheimer et al., 2017c).

As defined in section 4.1, several end-users can offer the same services. Therefore, they have to register the individual instance of their services (specific implementation of a service offered by an end-user) with the $S A R$ as shown in Figure 6.24. Similar services in this context means that the services have identical IFDs and are only accessible via different URIs. Identical services must therefore be registered under an identical serviceID. Conversely, this means that different services also have different IFDs (e.g. different in Input/Output parameters or accessControlPolicies) and must therefore be registered separately using a different service-ID.

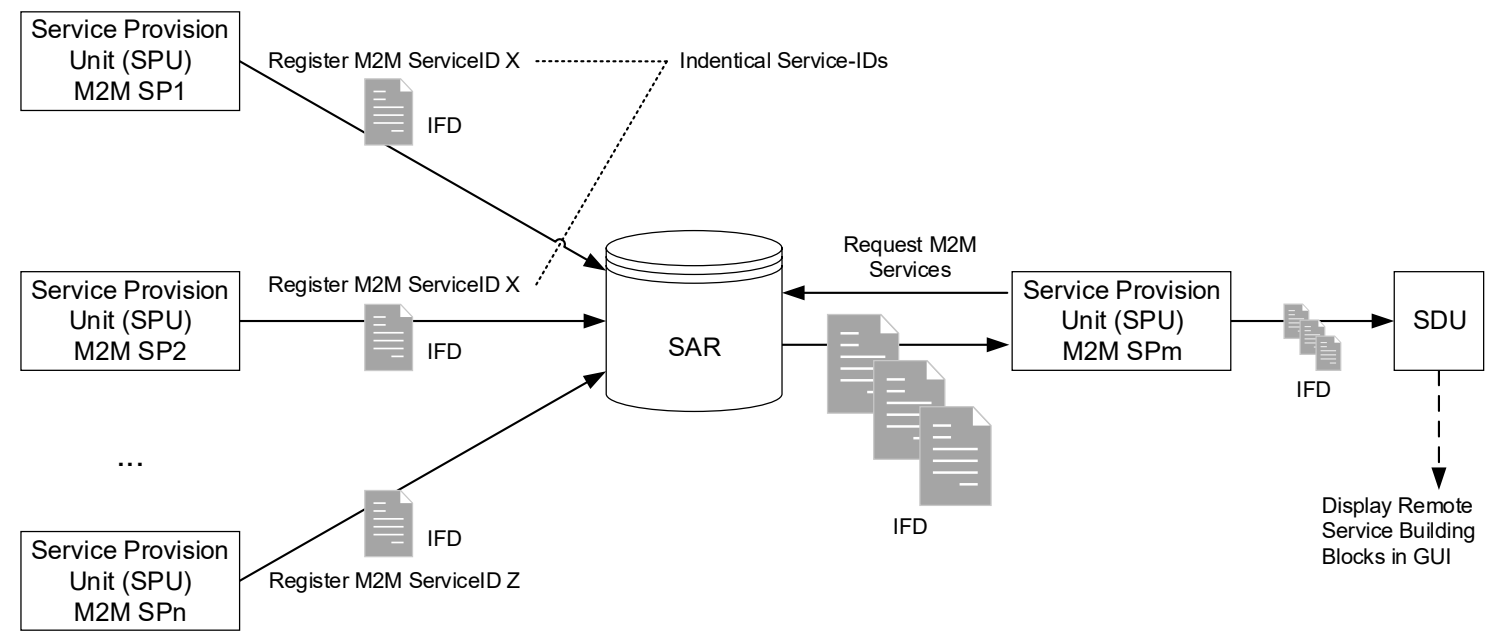

Figure 6.24: M2M Services Registration and Request

To be able to use a service (request a specific instance), the platform that wants to use the service has to make a request to the $S A R$ (specifying the service-ID of the desired service). The $S A R$ then provides a specific service IFD for the requested service-ID, containing the 
specific addressing information of the M2M SP (or multiple M2M SPs), so that the local $\mathrm{M} 2 \mathrm{M}$ platform can request the service on the remote $\mathrm{M} 2 \mathrm{M}$ platform.

To obtain an overview of all registered services, the Service Provision Unit (SPU) of a local M2M platform makes a request to the $S A R$ for all registered services, which then returns the registered IFDs. The resulting IFDs are forwarded by the querying $S P U$ to the Service Design Unit (SDU), which in turn generates remote service building blocks and displays them in the GUI (see Figure 5.14).

\subsubsection{Principles of distributed Data Storage}

As introduced in section 4.2 the $P 2 P$ Overlay Layer realises the functionality of distributed data storage by forming the P2P overlay network out of all existing end-user nodes. Distributed data storage means that data is not stored in one place, but stored in a network, spread across multiple instances (peers). The aim of the presented approaches is to entirely avoid central entities; therefore central data storage is declared as not possible.

Also RELOAD (IETF RFC 6940, 2014) and the Distributed Resource Directory Architecture for M2M Applications (DRD4M) Project (Liu et al., 2013) propose to use a P2P overlays for registration and lookup of resources.

According to (Liu et al., 2013) registration of resources means storing the resource description to the overlay (containing the IP, path, type, content type and name of the resource). Lookup of resources means searching for a specific resource to request the content of the resource. DRD4M also proposes to cache the resources in the overlay to 
reply the resource descriptions and resource content if devices are in sleep mode. To connect resources, the peers (resources) store their resource content in the overlay and peers that want to consume the resources, request the content from the overlay.

As mentioned in section 6.2 .1 additionally to $\mathrm{P} 2 \mathrm{P}$ message routing functionality RELOAD provides according to (IETF RFC 7374, 2014) and (IETF RFC 6940, 2014) the functionality for distributed storage of data items inside the overlay using the selected P2P overlay algorithm.

The approaches of distributed resource registration has been derived and applied to the concept for distributed M2M service provision presented in this research.

DRD4M uses the structured overlay algorithm Chord (Stoica et al., 2001) to implement the overlay (Liu et al., 2013). RELOAD also defines the usage of Chord as one mandatory integrated P2P overlay algorithm. RELOAD enables that other structured or unstructured P2P overlay algorithms could be used additionally to Chord to extend a RELOAD system (IETF RFC 6940, 2014).

Since the approaches of structured and unstructured P2P overlays are fundamentally different, the following section examines how a service registration could be realised using the respective approaches. The following topics should be clarified:

- Storing Service IFD in the Overlay - The specific information about a service (e.g. SP URI, interface parameters) is stored in the service IFD. To make it available, it must be stored in the overlay.

- Retrieving a Service IFD - To obtain the information about a service the IFD must be retrievable from the overlay. 
- Generate an overview of all existing services or service IFDs - If services are to be used by other end-users, they must know which services exist at all. Therefore, a list of all services is required (M2M Service Reference List).

\section{IFD Registration using structured P2P Overlays}

In a structured P2P overlay, the data sets are stored in a Distributed Hash Table (DHT) and are identified by a unique key (refer to section 2.2). The IFD must therefore be stored as a unique identifiable data item in the DHT. How the storage is carried out is described below. The corresponding message exchange between the involved peers is shown in Figure 6.25 .

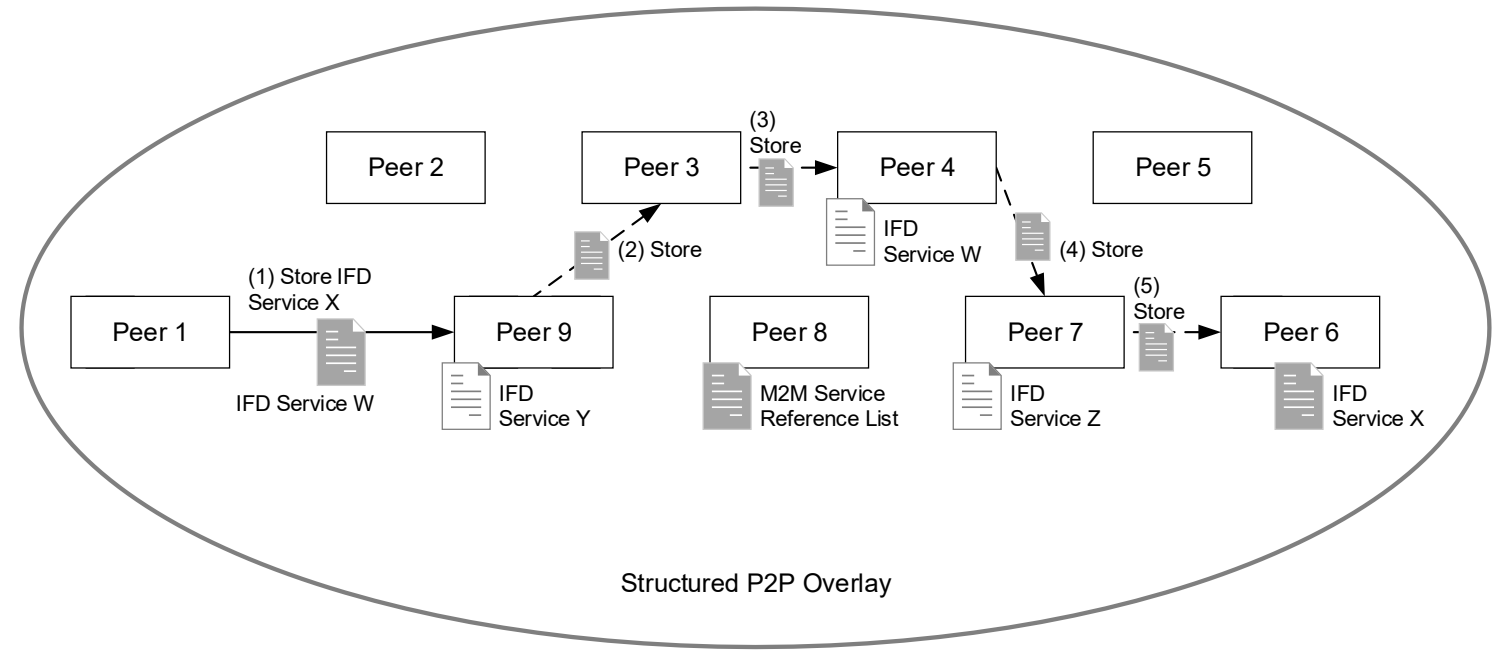

Figure 6.25: Store IFD in structured P2P Overlay

To store an IFD in the P2P overlay the key under which the IFD should be stored and later searched must be defined. As described in section 6.3.1, an M2M service is uniquely identified using the service-ID, which is therefore used as a key identifier for the data item in the DHT. Afterwards, a store request must be sent to the $\mathrm{P} 2 \mathrm{P}$ overlay, specifying the key and the IFD (step 1). The P2P overlay algorithm is used to determine the peer in 
the DHT that is responsible for storing the data item. The IFD is then transferred to the determined peer (step 2 - 5), which saves this IFD as a data item.

It is still necessary to check whether an IFD for an M2M service already does exist. This is the case if several peers offer the same service. The verification can be realised sending a request to the $\mathrm{P} 2 \mathrm{P}$ overlay with the service-ID as a key. If the overlay already contains an associated data record this is returned indicating that a service IFD has already been stored in the SAR. Then peers can register another instance of that service by adding their URI to the list of already contained M2M SP URIs in the IFD (pointOfAccess). After adding the entry, peers save the modified IFD back to the P2P overlay.

Figure 6.26 illustrates the process to retrieve an IFD from the P2P overlay. A peer requesting an IFD sends a request message to the DHT specifying the service-ID as identifier for the searched IFD (step 1). The DHT then routes the request according to the P2P overlay algorithm used to the peer storing the data item (step 2-5). The peer that stores the record returns it directly to the requesting peer without involving the other peers (step 6). After the IFD has been received, the information defined in it can be further processed (e.g., by displaying corresponding M2M service building blocks in the GUI). 


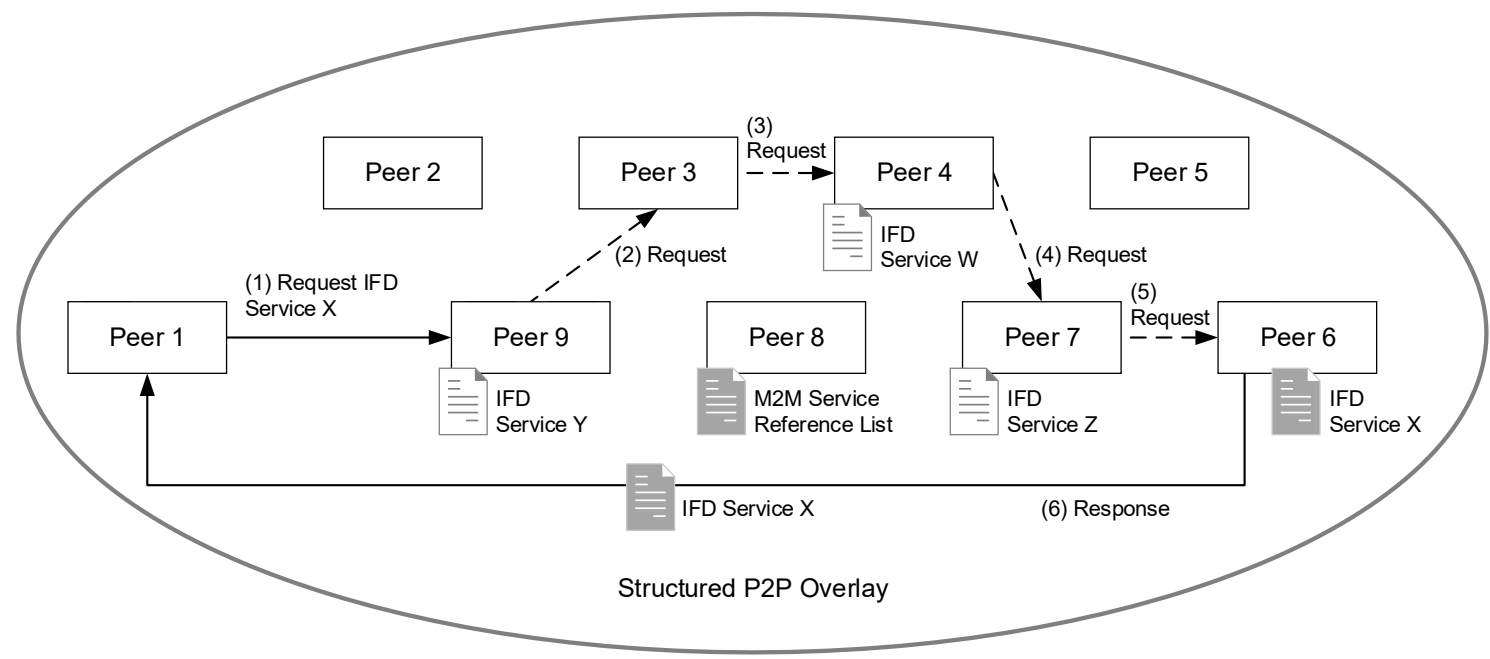

Figure 6.26: Request IFD from structured P2P Overlay

DHTs does not enable to determine all keys contained in it (because requests require specific keys for data items). Therefore, it is necessary for generating an overview of all existing M2M services that a list of service-IDs is managed, which is stored under a single record listing all existing services. For this purpose, the M2M Service Reference List has been introduced (see Peer 8 in Figure 6.25 and Figure 6.26). Peers that want to register a service load this M2M Service Reference List from the P2P overlay (similar to querying a service IFD), add the service-ID of the service that should be registered and store the M2M Service Reference List back to the P2P overlay. Each peer requested the M2M Service Reference List has an overview of the registered service-IDs and can query the corresponding IFDs.

\section{IFD Registration using unstructured P2P Overlays}

In unstructured $\mathrm{P} 2 \mathrm{P}$ overlays, in contrast to structured overlays, the records are stored locally at the peers offering (i.e. insert) the data sets (see Figure 6.27). The IFD is thus stored and managed locally by the peers who offer an M2M service. A data item (i.e., the 
IFD) is stored as a file in the filesystem of the peer. For identifying the IFD the serviceID of the M2M service is also selected as the identifier for the IFD file.

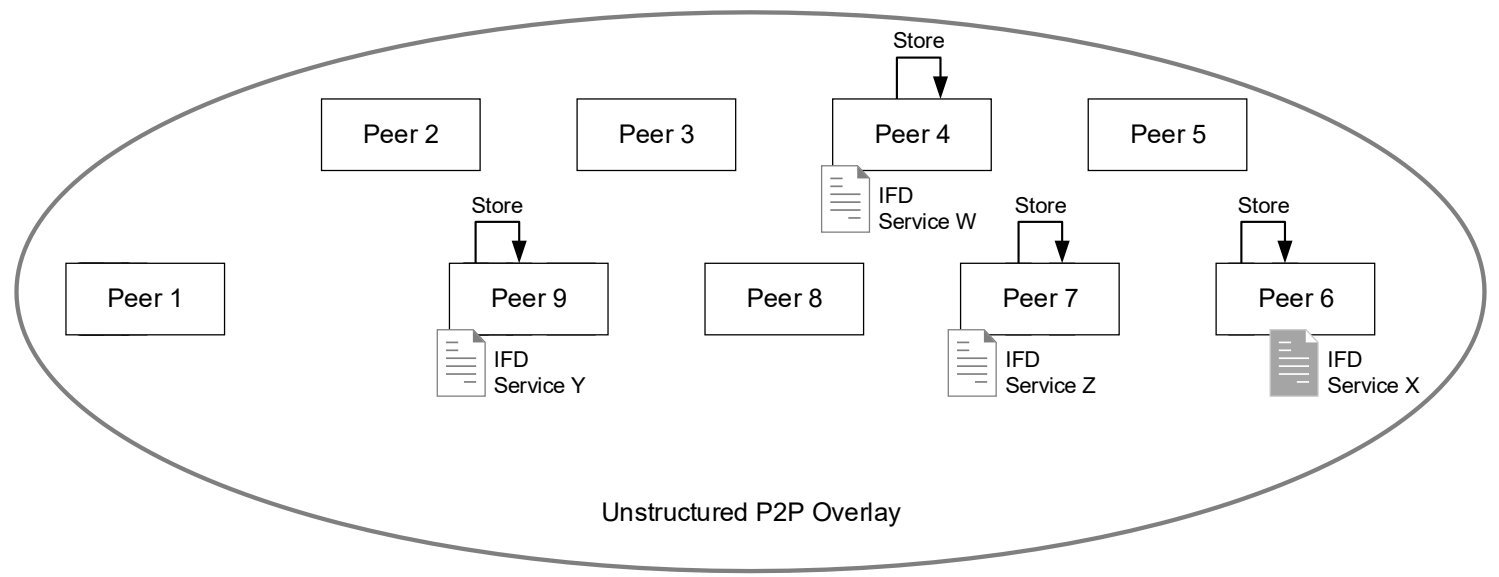

Figure 6.27: Store IFD in unstructured P2P Overlay

Communication between peers during file storage process is not necessary and takes place mainly during the search process for the IFD. It is required to manage a separate IFD for each instance of a service since it is not possible to store files in the filesystems of other peers (because missing write access). So peers that aim to register a service instance generate the IFD containing their service endpoint URI and store it in their local filesystem.

The search mechanism in unstructured P2P overlays (see Figure 6.28, refer to section 2.2) is also significantly different from the search mechanism in structured P2P overlays. Unstructured P2P algorithms cannot determine the localisation of data items independently. Instead, the P2P overlay is flooded by sending a query for an IFD (corresponding to a specified service-ID) to all neighbouring peers (step 1). The neighbouring peers also forward the query to their neighbouring peers until the defined Time-To-Live (TTL) value of the search query has been reached. If a data item is found, 
the response message from the peer that stores the M2M service IFD is returned to the requesting peer via the same route (step 2).

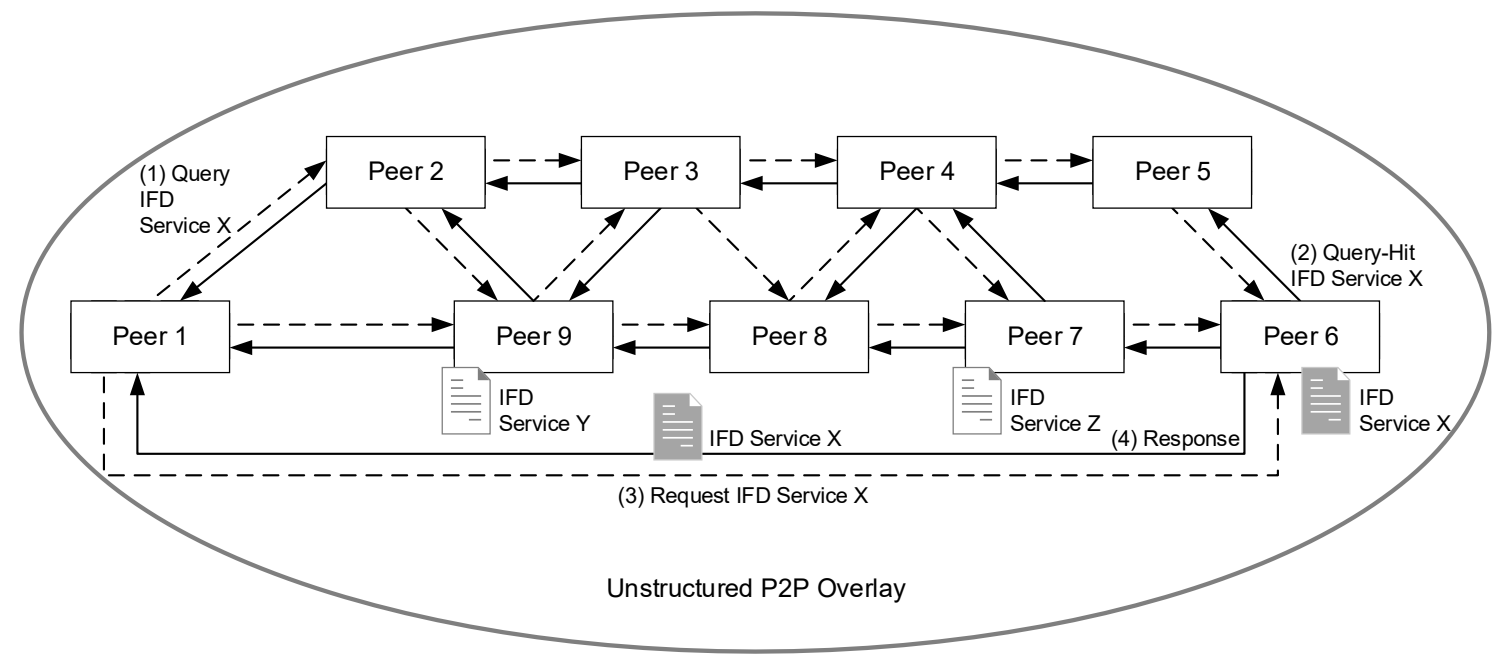

Figure 6.28: Request IFD from unstructured P2P Overlay

The query of the IFD is then made by directly information exchange between the peer that triggered the search query (step 3) and the peer storing the IFD. This then transfers the service IFD file to the requesting peer (step 4).

An unstructured P2P overlay allows complex search requests. This means that the ID of the data item does not necessarily have to be exactly known for locating data items. It may be possible, e.g. to search only for a part of the data item-ID and peers that store data items corresponding to the searched pattern would respond to the query. Furthermore, a query can be equipped with metadata in an unstructured overlay, which specifies a searched data item more detailed. Thus, the requesting peer could e.g. define filter criteria, such as accessControlPolicy or certain keywords, which specify the search for a service IFD more detailed. 
It has to be considered that unstructured P2P overlays do not guarantee reliable location of data items because the flooding mechanism of unstructured $\mathrm{P} 2 \mathrm{P}$ overlays for search requests. The TTL value of search queries defines the maximum number of hops to which the query is forwarded. If a record is not found within this range, the search request would be unsuccessful, although a suitable IFD exists.

Compared to the use of a structured P2P overlays, it is not necessary (and not possible) to have a separate list of all registered M2M services when using an unstructured $\mathrm{P} 2 \mathrm{P}$ overlay. This would be useless anyway, since it cannot be guaranteed that such a list would be found. To get an overview of all registered services and service instances, peers need to send a search query to the P2P overlay and query all existing service IFDs (e.g. via search for "Service*"). Locally, the peers then process all service IFDs (e.g. to display corresponding remote M2M Service building blocks in the GUI).

\subsubsection{Analysis of structured and unstructured Overlay Architectures}

RELOAD mentions the use of structured and unstructured P2P overlay networks for the storage of data sets, but does not make any statement about the performance and deployment scenario of different overlay algorithms. Therefore, an analysis of multiple overlay algorithms is given subsequently.

According to (Malatras, 2015), (Steinmetz and Wehrle, 2005) and (Lua et al., 2005) following common algorithms for structured P2P overlays exist: Pastry (Rowstron and Druschel, 2001), Tapestry (Zhao et al., 2006), Chord (Stoica et al., 2001), CAN (Ratnasamy et al., 2001), Kademlia (Maymounkov and Mazieres, 2002), and Viceroy (Malkhi et al., 2002). Furthermore, following common algorithm for unstructured P2P 
overlays exist: Gnutella 0.4 (Clip2, 2001), Freenet (Clarke, 2000; Freenet, 2000), FastTrack (Liang et al., 2006), and BitTorrent (Piatek et al., 2007). FastTrack and BitTorrent contain according to (Lua et al., 2005) centralised entities in their topologies such as super nodes or central trackers, which violates the requirement of avoiding central entities in the overall system architecture. Therefore, they cannot be considered as appropriate candidates to form a P2P overlay, the others are compared subsequently.

First, the essential criteria are listed below that should be considered according to (Malatras, 2015), (Lua et al., 2005), and (Steinmetz and Wehrle, 2005) for evaluation of P2P overlay algorithms.

- Lookup Performance - Defines the performance of the algorithm for routing lookup requests in the overlay (Lua et al., 2005). It specifies the efficiency for discovering the location (node) in the $\mathrm{P} 2 \mathrm{P}$ overlay where a specific data item is stored. Additionally, the Lookup Performance specifies the efficiency of determining the location where to store specific data items (Steinmetz and Wehrle, 2005).

- Churn Performance - Describes the performance of P2P overlay systems when churn or self-organisation mechanisms of the P2P overlay occurs (Lua et al., 2005). A single churn process according to (Binzenhöfer and Leibnitz, 2007) is defined as a peer joins or leaves the overlay. High churn rates represent large number of peers joining and leaving the overlay. The churn effect according to (Stutzbach and Rejaie, 2006) characterises a P2P overlay by describing the behaviour of the P2P overlay system for large number of peers that perform the 
“join-participate-leave cycle" collectively, which means that many nodes in parallel join the $\mathrm{P} 2 \mathrm{P}$ system, make a request and leave the $\mathrm{P} 2 \mathrm{P}$ system again.

- Success Guaranty - Defines if the P2P overlay algorithm can guarantee to find and retrieve a data item (if stored in the overlay).

- Fuzzy Search - Possibility to search for a data item whose name is not defined explicitly. E.g., search for a data item requesting sub-strings of data item identifier or keyword-based search queries.

Table 6.7 shows the evaluation of the structured and unstructured P2P overlays regarding the evaluation criteria defined above. The performance parameters define the complexity class into which the algorithms are assigned and represent the number of nodes involved (or messages between nodes) for specific operation or effect. The parameter "N" specifies the number of nodes within the P2P overlay system. 
Table 6.7: Evaluation P2P Algorithms acc. (Lua et al., 2005; Steinmetz and Wehrle, 2005; Malatras, 2015)

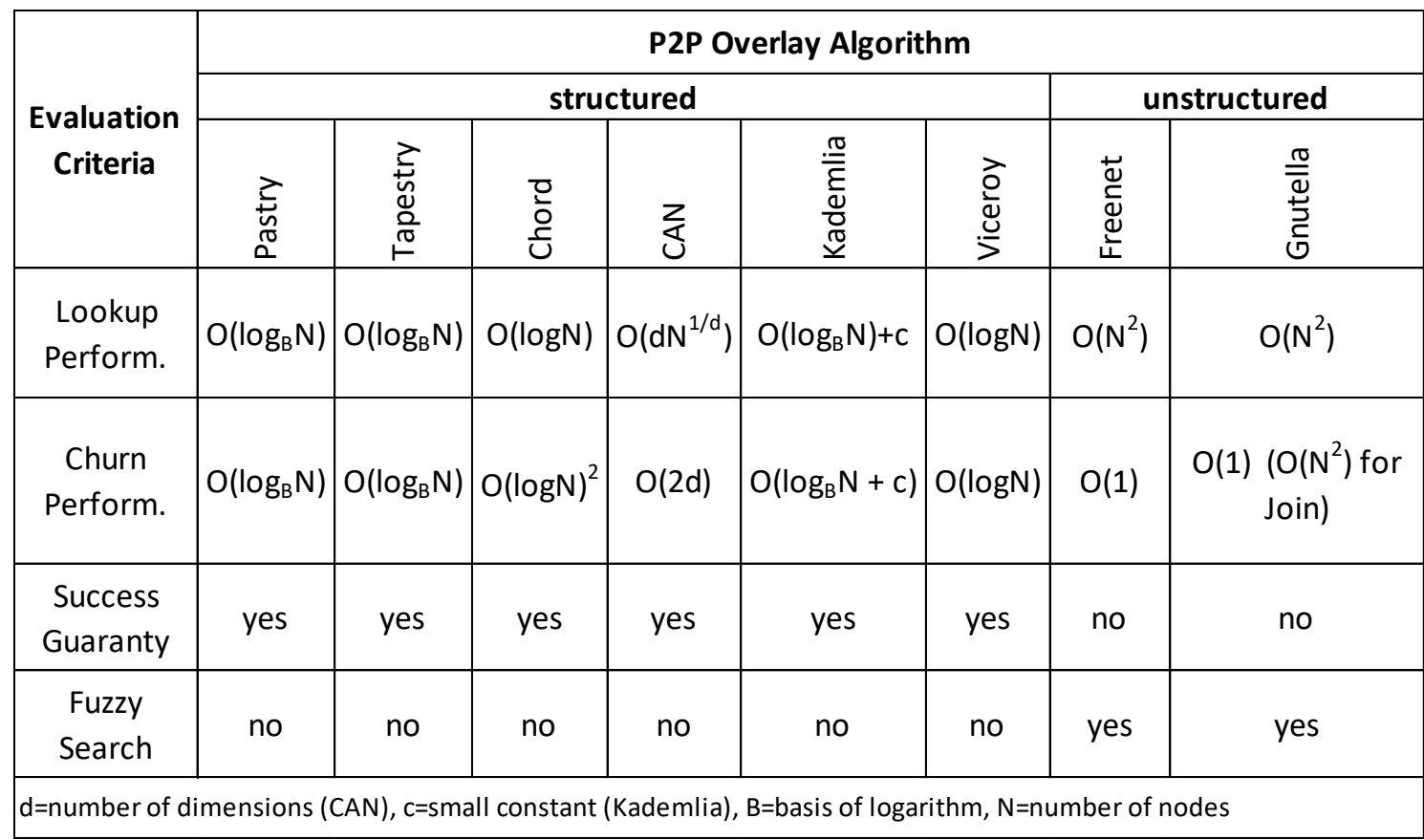

To illustrate the lookup and churn performance of different P2P overlay algorithms, Table 6.8 shows the lookup costs and Table 6.9 shows the churn costs for different P2P overlay algorithms with increasing number of peers.

Table 6.8: Lookup Costs P2P Overlay Algorithms acc. (Lua et al., 2005; Steinmetz and Wehrle, 2005; Malatras, 2015)

\begin{tabular}{|c|c|c|c|c|c|c|c|c|}
\hline \multirow{3}{*}{$\begin{array}{c}\text { Number of } \\
\text { Nodes }\end{array}$} & \multicolumn{8}{|c|}{ P2P Overlay Algorithm } \\
\hline & \multicolumn{6}{|c|}{ structured } & \multicolumn{2}{|c|}{ unstructured } \\
\hline & 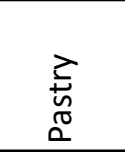 & 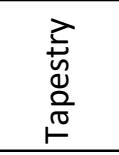 & 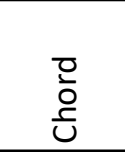 & 孞 & 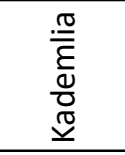 & $\begin{array}{l}\text { ò } \\
\stackrel{0}{J} \\
j\end{array}$ & $\begin{array}{l}+\overleftarrow{d} \\
\frac{c}{d} \\
\frac{1}{4} \\
\end{array}$ & $\begin{array}{l}\stackrel{0}{\overline{0}} \\
\stackrel{ \pm}{J} \\
\stackrel{5}{0}\end{array}$ \\
\hline 5 & 0,6989 & 0,6989 & 0,6989 & 5,1299 & 0,2902 & 0,6989 & 25 & 25 \\
\hline 10 & 1 & 1 & 1 & 6,4633 & 0,4152 & 1 & 100 & 100 \\
\hline 25 & 1,3979 & 1,3979 & 1,3979 & 8,772 & 0,5804 & 1,3979 & 625 & 625 \\
\hline 50 & 1,6989 & 1,6989 & 1,6989 & 11,052 & 0,7054 & 1,6989 & 2500 & 2500 \\
\hline 100 & 2 & 2 & 2 & 13,9247 & 0,8304 & 2 & 10.000 & 10.000 \\
\hline 250 & 2,3979 & 2,3979 & 2,3979 & 18,8988 & 0,9957 & 2,3979 & 62.500 & 62.500 \\
\hline 500 & 2,6989 & 2,6989 & 2,6989 & 23,811 & 1,1207 & 2,6989 & 250.000 & 250.000 \\
\hline 1000 & 3 & 3 & 3 & 30 & 1,2457 & 3 & $1 \mathrm{~m}$ & $1 \mathrm{~m}$ \\
\hline \multicolumn{9}{|l|}{$\mathrm{m}=$ million } \\
\hline
\end{tabular}


6 Cooperative M2M Application Service Provision

Table 6.9: Churn Costs of P2P Overlay Algorithms acc. (Lua et al., 2005; Steinmetz and Wehrle, 2005; Malatras, 2015)

\begin{tabular}{|c|c|c|c|c|c|c|c|c|}
\hline \multirow{3}{*}{$\begin{array}{c}\text { Number of } \\
\text { Nodes }\end{array}$} & \multicolumn{8}{|c|}{ P2P Overlay Algorithm } \\
\hline & \multicolumn{6}{|c|}{ structured } & \multicolumn{2}{|c|}{ unstructured } \\
\hline & 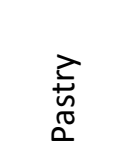 & 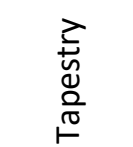 & $\begin{array}{l}\frac{0}{0} \\
\frac{0}{U}\end{array}$ & 过 & 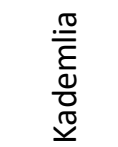 & $\begin{array}{l}\text { তे } \\
\frac{0}{\square} \\
\stackrel{\nu}{>}\end{array}$ & 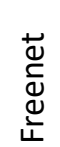 & $\begin{array}{l}\stackrel{0}{\bar{V}} \\
\mathbb{U} \\
\bar{E}\end{array}$ \\
\hline 5 & 0,6989 & 0,6989 & 0,4885 & 6 & 0,2902 & 0,6989 & 1 & $1(25)$ \\
\hline 10 & 1 & 1 & 1 & 6 & 0,4152 & 1 & 1 & $1(100)$ \\
\hline 25 & 1,3979 & 1,3979 & 1,9542 & 6 & 0,5804 & 1,3979 & 1 & $1(625)$ \\
\hline 50 & 1,6989 & 1,6989 & 2,8864 & 6 & 0,7054 & 1,6989 & 1 & $1(2500)$ \\
\hline 100 & 2 & 2 & 4 & 6 & 0,8304 & 2 & 1 & $1(10.000)$ \\
\hline 250 & 2,3979 & 2,3979 & 5,7501 & 6 & 0,9957 & 2,3979 & 1 & $1(62.500)$ \\
\hline 500 & 2,6989 & 2,6989 & 7,2844 & 6 & 1,1207 & 2,6989 & 1 & $1(250.000)$ \\
\hline 1000 & 3 & 3 & 9 & 6 & 1,2457 & 3 & 1 & $1(1.000 .000)$ \\
\hline
\end{tabular}

Considering the lookup costs for structured P2P overlays (see Figure 6.29), the effort involved in the use of the CAN algorithm is noticeably higher compared to the other structured P2P overlay algorithms. Therefore, Figure 6.30 shows the comparison of the lookup costs without CAN again.

Lookup Costs Comparison Structured P2P Overlay

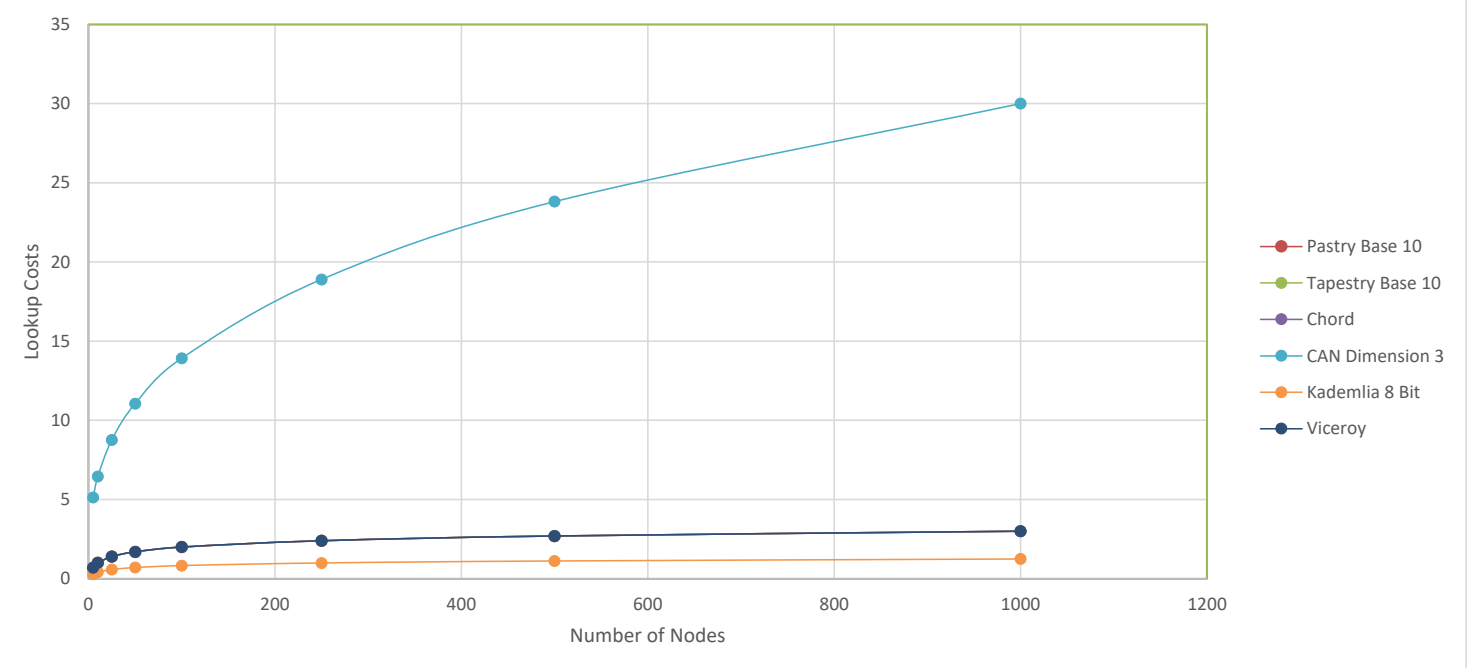

Figure 6.29: Comparison Lookup Costs structured P2P Overlays 
Lookup Costs Comparison Structured P2P Overlay (excl. CAN)

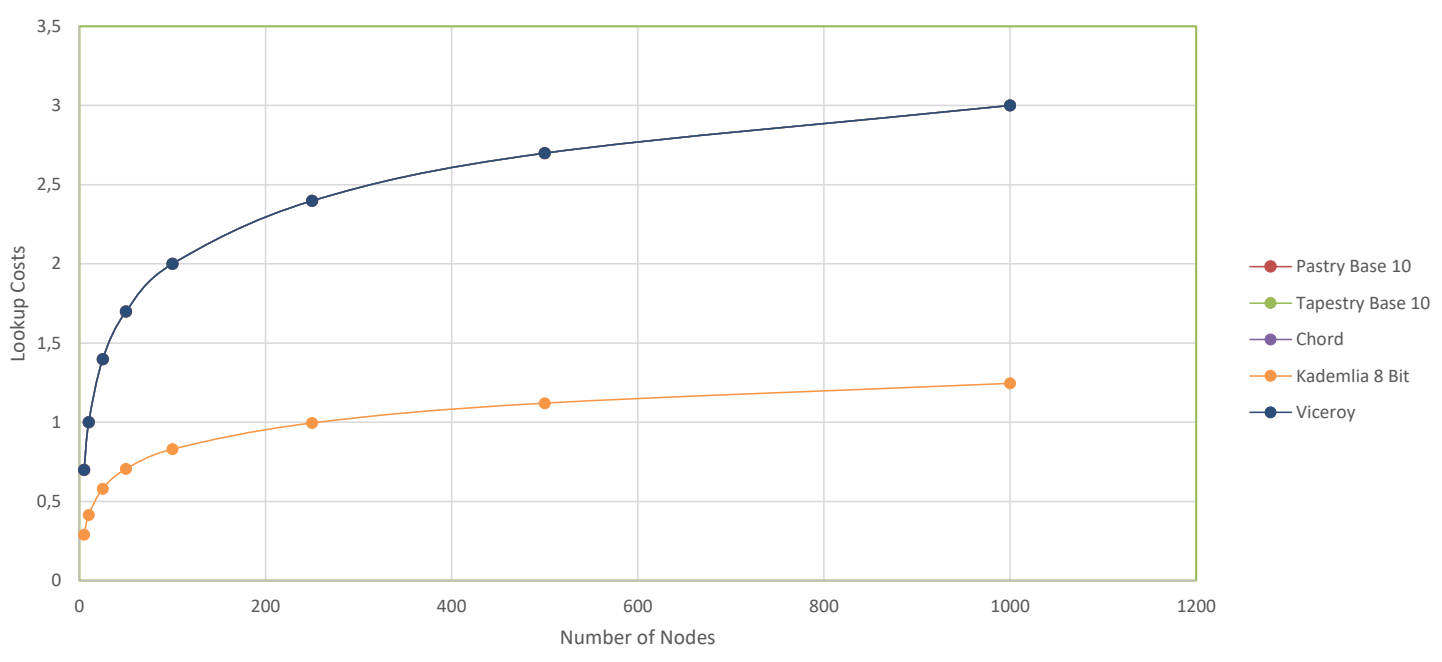

Figure 6.30: Comparison Lookup Costs structured P2P Overlays (excl. CAN)

Considering the lookup costs for structured P2P overlays without the CAN algorithm it shows that Kademlia requires half as much effort for locating a data item as the other algorithms.

Figure 6.31 illustrates the effort for search queries in unstructured P2P overlays.

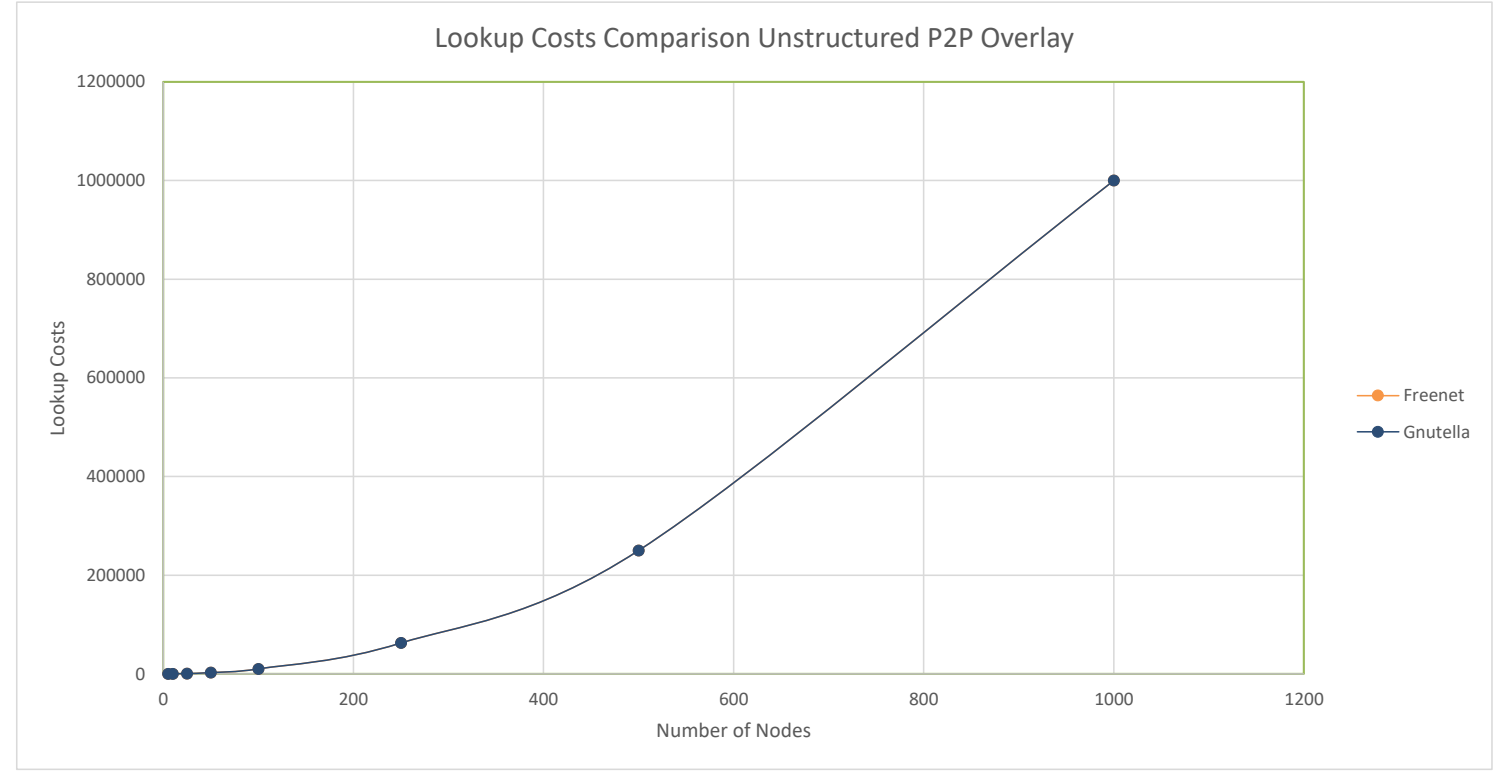

Figure 6.31: Comparison Lookup Costs unstructured P2P Overlays 
Unstructured P2P algorithms use a flooding mechanism when nodes sending search queries for data items to their neighbours (see section 2.2). This results according to (Steinmetz and Wehrle, 2005) in a massive effort of greater than $\mathrm{O}\left(\mathrm{N}^{2}\right)$.

Figure 6.32 illustrates the churn costs of structured P2P overlay algorithm networks as specified in Table 6.7 .

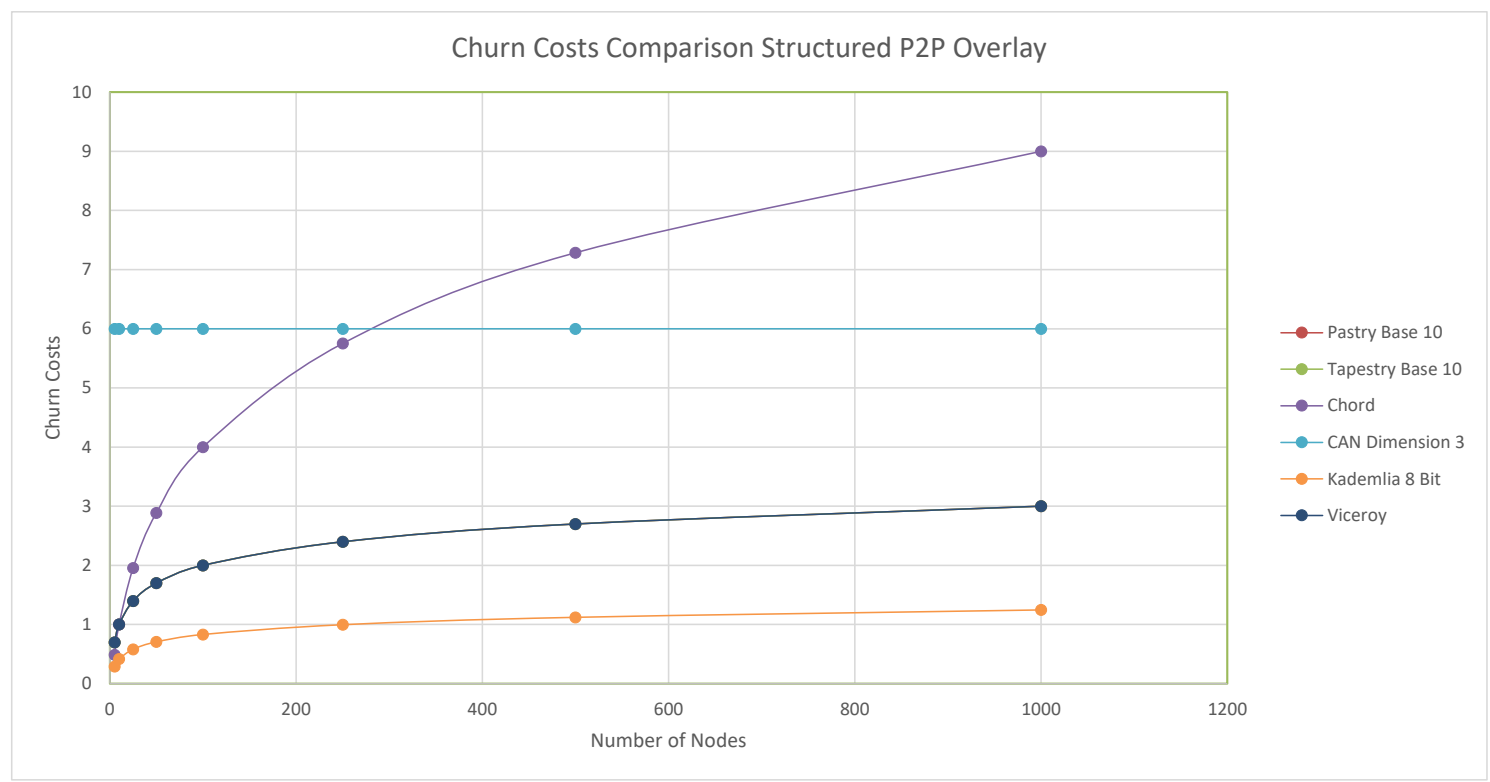

Figure 6.32: Comparison Churn Costs structured P2P Overlays

It can be seen that the churn performance is the best for Kademlia, and Chord has the worst churn performance. 
Figure 6.33 illustrates the churn costs for unstructured P2P overlays.

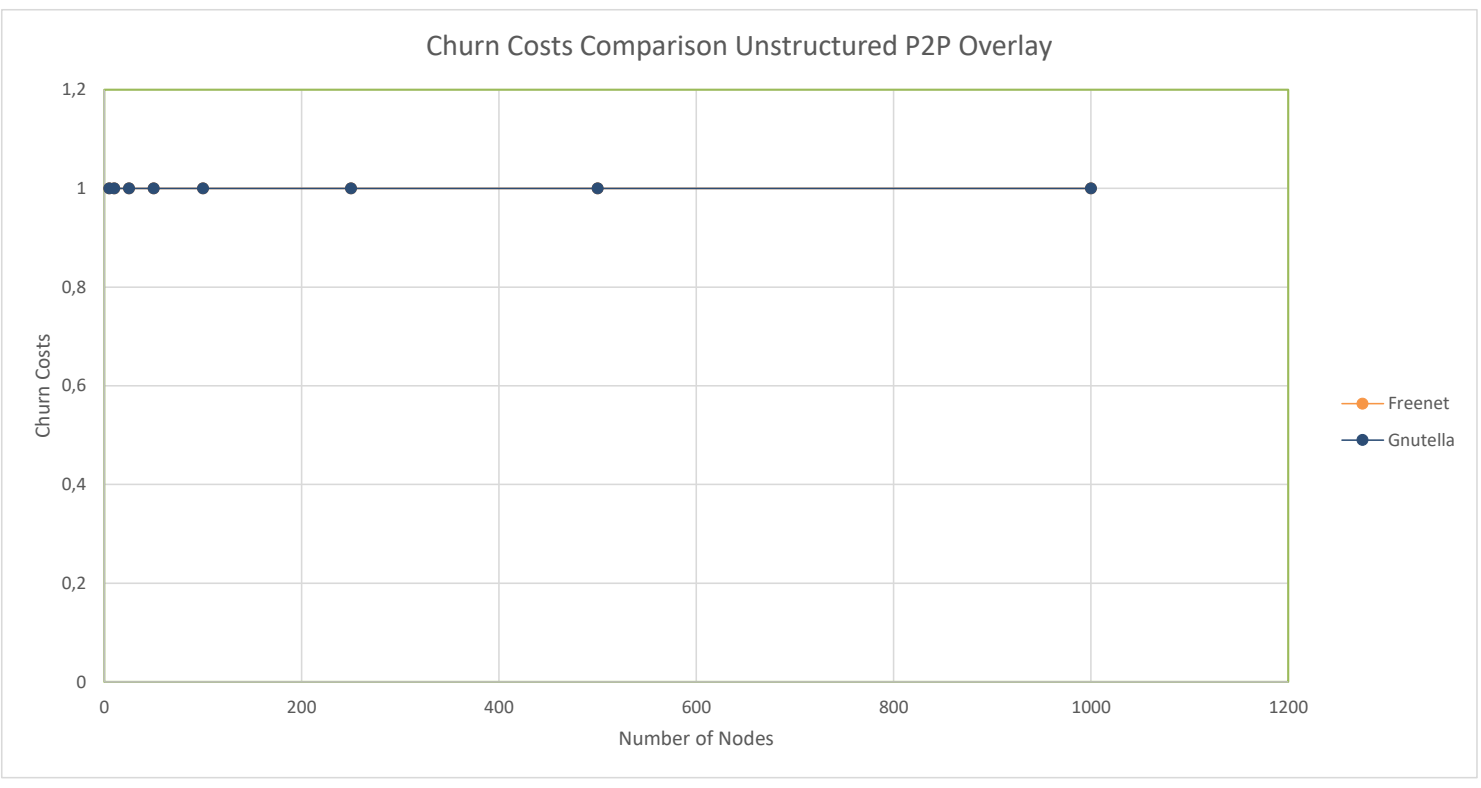

Figure 6.33: Comparison Churn Costs unstructured P2P Overlays

The churn costs of unstructured P2P overlays are equal and constant according (Steinmetz and Wehrle, 2005) O(1). This is because the nodes only connect to their direct neighbours and only manage their own records. The topology of the overlay does not need to be restructured when a new node connects to the overlay or leaves it.

Derived from (Malatras, 2015) the effort when entering into a Gnutella overlay should be $\mathrm{O}\left(\mathrm{N}^{2}\right)$ and when leaving the effort stays at $\mathrm{O}(1)$. This is because the fact that a node, after entering a Gnutella overlay, performs a so-called discovery operation. Such a discovery operation (node lookup by PING, PONG message) is performed to identify other nodes when joining the overlay. The discovery operation that uses flooding mechanism is initiated periodically and therefore the costs should be set to $\mathrm{O}\left(\mathrm{N}^{2}\right)$ (same as for lookup costs). The Freenet algorithm does not perform any operation when nodes join the overlay, but nodes only connect to their identified neighbours. 
In a direct comparison of structured and unstructured $\mathrm{P} 2 \mathrm{P}$ overlays with respect to the lookup costs (illustrated in Figure 6.34) it becomes clear that in an unstructured P2P overlay there is a massive increase of effort for searching data items. This is especially important when many search queries are directed to the P2P overlay. Structured P2P overlay algorithms remain in the complexity class $\mathrm{O}(\log \mathrm{N})$ for the search of data items. Thus, if many search requests are sent to a P2P overlay, a structured P2P overlay has the advantage that the load on the entire overlay is significantly lower in terms of number of messages exchanged between the nodes, but also in terms of the load on the involved peers. In the case of unstructured P2P networks, all peers that pass the search queries in the context of the flooding process are integrated into the communication. The same peers are involved to route the corresponding response message back to the requesting peer.

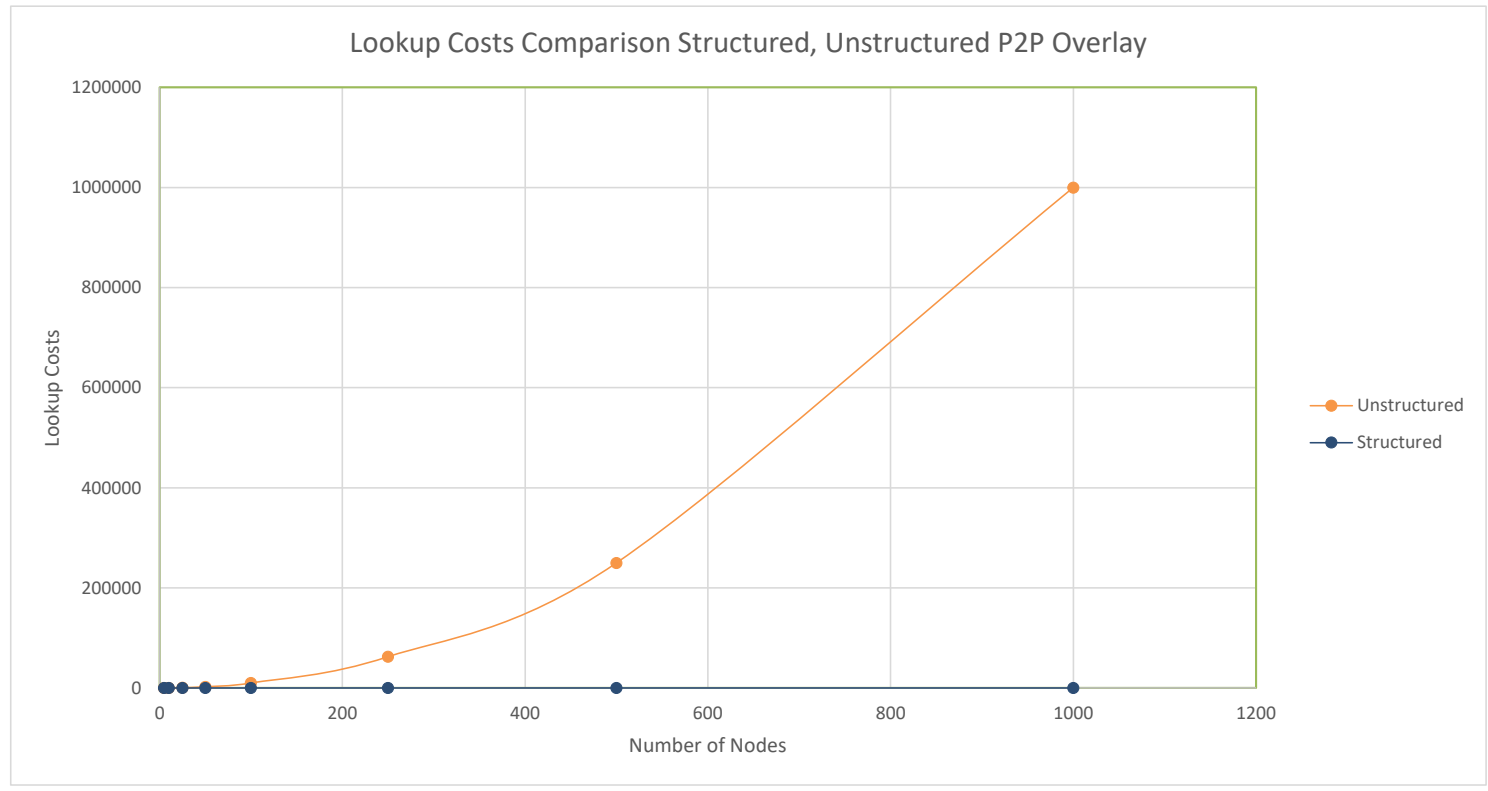

Figure 6.34: Comparison Lookup Costs structured and unstructured P2P Overlays

Comparing the churn costs, it can be identified, that structured $\mathrm{P} 2 \mathrm{P}$ overlay algorithms have $\log$ arithmic costs, $\mathrm{O}(\log \mathrm{N})$ for churn (illustrated in Figure 6.35) and unstructured P2P overlays have cost of $\mathrm{O}(1)$. If one considers the additional costs of structured P2P 
overlays compared to unstructured P2P overlays, it can be seen that the increase of the overhead is around 2-3 nodes. The small additional cost should not be relevant in the evaluation of efficiency.

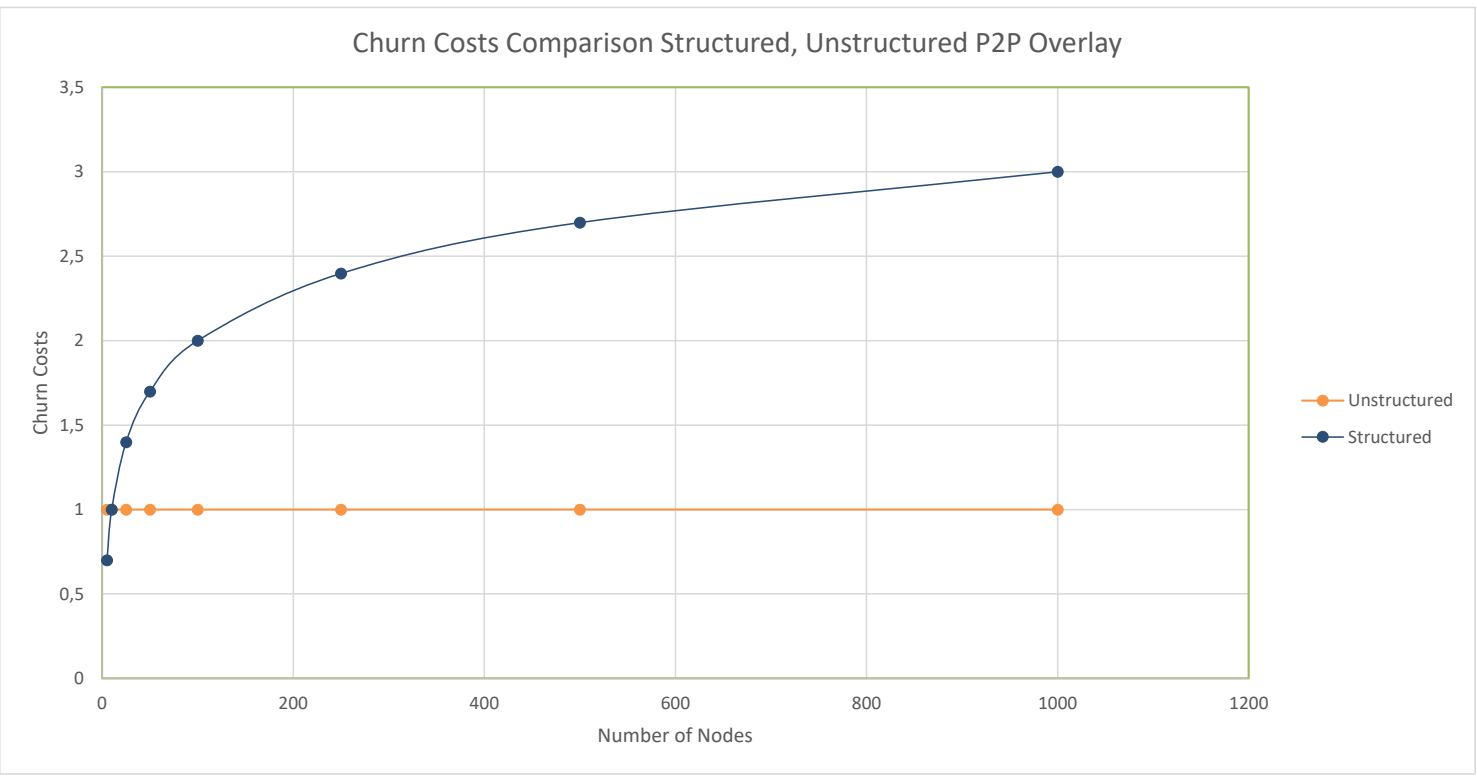

Figure 6.35: Comparison Churn Costs structured and unstructured P2P Overlays

Although the effect for churn in structured P2P overlays is only a little higher, (Steinmetz and Wehrle, 2005) states that unstructured P2P overlays are for better use in mobile networks with large movement of nodes. This is due to the fact that if peers often join or leave the P2P overlay, the load on the overlay is significantly higher with a structured overlay because the churn costs are aggregated. With an unstructured overlay, on the other hand, the effort for connecting and leaving is constantly low. Furthermore, according to (Binzenhöfer and Leibnitz, 2007) high churn rates in structured P2P overlays can also cause routing errors or destroy the overlay. Therefore, an unstructured P2P overlay is more advantageous at high churn rates. 
When using a P2P overlay to manage the IFDs it is important that the data records can be located. This is the case in an unstructured overlay because it guarantees that a record is found. With an unstructured overlay, on the other hand, this cannot be guaranteed, since due to the limited forwarding, it is not guaranteed that the search query will also reach the peer who stores the IFD. Therefore, structured overlays are preferable for this scenario.

Structured overlays enable peers not to store the IFD themselves, but only a reference to the data set. Nodes storing an IFD could therefore be temporarily unavailable. The peer who requested the reference to the IFD could contact the unavailable node at a later time to request the IFD without having to request the reference to the IFD again via the overlay. If, on the other hand, in an unstructured overlay, a node storing the IFD is temporarily unavailable, then a search query has to be sent again via the overlay, which would again stress the network. In an unstructured overlay, the information about offered services is therefore only available if the peers offering the service are also reachable.

In structured P2P overlays the ID of the IFD must have been uniquely determined when querying it. Therefore, a search request can only be successful if the service-ID of the IFD is known. In an unstructured P2P overlay, however, the search does not need to be based on a specific key. This makes it possible to search IFDs whose exact service-ID are unknown during search requests. Furthermore, in an unstructured P2P overlay, searching for a set of IFDs is possible, such as all services belonging to a specific group and contains specific keywords in their names. These types of search queries can only be realised with unstructured P2P overlays. 
Table 6.10 summarises the comparison of structured and unstructured P2P overlays in terms of their performance and structure-related search properties.

Table 6.10: Summary Evaluation of structured and unstructured P2P Overlay Algorithms

\begin{tabular}{|l|c|c|}
\hline & Structured P2P Overlay & Untructured P2P Overlay \\
\hline Many search requests & + & + \\
\hline Large churn rates & 0 & 0 \\
\hline Success guaranty required & + & + \\
\hline Complex data search required & - & \\
\hline +: advantageous; o: moderate; -: disadvantageous
\end{tabular}

As a summary result of the evaluation, it can be stated that because the massive contrasting performance behaviour and properties of the P2P overlay algorithms the choice of an adequate $\mathrm{P} 2 \mathrm{P}$ algorithm depends on the application scenarios of the framework. Nevertheless, since locating an IFD should be ensured, the use of a structured overlay is preferable. The significantly lower effort for a search query also argues for the use of a structured overlay.

\subsection{Cooperative M2M Application Service Provision}

In section 4.1, the definition of the Decentralised Cooperative M2M Application Service Provision (DCASP) model introduced the approach that in the framework for the provision of $\mathrm{M} 2 \mathrm{M}$ applications defined in this research, different end-users can combine their individually offered services and thus act as a cooperative M2M ASP for other endusers or organisations/companies. 
Section 6.4.1 describes the principles of cooperative M2M application service provision as well as the differences to the pure integration of remote M2M services into local M2M applications (refer to section 6.1.3). Section 6.4.2 describes the basics of the automatic configuration process of distributed $\mathrm{M} 2 \mathrm{M}$ applications required for cooperative application provision. Section 6.4 .2 describes the process how a distributed cooperative $\mathrm{M} 2 \mathrm{M}$ application is executed. Section 6.4 .3 deals with invalid configurations that may occur during the configuration process of a distributed M2M application even though the application has been correctly configured from the perspective of individual peers. For this purpose, an algorithm is introduced that enables each peer to independently detect and resolve invalid application configurations.

\subsubsection{Principles of cooperative M2M Application Service Provision}

In cooperative (distributed) application provisioning, the M2M services of different endusers are combined to form a complex distributed M2M application by connecting the output parameters of M2M services to the input parameters of the other M2M services. The design and formal description of such an application is done by defining an SMbased application model (refer to section 6.1.3), but instead of combining remote M2M services with local M2M devices/MMSCs, here remote M2M services are combined with each other. The services are linked by information-processing peers independently requesting the output parameters from the service-providing peers through a request message (refer to section 6.1.3). This enables the realisation of completely decentralised M2M application solutions consisting of individual distributed M2M services without the need for a central coordinator or a central MSP to manage the linking of the services. 
While the mechanism of requesting a service from another peer is the same as described in section 6.1.3, there is a difference in the architecture of the applications. To illustrate the difference, Figure 6.36 shows the principle to integrate a remote M2M service in a local application. The service request is only executed by a (local) M2M platform, and all returned information (data) is processed by this platform. The SM generated from the formal application description is thus executed and coordinated locally.

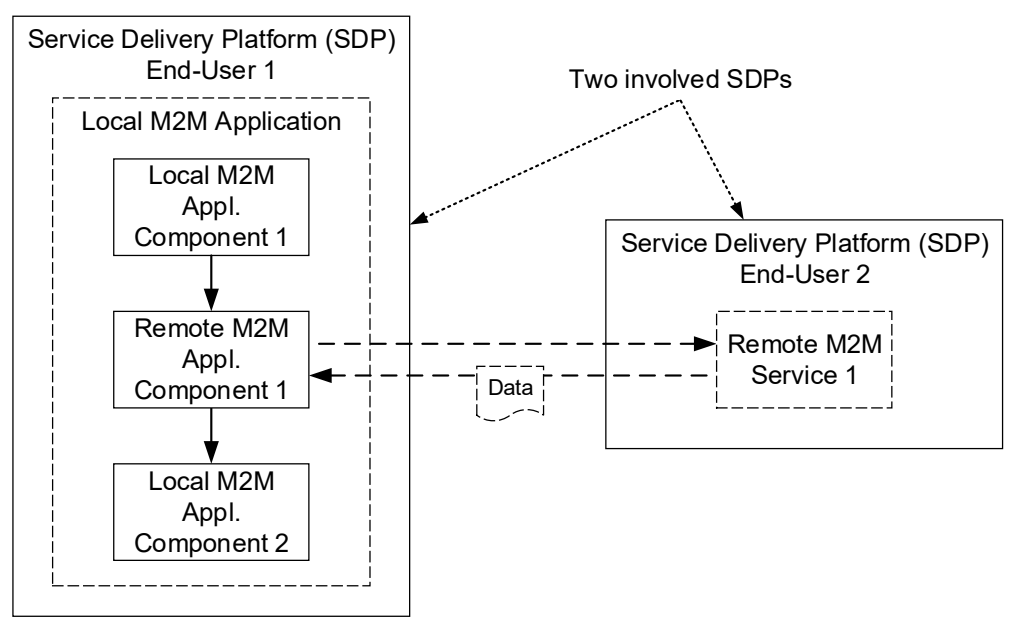

Figure 6.36: Architecture of remote M2M Service Integration

The principle of remote M2M service combinations is illustrated in Figure 6.37. The difference to the application architecture shown in Figure 6.36 is that the services pass the information among themselves, thus providing a concatenation of different services instead of the pure integration of remote M2M services into local M2M applications.

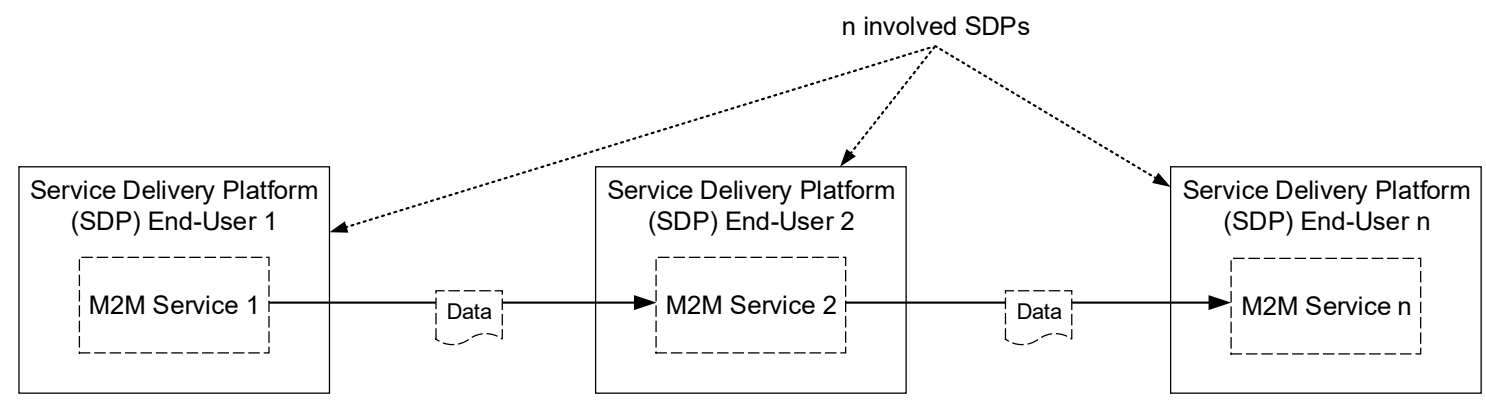

Figure 6.37: Architecture of remote M2M Service Combinations 
When concatenating services, the involved M2M services provide their information to other M2M SPs, which process the information and, if necessary, provide information to another M2M SP. There is no instance of a SM that is executed or coordinated locally in one single platform, but the different distributed peers realise the distributed SM logic.

As described in section 4.1, two variants have been introduced according to (Steinheimer et al., 2015a) for the cooperative M2M application service provision (illustrated in Figure $6.38)$.

M2M Service 1
End User 1

a) M2M Service Composition

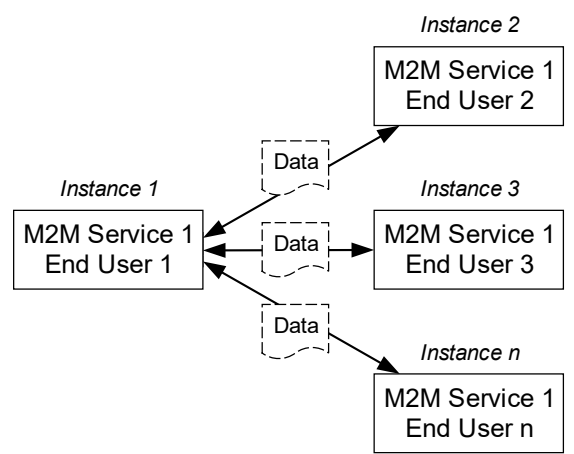

b) M2M Service Aggregation

Figure 6.38: Variants of cooperative M2M Service Combinations

The first option is the previously described composition of M2M services (Figure 6.38a). The second option is service aggregation (Figure 6.38-b). In this case, end-users can each offer the same service (different service instances) and exchange information among themselves for this one service.

Figure 6.39 shows the exchange of messages for the variant of M2M service compositions, respectively the service requests for the combination of services that realise a service chain. In this case, the services/M2M platforms subscribe with the preceding service in the service chain, since the services should consume the information of that preceding service. 


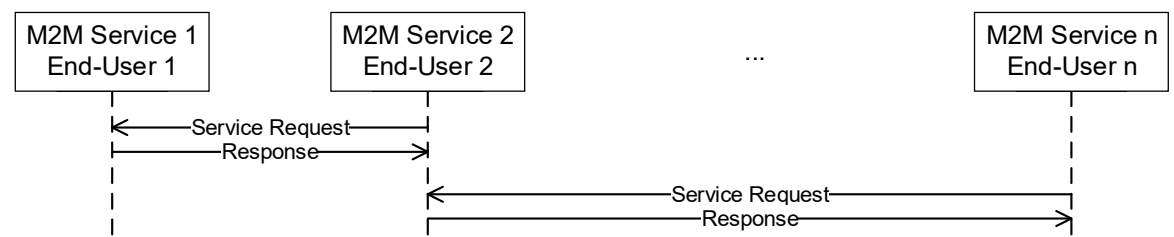

Figure 6.39: M2M Service Composition Message Exchange

Figure 6.40 shows the message exchange for the variant of M2M service aggregations. Since all services require the information of the other services, each service subscribes with the other services to obtain their data information.

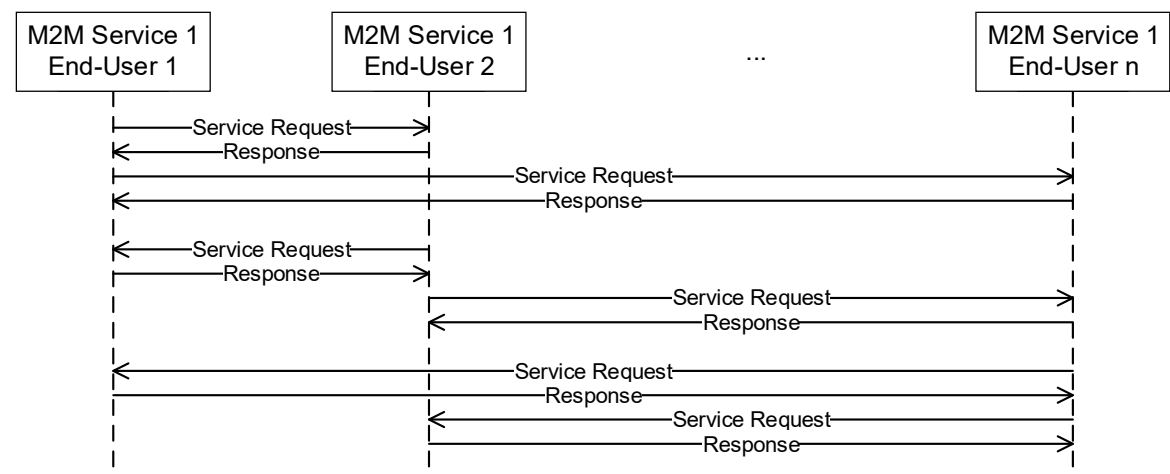

Figure 6.40: M2M Service Aggregation Message Exchange

The semantics for modelling a service composition have already been defined (linking services by defining a transition from a state representing the service to another state that represents the service to receive the information, refer to section 5.5). No corresponding semantics have yet been defined for service aggregation. Therefore, it is defined at this point that a service aggregation is described by the fact that a state receives a reference to itself, i.e. as the target of the transition, the same service-ID is specified as for the state from which the transition originates. In the case of a service composition, the peer who wants to use the corresponding service requests only one instance of a service. In the case of a service aggregation, however, all instances of the same service are requested and information about the entire application is exchanged with these instances. 
To illustrate the above-described variants of the cooperative M2M application service provision, two examples are described below. These examples correspond to Use Case 3 (Building Surveillance) and Use Case 4 (Energy Optimisation) described in section 2.4.

Figure 6.41 illustrates the cooperative M2M application service described by Use Case 3 as representation of an $\mathrm{M} 2 \mathrm{M}$ service composition.

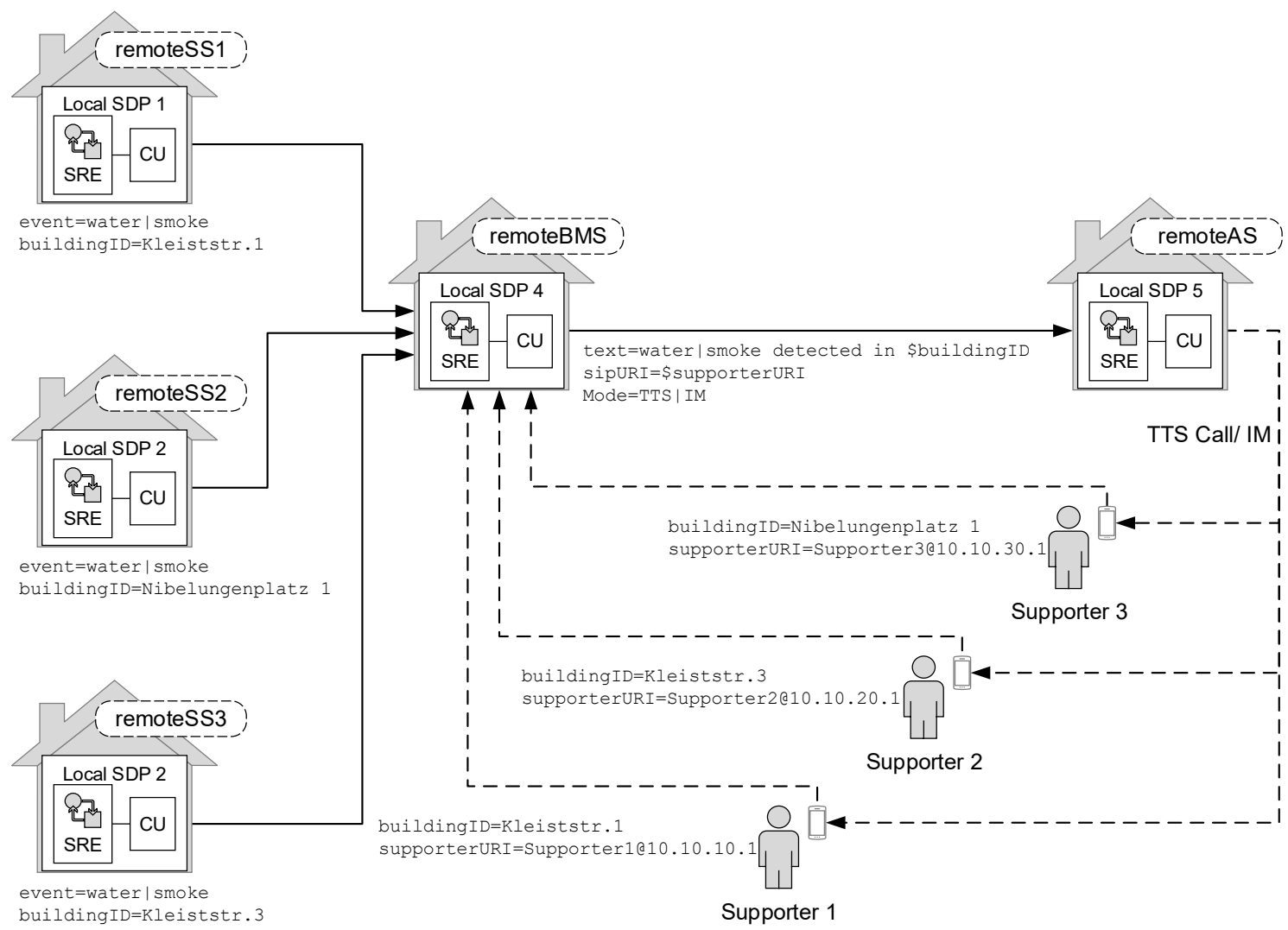

Figure 6.41: Representation of Use Case 3 (Building Surveillance)

Figure 6.41 shows the information exchange between the participating M2M services and end-users. The distributed M2M application consists of a total of five M2M services, each of which is provided by different peers. The following describes the functionality of the individual services that are integrated into the service composition and thus represent the functionality of the M2M application. 
- Remote Sensor Service (remoteSS) 1-3 - RemoteSS (1-3) are each different services which have similar functionality. They read the status of local sensors (water sensor and smoke detector). If one of the two sensors has the value "water" (indicates water detected) or "smoke" (indicates smoke detected), the service generates an event and forwards it to the Remote Building Monitoring Service (remoteBMS) together with the building-ID of the respective building. RemoteSS (1-3) differ only by the assigned building-ID at their output interfaces and thus monitor the status of different buildings.

- Remote Building Monitoring Service (remoteBMS) - RemoteBMS provides the functionality to aggregate the monitoring of different buildings and to manage the supporters associated with the buildings. The remoteBMS receives the event that has occurred in a building and determines the supporter responsible for this building. Depending on the event, the remoteBMS triggers a Remote Alarm Service (remoteAS). By the transmitted information, the latter is instructed to inform the registered supporter about the event, either by Text-to-Speech (TTS) call (in case of smoke detected) or by IM (in case of water detected).

- Remote Alarm Service (remoteAS) - The remoteAS provides the functionality to send an IM or to initiate a call and announce a text. This service expects as input parameters the destination SIP URI to which the message should be sent and the text which should be contained in the IM or which should be announced. Which of the two notifications to select is defined by the input parameter "mode". If "mode" parameter has the value "IM", an IM is generated. If "mode" parameter has the value "TTS", a TTS call is initiated. 
The supporters offer the service to take care of a specific building. This service is only loosely coupled to the M2M application service by not being directly part of the application, but only using the M2M application service functionality to provide the service. To utilise the Building Surveillance application, the supporter registers with the remoteBMS. During this registration, the supporters will tell the remoteBMS which building they are supporting and what is their contact information.

Because the flexibility of the introduced formal description language, multiple variants are possible to realise the formal application description (AD) for the scenario of Use Case 3:

- Separate ADs per Building - Definition of an individual AD for each building to monitor. Here the first state corresponds to the remoteSS of the building to monitor.

- One AD for all Buildings - Define one AD for all buildings to monitor. In this case, the first state of the AD can be modelled as parallel state element containing one section per remoteSS of the buildings to monitor.

The first variant (separate ADs) has been selected to show an extract of the formal AD (see Figure 6.42) represented as Statechart, since it has the advantage that an already existing $\mathrm{AD}$ could be reused by simple modifying the parameters corresponding to the building in focus. Listing C.10 illustrates the corresponding SCXML pattern. In the second variant, the structure of the AD would have to be customised by adding new states in the parallel state element and additionally does not hide other monitored buildings in the AD. 


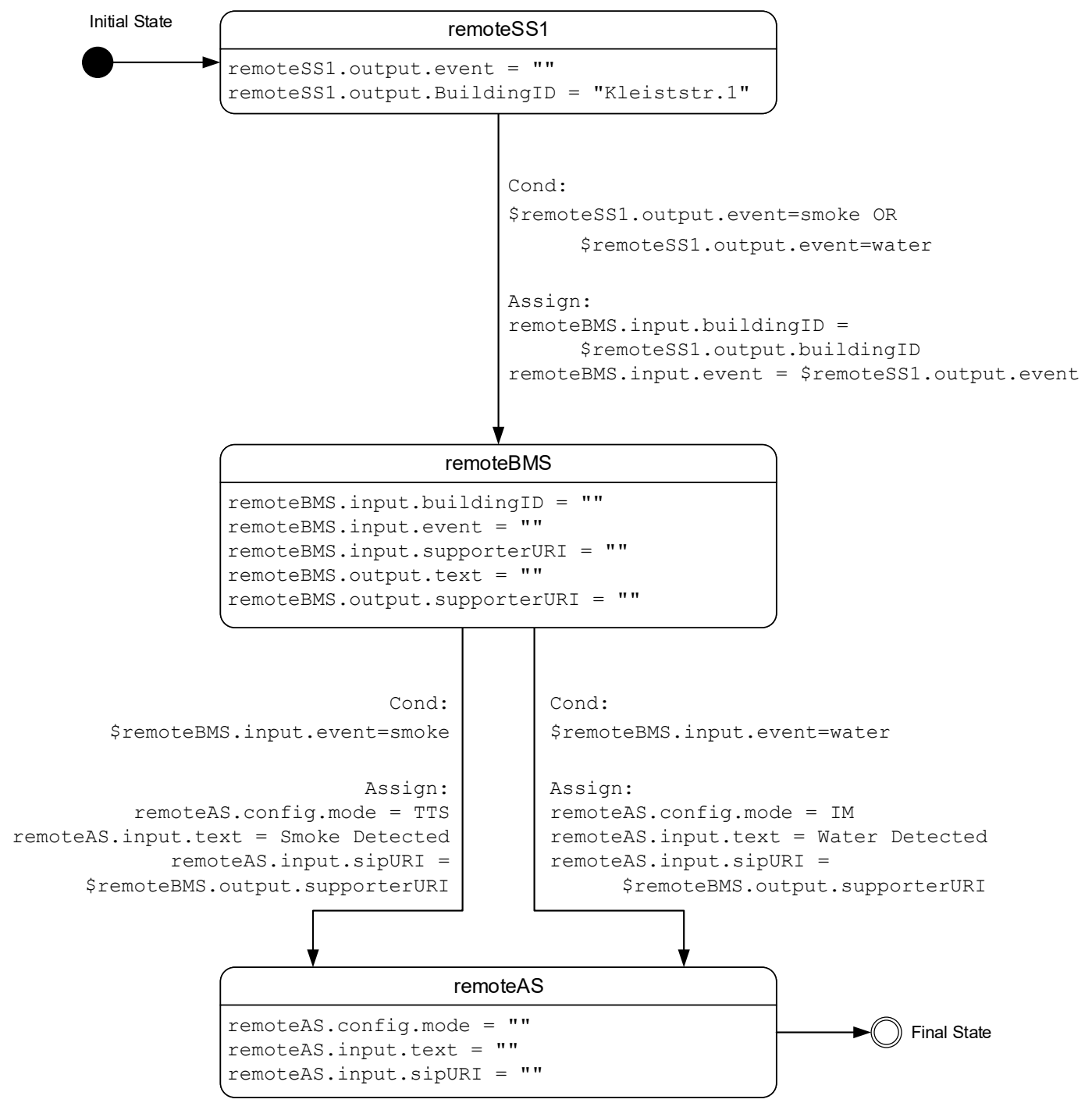

Figure 6.42: Statechart Representation AD of cooperative M2M Application Service for Use Case 3

Figure 6.43 illustrates the cooperative M2M application service described by Use Case 4 as representation of an $\mathrm{M} 2 \mathrm{M}$ service aggregation. The distributed application consists of two services. The remote Distribution Grid Parameter Provision Service (remoteDGPPS) is only present as a single instance in the example shown below. RemoteDGPPS is loosely coupled to the M2M application service by not being directly part of the application, since it requests the cooperative $\mathrm{M} 2 \mathrm{M}$ application service functionality as a service and does not actively participate in application execution. 


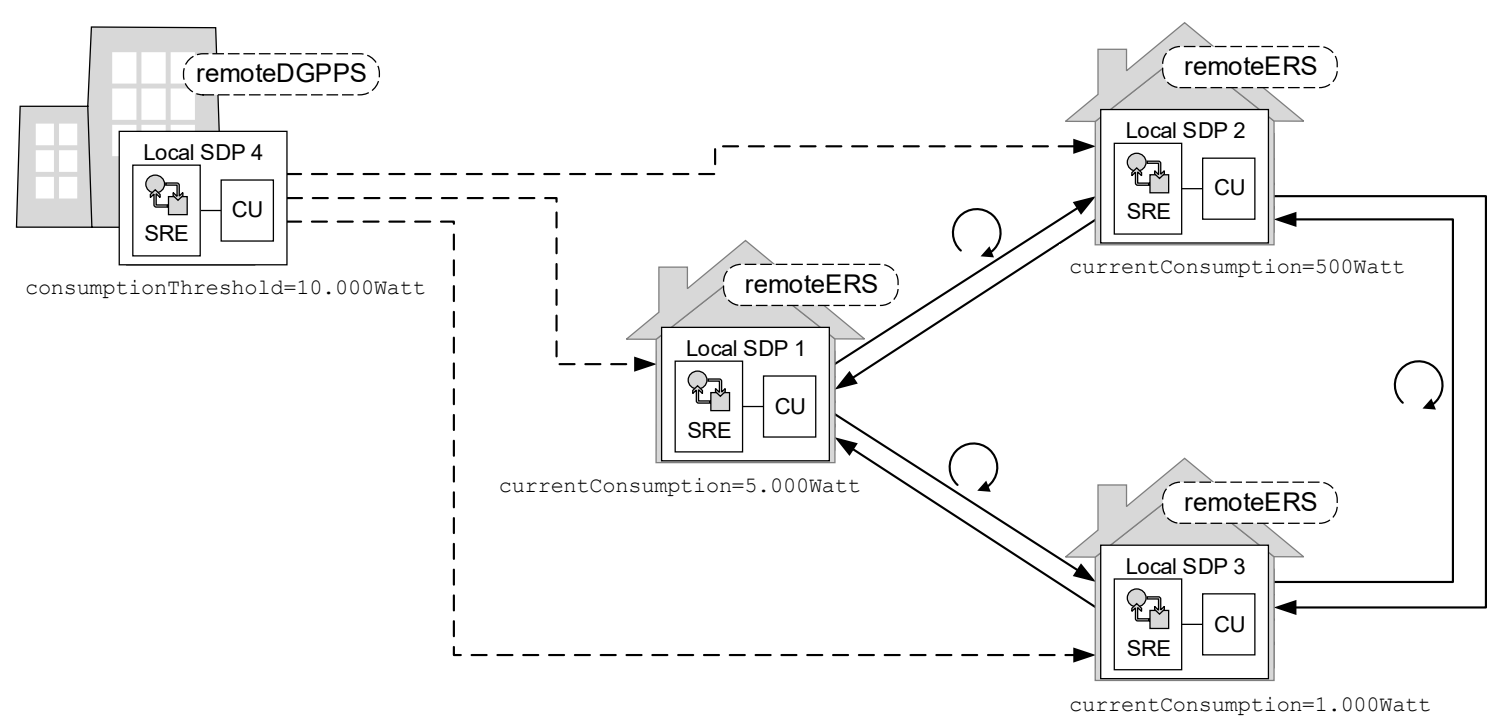

Figure 6.43: Representation of Use Case 4 (Energy Optimisation)

The remote Energy Reduction Service (remoteERS) has multiple instances, each of which is implemented by a different peer. Subsequently the functionality of these two services is described that represent the functionality of the total cooperative M2M application service. Figure 6.44 shows an extract of the corresponding formal AD of Use Case 4 represented as Statechart. Listing C.11 illustrates the corresponding SCXML pattern.

- Remote Distribution Grid Parameter Provision Service (remoteDGPPS) - The remoteDGPPS provides a limit value for the maximum peak load in a distribution grid via its output interface. This information the remoteDGPPS distributes to all instances of the service described below.

- Remote Energy Reduction Service (remoteERS) - The remoteERS offers the functionality to reduce the local energy consumption. The service receives the defined upper limit for the maximum peak load via its input interface (remoteERS.input.consumptionThreshold). Furthermore, the remoteERS calculates the local energy consumption. The service sends the calculated energy 
consumption to all other instances of the service. The other instances of the service perform the same and send their energy consumption back to all other instances. The energy consumption of the other instances of the service is received via a separate parameter of the service interface (remoteERS.input.remoteConsumption). The remoteERS calculates the total consumption of all participating service instances from the received consumption values. The service compares the calculated total consumption with the maximum peak load value obtained from the remoteDGPPS. If the limit is exceeded by the sum of all consumptions, the service reduces the local consumption (if possible). The remoteERS then redistributes the new calculated energy consumption to all other instances of the service.

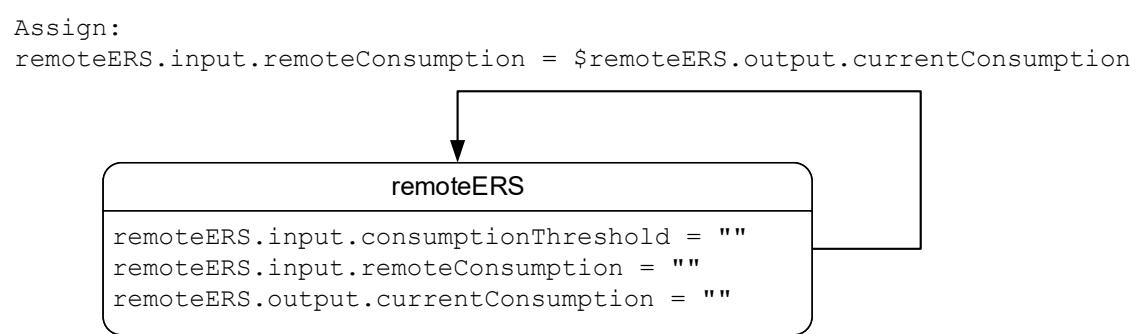

Figure 6.44: Statechart Representation AD of cooperative M2M Application Service for Use Case 4

For being able to use a cooperative $\mathrm{M} 2 \mathrm{M}$ application service an interface must also be defined for this purpose. As is apparent from the examples above, the interface to a cooperative M2M application service does not necessarily have to be the first service in the service combination. The interface that must be served for service utilisation can also be provided by a service that is located within the service chain. Therefore, additionally to the derivation of the IFD in section 6.1.1, it is defined that an interface to an M2M application service can also be specified addressing interface parameter of a service within the service combination. Additionally, an interface does not have to contain all 
interface parameters that the service to be addressed has. Therefore, it is further defined that the IFD of a cooperative M2M application service can only contain a subset of the interface parameters of all the services involved.

\subsubsection{Cooperative M2M Application Configuration and Execution Phase}

The application configuration phase has been designed as an automated and autonomous configuration process of the distributed M2M application (Steinheimer et al., 2017a). This configuration process is preceded of the actual application execution to connect the specific instances of the services involved. The connection of the services to each other represents the modelled SM and thus defines the exchange of information between the services.

The connections between the individual peers, respectively interconnection of the services, cannot be coordinated centrally since no central entities may be included in the M2M service architecture according to the requirements specification. This means that no central entity exists which directs the peers to which specific instances of a service they should connect to realise a distributed SM. Therefore, a mechanism is required enabling the peers to integrate themselves into the service chain. This means that the peers must independently determine the instances of the services with which they should connect and forward the information according to the application logic to the service succeeding to them (defined as transition between M2M services). 
Following the approach of processing a SM, the decision to forward information to the requesting service, i.e. to send a corresponding response message to the requesting peer (if defined as a transition condition), must be made in the service providing the information. For this purpose, the introduced concept defines that the distributed M2M application is initially configured (configuration phase), i.e. the services are linked together, before the actual application logic is executed (execution phase).

To enable peers to configure their individual parts of the application during the configuration phase independently, they need corresponding information defined in the formal AD. Peers can determine this information by automatically parsing the $\mathrm{AD}$ and extracting this information. It is sufficient if each peer only receives the segment of the $\mathrm{AD}$ containing the information necessary to configure their individual service and insert themselves into the overall context of the application according to the AD.

The following describes the information required by peers to embed themselves in the application context and from which elements of the formal AD this information can be extracted.

- Service to request - Information about which service (defined by its service-ID) a peer has to request from another peer. This information can be determined via the preceding state. The preceding state is identifiable since it has a transition targeting on its own state. The state-ID corresponds to the service-ID and thus provides the information about the service that should be requested.

- Parameter to request - Information about which parameters should be requested or set at the remote service. The data model of the preceding states (see section 5.5.3) specifies these parameters containing their identifier and (if necessary) 
initialise them with a value specifying which parameter content should be transmitted.

These parameters form the basis for determining the preceding service in the service chain. The following parameters form the basis for deciding when and which information should be transmitted to a succeeding service.

- Condition when to response - Information about the prerequisites for transmitting information to a peer who requested this information. This is defined by the transition condition of the own state. Thus, the decision is made as to when the distributed SM moves to another state.

- Data to response - Information about which data to transmit to the succeeding state. There are two possibilities for this: 1. the data being transmitted was defined via the request message, or 2 . the $<$ assign $>$ element of the (own) transition defines which data is sent to which input interface of a succeeding service (see section 5.5.3).

In addition to this information for embedding in the application context, peers need the following additional information, which they also get from the AD.

- Start Point of M2M application - Information about the entry point of the distributed M2M application, i.e. the first service in the service chain. The first service is labelled as an initial state in the formal AD and can thus be identified.

- End Point of M2M application - Information about the end point of the distributed M2M application, i.e. the last service in the service chain. This is defined as final state in the $\mathrm{AD}$. 
Information about the initial and final state is required to validate a correct application configuration (refer to section 6.4.3).

After peers involved in the distributed M2M application service have extracted the abovespecified configuration information by automated parsing of the formal $\mathrm{AD}$, they integrate their services autonomously into the overall context of the application. Figure 6.45 illustrates the application configuration process.

First, each peer determines the specific instances of the services (contact information, URI) to connected to, i.e. determines a list (SP list) of peers offering the service based on the service-ID. This list is integrated in the IFD. Therefore, peers request the IFD from the SAR.

The connection to a specific M2M service instance is performed by sending a request message to the SPs inside the SP list containing the Request Primitive including the parameter that should be either set (Input/Config parameter) or requested (Output parameter). If the requested service is available, the requested peer confirms the service request with a positive response. If the service is not available, the requested peer responds with a negative response. The requested peer holds a local Requestor List (RL) to store the requestors contact information and corresponding requested service parameter. 


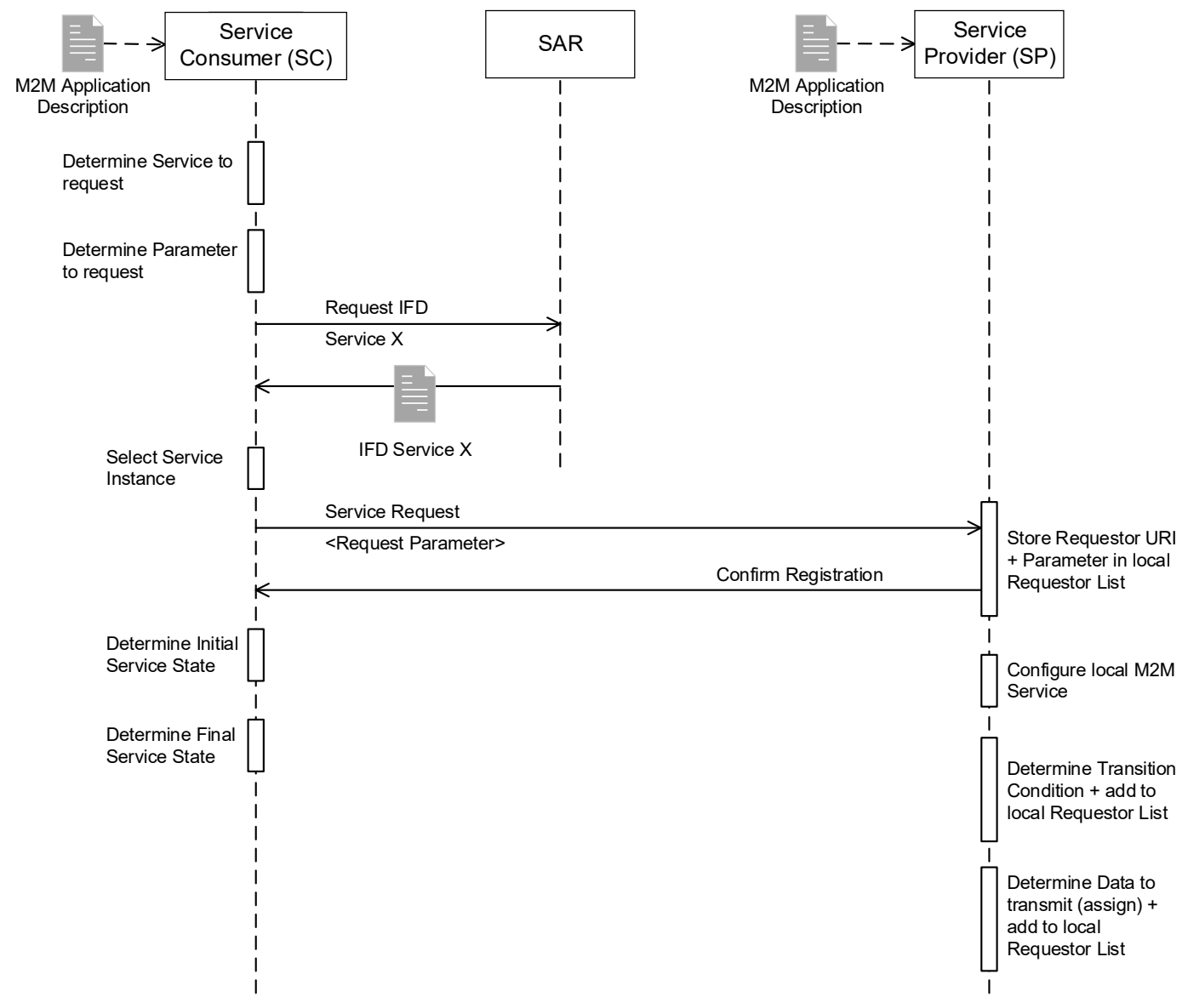

Figure 6.45: M2M Application Configuration Process

In this RL the service providing peer inserts the requested parameter and corresponding contact information after receiving the service request (if confirmed). If the service request contains Input/Config parameter data, the service providing peer assigns the corresponding values to the Input/Config parameter of the local service (e.g. for configuration of the service). After confirmation of the service request the SP adds the transition condition to the corresponding entry in RL for later usage to evaluate whether a response message should be send to the requestor. Additionally, the SP adds the information about the data that should be transmitted in response message (if defined using assign element of the transition). 
Through this configuration process, a connection between an M2M SP and an M2M SC was successfully established and it has been defined under which prerequisites specific information should be transferred from the SP to the SC.

After the configuration of the distributed $\mathrm{M} 2 \mathrm{M}$ application is finished, the execution phase of the application starts (illustrated in Figure 6.46) (Steinheimer et al., 2017a).

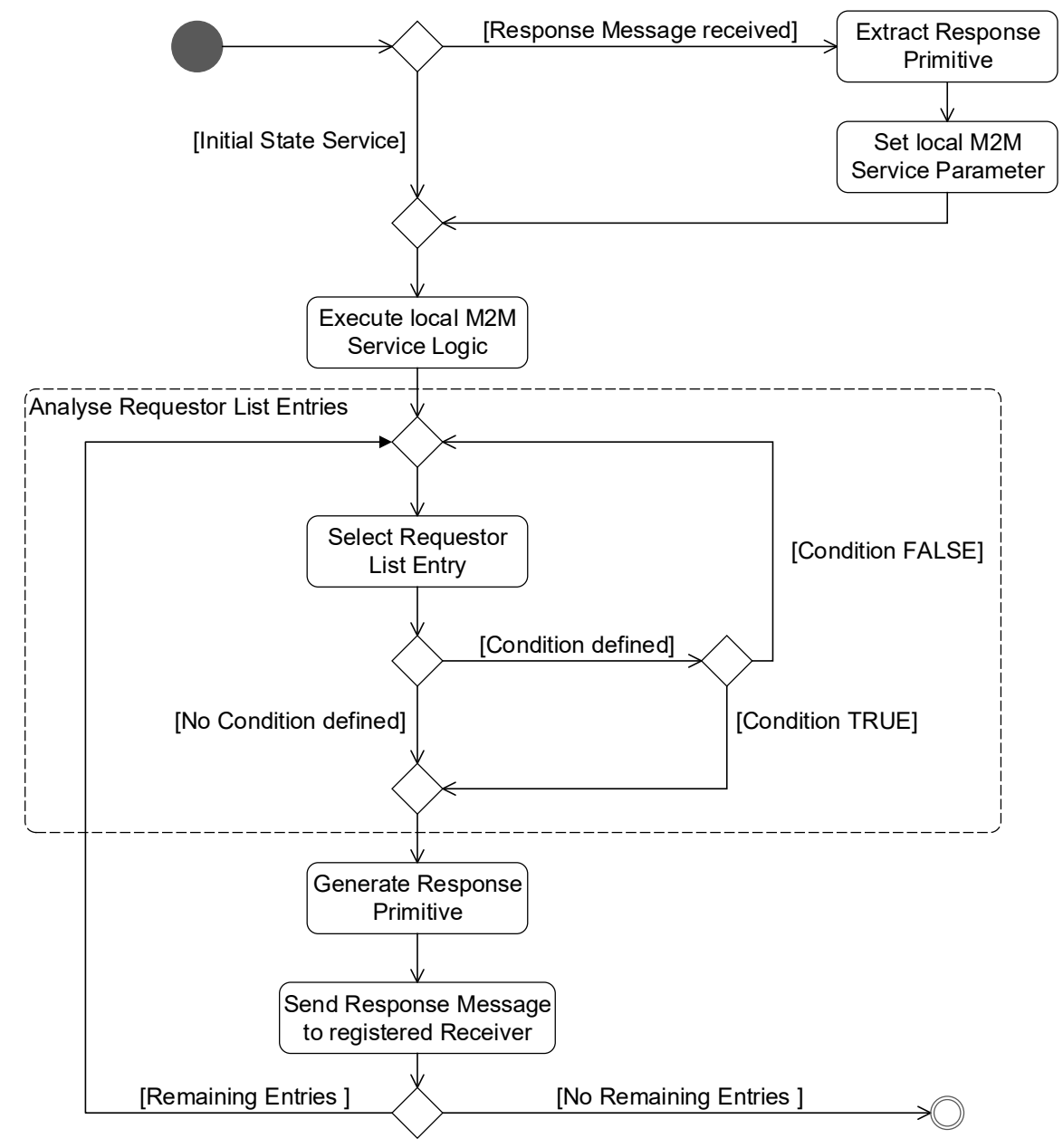

Figure 6.46: M2M Application Execution Process

During execution phase, the peers must distinguish whether their service is the first service in the service chain and thus starts the execution of the M2M application, or whether their service is a service within the service chain. If it is the first service in the 
service chain, the local service logic is executed directly. All other peers are triggered by a response (notify) message. After receiving a response message, they first extract the received Response Primitives and (if necessary) set local parameters defined for their service. Local service logic is then executed. After executing the local service logic, the peers analyse the RL. For this, they check for each entry whether a condition is defined and fulfilled. If the condition is fulfilled or no condition has been defined, a Response Primitive is generated with the parameters and values that are defined in the RL. These Response Primitives are sent to the receivers also stored in the RL, which in turn execute the local application logic in the same way and (if appropriate) send a response message to the peers succeeding of them. The execution of the application ends as soon as the M2M service defined as the final state in the $\mathrm{AD}$ has been reached and has executed its local application logic.

\subsubsection{Cooperative M2M Application Validation Algorithm}

Since multiple peers can offer different instances of the same M2M service, it is possible to create redundant configurations of a cooperative $\mathrm{M} 2 \mathrm{M}$ application by creating redundant connections between the instances of the services. Thus, individual connections between SPs and SCs could fail without affecting the functionality of the entire M2M application. To illustrate this, it is assumed that the following service chain was defined by a formal AD (see Figure 6.47).

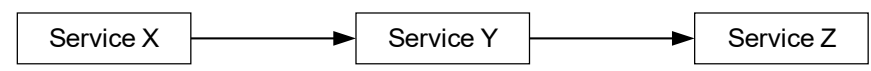

Figure 6.47: M2M Service Connections to demonstrate redundant Application Configurations 
During configuration phase, peers identify to which services to connect and determine a list of corresponding SPs (refer to section 6.3.1). Peers could connect to multiple SPs in parallel instead of selecting a single SP from the list (of SP URIs) included in the IFD. Figure 6.48 illustrates exemplarily the redundant connection of service instances resulting of optimal service availability.

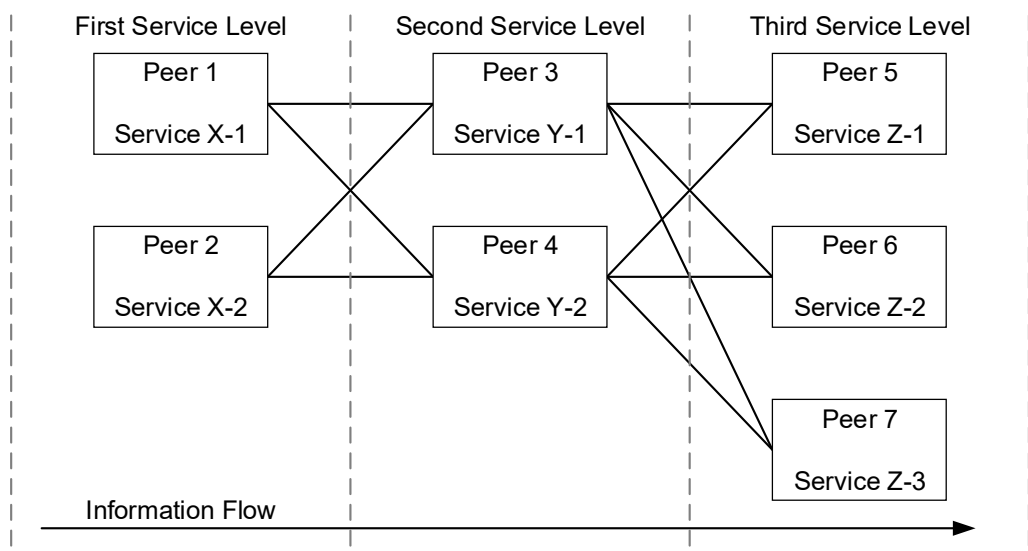

Figure 6.48: Distributed M2M Application with redundant Service Instances

The peers are represented with their identifiers (Peer 1 - Peer $n$ ) and the respective M2M service instance they provide (e.g. Service $X-1$ specifies the first instance of Service $X$ ). Figure 6.48 represents the concatenation of services (i.e. information flow), whereby the services are assigned to different service levels. A service level defines the location of a service in the sequential arrangement of the services. The first service in a service chain is located at the first service level, the second service at second service level etc. The last service in the service chain is on the most right service level. Different instances of a service, i.e. same services provided by different peers are on the same service level. Because all service instances on the same service level connect with all service instances located on the service level besides them, an extremely redundant configuration of the 
distributed M2M application arises since n-1 service instances on each service level can fail without failing of the application itself.

Figure 6.49 illustrates the application configuration and execution phase and shows the multiple instances of the services provided by different peers. In addition to a complete configuration of an application, Figure 6.49 illustrates that invalid configuration of the M2M application could be generated during configuration phase, although some individual peers have configured their service connection correctly. Invalid configurations can be created whenever several service instances exist and should therefore be considered during the configuration phase.

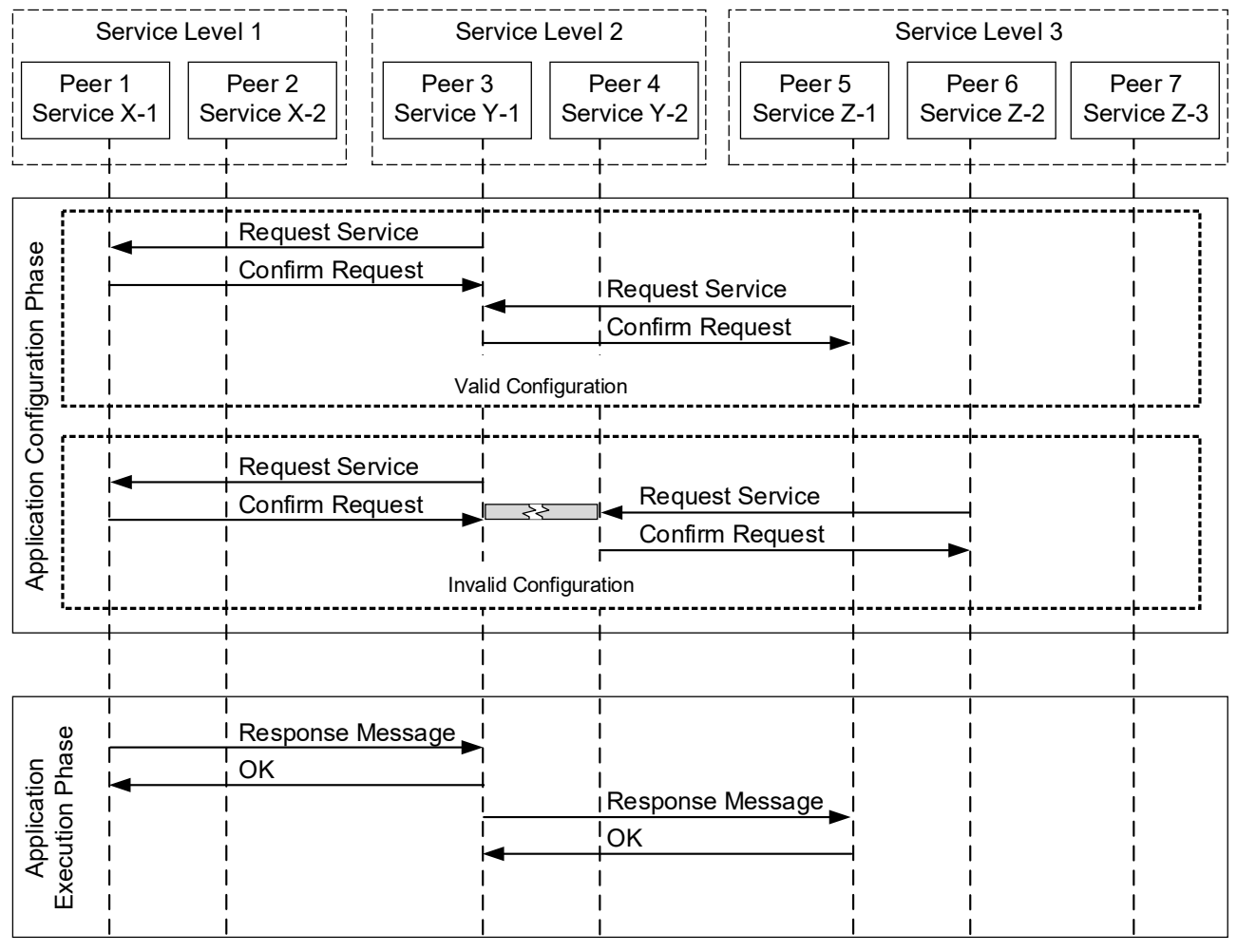

(C) 2017 IEEE

Figure 6.49: M2M Application Configuration and Execution Phase incl. multiple Service Instances

Connections could arise which do not result in any valid configuration of the application, since instances of services are connected with each other, which do not guarantee a 
continuous flow of information through all services. If a peer sends a service request message to another peer the requested peer could reject the service request, e.g. because invalid access control policies or simply because the service is currently unavailable, then the connection to a specific service instance is not established. Figure 6.50 illustrates exemplarily the connections between service instances that only partly exist redundantly.

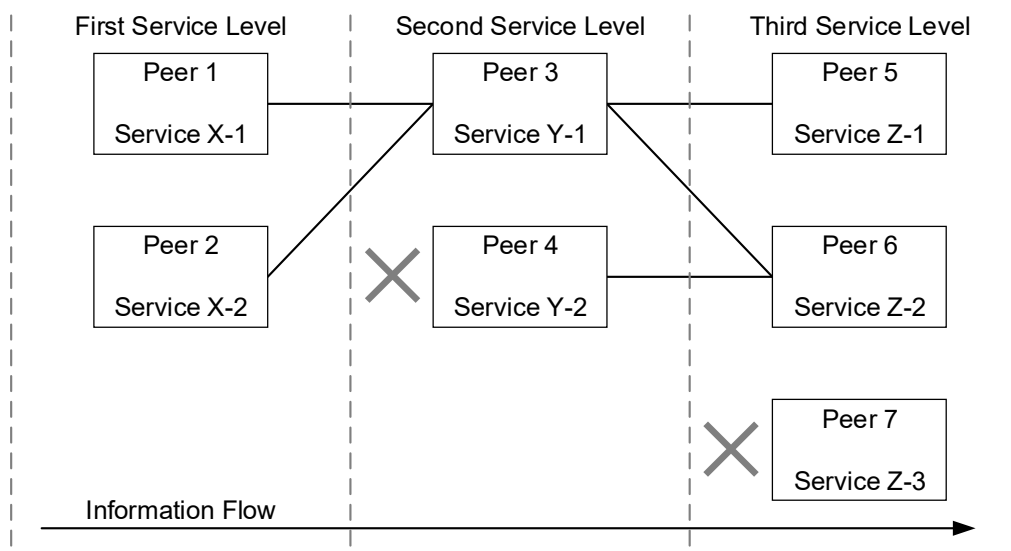

Figure 6.50: M2M Application Configuration with partly redundant Service Instance Connections

Figure 6.50 shows that no path exists between all instances of the first service in service chain (initial state, service at most left service level) and the last services (final states, service at most right service level). This means that the peers have correctly configured the configuration for their own service according to the $\mathrm{AD}$, but the application was not configured correctly in total. A fully configured application has at least one closed path, through all service levels, starting at an instance at first service level and ending at least one instance of each service at the most right service level. This means that the sequential concatenation of the services as defined in the application service description has been fully configured.

The following concatenations of the services illustrated in Figure 6.50 have been set up correctly according to the application service description (defined in Figure 6.47): 
1. Service $\mathrm{X}-1 \rightarrow$ Service $\mathrm{Y}-1 \rightarrow$ Service $\mathrm{Z}-1$

2. Service $\mathrm{X}-1 \rightarrow$ Service $\mathrm{Y}-1 \rightarrow$ Service $\mathrm{Z}-2$

3. Service $\mathrm{X}-2 \rightarrow$ Service $\mathrm{Y}-1 \rightarrow$ Service $\mathrm{Z}-1$

4. Service $\mathrm{X}-2 \rightarrow$ Service $\mathrm{Y}-1 \rightarrow$ Service $\mathrm{Z}-2$

The following concatenation do not result in any valid configuration according to the AD:

\section{Service $\mathrm{Y}-2 \rightarrow$ Service Z-2}

Because the invalid configurations are unusable for the application execution, they should first be identified as described subsequently and then removed again.

For enabling peers to identify independently whether they have established an invalid connection to another peer, the peers need an overview of the overall configuration of the application. I.e. they need an overview of service instances included in the specific application service configuration and connections between them (i.e. a representation of the information included in Figure 6.50). To provide such an overview of the overall configuration, the peers must document to which other peers they established a connection. This documentation must then be made available to all other peers, which in turn use the documentation to identify whether they have embedded themselves in an invalid application configuration. The SAR is a shared database that could be used to store this connection documentation using the application-ID as identifier.

The connections of the services can be mapped to a directed graph by representing the peers as nodes and the connections between the peers as edges between the nodes. Arithmetic operations can be applied to a graph to determine whether a closed connection exists from an initial node (first service level of the graph) to an end node (last service 
level of the graph). All nodes whose connections are not in the path between the start and end nodes have invalid connections and should remove them.

The structure of a graph is representable according to (Turau, 2009) as an adjacency matrix, which could be stored as a data structure in the SAR. The structure of the adjacency matrix describing the connection graph is designed as follows: The nodes of a graph are specified as row and column names in the adjacency matrix, and the connections between the nodes as entries in the associated fields in the adjacency matrix. Because it is a directed graph, the nodes from which a connection originates are specified in the row names, and the destination nodes of these links in the column entries. A unified notation of the column and row entries is defined as illustrated in Equation 6.1.

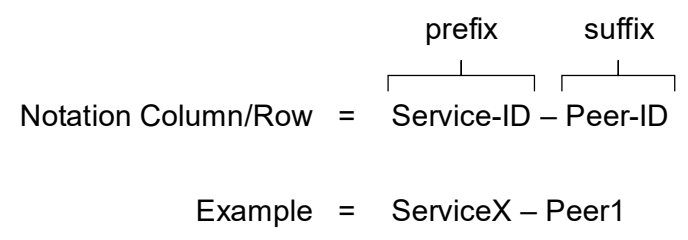

Table 6.11 shows the adjacency matrix describing the graph from Figure 6.50. For a clearer illustration, the table does not label columns and rows as defined above, but as abbreviation the format SxPy is used where Sx represents the service-ID and Py represents the peer-ID.

A directed connection between the nodes in the graph is represented by the entry "1" in the adjacency matrix. If there is no connection between the nodes, the corresponding entry in the adjacency matrix contains the entry "0". 
Table 6.11: Adjacency Matrix of Graph illustrated in Figure 6.50

\begin{tabular}{|c|c|c|c|c|c|c|c|}
\hline & SX-P1 & SX-P2 & SY-P3 & SY-P4 & SZ-P5 & SZ-P6 & SZ-P7 \\
\hline SX-P1 & 0 & 0 & 1 & 0 & 0 & 0 & 0 \\
\hline SX-P2 & 0 & 0 & 1 & 0 & 0 & 0 & 0 \\
\hline SY-P3 & 0 & 0 & 0 & 0 & 1 & 1 & 0 \\
\hline SY-P4 & 0 & 0 & 0 & 0 & 0 & 1 & 0 \\
\hline SZ-P5 & 0 & 0 & 0 & 0 & 0 & 0 & 0 \\
\hline SZ-P6 & 0 & 0 & 0 & 0 & 0 & 0 & 0 \\
\hline SZ-P7 & 0 & 0 & 0 & 0 & 0 & 0 & 0 \\
\hline
\end{tabular}

If peers established a connection according to the $\mathrm{AD}$ to other peers, respectively have associated a service offered by them with another service, they execute the algorithm shown in Figure 6.51 to document the established connection in the adjacency matrix (Steinheimer et al., 2017a).

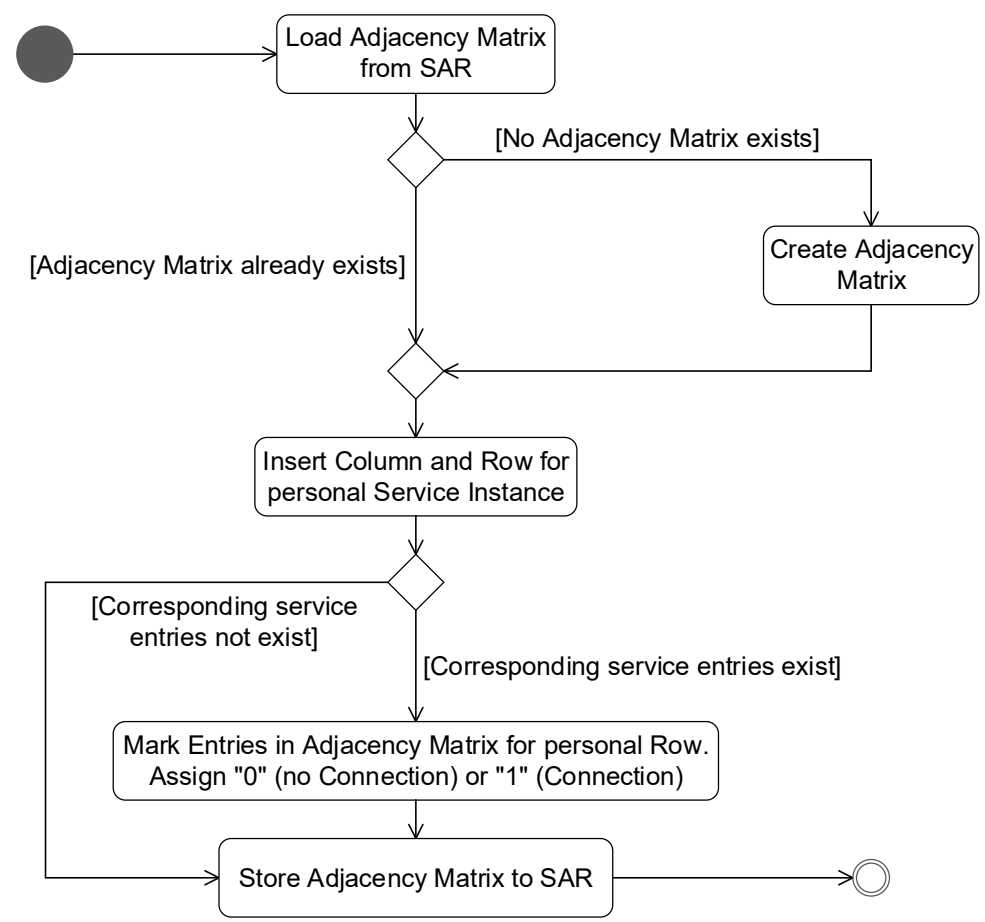

Figure 6.51: Algorithm for Documentation of Connection Establishments 
First, peers load the adjacency matrix of the connection graph from the SAR. If the SAR does not return the adjacency matrix, it can be interpreted that the adjacency matrix has not been created yet. In this case peers initialise the adjacency matrix. Then, they insert a column and a row entry for their own service instance. Afterwards peers validate if the corresponding service they connected to is already documented in the connection graph (column/row entry exists). If the corresponding entries do not exist, the peer stores back the adjacency matrix to the SAR. If the corresponding entries exist, then the entries in the adjacency matrix for the columns/rows assigned to the peer are marked with "0" for no connection to other peers and "1" for a connection to other peers. Afterwards the peer stores the customised adjacency matrix back to the SAR and finishes the algorithm for documentation of established connections.

Since each peer has executed the steps described above for connection documentation, a complete connection overview of the graph, respectively of the application, has been generated and is accessible to all peers contained in the application configuration.

The following describes how peers can use the connection overview to identify and remove invalid connections.

On graphs arithmetic operations and algorithms can be applied which allow an evaluation of characteristics of a graph. The Transitive Closure of a binary relation in a graph (link between nodes), calculated e.g. by the algorithm of Warshall (Warshall, 1962), indicates which node pairs are mutually accessible. This information determines whether a destination node is reachable from a start node, possibly with the inclusion of other nodes (Turau, 2009). The following Figure 6.52 shows the transitive closure of Peer 1 and Peer 4 from application configuration illustrated in Figure 6.50. 


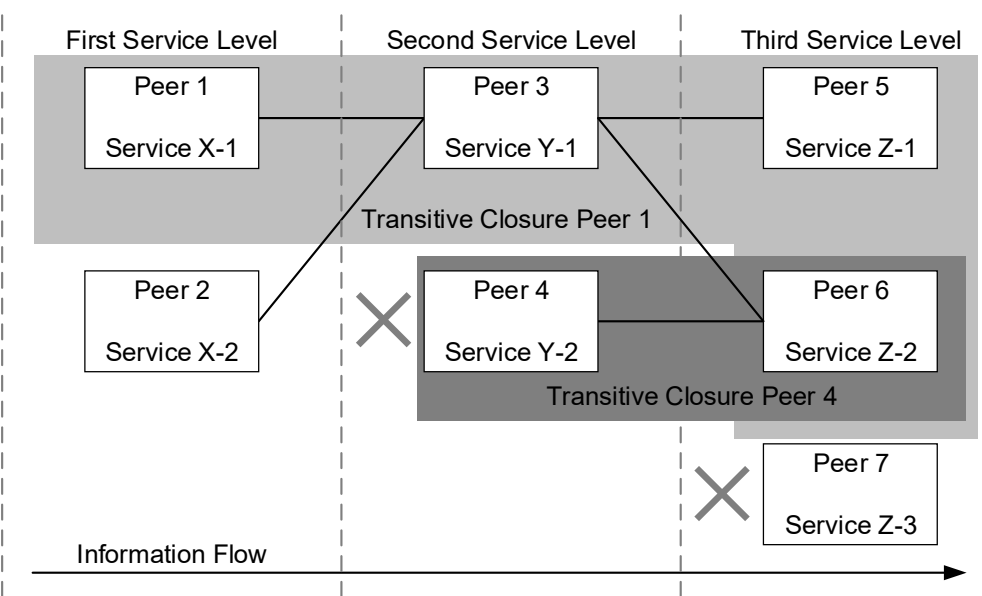

Figure 6.52: Transitive Closure of Peer 1 and Peer 4 based on Figure 6.50

All peers contained in the transitive closure of a peer can be accessed from the associated peer. The Transitive Closure of Peer 1 indicates that the nodes Peer 3, Peer 5, and Peer 6 can be reached from the node Peer 1 . The node Peer 3 is directly accessible and the other nodes indirectly via other nodes. The node Peer 7 is e.g. not in the Transitive Closure of Peer 1 and thus not reachable starting from the node Peer 1. Analogously, the Transitive Closure of Peer 4 indicates that only node Peer 6 is reachable starting from node Peer 4.

Since the connection graph is stored in the SAR, all peers can request and use it to compute the Transitive Closure of the connection graph autonomously. Thus, each peer gets an overview of which peers are reachable among each other.

The transitive closure can also be described as a matrix that indicates which nodes are mutually accessible (Turau, 2009). The matrix shown in Table 6.12 describes the complete transitive closure of the exemplary graph from Figure 6.50. Node pairs that can be reached are marked with "1" as an entry in the associated field. Node pairs that cannot be reached are marked with "0". 
Table 6.12: Transitive Closure based on Figure 6.50

\begin{tabular}{|c|c|c|c|c|c|c|c|}
\hline & SX-P1 & SX-P2 & SY-P3 & SY-P4 & SZ-P5 & SZ-P6 & SZ-P7 \\
\hline SX-P1 & 0 & 0 & 1 & 0 & 1 & 1 & 0 \\
\hline SX-P2 & 0 & 0 & 1 & 0 & 1 & 1 & 0 \\
\hline SY-P3 & 0 & 0 & 0 & 0 & 1 & 1 & 0 \\
\hline SY-P4 & 0 & 0 & 0 & 0 & 0 & 1 & 0 \\
\hline SZ-P5 & 0 & 0 & 0 & 0 & 0 & 0 & 0 \\
\hline SZ-P6 & 0 & 0 & 0 & 0 & 0 & 0 & 0 \\
\hline SZ-P7 & 0 & 0 & 0 & 0 & 0 & 0 & 0 \\
\hline
\end{tabular}

The AD specifies which service is on the first service level (initial state) and which services are on the last service level (final states). Each peer can use the transitive closure and the information of services on the first and last service level independently to determine which connections (according to the AD) are valid or invalid and therefore have to be removed again.

For checking the complete configuration of the application, each peer first determines for its own node in the connection graph whether at least one instance (node) of each service on the last service level is reachable from itself (marked by " 1 " in the associated field of the matrix). In the above example, nodes Peer 5 and Peer 6 are accessible from the perspective of node Peer 3. Thus, from the node Peer 3, there is at least one continuous connection to a node on the last service level.

In the next step, each peer checks whether the own node in the connection graph is accessible by at least one node on the first service level. If this is also the case, there is a 
continuous connection from the first service level to the last layer and therefore a complete configuration of the application.

Incomplete configuration of the application exists when:

1. The own node cannot reach at least one instance of a service on the last service level of the connection graph and/or

2. the own node cannot be reached by at least one instance of the service at the first service level of the connection graph.

If peers detect that they are within an incomplete configuration of the application, they should disconnect the peers to which they previously connected. Figure 6.53 describes the algorithm according to (Steinheimer et al., 2017a) for verifying a complete configuration of an application which is executed autonomously by each peer.

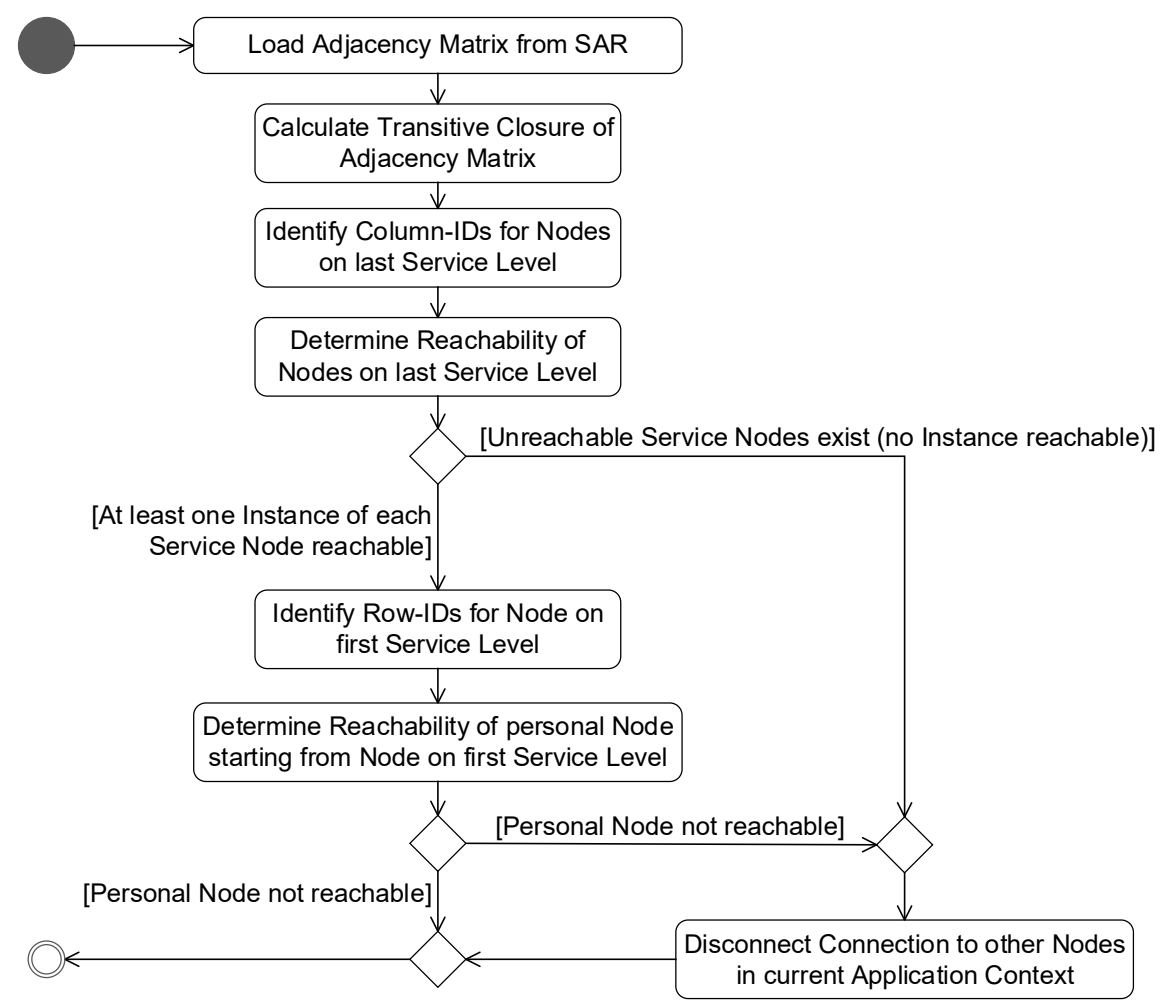

Figure 6.53: Algorithm for Determination of invalid Application Service Configuration 
First, the peer queries the complete adjacency matrix of the connection graph from the SAR. For the resulting connection graph, the peer then calculates the Transitive Closure. Thus, the peer has an overview of which nodes are mutually accessible. The column entries for the nodes on the last service level are determined afterwards. The determination of the services at the last service level is made possible since the last services in the formal AD are marked as "final state". This determines the prefix of the column description. The calculating peer does not know the suffix, but by the uniformly defined format of the column names enables the determination of the column name without knowledge about the suffix. Afterwards, peers check whether these nodes, starting from the own node, are reachable. This is the case if the corresponding entry in the matrix of the Transitive Closure is marked with " 1 ". If there is not at least one instance of each service at the last service level, an invalid configuration of the application exists and the connection to the peers must be removed again. If connections to the instances at the last service level exist, the next step checks whether the own node is accessible by an instance of a service on the first service level. For this purpose, the row-ID of the nodes on the first service level is first determined. This determination is made possible by marking the first service in the formal AD as "initial state". This determines the prefix of the line notation and enables to identify the row-IDs of nodes on the first service level. Afterwards, peers check whether the own node is accessible from one of these nodes. If this is the case, a continuous connection from the first to the last service level exists. If the own node is unreachable, the connection to other peers has to be removed because no valid configuration of the application has been established. Removing a connection between the peers is done by sending a request to the connected peer with the request to disconnect (unsubscription). 
This section introduced an approach for autonomous and distributed administration of the entities involved in an application. The approach allows the involved peers to generate an overview of all service instances integrated in an application configuration. This overview allows the peers to determine independently whether they have embedded themselves in valid application configuration. Invalid application configurations are automatically identified and removed, which ensures that a configuration of an application always takes place according to the $\mathrm{AD}$.

An alternative to the presented approach for application validation would be if each peer sends a message to the service instances at the first or last service level for connection control (something like a "PING message"). They could send this message to the peers connected to them, which in turn forward the message to their neighbours. The peer that is arranged in the last or first service level could then send back a reply message directly to the initiating peer or return a message on the same path as it has received it. However, this approach has the disadvantage that if peers were temporarily unavailable, although they are embedded correctly in the application context, the link control message would not be forwarded. As a result, the path to be checked between initiating and target peers would be declared invalid. Therefore, a connection-independent management of the connections between the M2M SPs should be preferred.

\subsection{M2M Community}

In section 4.2 it was already introduced that the framework presented in this thesis contains the community approach presented in (Steinheimer et al., 2012b) or (Steinheimer 
et al., 2013e). The M2M community is a social network that is built between users of the MSP.

In essence, the M2M Community is used for the following:

- Linking of end-users at interest-level - The community element of the presented framework enables to manage various interests the end-users using the MSP. Through the formation of interest groups, the end-users are grouped. To realise the different groupings, different sub-communities are provided. End-users who share the same interests enter the same sub-community. The community approach, enables that services are only released for certain user groups or restricted to specific geographic areas. If these services should be used, the SC must be assigned to the relevant sub-community.

- Legal Basis - Through the voluntary participation in the M2M community and the resulting consent for platform utilisation, a basis for the legal certainty can be created. This is intended to address the topic that in principle no one is authorised to act within the personal area of an end-user (e.g. trigger control operations) or to process data (monitor sensor data) from it. For avoiding the need to conclude appropriate contracts between the end-users or between end-users and companies/organisations, the voluntary participation and the acceptance of the usage conditions approves the data processing.

To organise the M2M community with its approaches the IFD can be used. This includes the element accessControlPolicy, which in turn contains the element privileges with the attribute access ControlOriginators. This parameter can be used to define a grouping, i.e. assignment to specific sub-communities. The accessControlPolicy is defined as part of 
the IFD during the generation of the IFD. Since the IFD is generated by SPs, they have the possibility to define the access ControlPolicy according to their ideas, which again corresponds to the concept that end-users are extensively involved in the application creation and have full control over their local M2M applications. Interface Description (AE)

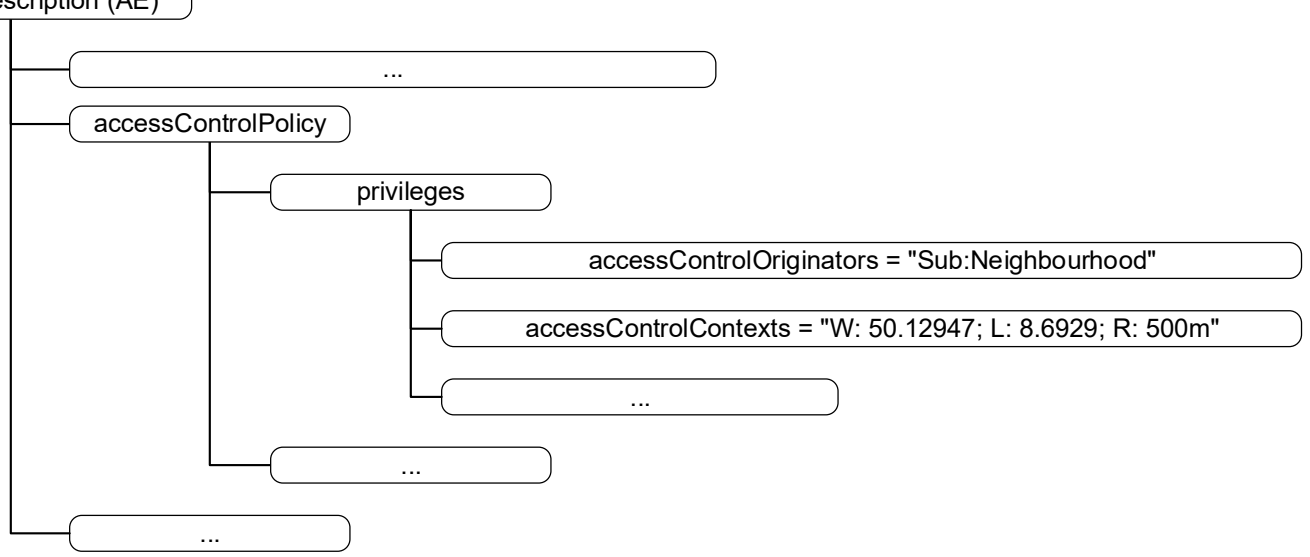

Figure 6.54 illustrates the extract from an IFD for the accessControlPolicy element. Listing C.12 illustrates the corresponding XML representation of the IFD element.

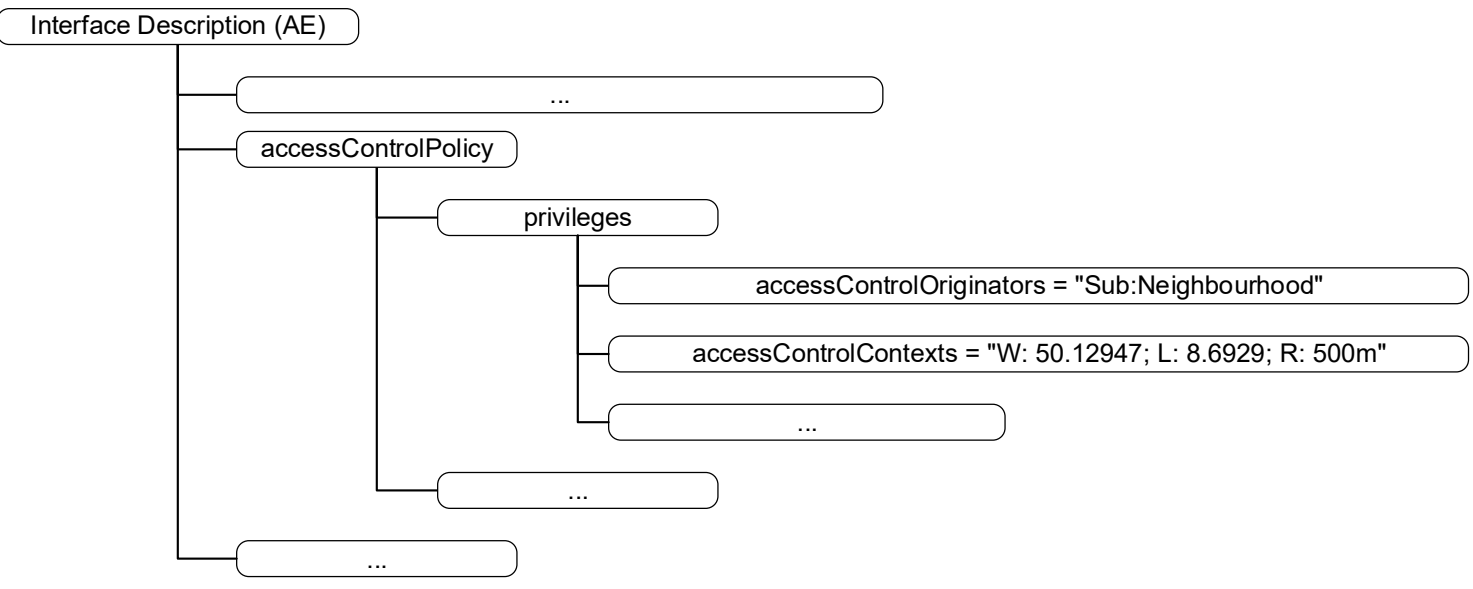

Figure 6.54: IFD Element AccessControlPolicy for Definition of (Sub-) Community Assignment

The accessControlOriginators parameter defines the sub-community (neighbourhood) in this section of the IFD. Additionally, the parameter accessControlContexts is also listed, which can be used to define in which area a service can be used (see section 6.1.1). The 
example shown above maps an IFD to the sub-community neighbourhood and defines that the associated service at the locality with the GPS coordinates $W: 50.12947 ; L$ : 8.6929 (Kleiststrasse 1, D-60318 Frankfurt, Germany) is available within a radius of 500 metres.

In order to make the M2M services available grouped according to the sub-communities, the GUI can be filtered accordingly. Therefore, it is possible that only the M2M services are displayed which are defined for the sub-community that belongs to an end-user.

Through the community approach and the capabilities of the presented decentralised MSP, it is possible to realise not only technical services, but also to use the platform functionality to offer social services (executed by fellow human beings).

Figure 6.55 shows exemplarily an IFD containing the interface parameters necessary for a service request. For the description of a social service, the prose description is particularly important, since this is the description of the service. Listing C.12 illustrates the corresponding XML representation of the IFD. 


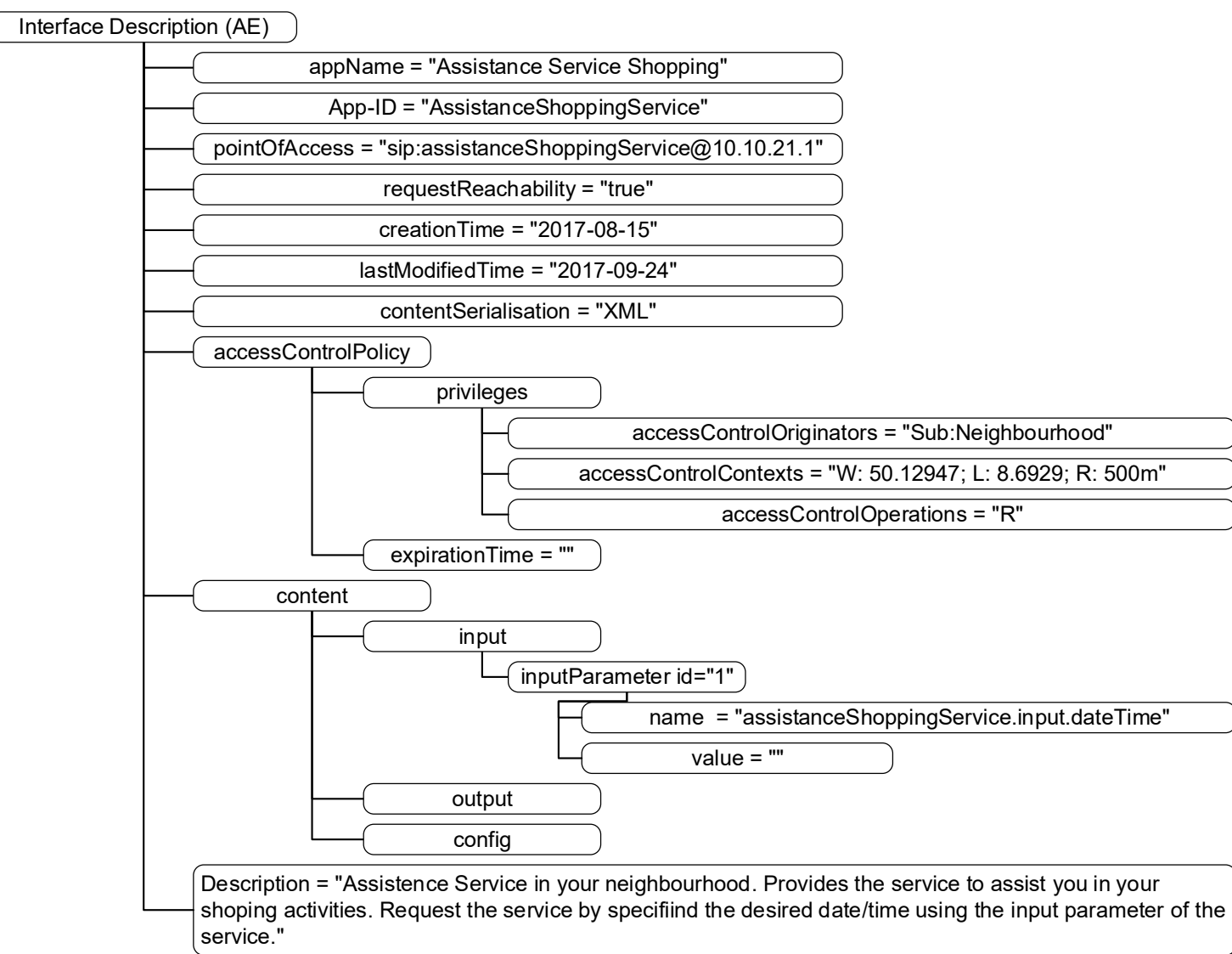

Figure 6.55: M2M application service IFD for Social Service

In the above example, a social service was specified, which is defined for the subcommunity neighbourhood and is only available in a certain place. The prose description defines the functionality of the service. The example shows an assistance service for shopping activities. (Social) Peers, i.e. people in the neighbourhood that register this service offer to assist other people in their shopping activities. Other possible services from this category are e.g. assistance service to go for a walk or assistance in gardening.

Through the designed framework and the social networking aspect integrated by the community approach, not only a decentralised MSP was designed, but also a decentralised social networking platform was created, which can be used for the administration and mediation of any (not only M2M) services. 


\subsection{Conclusion}

This chapter introduced the mechanisms and approaches for cooperative M2M application service provision. Section 6.1 specified a mechanism to make the local M2M applications and $\mathrm{M} 2 \mathrm{M}$ devices available as a service to other end-users and integrate them into local M2M applications. For this purpose, an IFD has been defined that is based on the specification of $\mathrm{M} 2 \mathrm{M}$ resources according to the oneM2M standard and has been extended with interface parameters for input/output/configuration of M2M applications. It has been illustrated how the IFD can be generated from the formal AD by extracting the essential interface parameters and inserting them into the IFD. It was shown how a remote $\mathrm{M} 2 \mathrm{M}$ application that was made available as a service could be used by sending a request message to the service, which possibly returns a response message. The exchange of information between the participating entities was carried out according to the requirements of the oneM2M standard by means of Request/Response Primitives. The SIU has been specified as part of the CU realising the communication between SP and SC. It was shown how remote M2M application services can be integrated into the graphical modelling of a local M2M application so that its functionality can be used within the local M2M application.

To enable communication between SPs and SCs, section 6.2 described the networking of them. It has been specified that the communication is done directly P2P between SP and SC node without involving further nodes at the application level. Different information exchange patterns were presented and evaluated in the context of this project. The result of the evaluation was that communication between the nodes according to the Subscribe/Notify principle is the optimal mechanism for information exchange. Different 
communication protocols for exchanging Primitive messages between the nodes were analysed and compared. The protocols CoAP and SIP were identified as optimal communication protocols. It was shown how the Primitive messages can be exchanged by means of CoAP in accordance with the oneM2M standard and additionally a SIP Protocol Binding was defined enabling to exchange Primitive messages by means of SIP.

Section 6.3 described how the service IFDs can be made accessible to other end-users, and how peers register the instances of their services. For this purpose, the SAR was introduced as a common database storing the IFDs. Peers who wish to use the IFD or an instance of a service, query the SAR using the service-ID as the key for the respective data record. To avoid a central entity responsible for data storage (as it would be the case with a central database), the distributed data storage was defined using P2P overlays as a mechanism for the realisation of the SAR. P2P overlays can be realised through structured or unstructured $\mathrm{P} 2 \mathrm{P}$ overlay algorithms. Because structured and unstructured $\mathrm{P} 2 \mathrm{P}$ overlays are fundamentally different in their structure, an approach has been presented for both types to manage the IFDs and service instances. To determine which P2P overlay mechanisms are best used in the context of the presented framework, common P2P overlay algorithms and architectural approaches were compared. The result of the evaluation was that, depending on the respective application scenario of the framework, structured or unstructured P2P overlays are advantageous. Structured overlays are advantageous when a large amount of SAR requests are made, or if the discovery of a record needs to be guaranteed. Unstructured P2P overlays are advantageous when large movement of peers exist or complex search queries should be made to the SAR. 
Section 6.4 specified the principles of the cooperative M2M application service provision that allows the services offered by end-users to be combined into a complex distributed M2M application consisting of a service composition or a service aggregation. The design of a cooperative M2M application takes place by definition of a SM-based application model defining the application semantics and is formally described by means of SCXML. A cooperative M2M application service is also made available via an IFD. Furthermore, it was specified how a cooperative M2M application is configured and executed. The configuration process of an application was decoupled from the execution phase of an application. The peers do the configuration of the services and the connection to the associated services fully autonomous, i.e. without a central control of the connection between SPs and SCs. To connect the services, the peer who is supposed to process information from another peer requests the information data at the information-providing peer. During the execution phase, the peers provide the information by sending a notification message to the peer following in the service chain. Through the autonomous configuration and execution as well as the information processing by the involved services a distributed SM according to the application description was realised that is executed completely independently. An autonomous configuration of a cooperative M2M application could create invalid links between the involved services. For this purpose, an algorithm was designed to enable each peer (also autonomously) to determine these invalid links so that they can be removed again.

Finally, section 6.5 described the details of the M2M community approach. Through the grouping via sub-communities it became possible to link the end-users at interest-level and create a legal basis for utilisation of the decentralised MSP. 
This chapter 6 presented the second part of the proposed concept for "Autonomous decentralised M2M Application Service Provision”. The concept of a decentralised MSP at the end-user level has been refined and an application architecture for cooperative M2M application provision has been introduced. The continuous concept has been extended by the fact that end-user can not only define applications for a local M2M platform but can also integrate remote offered M2M services into their individual applications. Furthermore, the concept has been extended in such a way that end-user can combine their M2M services and thus the possibility exists to appear as a cooperative M2M application service provider.

By providing local $\mathrm{M} 2 \mathrm{M}$ application functionality as a service to other end-users, resources become available that were previously not addressable. These resources can now be integrated into other M2M applications. The control and monitoring of $\mathrm{M} 2 \mathrm{M}$ devices are carried out exclusively according to the end-user's requirements, which means that end-users have full control over the performed activities in their personal environment. Because the large number of functionalities provided by other local M2M applications, many new applications can be realised. For example, no longer all M2M devices have themselves to be present locally, but end-user can integrate the functionality of other remote $\mathrm{M} 2 \mathrm{M}$ devices into their own applications. This extends the available possibilities to build up a local "smart environment" and at the same time reduces the costs for the required hardware.

The communication between distributed M2M platforms for provision and integration of the application functionality as a service is realised again consistently with the RESTful communication principle. According (Bayer, 2002) the generic interfaces defined in this 
way make it possible to implement and offer generic services. The IFD can be generated automatically from the formal application description. The unified interface cannot just be used to provide a local M2M application for others. When generalising the principle of service provision and defining a corresponding interface (e.g. via the MMSCs), nontechnical services, such as supporting services can also be provided via the defined interface.

Through the fully decentralised structure and the defined decentralised M2M application architecture, the presented approaches follow continuously the requirement not to contain any central entity in the MSP architecture. There is therefore no dependency on stakeholders and single point of failures are avoided. Because the distributed and decentralised approach, especially through the application of P2P mechanisms for data management and communication, a high degree of scalability of the MSP is already achieved in the approach. By specifying different mechanisms to use different types of P2P overlays, a high degree of flexibility is achieved for realising the MSP. Avoiding central entities achieves data security and end-user privacy, since only SPs and SCs are involved in the communication and the data are not stored together in a central location. The use of SIP as a communication protocol between the peers enables communication with existing technologies and reuse of existing multimedia communication interfaces at the end-users' ${ }^{6}$ environment.

Through the combination of distributed resources, the presented concept enables endusers to cooperate and provide complex and fully distributed M2M application services. Each peer in the context of an application acts autonomously (both in the configuration 
of an application and during the execution of an application). This avoids the use of a central component for controlling the application coordination.

The principle of application creation by the end-users is followed so that the end-users continue to have control over the modelled applications. The modelling is also graphical and thus intuitive. Thus, even complex distributed applications can be realised simply, according to the same principle as local M2M applications. The approach to define a cooperative $\mathrm{M} 2 \mathrm{M}$ application follows the principle of describing the application semantics using the specified formal description language. This means that the defined application is independent of the implementation of the execution environment because only the application description has to be parsed, instead of implementing the application logic in possibly different programming languages on a platform-dependent basis. Thus, different local platform technologies can be used, which are linked by the formal application description to a higher abstraction level. The application logic can be easily exchanged between platforms, so already defined applications can be used as a template for new applications.

Through the M2M community element of the proposed framework the flexible MSP could be enhanced by social networking aspects for linking end-users and enabled a decentralised social networking platform that can be used also for administration and mediation of social service. 



\section{Research Prototype and Framework}

\section{Evaluation}

This chapter introduces the research prototype and evaluates the proposed framework components. Section 7.1 analyses the defined requirements and describes how they are fulfilled by the proposed framework aspects. Section 7.2 describes the architecture of the developed prototype with its components and describes their functionality and how they were implemented. Section 7.3 discusses the utilisation of the prototype by means of exemplary M2M application service to evaluate the functionality of the proposed framework components.

\subsection{Evaluation of Framework Requirements}

This section evaluates the proposed framework for "Autonomous decentralised M2M Application Service Provision" in relation to the requirements defined in section 3.2. In the following, each requirement is evaluated with regard to its fulfilment in the presented framework.

- End-user environment integration - End-user environment integration into the M2M service platform (MSP) is supported through integration of M2M devices residing in end-users' personal environments. These M2M devices can be used to include their functionality in the application logic to realise an application that 
processes detected M2M device data or triggers control actions on the devices (see section 5.4). The M2M devices can be integrated regardless of their M2M device technology due to defined M2M device technology abstraction mechanism.

- End-user integration - The requirement of end-user integration is fulfilled since end-users are in focus of interest and continuously integrated in all aspects of the proposed framework. End-users can participate in application definition by graphically modelling of the application behaviour according their individual requirements (see section 5.2). They also participate in MSP provisioning by providing their local equipment used as Application Execution Environment (AEE) for hosting the MSP executables. End-users are additionally integrated essentially when providing/consuming social end-user services (see section 6.5).

- End-user service provision and utilisation - The requirement end-user service provision is fulfilled because end-users have the possibility to provide their local M2M applications as a service to others. For this purpose, a unified interface has been defined which contains information on the description of a service, such as service provider (SP) or time restrictions and specifies the service parameters (see section 6.1). End-user service provision/utilisation is enabled by the unified mechanism to request services and exchange information data using Subscribe/Notify Information exchange Pattern (see section 6.2). Remote M2M service parameters can be integrated into local M2M applications by processing their parameters or calling provided method functionality.

- Cooperative end-user service provision - The ability of cooperative end-user service provision is supported through the aspect of the proposed framework to 
combine end-user services by defining choreographies of end-user services (see section 6.4). This makes it possible to realise complex M2M applications that are executed in the end-user environments and that combine the resources provided by different end-users.

- Decentralised system architecture - The requirement of decentralised system architecture is fulfilled because all components of the proposed framework are completely decentralised. The services are created locally via the Service Design Unit (SDU). The application logic as well as all components of the framework are executed locally on end-user equipment (e.g. IAD) and not on a remote server or in the Cloud. The communication between SP and service consumer (SC) is P2P without intermediary entities on the application layer (see section 6.2). By using P2P overlays, the necessary data storage is decentralised (see section 6.3). The possibility that services can be offered multiple times means that there is no dependency on a central SP. The decentralised integration of different M2M devices provides a high degree of flexibility, so that M2M applications are not limited to a specific field of application predefined by the MSP. End-users together provide the MSP so that there is no dependency on a central stakeholder or central components for MSP provision.

- M2M device technology abstraction - The ability to abstract M2M device communication is provided by the Abstraction Layer (AL) component of the proposed framework (see section 5.4). The AL abstracts the communication between devices and the application by communicating with unified M2M device representations within the platform. The AL translates the uniform commands into 
technology-specific commands which are then sent to the devices by the corresponding technology interfaces and vice versa.

- Multimedia communication - The multimedia communication aspect of the proposed framework is supported through the specification of Multimedia Service Components (MMSCs) enabling to interact with the MSP using existing telephony equipment (see section 5.1). These MMSCs can be integrated into the M2M application flow to serve as an input/output interface for audio/video/text communication.

- Device/service lookup mechanism - The prerequisite for this ability is the provision of a shared database. The aspect is supported through the Service and Application Registry (SAR) realised by a P2P overlay (see section 6.3). This can be used to register M2M services and applications. For this purpose, an Interface Description Model (IFD) was defined containing the parameters and descriptions required for the service utilisation (see section 6.1). Instantiations of that IFDs describing specific M2M services can be requested by the Service Delivery Platforms (SDPs) of other end-users for e.g. displaying it in the SDU or detect specific M2M service instances. Within a local SDP, the M2M devices are registered and managed in the M2M Device Registry within the Communication Unit (CU), so that they can be queried by the SDU and, for example, listed in the GUI (see section 5.4). To describe the devices (and MMSCs), Device Capability Models (DCMs) have been specified which define the properties and parameters of the devices.

- Simplicity - The requirement of simplicity is fulfilled through the graphical modelling methodology of an M2M application (see section 5.2 and section 5.3). 
The modelling of a state machine (SM) describing the behavior of an application can be intuitively modelled by the end-user. The unified structure of an M2M application (connecting building blocks with input/config/output parameters supports the simple modelling of an application. Apart from modelling the application, no further steps are required that need to be done manually. The generation of the formal application description, the configuration of the M2M application and the execution of the application logic is done automatically (see section 5.5 and section 5.6).

- Minor hardware requirements - The aspects of minor hardware requirements is supported because the software executables of the framework components have low footprints and do not require heavy-weight application servers to execute them. The framework components could by executed locally on existing end-user equipment, which is powerful enough to run the executables. The entire AEE could be operated within the IAD, eliminating the need for additional resourceintensive AEEs such as high-performance servers. It can be assumed that a singleuser application running locally requires significantly less resources than a remote multi-user application environment. The runtime environment for the prototype requires little resources: Java SE 8: 124MB RAM, 128MB Disk (Oracle, 2017d); Java SE Embedded 8: 32MB RAM, 50MB Disk (Oracle, 2017e). The entire runtime environment has 190MB (Karaf: 60MB, Spring Boot Jar: 10MB, Docker Container: $120 \mathrm{MB})$. Due to their small size, they can be deployed on an IAD.

- Scalability - The requirement for scalability is fulfilled because the P2P mechanisms integrated in the proposed framework are naturally very scalable. Since the service provision and communication between the nodes as well as the 
data storage are $\mathrm{P} 2 \mathrm{P}$, the entire MSP is scalable. The defined framework enables the same services to be offered by several peers at the same time. The scalability of service utilisation therefore depends on the load distribution among the peers. Assuming that the load is equally distributed between the peers, then the load on the individual peers and subnets is low and the service utilisation scales.

- Platform independency - The requirement for platform independency of the proposed framework is supported through multiple aspects. When designing the concept, attention was paid to using standardised mechanisms and protocols. An application is specified by defining a formal application description independent of the execution environment (see section 5.5). The standardised SCXML was used as the formal description language. The application description (and also the modelling process) is independent of the system that interprets the application description and is therefore platform-independent. The interface description was also defined platform-independently in XML (see section 6.1). Only the algorithms used for P2P overlay are not standardised. Here, however, with Chord and Gnutella two widely used algorithms were used. There is also platform independence at the communication level. Communication between the platform components as well as between SP and SC takes place by means of service layer messages (Primitives) standardised by oneM2M. The communication between SP and $\mathrm{SC}$ is also established using the standardised protocols CoAP or SIP, so that no proprietary protocols are used. The prototypical implementation was done with platform-independent concepts. By using Java as a programming language, platform independence at operating system level is achieved. Through the 
additional use of Docker, a complete system independence was achieved, so that the entire SDP can be deployed as a container on the executing platform.

- Data safety, end-user privacy - The aspect of data safety and end-user privacy is supported through the avoidance of central entities storing data. Data is not stored in a central location, but rather decentralised by the users who do not have access to the entire data sets. Due to the cooperative platform provisioning at the enduser level, there is no binding to a central provider that could possibly misuse the data. The ability to define access control policies includes a mechanism for controlling access rights. It is possible to define access rights at user/group/time/ or location level.

The essential parts of the proposed framework have been implemented for the proof of concept. The following section presents the architecture of the research prototype.

\subsection{Research Prototype Architecture and Implementation}

To demonstrate the essential functionalities of the proposed framework for "Autonomous decentralised M2M Application Service Provision", a research prototype has been developed. The research prototype implements most of the components described in the framework architecture with required functionality for the proof-of-concept.

Beside the graphical user interface SDU with basic functionalities, trimmed versions of the Service Creation Unit (SCU) for transforming the graphical application model into formal application description, and the Service Runtime Environment (SRE) with Application Description Parser (ADP), Application Description Interpreter (ADI), State 
machine Repository (SM Repo), Service Execution Engine (SEE), and Instant Message Multimedia Service Component (IMMMSC) have been implemented. Also the CU with AL have been implemented with communication modules for Session Initiation Protocol (SIP), Constraint Application Protocol (CoAP), TC IP 1 Control Protocol (TC IP 1, 2013), and Bidirectional Communication Standard BidCos (eQ-3 BidCos, 2016). Furthermore basic functionality of Service Provision Unit (SPU) with overlay modules for Chord and Gnutella and integrated TCP fileserver have been implemented. Additionally as end-user communication interface a SIP Instant Message Client (representing end-user smartphone) have been implemented in both GUI variant as well as console-based variant. To make local M2M devices and MMSCs available to the SDU M2M Device/Service Capabilities have been specified for localRainSensor, localSmokeDetector, localWindowSensor, localWaterSensor, and IMMMSC as well as interface descriptions for remoteSensorService, remoteBuildingMonitoringService, remoteRainSensorService, remoteAlarmService, remoteEnergyReductionService.

Figure 7.1 shows the structure of the research prototype execution environment.

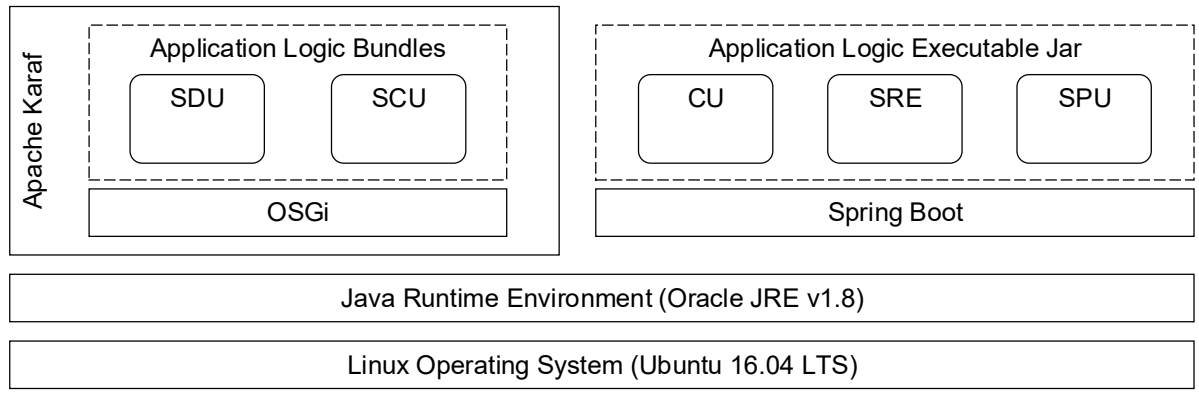

Figure 7.1: Research Prototype Application Architecture

The research prototype was developed with the Java programming language (Oracle, $2017 \mathrm{~b}$ ) as this is a common programming language for the development of software 
applications. Java is also known as a platform-independent language, which supports the implementation of the prototype on different platforms. In addition to Java as the basis for the implementation, the frameworks OSGi (OSGi Alliance R5, 2012) and Spring Boot (Spring Boot R1.5.8, 2017) were used. The use of the two different frameworks demonstrates the flexibility of the developed prototype. Depending on the preferences, it is therefore possible to extend the prototype using one or the other framework. The part responsible for creating the application description was developed with OSGi and the part responsible for application execution was developed with Spring Boot.

The focus in application development was on the development of loosely coupled application components to facilitate the simple extensibility of the prototype. This characteristic is supported by both frameworks. OSGi implements the loose coupling of application components by a so-called Bundle concept. The application components are deployed in the form of independent modules (Bundles) in an OSGi container. Apache Karaf (Apache Karaf, 2017) was used as OSGi container for the prototype implementation. However, other implementations such as Apache Felix (Apache Felix, 2017) or Eclipse Equinox (Eclipse Equinox, 2017) can also be used as OSGi containers, so that the implementation is not limited to a specific container environment. The Bundles are linked by the OSGi Container. This principle of component interconnection is called Inversion of Control (IOC). IOC is also an essential aspect of Spring Boot, whereby modularity is implemented by Dependency Injection (DI). Unlike OSGi-based platforms, running a Spring Boot-based application does not require a separate runtime environment (OSGi container) for the application modules. Spring Boot applications are deployed and executed as a single Jar executable. A comparison of these two frameworks is not in the focus of this work, but it is mentioned here briefly that OSGi has the advantage that 
Bundles can be exchanged during runtime. Spring Boot does not have this aspect, but has the advantage that no additional container is required to run the application, which has to be deployed and maintained in the target environment. To achieve not only independence from the operating system, but also a complete platform independence (also from the executing hardware), the container virtualisation technology Docker (Docker 17.06.2-ce, 2017) was used additionally to the mentioned frameworks. All the dependencies required for running an application (up to the operating system) are integrated into a container, which is then deployed as the only element on the target platform. The use of Java, OSGi, Spring Boot and Docker enables a complete platform-independence using up-to-date technologies.

The presented framework can be classified roughly into a part that can be validated locally and a part that can only be validated on several distributed systems. Figure 7.2 shows the system architecture created for the prototype.

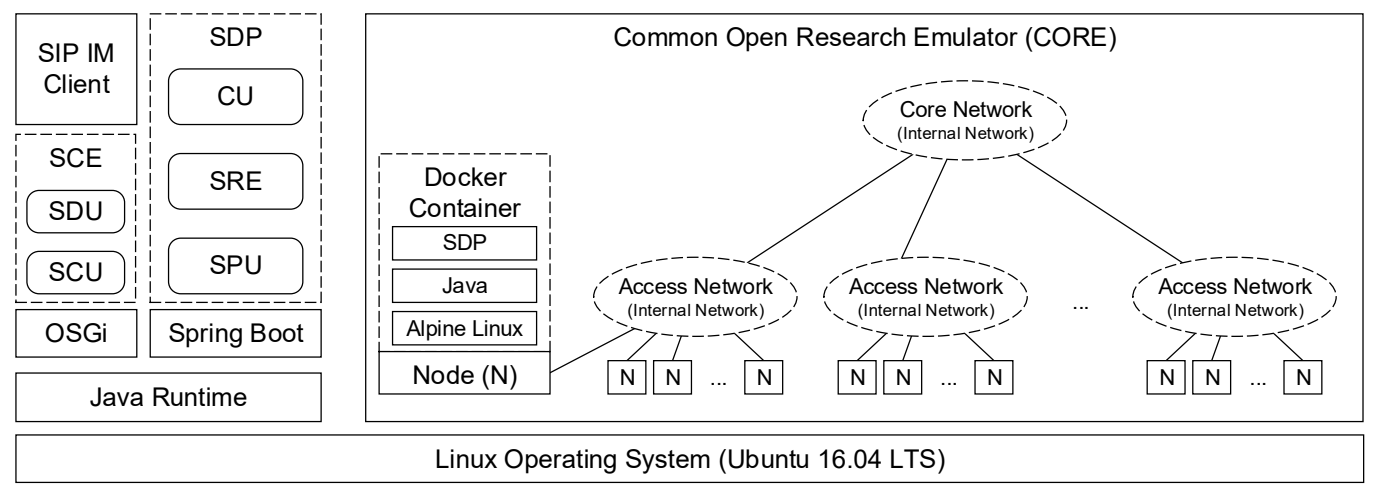

Figure 7.2: Research Prototype Emulation System Architecture

In order to validate the locally executable parts (e.g. application modelling, creation of formal application description, parsing of formal description), these parts of the research prototype were executed locally on the system. The Common Open Research Emulator (CORE) (CORE, 2017) was used to validate the implementation of scenarios that require 
a distributed system architecture. CORE allows to create different virtual networks and emulate their behaviour. These virtual networks can be used to connect different nodes (representing peers) that communicate with each other via virtual networks. It is also possible to execute application logic on the nodes using CORE. To deploy the application logic to the respective nodes, the local execution environment (SDP) was packaged into a Docker container, which was then deployed on the virtual nodes of CORE. Figure 7.3 shows the GUI of CORE with the created networks and nodes for the emulation environment.

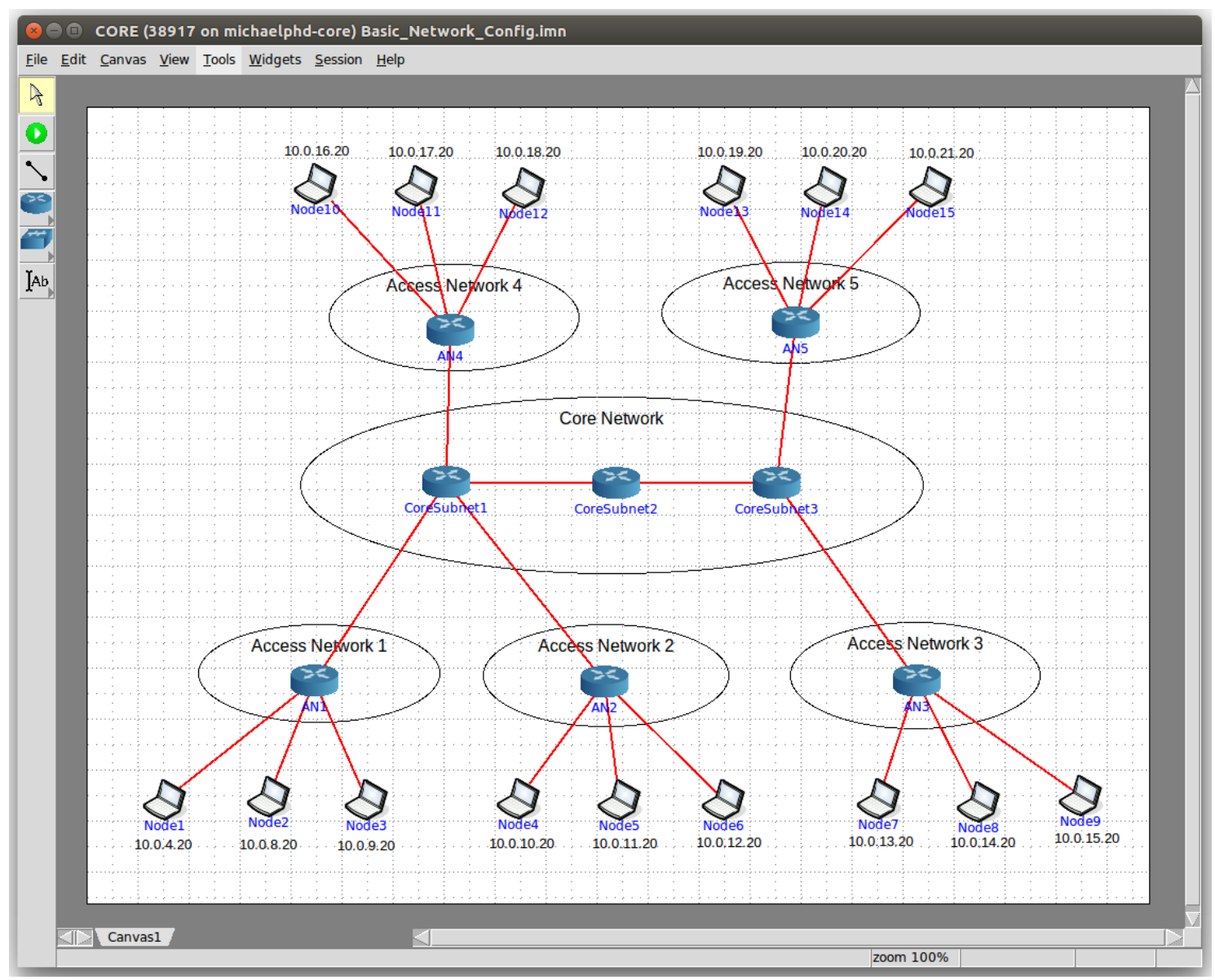

Figure 7.3: Research Prototype Emulation System Architecture

Coming back to the application architecture of the framework prototype, the following Figure 7.4 illustrates the application architecture of the framework prototype. The 
architecture depicts the individual modules of the prototype and how they are linked with each other. The modules are classified by name and the implementing Java package. The communication between the individual modules is represented by arrows. The numbering on the arrows defines the individual steps in the application creation and execution process. The different modules are described below and the interaction between them is explained. The modules are all loosely coupled, so they can be easily extended/replaced.

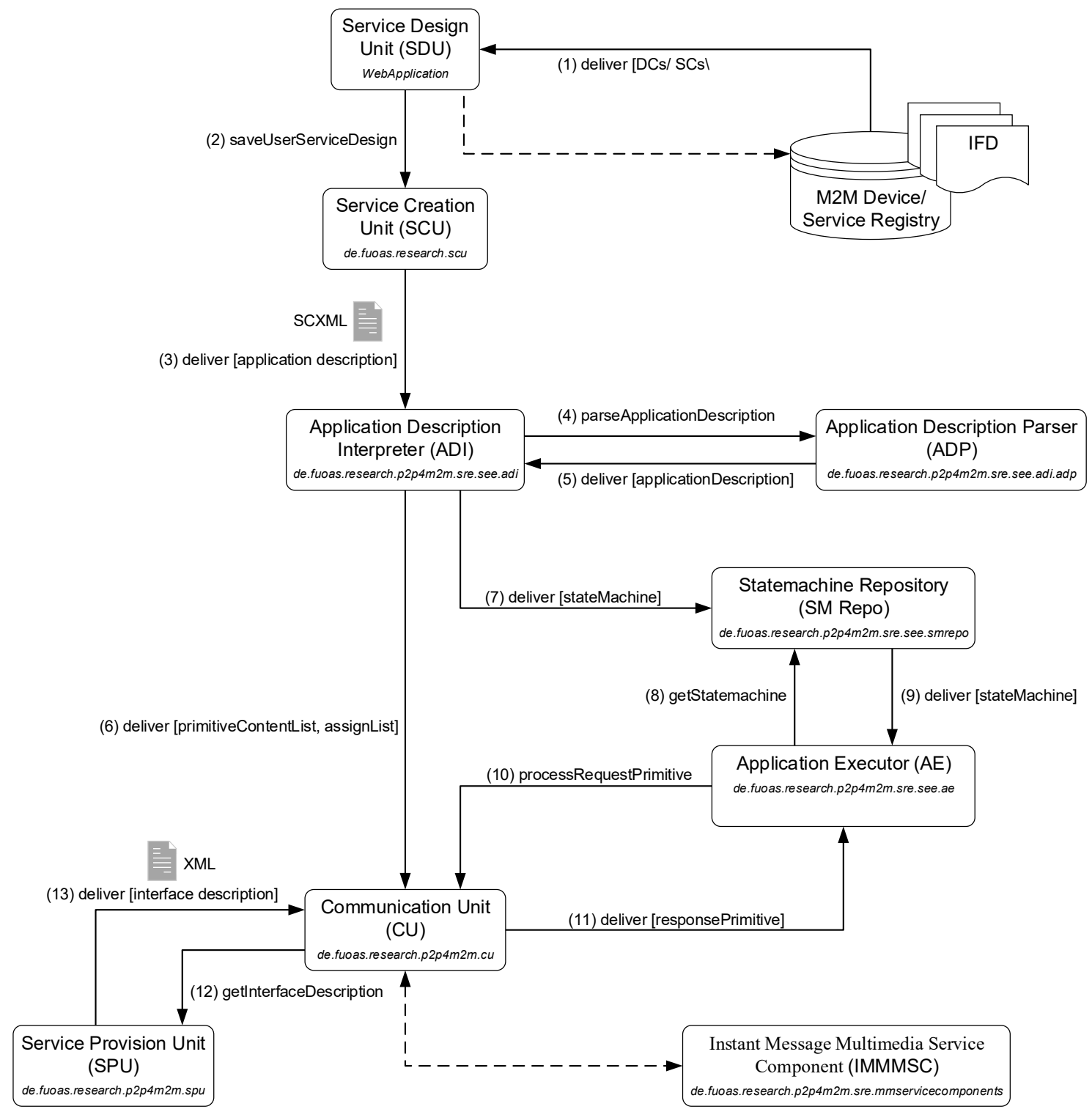

Figure 7.4: Research Prototype Architecture Components (illustrated as Packages) 
Application creation starts with modelling the application. The $S D U$ module has been developed in the form of a GUI which is illustrated in Figure 7.5.

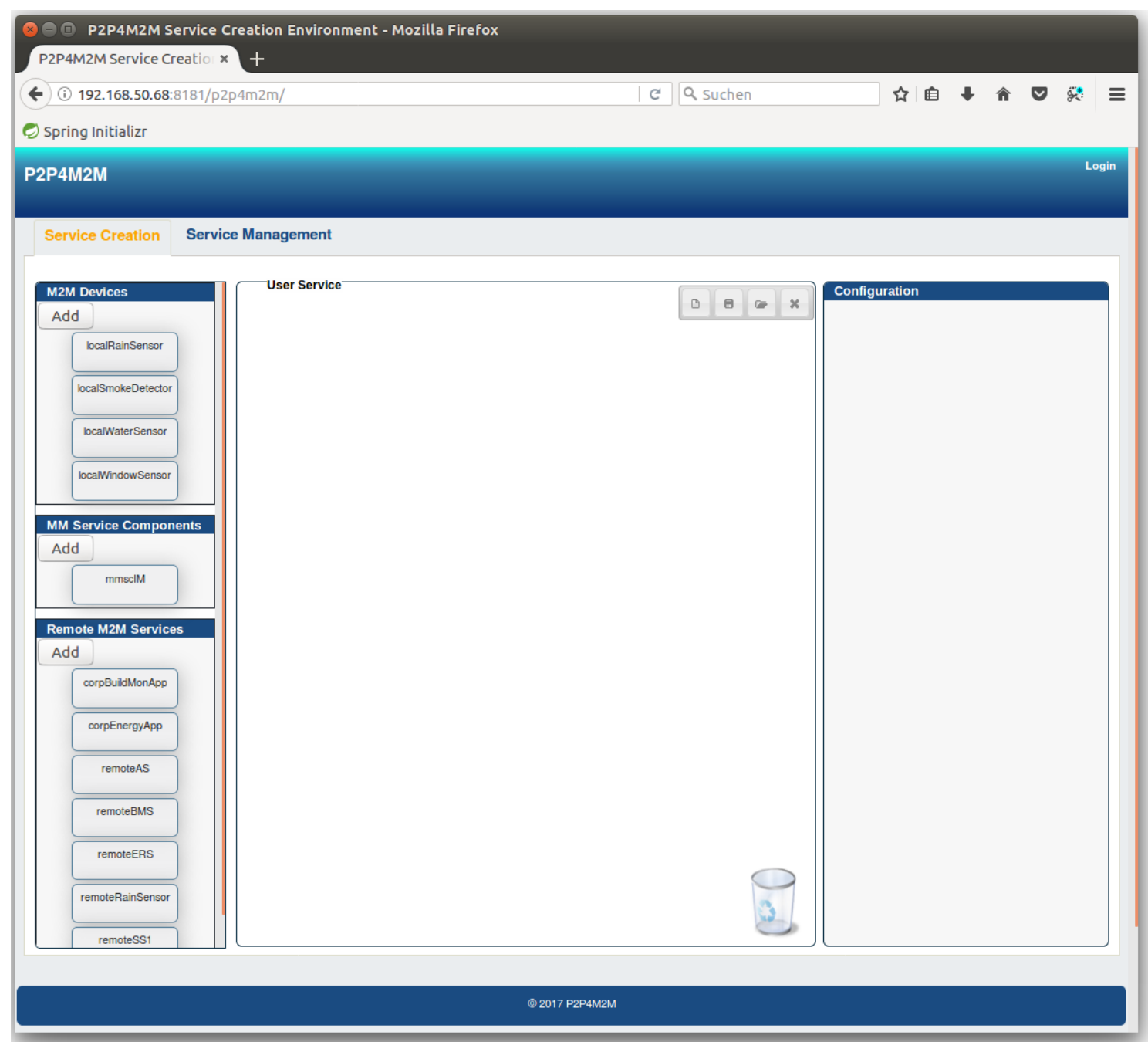

Figure 7.5: Screenshot of Service Design Unit GUI Web Application

The GUI is provided as a web application enabling the end-user to model the behaviour of an application as a SM. The WebContainer "Jetty" (Eclipse Jetty, 2017) integrated in Apache Karaf was used to provide the web application. The SM is modelled by dragging the building blocks representing M2M devices or M2M services into the workspace and connecting them to each other. Each building block (state) and the connections between the building blocks can be configured as described in section 5.5.3. The JavaScript library 
"JsPlumb" (JsPlumb, 2017) was used in Community Edition v1.5.5 for the implementation of the graphical modelling. JsPlumb allows to graphically model flow chart diagrams within a web application. To obtain an overview of the available M2M devices/services, the $S D U$ receives a HashMap with capability objects representing the device/service capabilities or interface descriptions of the remote M2M services (step 1). The capability representations are also used to display the configuration area for the states as an input mask (see Figure 7.6). This defines the configuration parameters of a state and displays the prose description of the M2M devices/service.

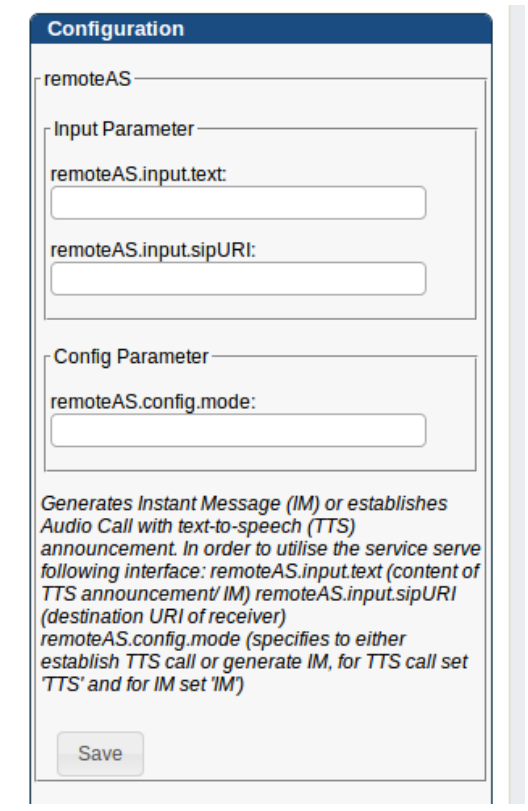

Figure 7.6: Screenshot of SDU GUI showing M2M Device/Service Configuration Section

Using JsPlumb, a data model is generated which describes the states and the connections between the states. The SDU generates two HashTables describing the states and transitions including the defined parameter configurations. By calling the saveUserServiceDesign method, these HashTables containing the application model and further information about initial and final state are passed to the $S C U$ as a JSON object (IETF RFC 7159, 2014) (step 2). The SCU module generates a Java object from the 
received data containing the application information. The $S C U$ then generates a formal description of a SM that represents the application logic according to the principles described in section 5.5.3 and transfers the SCXML document to the ADI module (step $3)$.

In addition to generating the graphical application model, the $S C U$ also generates the interface description for a modelled application. The content of the interface description described in section 6.1.1 (e.g. input parameter, AppID, prose description) is defined by the application creator using an input mask in the $S D U$ (see Figure 7.7). The $S D U$ passes this information back to the $S C U$ as a JSON object which then generates an interface description in XML format. This is done by the $S C U$ converting the JSON object with the interface description information into a Java object. Both, generation of the SCXML description and interface description is done with JAXB (Oracle, 2017c). This API enables to import XML documents and process them as Java objects, as well as to generate an XML document from a Java object.

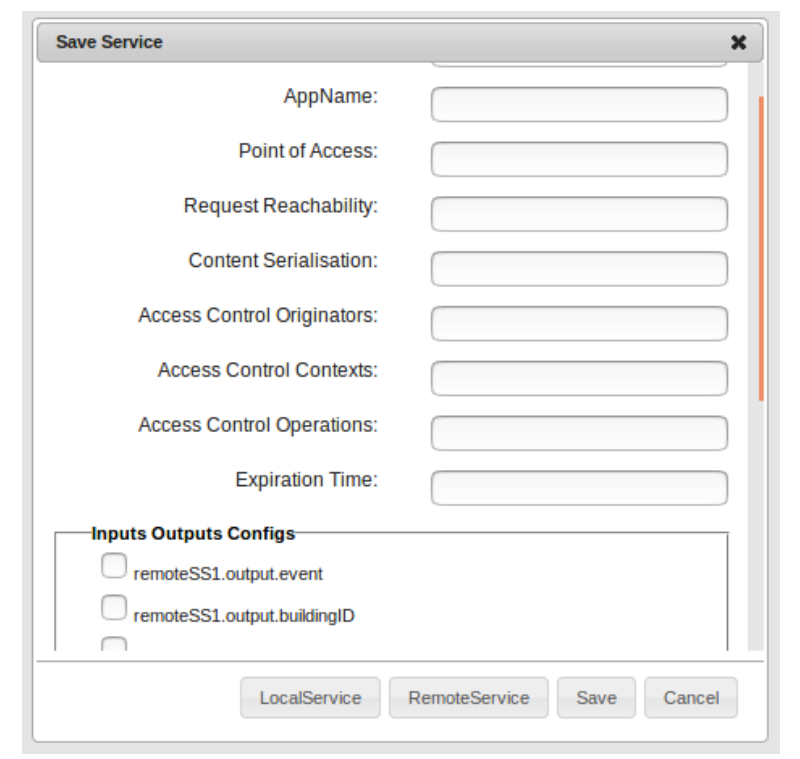

Figure 7.7: Screenshot of SDU GUI showing IFD Specification Form 
In order for the $A D I$ to be able to process the application logic defined in the application description, the $A D I$ requires it in a compatible format. To obtain this, the $A D I$ invokes the method parseApplicationDescription of its internal component ADP (step 4). The $A D P$ returns the formally described application logic to the $A D I$ in the form of an object (step 5). The generation of the Application Description Object is done by the ADP reading and parsing the SCXML representation of the application. Parsing is done using the Java library "Apache Commons SCXML" (Commons SCXML, 2016) which allows to get a representation of the statechart described with SCXML in the form of Java objects. The $A D P$ analyses the generated Java objects and transfers the information on the application logic (such as applicationID, initialStateID, finalStateID) into an internal application description model (ADM). Furthermore, the $A D P$ analyses all defined states and transfers the information defined such as stateDataModel, transitions, assigns, targetStates, predecessorStates into the ADM.

The $A D I$ now analyses the ADM and interprets the application logic. The $A D I$ must differentiate between a distributed application and a local application that integrates remote services. In the case of a local application, the $A D I$ first determines whether remote services are to be integrated into the local application. This is the case if the ADM includes states that refer to a remote service or attributes of a remote service are contained in transition conditions or assignments. The $A D I$ registers remote services to be integrated in a primitiveContentList which it transfers to the $C U$ module (step 6). In the next step, the $A D I$ generates a local SM from the ADM, which is represented by an internal SM model. To create the SM model, the $A D I$ analyses all state information defined in the $\mathrm{ADM}$ and creates an entry for each of the states in the SM. The $A D I$ adds information about the state transitions (e.g. targetState, condition, stateConfiguration, 
targetStateConfig) to these entries, which it also extracts from the ADM. Furthermore, the information about initialState and finalState as well as the currentState of a SM are added. The generated stateMachine object is passed to the SM Repo module, which manages the generated SMs in an internal HashMap allStateMachines (step 7). If it is a distributed application provided by multiple peers, no local SM is created. Instead, additional information is required for linking to other peers. The $A D I$ also determines this information by analysing the ADM. In this case, the state within the application description is in focus that represents the local peer or the service offered by the local peer. It is necessary to determine which other services a peer should connect to. The $A D I$ receives this information by determining a list of predecessorStates. Additionally, it is necessary to determine which parameters of a predecessorStates are to be requested if necessary. For all states in the predecessorStateList, the ADI therefore determines whether output parameters are contained in their assign definitions and registers them in an outputParameterList. Furthermore, the $A D I$ will determine the information to be sent to a connected service. This is done by analysing the stateTransitionList of its own state. This creates a list (assignList) which contains the condition and remote parameter assignments for each successorStateID. The $A D I$ has thus determined all the information necessary for linking with other peers and transmits this information to the $C U$ module (step 6).

The module $A E$ executes the local SM. For this purpose, the $A E$ first requests a list of all existing SMs from the SM Repo. The $A E$ periodically traverses the list of all SMs and loads the respective SM from the SM Repo using the getStateMachine method (step 8). The SM Repo provides the $A E$ with the requested SM (step 9), which is executed by the $A E$. This is done by the $A E$ determining and analysing the currentState of the SM via the 
currentState attribute. For this active state, the $A E$ sets the stateConfiguration and checks if the condition (if defined) for the transition to the next state is true. If the condition is true, the $A E$ sets the parameters defined via targetStateConfig and sets the targetState as new currentState. To check the condition and to set stateConfiguration and targetStateConfig, the $A E$ requests the $C U$ via a RequestPrimitive object (step 10) and receives the parameter value that is required for checking the condition via a ResponsePrimitive object (step 11).

The $C U$ is responsible for communication with local M2M devices and MMSCs or for communication with other peers. For communication via CoAP and SIP, communication modules have been integrated into the $C U$ for both protocols. For this purpose, the Java libraries "Californium" (Eclipse Californium, 2017) were used as CoAP stack and JAIN SIP (JAIN SIP, 2017) as SIP stack, which enable generating and receiving of CoAP/SIP messages via local network interface. For remote service requests using CoAP and SIP, the communication modules enable the query scenarios as defined in section 6.2.3 (onetime and continuous subscription of output parameter, termination of subscriptions and input/config requests). When remote services are requested, the $C U$ traverses the primitiveContentList to request parameters from the SP. To do this, the $C U$ generates a service request message as defined in section 6.2.3. To manage subscriptions, the $C U$ includes the SubscriptionManager component. All active subscriptions are managed in SubscriptionManager (activeSubscriptionsList). The SubscriptionManager manages besides the activeSubscriptions also a list of activeNotifications. This stores the requests for services generated by other peers. If the $C U$ receives a service request from another peer, it extracts the information defined in it, creates a requestPrimitive object and transfers it to the $A L$ component for processing. If a parameter is queried by the service 
request, the $C U$ generates a Request Primitive message and replies it to the requesting peer. If the service request is a continuous subscription, the $C U$ saves the generated responsePrimitive object and a defined condition in the activeNotification list. The component notificationAutomator of the $C U$ runs periodically through the activeNotifications list and checks if defined conditions are true. If this is the case, or if no condition has been defined, the notificationAutomator triggers the sending of a Request Primitive message to the defined receiver via the CoAP or SIP communication module.

The $A L$ component of the $C U$ is implemented using an internal $m 2 m$ DeviceServiceCache. This contains a representation of the parameters defined via the Device Capability Model. Parameters that are to be set or queried for an M2M device or an MMSC are processed by this component. To determine the addressing information of an M2M application service provider, the $C U$ requests the interface description from the $S P U$ module using the getInterfaceDescription method (step 12), which then returns the InterfaceDescription for a specific service (step 13).

The SPU implements the connection to a P2P overlay for distributed management of interface descriptions. The SPU has the functionality to join a Gnutella overlay or a Chord overlay. To realise the Gnutella overlay functionality, the Java Library “JTella” (JTella, 2016) was used, which is an implementation of the unstructured P2P overlay Gnutella. To implement the Chord overlay functionality, the Java library "Open Chord" (Open Chord, 2015) was used, which is an implementation of the Chord DHT. The SPU saves the URL of an interface description within the overlay and can also query it in the overlay. The URL of the interface description specifies the peer that stores the interface description 
as XML document. To download the interface description from the peer, the $S P U$ includes a TCPfileServer and a TCPfileClient. The TCPfileClient requests the TCPfileServer of the target platform with the service-ID, which then returns the interface description document. To transfer the interface description, it must first be serialised and transmitted as TCP stream. On the TCPfileClient side, the serialised interface description must be deserialised again. Serialisation and deserialization is done again using JAXB.

In addition to the modules described above, the IMMMSC was implemented. This MMSC is considered to be a representative of the MMSCs defined in section 5.1. IMMMSC enables receiving and sending IMs. IMMMSC uses the SIP communication module of the $C U$ as well as the $A L$. The $A L$ contains a representation of the capability description of the IMMMSC and manages its input and output parameters. The $A E$ can therefore use this interface to generate an IM or to process the text that has been sent to the platform via an IM. To provide an input and output interface for the end-user, an IM client has also been implemented as representation of a mobile phone interface (see Figure 7.8).

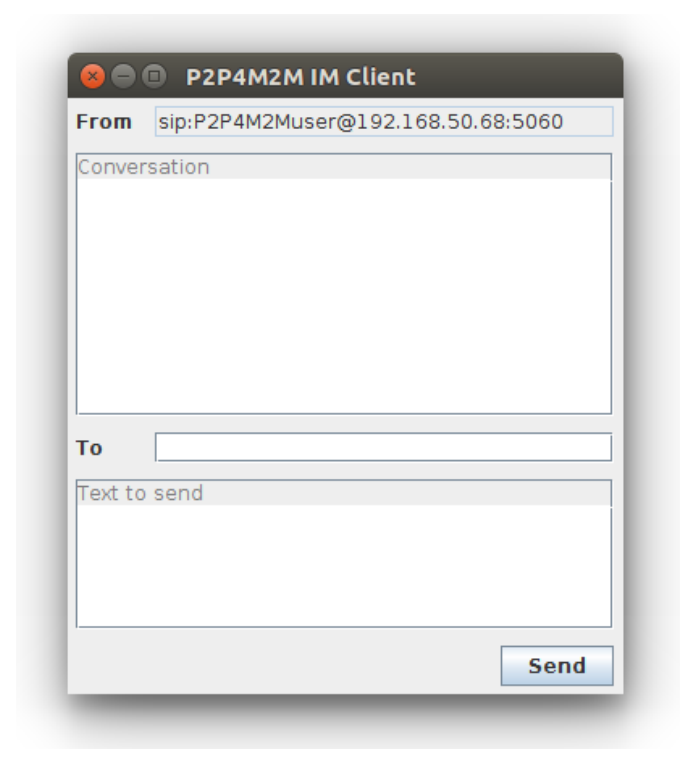

Figure 7.8: Screenshot of Instant Message Client 
In addition to the communication modules for CoAP and SIP, the $C U$ has also implemented communication modules for the TC IP 1 control protocol and for BidCos. The physical integration of M2M devices using TC IP 1 Control Protocol and BidCos were implemented in the associated e-SCHEMA research project (Steinheimer et al., 2013b; e-SCHEMA, 2015). The TC IP 1 communication module allows to control several energy manager devices and to read out the parameters provided by their sensors. The communication with the devices is realised via datagramSockets, which can be addressed natively from the Java programming language. To implement the communication via BidCos it was necessary to use an M2M gateway (so-called Common Control Unit, CCU) which implements the wireless communication with the M2M devices. The BidCos communication module communicates with the $C C U$ via XML RPC (XML-RPC, 1999) to transmit the control commands to the $\mathrm{M} 2 \mathrm{M}$ devices or to query parameters from the M2M devices. To establish a communication channel between the BidCos control module and the $C C U$, the communication module must register with the XML-RPC server of the $C C U$. Furthermore, it is necessary that an XML RPC server is also used in the BidCos communication module, so that the $C C U$ can send push messages to the BidCos control module. Both, the communication with the $C C U$ via XML-RPC and the operation of a local XML-RPC server is realised using the Java library "Apache XML-RPC" (Apache XML-RPC, 2017).

\subsection{Proof of Framework Concepts}

After the architecture and implementation of the research prototype was presented based on the novel concepts of the framework for "Autonomous decentralised M2M 
Application Service Provision", this section deals with the proof of concept and the evaluation of the underlying approaches. The research prototype was developed to demonstrate the essential aspects of the presented framework. To cover as many aspects of the presented framework as possible, the following use cases from the use cases presented in section 2.4 were selected:

- Use Case 2: Neighbourhood Weather Station - Local execution of SM-based application logic with remote $\mathrm{M} 2 \mathrm{M}$ service integration. A remote $\mathrm{M} 2 \mathrm{M}$ service provides the values of a rain sensor. A local M2M application integrates these values. If rain is detected and the window is open at the same time, an IM will be sent with a corresponding message.

- Use Case 3: Building Surveillance - Cooperative M2M application service provision based on horizontal M2M application service composition. A remote M2M service monitors water and smoke sensor in a building and reports them (in case of corresponding event occurred) to a remote building monitoring service that manages supporters of that building. The building monitoring service triggers another remote alarm service to send an IM with a specific text (depending on the event) to end-users taking care of the corresponding building.

For both scenarios, the M2M applications must be modelled and transferred to the formal application description. The application description must be interpreted and the local system configuration must be performed according to the defined logic. The M2M application must then be executed. The following section 7.3.1 demonstrates these steps for Use Case 2 and section 7.3.2 for Use Case 3. Since both contain the service request from other nodes, this scenario is considered separately in section 7.3.3. 


\subsubsection{Local M2M Application Execution with remote M2M Service}

\section{Integration}

First, the M2M application for Use Case 2 is graphically modelled. Figure 7.9 shows the graphical model and a section of the automatically generated formal M2M application description.

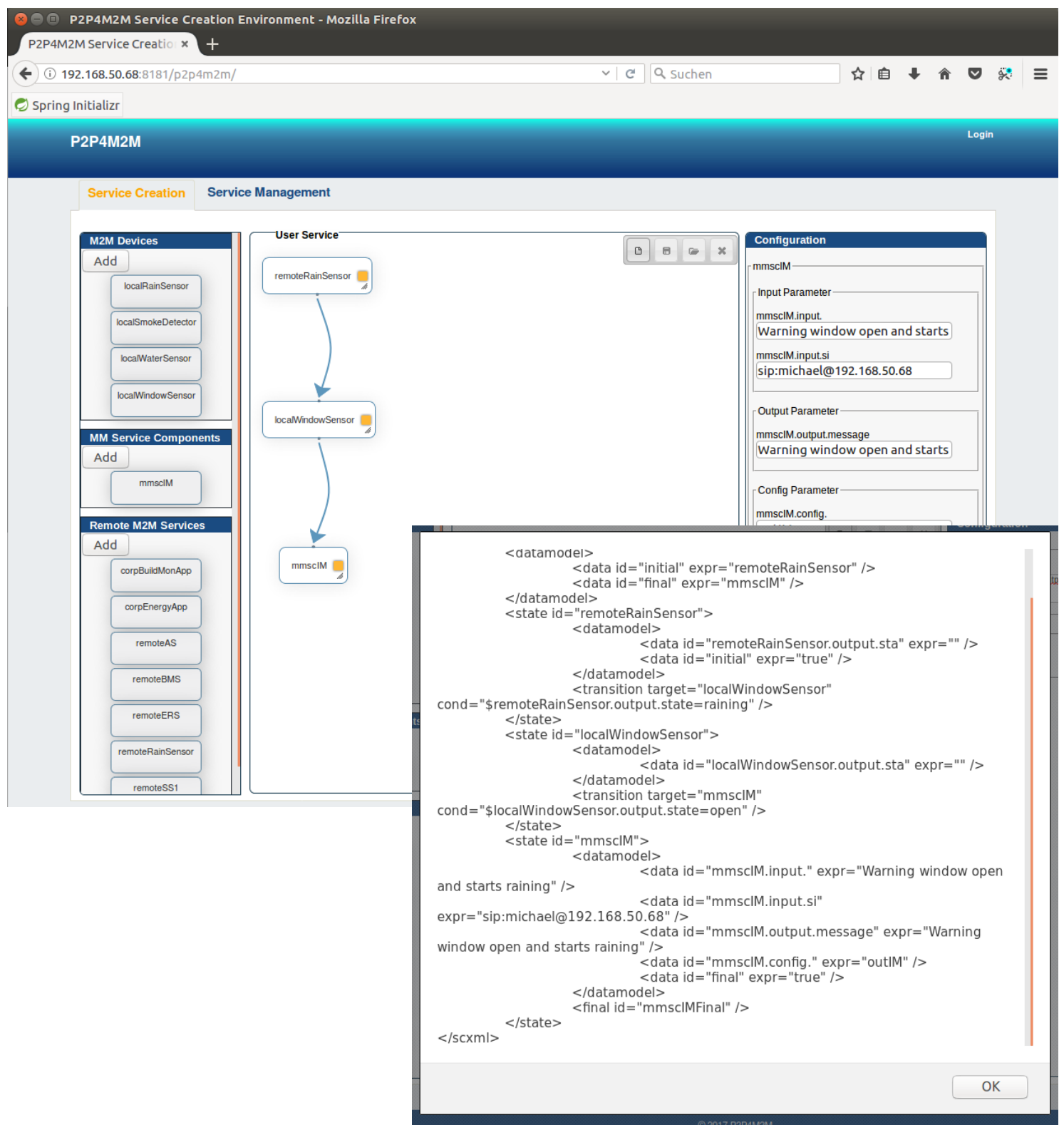

Figure 7.9: Screenshot of SDU GUI Web Application with SM Model Use Case 2 
The building blocks remoteRainSensor (remote M2M service providing rain sensor information data), localWindowSensor (provides the state of a window sensor located in local environment) and mmscIM (MMSC enabling sending of instant messages) are used in this scenario. These building blocks are placed in the workbench and connected with each other. States and transitions are configured as shown in Table 7.1 and Table 7.2, thus completing the definition of the application logic.

Table 7.1: State Configuration Use Case 2

\begin{tabular}{|l|l|l|}
\hline $\begin{array}{l}\text { State (Device/MM/M2M Service } \\
\text { Building Block) }\end{array}$ & \multicolumn{2}{|c|}{ Configuration } \\
\hline remoteRainSensor & \multicolumn{2}{|c|}{ No configuration required } \\
\hline localWindowSensor & \multicolumn{2}{|c|}{ No configuration required } \\
\hline \multirow{3}{*}{ mmscIM } & Parameter & Value \\
\cline { 2 - 3 } & mmscIM.input.text & Warning window open and starts raining \\
\cline { 2 - 3 } & mmscIM.input.sipURI & sip:michael@192.168.50.68 \\
\cline { 2 - 3 } & mmscIM.config.mode & outIM \\
\hline
\end{tabular}

Table 7.2: Transition Configuration Use Case 2

\begin{tabular}{|l|l|l|}
\hline $\begin{array}{l}\text { Transition (connection between } \\
\text { building blocks) }\end{array}$ & \multicolumn{2}{|c|}{ Configuration } \\
\hline $\begin{array}{l}\text { remoteRainSensor --> } \\
\text { localWindowSensor }\end{array}$ & Expression & \$remoteRainSensor.output.state=raining \\
\hline localWindowSensor --> mmsclM & Expression & \$localWindowSensor.output.state=open \\
\hline
\end{tabular}

Saving the M2M Application Model automatically generates the formal description of the M2M application in SCXML format (illustrated in Figure 7.10). The formal description can also be viewed via the GUI (see Figure 7.9). 


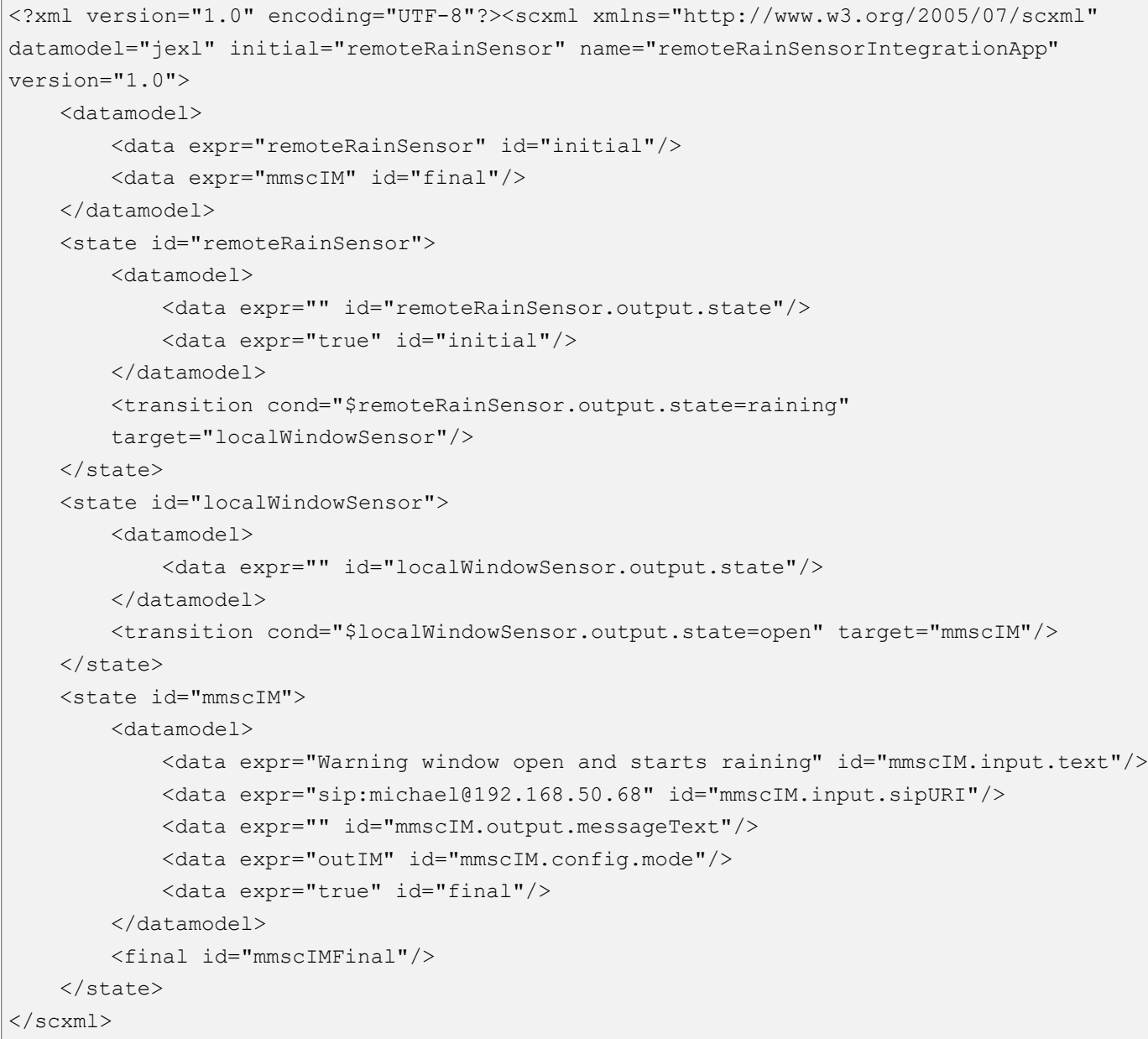

Figure 7.10: Use Case 2 M2M Application Description

Once the application has been formally described, the application description is transferred to the ADI. The ADI triggers the ADP which then imports the application description. Figure 7.11 shows the information imported by ADP into an internal application description object. 


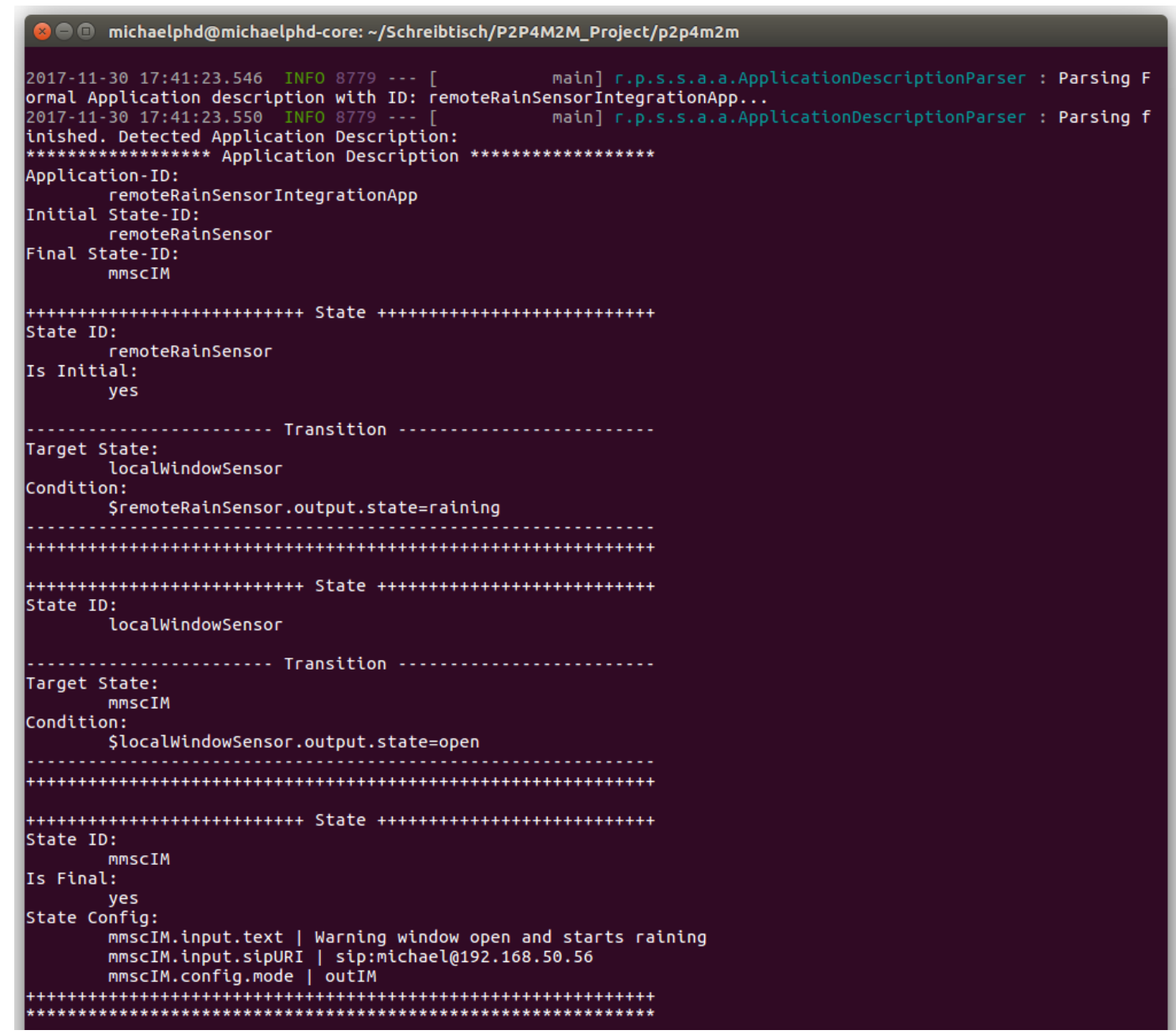

Figure 7.11: Screenshot of Terminal Output ADP Use Case 2

The ADP returns the application description object to the ADI which processes the information defined in it to configure the application locally. In this case, this means that the ADI generates a local SM as shown in Figure 7.12 and stores it locally. Figure 7.13 shows the SM object generated by the ADI.

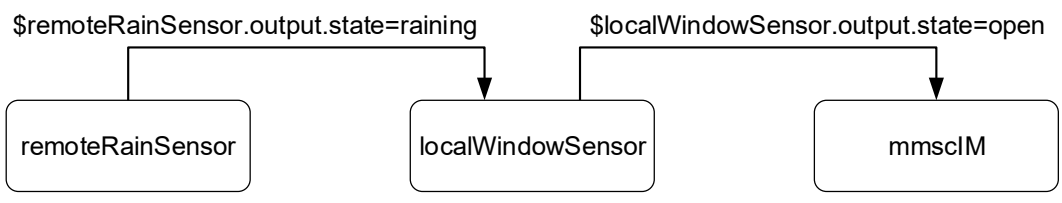

Figure 7.12: SM generated by ADI Use Case 2 


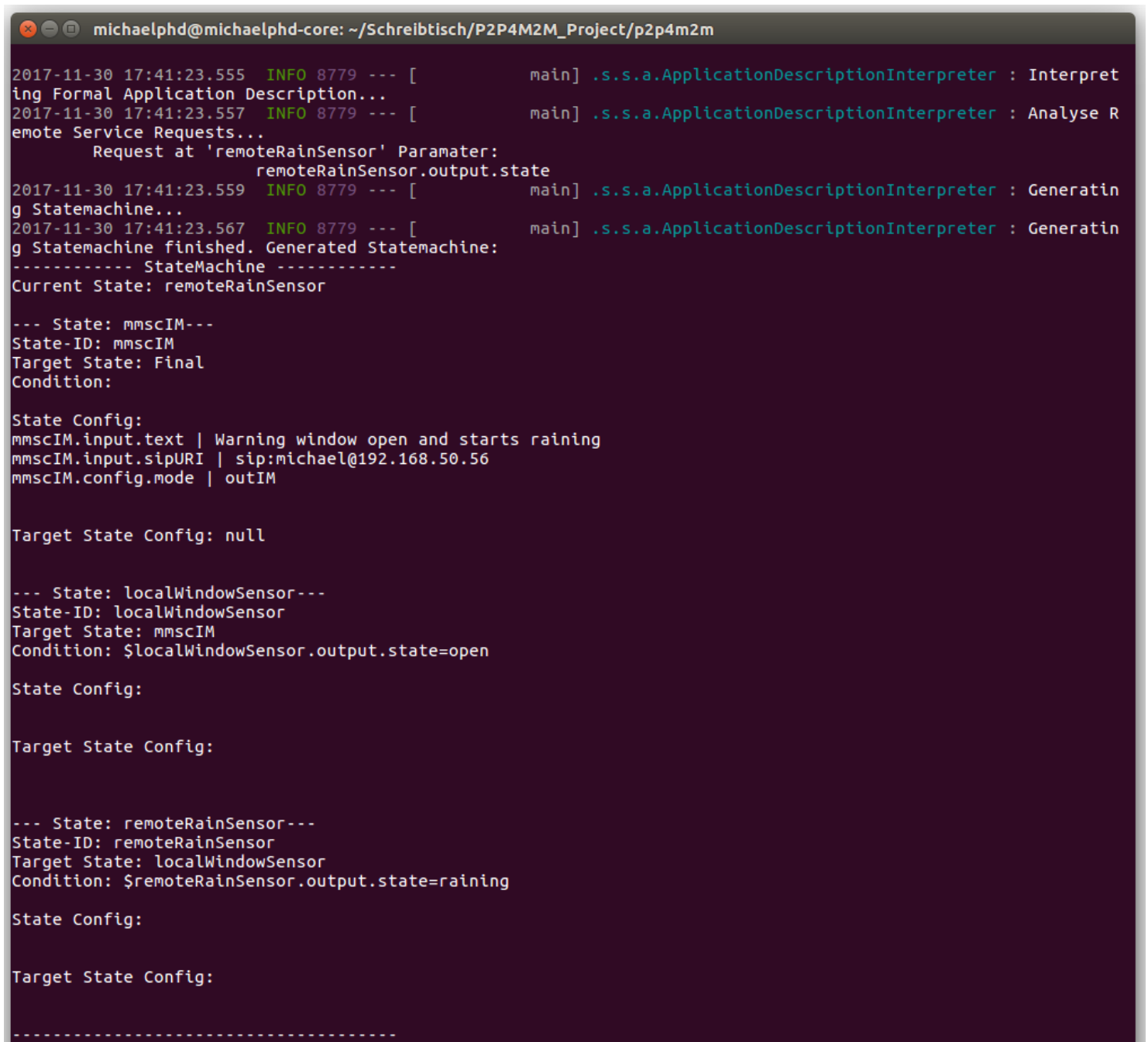

Figure 7.13: Screenshot of Terminal Output ADI Use Case 2

The outlined scenario integrates a remote $\mathrm{M} 2 \mathrm{M}$ service that provides the values of a rain sensor. To use this service, it must be requested from the SP. The following sequence chart (see Figure 7.14) illustrates the service request and information delivery process. 


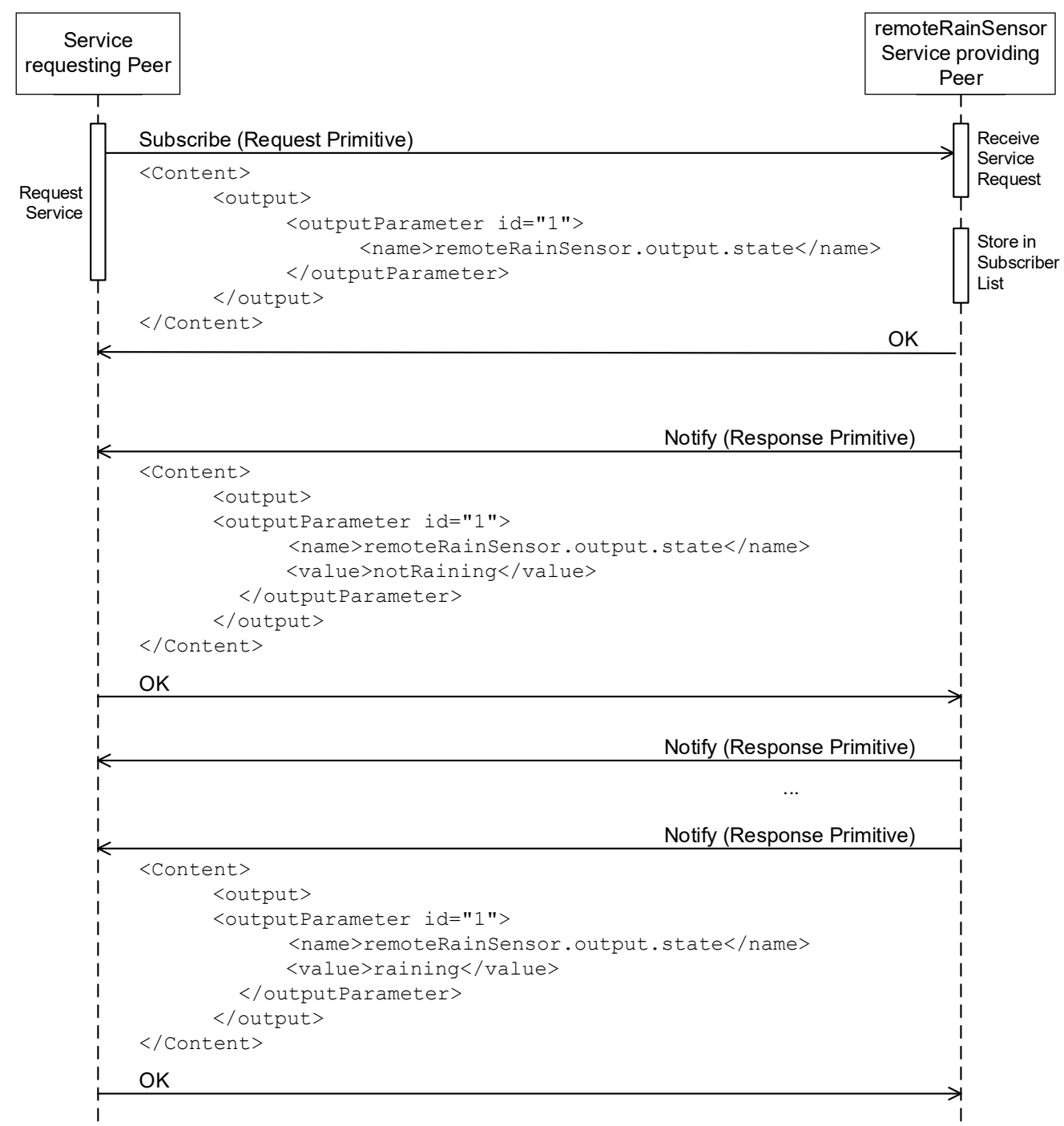

Figure 7.14: SM generated by ADI Use Case 2

After the SP has received the service request, it continuously sends the rain sensor values to the SC, which stores them locally.

The Application Executor (AE) component periodically executes the SM by checking the current state. As soon as the value of the remote rain sensor changes to status "raining", the AE initiates the transition to the next state. If the output value of the local window sensor now has the value "open", a state transition is also triggered which causes the local 
MMSC for sending Instant Messages (mmscIM) to send an IM to the specified user (see Figure 7.15).

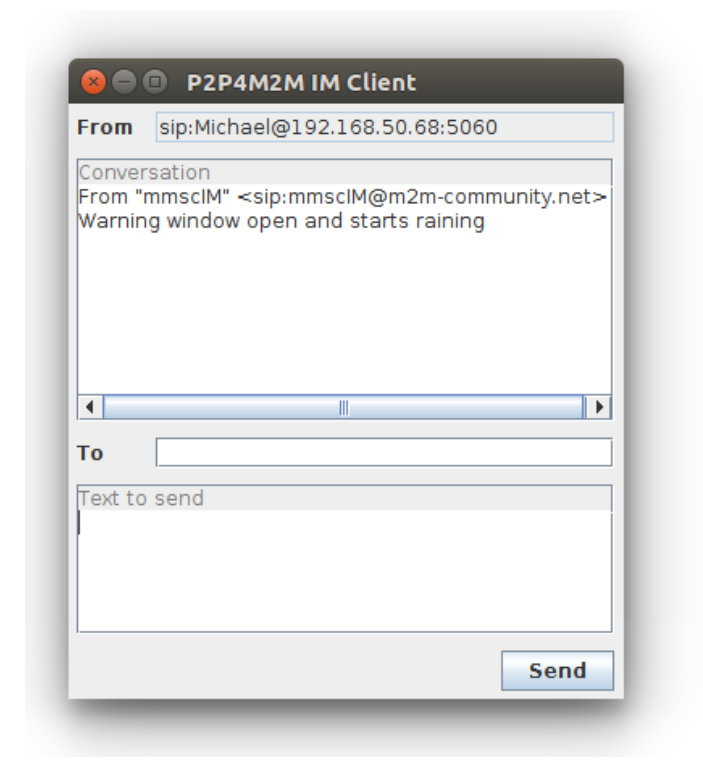

Figure 7.15: Screenshot of IM Client Use Case 2

This section demonstrated the local M2M application creation and execution processes. The following section demonstrates the cooperative M2M application service provision and execution.

\subsubsection{Cooperative M2M Application Service Provision}

The second scenario to demonstrate (Use Case 3) also starts with modelling the M2M application description in the same way as the previous one (refer to Figure 7.16). In this scenario the following building blocks are used: remoteSS1 (remote M2M service that monitors a building and generates information about detected events), remoteBMS (remote M2M service managing supporter registered for specific buildings), and remoteAS (remote M2M service providing the ability to send Instant messages). 


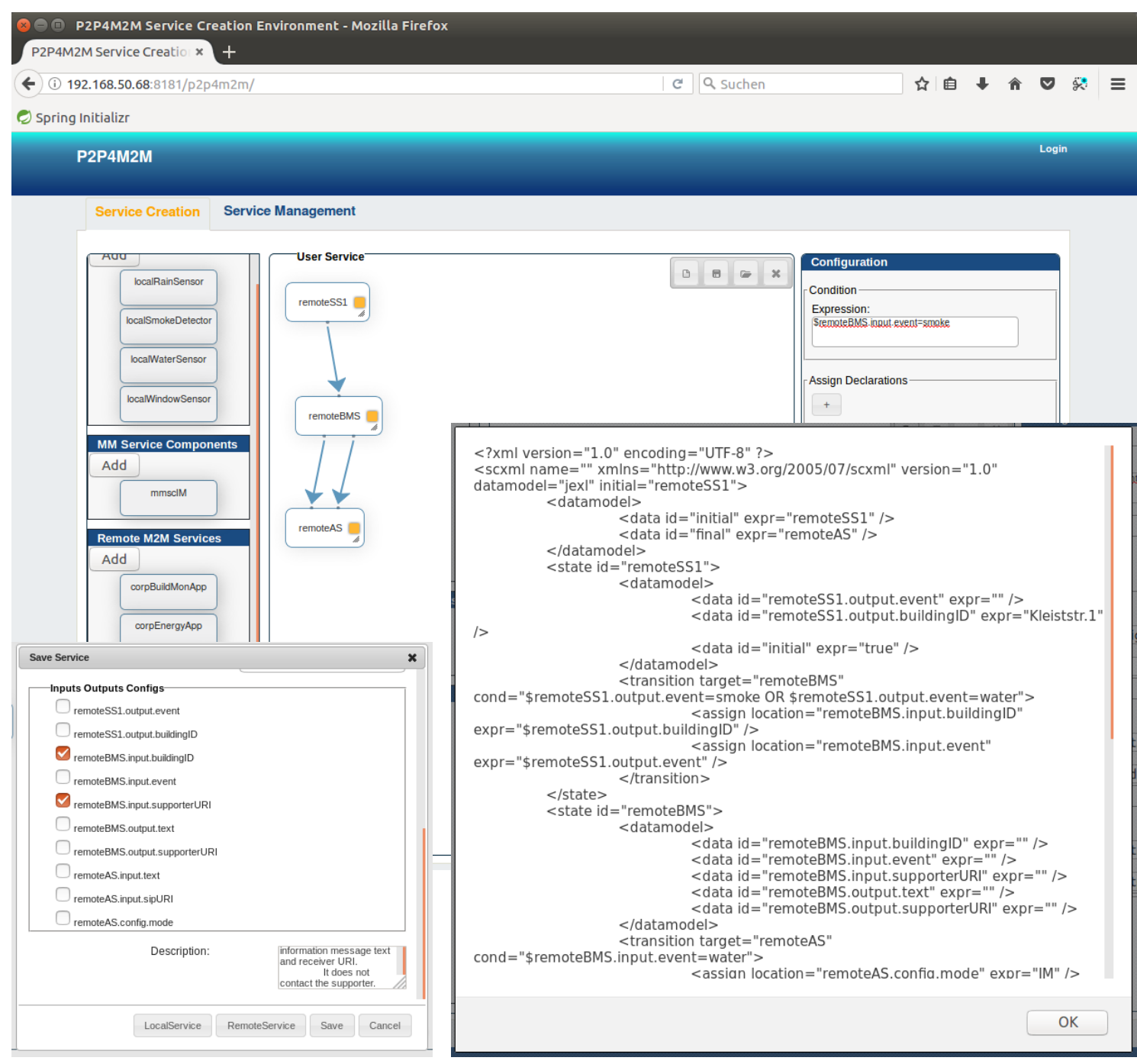

Figure 7.16: Screenshot of SDU GUI Web Application with SM Model Use Case 3

The configuration of the states and the transitions is illustrated in Table 7.3 and Table 7.4.

Table 7.3: State Configuration Use Case 3

\begin{tabular}{|l|l|l|}
\hline $\begin{array}{l}\text { State (Device/MM/M2M } \\
\text { Service Building Block) }\end{array}$ & \multicolumn{2}{|c|}{ Configuration } \\
\hline \multirow{2}{*}{ remoteSS1 } & Parameter & Value \\
\cline { 2 - 3 } & remoteSS1.output.buildingID & Kleiststr.1 \\
\hline remoteBMS & \multicolumn{2}{|c|}{ No configuration required } \\
\hline remoteAS & \multicolumn{2}{|c}{ No configuration required } \\
\hline
\end{tabular}


7 Research Prototype and Framework Evaluation

Table 7.4: Transition Configuration Use Case 3

\begin{tabular}{|c|c|c|c|}
\hline Transition & \multicolumn{3}{|c|}{ Configuration } \\
\hline \multirow{4}{*}{$\begin{array}{l}\text { remoteSS1 --> } \\
\text { remoteBMS }\end{array}$} & Expression & \multicolumn{2}{|c|}{ \$remoteSS1.output.event=smoke OR \$remoteSS1.output.event=water } \\
\hline & & Location & Expression \\
\hline & Assign (1) & remoteBMS.input.buildingID & \$remoteSS1.output.buildingID \\
\hline & Assign (2) & remoteBMS.input.event & \$remoteSS1.output.event \\
\hline \multirow{5}{*}{$\begin{array}{l}\text { remoteBMS --> } \\
\text { remoteAS (1) }\end{array}$} & Expression & \multicolumn{2}{|c|}{ \$remoteBMS.input.event=water } \\
\hline & & Location & Expression \\
\hline & Assign (1) & remoteAS.config.mode & IM \\
\hline & Assign (2) & remoteAS.input.text & Water detected \\
\hline & Assign (3) & remoteAS.input.sipURI & \$remoteBMS.output.supporterURI \\
\hline \multirow{5}{*}{$\begin{array}{l}\text { remoteBMS --> } \\
\text { remoteAS (2) }\end{array}$} & Expression & \multicolumn{2}{|c|}{ \$remoteBMS.input.event=smoke } \\
\hline & & Location & Expression \\
\hline & Assign (1) & remoteAS.config.mode & IM \\
\hline & Assign (2) & remoteAS.input.text & Smoke detected \\
\hline & Assign (3) & remoteAS.input.sipURI & \$remoteBMS.output.supporterURI \\
\hline
\end{tabular}

This configuration defines that if a an event in the building monitored by remoteSS1 occurs that is "water" or "smoke", remoteSS1 should send the event occurred to remote BMS. RemoteBMS should invoke another service remoteAS for sending an alarm message to supporters registered for the building to inform them about the event. Saving the M2M Application Model again automatically generates the formal description of the M2M application (illustrated in Figure 7.17). This formal description then is transferred to the service providing peers included in the service composition. Here it should be again highlighted that all participating peers stay at different locations. 


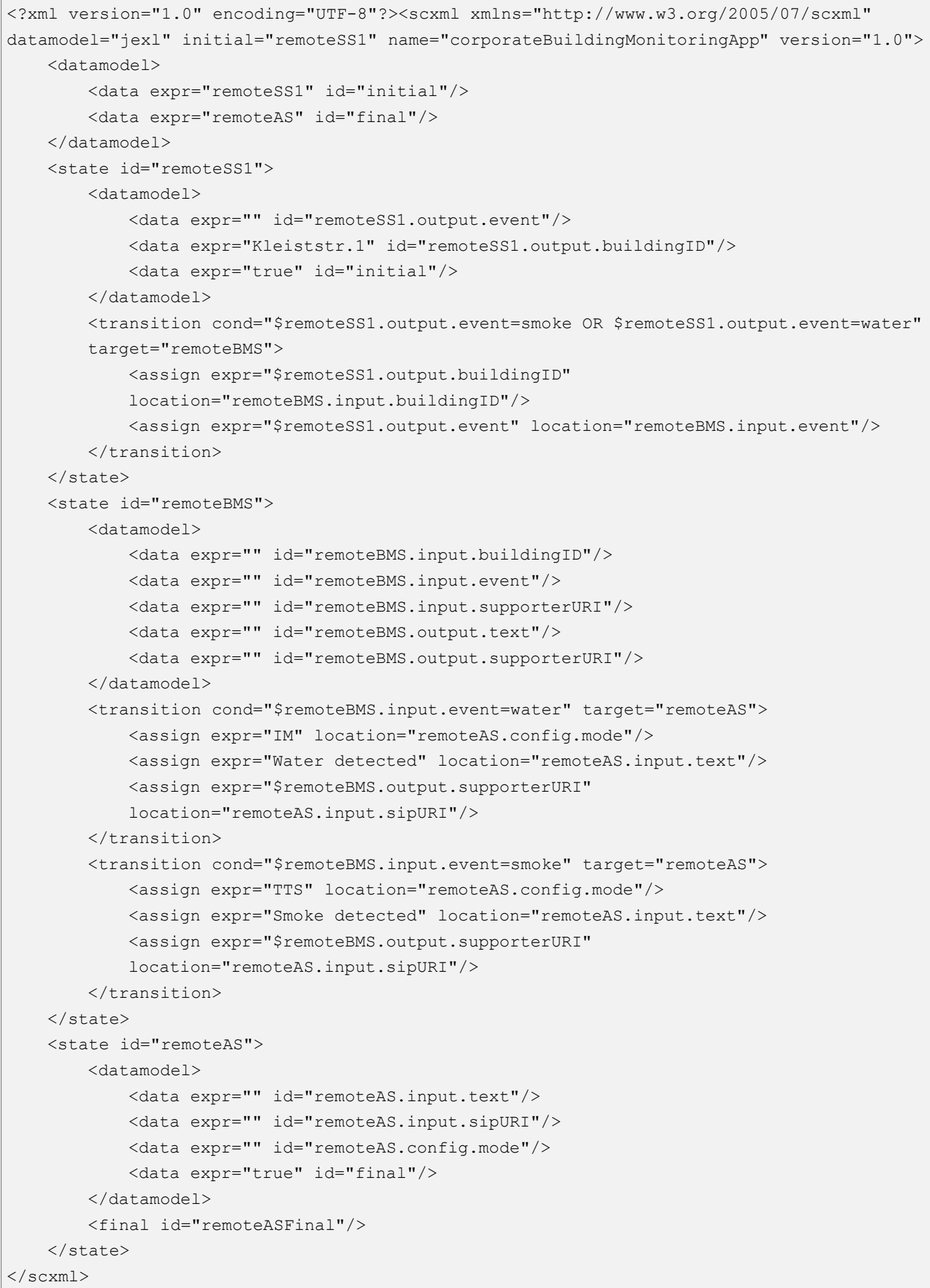

Figure 7.17: Use Case 3 M2M Application Description

Since it is an M2M application, which should be used by other peers (Building supporter, which want to register for a specific building), the designed cooperative M2M application 
needs to be equipped with an application IFD. This IFD is generated using the SDU GUI (illustrated in Figure 7.16) and afterwards stored in the P2P overlay. Figure 7.18 shows the automatically generated IFD.

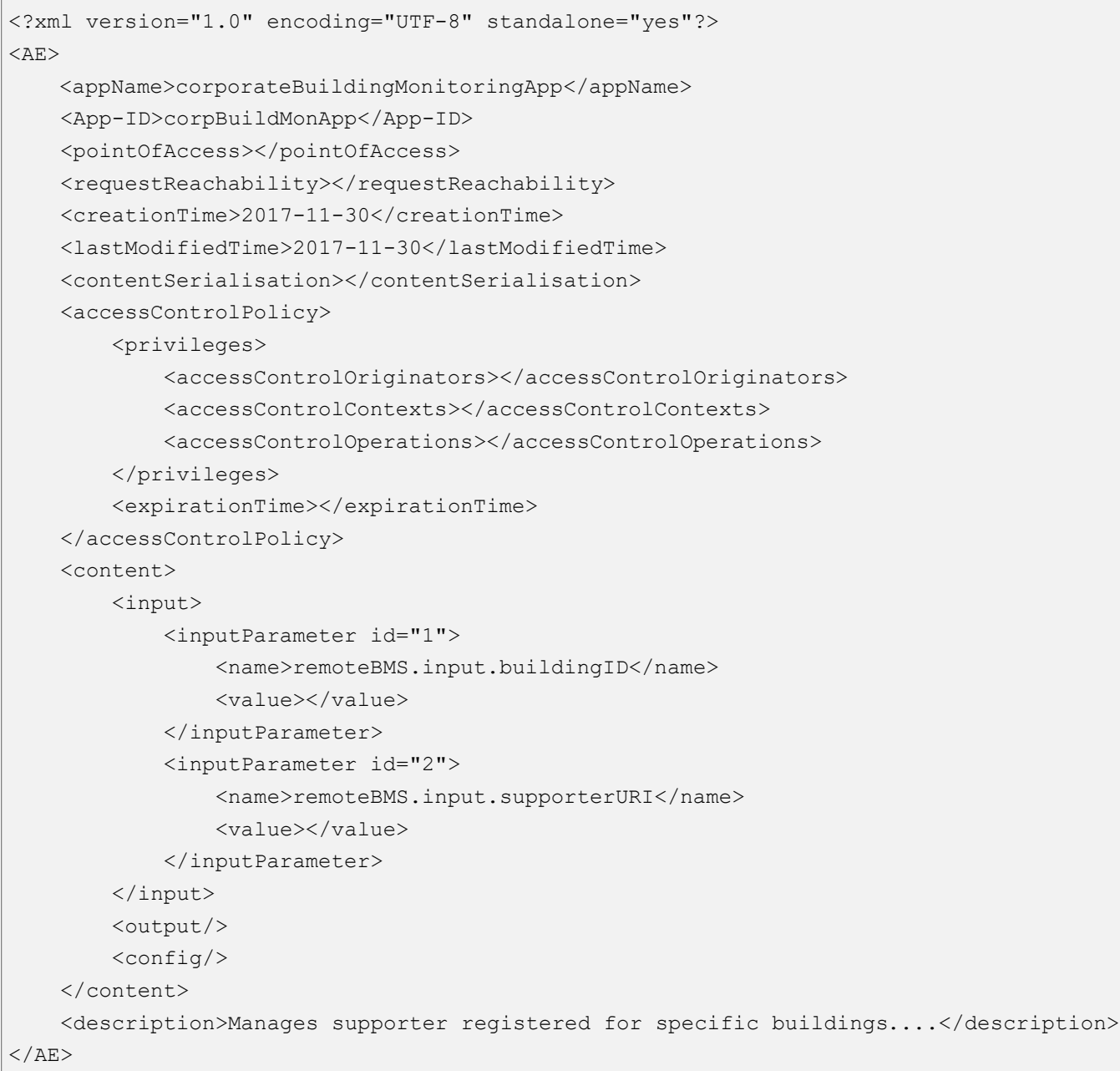

Figure 7.18: Use Case 3 corporateBuildingMonitoringApp IFD

The ADI of each peers interprets the formal application description by means of the ADP providing the internal application description object. This step is illustrated in Figure 7.19 once for all peers. 


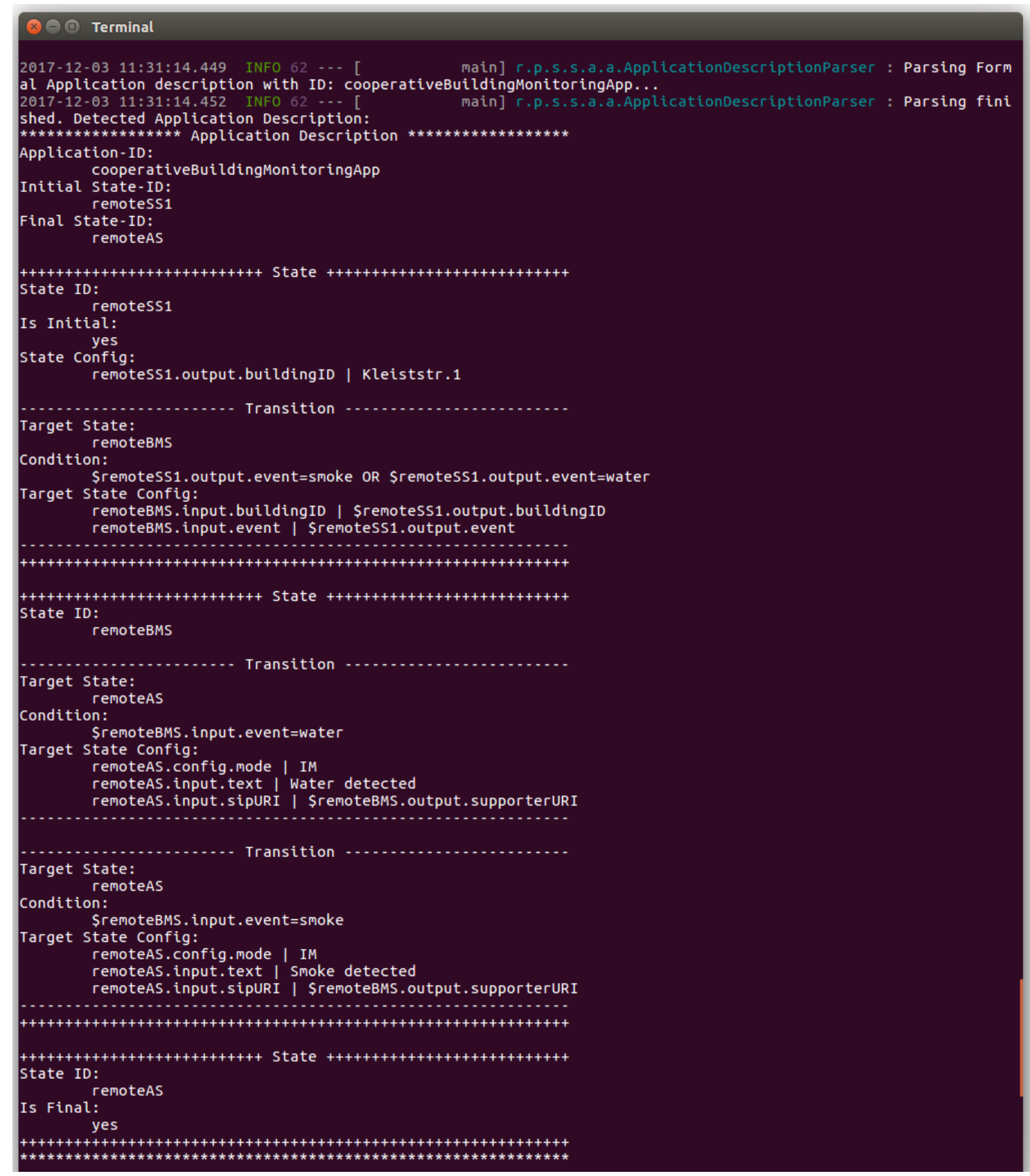

Figure 7.19: Screenshot of Terminal Output ADP Use Case 3

After generation of the application description object the peers process it to perform their individual configurations. Peers only process the information related to themselves to determine the information required to embed them into the distributed SM described by the formal description. Figure 7.20, Figure 7.21, and Figure 7.22 show the determined information by the individual peers. 


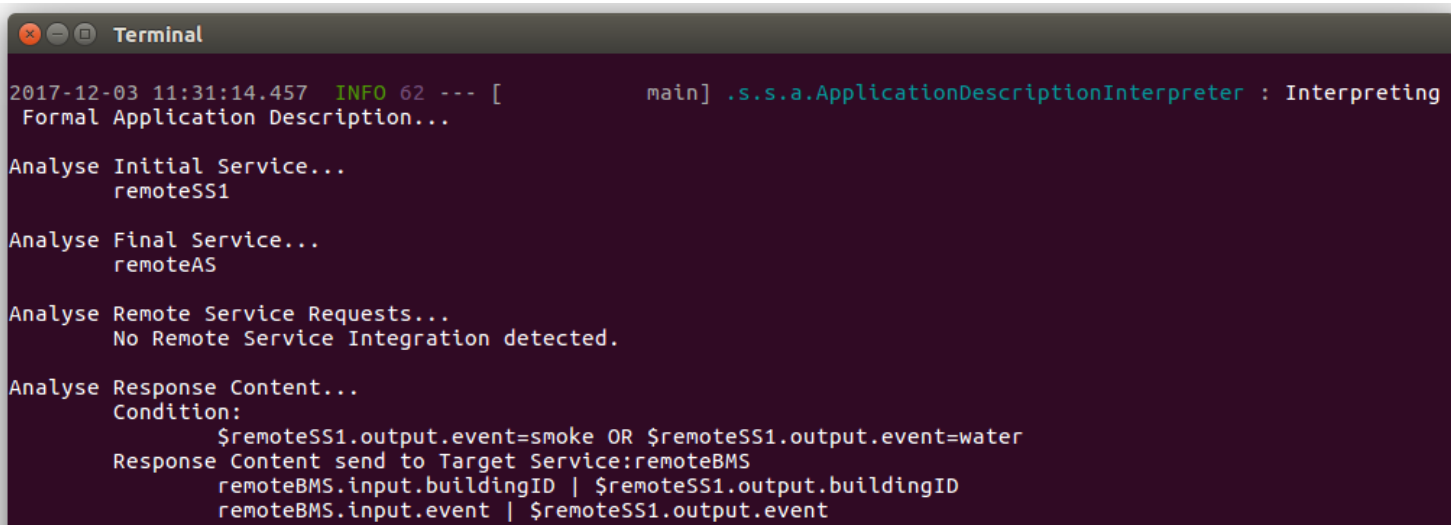

Figure 7.20: Screenshot of Terminal Output ADI Use Case 3 remoteSS1

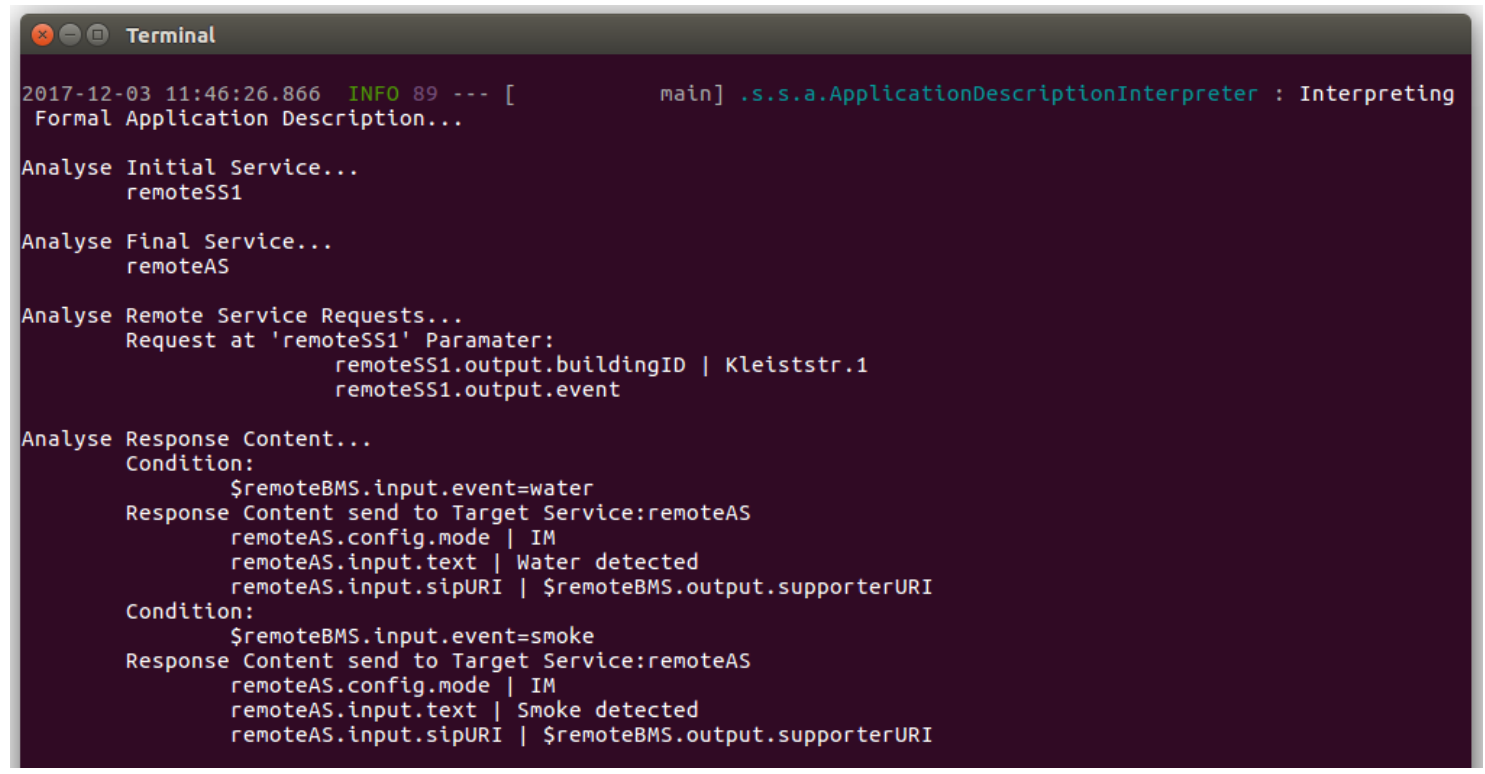

Figure 7.21: Screenshot of Terminal Output ADI Use Case 3 remoteBMS

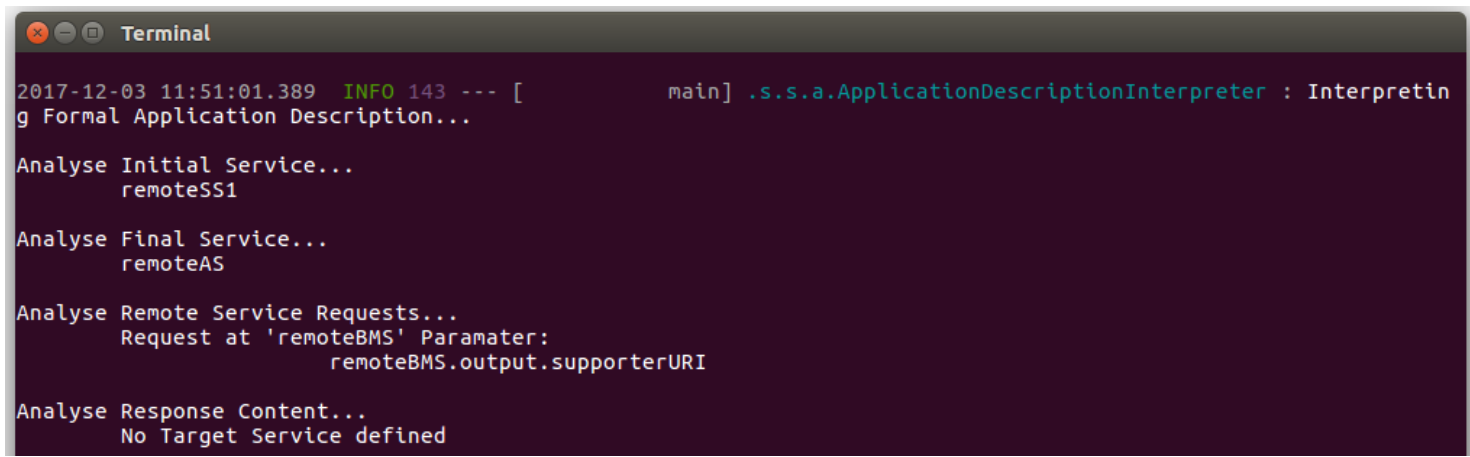

Figure 7.22: Screenshot of Terminal Output ADI Use Case 3 remoteAS 
The distributed SM that is to be generated after all peers have automatically connected with each other is illustrated in Figure 7.23.

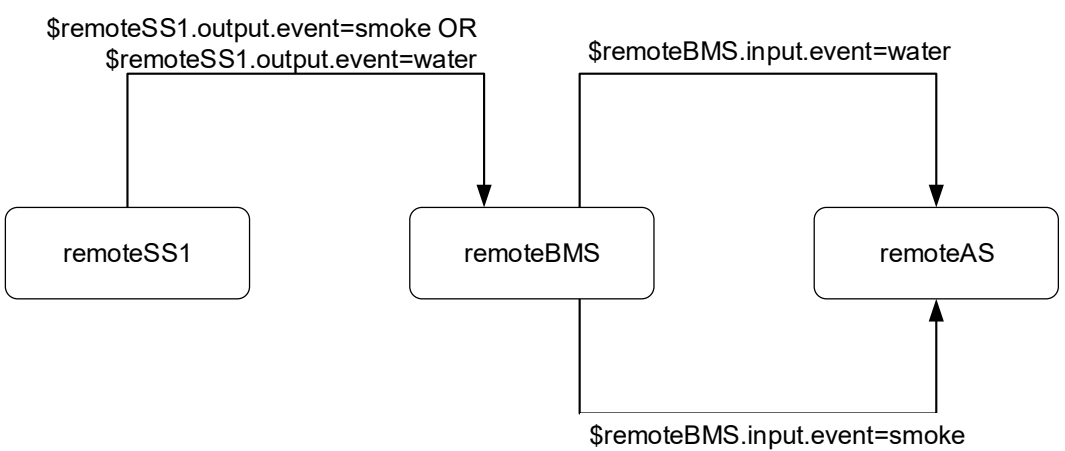

Figure 7.23: Distributed SM Use Case 3

To generate this distributed M2M application the signalling illustrated in Figure 7.24 is performed by the peers. After the signalling process is finished, the distributed cooperative $\mathrm{M} 2 \mathrm{M}$ application has been successfully configured and is ready for execution. Here it should be again mentioned, that the peers perform their individual part of the SM logic by checking the condition locally and send the response message (i.e. perform the state transition) only if the defined condition becomes TRUE.

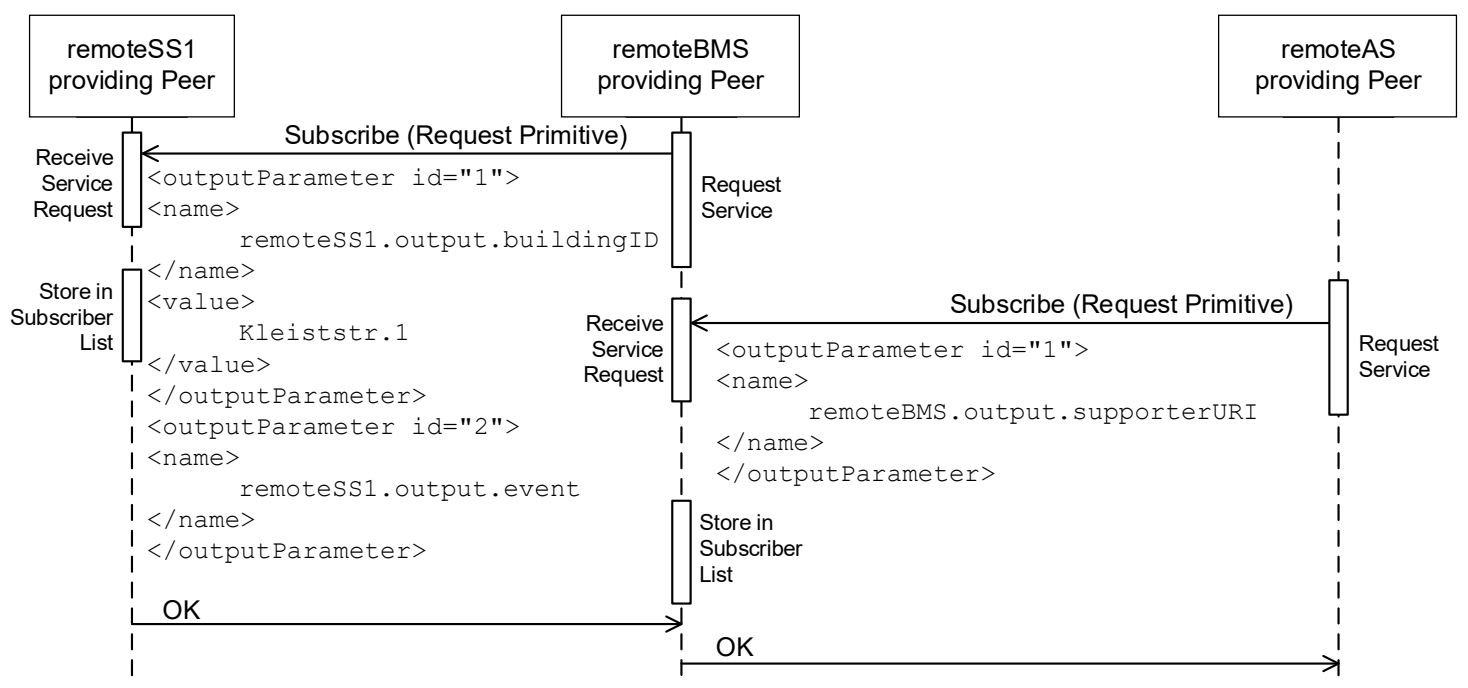

Figure 7.24: M2M Application Configuration Phase Use Case 3 
In the next step, supporter register for the buildings they take care of by requesting the M2M application service at the remoteBMS providing peer (illustrated in Figure 7.25).

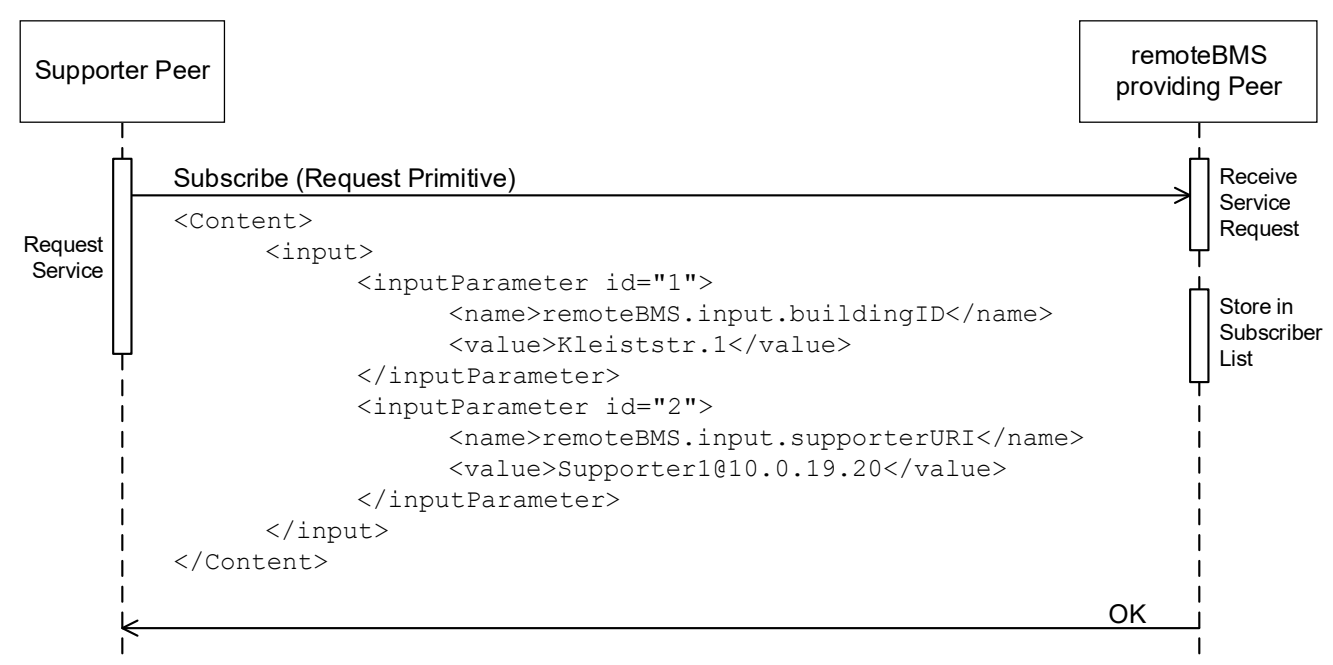

Figure 7.25: Service Request Process Supporter at remoteBMS Use Case 3

As soon as events "water" or "smoke" occur in the building monitored by remoteSS1 the M2M application execution starts. The performed message exchange is illustrated in Figure 7.26.

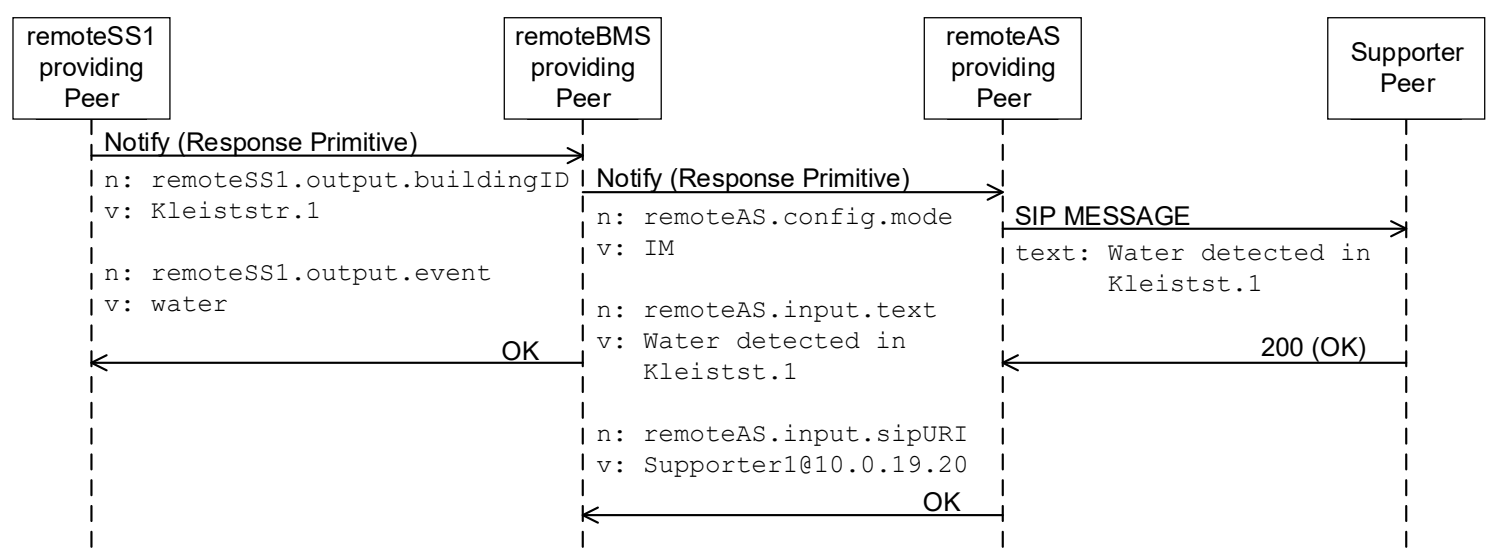

Figure 7.26: M2M Application Execution Process Use Case 3

RemoteSS1 generates a NOTIFY message and sends it to the remoteBMS providing peer. This determines the supporter of the building and generates the alarm message text in 
dependence of the occurred event. Afterwards the remoteBMS sends a NOTIFY message to the remoteAS providing peers, which generated a SIP IM and sends it to the building supporting peer (illustrated in Figure 7.27).

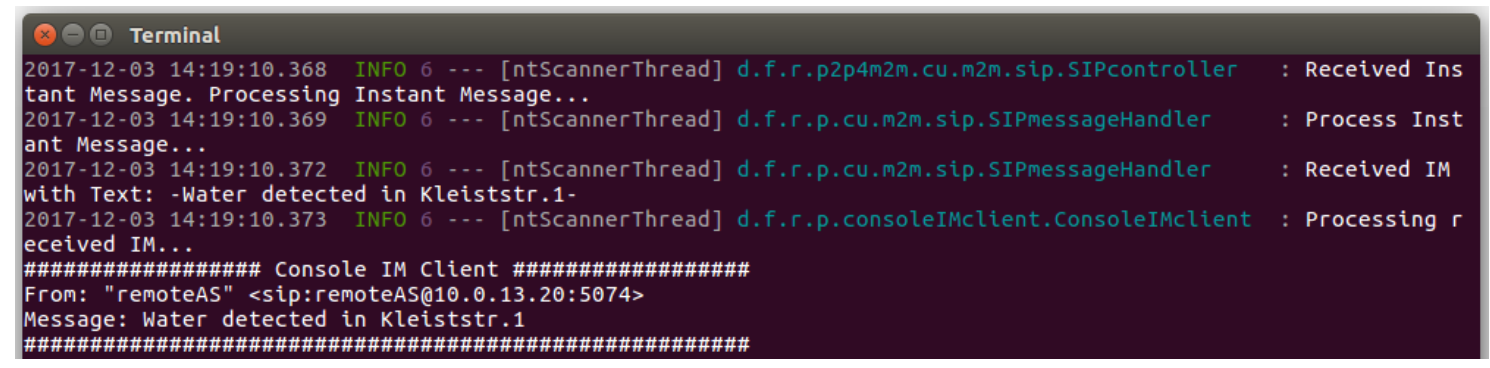

Figure 7.27: Screenshot of Terminal Output Supporter receiving Alarm Message Use Case 3

This section demonstrated the distributed cooperative M2M application creation and execution processes. The following section will demonstrate storing and requesting IFDs in a $\mathrm{P} 2 \mathrm{P}$ overlay.

\subsubsection{Communication Scenarios for M2M Service Requests}

To demonstrate the processes for the service request and the corresponding response messages, the following two scenarios are presented.

- One-time subscription via CoAP - A SC requests once for an M2M service/resource at an $\mathrm{M} 2 \mathrm{M}$ service providing peer using the communication protocol CoAP.

- Continuous subscription via SIP - A SC requests for an M2M service/resource at an M2M service providing peer using the communication protocol SIP. Requested resource is continuously sent to the requesting peer. 
To request a service from a service providing peer, a subscription request is sent to that service (see Figure 7.28). The requested parameter is defined in the message body of the message. The service request can also contain parameters that are to be set for the service (input/config parameter). It can be seen that each message is answered with an acknowledgement message (ACK).

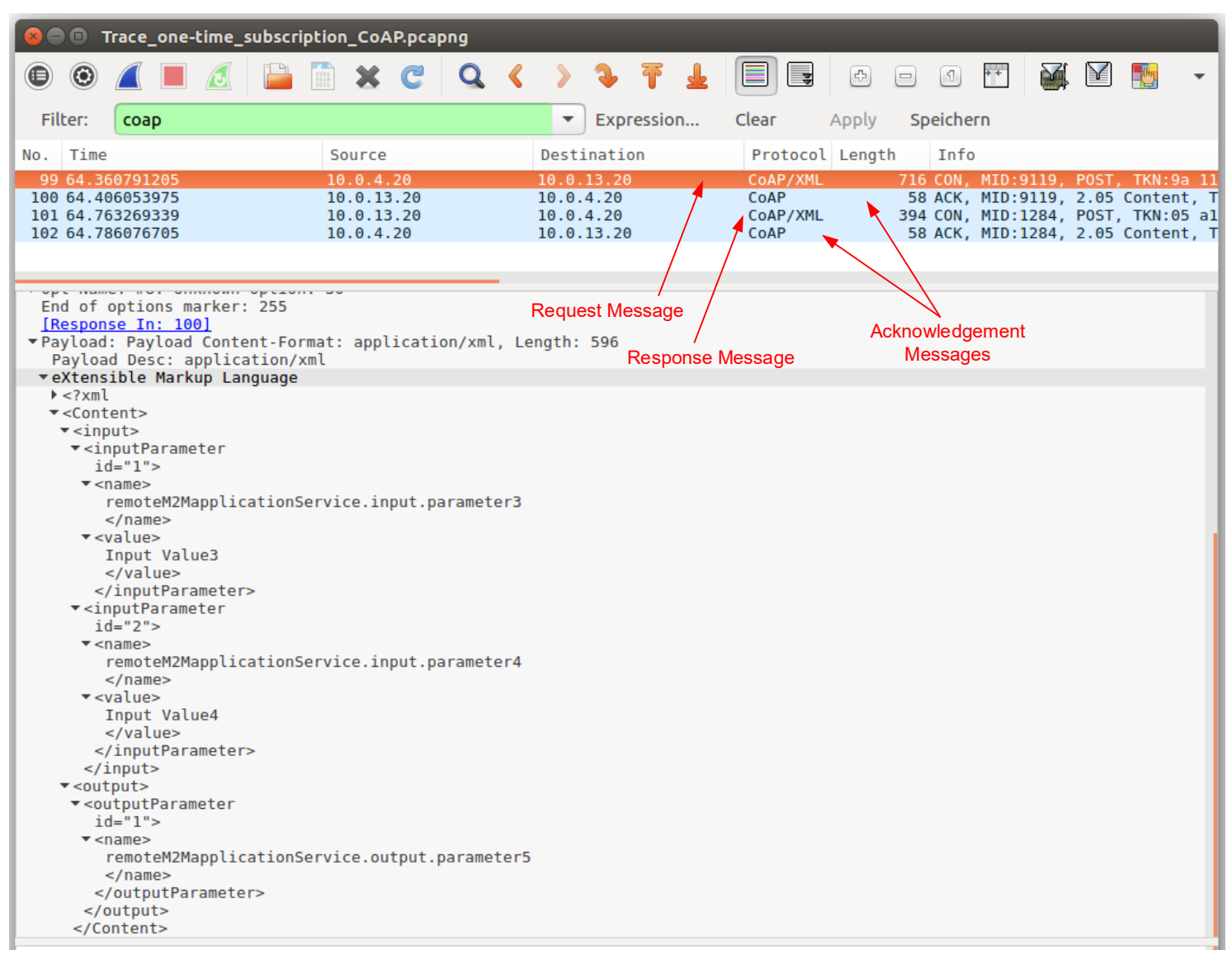

Figure 7.28: Screenshot of Wireshark Trace Request Message via CoAP one-time Subscription

Peers that receive service request messages generate Request Primitive messages from the received messages which are then further processed (see Figure 7.29). 


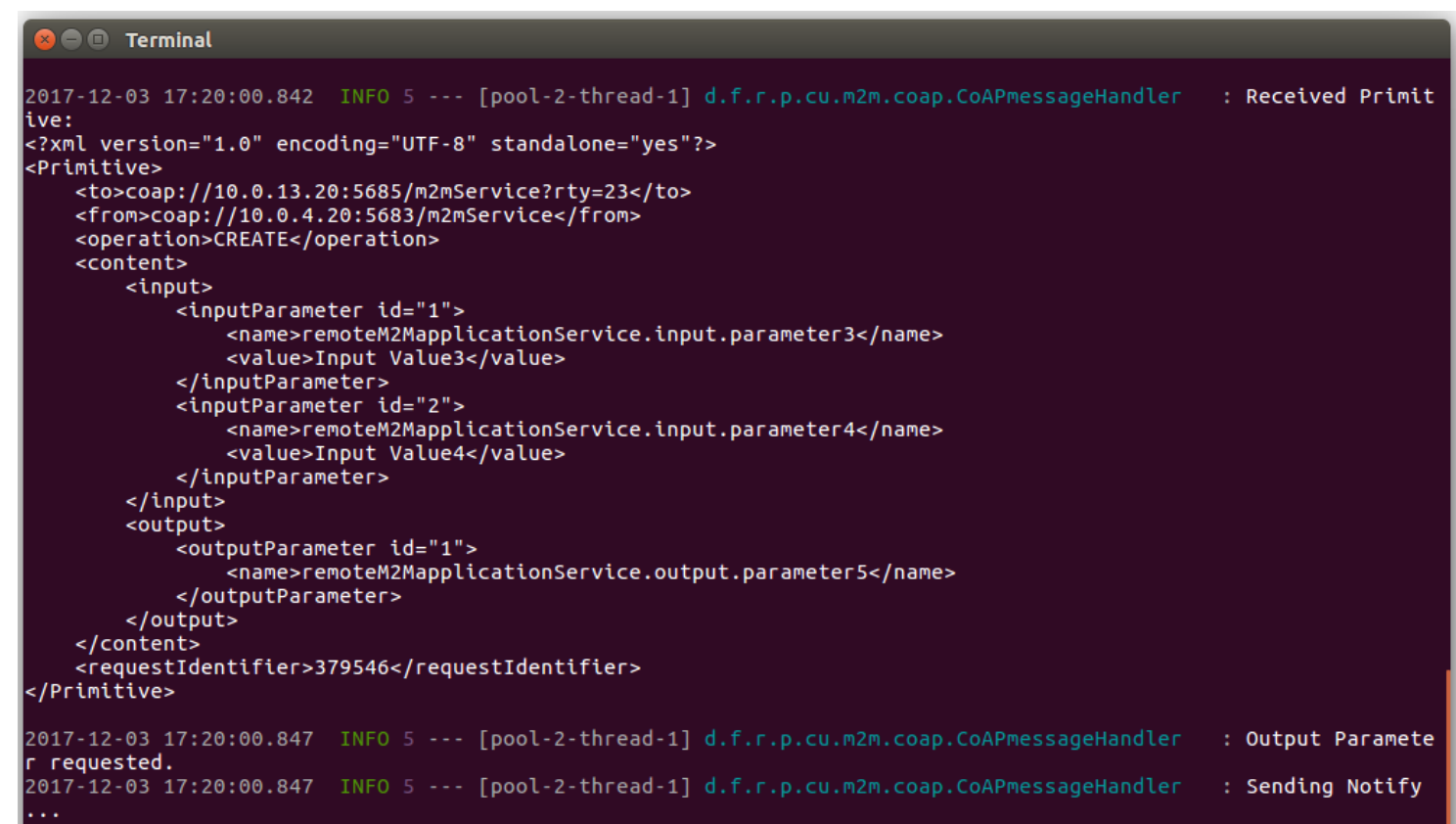

Figure 7.29: Screenshot of Terminal Output received Request Primitive via CoAP

Since the service request shown is a one-time subscription request of a resource, a single response message is sent back to the requesting peer (see Figure 7.30).

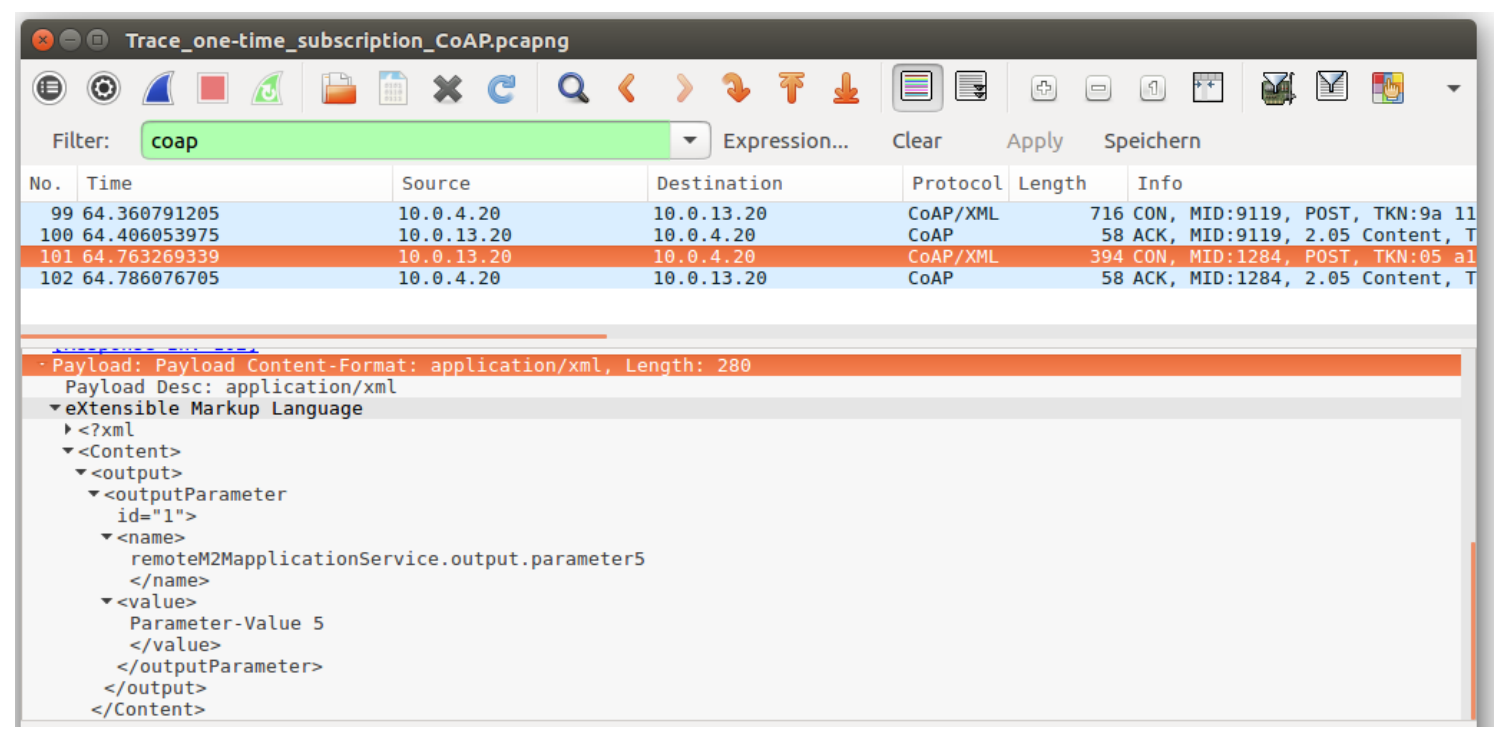

Figure 7.30: Screenshot of Wireshark Trace Response Message via CoAP 
The response message contains only the requested output parameters of a service request. After the service requesting peer has received the response message, it generates a Request Primitive for further processing containing the requested parameter values (see Figure 7.31).

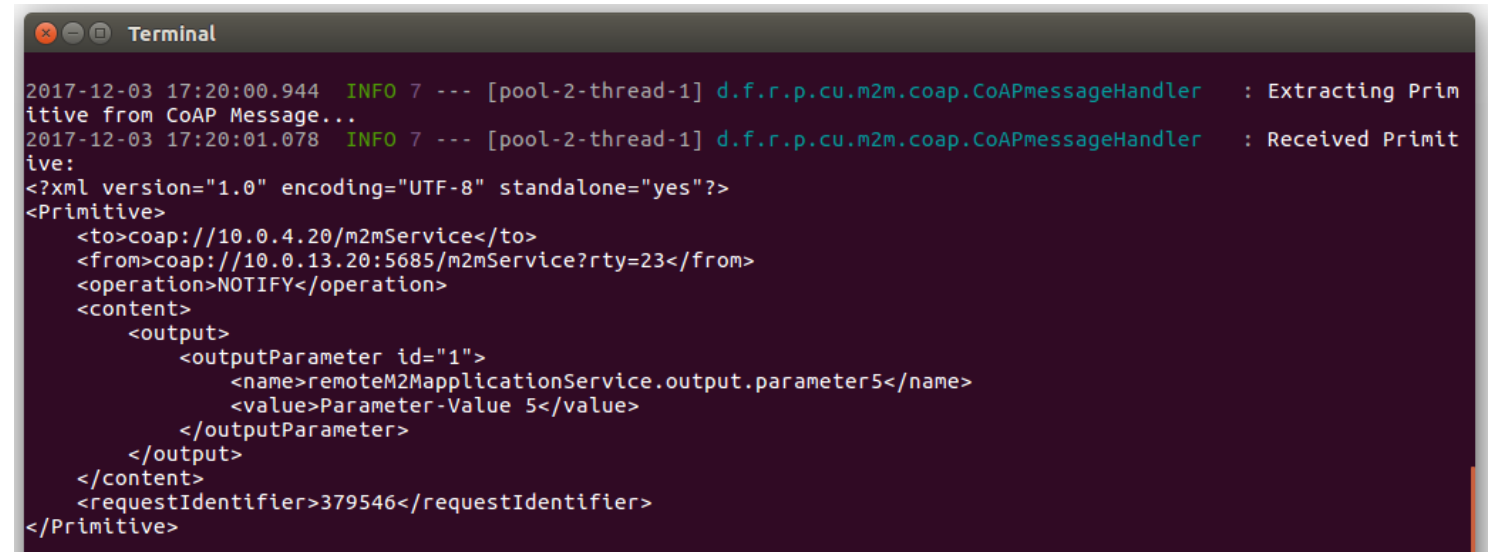

Figure 7.31: Screenshot of Terminal Output received Response Primitive via CoAP

The following Figure 7.32 shows a service request message transmitted via SIP.

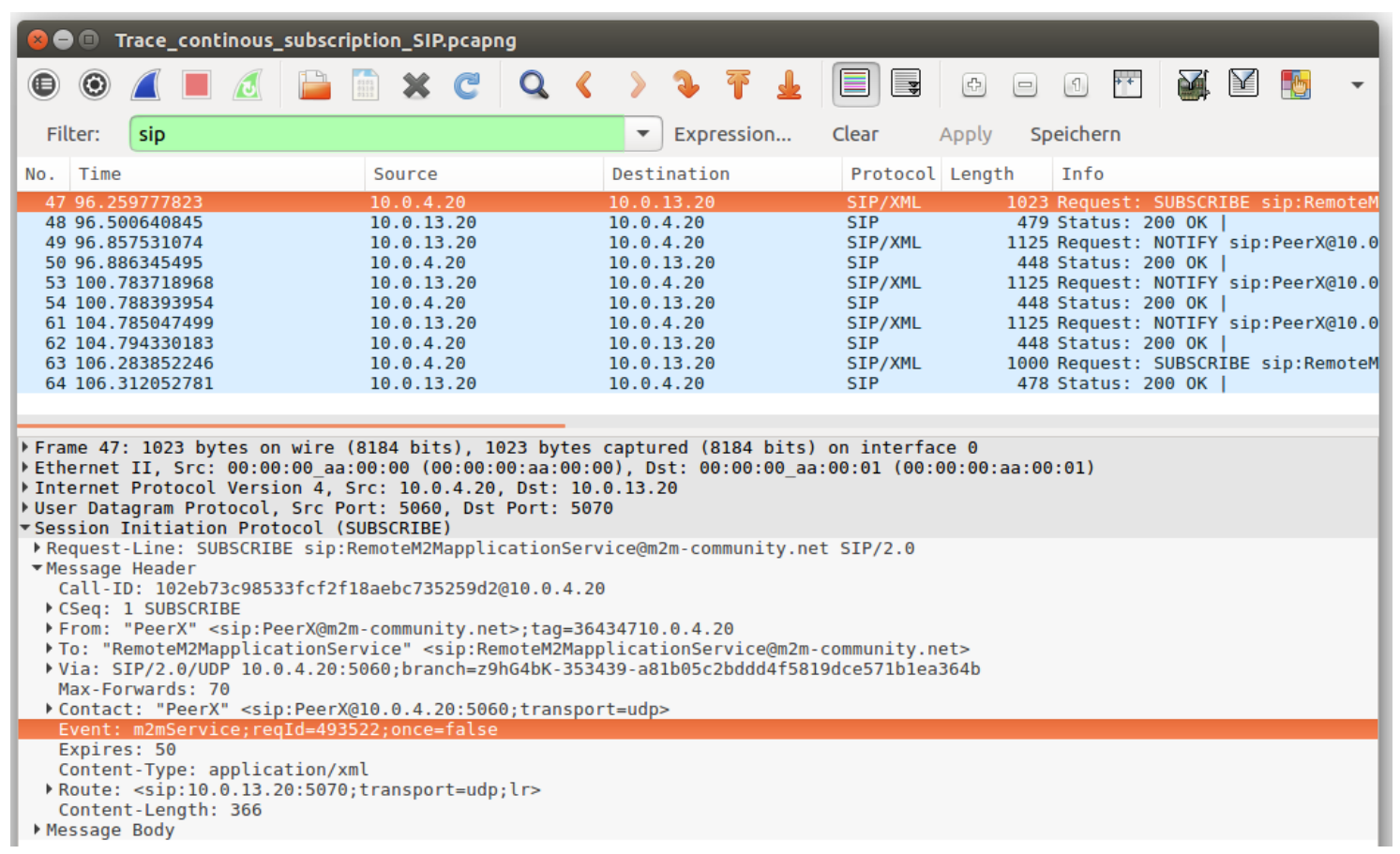

Figure 7.32: Screenshot of Wireshark Trace Request Message via SIP continuous Subscription 
In a continuous subscription, the requested parameter is also specified in the message body. The information about the continuous request is given in the shown example in the message header. This causes the SP to send the requested information continuously until the subscription is terminated (Expires: 0) (see Figure 7.33).

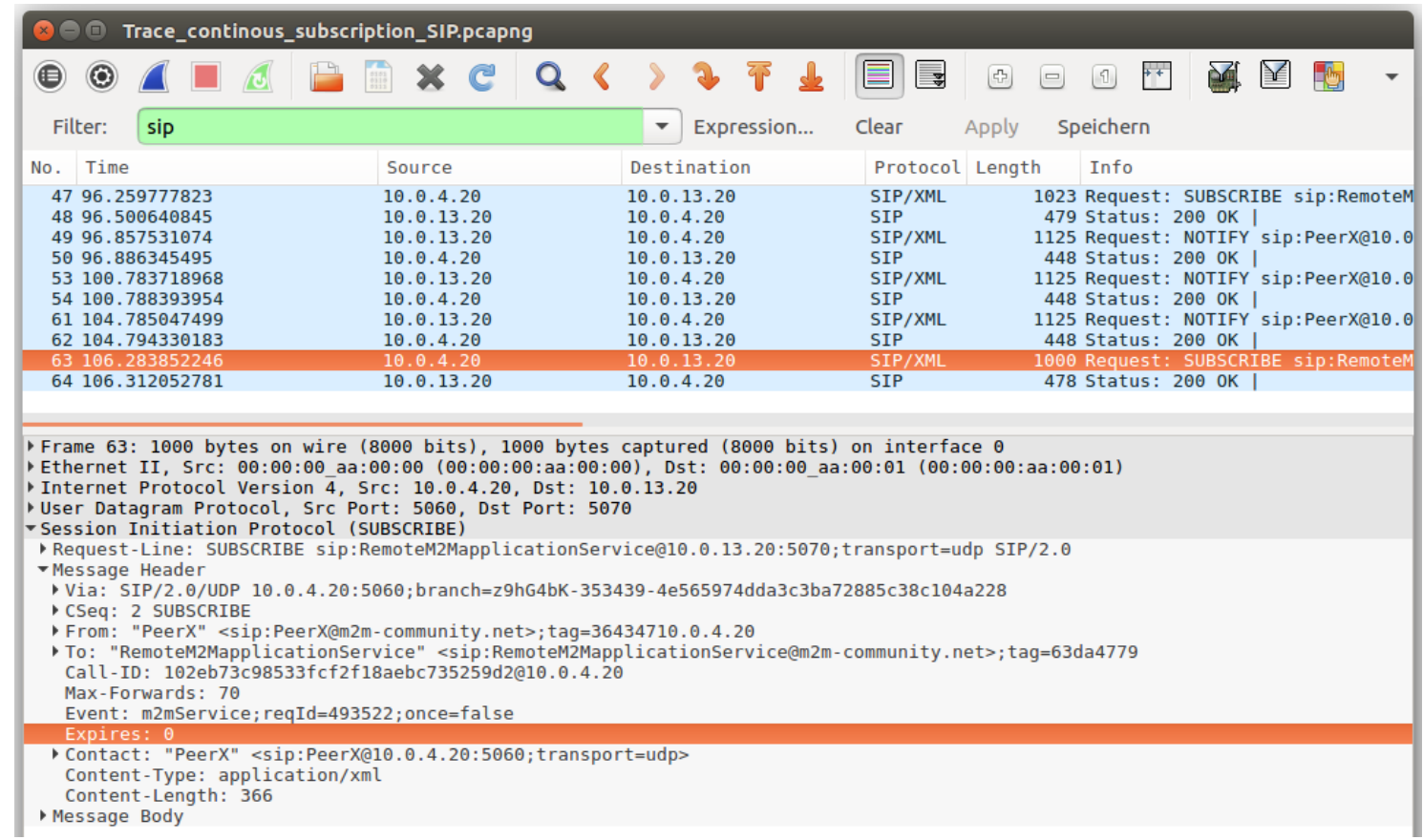

Figure 7.33: Screenshot of Wireshark Trace Terminate Request Message via SIP

In summary, it can be concluded that the framework and the prototype implementation could be evaluated by implementing different scenarios. The scenarios demonstrate the implementation of application and framework components without central entities and the central integration of the end-user into application creation. It could be shown that by applying the new concepts, M2M applications can be conveniently created and formally described with distributed provision and execution. 


\subsection{Performance Evaluation}

As shown in the proof-of-concept, an M2M application is based on the linking of distributed services through signalling based on the service request and transmitting service result via notification messages.

Assuming that when using a central MSP, services are also linked by the same signalling principle, the effort for the required signalling messages can be compared. The following scenarios (as illustrated in Figure 7.35 and Figure 7.36) are defined for the comparison:

- $\quad$ Services in same Subnet - N services that are to be connected to each other are located in the same access network (subnet) and on different nodes.

- Two Services in different Subnets - Two services to be linked are located in different subnets that are not connected to each other by a direct link.

- Two Services in different Subnets (direct link) - Two services to be linked to each other are located in different subnets connected by a direct link.

Furthermore, a uniform data basis for message exchange via CoAP and SIP is required. For this purpose, the service request message shown in Figure 7.28 is used as CoAP data basis. The same scenario has been performed via SIP as illustrated in Figure 7.34.

The signalling effort for the request of a service shown in Table 7.5 can be determined from the displayed messages. To determine the message volume in relation to the number of messages, the average message size consisting of request and response messages is used. Derived from Figure 7.28 and Figure 7.34, the average message size for CoAP is therefore exemplarily set to 306Byte and for SIP 781Byte. 


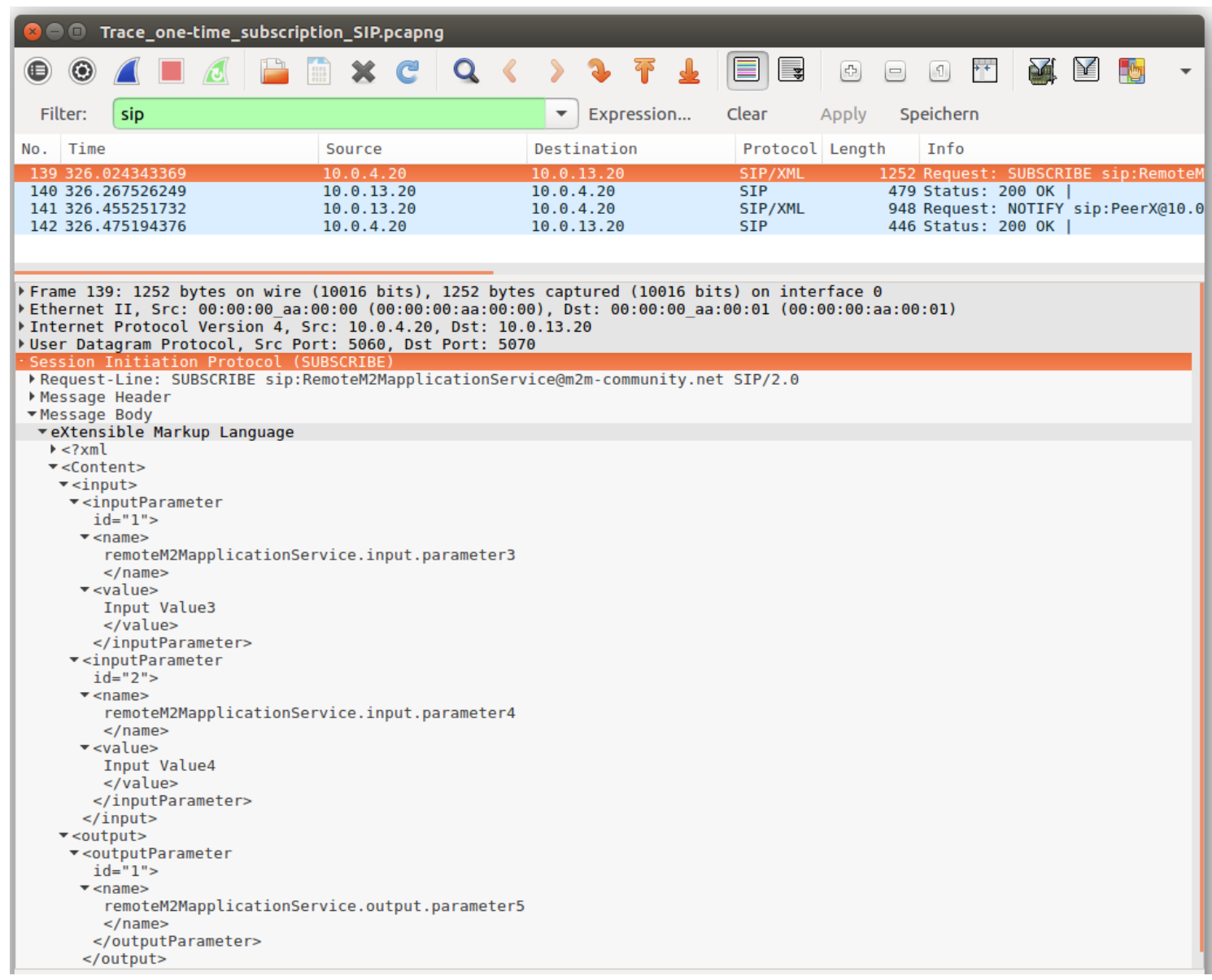

Figure 7.34: Screenshot of Wireshark Trace Request/Response Messages via SIP one-time Subscription

Table 7.5: Signalling Effort for Service Request and Service Response

\begin{tabular}{|l|c|c|c|}
\hline \multicolumn{2}{|c|}{} & \multicolumn{2}{c|}{ M2M Communication Protocol } \\
\hline Category & Description & SIP & CoAP \\
\hline $\begin{array}{l}\text { Service Request/ } \\
\text { Response }\end{array}$ & Service Request (Request + Acknowledgement) & 1731 Byte & 774 Byte \\
\cline { 2 - 4 } & Service Response (Notify + Acknowledgement) & 1394 Byte & 452 Byte \\
\hline
\end{tabular}

Based on the defined scenarios and using a central MSP, Figure 7.35 shows the messaging effort. It is assumed that the nodes are each connected to an Access Network (AN), which in turn is connected to the Core Network. The Core Network connects the ANs with each other and also consists of several Core Subnets (CSNs). 


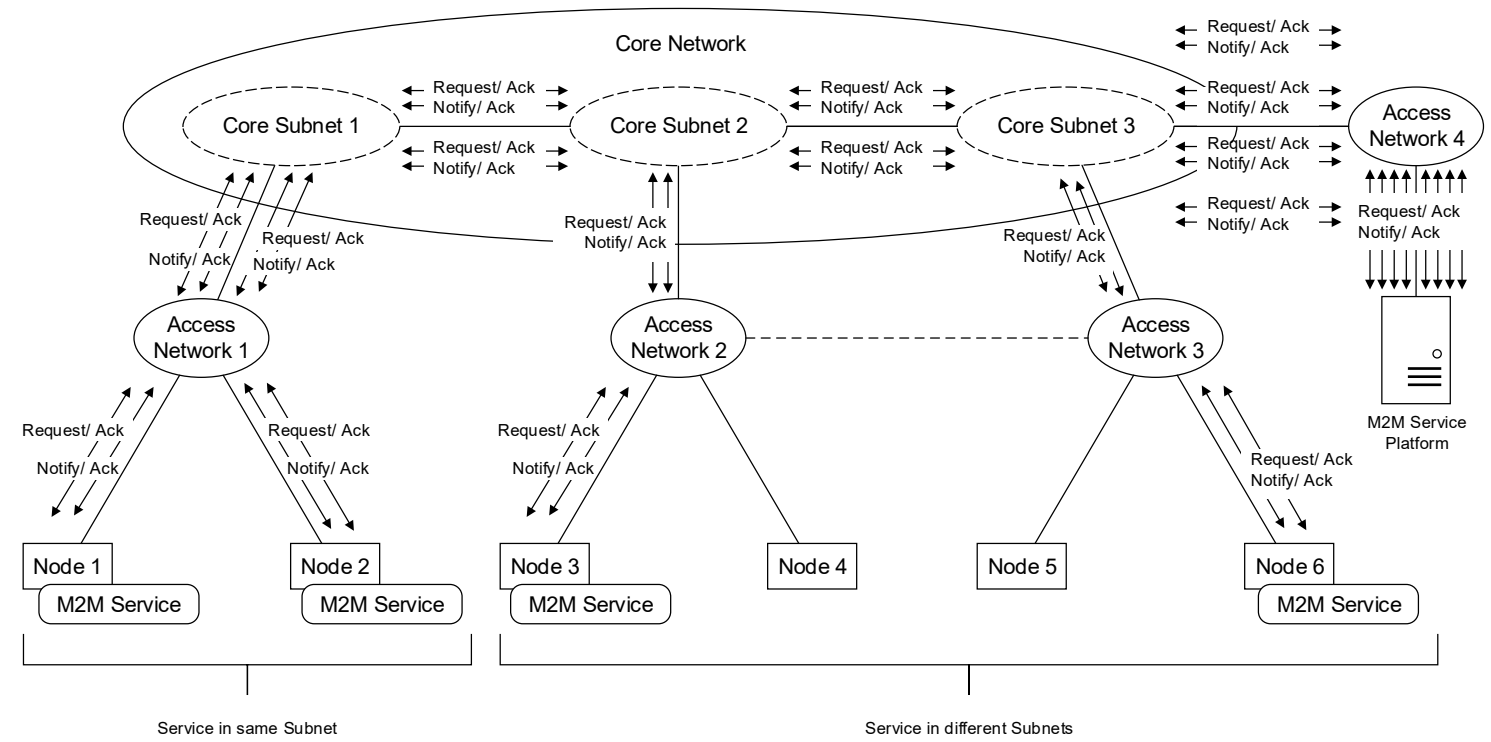

Figure 7.35: Signalling Effort using centralised M2M Service Platform (MSP)

A central MSP must always request all services to be linked to each other and receives a response from these services. Derived from the signalling behaviour shown in Figure 7.35 , the message effort for requesting $\mathrm{n}$ services that are located in the same subnet can be derived as shown in Equation 7.1. The variable " $\mathrm{m}$ " corresponds to the number of mediating CSNs located between the CSNs to which the ANs of the service nodes and the MSP are connected.

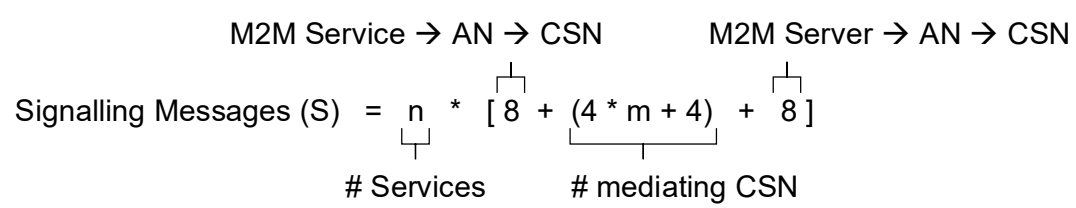

For the messaging effort of requesting two services located in different subnets, the signalling effort shown in Equation 7.2 can be derived. Since a different number of mediating CSNs can exist in this scenario, this is taken into account by the variable " $m_{1} "$ and " $\mathrm{m}_{2}$ ". The messaging effort for the scenario in which the ANs are connected to each 
other is identical, since the services are requested by the central MSP and therefore there would be no advantage by connecting ANs.

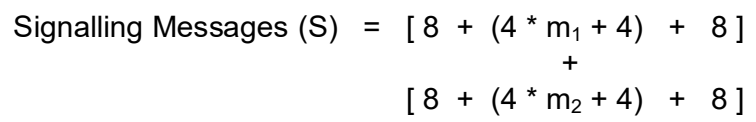

Table 7.6 shows the messaging effort for signalling using a central MSP for scaling services (n) and number of mediating CSNs (m), assuming that $\mathrm{m}_{1}$ is equal to $\mathrm{m}_{2}$ and that in the scenario where $n$ services are connected to each other $m$ is equal to 1 (as shown in Figure 7.35).

Table 7.6: Performance Analysis central M2M Service Platform for Service Requests

\begin{tabular}{|c|c|c|c|c|c|c|}
\hline \multirow[t]{2}{*}{ Scenario } & \multicolumn{6}{|c|}{ Central M2M Service Platform } \\
\hline & & \multicolumn{2}{|c|}{ \# Messages } & \multicolumn{3}{|c|}{ Total Byte } \\
\hline \multirow{4}{*}{$\begin{array}{l}\text { N services same } \\
\text { subnet }\end{array}$} & \multirow{4}{*}{$n *[8+(4 * m+4)+8]$} & $n$ & & $n$ & COAP & SIP \\
\hline & & 2 & 48 & 2 & 14688 & 37488 \\
\hline & & 10 & 240 & 10 & 73440 & 187440 \\
\hline & & 100 & 2400 & 100 & 734400 & 1874400 \\
\hline \multirow{4}{*}{$\begin{array}{l}2 \text { services } \\
\text { different subnets }\end{array}$} & \multirow{4}{*}{$\begin{array}{c}{\left[8+\left(4 * \mathrm{~m}_{1}+4\right)+8\right]} \\
+ \\
{[8+(4 * \mathrm{~m} 2+4)+8]}\end{array}$} & $m$ & & $m$ & COAP & $S I P$ \\
\hline & & 2 & 56 & 2 & 17136 & 43736 \\
\hline & & 5 & 80 & 5 & 24480 & 62480 \\
\hline & & 10 & 120 & 10 & 36720 & 93720 \\
\hline
\end{tabular}

In comparison, Figure 7.36 shows the message effort using signalling approaches from the proposed framework. 


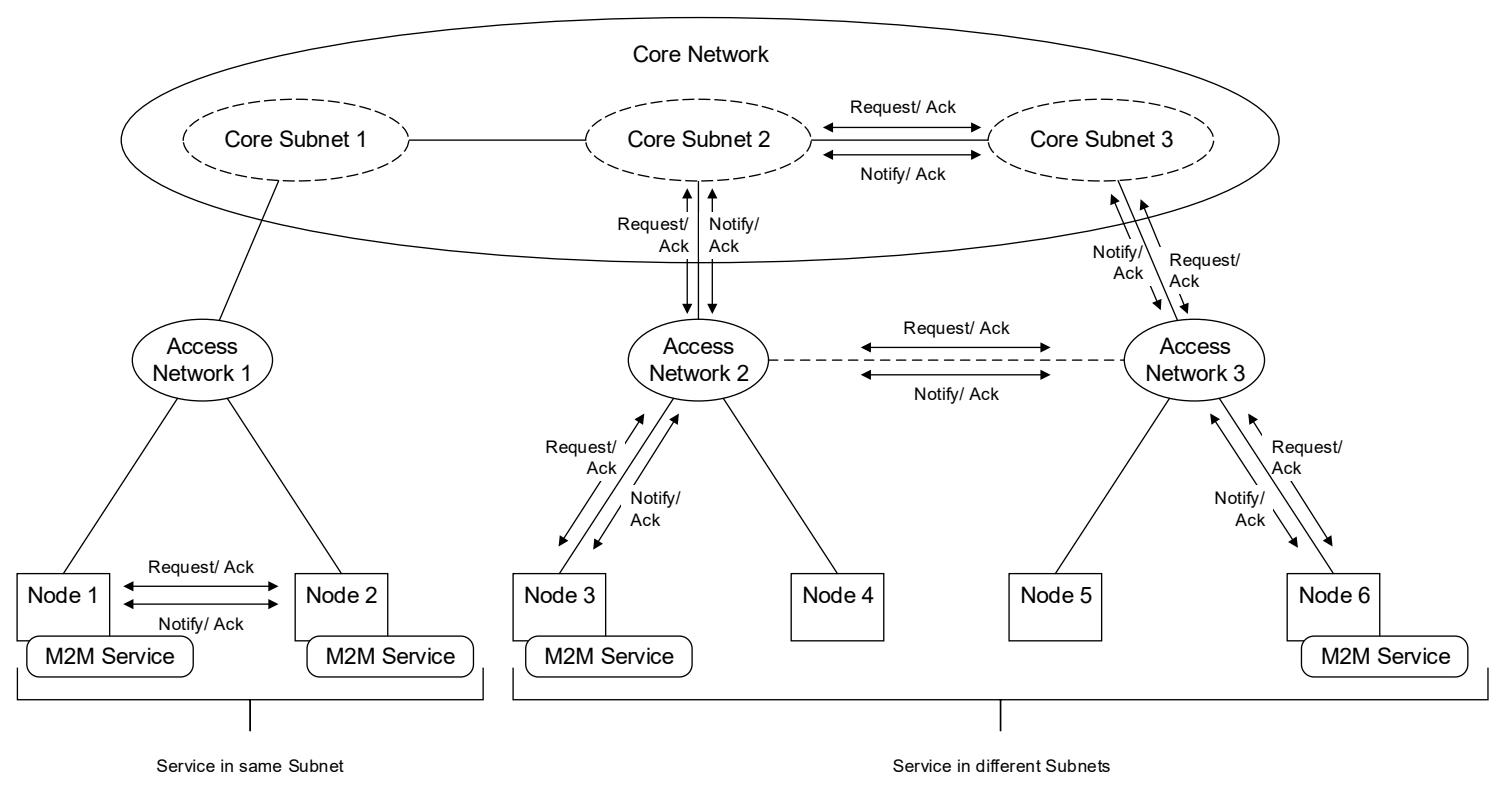

Figure 7.36: Signalling Effort using proposed Framework

Derived from the signalling behaviour shown in Figure 7.36, the messaging effort shown in Equation 7.3 or requesting n services within the same subnet can be derived. Since the services are connected $\mathrm{P} 2 \mathrm{P}$, only signalling between the nodes in the same AN is necessary.

$$
\begin{aligned}
\text { Signalling Messages }(\mathrm{S}) & =\underset{\uparrow}{(n * 4)-4} \\
& \text { \# Services }
\end{aligned}
$$

For requesting two services located in different subnets, the signalling effort shown in the following Equation 7.4 can be derived.

$$
\begin{aligned}
& \text { M2M Service } \rightarrow \mathrm{AN}_{1} \rightarrow \mathrm{CSN} \quad \text { M2M Service } \rightarrow \mathrm{AN}_{2} \rightarrow \mathrm{CSN} \\
& \text { Signalling Messages }(S)=\left[\begin{array}{l}
(4 \\
(4 * m+4)+\not{b}
\end{array}\right] \\
& \text { \# mediating CSN }
\end{aligned}
$$

If the ANs are connected to each other by a direct link, the connection via the CSNs can be reduced. In this way, the signalling effort shown in Equation 7.5 can be derived. 


$$
\begin{aligned}
& \text { M2M Service } \rightarrow \mathrm{AN}_{1} \quad \text { M2M Service } \rightarrow \mathrm{AN}_{2} \\
& \text { Signalling Messages }(S)=[4+4+4] \\
& \mathrm{AN}_{1} \rightarrow \mathrm{AN}_{2}
\end{aligned}
$$

Table 7.7 shows the messaging effort for signalling using the proposed framework for

\begin{tabular}{|c|c|c|c|c|c|c|}
\hline \multirow[t]{2}{*}{ Scenario } & \multicolumn{6}{|c|}{ Distributed M2M Service Platform Concept } \\
\hline & & \multicolumn{2}{|c|}{ \# Messages } & \multicolumn{3}{|c|}{ Total Byte } \\
\hline \multirow{4}{*}{$\begin{array}{l}\text { N services same } \\
\text { subnet }\end{array}$} & \multirow{4}{*}{$(n * 4)-4$} & $n$ & & $n$ & COAP & SIP \\
\hline & & 2 & 4 & 2 & 1224 & 3124 \\
\hline & & 10 & 36 & 10 & 11016 & 28116 \\
\hline & & 100 & 396 & 100 & 121176 & 309276 \\
\hline \multirow{4}{*}{$\begin{array}{l}2 \text { services } \\
\text { different subnets }\end{array}$} & \multirow{4}{*}[8+(4*m+4)+8]{} & $m$ & & $m$ & COAP & $S I P$ \\
\hline & & 2 & 28 & 2 & 8568 & 21868 \\
\hline & & 5 & 40 & 5 & 12240 & 31240 \\
\hline & & 10 & 60 & 10 & 18360 & 46860 \\
\hline \multirow{2}{*}{$\begin{array}{l}2 \text { services } \\
\text { different subnets } \\
\text { (direct link) }\end{array}$} & \multirow{2}{*}{$12(4+4+4)$} & \multirow{2}{*}{\multicolumn{2}{|c|}{12}} & \multicolumn{2}{|r|}{ CoAP } & SIP \\
\hline & & & & 3672 & & 9372 \\
\hline
\end{tabular}
scaling services (n) and number of mediating CSNs (m).

Table 7.7: Performance Analysis distributed M2M Service Platform Concept for Service Requests

The comparison visualised in Figure 7.37 shows the significantly lower signalling effort using the presented framework. The number of messages required for linking $\mathrm{n}$ services in the same subnet is about one-fifth with the proposed framework. Only half of the messages are needed to connect two services in different subnets (without a direct link). If the subnets are connected by a direct link, on the other hand, only 12 messages are needed constantly. 


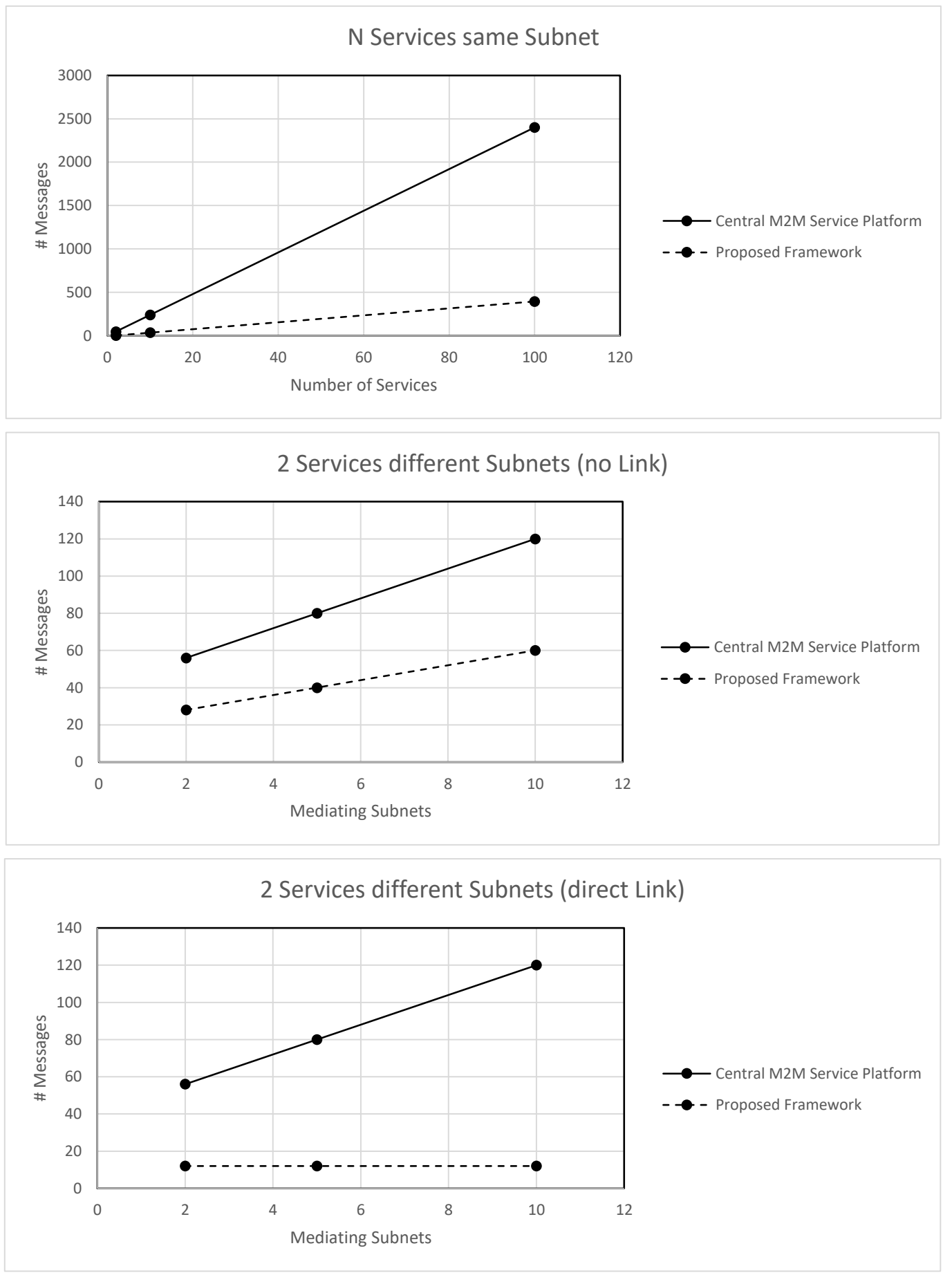

Figure 7.37: Comparison Signalling Effort (\# Messages)

Figure 7.38 visualises the comparison under consideration of the required data volume. Again, it can be seen that the effort required by the proposed framework is significantly lower. 


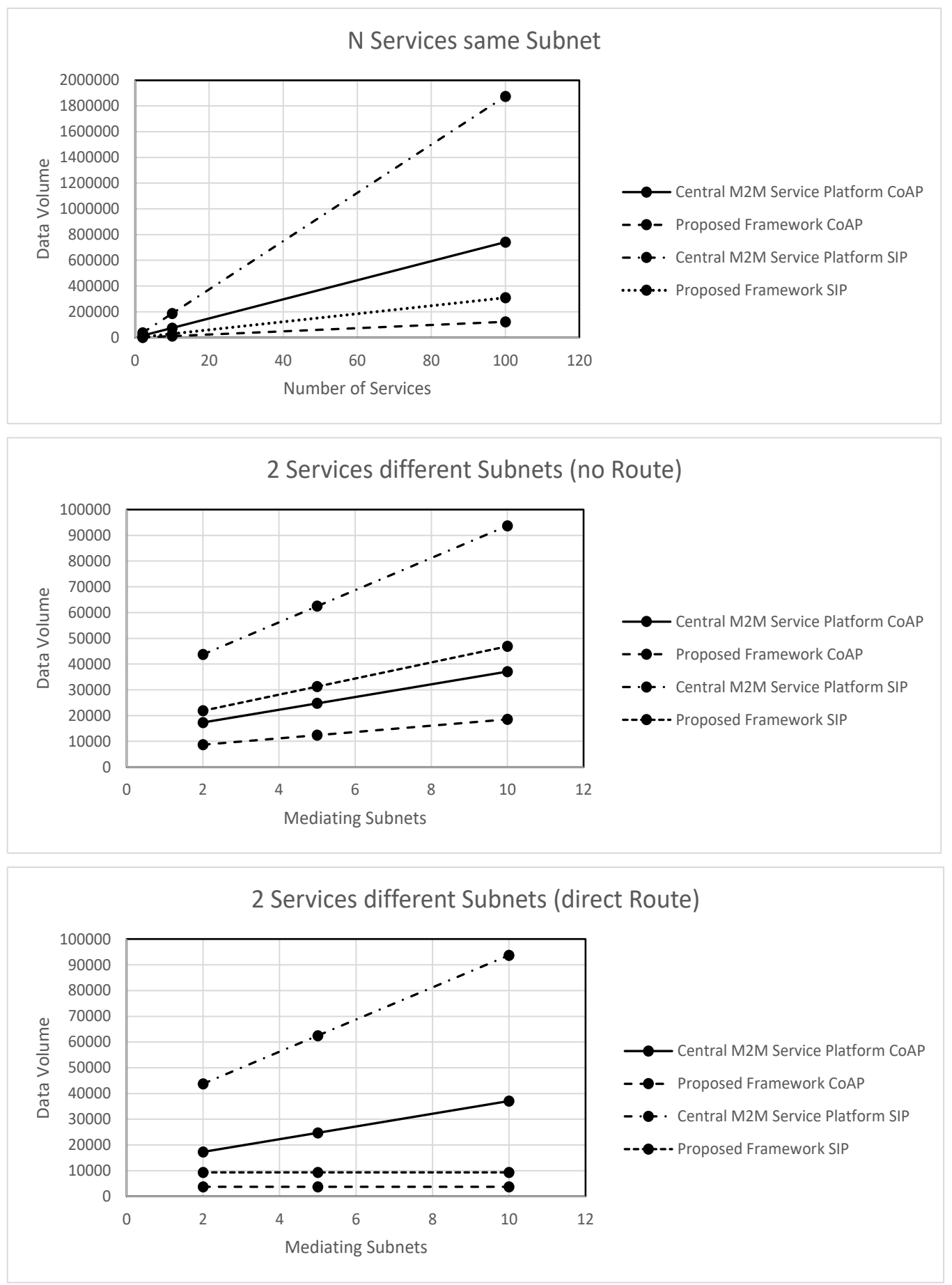

Figure 7.38: Comparison Signalling Effort (Data Volume)

In particular, it should be noted that the load on the central MSP and the AN to which it is connected is a multiple higher, since the messages all have to be processed in a single 
place, whereas the load in the presented framework is distributed among several nodes and ANs.

\subsection{Conclusion}

This chapter presented the evaluation of the proposed framework for "Autonomous decentralised M2M Application Service Provision" (section 7.1). The framework has been analysed with regard to the requirements defined in section 3.2 and determined whether or not these requirements are met. All the requirements for the framework were met by the concepts and the M2M system architecture presented.

Section 7.2 presented the research prototype architecture and implementation. The relevant developed components were described and the interaction between the framework components was discussed.

The research prototype was successfully implemented for the proof-of-concept evaluation of the proposed framework (section 7.3). This demonstrated the main functionality of the framework and proved its applicability. The proof-of-concept was demonstrated using previously defined use cases that illustrate the essential aspects of the framework (enduser integration, formal description of application logic, decentralised SM-based service execution, combination of distributed M2M application services, and cooperative M2M application service provision). All the relevant steps for modelling, generating and executing an M2M application service were illustrated using the respective use cases.

Finally, in section 7.4 the signalling effort for service requests and transmission of service results required for linking services was considered. The connection of a scaling number 
of services in the same AN was analysed. Furthermore, the connection of two services in different (possibly directly linked) ANs was analysed, which are interconnected by a scalable number of mediating CNs. The number of messages was determined and based on exemplary message sizes the required data volume was calculated. The comparison of the distributed MSP system architecture introduced in this project with a centrally organised system architecture has shown that the presented approach requires significantly less effort for signalling (services in the same AN 80\%, different ANs 50\%, directly linked ANs constant 12 messages). 


\section{Conclusion and Future Work}

This chapter concludes the research project. Section 8.1 summarises the main achievements. Section 8.2 discusses limitations of the project. Section 8.3 suggests scopes and ideas for further research.

\subsection{Achievements of the Research}

The research performed in this project was dedicated to the design of a novel approach for $\mathrm{M} 2 \mathrm{M}$ application service provision. A framework has been designed enabling the realisation of a distributed M2M service platform (MSP). This showed an alternative to traditional approaches of MSPs. The proposed MSP eliminates certain disadvantages of traditional MSPs such as dependencies on central system components or stakeholders. The MSP, as well as the M2M applications, can be operated autonomously with existing equipment. The framework is an integrated solution for decentralised M2M application service provision with native end-user integration. It describes all necessary steps from application modelling to execution and automated configuration of $\mathrm{M} 2 \mathrm{M}$ applications.

The analysis of currently existing approaches in the field of MSPs was presented (refer to section 3.2). For this purpose, the oneM2M standard and approaches from the research field were considered. Requirements for a new framework for "Autonomous decentralised M2M Application Service Provision" were determined on the basis of their advantages and deficits (refer to section 3.2). It has been analysed whether one of the 
considered projects fulfils the requirements with the result that none of them fulfils the overall requirements catalogue, since the existing approaches for MSPs usually comprise individual solutions or do not include essential aspects for decentralisation or end-user integration.

A new framework has been designed based on the requirements resulting from the deficits and advantages of related projects (refer to chapter 4). The designed framework architecture contains new conceptual models for M2M system architectures enabling decentralised, horizontal and cooperative M2M application service provision.

The Decentralised M2M Service Provision (ADSP) model was introduced as the first new model. With the introduced framework, end-users have the possibility to create their individual applications with the resources existing in their personal environment and execute them locally. In the related projects, M2M applications for supporting specific business processes are created by specialised developers and are executed centrally on M2M application servers. Often the MSPs are limited to specific application fields and therefore not flexible enough to cover the end-user's individual field of application. By integrating end-users into the application creation they can create applications that meet their individual requirements. Since the end-users themselves provide the execution environment, they are not dependent on a central platform operator or central system components that they cannot manage. Additionally, end-users are not dependent on the functionality of a central MSP, but use the functionality that exists in their personal area for which the application is intended. By distributing the load across different execution environments, the platforms that provide the execution environment can have significantly less system resources than if all applications were running on a central 
platform. A further advantage of the decentralised platform architecture and the avoidance of central stakeholders is that there are no dependencies on them and there is no central data storage that could compromise end-users' privacy.

The Horizontal M2M Service Provision and Utilisation (HSPU) model was introduced as the second new conceptual model. The HSPU model allows end-users to provide their local resources and M2M applications end-to-end as a service to other end-users, but also to external service providers (SPs) who can then integrate them into their own M2M applications. As a result of this facility, the end-user is no longer only in the role of a service consumer (SC), but can also act as a SP. The HSPU model enables external access to the end-user's personal environment, which is not intended for most existing MSPs. The integration of resources from the domain of other end-users in M2M applications can extend their functionality or be integrated into certain business processes.

The third newly introduced Decentralised Cooperative M2M Application Service Provision (DCASP) model allows end-users to connect their distributed M2M services. Thereby, the functionality and effectiveness of an M2M application can be extended by integrating the personal environments of other end-users that were previously not addressable.

To enable decentralised and autonomous M2M application provisioning, several approaches and algorithms have been designed enabling the most automatic application generation and execution (refer to chapter 5).

The prerequisite for M2M application provisioning where the end-user is in focus is the integration of the end-user into application creation. This is one of the key novelties of 
this research. A unified structure of an M2M application was designed and based on it a concept for the graphical modelling of an M2M application was introduced that is independent of the execution environment. With the proposed new approach of application creation, the end-user has the possibility to graphically model the behavior of an application in an intuitive way as a state machine. Compared to other related approaches, where the application logic has to be implemented programmatically, no expert knowledge is required for this kind of application development.

Another key novelty of this research relates directly to the previous one. An approach has been designed that maps the graphically modelled application logic into a formal language. For this purpose, different standardised modelling languages were examined and based on a defined catalogue of requirements, UML StateMachine Diagrams were selected as the basis for the formal description. Using SCXML as a standardised formal language, a new description language for $\mathrm{M} 2 \mathrm{M}$ applications has been introduced describing the semantics of the M2M application.

The advantage that the application logic is described in a formal language defines another novelty of the described framework in the context of MSPs. Due to the formal description, the application logic is machine-readable and can be executed on other systems that contain a corresponding parser. New algorithms have been designed with which the formal description can be generated automatically and to generate an executable state machine which is executed by the local M2M platform.

Reusable Multimedia Service Components (MMSCs) have been defined which can be integrated into M2M applications and serve as an input/output interface. This enables to interact with the M2M platform or M2M applications via a comfortable interface (natural 
language or textual) using existing multimedia communication equipment. Although the principles of these MMSCs are already common communication methods, the integration into an MSP was not considered in any of the related projects. Through this the scope of traditional MSPs have been extended to the M2M application field that (Decker, 2012) classifies as 2nd generation M2M (M2M end-user/devices/environment interaction) and 3rd generation M2M (Social M2M, integration of people and networking of people).

The basis for decentralised and cooperative application provisioning is the networking of SP and SC as well as the composition of a distributed M2M application. For this purpose, several approaches and algorithms have been defined, which again focus on automated execution (refer to chapter 6).

Another key novelty of this research is the provision of local resources and applications for other end-users and their integration into M2M applications. For this purpose, a unified interface description for M2M device/application services based on the resource descriptions defined by oneM2M has been introduced containing all necessary parameters for service utilisation. An approach was defined how M2M service requests can be requested and integrated into other $\mathrm{M} 2 \mathrm{M}$ applications by exchanging service layer messages in a standard-compliant manner. Due to the uniformly defined structure of an $\mathrm{M} 2 \mathrm{M}$ application, it is possible to integrate remote $\mathrm{M} 2 \mathrm{M}$ services with the same semantics as local M2M devices into the application logic. The designed parsing algorithm determines whether the resource is a local or remote resource and requests it from the SP. 
As a central concept of the presented framework and thus as a new approach for the realisation of an MSP, central components in the platform architecture were completely avoided. To achieve this, a number of new approaches have been designed.

Networking between SPs and SCs is end-to-end. This means that no intermediary entities on the application layer are involved in the communication. To achieve this, the $\mathrm{P} 2 \mathrm{P}$ networking approaches were applied to the networking of SPs and SCs. To define an adequate approach for the exchange of messages, different Information Exchange Patterns (IxP) have been analysed with the result that the Subscribe/Notify IxP is the most appropriate pattern. To define an optimal P2P communication protocol, the protocols suggested by oneM2M were analysed. The evaluation has shown that CoAP is the only suitable of the proposed protocols considering the defined framework requirements. As an alternative, SIP was proposed as communication protocol, since the technical requirements for communication via SIP are already present in the end-user environment. A new SIP Protocol Binding was specified to enable oneM2M standards-compliant communication between SP and SC for service utilisation. The comparison of CoAP and SIP showed that although CoAP has the lower message overhead, SIP should be regarded as more advantageous, in particular through the newly proposed integration of multimedia communication in $\mathrm{M} 2 \mathrm{M}$ service platforms.

The use of a $\mathrm{P} 2 \mathrm{P}$ overlay was proposed to realise decentralised data management for the administration of the service interface descriptions. Different algorithms for managing structured and unstructured P2P overlays were analysed in the context of the presented framework. As a result, structured overlays were found to be advantageous for many 
search queries and a required guarantee of success. Unstructured overlays have the advantage of supporting a high fluctuation of peers.

Another key novelty of this research work is the cooperative M2M application service provision approach. Approaches and algorithms were defined to carry out a decentralised composition of the distributed M2M application. The application modelling and the formal description is also done by modelling a state machine. A mechanism has been designed to realise a distributed state machine which corresponds to the described application logic and to build a service overlay network. In this completely new approach, all nodes involved are able to embed themselves autonomously in the application context by analysing the relevant part of the formal description. Since the autonomous embedding in the application context can lead to invalid interconnections, an algorithm has been designed enabling each peer to recognise independently whether an invalid configuration exists. This new approach enables realise distributed M2M applications, which in the existing MSP approaches is only possible with the help of a central service orchestrator.

The new approach for the decentralised M2M application service provision is completed by the introduction of a new M2M community. The introduction of an M2M community created the ability for end-users to interlink with each other on the basis of a social network for offering and utilising services.

The final chapter 7 described the evaluation of the defined requirements (refer to section 3.2) with the result that all the specified requirements are fulfilled by the presented framework. The verification of the main framework functionalities has been established by the development of a research prototype. The research prototype has been successfully adopted for the proof of concept of the proposed framework by demonstrating exemplary 
use cases for local M2M application modelling and execution with remote M2M service integration as well as for cooperative M2M application service execution.

Several publications on the different aspects of the research outcomes were presented at related conferences, which received positive comments from the reviewers and delegates.

\subsection{Limitations of the research}

Although the overall objectives of research were achieved, some decisions had to be made that limited the work of this research. In principle, these decisions were made for practical reasons, to limit the expenditure in areas where no new findings were expected or were outside the scope of this research. The main restrictions are summarised below.

1. Only the IMMMSC of the defined MMSCs was implemented in the framework prototype, since its interfaces can be operated with the same client application. However, the implementation of the other MMSCs would not have been relevant, since the IMMMSC already serves both input and output interface.

2. The Service Design Unit does not support parallel state elements. This is because the JsPlumb library does not support nested state elements in the used and freely available version. However, the modelling of AND-State elements has been described in detail, so that this functionality can be easily implemented when the library functionality is available. Furthermore, the conditions for the state transitions must be defined statically. Here, the definition could be done via selection lists, but this is only a design aspect of the GUI. The basic functionality 
of dynamic conditions (defined in JEXL format) and their evaluation have been validated so that it can be implemented easily.

3. The implementation of structured and unstructured overlays for Gnutella and Chord algorithms were integrated into the prototype implementation. However, the performance is highly dependent on the library implementation and its configuration options (e.g. stabilisation processes, redundancies or update intervals), so that no reliable analysis can be performed with the prototype implementation. However, the performance of the different overlay algorithms was evaluated based on literature research results. A modular design of the prototype makes it easy to replace the overlay algorithm.

4. The application validation algorithm has not been implemented. The mathematical principles used have already been sufficiently proven, so that it can be assumed that the algorithm is valid. Implementation in the prototype would not have brought much added value.

5. The integration of the $\mathrm{P} 2 \mathrm{P}$ overlay layer into the system architecture enables completely decentralised data storage, which meets the requirements of avoiding central entities. Distributed data storage, however, creates additional traffic for overlay management and searching datasets.

6. The security aspect of the proposed platform architecture is limited to distributed data storage and the definition of access rights via the IFDs. This prevents data from being stored at central locations and enables applications to be restricted to specific user groups. Security aspects such as trustworthiness of applications provided by end-users, access protection for the overlay or authentication of users to use M2M applications were not considered. 
7. The presented framework does not include any approaches to validate the provided M2M applications in terms of functionality, reliability or availability. These aspects were not the focus of this project and could be part of further research.

Despite this restrictions, this research project made valid contributions to knowledge and provided sufficient evidence of the concept for the proposed approaches.

\subsection{Suggestions and Future Work}

This research has expanded the field of MSPs by introducing new approaches and ideas for the decentralised realisation of MSPs with native end-user integration. However, some areas for future research have been identified during this research. Possible extensions of this research are listed subsequently.

1. Further research can be undertaken in the area of application modelling. Since application semantics modelling is independent of the executing component, alternative approaches for generating the application model could be designed. Possible are, e.g. the definition of an application model by means of natural language, in which the connections of the devices and services are described verbally.

2. Because of the independence from central components that are located somewhere in the Cloud, it can be investigated whether the described concepts can be used advantageously in networks that are locally limited in their extent and are not 
connected to public networks, e.g. Mobile Ad-hoc networks or Wireless Mesh Networks.

3. The distributed provision of M2M applications, especially in the end-user domain, results in new challenges. It can be investigated how the functionality of a distributed application can be tested. Since according to the described concept there is no central entity that would test applications as in traditional systems, it is possible to research how a distributed autonomous test approach could be realised. Another aspect of testing that can be considered is the compatibility of services with each other.

4. While some of the aforementioned security restrictions can be solved by development activities (e.g. access protection), the aspect of trust could be further examined. Since M2M services are provided by end-users, a mechanism could be designed that allows a distributed trust determination, so that only trusted M2M services can connect to each other.

5. In this approach, the application developers define the interface descriptions. Since this contains many freely definable fields, semantics and ontologies can be examined for their application in describing M2M services to specify them uniquely and also to get alternative services offered during the search process. 



\section{References}

1. 3GPP TS 23.002 v8.7.0 (2010), Technical Specification, "Network Architecture (Release 8)", 3GPP

2. 3GPP TS 23.228 v5.15.0 (2006), Technical Specification, "IP Multimedia Subsystem (IMS); Stage 2 (Release 5)", 3GPP

3. 3GPP TS 23.682 v14.2.0 (2016), Technical Specification, “Architecture enhancements to facilitate communications with packet data networks and applications (Release 14)", 3GPP

4. Amaral, L. A.; Tiburski, R. T.; Matos, E.; Hessel, F. (2015), "Cooperative Middleware Platform as a Service for Internet of Things Applications", Proceedings of the $30^{\text {th }}$ Annual ACM Symposium on Applied Computing (SAC'15), pp. 488-493, Salamanca, Spain, ACM

5. Apache ANT (2017), "The Apache ANT Project", Available at: http://ant.apache.org/, [accessed $19^{\text {th }}$ February 2017]

6. Apache Felix (2017), “Apache Felix", Available at: http://felix.apache.org/, [accessed $07^{\text {th }}$ November 2017]

7. Apache Karaf (2017), “Apache Karaf”, Available at: http://karaf.apache.org/, [accessed 07 $7^{\text {th }}$ November 2017]

8. Apache Struts (2017), Apache Foundation, "Apache Struts", Available at: https://struts.apache.org/[accessed $1^{\text {st }}$ February 2017]

9. Apache XML-RPC (2017), “Apache XML-RPC", Available at: https://ws.apache.org/xmlrpc/, [accessed 09 ${ }^{\text {th }}$ November 2017]

10. Appcelerator (2017), Appcelerator Inc., "Titanium - Cross-platform mobile app development using JavaScript", Available at: http://www.appcelerator.com/mobile-app-development-products/[accessed $15^{\text {th }}$ February 2017]

11. Arndt, M. and Koss, J. (2014), "ETSI M2M Horizontal Platform Strategy", DG Connect \& ETSI Workshop on Smart Appliances, ETSI

12. Bahga, A. and Madisetti, V. (2014), "Internet of Things (A Hands-onApproach)", USA, ISBN: 978-0996025515

13. Bayer, T. (2002), "REST Web Services", Whitepaper, Orientation in Objects $\mathrm{GmbH}$, Available at: http://www.oio.de/public/xml/rest-webservices.pdf, [accessed 18 $8^{\text {th }}$ June 2017]

14. Binzenhöfer, A. and Leibnitz, K. (2007), "Estimating Churn in Structured P2P Networks", Proceedings of the $20^{\text {th }}$ International Teletraffic Congress (ITC20 2007), pp. 630-641, June 2007, Ottawa, Canada 
15. Böckenhauer, H. and Hromkovic, J. (2013), "Formale Sprachen" (translated title: "Formal Languages"), Springer, Wiesbaden, Germany, ISBN: 3-658-00725-7, 2013

16. Bonomi, F.; Milito, R.; Zhu, J.; Addepalli, S. (2012), "Fog Computing and Its Role in the Internet of Things", Proceedings of the First Edition of the MCC Workshop on Mobile Cloud Computing (MCC '12), pp. 13-16, Helsinki, Finland, ISBN: 9781-4503-1519-7, ACM

17. Boswarthick, D., Elloumi, O., Hersent, O. (2012), "M2M Communications: A Systems Approach", Wiley, Chichester, West Sussex, United Kingdom, ISBN: 978-1-119-99475-6

18. Carreiro, A. M.; López, G. L.; Moura, P. S.; Moreno, J. I.; Almeida, A. T.; Malaquias, J. L. (2011), "In-house monitoring and control network for the Smart Grid of the future", Proceedings of the $20112^{\text {nd }}$ IEEE PES International Conference and Exhibition on Innovative Smart Grid Technologies, pp. 1-7, IEEE

19. Carrez, F.; Bauer, M.; Boussard, M.; Bui, N.; Jardak, C.; De Loof, J.; Magerkurth, C.; Meissner, S.; Nettsträter, A.; Olivereau, A.; Thoma, M.; Walewski, J. W.; Stefa, J.; Salinas, A. (2013), Technical Report, "Deliverable D1.5 - Final architectural reference model for the IoT v3.0", The Internet of Things Architecture, IoT-A (257521)", IoT-A

20. Clarke, I.; Sandberg, O.; Wiley, B.; Hong, T. W. (2000), "Freenet: a distributed anonymous information storage and retrieval system", Proceedings of the International workshop on Designing privacy enhancing technologies: design issues in anonymity and unobservability, pp. 46-66

21. Clayman, S. and Galis, A. (2011), "INOX: A Managed Service Platform for InterConnected Smart Objects", Proceedings of the Workshop on Internet of Things and Service Platforms (IoTSP 2011), Tokyo, Japan, ACM

22. Clip2 Distributed Search Services (2001), "The Gnutella Protocol Specification v0.4”, Available at: http://web.stanford.edu/class/cs244b/gnutella_protocol_0.4 .pdf, [accessed 28 ${ }^{\text {th }}$ April 2014]

23. CloudFoundry (2017), Cloud Foundry Foundation, "Cloud Foundry Platform", Available at: https://www.cloudfoundry.org/[accessed 15 ${ }^{\text {th }}$ February 2017]

24. Commons JEXL (2017), "Java Expression Language (JEXL)", Available at: http://commons.apache.org/proper/commons-jexl/, [accessed $19^{\text {th }}$ February 2017]

25. Commons SCXML (2016), "Apache Commons SCXML", Available at: http://commons.apache.org/proper/commons-scxml/, [accessed $13^{\text {th }}$ January 2016]

26. CORE (2017), U.S. Naval Research Laboratory, "Common Open Research Emulator (CORE)", Available at: https://www.nrl.navy.mil/itd/ncs/products/core [accessed $08^{\text {th }}$ February 2017]

27. Damour, N. (2014), "oneM2M Taking a Look Inside", Webcast, Available at: http://www.etsi.org/news-events/events/831-2014-10-taking-a-look-insideonem $2 \mathrm{~m}$ [accessed $16^{\text {th }}$ December 2016], ETSI, oneM2M 
28. Danila, I.; Dobrescu, R.; Popescu, D.; Marcu, R.; Ichim, L. (2015), “M2M service platforms and device management", Proceedings of the $9^{\text {th }}$ International Symposium on Advanced Topics in Electrical Engineering (ATEE), pp. 67-72, IEEE

29. Dawaliby, S.; Bradai, A.; Pousset, Y. (2016), "In depth performance evaluation of LTE-M for M2M communications", Proceedings of the 2016 IEEE $12^{\text {th }}$ International Conference on Wireless and Mobile Computing, Networking and Communications (WiMob), pp. 1-8, December 2016, New York, USA, IEEE

30. Dawy, Z.; Saad, W.; Ghosh, A.; Andrews, J. G.; Yaacoub, E. (2017), “Toward Massive Machine Type Cellular Communications", IEEE Wireless Communications, Vol. 24, no.1, pp. 120-128, IEEE

31. De Boever, J. (2007), "Peer-to-Peer Networks as a Distribution and Publishing Model", Proceedings of the ELPUB2007 Conference on Electronic Publishing, pp. 175-187, Vienna, Austria

32. Decker, P. (2012), "Facebook of Things - The challenge of Endusers within M2M Systems", Symphony Teleca Corporation, M2M Summit 2012, Düsseldorf, Germany

33. Docker 17.06.2-ce (2017), Docker Inc., "Docker", Version 17.06.2-ce, Available at: https://www.docker.com/[accessed 05 ${ }^{\text {th }}$ November 2017]

34. Doukas, C. and Antonelli, F. (2013), "COMPOSE: Building smart \& contextaware mobile applications utilizing IoT technologies", Proceedings of the Global Information Infrastructure Symposium (GIIS 2013), pp. 1-6, IEEE

35. Doukas, C. and Antonelli, F. (2014), "A full end-to-end platform as a service for smart city applications" Proceedings of the 2014 IEEE $10^{\text {th }}$ International Conference on Wireless and Mobile Computing, Networking and Communications (WiMob), pp. 181-186, IEEE

36. Doukas, C.; Capra, L.; Antonelli, F.; Jaupaj, E.; Tamilin, A.; Carreras, I. (2015), "Providing generic support for IoT and M2M for mobile devices", Proceedings of the 2015 IEEE RIVF International Conference on Computing \& Communication Technologies - Research, Innovation, and Vision for Future (RIVF), pp. 192-197, IEEE

37. Doukas, C. and Antonelli, F. (2015), "Developing and deploying end-to-end interoperable \& discoverable IoT applications," Proceedings of the 2015 IEEE International Conference on Communications (ICC), pp. 673-678, IEEE

38. Eclipse Californium (2017), “CoAP in Java”, Available at: https://www.eclipse.org/californium/, [accessed $08^{\text {th }}$ November 2017]

39. Eclipse Equinox (2017), "Equinox OSGi”, Available at: http://www.eclipse.org/equinox/, [accessed 0 $7^{\text {th }}$ November 2017]

40. Eclipse Jetty (2017), "Jetty://", Available at: https://www.eclipse.org/jetty/, [accessed $10^{\text {th }}$ November 2017] 
41. Elloumi, O. (2014), oneM2M, “oneM2M Service Layer Platform - Initial Release", Available at: http://www.onem2m.org/onem2m-showcase/showcasepresentations [accessed $18^{\text {th }}$ August 2017]

42. e-SCHEMA (2015), Steinheimer, M.; Trick, U.; Wacht, P.; Fischer, M.; Hölker, D.; Tönjes, R., "e-SCHEMA Schlussbericht" (translated title: "e-SCHEMA Final Report"), Research Project: easy- Service Creation for Home and Energy Management (e-SCHEMA), Federal Ministry of Education and Research (BMBF), Federal Republic of Germany, 2012-2015, Grant Number 17018B11

43. ETSI (2014), "Mobile-Edge Computing-Introductory Technical White Paper", Available at: https://portal.etsi.org/Portals/0/TBpages/MEC/Docs/Mobileedge_Computing_-_Intr oductory_Technical_White_Paper_V1\%2018-09-14.pdf, ETSI

44. ETSI ES 201 235-1 V1.1.1 (2000), ETSI Standard, "Specification of Dual Tone Multi-Frequency (DTMF); Transmitters and Receivers Part 1: General”, ETSI

45. ETSI TR 101584 V2.1.1 (2013), ETSI Technical Report, "Machine-to-Machine Communications (M2M); Study on Semantic support for M2M Data", ETSI

46. ETSI TR 102725 V1.1.1 (2013), ETSI Technical Report, "Machine-to-Machine communications (M2M); Definitions", ETSI

47. ETSI TR 102966 V1.1.1 (2014), ETSI Technical Report, "Machine-to-Machine Communications (M2M); Interworking between the M2M Architecture and M2M Area Network technologies", ETSI

48. ETSI TS 102690 V2.1.1 (2013), ETSI Technical Specification, "Machine-toMachine communications (M2M); Functional architecture", ETSI

49. ETSI TS 123228 V11.10.0 (2013), ETSI Technical Report, "IP Multimedia Subsystem (IMS); Stage 2; Release 11", ETSI

50. eQ-3 BidCos (2016), eQ-3 AG, "BidCoS: Funkstandard bei HomeMatic", Available at: https://www.homeandsmart.de/bidcos-funkstandard-eq-3hausautomation [accessed $7^{\text {th }}$ June 2016]

51. Fielding, R. T. (2000), "Architectural Styles and the Design of Network-based Software Architectures", Ph.D. Thesis, University of California, Irvine, USA

52. Foschini, L.; Taleb, T.; Corradi, A.; Bottazzi, D. (2011), "M2M-based metropolitan platform for IMS-enabled road traffic management in IoT", IEEE Communications Magazine, Vol. 49, no. 11, pp. 50-57, IEEE

53. Freenet (2000), “The Freenet Project", Available at: https://freenetproject.org/pages/documentation.html, [accessed 10 ${ }^{\text {th }}$ March 2017]

54. Geambasu, C. V. (2012), "BPMN vs. UML Activity Diagram for Business Process Modeling", Journal of Accounting and Management Information Systems, Vol. 11, No. 4, pp. 637-651

55. Grandison, T.; Maximilien, E. M.; Thorpe, S.; Alba, A. (2010), "Towards a Formal Definition of a Computing Cloud", Proceedings of the 2010 IEEE $6^{\text {th }}$ World Congress on Services, pp. 191-192, IEEE 
56. Harel, D. (1987), "Statecharts: a visual formalism for complex systems", Science of Computer Programming, Vol. 8, No. 3, pp. 231-274

57. Harel, D. and Kugler, H. (2004), "The RHAPSODY Semantics of Statecharts (or, On the Executable Core of the UML)", Integration of Software Specification Techniques for Applications in Engineering, Vol. 3147, pp. 325-354, Springer

58. Harel, D. and Naamad, A. (1996), "The STATEMATE Semantics of Statecharts", Transactions on Software Engineering and Methodology (TOSEM), Vol. 5, Issue 4, pp. 293-333, ACM

59. Harel, D. and Politi, M. (1998), "Modeling Reactive Systems with Statecharts: The Statemate Approach", McGraw-Hill, New York, USA, ISBN: 0-07-026205-5

60. Hauswirth, M. and Dustdar, S. (2005), "Peer-to-Peer: Grundlagen und Architektur" (translated title: "Peer-to-Peer: Fundamentals and Architecture"), DatenbankSpektrum, Vol. 13, pp. 5-13

61. Hibernate (2017), Red Hat Inc. "Hibernate", Available at: http://hibernate.org/[accessed $1^{\text {st }}$ February 2017]

62. Holler, J.; Tsiatsis, V.; Mulligan, C.; Avesand, S.; Karnouskos, S.; Boyle, D. (2014), "From Machine-to-Machine to the Internet of Things", Elsevier, Waltham, USA, ISBN: 978-0-12-407684-6

63. IETF RFC 768 (1980), Request For Comments, "User Datagram Protocol”, IETF

64. IETF RFC 791 (1981), Request For Comments, "Internet Protocol”, IETF

65. IETF RFC 793 (1981), Request For Comments, "Transmission Control Protocol”, IETF

66. IETF RFC 2068 (1997), Request For Comments, "Hypertext Transfer Protocol -HTTP/1.1", IETF

67. IETF RFC 2326 (1998), Request For Comments, "Real Time Streaming Protocol (RTSP)", IETF

68. IETF RFC 3261 (2002), Request For Comments, "SIP: Session Initiation Protocol", IETF

69. IETF RFC 3265 (2002), Request For Comments, "Session Initiation Protocol (SIP)-Specific Event Notification", IETF

70. IETF RFC 3428 (2002), Request For Comments, "Session Initiation Protocol (SIP) Extension for Instant Messaging", IETF

71. IETF RFC 3550 (2003), Request For Comments, "RTP: A Transport Protocol for Real-Time Applications", IETF

72. IETF RFC 3629 (2003), Request For Comments, "UTF-8, a transformation format of ISO 10646", IETF

73. IETF RFC 3665 (2003), Request For Comments, "Session Initiation Protocol (SIP) Basic Call Flow Examples", IETF 
74. IETF RFC 3903 (2004), Request For Comments, "Session Initiation Protocol (SIP) Extension for Event State Publication", IETF

75. IETF RFC 4566 (2006), Request For Comments, "SDP: Session Description Protocol", IETF

76. IETF RFC 4975 (2007), Request For Comments, "The Message Session Relay Protocol (MSRP)", IETF

77. IETF RFC 6086 (2011), Request For Comments, "Session Initiation Protocol (SIP) INFO Method and Package Framework", IETF

78. IETF RFC 6141 (2011), Request For Comments, "Re-INVITE and Target-Refresh Request Handling in the Session Initiation Protocol (SIP)", IETF

79. IETF RFC 6234 (2011), Request For Comments, "US Secure Hash Algorithms (SHA and SHA-based HMAC and HKDF)”, IETF

80. IETF RFC 6314 (2011), Request For Comments, "NAT Traversal Practices for Client-Server SIP”, IETF

81. IETF RFC 6455 (2011), Request For Comments, “The WebSocket Protocol”, IETF

82. IETF RFC 6940 (2014), Request For Comments, "REsource LOcation And Discovery (RELOAD) Base Protocol", IETF

83. IETF RFC 7159 (2014), Request For Comments, “The JavaScript Object Notation (JSON) Data Interchange Format", IETF

84. IETF RFC 7252 (2014), Request For Comments, "The Constrained Application Protocol (CoAP)", IETF

85. IETF RFC 7374 (2014), Request For Comments, "Service Discovery Usage for REsource LOcation And Discovery (RELOAD)", IETF

86. IETF RFC 7650 (2015), Request For Comments, "A Constrained Application Protocol (CoAP) Usage for REsource LOcation And Discovery (RELOAD)", IETF

87. IETF RFC 7890 (2016), Request For Comments, "Concepts and Terminology for Peer-to-Peer SIP (P2PSIP)", IETF

88. IETF RFC 7904 (2016), Request For Comments, “A SIP Usage for REsource LOcation And Discovery (RELOAD)", IETF

89. ISO/IEC 19514 (2017), International Standard, "Information Technology - Object Management Group Systems Modeling Language (OMG SysML), First Edition, ISO/IEC

90. ISO IEC 20000-1:2011 (2013) "Part 1: Service management system requirements", IEEE

91. ITIL V3.1.24 (2007), "Glossary of Terms, Definitions and Acronyms”, ITIL

92. ITU-T E.4110 (2010), Recommendation, "Framework for operations requirements of next generation networks and services", ITU-T 
93. ITU-T Q.23 (1993), Recommendation, "Technical Features of Push-Button Telephone Sets", ITU-T

94. ITU-T Y.101 (2000), Recommendation, "Global Information Infrastructure terminology: Terms and definitions", ITU-T

95. ITU-T Y.110 (1998), Recommendation, "Global Information Infrastructure principles and framework architecture", ITU-T

96. ITU-T Y.2001 (2004), Recommendation, “General overview of NGN”, ITU-T

97. ITU-T Y.2012 (2006), Recommendation, "Functional requirements and architecture of the NGN release 1", ITU-T

98. ITU-T Y.2301 (2013), Recommendation, "Network intelligence capability enhancement - Requirements and capabilities", ITU-T

99. JAIN SIP (2017), "JSR 32: JAIN SIP API Specification", Available at: https://jcp.org/en/jsr/detail?id=32, [accessed $09^{\text {th }}$ November 2017]

100. JsPlumb (2017), jsPlumb Pty Ltd, “jsPlumb Toolkit”, Available at: https://jsplumbtoolkit.com/, [accessed 07 $7^{\text {th }}$ November 2017]

101. JTella (2016), "JTella, a Java API for GNUTella”, Available at: https://osdn.net/projects/sfnet_jtella/, [accessed $07^{\text {th }}$ January 2016]

102. Kim, E. K.; Kim, Y.; Chong, I. (2009), "Architectural model and service scenario of dynamic service overlay network (DSON)", Proceedings of the 2009 First International Conference on Ubiquitous and Future Networks, pp. 52-55, IEEE

103. Kim, Y.; Kim, E.; Chong, I. (2010), "An architectural view of service overlay networking platform for future user-centric networking", Proceedings of the 2010 Second International Conference on Ubiquitous and Future Networks (ICUFN), pp. 22-26, IEEE

104. Kim, E. K.; Kim, Y. J.; Chong, I. (2011), "Architecture of service overlay network platform for web-based multimedia applications", Proceedings of the 2011 Third International Conference on Ubiquitous and Future Networks (ICUFN), pp. 387392, IEEE

105. Kim, Y. J.; Kim, E. K.; Nam, B. W.; Chong, I. (2012), "Service composition using new DSON platform architecture for M2M service," Proceedings of the International Conference on Information Network 2012, pp. 114-119, IEEE

106. Kim, J.; Lee J.; Kim, J.; Yun, J. (2014), "M2M Service Platforms: Survey, Issues, and Enabling Technologies", Proceedings of the $16^{\text {th }}$ IEEE Communications Surveys \& Tutorials, pp. 61-76, IEEE

107. Kitagami, S.; Miyanishi, Y.; Urano, Y.; Shiratori, N. (2014), "Proposal of a Distributed Cooperative M2M System for Flood Disaster Prevention", Proceedings of the 2014 IEEE $11^{\text {th }}$ Intl Conf on Ubiquitous Intelligence and Computing and 2014 IEEE $11^{\text {th }}$ Intl Conf on Autonomic and Trusted Computing and 2014 IEEE $14^{\text {th }}$ Intl Conf on Scalable Computing and Communications and Its Associated Workshops, pp. 637-642, IEEE 
108. Labiak, G. and Miczulski, P. (2004), "UML statecharts and Petri nets model comparison for system level modelling", Mezdunarodnyj sbornik naucnych trudov: Progressivnye technologii i sistemy masinostroenija, Vol. 27, pp. 310-314

109. LeClair, D. (2014), Innovation Insights, "The Edge of Computing: It's Not ALL About the Cloud", Available at: http://insights.wired.com/profiles/blogs/the-edgeof-computing-it-s-not-all-about-the-cloud\#axzz3rC9fmJ23 [accessed $\quad 11^{\text {th }}$ November 2015]

110. Liang, J.; Kumar, R.; Ross, K. W. (2006), "The FastTrack overlay: a measurement study", The International Journal of Computer and Telecommunications Networking - Overlay distribution structures and their applications, Vol. 50, no.6, pp. $842-858$

111. Liu, M.; Leppänen, T.; Harjula, E.; Ou, Z.; Ramalingam, A; Ylianttila, M.; Ojala, T. (2013), "Distributed resource directory architecture in Machine-to-Machine communications", 2013 IEEE $9^{\text {th }}$ International Conference on Wireless and Mobile Computing, Networking and Communications (WiMob), pp. 319-324, October 2013, Lyon, France, IEEE

112. López, G.; Moura, P.; Moreno, J. I.; Almeida, A. (2011a), "ENERsip: M2M-based platform to enable energy efficiency within energy-positive neighbourhoods", Proceedings of the 2011 IEEE Conference on Computer Communications Workshops (INFOCOM WKSHPS), pp. 217-222, IEEE

113. López, G.; Moura, P.; Sikora, M.; Moreno, J. I.; Almeida, A. T. (2011b), "Comprehensive validation of an ICT platform to support energy efficiency in future smart grid scenarios", Proceedings of the 2011 IEEE International Conference on Smart Measurements of Future Grids (SMFG), pp. 113-118, IEEE

114. López, G.; Moura, P. S.; Custodio, V.; Moreno, J. I. (2012), "Modeling the Neighborhood Area Networks of the Smart Grid", Proceedings of the 2012 IEEE International Conference on Communications (ICC), pp. 3357-3361, IEEE

115. López, G.; Almeida, A.; Moura, P.; Pérez, M.; Moreno, J.; Blanco, L. (2013), "Monitoring system for the local distributed generation infrastructures of the Smart Grid", Proceedings of the $22^{\text {nd }}$ International Conference and Exhibition on Electricity Distribution (CIRED 2013), pp. 1-4, IEEE

116. Lua, E. K.; Crowcroft, J.; Pias, M. (2005), "A Survey and Comparison of Peer-ToPeer Overlay Network Schemes", Journal IEEE Communications Surveys \& Tutorials, Vol. 7, no.2, pp. 72-93, IEEE

117. Mackenzie, M. (2014), Analysis Mason, "M2M device connections and revenue: worldwide forecast 2014 - 2024", Available at: http://www.analysysmason.com/Research/Content/Reports/M2M-forecast-2014Nov2014-RDME0/[accessed $18^{\text {th }}$ April 2017]

118. Magedanz, T. and de Gouveia, F.C. (2006), "IMS - the IP Multimedia System as NGN Service Delivery Platform”, Elektrotechnik \& Informationstechnik, Vol. 123, Issue 7-8, pp. 271-276, Springer 
119. Malatras, A. (2015), "State-of-the-art survey on P2P overlay networks in pervasive computing environments", Journal of Network and Computer Applications, Vol. 55, pp. $1-23$

120. Malkhi, D.; Naor, M.; Ratajczak, D. (2002), "Viceroy: a scalable and dynamic emulation of the butterfly", Proceedings of the ACM PODC '02 twenty-first annual symposium on Principles of distributed computing, pp. 183-192, ACM

121. Mandler, B.; Antonelli, F.; Kleinfeld, R.; Pedrinaci, C.; Carrera, D.; Gugliotta, A.; Schreckling, D.; Carreras, I.; Raggett, D.; Pous, M.; Villares, C. V.; Trifa, V. (2013), "COMPOSE -- A Journey from the Internet of Things to the Internet of Services" Proceedings of the $201327^{\text {th }}$ International Conference on Advanced Information Networking and Applications Workshops, pp. 1217-1222, IEEE

122. Maymounkov, P. and Mazieres, D. (2002), "Kademlia: A Peer-to-Peer Information System Based on the XOR Metric", Proceedings of the IPTPS '01 International Workshop on Peer-to-Peer Systems, pp. 53-65

123. Mehmood, Y.; Görgn C.; Muehleisen, M.; Timm-Giel, A. (2015), "Mobile M2M communication architectures, upcoming challenges, applications, and future directions", EURASIP Journal on Wireless Communications and Networking, Springer

124. Milojicic, D.; Kalogeraki, V.; Lukose, R.; Nagaraja, K.; Pruyne, J.; Richard, B.; Rollins, S.; Xu, Z. (2002), "Peer-to-Peer Computing", Technical Report, HP Laboratories Palo Alto, Available at: http://www.hpl.hp.com/techreports/2002/HPL-2002-57R1.pdf [accessed $3^{\text {rd }}$ September 2017]

125. Monteil, T. and Alaya, M. B. (2014), "OM2M: A flexible ETSI-compliant service platform for M2M", Eclipse IoT Day, Grenoble, France

126. NodeRed (2017), JS Foundation, "Node-RED Flow-based programming for the Internet of Things", Available at: http://nodered.org/[accessed $15^{\text {th }}$ February 2017]

127. OASIS SOA-RM-V1.0 (2006), "Reference Model for Service Oriented Architecture 1.0", OASIS

128. OASIS (2007), OASIS Standard, "Web Services Business Process Execution Language Version 2.0", OASIS

129. OASIS mqtt-v3.1.1 (2014), "MQTT Version 3.1.1”, OASIS

130. Object Management Group, OMG (2011), "Business Process Model and Notation (BPMN)", Version 2.0, Boston, USA, January 2011, OMG

131. Object Management Group, OMG (2014), "Model Driven Architecture (MDA) MDA Guide rev. 2.0", Version 2.0, Boston, USA, June 2014, OMG

132. Object Management Group, OMG (2015), "OMG Unified Modeling Language (OMG UML)", Version 2.5, Boston, USA, March 2015, OMG

133. Object Management Group, OMG (2017a), "OMG Model Driven Architecture", Available at: http://www.omg.org/mda/[accessed $25^{\text {th }}$ January 2017], OMG 
134. Object Management Group, OMG (2017b), "Information Technology - Object Management Group Systems Modeling Language (OMG SysML)", Boston, USA, Mai 2017, OMG

135. ODE (2017), “Apache ODE”, Available at: http://ode.apache.org/, [accessed $8^{\text {th }}$ June 2017]

136. OM2M (2016), The Eclipse Foundation, "OM2M Connecting Things", Available at: http://www.eclipse.org/om2m/[accessed 22 $2^{\text {th }}$ December 2016]

137. oneM2M (2015), “The Interoperability Enabler for entire M2M and IoT Ecosystem [White Paper]", oneM2M

138. oneM2M TR-0001-V2.4.1 (2016), Technical Report, "Use Cases Collection”, oneM2M

139. oneM2M TR-0007-V1.0.0 (2014), Technical Report, "Study of Abstraction and Semantics Enablements", oneM2M

140. oneM2M TR-0007-V2.11.1 (2016), Technical Report, "Study of Abstraction and Semantics Enablements", oneM2M

141. oneM2M TR-0025-V1.0.0 (2016), Technical Report, “Application Developer Guide", oneM2M

142. oneM2M TS-0001-V1.13.1 (2016), Technical Specification, "Functional Architecture", oneM2M

143. oneM2M TS-0001-V2.10.0 (2016), Technical Specification, "Functional Architecture", oneM2M

144. oneM2M TS-0002-V1.0.1 (2015), Technical Specification, "Requirements", oneM2M

145. oneM2M TS-0002-V2.7.1 (2016), Technical Specification, "Requirements", oneM2M

146. oneM2M TS-0004-V-2014-08 (2014), Technical Specification, "Service Layer Protocol Core Specification", oneM2M

147. oneM2M TS-0004-V2.7.1 (2016), Technical Specification, "Service Layer Protocol Core Specification", oneM2M

148. oneM2M TS-0007-V2.0.0 (2016), Technical Specification, "Service Components", oneM2M

149. oneM2M TS-0008-V1.0.1 (2015), Technical Specification, "CoAP Protocol Binding”, oneM2M

150. oneM2M TS-0009-V2.6.1 (2016), Technical Specification, "HTTP Protocol Binding TS", oneM2M

151. oneM2M TS-0010-V2.4.1 (2016), Technical Specification, "MQTT Protocol Binding", oneM2M

152. oneM2M TS-0011-V1.2.1 (2015), Technical Specification, "Common Terminology", oneM2M 
153. oneM2M TS-0011-V2.4.1 (2016), Technical Specification, "Common Terminology", oneM2M

154. oneM2M TS-0020-V2.0.0 (2016), Technical Specification, "WebSocket Protocol Binding TS", oneM2M

155. Open Chord (2015), “Open Chord", Available at: https://sourceforge.net/projects/open-chord/, [accessed $8^{\text {th }}$ November 2015]

156. Oracle (2017a), Oracle Corporation, “Oracle BPEL Process Manager”, Available at: http://www.oracle.com/technetwork/middleware/bpel/overview/index.html, [accessed $8^{\text {th }}$ June 2017]

157. Oracle (2017b), Oracle Corporation, “Java Platform, Standard Edition”, Available at: $\quad$ http://www.oracle.com/technetwork/java/javase/downloads/index.html [accessed 05 $5^{\text {th }}$ November 2017]

158. Oracle (2017c), Oracle Corporation, "Java Architecture for XML Binding (JAXB)", Available at: http://www.oracle.com/technetwork/articles/javase/index140168.html [accessed $09^{\text {th }}$ November 2017]

159. Oracle (2017d), Oracle Corporation, "Windows System Requirements for JDK and JRE", Available at: https://docs.oracle.com/javase/8/docs/technotes/guides/ install/windows_system_requirements.html\#BABHGIJF [accessed $22^{\text {th }}$ November 2017]

160. Oracle (2017e), Oracle Corporation, "JAVA SE EMBEDDED SYSTEM REQUIREMENTS", Available at: http://www.oracle.com/technetwork/java/ embedded/embedded-se/documentation/javase-embedded-sysreq-2043454.html [accessed 22 $2^{\text {th }}$ November 2017]

161. Oram, A. (2001), “Peer-to-Peer: Harnessing the Power of Disruptive Technologies (1st edition)", O’Reilly \& Associates, Inc., Sebastopol, USA, ISBN: 9780596001100

162. Osanaiye, O.; Chen, S.; Yan, Z.; Lu, R.; Choo, K.; Dlodlo, M. (2017), "From cloud to fog computing: A review and a conceptual live VM migration framework", IEEE Access Journal Issue: 99, IEEE

163. OSGi Alliance R5 (2012), "OSGi Core Release 5”, OSGi specification, Version 5.0.0

164. P2P4M2M (2016), Steinheimer, M.; Shala, B.; Trick, U.; Ghita, B., "P2P4M2M Zwischenbericht" (translated title: "P2P4M2M Preliminary Report"), Research Project: Optimierte P2P-Dienstearchitektur für hochverfügbare M2MApplikationen (P2P4M2M) (translated title: Optimised P2P Service Architecture for highly available M2M Applications (P2P4M2M)), Federal Ministry of Education and Research (BMBF), Federal Republic of Germany, 2015-2019, Grant Number 03FH022IX5

165. Padilla, J. E. V.; Lee, J. O.; Kim, J. H. (2013), "A M2M horizontal services platform implementation over IP multimedia subsystem (IMS)," Proceedings of the $201315^{\text {th }}$ Asia-Pacific Network Operations and Management Symposium (APNOMS), pp. 1-3, IEEE 
166. Pedrinaci, C.; Liu, D.; Maleshkova, M.; Lambert, D.; Kopecky, J.; Domingue, J. (2010), "iServe: a linked services publishing platform", Proceedings of the Ontology Repositories and Editors for the Semantic Web Workshop at the $7^{\text {th }}$ Extended Semantic Web Conference, Heraklion, Greece.

167. Petrasch, R. and Meimberg, O. (2006), "Model Driven Architecture", dpunkt.verlag, Heidelberg, Germany, ISBN: 978-3-89864-343-6, 2006

168. Piatek, M.; Isdal, T.; Anderson, T.; Krishnamurthy, A.; Venkataramani, A. (2007), "Do incentives build robustness in bit torrent", Proceedings of the NSDI'07 $4^{\text {th }}$ USENIX conference on Networked systems design \& implementation, Cambridge, USA

169. Poikselkä, M. and Mayer, G. (2009), "The IMS: IP Multimedia Concepts and Services $\left(3^{\text {rd }}\right.$ edition)", John Wiley \& Sons Inc, Hoboken, USA, ISBN: 978-04707-2196-4

170. Ratnasamy, S.; Francis, P.; Handley, M.; Karp, R.; Shenker, S. (2001), “A scalable content-addressable network", Proceedings of the ACM SIGCOMM 2001 conference on Applications, technologies, architectures, and protocols for computer communications, pp. 161-172, ACM

171. Rayes, A and Salam, S. (2017), "Internet of Things From Hype to Reality", Springer, Heidelberg, Germany, ISBN: 978-3-319-44858-9

172. Rodrigues, L.; Guerreiro, J.; Correia, N. (2016), "RELOAD/CoAP architecture with resource aggregation/disaggregation service", 2016 IEEE $27^{\text {th }}$ Annual International Symposium on Personal, Indoor, and Mobile Radio Communications (PIMRC), pp. 1-6, September 2016, Valencia, Spain, IEEE

173. Rowstron, A. I. T.; Druschel, P. (2001), "Pastry: Scalable, Decentralized Object Location, and Routing for Large-Scale Peer-to-Peer Systems", Proceedings of the IFIP/ACM International Conference on Distributed Systems Platforms, pp. 329350, Heidelberg, Germany, ISBN: 3-540-42800-3, ACM

174. Rupp, C.; Queins, S.; Zengler, B. (2007), "UML Glasklar - Praxiswissen für die UML-Modellierung ( $3^{\text {rd }}$ edition)" (translated title: "UML Crystal Clear - Practical Knowledge for UML Modelling ( $3^{\text {rd }}$ edition)"), Hanser, Munich, Germany, ISBN 978-3-446-41118-0, 2007

175. Samaniego, M. L.; Yelmo, I. M.; Sanchez, R. G. (2013), "Analysis of relod.net, a basic implementation of the RELOAD protocol for peer-to-peer networks", Actas de las Jornadas de Ingeniería Telemática 2013, pp. 147-154, ISBN: 978-84-6165597-7

176. Sarigiannidis, P.; Zygiridis, T.; Sarigiannidis, A.; Lagkas, T. D.; Obaidat, M.; Kantartzis, N. (2017), "Connectivity and coverage in machine-type communications", Proceedings of the 2017 IEEE International Conference on Communications (ICC), pp. 1-6, May 2017, Paris, France, IEEE

177. Savaglio, C. and Fortino, G. (2015), "Autonomic and Cognitive Architectures for the Internet of Things", Proceedings of the $8^{\text {th }}$ International Conference on Internet and Distributed Computing Systems (IDCS 2015), pp. 39-47, Windsor, UK 
178. Shala, B.; Wacht, P.; Trick, U.; Lehmann, A.; Ghita, B.; Shiaeles, S. (2017a), "Trust Integration for Security Optimisation in P2P-based M2M Applications", Proceedings of the 2017 IEEE Trustcom/BigDataSE/ICESS, pp. 949-954, August 2017, Australia, IEEE

179. Shala, B.; Wacht, P.; Trick, U.; Lehmann, A.; Ghita, B.; Shiaeles, S. (2017b), "Ensuring Trustworthiness for P2P-based M2M Applications ", Proceedings of the Seventh International Conference on Internet Technologies and Applications (ITA 17), pp. 58-63, September 2017, Wrexham, UK, IEEE

180. Shi, J.; Wan, J.; Yan, H.; Suo, H. (2011), “A Survey of Cyber-Physical Systems", Proceedings of the Int. Conf. on Wireless Communications and Signal Processing, November 2011, Nanjiing, China

181. Shiratori, N.; Inaba, T.; Nakamura, N.; Suganuma, T. (2012), "Disaster-resistant Green-oriented Never Die Network", Journal of Information Processing Society of Japan, Vol. 53, no. 7, pp. 1821-1831

182. Spring (2017), Pivotal Software, "Spring Framework", Available at: https://spring.io/[accessed $1^{\text {st }}$ February 2017]

183. Spring Boot R1.5.8 (2017), Pivotal Software, "Spring Boot Reference Guide 1.5.8.RELEASE", Release 1.5.8

184. Steinheimer, M.; Trick, U.; Ruhrig, P. (2012a), "New approaches for energy optimisation in Smart Homes and Smart Grids by automated service generation and user integration", Proceedings of the Collaborative European Research Conference (CERC), pp. 111-119, April 2012, Darmstadt, Germany

185. Steinheimer, M.; Trick, U.; Ruhrig, P. (2012b), "Energy communities in Smart Markets for optimisation of peer-to-peer interconnected Smart Homes", Proceedings of the $20128^{\text {th }}$ International Symposium on Communication Systems, Networks \& Digital Signal Processing (CSNDSP), pp. 1-6, July 2012, Poznan, Poland, IEEE

186. Steinheimer, M.; Trick, U.; Ruhrig, P.; Wacht, P.; Tönjes, R.; Fischer, M.; Hölker, D. (2013a), "SIP-basierte P2P-Vernetzung in einer Energie-Community" (translated title: "SIP-based P2P Networking inside an Energy-Community"), Proceedings of the Eighteenth VDE/ITG Mobilfunktagung, pp. 64-70, Mai 2013, Osnabrück, Germany, VDE

187. Steinheimer, M.; Trick, U.; Ruhrig, P.; Fuhrmann, W.; Ghita, B. (2013b), "Load Reduction in Distribution Networks through P2P networked Energy-Community", Proceedings of the Fifth International Conference on Internet Technologies and Applications (ITA 13), pp. 90-97, September 2013, Wrexham, UK

188. Steinheimer, M.; Trick, U.; Wacht, P.; Ruhrig, P. (2013c), "Decentralised optimisation solution for Smart Grids using Smart Market aspects and P2P internetworked Energy-Community“, VDE/IEC World Smart Grid Forum 2013, September 2013, Berlin, Germany, VDE

189. Steinheimer, M.; Trick, U.; Wacht, P.; Ruhrig, P.; Fuhrmann, W.; Ghita, B. (2013d), "Decentralised optimisation solution for provision of value added services 
and optimisation possibilities in Smart Grids", 2013 Collaborative European Research Conference (CERC), October 2013, Cork, Ireland

190. Steinheimer, M.; Trick, U.; Fuhrmann, W.; Ghita, B. (2013e), "P2P-based community concept for M2M Applications“, Proceedings of the Second International Conference on Future Generation Communication Technologies (FGCT 2013), pp. 114-119, November 2013, London, UK, IEEE

191. Steinheimer, M.; Trick, U.; Fuhrmann, W.; Ghita, B. (2015a), "P2P based service provisioning in M2M networks", Proceedings of the Sixth International Conference on Internet Technologies and Applications (ITA 15), pp. 132-137, September 2015, Wrexham, UK, IEEE

192. Steinheimer, M.; Trick, U.; Fuhrmann, W.; Ghita, B. (2015b), "P2P based service provisioning in M2M networks", Second Spanish-Geman Symposium on Applied Computer Science (SGSOACS), Invited Talk, December 2015, Frankfurt, Germany

193. Steinheimer, M.; Trick, U.; Fuhrmann, W.; Ghita, B. (2016), "P2P-based M2M Community Applications“, Proceedings of the Eleventh International Network Conference (INC 2016), pp. 115-120, July 2016, Frankfurt, Germany

194. Steinheimer, M.; Trick, U.; Fuhrmann, W.; Ghita, B.; Frick, G. (2017a), “M2M Application Service Provision: An autonomous and decentralised Approach", Journal of Communications, Vol. 12, no. 9, pp. 489-498, 2017

195. Steinheimer, M.; Trick, U.; Fuhrmann, W.; Ghita, B. (2017b), "Decentralised System Architecture for autonomous and cooperative M2M Application Service Provision", Proceedings of the 2017 IEEE International Conference on Smart Grid and Smart Cities (ICSGSC 2017), pp. 312-317, July 2017, Singapore, IEEE

196. Steinheimer, M.; Trick, U.; Fuhrmann, W.; Ghita, B. (2017c), “Autonomous decentralised M2M Application Service Provision", Proceedings of the Seventh International Conference on Internet Technologies and Applications (ITA 17), pp. 18-23, September 2017, Wrexham, UK, IEEE

197. Steinmetz, R. and Wehrle, K. (2004), "Peer-to-Peer Networking \& -Computing", Informatik-Spektrum, Vol. 27, Issue 1, pp. 51-54, Springer

198. Steinmetz, R and Wehrle, K. (2005), "Peer-to-Peer Systems and Applications", Springer, Heidelberg, Germany, ISBN: 978-3-540-29192-3, 2005

199. Stoica, I.; Morris, R.; Karger, D.; Kaashoek, M. F.; Balakrishnan, H. (2001), "Chord: A Scalable Peer-to-peer Lookup Service for Internet Applications", Proceedings of the ACM SIGCOMM 2001 conference on Applications, technologies, architectures, and protocols for computer communications, pp. 149$160, \mathrm{ACM}$

200. Stutzbach, D. and Rejaie, R. (2006), "Understanding churn in peer-to-peer networks", Proceedings of the $6^{\text {th }}$ ACM SIGCOMM conference on Internet measurement, pp. 189-202, ACM

201. TC IP 1 (2013), UDP Control Protocol for TC IP 1 Energy Manager, Available at: http://www.rutenbeck.de/downloads/softwarefirmwareupdates.html, [accessed $10^{\text {th }}$ July 2013] 
202. Terpak, J.; Horovcak, P.; Lukac, M. (2016), "Mathematical models creation using orchestration and choreography of web services", Proceedings of $17^{\text {th }}$ International Carpathian Control Conference (ICCC), pp. 739-742, IEEE

203. Thakar, U.; Tiwari, A.; Varma, S. (2016), "On Composition of SOAP Based and RESTful Services", 2016 IEEE $6^{\text {th }}$ International Conference on Advanced Computing, pp. 500-505, August 2016, Bhimavaram, India, IEEE

204. Trick, U. and Weber, F. (2015), "SIP und Telekommunikationsnetze (5. Auflage)" (translated title: "SIP and Telecommunication Networks ( $5^{\text {th }}$ edition)"), De Gruyter Oldenbourg, Berlin, Germany, ISBN: 3-486-77853-3

205. Turau, V. (2009), “Algorithmische Graphentheorie (3. Auflage)" (translated title: "Algorithmic Graph Theory ( $3^{\text {rd }}$ edition)"), Oldenbourg Wissenschaftsverlag, Munich, Germany, ISBN: 978-3-486-59057-9

206. UC Santa Cruz (2017), “Is It a Service?”, University of California Santa Cruz, Available at: https://its.ucsc.edu/itsm/service.html [accessed $20^{\text {th }}$ February 2017]

207. Vaquero, L. M. and Rodero-Merino, L. (2014), "Finding Your Way in the Fog: Towards a Comprehensive Definition of Fog Computing", ACM SIGCOMM Journal of Computer Communication Review, Vol. 44, no. 4, pp. 27-32, ACM

208. Vossen, G. and Witt, K. (2016), "Grundkurs Theoretische Informatik" (translated title: "Basic Course Theoretical Computer Science"), Springer, Wiesbaden, Germany, ISBN: 978-3-8348-1770-9, 2016

209. W3C (2000), Recommendation, "Simple Object Access Protocol (SOAP) 1.1", W3C

210. W3C (2001), Recommendation, "Web Services Description Language (WSDL) $1.1 ", \mathrm{~W} 3 \mathrm{C}$

211. W3C (2008), Recommendation, "Extensible Markup Language (XML) 1.0 (Fifth Edition)", W3C

212. W3C (2015), Recommendation, "State Chart XML (SCXML): State Machine Notation for Control Abstraction", W3C

213. Wagenknecht, C. and Hielscher, M. (2015), "Formale Sprachen, abstrakte Automaten und Compiler" (translated title: "Formal Languages, abstract Finite State Machines and Compiler"), Springer, Wiesbaden, Germany, ISBN 3-65802692-8, 2015

214. Wan, J.; Chen, M.; Xia, F.; Li, D.; Zhou, K. (2013), "From Machine-to-Machine Communications towards Cyber-Physical Systems", Computer Science and Information Systems, Vol. 10, no. 3, pp. 1105-1128

215. Warshall, S. (1962), "A Theorem on Boolean Matrices", Journal of the ACM (JACM), Vol. 9, Issue 1, pp. 11-12, ACM

216. Xiaocong, Q. and Jidong, Z. (2010), "Study on the structure of "Internet of Things(IOT)" business operation support platform", Proceedings of the $12^{\text {th }}$ International Conference on Communication Technology, pp. 1068-1071, IEEE 
217. XML-RPC (1999), XML-RPC Specification, Available at: http://xmlrpc.scripting.com/spec.html, [accessed 19 ${ }^{\text {th }}$ February 2017]

218. Zhang, S. K.; Zhang, J. W.; Li, W. (2010), "Design of M2M Platform Based on J2EE and SOA", 2010 International Conference on E-Business and EGovernment, pp. 2029-2032, IEEE

219. Zhao, B. Y.; Huang, L.; Stribling, J.; Rhea, S. C.; Joseph, A. D.; Kubiatowicz, J. D. (2006), "Tapestry: a resilient global-scale overlay for service deployment", IEEE Journal on Selected Areas in Communications, Vol. 22, no.1, pp. 41-53, IEEE 


\title{
Appendix A - Abbreviations
}

\author{
3/4G $\quad 3^{\text {rd }} / 4^{\text {th }}$ Generation Mobile Network \\ 3GPP Third Generation Partnership Project
}

A

AD Application Description

ADI Application Description Interpreter

ADM Application Description Model

ADN Application Dedicated Node

ADP Application Description Parser

ADSP Autonomous Decentralised M2M Service Provision

AE Application Entity, Application Executor

AEE Application Execution Environment

AL Abstraction Layer

AS Application Server

ASN Application Service Node

ASP Application Service Provider

AV Audio/Video

B

BPMN Business Process Model and Notation

BSPU Bottom-Up M2M Service Provision and Utilisation

C

CoAP Constrained Application Protocol 
CPS Cyber Physical System

CR Common Resource

CRUD Create Request Update Delete

CSE Common Service Entity

CSF Common Service Function

CU Communication Unit

D

DCASP Decentralised Cooperative M2M Application Service Provision

DCM Device Capability Model

DHT Distributed Hash Table

DI Data Item

DNS Domain Name System

DRD4M Distributed Resource Directory Architecture for M2M Applications

DTLS Datagram Transport Layer Security

DTMF Dual-tone multi-frequency

$\mathbf{E}$

ETSI European Telecommunications Standards Institute

$\mathbf{F}$

FSM Finite State Machine

G

GUI Graphical User Interface

GW Gateway 
$\mathbf{H}$

HFC Hybrid Fibre Coaxial

HSPU Horizontal M2M Service Provision and Utilisation

HTTP Hypertext Transfer Protocol

I

IAD Integrated Access Device

ICT Information and Communications Technology

ID Identifier

IEC International Electrotechnical Commission

IEEE Institute of Electrical and Electronics Engineering

IETF Internet Engineering Task Force

IF Interface

IFD Interface Description

IM Instant Message

IMMMSC Instant Message Multimedia Service Component

IMS IP Multimedia Subsystem

IN Infrastructure Node

IoT Internet of Things

IP Internet Protocol

ISO International Organization for Standardization

ISP Internet Service Provider

ITU International Telecommunication Union

IxP Information Exchange Pattern 
$\mathbf{J}$

JDBC Java Database Connectivity

JEXL Java Expression Language

JSON JavaScript Object Notation

$\mathbf{L}$

LTE Long Term Evolution

M

M2M Machine-to-Machine Communication

M2M ADisp Application Dispatcher

M2M DCM M2M Device Capability Model

M2M TI M2M Technology Interface

MDA Model Driven Architecture

MM Multimedia

MMSC Multimedia Service Component

MN $\quad$ Middle Node

MQTT Message Queue Telemetry Transport

MSP M2M Service Platform

MSRP Message Session Relay Protocol

$\mathbf{N}$

NGN Next Generation Networks

NSE Network Services Entity 
$\mathbf{O}$

OASIS Organization for the Advancement of Structured Information Standards

OMA Open Mobile Alliance

OMG Object Management Group

ORM Object Relational Mapping

$\mathbf{P}$

P2P Peer-to-Peer

$\mathbf{R}$

RELOAD Resource Location and Discovery Protocol

RemoteAS Remote Alarm Service

RemoteBMS Remote Building Monitoring Service

RemoteDGPPS Remote Distribution Grid Parameter Provision Service

RemoteERS Remote Energy Reduction Service

RemoteSS Remote Sensor Service

REST Representational State Transfer

RFC Request for Comments

RL Requestor List

RTP Real-Time Transport Protocol

RTSP Real-Time Streaming Protocol

S

SAR Service/Application Registry

SC Service Consumer

SCE Service Creation Environment 
SCU Service Creation Unit

SCXML State Chart extensible Markup Language

SDP Service Delivery Platform, Service Description Protocol

SDU Service Design Unit

SEE Service Execution Engine

SIP Session Initiation Protocol

SIU Service Interface Unit

SM State Machine

SM Repo State Machine Repository

SOA Service Oriented Architecture

SP Service Provider

SPU Service Provision Unit

SR Speech Recognition

SRE Service Runtime Environment

$\mathbf{T}$

TLS Transport Layer Security

TTL Time-to-Live

TTS Text-to-Speech

TURN Traversal Using Relays around NAT

TCP Transmission Control Protocol

$\mathbf{U}$

UA User Agent

UAC User Agent Client

UAS User Agent Server 
UDP User Datagram Protocol

UML Unified Modelling Language

UML AD UML Activity Diagram

UML SMD UML Statemachine Diagram

URI Uniform Resource Identifier

V

VAS Value-added Service

VM Virtual Machine

VoIP Voice over Internet Protocol

W

W3C World Wide Web Consortium

Wi-Fi Wireless Local Area Network

WS Webservice

WSBPEL Webservice Business Process Execution Language

WSDL Webservice Description Language

WSN Wireless Sensor Network

$\mathbf{X}$

xDSL Digital Subscriber Line (all variants)

XML Extensible Markup Language 

Appendix B - Own Publications 
Published in Proceedings of the Collaborative European Research Conference (CERC), pp. 111-119, April 2012, Darmstadt, Germany

\title{
New approaches for energy optimisation in Smart Homes and Smart Grids by automated service generation and user integration
}

\author{
M.Steinheimer ${ }^{1,2}$, U.Trick ${ }^{1}$, P.Ruhrig 1 \\ ${ }^{1}$ Research Group for Telecommunication Networks, University of Applied Sciences \\ Frankfurt/M., Frankfurt/M., Germany \\ ${ }^{2}$ Centre for Security, Communications and Network Research, University of \\ Plymouth, Plymouth, United Kingdom \\ E-mail: steinheimer@e-technik.org
}

\begin{abstract}
This paper describes approaches for a framework to design and generate value-added services for management Smart Grids and Smart Homes. It offers users the possibility to design services for managing decentralised energy devices and resources as well as optimise the energy consumption by intelligent energy management. New approaches are introduced that offer automated service generation and optimisation. This new approaches are based on interconnection of households and algorithms for automated optimisation of energy consumption in single households or whole regions, without assistance of third parties. The interconnection of the households rests upon the peer-to-peer principles for communication and automated optimisation as well as forming communities between the participated peers.
\end{abstract}

\section{Keywords}

Smart Grid, Smart Homes, Energy Management, Service Creation, Peer-to-Peer

\section{Introduction}

Today the power supply system is static and consists of dedicated categories. It has been defined where energy is produced and where energy is supplied to the grid. The energy flow basically takes place only in one way: from power generator in the maximum voltage grid to the energy consumer in the Local Grid. Primary large power plants like coal-fired and nuclear power plants supply companies and households with energy. Energy generation is focussed on central appointments in the power system. The transfer of energy occurs with as much as possible electric tension because of the meanest loss rate. Figure 1 shows the structure of the power supply system today.

The power supply system is separated into four levels of electric tension which are used for transfer and distribution of the energy: Transmission Grid (maximum voltage), Primary Distribution Grid (high voltage), Secondary Distribution Grid (mean voltage), and Local Grid (low voltage). In order to reduce the electric tension 
to transfer or rather consume the energy on the next lower level, transformers are used at the transition points.

The supply of energy by the central power plants takes place at several levels of electric tension, according to the amount of produced energy. Large power plants like nuclear power plants supply at maximum voltage level, wind parks at high voltage level, small wind power plants at mean voltage grid and sporadically, e.g. photovoltaic power plants at the Local Grid.

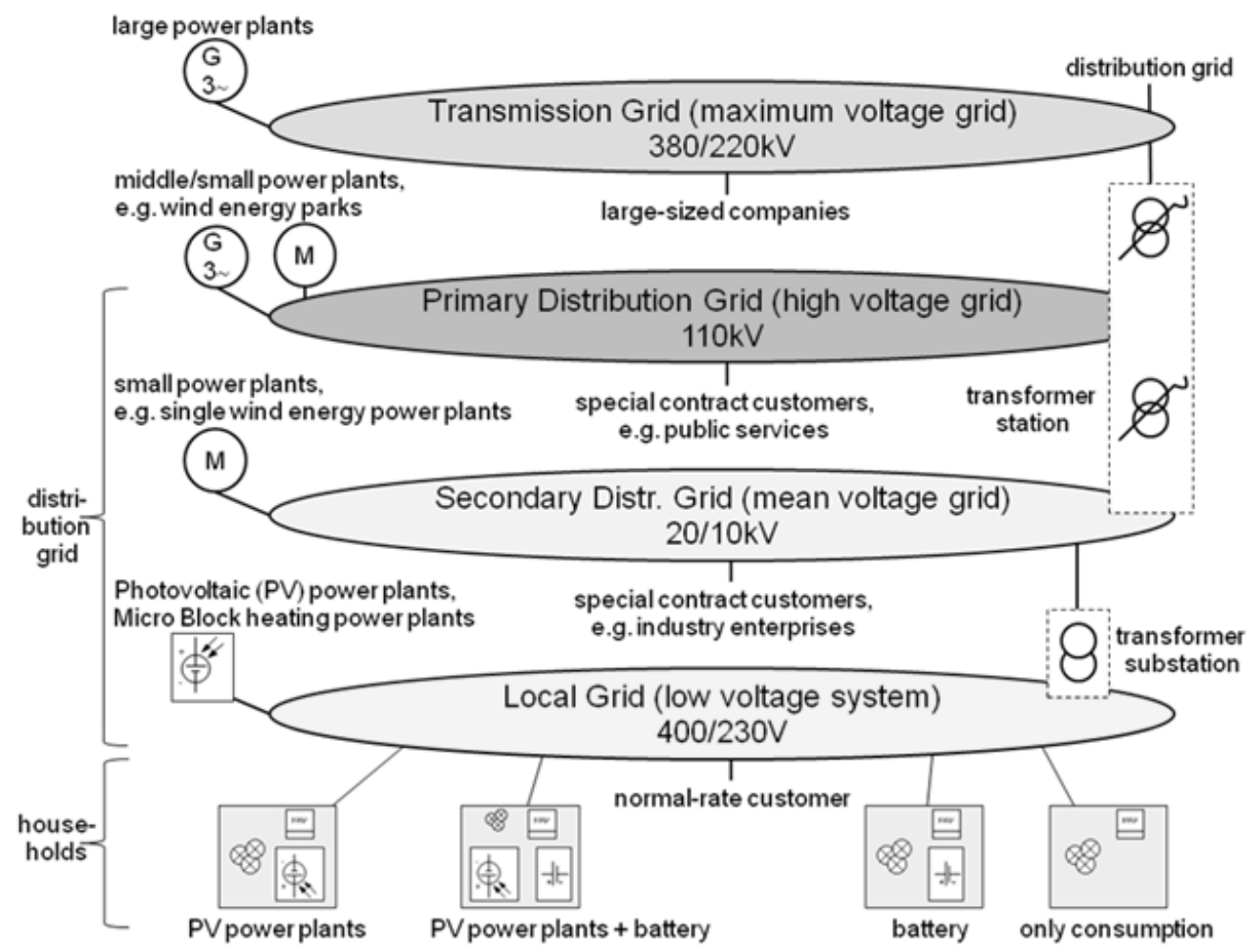

Figure 1: Structure of the power supply system today

The connection of the customers to the different level depends on their demand for energy. Large companies with very high demand for energy are directly connected to the maximum voltage grid. Companies with an average demand are connected to the mean voltage grid and the normal customer is connected to the Local Grid.

In the power supply system a balance of electric current is very important. This implies that the produced and supplied energy has to be equal to the consumed energy. If this is not the case, adjustment control is necessary. Adjustment control today occurs mostly by the central placed power plants. In the Local Grid, where most adjustment is necessary (because the demand for energy is different in specific areas), insufficient possibilities for adjustment exist. The frequency in the grid is one basic indicator to detect the balance of electric current. The $50 \mathrm{~Hz}$ frequency is measured and corrected on demand. The type of energy used for adjustment is called 
regulating energy. This energy is very expensive because it has to be provided permanently. Using forecasts allows to detect how much energy will be needed. The more correct the forecast the less adjustment is required and the more convenient is the power production.

In the future the number of decentralised power plants will increase and the central power plants will be reduced. That leads to much more households installing a photovoltaic plant or a micro block heating power plant (BHPP). Furthermore, the number of wind power plants in communes will increase, as well as power generation by biogas. Because the large power plants will be reduced, the still needed adjustment of the power system cannot only occur in the maximum voltage grid. Adjustment in the Distribution Grid is recommended by controlling and monitoring the decentralised power plants and energy consumer. Controlling of these decentralised devices requires fusion of information- and communication technology and energy technology. This will lead to a Smart Grid.

"A Smart Grid is an electricity network that can intelligently integrate the actions of all users connected to it - generators, consumers and those that do both - in order to efficiently deliver sustainable, economic and secure electricity supplies"(GSGF 2012). As a first step towards Smart Grids energy suppliers already install smart meters.

Comprehensive possible solutions for Smart Grids are:

1. The central adjustment (monitoring and control) of distributed generators, storages, and consumers in so-called virtual power plants. This is the preferred solution on the current state of research. In this approach for example, distributed wind power, biogas, photovoltaic plants supplying energy to the primary or secondary distribution Grid can be controlled by the energy provider as well as large energy consumers are switched off for reducing the load. Furthermore, the problem is that the small energy consumers or the power generators and storages in the local grid (households of the customers) cannot be involved in a central control, because the legal basis and technical solutions for controlling of any devices in the user's sovereign territory are missing. Therefore, a more decentralised solution is necessary for optimisation of the energy consumption and energy supply in Local Grids.

2. Decentralised solution for the integration of households in energy management. Here, the energy consumption and the energy purchase are adjusted, controlled and predicted for every household. This approach requires the integration of the customer (in the following "user") and the consideration of their needs. But this is hardly possible because their needs are not known.

One option is energy management or develop/design solutions through value-added services which the user can design active. In addition, the user defines certain boundary conditions and criteria which are used to achieve an added value for the user to optimise energy consumption. 
Following approaches will be introduced to allow a control of distributed energy management and monitoring of the power system. With value-added services and the integration of the user in the development and definition of value-added services, a new means of regulating the power grid and controlling the Smart Homes is presented. This approaches will be extended by the automated generation of valueadded services and the optimisation of single households. Finally, approaches are presented to achieve optimisation in a Local Grid, through the peer-to-peer internetworking of one or several regions of households (peers) and forming communities between this peers.

\section{Services and service platform for energy management}

In Smart Home domain some approaches exist to use web services for energy management and connecting heterogeneous systems. (Verschueren et al. 2010, Jisun et al. 2010) adopt service platforms for Smart Homes where services are provided by external service providers. (Shudong et al. 2010) also proposes using web services extended for service orchestration. For fast and easy service creation literature proposes orchestration of web services and composition of services out of reusable building blocks using a service creation environment (SCE). The LOMS-, and the MAMS-project and the open source initiative SPAGIC expose the advantages of graphical service development (Keiser et al. 2008, Freese et al. 2007, Spagic 2012). To solve the permanent rising requirements for services and the underlying heterogeneous communication and execution layer, the projects SeCSE and PLASTIC propose self adaptive service oriented applications (Baresi et al. 2006, Autili et al. 2007).

As described above, several approaches exist in different areas to generate and orchestrate services. Currently, no approach exists where users get the possibility to design personalised services or workflows for energy management in the household, fulfilling their personal needs, including automatic service generation and deployment. Additionally, the approaches are focused on concrete target groups or concrete fields of application, mostly in business field, and are not applicable for users in simple households. Therefore, design and orchestration requires expert knowledge. Currently, there are no known publications that name automated solutions for provisioning of user composed services in Smart Grids and Smart Homes.

Therefore, a continuous solution is needed, oriented at the personal needs of users, to offer cost-efficient energy management, according to user personal needs, and integration within their house automation.

In order to involve users into Smart Grid or Smart Home management a service creation environment ( $\mathrm{SCE}$ ) is needed, which brings the possibility to design and configure value-added services, according to the personal needs of the users, to offer cost efficient energy management and optimised usage of energy networks. A service delivery platform (SDP) is needed to provide automated solutions for service provisioning, service controlling and service management. The following will figure out new approaches for a Service Management Framework (SMF) consisting of SCE 
and SDP that allows centralised controlling and management of resources and devices in Smart Grids and Smart Homes.

\subsection{Energy management in the Local Grid}

Monitoring and control respectively adjustment of decentralised power generators and consumers is currently possible on the upper layers of the power grid by the large power plants. Despite the reduction of large power plants still a regulation is required. The control must be in Distribution and Local Grid, where the local power generators supply the current to the power system. The decentralised generators and consumers must therefore be mandatory involved in the adjustment.

Figure 2 shows the increasing decentralised power generators in the Local Grid, and the Service Management Framework which is used for controlling and cross linking of the decentralised generators and consumers in households in the Local Grid as well as decentralised generators in the Distribution Grid.

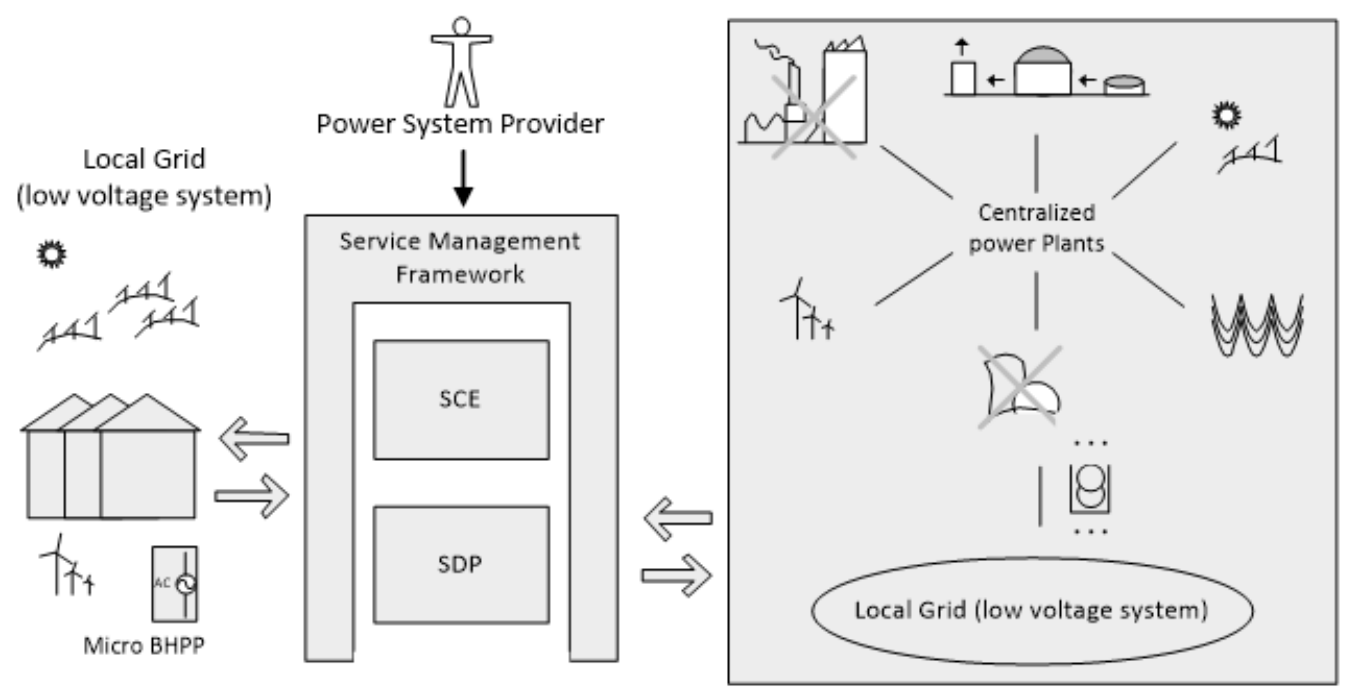

Figure 2: SMF for controlling centralised and decentralised energy resources

The approach through the provision of communication and control services through a Service Management Framework offers power system providers the possibility to implement a central control and monitoring solution in Transmission and Distribution Grids as well as in Local Grids. Furthermore, it is possible to predict the energy demand: whereby the energy operator gets the advantage of providing energy resources timely and with reasonable limits.

As described above, today the consumers, generators and storages in the households cannot be involved in a central control because the legal basis is missing. Therefore in next sections approaches for decentralised solutions are presented by integration of users in the service design. 


\subsection{Services for Smart Home inclusive energy management}

The TeamCom SCE offers the possibility to describe the application flow of a service in a graphical SCE with BPEL (Business Process Execution Language) as service description language. The service described with BPEL will be compiled and deployed on a JAIN SLEE (Java API for Intelligent Network Service Logic Execution Environment) Application Server (Eichelmann et al. 2009, Lasch et al. 2009). This approach comes with the possibility for fast service creation, but recommends knowledge in BPEL and offers no mechanism to integrate these services in Smart Grids or Smart Homes.

The SCE contained in the proposed Service Management Framework makes it possible for the user to design services for its Smart Home in a simple way. Designing services for smart homes requires networking and controllable devices (up to the final consumer). This leads to the connection of electrical devices in households with IT technologies.

Figure 3 shows different energy consumers and energy generators in a smart home, which are connected to each other through various communication networks and controlled by the Service Management Framework.

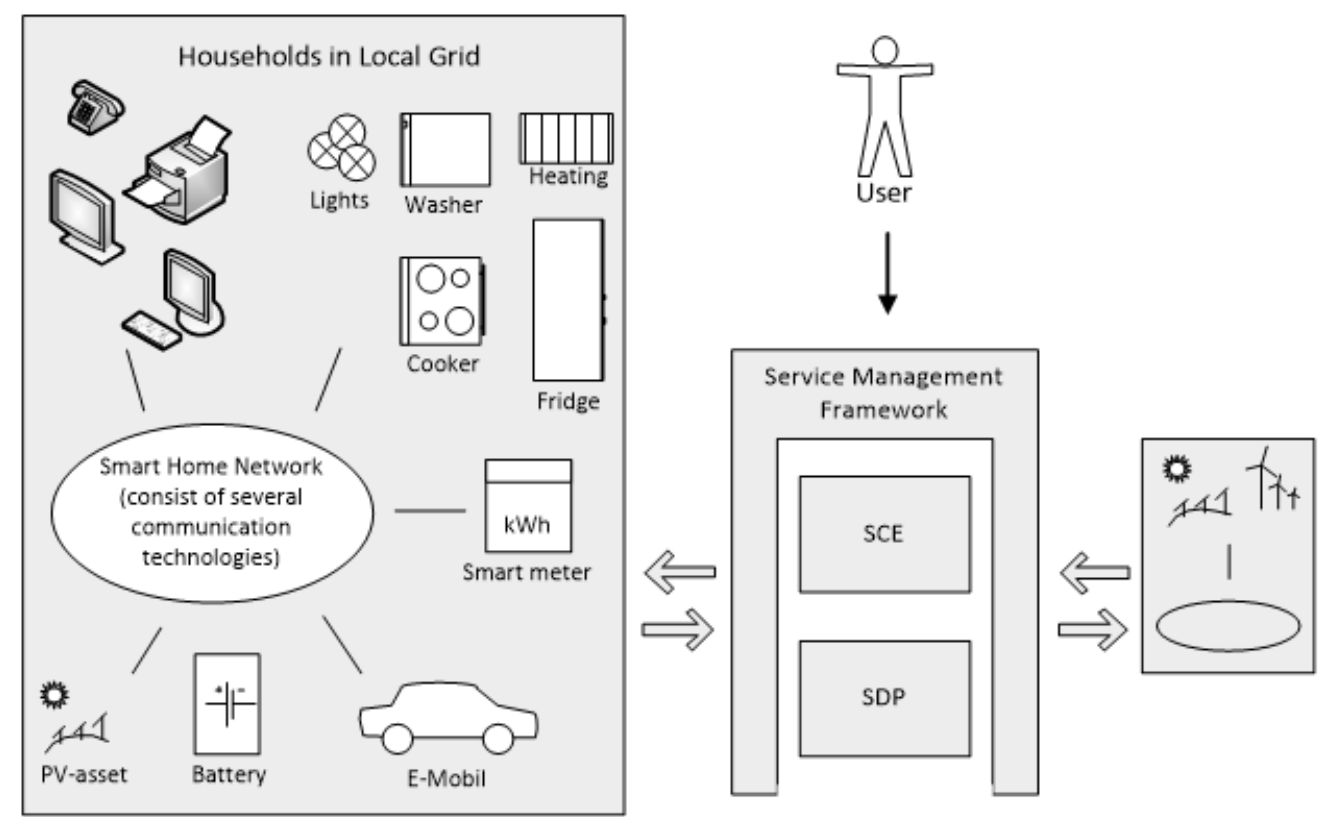

Figure 3: SMF for controlling decentralised energy devices

Power generators and consumers provide device-specific functionality (e.g. timer for start-up and shutdown of equipment or controller for the power supply of power generating facilities). These functionalities can be configured and invoked by the user via the SCE and integrated in the designed service. Generators and consumers 
are represented graphically in the SCE and form the design interface between the user and the devices. The SCE gives the user the possibility to design own services. In addition, the user can obtain and integrate pre-configured services in the SCE. These services can be offered e.g. as apps from the device manufacturer or a service provider. Thus, for example services can be produced which improve the comfort of the user, reduce the energy costs or increase security. Examples for those services: Increase security by disabling certain devices when the user leaves the apartment (e.g. ensuring that the cooker is switched off). Increase comfort by scheduling starting and stopping of equipment, so they finished their running until defined end points (e.g. washing machine ready when the user comes home). Reduce energy costs by charging the local power storage when energy rate is convenient and using the local power storage as energy resource in inconvenient periods. Also, a service can be designed in order to supply locally produced energy at the best terms to the power system.

In this approach the task of the SCE is to provide an interface for service design and configuration. Additionally, the task contained in the SDP is the execution of these services and the abstraction of communication between the devices in a smart home.

Another function of the SCE is to give users a graphical overview of the energy consumption in its households, the energy consumed by the devices, based on the past and for future planned services. The visualisation of energy consumption also strengthens the awareness of users to use energy more efficiently.

According to the actual technologies, only isolated devices exist which offer the possibility for remote configuration and control. In order to communicate and interconnect devices no unified standard exists. According to the manufacturer devices use proprietary protocols which are incompatible with each other, so networking between devices of different manufacturers currently is hardly possible. To rudimentary activate and deactivate devices, at present approaches are existing using intelligent sockets, which can be predefined to interrupt the power supply. In this new approach, the SMF will offer the possibility for unified communication between the interconnected devices and the integration of device functionalities in user designed services.

\subsection{Automated service creation and optimisation}

As described above the Service Management Framework will enable the user to create services to control the devices in the Smart Home. This functionality is extended to the automated service creation and optimisation of services and energy consumption. For this, the user can define boundary conditions for energy supply and optimisation, under whose account the SCE generates an optimised service automatically. The user can e.g. define criteria for the price, which advises the SCE not to exceed certain price limit for energy purchases. Or the user defines a lower threshold so that devices only start if the price is below this threshold. Furthermore, users can define criteria for device control and thus define the earliest starting times and latest end times. Similarly, the user can e.g. set the type of power generation for its purchased energy (wind power, hydropower, etc.). 
Considering the above-mentioned conditions a service will be designed by the SCE and run by the SDP, which satisfies these criteria and also represents an optimal solution with respect to energy consumption. By scheduling the devices the most continuous energy demand is generated in a household, so that peak loads can be reduced or avoided. An application can e.g. consist of the optimised implementation for the procurement of electricity, where supply, storage and consumption are optimised that the load curve of a household is as flat as possible.

The optimisation of energy consumption by optimal load distribution reduces the load in users' households (scheduling, eliminating temporal peak loads by using batteries for power supply). Forecasts for locally generated and consumed energy calculated by the SCE and sent to the energy vendor enables to keep power plant capacity available within reasonable limits to avoid over- or under-capacity.

Figure 4 shows a standard load profile of an average household (continuous line), and the estimated load curve of a household has been optimised by the SCE (PVU Netze 2012).

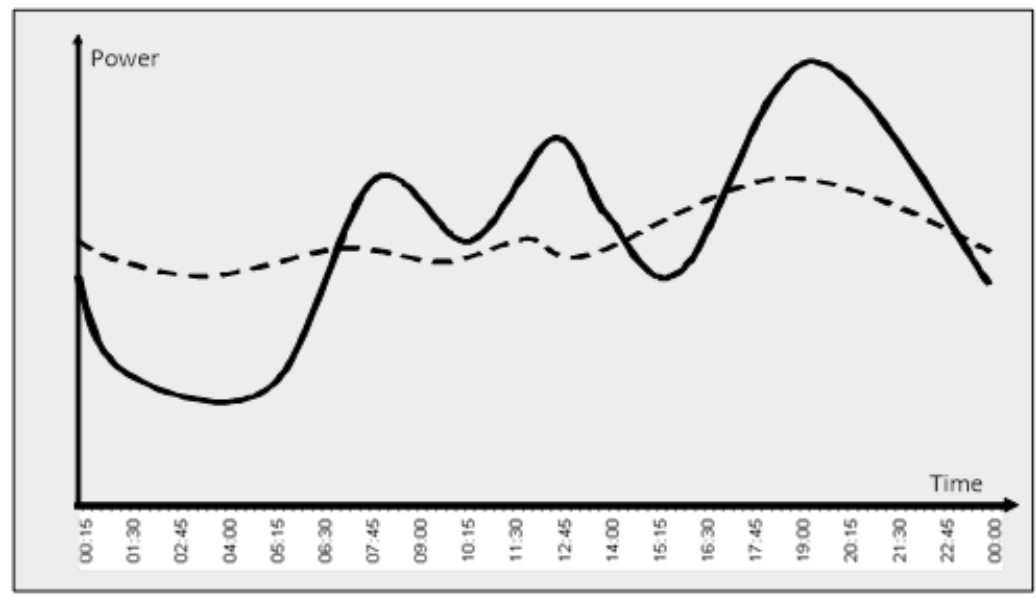

Figure 4: Smoothed load curve in households based on Value-added Services

The maxima, which represent the peak load, can be smoothed considerably, achieved by optimising the load (dashed line). The Optimisation can take place by disarranging electrical consumers from ranges with high load to ranges with little load. Another possibility for smoothing the load curve is to use stored energy in households (e.g. in batteries) during peak loads. The result is that for these periods no extra power plant capacity will be kept.

\section{Peer-to-Peer internetworking and energy community}

The approach to optimise a single household is critical because smoothing of the load curve is not sufficient enough to influence the power system without limitation of users comfort. A central control of all households by the energy operator cannot be implemented, caused by legal reasons. The following additional approaches will 
be introduced to achieve an impact on the power system by simultaneous optimisation of households. Since a central control is not feasible, a decentralised solution is needed.

\subsection{Peer to Peer internetworking}

A simultaneous optimisation of households requires a network of households. Therefore, communication between the households must exist for exchanging information, which can be used to adjust the energy consumption of a single household on the energy consumption in the region concerned. Figure 5 shows a cross-linking of the households by the Peer-to-Peer principle.

In this approach, the Peer-to-Peer (P2P) networking is chosen because no central authority for control is integrated. Each household will be considered as a peer in the network, which communicates anonymously with other peers. In addition, the other players in the power generation and distribution, e.g. power supplier, distribution system operators, etc., are also considered and integrated as peers in the network.

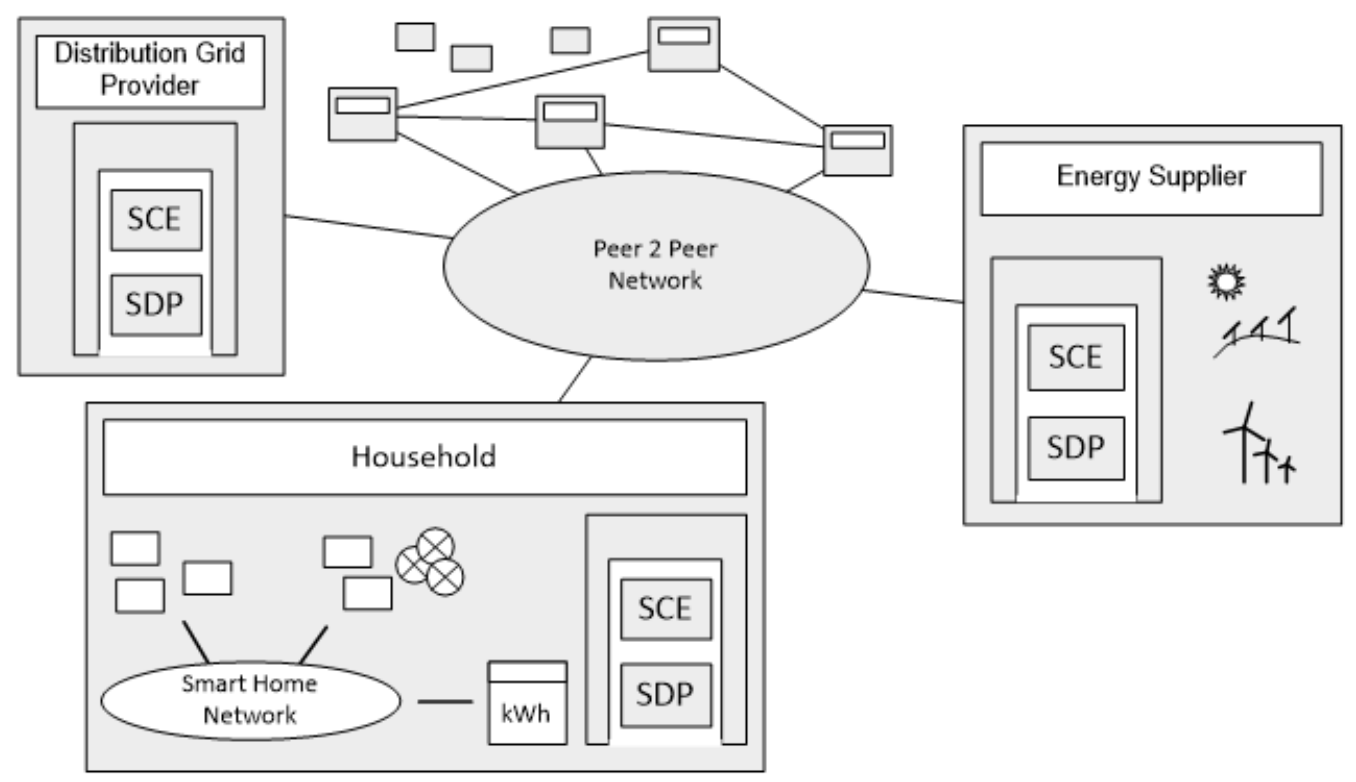

Figure 5: Peer-to-Peer connected households

A self sustained optimisation of every household in a region should lead to an optimisation of the energy requirement in this region. This new approach provides a special algorithm for optimising a single household and the whole region, without assistance of the energy provider, by management and control of the consumers, especially of the generators and energy storages. For the optimisation the exchanged information between peers and the knowledge concerning the own household is used. The exchanged information may include current consumption, forecast of consumption, possible power supply etc. 
To ensure the data security and protection of privacy the communication between households has to be anonymous and encrypted. While sharing information between the participating systems, it must be ensured that a third party (e.g. a neighbour) cannot assign this information to a specific household for example, the information about the planned power consumption. To ensure the anonymity, the communication occurs via the Service Management Framework and is not transparent for the users of the respective peers.

In the course of this project, the possible approaches for a P2P internetworking are examined (e.g. Trusted Proxy Provider, Certificate-based P2P networking, etc.). Additionally the different peer-to-peer models for the provisioning of value-added services: Hybrid P2P, Super P2P, Pure P2P (Lehmann et al. 2008) will be analysed for their application in Smart Grids and Smart Homes.

\subsection{Energy Community}

The approach of the $\mathrm{P} 2 \mathrm{P}$ networking will be optimised by extending to the mechanisms of social networks to form a community between households, which have common shared interests and follow the same economic goals. This may include: energy saving, cost reduction, environmental conservation. In a region (e.g. households in a street, city districts) sub-communities can be formed, which appear as part of the whole community. The previously described optimisation of households in this approach occurs between members of the community or within sub-communities. Figure 6 shows the concentration of households in a community consisting of various sub-communities.

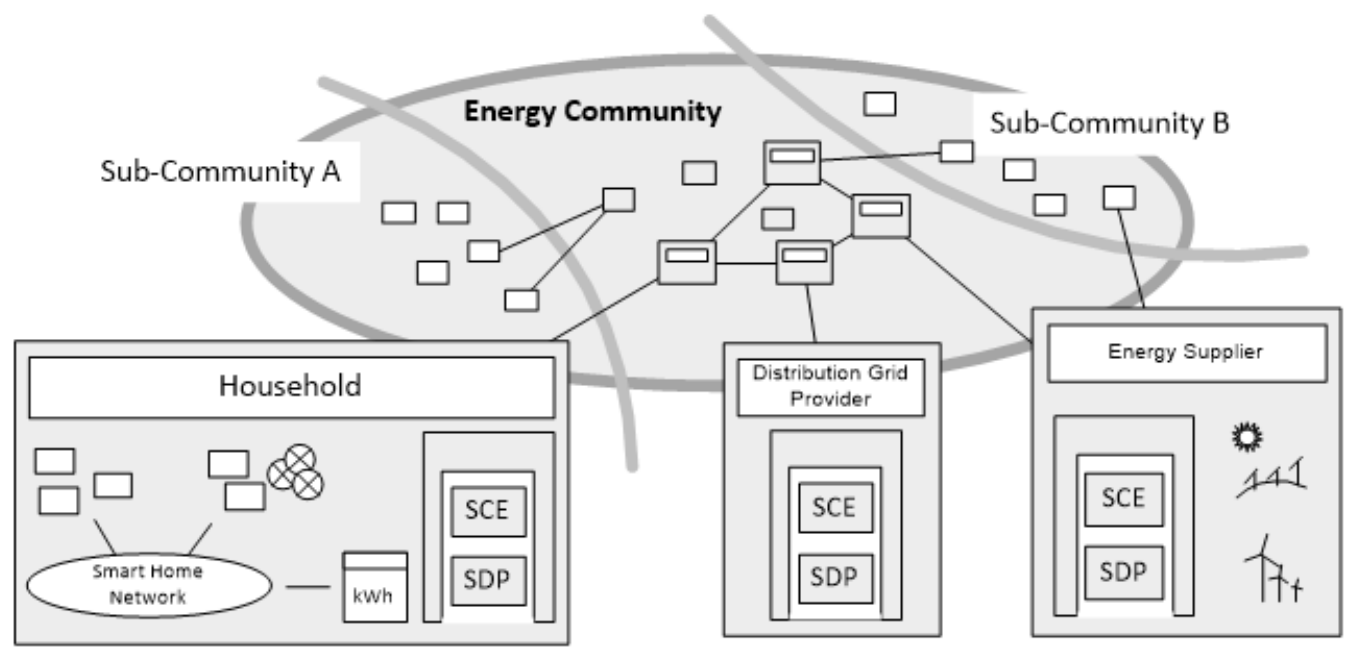

Figure 6: Energy Community with Sub-Communities

According to the principle of a social network, the community may grow independently and every participant may accede to his free will. The possibility to join on a sub-community voluntarily, which matches users' interests promotes the willingness to participate in this system and creates a personal benefit. 
Through sub-communities the different interests of the members are considered. Members sharing the same interests come together to achieve their personal goals regardless of other members in the community. Thus, a variety of targets can be achieved e.g. to save energy costs, to reduce $\mathrm{CO}_{2}$ emissions or to protect the environment. The target may also be to have lower energy consumption than other members of the community. The sub-communities can interact with each other and compare the fuel economy of different regions e.g. challenges may arise with the target to find out which sub-community achieved the lowest energy consumption (or the largest monthly reduction in energy consumption relative to the previous month).

The energy suppliers and power system operators can also occur as part of this community and offer, e.g. for the members of a community, special billing models. Thinkable are flexible tariffs with the possibility to change tariffs and providers dynamically or prepaid billing models. A sub-community can also occur as a service provider for the control area provider by offering adjustment, active and reactive power. Services that are implemented for a sub-community could include the optimisation of power consumption and purchasing. Furthermore, smoothing the load profile for a community which in turn can be honoured by the energy provider with a better deal.

\section{Conclusion}

This paper presents approaches for a service management framework to control and monitor decentralised energy consumers, storages and generators. In this new approaches the user is integrated in design and configuration of the services for energy management which offer the possibility to follow its personal needs.

The algorithms for automated optimisation and generation of services by controlling decentralised energy consumers, storages and generators, offers the possibility to reduce the energy consumption in households or whole regions. This leads to a possible reduction of costs or environmental benefits. In addition, the power supply system is discharged by reducing load peaks and smoothing the load curve of households and regions. In order to communicate and interconnect the Service Management Framework is used, thus the integration of different devices gets possible und the privacy of involved users is ensured. On the first time approaches point out the peer-to-peer interconnection of households in Smart Grids and build up communities for decentralised optimisation of energy consumption.

In the next research topics algorithms for optimisation of energy consumption between peer-to-peer interconnected households are examined, as well as techniques for abstraction energy devices.

\section{References}

Autili, M., Berardinelli, L., Cortellessa, V., Marco, A., Ruscio, D., Inverardi, P., Tivoli, M. (2007), "A Development Process for Self-adapting Service Oriented Applications", proceeding of ICSOC, pp442-448. 
Baresi, L., Di Nitto, E., Ghezzi, C. (2006), "Toward Open-World Software: issues and challenges", IEEE Computer, Volume 39, No. 10, pp36-43.

Eichelmann, T., Fuhrmann, W., Trick, U., Ghita, B. (2009), "Support of parallel BPEL activities for the TeamCom Service Creation Platform for Next Generation Networks", Proc. of SEIN 2009, pp69-80.

Freese B., Staiger, U., Stein, H. (2007), "Multi-Access Modular-Services Framework Whitepaper", Deutsche Telekom Laboratories.

GSGF Corporation Web Site (2012), "What is the Definition of Smart Grid", http://www.globalsmartgridfederation.org/smartgriddef.html\#Definition, (Accessed 2 January 2012).

Jisun, L., Dae-Kyo, J., Yoonkee, K., Young-Woo, L., Young-Myuong, K. (2010), "Smart Grid Solutions, Services, and Business Models Focused on Telco", IEEE/IFIP Network Operations and Management Symposium, pp323-326.

Keiser, J., Kriengchaiyapruk, T. (2008), "Bringing Creation of Context-Aware Mobile Services to the Masses", IEEE SOA Industry Summit (SOAIS), pp105-106.

Lasch, R., Ricks, B., Tönjes, R., Lehmann, A., Eichelmann, T., Trick, U. (2009), "TeamCom: A Service Creation Platform for Next Generation Networks", The Fourth International Conference on Internet and Web Applications and Services ICIW 2009.

Lehmann, A., Fuhrmann, W., Trick, U., Ghita, B. (2008), " New possibilities for the provision of value-added services in SIP-based peer-to-peer networks", Proc. of SEIN 2008, pp167-176.

PVU Netze Web Site (2012), "Standardlastprofil VDEW", http://www.pvunetze.de/media/Standardlastprofil\%20VDEW.pdf, (Accessed 10 January 2012).

Shudong, C., Lukkien, J., Liang, Z. (2010), "Service-oriented Advanced Metering Infrastructure for Smart Grids", IEEE Power and Energy Engineering Conference (APPEEC), pp1-4.

Spagic Corporation Web Site (2012), "Spagic, the universal middleware for the development of SOA/BPM extensible solutions and governance of services", http://www.spagoworld.org/xwiki/bin/view/Spagic/, (Accessed 20 January 2012).

Verschueren, T., Haerick, W., Mets, K., Develder, C., De Turck, F., Pollet, T. (2010) "Architecture for smart end-user services in the power grid", IEEE/IFIP Network Operations and Management Symposium, pp316-322. 


\title{
Energy communities in Smart Markets for optimisation of peer-to-peer interconnected Smart Homes
}

\author{
M.Steinheimer ${ }^{* * *}$, U.Trick$^{*}$, P.Ruhrig ${ }^{*}$ \\ - Research Group for Telecommunication Networks, University of Applied Sciences Frankfurt/M., \\ Frankfurt/M., Germany \\ "Centre for Security, Communications and Network Research, University of Plymouth, \\ Plymouth, United Kingdom \\ e-mail steinheimer@e-technik.org, trick@e-technik.org, ruhrig@e-technik.org
}

\begin{abstract}
Energy management in Smart Grids and Smart Homes will play a decisive role in future power system. Due to the rising decentralised power generation and its supply to the local grid, the individual households have to become more and more a participant in the Smart Market. Therefore approaches are presented for a framework to design and generate value-added services for management Smart Grids and Smart Homes. It offers users the possibility to design services for managing decentralised energy devices and resources as well as optimise the energy consumption by intelligent energy management and automated service generation and optimisation. These new approaches are based on interconnection of households and algorithms for automated optimisation of energy consumption in single households or whole regions, without assistance of third parties. The interconnection of the households is based upon the peer-to-peer principles for communication and automated optimisation as well as forming a social network, as a so called energy community between the participated peers.
\end{abstract}

\section{INTRODUCTION}

\section{A. Adjustment in future power supply system}

Today the power supply system is static. Primary large central power plants like coal-fired and nuclear power plants supply companies and households with energy. In the future the number of decentralised power plants will increase and the central power plants will be reduced. That leads to much more households installing a photovoltaic plant or a micro block heating power plant (BHPP). Furthermore, the number of wind power plants in communes will increase, as well as power generation by biogas. Due to the fact that large power plants will be reduced, the still needed adjustment of the power system cannot only occur in the maximum voltage grid. Adjustment in the Distribution Grid is recommended by controlling and monitoring the decentralised power plants and energy consumer. Controlling of these decentralised devices requires fusion of information- and communication technology and energy technology. This will lead to a Smart Grid.

\section{B. Smart Grid}

"A Smart Grid is an electricity network that can intelligently integrate the actions of all users connected to it - generators, consumers and those that do both - in order to efficiently deliver sustainable, economic and secure electricity supplies" [6]

\section{Smart Market}

Separating Smart Grid and Smart Market is mainly based on the consideration of whether quantities of energy flows (market sphere) or capacity (power sphere). Primary subjects of Smart Grid considerations are not the amounts of regenerative energy produced fortunately, but Smart Grids consider the requirements for capacity resulting to the amounts of regenerative energy. Because the core business of energy network operators is to deploy, maximize, and optimise the network capacity. All actors who provide or consume energy can be participants in the Smart Market. They can cause a temporary reduction as well as an increase of the load according to price signals. The energy consumption and the energy supply will be driven by variable energy rates and/or variable energy network access fees. The user becomes a participant of the energy market which consumes, supplies and stores energy optimised by economical aspects. Smart Grids and Smart Markets should help to increase the usable capacity of conventional power lines through intelligent solutions (smart grid aspect). Furthermore Smart Grids and Smart Markets should utilise the available capacity of existing networks and improve its average load by supply and load shifting (Smart Market aspect). Both will be able to make economically optimal network expansion [3].

\section{Solutions for Smart Grid and Smart Markets}

Comprehensive possible solutions for Smart Grids are: 1. The central adjustment (monitoring and control) of distributed generators, storages, and consumers in socalled virtual power plants. This is the preferred solution on the current state of research. In this approach for example, distributed wind power, biogas, photovoltaic plants supplying energy to the primary or secondary distribution Grid can be controlled by the energy provider as well as large energy consumers are switched off for reducing the load. Furthermore, the problem is that the small energy consumers or the power generators and storages in the local grid (households of the customers) cannot be involved in a central control, because the legal basis and technical solutions for controlling of any devices in the user's sovereign territory are missing. Therefore, a more decentralised solution is necessary for optimisation 
of the energy consumption and energy supply in Local Grids.

2. The decentralised adjustment of distributed generators, storages, and consumers by integration of households in energy management. Here, the energy consumption and the energy purchase are adjusted, controlled and predicted for every household. This approach requires the integration of the customer (in the following "user") and the consideration of their needs. But this is hardly possible because their needs are not known.

One option is energy management or develop/design solutions through value-added services which the user can design active. In addition, the user defines certain boundary conditions and criteria which are used to achieve an added value for the user to optimise energy consumption.

Following approaches will be introduced to allow a control of distributed energy management and monitoring of the power system. With value-added services and the integration of the user in the development and definition of value-added services, a new means of regulating the power grid and controlling the Smart Homes is presented. These approaches will be extended by the automated generation of value-added services and the optimisation of single households. Finally, approaches are presented to achieve optimisation in a Local Grid, through the peer-topeer internetworking of one or several regions of households (peers) and forming a special energy community between these peers.

\section{SERVICES AND SERVICE PLATFORM FOR ENERGY} MANAGEMENT

In Smart Home domain some approaches exist to use web services for energy management and connecting heterogeneous systems. [7, 14] adopt service platforms for Smart Homes where services are provided by external service providers. [12] also proposes using web services extended for service orchestration. For fast and easy service creation literature proposes orchestration of web services and composition of services out of reusable building blocks using a service creation environment (SCE). The LOMS-, and the MAMS-project and the open source initiative SPAGIC expose the advantages of graphical service development $[5,8,13]$. To solve the permanent rising requirements for services and the underlying heterogeneous communication and execution layer, the projects SeCSE and PLASTIC propose self adaptive service oriented applications $[1,2]$.

As described above, several approaches exist in different areas to generate and orchestrate services. Currently, no approach exists where users get the possibility to design personalised services or workflows for energy management in the household, fulfilling their personal needs, including automatic service generation and deployment. Additionally, the approaches are focused on concrete target groups or concrete fields of application, mostly in business field, and are not applicable for users in simple households. Therefore, design and orchestration requires expert knowledge. Currently, there are no known publications that name automated solutions for provisioning of user composed services in Smart Grids and Smart Homes.

Therefore, a continuous solution is needed, oriented at the personal needs of users, to offer cost-efficient energy management, according to user personal needs, and integration within their house automation.

In order to involve users into Smart Grid or Smart Home management a service creation environment (SCE) is needed, which brings the possibility to design and configure value-added services, according to the personal needs of the users, to offer cost efficient energy management and optimised usage of energy networks. A service delivery platform (SDP) is needed to provide automated solutions for service provisioning, service controlling and service management. The following will figure out new approaches for a Service Management Framework (SMF) consisting of SCE and SDP that allows centralised controlling and management of resources and devices in Smart Grids and Smart Homes.

\section{A. Energy management in the Local Grid}

The adjustment of the power supply system is currently only possible on the upper layers of the power grid through the centralized large power plants by regulating the power generation and supply. Despite the reduction of large power plants still a adjustment is required. The control must be in Distribution and Local Grid, where the local power generators supply the current to the power system. The decentralised generators and consumers must therefore be mandatory involved in the adjustment.

Figure 1 shows the increasing decentralised power generators in the Local Grid, and the Service Management Framework which is used for controlling and cross linking of the decentralised generators and consumers in households in the Local Grid as well as decentralised generators in the Distribution Grid.

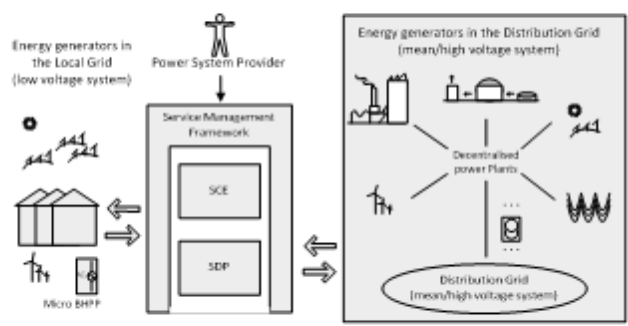

Figure 1. SMF for controlling centralised and decentralised energy resources

The approach through the provision of communication and control services through a Service Management Framework offers power system providers the possibility to implement a central control and monitoring solution in Distribution Grids in general as well as in the Local Grids. Furthermore, it is possible to predict the energy demand: whereby the power system provider gets the advantage of providing energy resources timely and with reasonable limits.

As described above, today the consumers, generators and storages in the households cannot be involved in a central control because the legal basis is missing and users perception of comfort would be affected. Therefore in next sections approaches for decentralised solutions are presented by integration of users in the service design. 


\section{B. Services for Smart Home inclusive energy management}

The TeamCom SCE offers the possibility to describe the application flow of a service in a graphical SCE with BPEL (Business Process Execution Language) as service description language. The service described with BPEL will be compiled and deployed on a JAIN SLEE (Java API for Intelligent Network Service Logic Execution Environment) Application Server [4, 9]. This approach comes with the possibility for fast service creation, but recommends knowledge in BPEL and offers no mechanism to integrate these services in Smart Grids or Smart Homes.

The SCE contained in the proposed Service Management Framework makes it possible for the user to design services for its Smart Home in a simple way. Designing services for smart homes requires networking and controllable devices (up to the final consumer). This leads to the connection of electrical devices in households with IT technologies.

Figure 2 shows different energy consumers and energy generators in a smart home, which are connected to each other through various communication networks and controlled by the Service Management Framework.

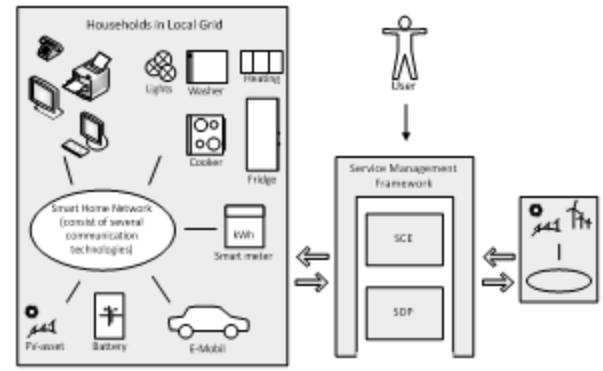

Figure 2. SMF for controlling decentralised energy devices

Power generators and consumers provide devicespecific functionality (e.g. timer for start-up and shutdown of equipment or controller for the power supply of power generating facilities). These functionalities can be configured and invoked by the user via the $\mathrm{SCE}$ and integrated in the designed service. Generators and consumers are represented graphically in the SCE and form the design interface between the user and the devices. The SCE gives the user the possibility to design own services. In addition, the user can obtain and integrate pre-configured services in the SCE. These services can be offered e.g. as apps from the device manufacturer or a service provider. Thus, for example services can be produced which improve the comfort of the user, reduce the energy costs or increase security. Examples for those services: Disabling certain devices when the user leaves the apartment (e.g. ensuring that the cooker is switched off). Remote monitoring users place or remote unlocking doors if children have lost their key. Increase comfort by scheduling starting and stopping of equipment, so they finished their running until defined end points (e.g. washing machine ready when the user comes home). Reduce energy costs by charging the local power storage when energy rate is convenient and using the local power storage as energy resource in inconvenient periods. Also, a service can be designed in order to supply locally produced energy at the best terms to the power system.

In this approach the task of the SCE is to provide an interface for service design and configuration. Additionally, the task contained in the SDP is the execution of these services and the abstraction of communication between the devices in a smart home.

Another function of the SCE is to give users a graphical overview of the energy consumption in its households, the energy consumed by the devices, based on the past and for future planned services. The visualisation of energy consumption also strengthens the awareness of users to use energy more efficiently.

According to the actual technologies, only isolated devices exist which offer the possibility for remote configuration and control. In order to communicate and interconnect devices no unified standard exists. According to the manufacturer devices use proprietary protocols which are incompatible with each other, so networking between devices of different manufacturers currently is hardly possible. To rudimentary activate and deactivate devices, at present approaches are existing using intelligent sockets, which can be predefined to interrupt the power supply. In this new approach, the SMF will offer the possibility for unified communication between the interconnected devices and the integration of device functionalities in user designed services.

\section{Automated service creation and optimisation}

As described above the Service Management Framework will enable the user to create services to control the devices in the Smart Home. This functionality is extended to the automated service creation and optimisation of services and energy consumption. For this, the user can define boundary conditions for energy supply and optimisation, under whose account the SCE generates an optimised service automatically. The user can e.g. define criteria for the price, which advises the SCE not to exceed certain price limit for energy purchases. Or the user defines a lower threshold so that devices only start if the price is below this threshold. Furthermore, users can define criteria for device control and thus define the earliest starting times and latest end times. Similarly, the user can e.g. set the type of power generation for its purchased energy (wind power, hydropower, etc.).

Considering the above-mentioned conditions a service will be composed by the SCE and run by the SDP, which satisfies these criteria and also represents an optimal solution with respect to energy consumption. By scheduling the devices the most continuous energy demand is generated in a household, so that peak loads can be reduced or avoided. An application can e.g. consist of the optimised implementation for the procurement of electricity, where supply, storage and consumption are optimised that the load curve of a household is as flat as possible.

The optimisation of energy consumption by optimal load distribution reduces the load in users' households (scheduling, eliminating temporal peak loads by using batteries for power supply). Forecasts for locally generated and consumed energy calculated by the SCE and sent to the energy vendor enables to keep power plant capacity available within reasonable limits to avoid overor under-capacity. 
Figure 3 shows a standard load profile of an average household (continuous line) [11], and the estimated load curve of a household has been optimised by the SCE.

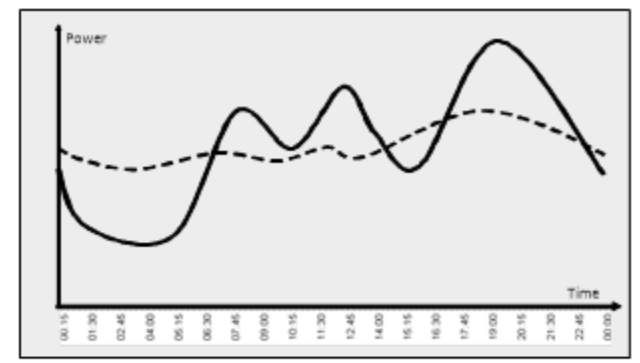

Figure 3. Smoothed load curve in households based on Valueadded Services

The maxima, which represent the peak load, can be smoothed considerably, achieved by optimising the load (dashed line). The result is that for these periods no extra costly power plant capacity will be kept.

\section{PEER-TO-PEER INTERNETWORKING AND ENERGY COMMUNITY}

The approach to optimise a single household is critical because smoothing of the load curve is not sufficient enough to influence the power system without limitation of users comfort. A central control of all households by the energy operator cannot be implemented, caused by legal reasons. Since a central control is not feasible, a decentralised solution is needed. The following additional approaches will be introduced to achieve an impact on the power system by simultaneous optimisation of many households combined. This offers the possibility to optimise the demand of limited regions (e.g. the area of local transformer substations) and adjustment of the voltage in this area by enabling or disabling distributed power generators or batteries (for solving the range of tolerance to $+/-10 \%$ ).

\section{A. Peer to Peer internetworking}

A simultaneous optimisation of households requires a network of households. Therefore, communication between the households must exist for exchanging information, which can be used to adjust the energy consumption of a single household on the energy consumption in the region concerned. Figure 4 shows a cross-linking of the households by the Peer-to-Peer principle.

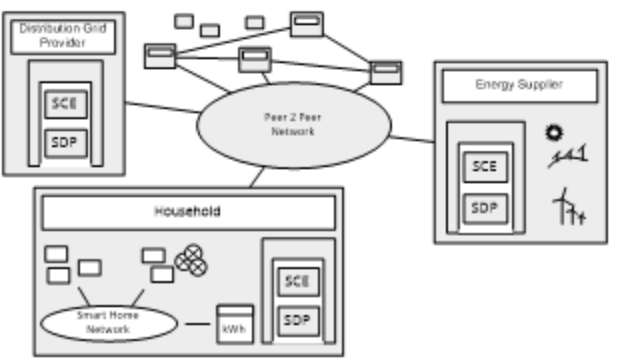

Figure 4. Peer-to-Peer connected households

In this approach, the Peer-to-Peer (P2P) networking is chosen because no central authority for control is integrated and the structure is mostly self organised as well as the data storage is distributed. Each household will be considered as a peer in the network, which communicates anonymously with other peers. In addition, the other players in the power generation and distribution, e.g. power supplier, distribution system operators, etc., are also considered and integrated as peers in the network.

A self sustained optimisation of every household in a region should lead to an optimisation of the energy requirement in this region. This new approach provides a special algorithm for optimising a single household and the whole region, without assistance of the energy provider, by management and control of the consumers, especially of the generators and energy storages. For the optimisation the exchanged information between peers and the knowledge concerning the own household is used The exchanged information may include current consumption, forecast of consumption, possible power supply etc.

To ensure the data security and protection of privacy the communication between households has to be anonymous and encrypted. While sharing information between the participating systems, it must be ensured that a third party (e.g. a neighbour) cannot assign this information to a specific household for example, the information about the planned power consumption. To ensure the anonymity, the communication occurs via the Service Management Framework and is not transparent for the users of the respective peers. The communication between the peers should occur based on IP-Technology because of existing mechanisms for security, availability and quality of service, preferable via Next Generation Networks.

\section{B. Constituent parts of communication}

The information exchanged between the peers include the quantity of estimated demand for energy of a household, the actual consumption, possibly capacity of energy supply, variable energy network access fees, and variable energy prices as well as the corresponding time slots, the kind of energy (regenerative energy or gas etc.) Further parts of the exchange between the peers are information about existing energy storages (batteries, their capacity, charge condition) and existing energy producers (e.g. kind of energy producers and deliverable power) as well as forecasts of load profiles in households (calculated by the SCE). Batteries in electrically powered cars are excellent energy storages. Because of their mobility, the information has to be provided that these energy storages have no static location.

\section{Approaches for Peer-to-Peer networking}

In the course of this project, the possible approaches for a P2P internetworking are examined (e.g. Trusted Proxy Provider, Certificate-based P2P networking, etc.) Additionally the different $\mathrm{P} 2 \mathrm{P}$ models for the provisioning of value-added services will be analysed for their application in Smart Grids and Smart Homes. Three different models were proposed by prior research as shown in Figure 5 [10]. The 1st model (Hybrid P2P model) is semi-centric with at least one centric checkpoint and client-server as well as $\mathrm{P} 2 \mathrm{P}$ communication. The 2 nd model (Pure P2P model) is fully distributed as well as structured (linked to a special topology) or non-structured 
organization of the peers. The 3rd model (Super P2P model) is an enhancement of the hybrid P2P model, centralized checkpoints are substituted by P2P network of super nodes and interaction between super nodes and normal peers is based on client-server model. Using the Hybrid P2P model with interconnected distributed servers is preferred, hosted for example by the Energy Community introduced in the next section.

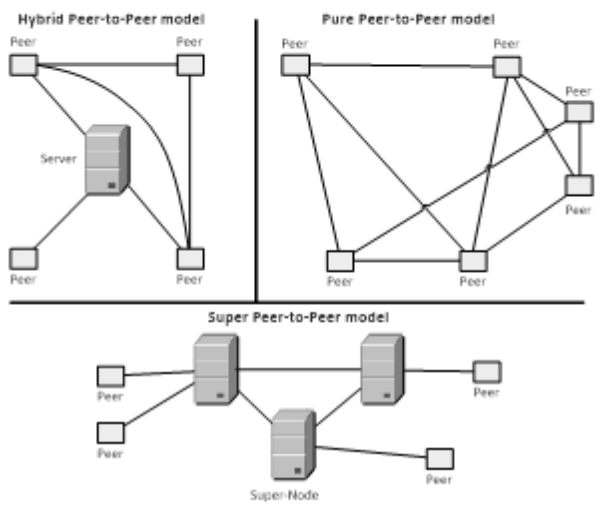

Figure 5. Different Peer-to-Peer models

\section{Energy Community}

The approach of the P2P networking will be extended by the mechanisms of social networks to form a community between households. This approach offers a solution to solve users rights and privacy restrictions and provides a legal construct for administration of a energy network (e.g. server in P2P network) as well as a mechanism for joining the network.

The member of the community have common shared interests and follow the same economic goals. This may include: energy saving, cost reduction, environmental conservation. In a region (e.g. households connected to a local transformer substation, city districts) subcommunities can be formed, which appear as part of the whole community. The previously described optimisation of households in this approach occurs between members of the community or within subcommunities. Figure 6 shows the concentration of households in a community consisting of various subcommunities.

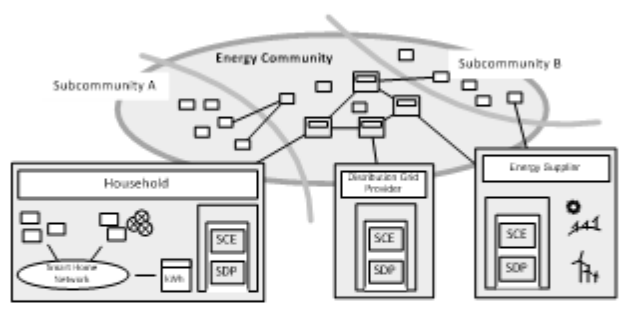

Figure 6. Energy Community with subcommunities

According to the principle of a social network, the community may grow independently and every participant may accede to his free will. The community is not limited to the electrical energy supply and consumption, e.g. other kinds of energy can also be included (e.g. gas). The possibility to join on a subcommunity voluntarily, which matches users' interests promotes the willingness to participate in this system and creates a personal benefit.

Through subcommunities the different interests of the members are considered. Members sharing the same interests come together to achieve their personal goals regardless of other members in the community. Thus, a variety of targets can be achieved e.g. to save energy costs, to reduce $\mathrm{CO}_{2}$ emissions or to protect the environment. The target may also be to have lower energy consumption than other members of the community. The subcommunities can interact with each other and compare the fuel economy of different regions e.g. challenges may arise with the target to find out which subcommunity achieved the lowest energy consumption (or the largest monthly reduction in energy consumption relative to the previous month).

The energy suppliers and power system operators can also occur as part of this community and offer, e.g. for the members of a community, special billing models. Thinkable are flexible tariffs with the possibility to change tariffs and providers dynamically, variable energy network access fees or prepaid billing models. Subcommunities can be formed for the area of local transformer substations.

Through this approach costs for local transformer substations can be reduced by avoiding energy network enhancement or automation of local transformer substations. Because the Local Grid still needs little intelligence the operational costs will be permanent marginal as well as the costs for automation and enhancement of the Local Grid can be saved by the Smart Market approach, presented in this paper.

Subcommunities can also occur as a service provider for the control area provider by offering adjustment, active and reactive power. Services that are implemented for a subcommunity could include the optimisation of power consumption and purchasing. Furthermore, smoothing the load profile for a community which in turn can be honoured by the energy provider with a better deal. The forecasted or determined load curve of the households can be used for smoothing this load curve or for optimizing the energy consumption of many households in the area of local transformer substations. Variable energy network access fees could be communicated to subcommunities by the distribution grid provider (e.g. low fees between $9 \mathrm{~h}$ and $10 \mathrm{~h}$, afterwards rising). The distribution grid provider (as part of the community) can deliver a aspired load profile to the community. If this load profile is solved on e.g. 20 days per month, they will get a bonus or no energy network access fees. Subcommunities try to reach this load profile by optimizing every member in collaboration of energy consumption and offering positive or negative regulating energy.

\section{CONCLUSION}

This paper presents approaches for a service management framework to control and monitor decentralised energy consumers, storages and generators. In these new approaches the user is integrated in design and configuration of the services for energy management which offer the possibility to follow its personal needs.

The algorithms for automated optimisation and generation of services by controlling decentralised energy consumers, storages and generators, offers the possibility 
to reduce the energy consumption in households or whole regions. This leads to a possible reduction of costs or environmental benefits. In addition, the power supply system is discharged by reducing load peaks and smoothing the load curve of households and regions. In order to communicate and interconnect the Service Management Framework is used, thus the integration of different devices gets possible und the privacy of involved users is ensured. On the first time approaches point out the peer-to-peer interconnection of households in Smart Grids. Also firstly energy communities were built up for decentralised optimisation of energy consumption as participants in the Smart Market.

In the next research topics algorithms for optimisation of energy consumption between peer-to-peer interconnected households are examined, as well as techniques for abstraction energy devices. In the first step energy consumer in households will be considered as a single block and the adjustment will occur by the local energy generators as well as the local energy storages.

\section{ACKNOWLEDGMENT}

The research project e-SCHEMA providing the basis for this publication is partially funded by the Federal Ministry of Education and Research (BMBF) of the Federal Republic of Germany under grant number 17018B11. The authors of this publication are in charge of its content.

\section{REFERENCES}

[1] M. Autili, et al., "A development process for self-adapting service oriented applications", proceeding of ICSOC, pp.442-448, 2007.

[2] L. Baresi, E. Di Nitto, C. Ghezzi, "Toward open-world software: issues and challenges", IEEE Computer, Volume 39, No. 10, pp. $36-43,2006$.

[3] Bundesnetzagentur, "Smart Grid und Smart Market Eckpunktepapier der Bundesnetzagentur zu den Aspekten des sich verändernden Energieversorgungssystems", Bonn, 2011.

[4] T. Eichelmann, W. Fuhrmann, U. Trick, and B. Ghita, "Support of parallel BPEL activities for the TeamCom service creation platform for next generation networks", Proc. of SEIN 2009, pp.69-80, 2009.

[5] B. Freese, U. Staiger, H. Stein, "Multi-access modular-services framework - whitepaper", Deutsche Telekom Laboratories, 2007.

[6] GSGF Corporation Web Site, "What is the definition of smart grid", http://www.globalsmartgridfederation.org/smartgriddef.htm \#Definition, 2012

[7] L. Jisun, J. Dae-Kyo, K. Yoonkee, L. Young-Woo, K. Young Myuong, "Smart grid solutions, services, and business models focused on telco", IEEE/IFIP Network Operations and Management Symposium, pp.323-326, 2010

[8] J. Keiser, T. Kriengchaiyapruk, "Bringing creation of contextaware mobile services to the masses", IEEE SOA Industry Summit (SOAIS), pp.105-106, 2008.

[9] A. Lehmann, T. Eichelmann, U. Trick, R. Lasch, B. Ricks, R. Tönjes, "TeamCom: a service creation platform for next generation networks", The Fourth Intemational Conference on Internet and Web Applications and Services ICIW 2009, pp.12-17, 2009.

[10] A. Lehmann, W. Fuhrmann, U. Trick, B. Ghita, "New possibilities for the provision of value-added services in SIP-based peer-to peer networks", Proc. of SEIN 2008, pp.167-176, 2008.

[11] PVU Netze Web Site, "Standardlastprofil VDEW", http://www. pvu-netze.de/media/Standardlastprofil\%20VDEW.pdf, 2012.

[12] C. Shudong, J. Lukkien, Z. Liang, "Service-oriented advanced metering infrastructure for smart grids", IEEE Power and Energy Engineering Conference (APPEEC), pp.1-4, 2010.
[13] Spagic Corporation Web Site, "Spagic, the universal middleware for the development of SOABPM extensible solutions and governance of services", http $/ /$ www.spagoworld.org/xwiki/bin view/Spagic/, 2012

[14] T. Verschueren, W. Haerick, K. Mets, C. Develder, F. De Turck, T. Pollet, "Architecture for smart end-user services in the power grid", IEEE/IFIP Network Operations and Management Symposium, pp.316-322, 2010. 
Published in Proceedings of the Eighteenth VDE/ITG Mobilfunktagung, pp. 64-70, Mai 2013, Osnabrück, Germany, VDE

\section{SIP-basierte P2P-Vernetzung in einer Energie-Community}

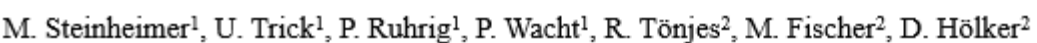

${ }^{1}$ Fachhochschule Frankfurt a. M., Kleiststraße 3, 60318 Frankfurt/M., steinheimer@e-technik.org

${ }^{2}$ Hochschule Osnabrück, Albrechtstraße 30, 49076 Osnabrück

\section{Kurzfassung}

Den Problemen, die sich aus der zunehmend volatilen und immer mehr dezentralen Energieerzeugung sowie den heutigen Verteilnetzen als „Black Box ${ }^{\iota}$ ergeben, wird hier durch die Einführung eines neuen Konzepts der Peerto-Peer-vernetzten Energie-Community begegnet. Ausgehend von den Erfahrungen mit Kommunikationsdiensten wird ein auf SIP und verteilter Datenhaltung basierendes P2P-Vernetzungskonzept für Haushalte und andere Marktteilnehmer vorgestellt und das Zusammenspiel mit einem Community Web Server beschrieben. Zum anderen wird ein Service Management Framework eingeführt. Es ermöglicht nicht nur die komfortable Vernetzung von Haushalten mit den Zielen Kostenersparnis, Vermeidung von Netzausbau, Energieeinsparung etc., sondern auch die Entwicklung und Bereitstellung personalisierter Dienste für das Smart Home und insbesondere das Energiemanagement inkl. der Einbindung von Multimediakommunikation. Darüber hinaus bietet es Funktionen für das Testen und für Simulationen.

\section{$1 \quad$ Einführung}

In den elektrischen Energieversorgungsnetzen der $\mathrm{Zu}$ kunft geht die Tendenz hin zu stärker volatiler regenerativer Energieerzeugung mittels Windkraft- und Photovoltaikanlagen und weg von der zentralen hin zur dezentralen Erzeugung und Einspeisung, auch im bisher nicht überwachten Ortsnetz. Um trotzdem eine stabile und qualitativ gute Energieversorgung sicherstellen zu können, muss das Netz ausgebaut und vor allem durch Informations- und Kommunikationstechnik intelligent gemacht werden. Diese Entwicklung wird allgemein durch den Begriff "Smart Grid" umschrieben. Ergänzende oder auch alternative Ansätze, die vor allem über variable Preissignale versuchen, das Verhalten der Energieverbraucher und -erzeuger zu beeinflussen und damit z.B. den Aufwand für Netzausbau zu minimieren, werden unter dem Oberbegriff „Smart Market" betrachtet [1].

Eine neue Smart Market-Lösung, die EnergieCommunity auf Basis vernetzter Haushalte, wurde in [2] und [3] eingeführt. Hierbei treten Haushalte, aber auch Energielieferanten und der Verteilnetzbetreiber als Peers einem sozialen Netzwerk für das Energiemanagement bei und kommunizieren und interagieren in der Folge mittels vernetzter, z.B. in DSL-Routern integrierter sog. Service Management Frameworks (SMF). Die durch jeweils eine SMF repräsentierten Peers tauschen energierelevante Informationen wie den aktuellen Verbrauch, die Prognose, Kennwerte vorhandener Energieerzeuger und -speicher sowie Strompreise und Netzentgelte aus. Für das Energiemanagement der gesamten Community oder auch nur einer Subcommunity (z.B. Region, Bereich einer Ortsnetzstation, Kunden eines Energielieferanten, Nutzer von Elektrofahrzeugen in einem Stadtteil) ist ein auf den SMFs dezentral laufender Algorithmus (spezieller Energiemanagementdienst) zuständig, der anhand gegebener Kriterien das energetische Verhalten der Community bzw. Subcommunity über die Steuerung von Energieverbrauchern (Demand Side Integration, DSI), -erzeugern und vor allem -speichern (optimale Zeiten für Laden, Speichern, Entladen ermitteln und Speicher entsprechend steuern) optimiert. Dabei wirken die Energielieferanten über die Strompreise, der Verteilnetzbetreiber über variable Netzentgelte oder sonstige Boni in ihrem Sinne mit. Der neu eingeführte Energie-CommunityBetreiber könnte als neue Marktrolle betrachtet werden, die z.B. von Städten oder Landkreisen im Rahmen von $\mathrm{Ma}$ nnahmen für den Klimaschutz- oder die regionale Förderung der Energiewende vorteilhaft übernommen werden könnte.

Diese Publikation beschreibt zum einen für die Cluster von Haushalten die Kommunikation und P2P-basierte (Peer-to-Peer) Vernetzung mittels SIP (Session Initiation Protocol) inkl. des Einsatzes von DHT (Distributed Hash Table) und Chord-Algorithmus für die verteilte Datenhaltung in der Community. Dabei wird auf die Mechanismen, die konkreten Protokolle sowie die Vorteile der vorgeschlagenen Kommunikationslösung eingegangen (Kapitel 2).

Zum anderen wird die zum Einsatz kommende Diensteplattform (SMF) im Hinblick auf die Anforderungen, die Funktionalität, die Software-Architektur und die Implementierung (auf Basis OSGi) vorgestellt (Kapitel 3). Das SMF besteht nicht nur aus einem Service Creation Environment (SCE), mit dessen Hilfe ein Nutzer mittels grafischer Oberfläche einfach personalisierte Dienste und/oder korrespondierende Tests für das Haus- und Energiemanagement designen kann, und einer Service Delivery Platform (SDP) zur Bereitstellung der Dienste inkl. ihrer Optimierung nach Vorgaben des Nutzers, sondern auch aus einem Simulation and Test Environment (STE) zur Simulation von Haushaltsszenarien und zum Test generierter Dienste 
(Kapitel 4). Kapitel 5 fasst die Ergebnisse zusammen und gibt einen Ausblick.

\section{SIP-basierte Peer-to-Peer- Vernetzung}

Wie in [2] beschrieben, führt die Optimierung eines einzelnen Haushaltes nicht zu einer Optimierung für eine Region, respektive zu einer Optimierung des Übertragungs- oder Verteilnetzes. Weitaus effektiver ist die gleichzeitige Optimierung mehrerer Haushalte. Der kritische Faktor ist demnach die statistische Aggregation von Haushalten $\mathrm{zu}$ einem Cluster von Haushalten, die gemeinsam optimiert werden. Um diese Aggregation zu erreichen, sind die Haushalte über die darin installierten Service Management Frameworks (SMF) miteinander verbunden und bilden ein SIP-basiertes Peer-to-Peer-Netzwerk [5]

Ein Haushalt kann dem P2P-Netzwerk, bzw. der Community beitreten, indem sich der Benutzer über seinen Webbrowser auf der Community Website einen Account anlegt. Nach erfolgter Authentifizierung registriert sich der Benutzer bei dem Community Webserver (siehe Bild 1).

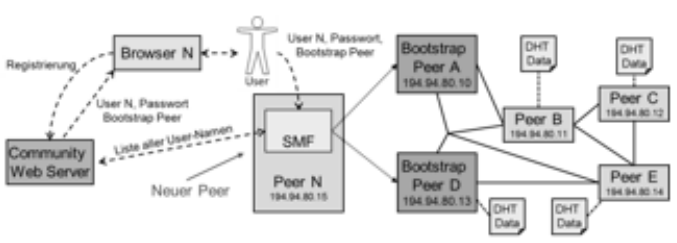

Bild 1 Beitritt zur Energie-Community

Nach der Registrierung erhält der Benutzer von dem Webserver die Zugangsdaten und die IP-Adressen von mindestens zwei Bootstrap Peers (Knoten im P2PNetzwerk zur ersten Kontaktaufnahme). Mit diesen Informationen konfiguriert der Benutzer seine SMF, welche wiederum einen verfügbaren Bootstrap Peer kontaktiert und somit den Beitritt zum P2P-Netzwerk erwirkt.

Um Informationen (betreffend des Energieverbrauchs, Einsparpotenzials und der Speichermöglichkeiten etc.) zwischen den Haushalten austauschen zu können, müssen die Haushalte miteinander vernetzt werden. Die Kommunikation zwischen den Haushalten und die Verbindung der Haushalte erfolgt mittels des Session Initiation Protokolls (SIP). SIP wurde als Protokoll für den Informationsaustausch gewählt, da es ein weit verbreitetes Protokoll zur Steuerung von Kommunikationssitzungen ist und die Anbindung von Telekommunikationsdiensten an die vorgestellte Plattform ohnehin die Verwendung von SIP erfordert. Das Nutzen von vorhandenen Synergien durch die Integration der Telekommunikationsdiente sowie die hierdurch bedingte Anbindung über ein IP-Netz (mit exis- tierenden Mechanismen für Sicherheit, Verfügbarkeit, und Quality of Service) unterstreicht den Einsatz von SIP zur Vernetzung der Haushalte.

Der Ansatz der Peer-to-Peer-Vernetzung der Haushalte und weiterer Instanzen (z.B. Verteilnetzbetreiber, Energielieferanten) wurde gewählt, da eine zentrale Vernetzung mit großem Aufwand und ggf. mit rechtlichen Einschränkungen verbunden ist und die Struktur eines P2P-Netzwerkes weitgehend selbstorganisiert ist. Schwierigkeiten bei einer zentralen Organisation würden bez. der Bereitstellung der Infrastruktur bestehen, der Übernahme der Betriebskosten, des Datenschutzes etc. Durch den P2P-Ansatz besteht die Möglichkeit, eine Vernetzung von Haushalten aufzubauen, für die keine zentrale Instanz erforderlich ist und welche eine hohe Verfügbarkeit und Skalierbarkeit bietet. [5] beschreibt die Bereitstellung von Mehrwertdiensten, welche in diesem Ansatz eine zentrale Rolle spielen, über ein P2P-Netzwerk. Hierdurch ist die Möglichkeit gegeben, Dienste, die nach dem in [5] beschriebenen Ansatz über SIP in einem P2P-Netzwerk bereitgestellt werden, in der entwickelten Plattform verfügbar bzw. anbindbar zu machen.

Jeder Haushalt wird als ein Peer innerhalb dieses Netzwerkes betrachtet, ebenso die Energielieferanten, Netzbetreiber und weitere Marktteilnehmer. Die Kommunikation zwischen den Peers erfolgt anonymisiert und verschlüsselt über das Internet, wobei jeder Peer nur die Informationen bereitstellt, welche der Benutzer autorisiert hat. Bild 2 zeigt den auf dieser Kommunikationslösung basierenden Austausch von Nachrichten zwischen den Peers, welche die für eine Optimierung relevanten Parameter beinhalten (z.B. aktueller oder geplanter Energiebedarf, Angaben zu vorhandenen energieerzeugenden oder energiespeichernden Anlagen (stationäre Energiespeicher, Elektroautos), Energiepreise und/oder Netzentgelte).

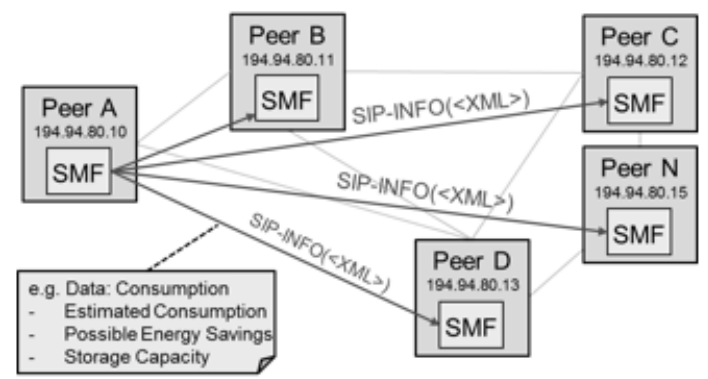

Bild 2 Versenden von Peer-Informationen

Die Informationen werden zwischen den Peers mittels SIP-INFO-Nachrichten ausgetauscht, welche eine Beschreibung der Energiedaten im XML-Format beinhalten. Die Versendung der Nachrichten erfolgt von jedem Peer an alle weiteren im P2P-Netzwerk angemeldeten Haushalte. Die Information über die Adressierung der Peers erhält die SMF des versendenden 
Peers durch Abrufen einer Liste mit allen Teilnehmern über den Community-Webserver. Hierüber ist die SMF in der Lage, die ständigen SIP URIs zur Kontaktaufnahme zu bilden. Die orts- bzw. subnetzabhängigen Kontaktadressen der Peers ermittelt die SMF über einen ebenfalls in dem P2P-Netzwerk implementierten Location Service (siehe unten).

Basierend auf den ausgetauschten Informationen stellt das SMF einen Algorithmus zur Verfügung, welcher automatisiert den aggregierten Energieverbrauch des gesamten P2P-Netzwerks optimiert (bis hin zum gesamten Verteilnetz), unter Berücksichtigung der Informationen von den anderen Peers. Die hierzu notwendige Datenhaltung ist realisiert, basierend auf Distributed Hash Tables (DHT) und dem ChordAlgorithmus [8].

In dem vorgestellten Ansatz wird eine verteilte $\mathrm{Da}$ tenhaltung für die Speicherung der für den Betrieb der Community und die energetische Optimierung erforderlichen Daten vorgesehen. Verteilte Datenhaltung bedeutet, dass Daten nicht an einer zentralen Stelle, sondern verteilt auf mehrere Instanzen (Peers) in einem Netzwerk gespeichert werden. Das Bestreben des vorgestellten Ansatzes ist es, gänzlich auf zentrale Instanzen zu verzichten, was demnach auch eine zentrale Datenhaltung ausschließt.

Eine Hash Table ist eine Struktur, um Daten abzulegen. Die Daten werden innerhalb dieser Struktur durch Key-Value-Wertepaare abgelegt. Ein konkretes Datenobjekt (z.B. die temporäre SIP URI eines Peers) wird unter einem definierten Schlüssel gespeichert. Mittels dieses Schlüssels kann auf das zugeordnete Datenobjekt zugegriffen werden. Eine Distributed Hash Table (DHT) ist eine Variante einer Hash Table, die es ermöglicht, die Datensätze verteilt auf verschiedene Knoten in einem Netzwerk abzulegen, und stellt somit eine Möglichkeit dar, verteilte Datenhaltung zu realisieren.

Ein Mechanismus, der die verteilte Datenhaltung mittels DHT beschreibt, ist der Chord-Algorithmus, entwickelt am MIT (Massachusetts Institute of Technology). "Chord ist ein einfaches, verteiltes Suchprotokoll furr Peer-to-Peer Systeme, das Schlüssel auf Knoten abbildet. " [9] Chord ermöglicht den Aufbau eines strukturierten P2P-Systems. Durch diesen Algorithmus lässt sich eine Topologie erzeugen, die aktiv auf Strukturänderungen (z.B. Wegfallen oder Hinzufügen von Knoten) reagiert. Die zu speichernden $\mathrm{Da}-$ ten werden redundant in den Knoten gespeichert, wodurch die Verfügbarkeit im Vergleich zu einer zentralen Datenhaltung drastisch erhöht wird. Die Identifikation eines Knotens erfolgt durch eine diesem zugeordneten Knotenschlüssel (Node ID). Die Identifikation eines Datensatzes erfolgt analog über einen Datensatzschlüssel (Key). Eindeutige Knoten- und
Datensatzschlüssel werden gebildet, indem z.B. die Hash-Funktion SHA1 auf die Node ID und den Key angewendet wird. „Ein Schlüssel k wird dem Knoten n zugewiesen, dessen ID grösser oder gleich der ID des Schlüssels $k$ ist ${ }^{\text {c }}$ [9]. Jeder Knoten kennt seinen Vorgänger (in der Chord-Topologie) und seinen Nachfolger. Durch Chord ist demnach eine Möglichkeit gegeben, zu einem gegebenen Schlüssel k die ID des Knotens zu ermitteln, welcher den Datensatz speichert. [9]

Bild 3 zeigt eine Chord-Topologie in ihrer einfachsten Struktur (Ring) und die Implementierung eines Location Service über eine mit dem Chord-Mechanismus realisierte DHT. Über den Location Service ist es möglich, die Zuordnung von den ständigen SIP URIs der Peers zu den temporären SIP URIs zu ermitteln Diese Information wird benötigt, um die Informationen, wie oben beschrieben, an alle Teilnehmer in dem P2P-Netzwerk zu senden.

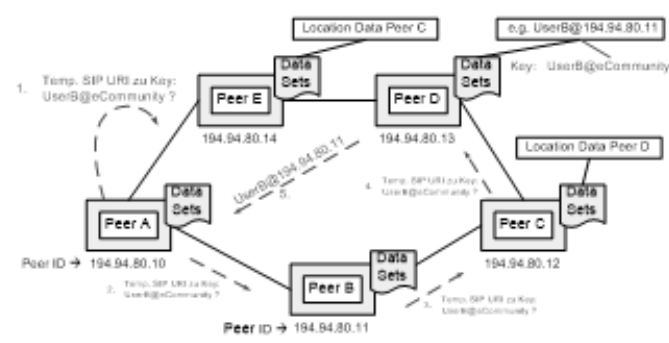

Bild 3 Chord-basierter Location Service

Die Ermittlung von einer temporären SIP URI erfolgt, indem ein Peer, bzw. dessen SMF, eine Anfrage (unter Angabe der ständigen SIP URI) an den Chord-Ring stellt. Hierbei erfolgt die Anfrage immer an den Knoten (Peer), welcher in der Topologie neben dem anfragenden Knoten angeordnet ist. Falls dem angefragten Knoten der zugehörige Datensatz vorliegt, liefert er diesen direkt an den anfragenden Knoten zurück. Liegt der Datensatz nicht vor, richtet dieser Knoten die Anfrage an den in der Topologie neben ihm angeordneten Knoten. Dieser Vorgang wird so lange wiederholt, bis der gesuchte Datensatz gefunden wurde.

Sobald Informationen zwischen den Peers ausgetauscht werden, muss sichergestellt werden, dass kein Teilnehmer in dem Netzwerk (z.B. der Nachbar) diese Informationen nutzen kann, um Rückschlüsse auf den Energieverbrauch eines spezifischen Haushaltes zu ziehen. Um die Anonymität zu gewährleisten, ist die Kommunikation zwischen den SMFs der Peers für die Nutzer nicht transparent. 


\section{3 Service Management Frame-
work (SMF)}

Im ersten Schritt dieser ganzheitlichen Lösung für das individuelle Energiemanagement im Smart Home/Grid erhält der Benutzer die Möglichkeit, eigenständig personalisierte Dienste für sein Smart Home und für das Energiemanagement in diesem zu erstellen. Dies wird durch die Bereitstellung eines Service Management Frameworks (SMF), bestehend aus Service Creation Environment (SCE) und Service Delivery Platform (SDP), ermöglicht, welches z.B. im DSL Router des Benutzers untergebracht sein kann Durch die Erstellung personalisierter Dienste ist es möglich, die persönlichen Bedürfnisse und Ansprüche des Benutzers zu berücksichtigen $[4,5]$.

Mittels des SCE erhält der Benutzer die Möglichkeit, Mehrwertdienste durch grafische Kombination von Bausteinen (Device/Service Blocks), welche durch den Geräte-/Diensthersteller geliefert werden, zu entwerfen. Die genutzten Device/Service Blocks beschreiben die Steuerungsmöglichkeiten der Geräte sowie die Funktionalität der Basisdienste und machen diese in der SCE verfügbar. Im einfachsten Fall konfiguriert der Benutzer die zeitliche Steuerung seiner Geräte abhängig von Preissignalen für den Strompreis für unterschiedliche Zeitabschnitte eines Tages. Der personalisierte Energiemanagementservice wird von der SDP ausgeführt. Die erforderlichen Messwerte werden von einem mit dem SMF verbundenen Smart Meter bereitgestellt. Hiervon ausgehend ist das SMF in der Lage, Informationen über den Energieverbrauch in der Vergangenheit sowie den geplanten zukünftigen Energieverbrauch zu ermitteln. Der Vorteil dieser Lösung zur Optimierung des Energieverbrauchs ist, dass die persönlichen Bedürfnisse des Benutzers in jedem Moment berücksichtigt werden. Nachteil ist, dass die Optimierung nur aus Sicht eines speziellen Kunden stattfindet.

In einem zweiten Schritt stellt das SMF einen speziellen Mechanismus bereit, welcher automatisiert Mehrwertdienste generiert, in Abhängigkeit von den durch den Benutzer definierten Randbedingungen (z.B. keine zeitliche Einschränkung für das Kochen, Minimierung der Energiekosten oder die Beschränkung des maximalen Energieverbrauchs). Das Ziel des generierten Dienstes ist es z.B., die Lastkurve für einen Haushalt zu glätten, in Abhängigkeit von den durch den Benutzer definierten Randbedingungen, um kostenintensive Lastspitzen zu reduzieren/vermeiden. Die Energieoptimierung erfolgt nicht nur aus der Sicht eines einzelnen Benutzers, sondern unter Berücksichtigung des gesamten Haushalts. Dies ist besonders effektiv, wenn in dem Haushalt nicht nur Energieverbraucher, sondern auch energieerzeugende Anlagen oder Energiespeicher installiert sind.
In beiden Fällen geht die Leistung des SMF über die existierender Lösungen für Smart Home-Steuerungen hinaus, da das SMF nicht nur das oben beschriebene Energiemanagement und IT-Dienste (z.B. Zugriff auf den Kalender vor dem Laden eines Elektroautos) beinhaltet, sondern auch Kommunikationsanwendungen (z.B. Instant Message oder Anruf, wenn die Waschmaschine fertig ist). Da das SMF über das InternetProtokoll (IP) mit externen Netzwerken, wie z.B. Internet, Fest- oder Mobilfunknetz, verbunden ist, bietet sich als Anwendungsprotokoll das standardisierte Session Initiation Protocol für die Signalisierung (vgl. Kapitel 2) an und z.B. das Realtime Transport Protocol (RTP) für die Übertragung von Nutzdaten [6]. Daher ist das SMF eine Kombination einer Lösung zur Smart Home-Steuerung und eines SIP Application Servers, dessen Realisierung auf dem OSGi Framework basiert [7]. Bild 4 zeigt eine Übersicht über die Komponenten des SMF.

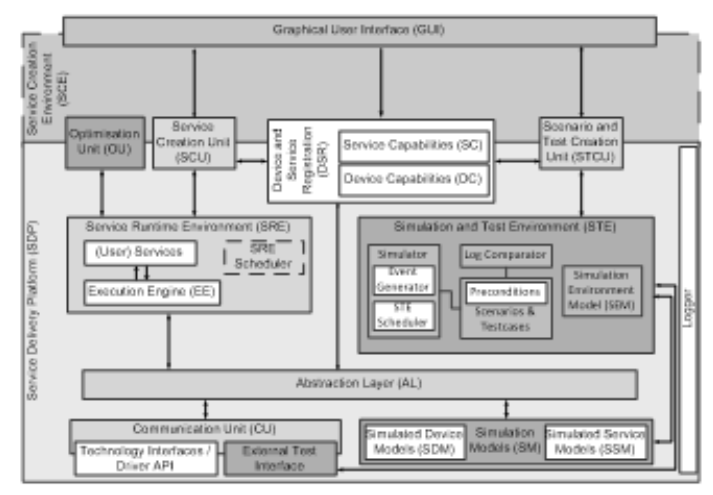

Bild 4 Übersicht über die Komponenten des SMF

Über das Graphical User Interface (GUI) erstellt und konfiguriert der Benutzer Dienste zur Steuerung seines Smart Home. Um diese Dienste zu erzeugen, verwendet der Benutzer Device/Service Blocks. Diese Device/Service Blocks beinhalten eine Beschreibung der Geräte und deren Funktionalitäten (Device/Service Capabilities). Diese Informationen werden genutzt, um die Geräte in der GUI zu repräsentieren und dem Benutzer als Teil des Dienstes verfügbar zu machen. Als einfaches Beispiel kann der Nutzer einen Dienst definieren, welcher die Aufladung eines Elektroautos (repräsentiert in der GUI über die Geräte- und Eigenschaftenbeschreibung des Elektroautos) startet, sobald der Strompreis unter einer bestimmten Obergrenze liegt. Der Benutzer definiert über diese GUI auch Randbedingungen und Ziele, welche bei der Optimierung berücksichtigt werden (z.B. kann der Benutzer Optimierungsziele definieren wie "Preisoptimierung", "Lastreduktion" etc.). Die Aufgabe der Service Creation Unit (SCU) ist es, dem Benutzer die GUI in Form einer Webseite zur Verfügung zu stellen. Weiterhin ist die Aufgabe der SCU, den definierten/konfigurierten Dienst mittels einer formalen Nota- 
tion zu beschreiben und an das Service Runtime Environment (SRE) zu übergeben.

Das SRE (bestehend aus Repository mit den erstellten Diensten, Execution Engine und SRE Scheduler) interpretiert diese Service Description (abgelegt im Service Repository) und führt die darin beschriebenen Aktionen aus. Hierfür kommuniziert das SRE mit dem Abstraction Layer (AL) (siehe nächsten $\mathrm{Ab}$ schnitt) und steuert hierüber die Aktionen in den verbundenen Geräten. Solche Aktionen sind z.B. die Deaktivierung von Geräten um Energie zu sparen, oder die Entladung eines Energiespeichers in einem Haushalt. Als weitere Funktionalität wurde die Multimediakommunikation integriert. Dies gestattet es, unter bestimmten Voraussetzungen, z.B. wenn ein Elektroauto vollständig aufgeladen ist oder eine Bewegung durch einen Bewegungssensor erkannt wurde (zur Anwendung als Einbruchserkennung), z.B. eine IM (Instant Message) zu versenden oder einen Videoanruf zu initiieren.

Für die Abstraktion der Kommunikation und der Anbindung von unterschiedlichen Bussystemen zur Smart Home-Steuerung (z.B. KNX, EnOcean, MBus, ZigBee, digitalSTROM) bzw. zur Anbindung von Multimediasystemen (z.B. SIP, RTP, HTTP) wurde ein AL konzipiert. Dieser ermöglicht es, verschiedene Smart Home- und Multimediakommunikationstechniken zu verknüpfen, da der AL die Kommunikation zwischen den Geräten, Teildiensten, Smart HomeBussystemen und der Multimediakommunikation vereinheitlicht. Der AL abstrahiert die Steuerung von verschiedenartigem Equipment und bietet daher die Möglichkeit, Dienste für eine Smart Home-Steuerung ohne Berücksichtigung der verwendeten Technologien zu definieren.

Um die Anbindung der verschiedenen Technologien zur Kommunikation und Steuerung in einem Smart Home umzusetzen, beinhaltet die SMF-Architektur eine Communication Unit (CU), welche direkt mit dem AL verbunden ist und die Kommunikation mit den spezifischen Bussystemen und Multimediatechnologien realisiert. Geräte-APIs (Application Programming Interface), bereitgestellt durch die $\mathrm{CU}$, werden von dem $\mathrm{AL}$ angesprochen und bilden die Schnittstelle zu den verschiedenen Smart HomeTechnologien. Alle verbundenen Geräte sind bei der DSR (Device and Service Registration) unter einer allgemeinen, in der Plattform eindeutigen ID registriert und werden durch die Dienste in einer abstrahierten Weise gesteuert. Die Umsetzung von abstrahierter in eine gerätespezifische ID erfolgt durch den AL.

Die Optimisation Unit (OU) implementiert die oben beschriebenen Optimierungen von Diensten durch Rekonfiguration der definierten Benutzerdienste. Die
Optimierung erfolgt unter Berücksichtigung der durch den Benutzer definierten Randbedingungen und Optimierungsziele. Die Optimierung kann u.a. durch die zeitliche Verschiebung der Gerätenutzung, z.B. Verschieben des Aufladens eines Elektroautos in einen kostengünstigeren Zeitraum (sofern der Benutzer als Ziel eine Kostenoptimierung ausgewählt hat) erfolgen. Eine weitere Aufgabe der OU ist es, die Koordination von mehreren Haushalten bez. Steuerung und Optimierung durchzuführen. Dies kann z.B. durch die koordinierte Disposition großer Energieverbraucher in verschiedenen Haushalten oder durch Entladen von Batteriespeichern, sobald die Last in einer Region eine definierte Grenze überschreitet, erfolgen.

\section{Simulation and Test Envi- ronment (STE)}

Nutzerdienste, die neu erstellt wurden und ungetestet mit realen Geräten genutzt werden, können an diesen Geräten irreparable Schäden hinterlassen oder sogar die Gesundheit der im Haushalt lebenden Personen gefährden. Das Simulation and Test Environment (STE) hat die Aufgabe, die neu erstellten Nutzerdienste zu testen, bevor sie mit realen Geräten genutzt werden. In Bild 5 ist eine Übersicht des STE mit den Simulationsmodellen und einer externen Testschnittstelle dargestellt.

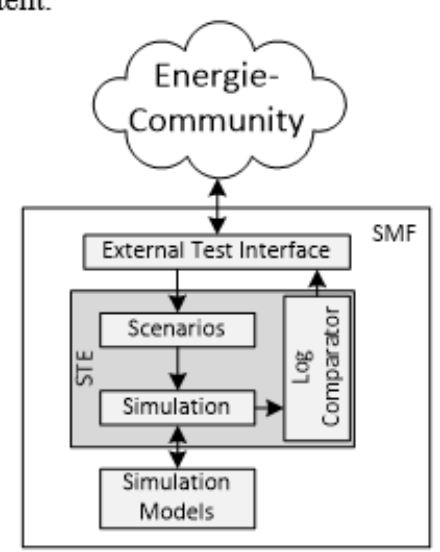

Bild 5 Komponenten für eine Simulation innerhalb einer Energie-Community

Szenarien beschreiben den Ablauf einer Simulation. Ein Szenario beschreibt dabei sämtliche Ereignisse innerhalb eines Haushaltes, z.B. den Verlauf des Strompreises oder das Nutzerverhalten durch Aktivierung und Deaktivierung von Geräten. Ein Szenario setzt sich dabei aus zwei Teilen zusammen:

1. Vorbedingungen: in den Vorbedingungen wird der Startzustand der Simulation definiert. Mögliche Einstellungen sind beispielsweise, ob das Elektroauto vorhanden ist oder der Füllstand des Batteriespeichers. 
2. Ablaufplan: Der Ablaufplan beinhaltet alle Ereignisse, die während der Simulation auftreten sollen. Diese umfassen neben dem Benutzerverhalten auch externe Einflüsse wie die Dargebotssituation für eine eigene PV-Anlage oder die Netzsituation, abgebildet durch den Strompreis. Hierdurch sollen die vom Benutzer erstellten Dienste stimuliert und somit aktiviert werden.

Neben den Simulationsszenarien gibt es noch Testszenarien, hier kommt ein dritter Punkt hinzu:

3. Erwartete Simulationsergebnisse: In diesem Teil eines Szenarios können Ereignisse definiert werden, die bei einer nach der Simulation folgenden Analyse zutreffen sollen. Dabei sind nicht die Ereignisse aus Teil 2 anzugeben, sondern die Reaktionen auf diese durch vom Benutzer erstellte Dienste. Weiterhin können hier Verläufe virtueller Messgrößen, beispielsweise der Gesamtenergieverbauch, überprüft werden.

Ein solches Simulations- oder Testszenario kann von einem Hersteller zusammen mit den Gerätebeschreibungen ausgeliefert werden. Da ein Szenario für einen zukünftigen Zeitraum häufig nicht perfekt ermittelt werden kann, ist es möglich, nicht nur exakte Werte, sondern auch mehrere Werte, Wertebereiche oder Varianzen einzelner Werte zu definieren.

Die Simulation ist, genau wie die Kommunikation zwischen den einzelnen Komponenten in der Plattform, eventbasiert (Diskrete Event-Simulation, DES [10]). Die Simulationsmodelle sind dafür verantwortlich, ihre zu sendenden Events in einen Scheduler einzutragen, welcher diese in passender Reihenfolge aussendet. Ein Simulationsfortschritt erfolgt bei der DES nicht zeitgesteuert, sondern durch abgearbeitete Events. Die Zeit schreitet demnach nicht kontinuierlich voran, sondern bleibt abhängig vom Aufkommen der Events länger an einem Zeitpunkt stehen oder überspringt größere Zeiträume. Dies hat den Vorteil dass die Simulation, besonders bei wenigen Events, deutlich schneller ablaufen kann.

Simulation Models spiegeln das Gegenstück zu realen Geräten und den in Benutzerdiensten verwendeten Basisdiensten wieder. Dies ist notwendig, da reale Geräte bei einem Test beschädigt werden oder Schaden verursachen könnten. Basisdienste hingegen sollen nachgebildet werden, um keine reellen Kosten durch die Nutzung innerhalb einer Simulation zu verursachen. Für das Testen sind die Simulationsmodelle von Bedeutung, da so Extremsituationen wie der Ausfall eines Gerätes oder die Überlastung des Stromnetzes nachgebildet werden können. Die realen Geräte werden durch sog. Simulated Device Models (SDM) und die Basisdienste durch Simulated Service Models (SSM) abgebildet. Diese verhalten sich der Plattform gegenüber annähernd identisch wie die realen Geräte bzw. Dienste. Dabei ist besonders der Energiever- brauch der Geräte von Interesse. Dieser wird der Plattform von jedem SDM bei einer Änderung per Event mitgeteilt. Im Logger können dann die genauen Verbrauchsdaten der einzelnen Geräte oder des gesamten Haushaltes abgefragt werden.

Durch das External Test Interface können Simulationsszenarien nicht nur von Benutzern erstellt oder von Herstellern mit den Geräten ausgeliefert werden, sondern auch von einem Mitglied der EnergieCommunity über eine von extern erreichbare Schnittstelle geladen werden. Dies ist insbesondere für einen Energieversorger interessant. Dieser kann verschiedene Szenarien in die Plattformen einspielen und mit den zurückgesendeten Simulationsergebnissen eine genauere Prognose über den zeitlichen Energieverlauf errechnen. Der Nutzer kann dabei einstellen, welche Daten er preisgeben möchte. Im Normalfall sollte eine diskrete Zeitreihe des hauseigenen Energieverbrauchs für den Energieversorger ausreichend sein. Auch ist es vorgesehen, dass nur einzelne Parameter für ein Szenario von extern bereitgestellt werden müssen (z.B. ausschließlich der Strompreis) und diese auf bestehende Szenarien des Haushaltes angewandt werden können. Dies hat den großen Vorteil, dass das Verhalten des Nutzers oder sonstige Besonderheiten berücksichtigt werden können.

Durch den Log Comparator kann, zusammen mit den erwarteten Simulationsergebnissen, nach der Ausführung die durch den Logger aufgezeichnete Simulation analysiert werden. Dabei kann untersucht werden, ob ein Event, passend zu einem erwarteten Simulationsergebnis aus dem Szenario, in der Simulation vorgekommen ist (z.B. „Laden des Elektroautos um 3:00 $\mathrm{Uhr}^{c *}$ ). Eine solche Erwartung kann negiert werden, wodurch angegeben wird, dass ein entsprechendes Event innerhalb der Simulation nicht vorkommen soll. Weiterhin können zeitliche Einschränkungen spezifiziert werden, um so Ruhezeiten oder ähnlichem Rechnung zu tragen (z.B. „Waschmaschine nicht zwischen 20:00 und 6:00 starten"). Darüber hinaus besteht die Möglichkeit, nach virtuellen Messgrößen wie Energiekosten zu suchen und Vergleiche mit vorhergehenden Simulationen oder dem Realbetrieb aufzustellen. Somit kann beispielsweise bestimmt werden, ob ein Benutzerdienst negative Auswirkungen auf den Energieverbrauch oder die Energiekosten hat. Dabei ist zu beachten, dass der Vergleich nicht zu einem festen Zeitpunkt, sondern über eine Zeitspanne aufzustellen ist. Bedingt durch die DSI sollte diese Zeitspanne mindestens eine Nacht umspannen.

\section{$5 \quad$ Zusammenfassung und Aus- blick}

Mit dem vorgestellten Konzept der P2P-Vernetzung von Haushalten innerhalb einer Energie-Community 
unter Nutzung eines OSGi-basierten Service Management Frameworks wird eine ganzheitliche und sehr leistungsfähige Lösung vorgestellt, um sowohl für einen einzelnen Haushalt als auch ein Cluster von Haushalten Energieoptimierung mit maximalem Nutzen für die Beteiligten zu erreichen (Lastsenkung, Kostenoptimierung etc.). Gleichzeitig werden die Komfort-, Kommunikations-, Test- und Simulationsbedürfnisse der Nutzer berücksichtigt. Dies erfolgt mittels P2P-Vernetzung auf Basis SIP und verteilter Datenhaltung mit DHTs und Chord-Algorithmus. Das beschriebene SMF bietet vorteilhafterweise nicht nur SCE- und SDP-, sondern auch STE-Funktionen. Erste prototypische Realisierungen wurden vorgenommen. Weitere Arbeiten zur Detaillierung der angesprochenen Netz- und Systemkomponenten, zu den Optimierungsalgorithmen, zu den Bereichen Test und Simulation sowie zu Sicherheit und Datenschutz sind noch notwendig.

Das dieser Publikation zugrunde liegende Vorhaben wird mit Mitteln des Bundesministeriums für Bildung und Forschung (BMBF) unter dem Förderkennzeichen 17018A/B11 gefördert. Die Verantwortung für den Inhalt dieser Veröffentlichung liegt bei den Autoren.

\section{$6 \quad$ Literatur}

[1] Bundesnetzagentur; "Smart Grid" und "Smart Market" - Eckpunktepapier der Bundesnetzagentur zu den Aspekten des sich verändernden Energieversorgungssystems, Bonn, Dezember 2011.

[2] M. Steinheimer, U. Trick, P. Ruhrig: Energy communities in Smart Markets for optimisation of peer-to-peer interconnected Smart Homes. Proc. CSNDSP 2012, Poznan, July 2012.

[3] U. Trick, M. Steinheimer, P. Ruhrig, R. Tönjes, D. Hölker: Smart Grid - Integration von Haushalten mittels Vernetzung und EnergieCommunity. VDE-Kongress 2012 - Smart Grid, Stuttgart, November 2012.

[4] T. Eichelmann et al., "Discussion on a framework and its service structures for generating JSLEE based value-added services", Proc. of ITA 2011, pp. 169-176, 2011.

[5] A. Lehmann et al., "New possibilities for the provision of value-added services in SIP-based peer-to-peer networks", Proc. of SEIN 2008, pp. 167-176, 2008.

[6] U. Trick et al., "Herausforderungen an die Kommunikationstechnik im Smart Home/Grid", VDE/ITG Fachtagung Mobilkommunikation 2012, 2012.

[7] A. Alves, "OSGi in Depth", Manning, 2011.
[8] I. Stoica et al., "Chord: A Scalable Peer-to-peer Lookup Service for Internet Applications", IEEE/ACM Transactions on Networking, Vol. 11, Issue 1, pp. 17-32, 2003.

[9] Humboldt Universität Berlin, FB Informatik: http://sarwiki.informatik.hu-berlin.de/Chord

[10] J. Banks et al., "Discrete-event system simulation." Prentice Hall, Englewood Cliffs, NJ, 2008. 
Published in Proceedings of the Fifth International Conference on Internet

Technologies and Applications (ITA 13), pp. 90-97, September 2013, Wrexham, UK

\title{
LOAD REDUCTION IN DISTRIBUTION NETWORKS THROUGH P2P NETWORKED ENERGY-COMMUNITY
}

\author{
Michael Steinheimer ${ }^{1,2}$, Ulrich Trick ${ }^{1}$, Patrick Ruhrig1, Woldemar Fuhrmann ${ }^{3}$, \\ Bogdan Ghita ${ }^{2}$ \\ ${ }^{1}$ Research Group for Telecommunication Networks, University of Applied Sciences \\ Frankfurt/M., Frankfurt/M., Germany \\ \{steinheimer|trick|ruhrig\}@e-technik.org \\ ${ }^{2}$ Centre for Security, Communications and Network Research, University \\ of Plymouth, Plymouth, UK \\ \{michael.steinheimer|bogdan.ghita\}@plymouth.ac.uk \\ ${ }^{3}$ Department for Computer Science, University of Applied Sciences Darmstadt, \\ Darmstadt, Germany \\ woldemar. fuhrmann@h-da.de
}

\begin{abstract}
The increasingly volatile and decentralised power generation as well as the lack of transparency within distribution networks raise a number of challenges for the current power distribution paradigm. This paper aims to address the lack of flexibility of the current architecture by introducing the concept of a peer-to-peer networked energy community. The proposed approach includes a service management framework based on the dynamics of value-added services to provide personalised services for the Smart Home, especially the energy management, and includes the integration of multimedia communications. In addition the concept provides a mechanism for convenient networking of households, energy suppliers, distribution network operators and others with the objectives cost savings, avoidance of grid expansion, energy savings, $\mathrm{CO}_{2}$ reduction etc. Approaches for optimisation algorithms for reducing the residual load offer a set of instruments for cost reduction in households, environmental protection or the assurance of energy supply. Initial simulations confirm the effectiveness of this new and powerful approach, most notably the benefits of battery storage in homes.
\end{abstract}

\section{KEYWORDS}

Smart Grid, Smart Homes, Energy Management, Service Creation, Peer-to-Peer, Energy Community

\section{INTRODUCTION}

\subsection{Adjustment in future power supply system}

In the electric power grids of the future, the likely trend will be to increase production of more volatile renewable energy using wind power and photovoltaic systems. Based on this trend, the proportion of centralised energy generation will decrease, to be replaced by distributed generation and supply; this shift has already started, within the non-monitored local energy grid. To still ensure stability and good quality in the power supply, the local network has to be expanded and especially has to get smarter through information and communication technology. This development is commonly described by the term "Smart Grid" [2]. To solve these problems, different, in part complementary approaches are discussed. Mentioned are the keywords "Grid Expansion" [2], "Virtual Power Plants" [2], "automation of distribution networks" [2], "forced shutdown of producers and/or consumers" [3], as well as "control 
through price signals" [4]. The German Federal Network Agency covers the last topic with the term "Smart Market" and describes it as the preferred solution [1].

Despite extensive research and development activities in the areas of Smart Grid and Smart Home in recent years, the published solutions outline the following gaps or deficiencies:

- The user of a Smart Home gets offered services for home automation and energy management [5], but the user gets no or only rudimentary support by communication, especially multimedia communication.

- Even the self-development of services for energy management by the users themselves is treated in projects [6], but they are limited to pure configuration and also do not allow communication services.

- While there are solutions for the automated optimisation of energy in a single household or a cluster of households, the intelligent control system typically uses a centralised or hybrid centralised/decentralised approach $[4,6]$. A completely decentralised solution, while it is more complex to establish, would have indisputable advantages in scalability and reliability as well as in networking based on private initiative. Only one such approach with limited scope was found in [7].

- Also not been considered yet is the independent clustering of households, i.e. how households join together to form clusters for energy management in a comfortable way. Previous solutions $[4,6]$ are based on a cluster organisation by a central authority. The advantage of the new approach here could be an interconnection through voluntary energy community to enable the self-organisation of the network, to address issues of data protection, to define common interests of households, etc.

To close the gaps mentioned above, in October 2011 the BMBF-project FHprofUnt 2011 "EasyService Creation for Home and Energy Management (e-SCHEMA)" was initiated $[8,9,10]$. Within this project four solutions on "households in Smart Grids" (based on each other) get connected. These solutions are described in the following. All of these solutions are assigned to the field of "Smart Market".

\section{SERVICE PLATFORM FOR HOUSEHOLDS}

In the first part of this new overall solution for individual home and energy management the user gets the possibility to develop personalised services for home and energy management on its own, according to its personal needs, using a Service Management Framework (SMF) which may be integrated in the DSL-Router $[12,13]$. Through a SCE (Service Creation Environment), the user becomes able to design value added services by graphical combining of device/service blocks, delivered by the manufacturer of home appliances. This device/service blocks describe the control functions of devices and basic services and make them available in the SCE. In the simplest case, the user configures the scheduling of home appliances in line with the corresponding price variations for energy at different time intervals in the day. The personalised energy management service is running on a SDP (Service Delivery Platform). The required measurement values for the services are provided by the connected Smart Meter. On this basis the SMF, consisting of SCE and SDP, delivers information about the energy demand in the future and in the past. The advantage of this solution is that users' needs and rights are continually considered. Disadvantage is that the optimisation of the power consumption takes place only from the perspective of the individual user.

In a second step, the SMF provides a special mechanism which composes automatically a value added service, according to restrictions of the user (allowing combining permissions and restrictions, such as no time limits for cooking, minimising the cost of electricity, or limit the tolerable energy consumption). The aim of the resulting service for example is to minimise the variations in the load curve of a household according to users' restrictions, to avoid expensive 
peak power. Here the energy optimisation is not only from the perspective of the user, but of the complete household. This is especially effective when not only energy consumers, but also producer and/or storage in the home are available.

In both cases, the performance of the SMF is much better than in current Smart Home control solutions because the SMF does not only integrate the described energy management and IT services (e.g. access to the calendar before charging the e-car), but also integrates communication applications (e.g. SMS or call if the washing machine has finished). Since the SMF is connected definitely via IP to external networks such as the internet or fixed and mobile networks, this offer as application layer protocols SIP (Session Initiation Protocol) for signalling and e.g. RTP (Real-time Transport Protocol) for user data [10]. Therefore, the SMF is a combination of a Smart Home control and a SIP Application Server, whereby the realisation is based on OSGi (Open Services Gateway initiative) [17]. Figure 1 shows an overview of the relevant parts of the SMF.

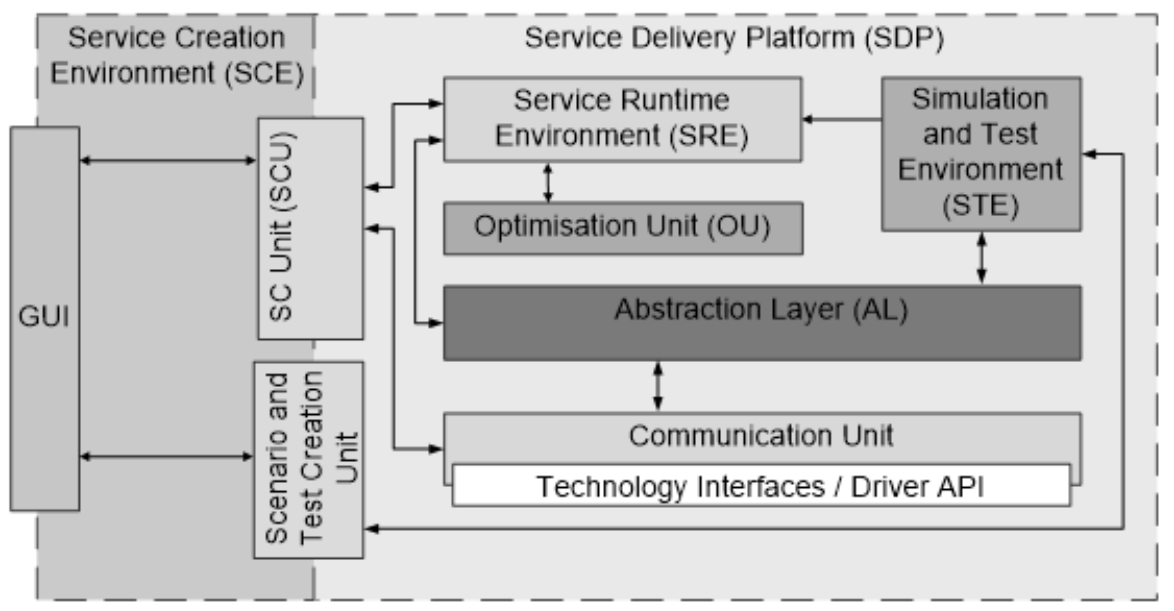

Figure 1. Overview of relevant parts of SMF

With the GUI (Graphical User Interface) the user creates and configures services to control its Smart Home. To create the service, the user applies device/service blocks that are provided by the device manufacturer. This device/service blocks include a description of the device and its capabilities. This information is used to represent the device in the GUI and make it available to the user as part of a service. As a simple example the user can define a service to start charging of an e-car (represented in the GUI using the device description and capabilities of the e-car) if the energy price is below a defined threshold. The user also defines, via this GUI, boundary conditions and goals, which are taken into account in the optimisation (e.g. for an optimisation service the user can define the optimisation goals like "price optimisation", "load reduction" etc.). The task of the SCU (Service Creation Unit) is to make the GUI available in the form of a website. Another task is to describe the service defined/configured by the user in a formal notation and deliver this description to the SRE (Service Runtime Environment).

The SRE interprets the service description and executes the actions described in the formal service notation. For this, the SRE communicates with the AL (Abstraction Layer) and thus controls the actions in the connected devices. Actions for example are activation or deactivation of devices to save energy or discharging existing energy storage in a household. As further functions the multimedia communication has been integrated. That allows, under defined conditions, e.g. if the e-car is fully charged or the motion detection device is triggered (in case 
of using for intrusion detection), for example to initiate a video call or to send an IM (Instant Message).

For abstraction of communication and connection of different bus systems for Smart Home controlling (e.g. KNX, EnOcean, MBus, ZigBee) an Abstraction Layer (AL) has been designed. This makes it possible to connect a variety of smart home and multimedia communication techniques, as the AL unifies communication between services, part services, Smart Home bus systems as well as elements for multimedia communications. This coordinates the control of different types of equipment and thus offers the possibility to define services for Smart Home control without depending on the technologies used.

To implement this integration of different communication and Smart Home systems, the architecture includes a CU (Communication Unit), which is connected directly to the $\mathrm{AL}$, allowing the device and technology specific control of devices. All connected devices are registered at the $\mathrm{AL}$ and are controlled within the services in an abstracted way. Devices APIs, provided by the $\mathrm{CU}$, are driven by the $\mathrm{AL}$ and form the interface to the various technologies (like $\mathrm{KNX}$, EnOcean, MBus, ZigBee, internet browsing, multimedia communication).

The OU (Optimisation Unit) implements the above described optimisation of services through re-configuration of defined services. This is done taking into account the constraints and optimisation objectives which the user has defined via the GUI. Optimisation can be done by rescheduling the devices, e.g. shift the charging of an e-car to time slots in which the energy price is low (if the user defined cost saving as optimisation goal). Another task of the OU is to organise the coordination of multiple households for control and optimisation. This can be done by coordinated scheduling of large consumers in different households or by discharging energy storages if the load in a region is about a defined threshold.

In order to test and simulate the effectiveness and accuracy of the created services based on definable test cases, the architecture also includes a Scenario and Test Creation Unit (for creation and presentation of test cases) as well as a STE (Simulation and Test Environment).

\section{P2P INTERCONNECTED HOUSEHOLDS AND ENERGY COMMUNITY}

The independent optimisation of individual households does not automatically lead to optimisation of the local or distribution grid; the critical factor that leads to optimisation is statistical aggregation through cooperation within a cluster of consumers. In order to provide this level of aggregation, the SMFs of individual households are connected using a SIP-based peer-to-peer (P2P) network [13] (Figure 2). The peers represent households, energy suppliers, distribution network operators and other market participants. The communication between the peers is anonymised and encrypted, also each peer only provides user authorised information. Based on this communication channel, the peers exchange a number of parameters, such as the current or forecasted consumption, key indicators for energy producers and storages (including electric vehicles) as well as energy prices and network charges. On this basis, the SMF provides an algorithm that automatically optimises the aggregated energy consumption for the whole P2P-network (up to complete local grid), taking into account the information from all other peers. The necessary data management is distributed based on DHT (Distributed Hash Table) and the Chord algorithm [18].

The method of joining the network on a comfortable and legal basis has not been resolved up to this point. Creation of an energy-community considers this issue and forms a new comfortable way to join the network, whereby the connection of households is done by joining the users to a social network for energy-peers. (Figure 2). Therefore, the SMF also provides functionality for the energy community and joining the P2P network. The members have the same interests and 
economic goals (e.g., energy consumption reduction, cost savings, environmental preservation), their profiles could include the energy consumption as well as existing storage and generation capacity. The formation of sub-communities is also possible (e.g. based on a street, geographical proximity, secondary substation, customers of an energy supplier or distribution network operator).

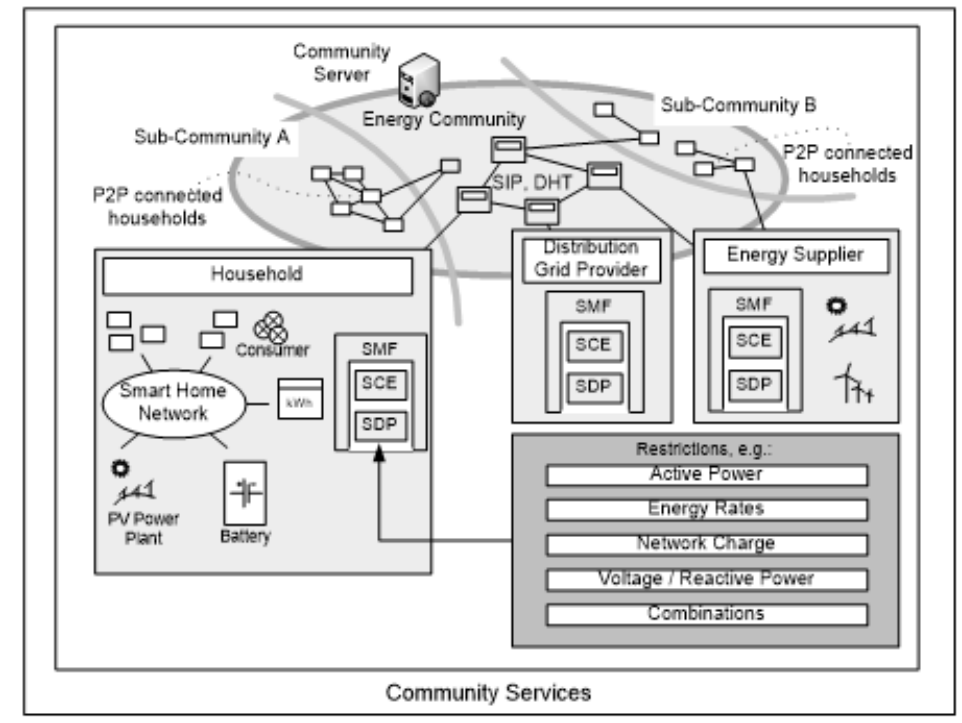

Figure 2. P2P connected households with energy-community

The whole community or a sub-community can appear together and offer e.g. regulation energy. Moreover, competition between sub-communities could be possible, for example aiming to reduce energy consumption, where comparable households exchange their consumption values. Another possibility is to compare energy consumption profiles with the building and user profiles of other participants. On this basis opportunities for optimisation can be presented to the participants of the community. The energy consumption optimisation is carried out from the perspective of the whole community and also across local network areas. Therefore, the two combined approaches (P2P network and energy-community) represent a comprehensive new solution for the creation of a Smart Market for households.

The community server provider could be third party, such as a city or county, which may have energy savings as part of their objectives or agenda. The introduced energy community with $\mathrm{P} 2 \mathrm{P}$ networking households is a very powerful instrument to optimise the energy distribution in the local grid, and additionally provides potential for price and cost reduction.

\section{LOAD REDUCTION BY INTELLIGENT CONTROL}

The households connected by an energy community or by sub communities, as introduced in section 3, can work together and implement application scenarios, such as avoiding peak loads, avoiding regulation energy, optimisation of the energy costs, incentives to save energy etc. (see [19]). They use the more flexible options that come up through the use of distributed storages, generators and/or time shifted activation of consumers (demand-side integration (DSI)). Depending on the use case, this may result in benefits to the distribution system operator, the balancing group managers, energy suppliers, consumers or the environment etc. In the following (Figure 3) a section of the complex algorithm is presented, which is used for a scenario for peak load reduction in a region. 
Firstly, each peer determines the residual load in the region concerned, which represents the difference between the energy demand and the supply by renewable energy. If the calculated residual load for the region exceeds the defined limit, energy storages present in this region will discharge and supply additional energy to the grid.

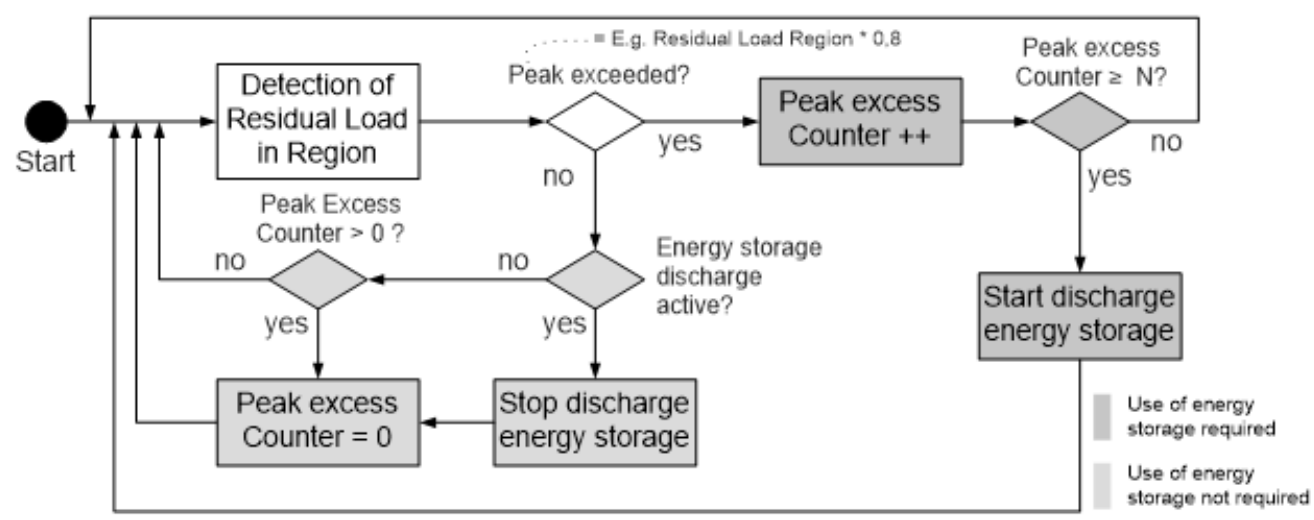

Figure 3. Section of algorithm for peak load reduction

The demand will be met locally through the local supply, preventing the supply of additional power from upper voltage level. Recharging the energy storage takes place in line with defined lower limits of the residual load.

Figure 4 shows the part of the algorithm responsible for the calculation of the residual load in a region. Each peer receives the energy requirements of all other peers. The required data is transmitted via SIP messaging, based on the internetworked households described in section 3 . After the energy demand of all households was received, the individual energy requirement is determined and distributed to other peers. The distribution of consumption data will be anonymous as described in section 3 . To determine the residual load, each peer adds its energy demand to the sum of the received energy demands of all peers. This determination of the total energy demand is the basis for the decision to discharge existing energy storage as described above.

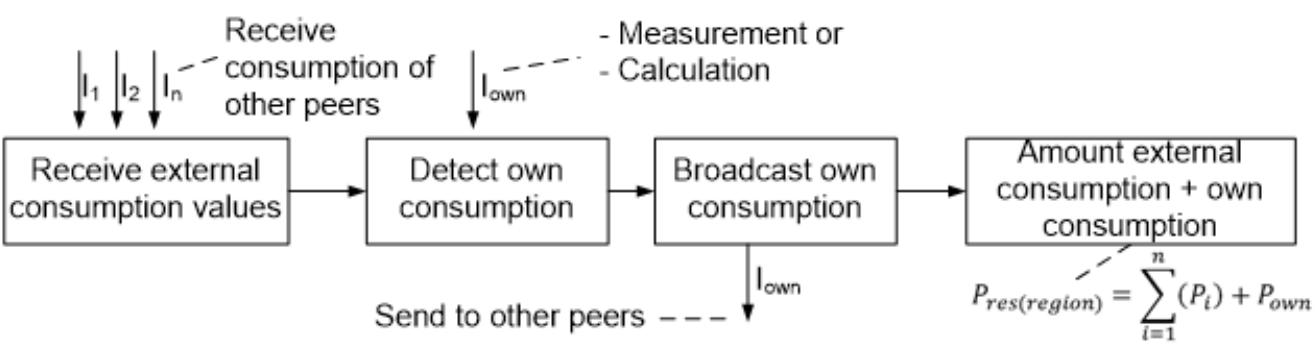

Figure 4. Responsible part of algorithm for calculation of the residual load

To demonstrate the effectiveness of the outlined energy community below two concrete, simulated application scenarios are discussed.

The simulations are based on the following parameters: households with 1-5 persons, based on determined or estimated values for consumers (cooking, washing, freezing, lighting, office equipment, consumer electronics, drying, water heating and miscellaneous e.g. ironing), energy generators like photovoltaic and energy storages as well as probability distributions to 
households, equipment availability and usage [14, 15]. As energy storage a commercial battery model is used with the following parameters: charge capacity: $1400-5400 \mathrm{Wh}$; maximum charge/discharge power: $4800 \mathrm{~W} / 4000 \mathrm{~W}$; efficiency: $97 \%$. The applied PV system delivers $4000 \mathrm{~W}$ peak power. Moreover it should also be noted that the simulations consider only 24 hours, i.e. devices can be activated from 0 a.m. and not be active for more than 24 hours, moreover, the storage capacity amounts $30 \%$ load at midnight.

To demonstrate the performance of the simulation, in a first step, the pure power consumption for 1000 households or 2024 individuals was determined, using a distribution of $39,7 \% 1$-, $34,6 \% 2-, 12,7 \% 3-, 9,6 \% 4-$ and 3,4\% 5-person households [14]. This simulation was compared with the German standard load profile $\mathrm{H} 0$ for a working day in the summer [16]. The result is shown in Figure 5 (left). The good agreement between the two curves shows the quality of the underlying simulation model, and the calculation for an infinite number of households reveals an even better match.
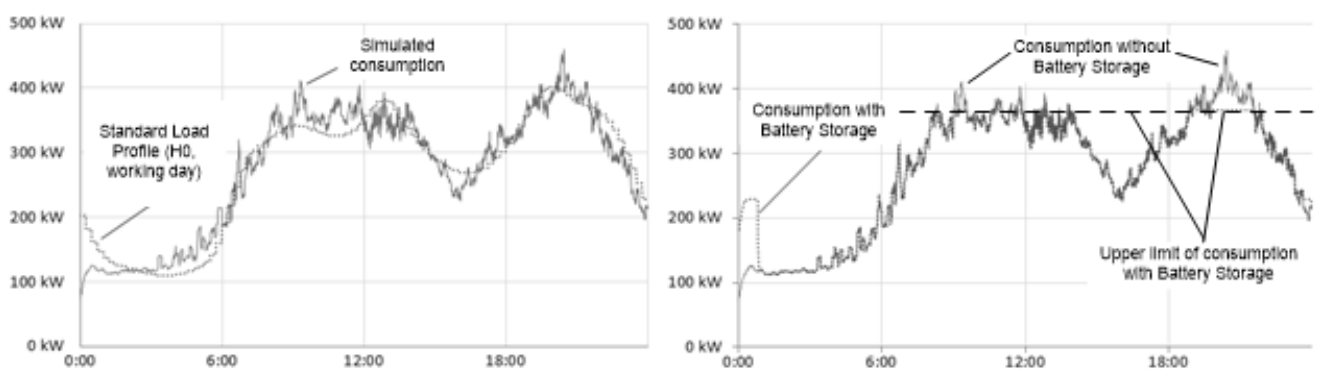

Figure 5. Comparison simulated consumption with standard load profile H0 (left), Consumption with Battery Storage (right)

The Scenario covers "avoidance of peak load" by using battery storages for a community of 1000 households. The results shown in Figure 5 (right) indicate that, for a $20 \%$ peak load reduction (from $45.9 \mathrm{~kW}$ to $36.7 \mathrm{~kW}$ ), already 23 battery storages are sufficient, that means only $2.3 \%$ of households would have to be equipped with one battery storage. Worth mentioning here is that not the storage capacity of the battery, but the maximum discharge power is the limiting factor.

The simulation shows that battery storages are very effective for peak load reduction. This result was also confirmed by the simulation of additional scenarios on the same topic "Avoiding peak load" (see [19]).

\section{Conclusions}

This publication presents a novel solution for optimisation of the aggregate energy usage. The concept of personalised services and above all the P2P networked energy-community forms a new and very powerful solution for the Smart Home and especially for the Smart Market. The presented solution may be successfully applied in the distribution grid, as confirmed by a preliminary set of simulation results. Apart from the optimisation service that it provides, the proposed solution also allows the user to individually create/compose control services for its Smart Home. At the same time it contributes to reduce the load of the distribution grid and thus reduce the costs. The solution takes into account the personal needs of the user and its defined optimisation goals at any time. Benefits are not only for the customers, but for all participants in the value chain. Approaches for an optimisation algorithm were presented to reduce the residual load in a region and can be extended in the future to consider both financial aspects as well as other optimisation objectives of grid view. Thus, a set of instruments for cost reduction in a 
household, environmental protection or the assurance of energy supply is available. Further studies, including the various application scenarios and their interdependencies as well as economic, security and privacy aspects are necessary.

\section{ACKNOWLEDGEMENTS}

The research project e-SCHEMA providing the basis for this publication is partially funded by the Federal Ministry of Education and Research (BMBF) of the Federal Republic of Germany under grant number 17018B11. The authors of this publication are in charge of its content.

\section{REFERENCES}

[1] Bundesnetzagentur, "Smart Grid und Smart Market - Eckpunktepapier der Bundesnetzagentur zu den Aspekten des sich verändernden Energieversorgungssystems", Bonn, 2011.

[2] N. Joo Teh, et al., "UK Smart Grid Capabilities Development Programme", Study Energy Generation \& Supply KTN, 2011.

[3] R. Gutzwiller, et al., "Lokales Lastmanagement - Stromverbrauch sinnvoll mit der Erzeugung koordinieren", Bulletin SEV/AES 22/23, pp. 9-13, 2008.

[4] A. Weidlich, et al., "Smart Houses Interacting with Smart Grids to achieve next-generation efficiency and sustainability", Deliverable D5.5 EU project Smart House/Smart Grid, 2011.

[5] Connected Living e.V., "Connected Living", www.connectedliving.org/ziele/sercho_technologie/, 2012.

[6] E. Hauser, et al., "Modellstadt Mannheim - Arbeitspaket 5: Untersuchung des technischen, energiewirtschaftlichen und regulatorischen Rahmens", BMWi-Projekt E-Energy, 2011.

[7] A. Kamper, "Dezentrales Lastmanagement zum Ausgleich kurzfristiger Abweichungen im Stromnetz", Dissertation Karlsruher Institut für Technologie, 2009.

[8] www.ecs.hs-osnabrueck.de/e-schema.html, 2011.

[9] M. Steinheimer, et al., "Energy communities in Smart Markets for optimisation of peer-to-peer interconnected Smart Homes", Proc. CSNDSP 2012, 2012.

[10] U. Trick, et al., "Herausforderungen an die Kommunikationstechnik im Smart Home/Grid", VDE/TTG Fachtagung Mobilkommunikation 2012, 2012.

[11] A. Lehmann, et al. "TeamCom: A Service Creation Platform for Next Generation Networks", Proc. of ICIW 2009, pp. 12-17, 2009.

[12] T. Eichelmann, et al., "Discussion on a framework and its service structures for generating JSLEE based value-added services", Proc. of ITA 2011, pp. 169-176, 2011.

[13] A. Lehmann, et al., "New possibilities for the provision of value-added services in SIP-based peer-to-peer networks", Proc. of SEIN 2008, pp. 167-176, 2008.

[14] Statistisches Bundesamt Deutschland, "Bauen und Wohnen - Mikrozensus - Zusatzerhebung 2010", Fachserie 5, Heft 1, 2012.

[15] D. Hölker, "e-SCHEMA - Simulation", Internal report Hochschule Osnabrück, 2012.

[16] E.ON Mitte AG, "Normierte Standardlastprofile H0, L0-L2, G0-G6 für Hessen", 2011.

[17] A. Alves, "OSGi in Depth", Manning, 2011.

[18] I. Stoica, et al., "Chord: A Scalable Peer-to-peer Lookup Service for Internet Applications", IEEE/ACM Transactions on Networking, Vol. 11, Issue 1, pp. 17-32, 2003.

[19] U. Trick, et al., " Smart Grid - Integration von Haushalten mittels Vernetzung und EnergieCommunity ", Proc. VDE-Kongress 2012, 2012. 
Published at VDE/IEC World Smart Grid Forum 2013, September 2013, Berlin, Germany, VDE

\title{
Decentralised optimisation solution for Smart Grids using Smart Market aspects and P2P internetworked Energy-Community
}

\author{
M. Steinheimer, U. Trick, P. Wacht, P. Ruhrig
}

Research Group for Telecommunication Networks, University of Applied Sciences Frankfurt/M., Frankfurt/M., Germany Email: steinheimer@e-technik.org

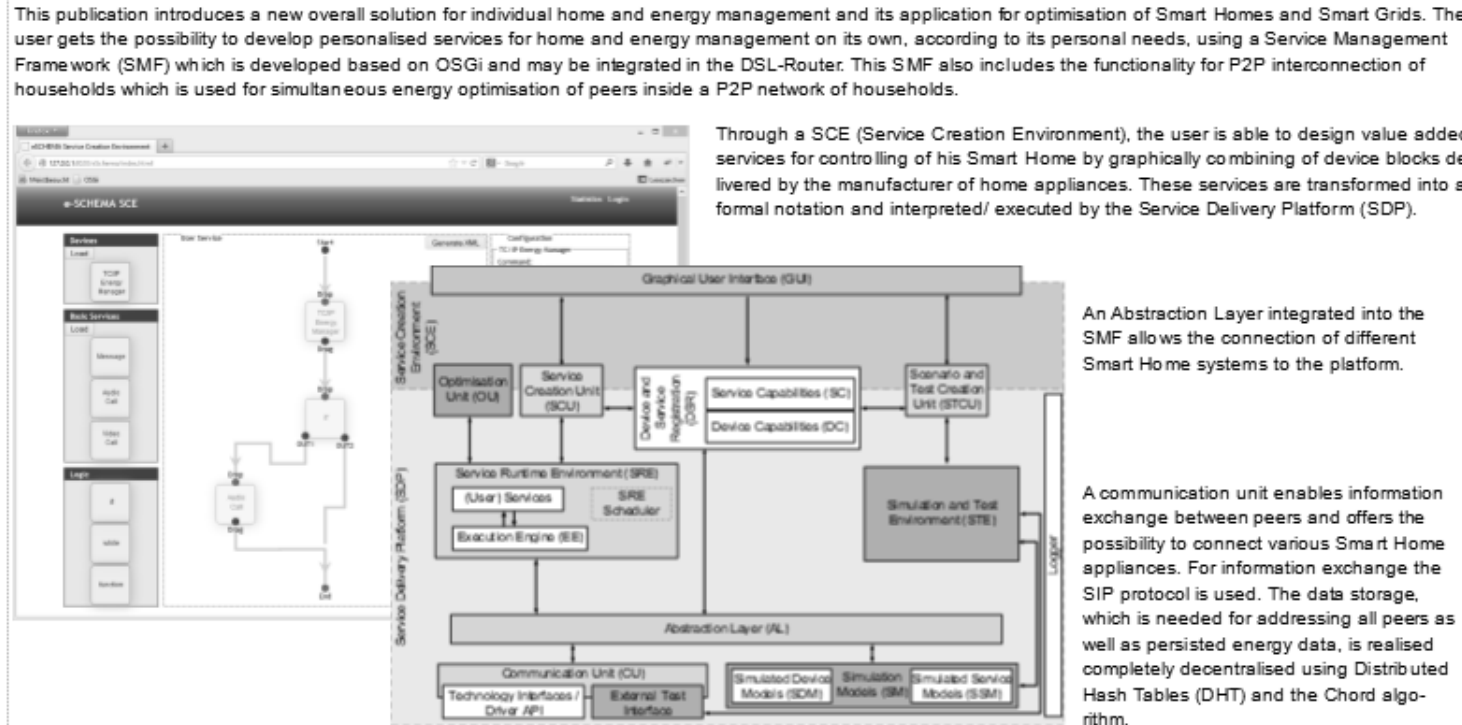

Based on constant exchange of information between peers in terms of energy consumption, production (e.g. photovoltaic power plant), storsge capscity and potentisl energy savings, all involved peers sutonomously optimise the energy consumption in their households.

The concept provides a mechs nism for convenient networking of households, energy suppliers, distribution network operators and others with the objec

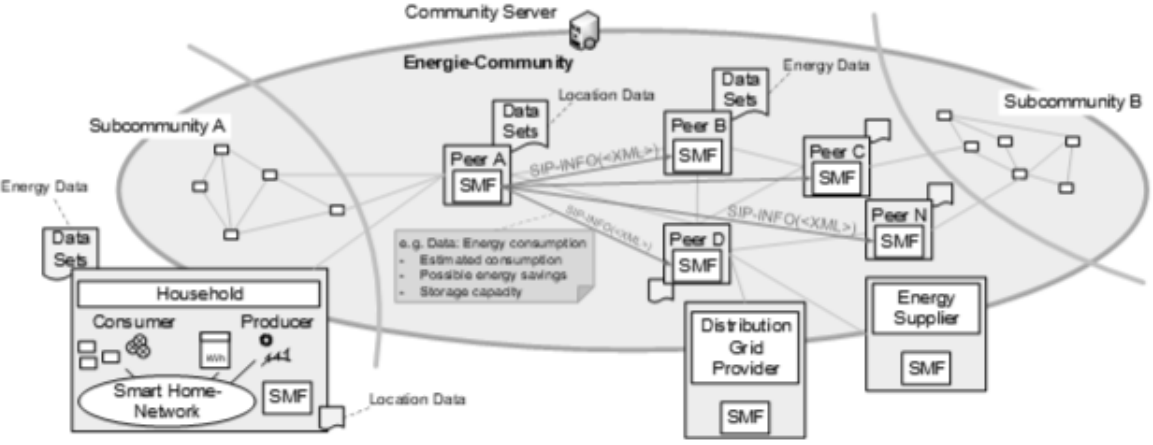
grid expansion, energy savings, $\mathrm{CO}_{2}$ reduction etc.

This network offers statistics aggregation and optimisatio through cooperation within s cluster of consumers.

An Energy-Community forms a new comfortable way to join the network, whereby the connection of households is done by joining the users to a social network for energy-peers.

Depending on the optimisstion gosl it is possible to schieve $a$ finsncial benefit for each participant as well as a relief of the energy grid through e.g. the reduction of the residusl losd.

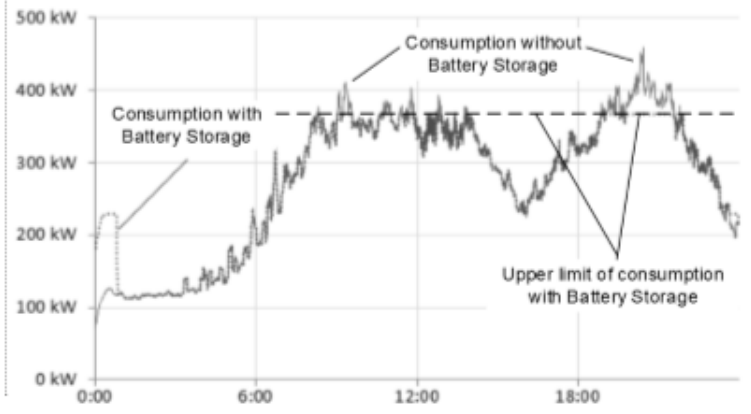

An exemplary simulated scenario covers "avoidance of peak logd" by using battery storages for a community of 1000 households. The results shown on the left side indicate that, for $\mathrm{a} 20 \%$ pesk bad reduction (from $45.9 \mathrm{~kW}$ to $36.7 \mathrm{~kW}$ ) glready 23 standard battery storgaes are sufficient, that means only $2.3 \%$ of households would have to be equipped with one battery storage.

References

[1] M. Seinhemer, a al., New approaches for energy optmistion in Smat Homes and Smat Grids by automated sevioe generationand user integration ", PROC. CERC 2012,2012 [2] U. Trick, et al, "Herausfordenungen an die Kommunkationstechnik im Smat Home/Grid". VDEITG Fachtagung Mobilkommunikation 2012, 2012

[3] M. Seinheimes, et al., "Energy communties in Smart Markets for optims ation of peer-to-pe interconnected Smart Homes. Proc. CSNDSP 2012, 2012

[4] U. Trok, et al, "Smatt Grid- Irtegrationvon Haushaten mittes Vernetzung und Energie Community", Proc. VDE-Kongress 2012, 2012

[5] M. Seinheimer, et al., "SIP-basiete P2P-Vernetzung in einer Energie-Community", VDEITT Fachtagung Mobikommunikation 2013, 2013 
Published at 2013 Collaborative European Research Conference (CERC), October 2013, Cork, Ireland

\section{Decentralised optimisation solution for provision of value added services and optimisation possibilities in Smart Grids

\author{
M.Steinheimer ${ }^{1,2}$, U.Trick ${ }^{1}$, P.Wacht ${ }^{1,2}$, P.Ruhrig ${ }^{1}$, W.Fuhrmann ${ }^{3}$, B.Ghita ${ }^{2}$
}

${ }^{1}$ Research Group for Telecommunication Networks, University of Applied Sciences Frankfurt/M., Frankfurt/M., Germany

${ }^{2}$ Centre for Security, Communications and Network Research, University of Plymouth, Plymouth, UK

${ }^{3}$ Department for Computer Science, University of Applied Sciences Darmstadt, Darmstadt, Germany Email: steinheimer@e-technik.org

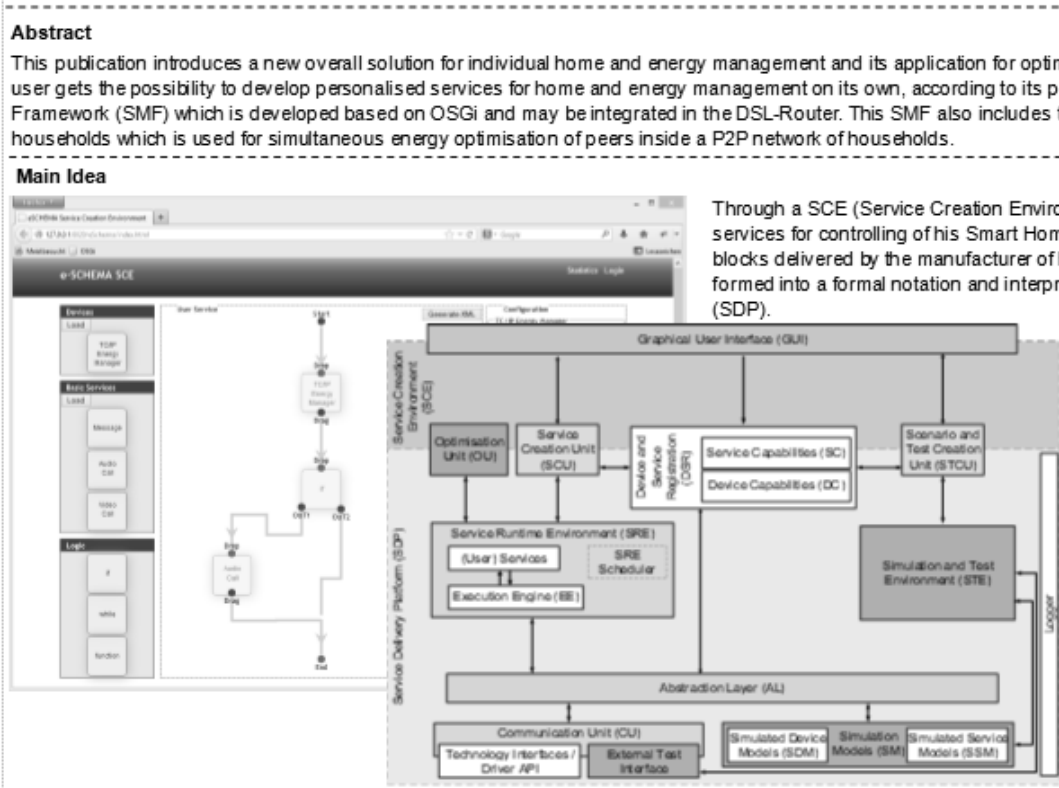

Based on exchange of information between peers in terms of energy consumption, storage capacity, and potential energy savings, all involved peers autonomously optimise the energy consumption in their households.

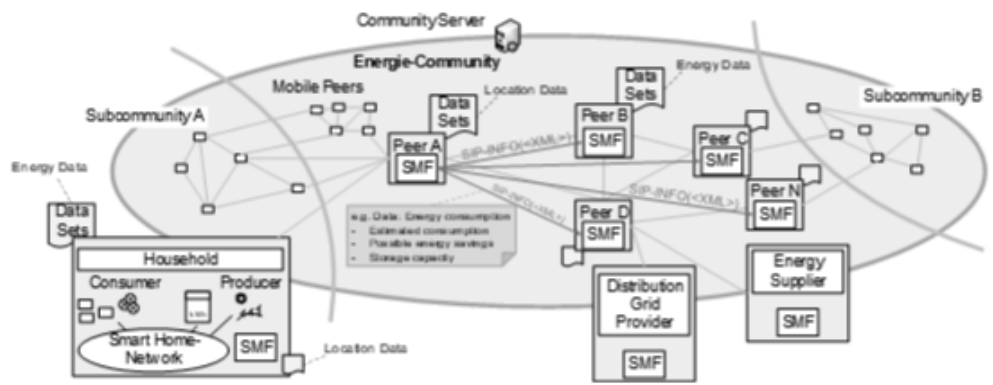

An Abstraction Layer integrated into the SMF allows the connection of different Smart Home systems to the platform using various interfaces (e.g. digitalStrom, KNX, ZigBee).

A communication unit enables information exchange between peers and offers the possibility to connect various Smart Home appliances. For information exchange the SIP protocol is used. The data storage needed for addressing all peers as well as persisted energy data is realised completely decentralised using Distributed Hash Tables (DHT) and the Chord algorithm.

The integration of mobile peers (via smartphone) allows e.g to provide value added service for the optimis value of the parking sitation formis tion of heparing siturion for electric vehides. Here, parking lots with charging option for electric vehicles are considered which are a scarce source because of the increasing number of electric vehicles. Through communication and exchange of information (capacity of battery and destination) between mobile peers an efficient discoven of available parkeficient discovery of allable parkpower consumption) is realised.

\section{Results and Conclusion}

The concept provides a mechanism for convenient networking of households, energy suppliers, distribution network operators and others with the objectives cost savings, avoidance of grid expansion, energy savings. $\mathrm{CO}_{2}$ reduction etc. This network offers stafistical aggregation and optimisation through cooperation within a cluster of consumers. An Energy-Community (community server available over internet and provided by any party) forms a new comfortable way to join the network, whereby the connection of households is done by joining the users to a social network for energy-peers. Depending on the optimisation goal it is possible to achieve a financial benefit for each participant, as well as a relief of the energy grid through e.g. the reduction of the residual load.

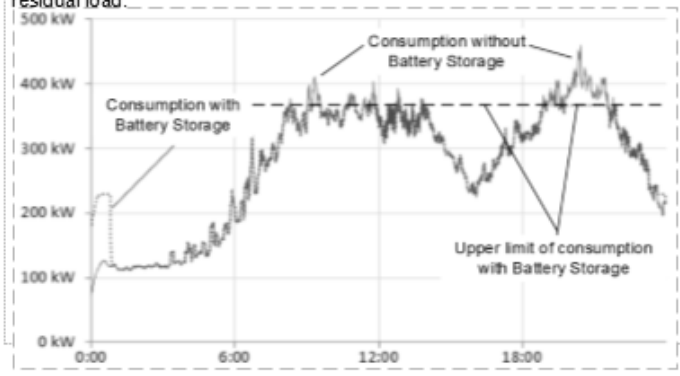

An exemplary Excel-simulated scenario covers "avoidance of peak load" by using battery storages for a community of 1000 hous eholds. The results shown on the left side indicate that, for a $20 \%$ peak load reduction (from $45.9 \mathrm{~kW}$ to $36.7 \mathrm{~kW}$ ), already 23 standard battery storages are sufficient, that means only $2.3 \%$ of hous eholds would have to be equipped with one battery storage. References

[1] M. Seinheimer, et al., New approaches for energy optmisation in Smat Homes and Smart Grids by automzted senvice generation and user irtegration". Proc. CERC 2012, 20 [2] U. Trick, et al, "Herausforderungen an die Kommunikationstechnik im Smart Home/Grid", 政

[3] M. Steinheimer, et al., Energy communties in Smart Markets for optimsation of peer-to-pee

[4] U. Trick, et al, "Smat Grid-Integration von Haushalten mittels Vernezzung und Energie [5] M. Steinheimer, et al., -SIR-basierte P2P-Vernetzung in einer EnergieCommunty". VDEITG 


\title{
P2P-based community concept for M2M Applications
}

\author{
M. Steinheimer, U. Trick \\ Research Group for Telecommunication Networks \\ University of Applied Sciences Frankfurt \\ Frankfurt, Germany \\ \{steinheimer, trick\}@e-technik.org
}

\author{
W. Fuhrmann \\ Department for Computer Science \\ University of Applied Sciences Darmstadt \\ Darmstadt, Germany \\ w.fuhrmann $@$ fbi.h-da.de
}

\author{
M. Steinheimer, B. Ghita \\ Centre for Security, Communications, and Network research \\ University of Plymouth \\ Plymouth, United Kingdom \\ \{michael.steinheimer, bogdan.ghita\}@plymouth.ac.uk
}

\begin{abstract}
This publication presents a novel concept to provide and use services in a Peer-to-Peer (P2P) connected MachineTo-Machine (M2M) community. Service compositions are presented that address several application fields like Ambient Assisted Living, traffic, energy, electro mobility, logistic or surveillance. The successfully implemented concept for optimisation of energy management in the local grid with P2P connected households became more generalised. Now it can be part of M2M application fields of $2^{\text {nd }}$ and $3^{\text {rd }}$ generation. The concept provides an overall solution for information exchange, extended by community mechanism, for convenient networking of peers to use and provide optimised services. Inside the community, the peers can operate as a single peer or operate as a cluster of peers to provide and use the services.
\end{abstract}

Keywords-Smart Grid; Smart Home; Energy Management Service Provisioning; Peer-to-Peer; Community; Machine-To Machine; Ambient Assisted Living; Logistic, Traffic Management

\section{INTRODUCTION}

In the electric power grids of the future, the trend will be to increase production of volatile renewable energy using wind power and photovoltaic systems. Based on this trend, the proportion of centralised energy generation will decrease and replaced by distributed generation and supply. This shift has already started within the non-monitored local energy grid. To still ensure stability and good quality in the power supply, the local grid has to be expanded and especially has to get smarter through information and communication technology. This development is commonly described by the term "Smart Grid" [6]. The published solutions to manage the Smart Grid outline several gaps or deficiencies, described in $[1 ; 4]$, and propose centralised or hybrid centralised/decentralised approaches for optimisation with no consideration of independent clustering of households. Because of these gaps a completely different solution, which follows the approach of "control through price signals" [7], is proposed in [1-5] to control and manage single Smart Homes as well as a cluster of Smart Homes. A concept of a P2P networked Energy-Community is presented that is novel solution for completely decentralised optimisation of the aggregated energy usage. This solution is introduced more detailed in chapter II and III. That concept offers the possibility to connect devices, service platforms, environment and people using IP-based communication networks and exchange information between them to make each of them more intelligent The possibility to create and provide services in a comfortable way can be defined for several application fields. This publication proposes a generalised concept for M2M applications, based on the solution for decentralised energy management with $\mathrm{P} 2 \mathrm{P}$ connected communities. The proposed concept is part of M2M application fields, that [8] classifies as $2^{\text {nd }}$ generation (M2M end user/ devices/ environment interaction) and $3^{\text {rd }}$ generation (Social M2M, integration of people and networking of people). In chapter IV and V the concept is proposed that uses the advantages of providing the infrastructure for different kind of services in different M2M application fields with same and cost efficient hardware and the independence of central service provider.

\section{CONCEPT OF P2P NETWORKED ENERGY-COMMUNITY}

In $[3 ; 4]$ a Service Management Framework (SMF), consisting of Service Delivery Platform (SDP) and Service Creation Environment (SCE), is proposed where the customer can develop optimisation services for energy management in an easy and convenient way using a web-based Graphical User Interface (GUI). The SMF provides the personalised valueadded services for the Smart Home, especially for energy management, and includes the integration of multimedia communications. In addition the framework provides a mechanism for convenient networking of households, energy suppliers, distribution network operators and others with the objectives cost savings, avoidance of grid expansion, energy savings, $\mathrm{CO} 2$ reduction, reduction of residual load etc.

Energy optimisation of single households does not have enough influence to the grid; therefore a simultaneous optimisation of households is required. Optimisation of multiple households requires the exchange of information between the households and in consequence the networking of the households to exchange information between each other. The approach, proposed in [1-5], is based on completely decentralised structure and forms a P2P network of households that exchange information for optimisation like e.g. current or forecasted consumption, information about existing energy generators and 
storages (batteries, electric vehicles), energy rates and/or network fee). The peers represent households, energy suppliers, and distribution grid provider.

To solve issues for networking, avoid legal restrictions, optimisation, missing mechanisms to form the P2P network or the fact that user aim different goals for optimisation (e.g. cost reduction, obtain only "green energy", most benefit for energy supply) the P2P networking approach was extended by community mechanism. The community-based approach forms a new comfortable way to join a network of peers, whereby the connection of peers is done by joining the users to a social network. Fig.1 shows the structure of the P2P connected households within an Energy-Community.

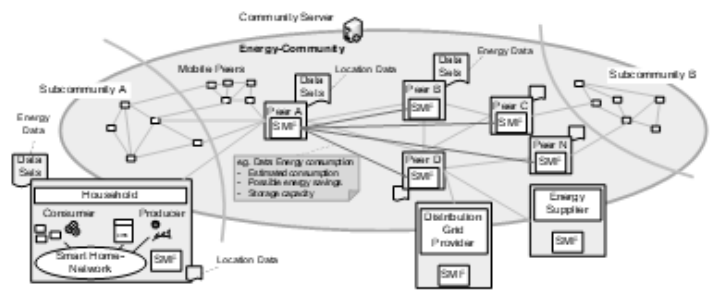

Fig. 1. P2P connected households within an Energy-Community

According to the principle of a social network, the community may grow independently and every participant may accede to his free will. Existing infrastructure can be used to provide different kind of services in different application field with same and cost efficient hardware. Using IP-based Information exchange guarantees the applicability by everyone that has access to the Internet. Therefore the realisation is possible with minimal costs. Inside that Energy-Community not only the single customer goals are considered, the goal is to reach a benefit for every participant in the community. In addition the participants can form sub-communities to address common shared interests (e.g. energy saving, cost reduction, obtain only "green energy").

The participants inside the Energy-Community have different roles (community operator/provider, energy supplier, household, distribution grid provider). The peer household can define boundary conditions for its personal optimisation (e.g. restrictions for Demand Side Management or definition of date/time when electric vehicles have to be fully charged). With consideration of these boundary conditions a special algorithm defines an optimised schedule for energy consumption and supply. The distribution grid provider and energy supplier can offer better rates for reaching a permitted load curve within a region (benefit for the distribution grid provider: load reduction in a region; benefit for the energy supplier: reach the forecast for energy supply).

The optimisation algorithm is implemented inside of every SMF and operates based on e.g. using battery storages that supply power to the grid or charge battery to reduce the load. The decision to supply energy is taken inside the local SMF, based on the information received from other peers. The data storage, required for the services, information exchange and optimisation is also completely decentralised using Distributed Hash Tables (DHT).

\section{REALISATION OF P2P NETWORKED ENERGY- COMMUNITY}

\section{A. Service platform for households}

The Service Management Framework (SMF) offers the possibility to the user to create services like e.g. start charging of an electric vehicle (EV) if the energy price is below a defined threshold (the process for service creation via the user interface is described in $[3 ; 4]$ ). Fig. 2 shows an overview of the relevant parts of the SMF.

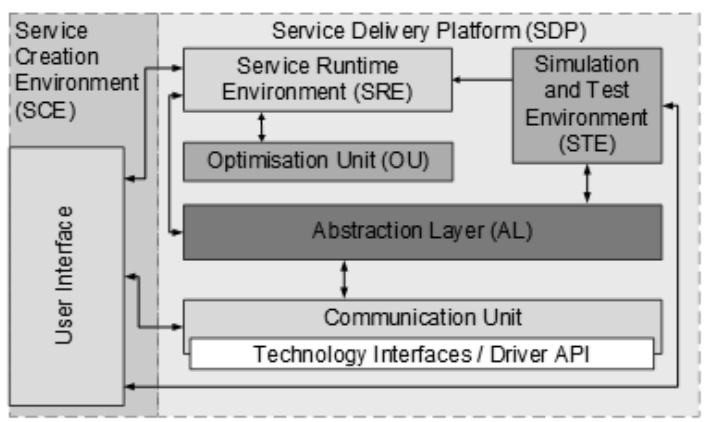

Fig. 2. Overview of relevant parts of SMF

The Service Runtime Environment (SRE) interprets the service description (formal service notation using State Chart XML, SCXML) and executes the actions described in it. For this, the SRE communicates with the Abstraction Layer (AL) and thus controls the actions in the connected devices. For abstraction of communication and connection of different bus systems for Smart Home controlling (e.g. KNX, EnOcean, MBus, ZigBee) the AL has been designed. This makes it possible to connect a variety of Smart Home and multimedia communication techniques, as the AL unifies communication between services, part services, Smart Home bus systems as well as elements for multimedia communications. To implement this integration of different communication and Smart Home systems, the architecture includes a Communication Unit (CU), which is connected directly to the $\mathrm{AL}$, allowing the device and technology specific control of devices. All connected devices are registered at the $\mathrm{AL}$ and are controlled within the services in an abstracted way. The Optimisation Unit (OU) implements the above described optimisation of services through reconfiguration of pre-defined services. This is done taking into account the constraints and optimisation objectives, which the user has defined via the user interface.

\section{B. Process for participation in Energy-Community}

To join the Energy-Community and the P2P network (illustrated in Fig. 3), the user registers via the Web Browser at the Energy-Community and joins his sub-community. After registration the user gets his access data which he configures at his SMF. The SMF requests a list of all participants in the commu- 
nity/sub-community and connects to the $\mathrm{P} 2 \mathrm{P}$ network (via the Bootstrap Peers)

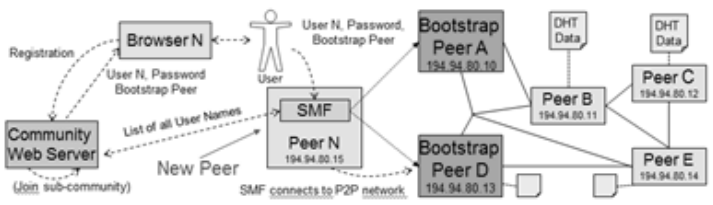

Fig. 3. Joining the P2P networked Energy-Community

\section{Information exchange in the $P 2 P$ network}

The information exchange between the peers is based on the Session Initiation Protocol (SIP) [12]. The SIP protocol is used because of its standard in multimedia communication, which was defined as a requirement for the platform, to offer total communication possibility. Also SIP has standardised functionality for transport of XML-data and mechanisms for reliable communication, realised in Application Layer that of fers the functionality to act in heterogeneous networks. In addition, Interactive Connectivity Establishment (ICE) [14] can be used to avoid Network Address and Port Translation (NAPT) or Firewall issues.

All peers observe the state of all other peers in the community. The observation of the state with the SIP protocol is done via a process that is specified in [11] as "SIP-Specific Event Notification". Every peer offers a service to provide his energy information. All peers send a SUBSCRIBE Message to all participants in the sub-community. This indicates to the receiving peers, that the requesting peer (service consumer) is interested in its energy information. At defined intervals (e.g. 15 minutes) the peers send its own energy information to all other peers in the sub-community (peers that subscribed for the event). Thi occurs via the SIP message NOTIFY. For description of the energy data, the XML format is used. Another option for SIPbased information exchange between the peers is to use SIP MESSAGE (specified in [13]) to push messages directly to peers.

As an alternative to SIP-based information exchange, the calculation methods of Distributed Hash Tables (DHT, described in section III.D) can be used to collect the energy data from all peers. Especially to collect data (e.g. summation like determination of residual load) DHT can be used. Every peer can store its energy information directly inside the DHT and every peer can request the total energy data of the subcommunity.

The option for information exchange (SIP or DHT) depends on whether it is a data- or a message-centred service SIP-based information exchange is advantageous if direct communication between the peers is required. Also if the peers have to exchange data that should not be send to the whole subcommunity but only to a certain peer or group of peers. To select the type of data exchange, the use case (message- or data-centred service) has to be determined and the resulting amount of data as well as the number of required messages has to be estimated. As optimisation SIP Compact Headers [12] or SIP over Websocket protocol [15] can be used. SIP Compact
Headers reduces the data volume. SIP over Websocket protocol can also avoid NAPT or Firewall issues.

\section{Distributed data storage in the $P 2 P$ network}

To avoid central authorities, achieve scalability and redundancy, the necessary data management is distributed based on DHT (Distributed Hash Table) and the Chord algorithm [9]. The DHT stores e.g. data of a location service to provide the address information of peers. The SMF of sending peer gets a list of all participants (usernames) from the Community Web Server (refreshed periodically). The SMF detects the contact address (temporal SIP URI) of the other peers via the Location Service, implemented in the P2P network. Fig. 4 shows a simple architecture of the chord topology.

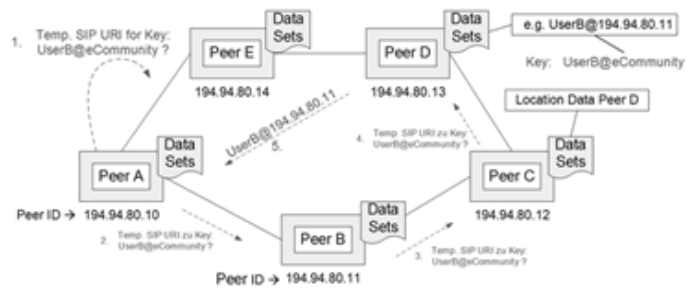

Fig. 4. Chord-based Location Service

If e.g. peer A requests for the temporary SIP URI of peer B then the SMF requests the corresponding data to the Chord topology. The request is forwarded from one peer to the next until the peer is detected that stores the dataset. This peer responses the dataset directly to the requesting peer.

\section{E. Optimisation of P2P networked households}

The households connected by an Energy-Community or by sub-communities, as introduced in section II, can work together and implement application scenarios, such as avoiding peak loads, avoiding regulation energy, optimisation of the energy costs, incentives to save energy etc. (see [2]). They use the more flexible options that come up through the use of distributed storages, generators and/or time shifted activation of consumers (demand-side integration (DSI)). Depending on the use case, this may result in benefits to the distribution system operator, the balancing group managers, energy suppliers, consumers or the environment etc.

Fig. 5 presents a section of the algorithm that is used to implement a scenario for peak load reduction in a region.

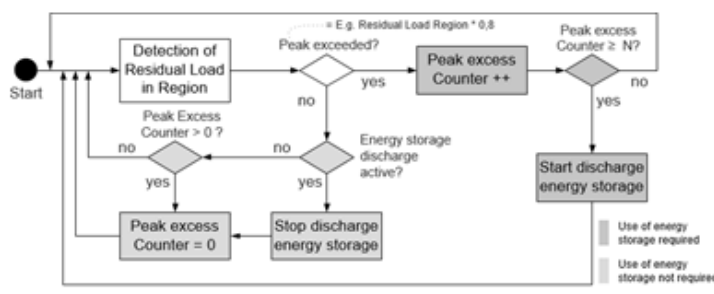

Fig. 5. Algorithm for peak load reduction 
Firstly, each peer determines the residual load in the region, which represents the difference between the energy demand and the supply by renewable energy. If the calculated residual load for the region exceeds the defined limit, energy storages present in this region will discharge and supply additional energy to the grid. The demand will be met locally through the local supply, preventing the supply of additional power from upper voltage level. Recharging the energy storage takes place in line with defined lower limits of the residual load. Fig. 6 shows the part of the algorithm that is responsible for the calculation of the residual load in a region.

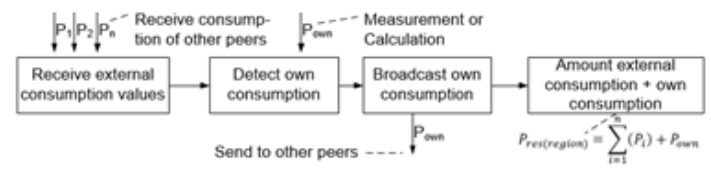

Fig. 6. Part of algorithm for calculation of residual load

Each peer receives the energy requirements of all other peers. The required data is transmitted via SIP messaging, based on the networked households described in section II. After the energy demand of all households was received, the individual energy requirement is determined and distributed to other peers. To determine the residual load, each peer adds its energy demand to the sum of the received energy demands of all peers. This determination of the total energy demand is the basis for the decision to discharge existing energy storage as described above.

To demonstrate the effectiveness of the outlined energy community below the result of a concrete, simulated application scenario of the proposed algorithm is presented. For more detailed description of the test bed and the validation of the simulation see [4]. The scenario covers "avoidance of peak load" by using battery storages for a community of 1000 households. The results shown in Fig. 7 indicate that, for a $20 \%$ peak load reduction (from $45.9 \mathrm{~kW}$ to $36.7 \mathrm{~kW}$ ), already 23 battery storages are sufficient, that means only $2.3 \%$ of households would have to be equipped with a battery storage.

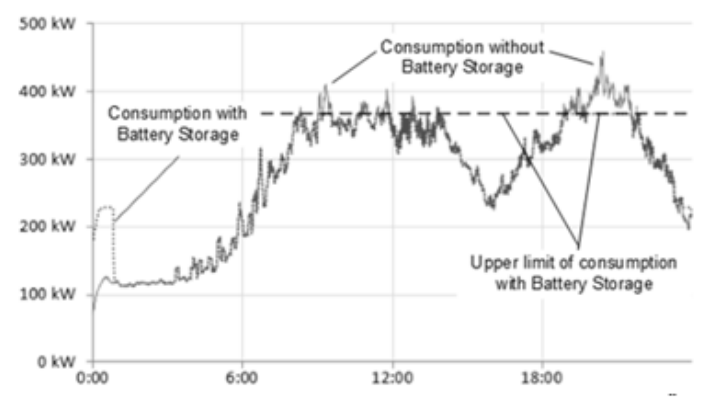

Fig. 7. Results peak load reduction using Battery Storages

Worth mentioning here is that not the storage capacity of the battery, but the maximum discharge power is the limiting factor. The simulation shows that battery storages are very effective for peak load reduction. This result was also confirmed by the simulation of additional scenarios on the same topic "Avoiding peak load" (see $[2 ; 4]$ ).

\section{P2P NETWORKED COMMUNITY INSIDE A M2M ENVIRONMENT}

The concept of P2P networked households within an Energy-Community presents an overall and very powerful solution for energy management and optimisation of households or cluster of households. The advantages of that solution are not limited to the energy sector, but can also be applied in various sectors of M2M applications.

The P2P networked community concept offers advantages in all application fields where decentralised challenges exist and central solutions for service provisioning or optimisation are not realisable (e.g. because of costs or infrastructural precondition). The joint optimisation is realisable, based on information exchange between the peers. On the one side information are exchanged from machine to machine (SMF to SMF, device to device, device to SMF, SMF to device) and from machine to process (optimisation algorithm and other services), one the other side information become available for customers (machine to human and human to human information exchange). The participants have the possibility to provide services and to use services according to their personal interests. The formation of sub-communities allows multiple peers to jointly offer and use services to achieve common interests. Thus, a variety of targets can be achieved in various sectors.

The challenges to M2M platforms or IoT (Internet of Things) mentioned in [10] (scalability, robustness, openness, flexibility etc.) are addressed/solved by the SMF and the P2P networked community concept. The SMF form a platform for automated information exchange (using communication networks) between devices, humans and interaction/networking of people. The SMF is the basis for exchange of information with limited human interaction. The integration of different house automation systems and communication technologies into SMF brings the possibility to build many services based on M2M. These services can be provided by one or many peers and used by one or many peers (service user and provider are linked in a $\mathrm{n}-\mathrm{n}$ constellation).

Using a mobile implementation of the SMF, the mobile phone (with e.g. Android as operating system) is the runtime environment and at the same time the interface to the customer. The implementation on mobile platforms offers possibilities for many applications and services, independent of the location, using location data and environment information.

The more generalised consideration and implementation of the P2P-based community approach offers many new application fields for community-based services and optimisations like energy, traffic, logistic, Ambient Assisted Living (AAL). A non-exhaustive overview is presented in Chapter V.

\section{P2P NETWORKED COMMUNITY SERVICES IN M2M ENVIRONMENTS}

The platform, described above, forms the main part in provision and use of $\mathrm{M} 2 \mathrm{M}$ services by connecting service providers and service users, using an overall solution for information 
exchange. The focus is the $\mathrm{P} 2 \mathrm{P}$ connection of all participants and the community mechanism. Based on that concept, different kind of services are realisable in different application fields. Fig. 8 describes the generalised structure for service provision and usage in $\mathrm{P} 2 \mathrm{P}$ networked communities.

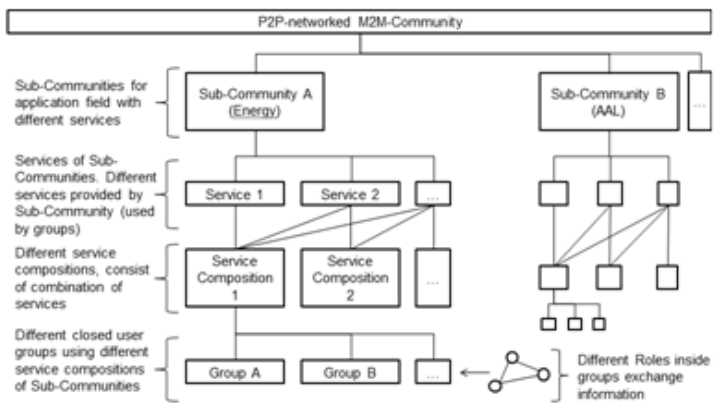

Fig. 8. Generalised structure of $\mathrm{P} 2 \mathrm{P}$ networked $\mathrm{M} 2 \mathrm{M}$ communitiy services

The concept identifies several elements in the structure The P2P networked M2M-Community itself, sub-communities for different application fields, different services provided by the sub-communities, different service compositions consisting of several services and different closed user groups that use the service compositions. Within the groups different roles exist that are central parts for the service realisation. This structure was used to apply the concept for several M2M applications like energy management, traffic management, electro mobility, logistic, Ambient Assisted Living. According to that structure, below several sub-communities are defined (with their community services, service compositions, groups and roles) that are already partly implemented.

\section{A. Sub-community for traffic management}

The sub-community for traffic management can provide services for local/urban public transport optimisation as well as private transport organisation.

Community service public transport: The public transport provider and transport customer act as peers. Also private transport providers are part of this community. They exchange information relating to current timetable, transport offers transport requests, rates, locations of the peers and route information, using the mobile SMF. As service composition dynamic and personalised time tables can be created, according to transport offers of the transport providers and the requests (subscriptions) of the customer. The integration of private transport provider enlarges the flexibility by flexible combination of public and private transport offers.

Community service share taxi: The private peers (customer) can subscribe for share taxi whereby the taxi control centre, the taxies and the customer act as peers. They exchange information about timetable, taxi requests, taxi positions, rates, special offers, stops and route information. As service composition the services are used to create a personalised timetable and create detailed navigation information to the relevant stops by matching requests and offers of all participating peers.
Community service car sharing: The car sharing provider, the car and the car-user act as peers. They exchange information about car availability, car positions, subscriptions for cars and route information, using mobile SMF. As service composition the personalised, timed and regional optimised allocation of vehicles can be displayed to the car sharing user, according to their subscriptions.

Community service lift-arranging agency: The provider of a trip as well as the user of a trip act as peers. The user of that service can be several groups with attendance of relatives and friends. They exchange information for requests for drives (user), offer for drives (provider), location information of peers, and route information. As service composition personalised, timed and regional optimised trips can be organised, according to requests and offers. In addition the communication between peers can be done directly via IM and audio call.

\section{B. Sub-community for electro mobility}

Community service electric vehicle (EV) charging: The EV and the provider of a charging station for EVs exchange information about location, route information, charge state of the EV and requests for charging. The EVs subscribe at the charging station provider. The charging station provider notifies the EV if charging is possible. If not, the charging station provider sends alternative locations of other charging stations to the EV. In extension an algorithm optimises the allocation of charging stations which generates optimal route information for all EVs that are currently located in an area. The distribution grid provider and the energy supplier can also be part of the community, whereby they publish information about current energy rates or residual load in a region. Using this information the optimisation of EV charging in a region (by e.g. optimal scheduling) is possible (cost and load optimisation).

\section{Sub-community for logistic}

Community service delivery tracking: The complex structure of supply chain is an issue for delivery tracking. During the delivery of goods no networking between logistic providers exists. The solution for information exchange inside the P2P networked community offers the possibility to create an overlay network which can detect and store the current position and additional information about the delivered goods. The tracking station subscribes for the state information of the goods and the good itself notifies the tracking station about its current location and state.

Community service parcel service: The SMF of parcel service provider and the customer exchange information about presence at delivery location. The provider subscribes for packet delivery and the customer notifies if at home or not. Advantage of that service is that unnecessary drives are avoided (in consequence preserve of environment and avoidance of costs and time).

Community service for relocation/cold transport companies: The relocation/cold transport companies communicate with other relocation/cold companies to inform about location and additional available transport capacity. The other company can use this capacity. This reduces drives on same route (saves money, time, and environment). The relocation/cold transport 
company can also offer free transport capacity (location-based) to other customers. The customer can subscribe for short-term transport options. The driver of the truck notifies customer for acceptance.

\section{Ambient Assisted Living (AAL) and surveillance community}

Community service Ambient Assisted Living: The parented person (e.g. senior citizen), the residential monitoring platform (as part of Smart Home), relatives, nursing service, emergency service, friends and other service providers act as peers. They exchange information according to the current state of the parented person (location information, medical status etc.). As service composition the residential monitoring platform can coordinate the order to inform the assistants of the parented persons (in case of emergency e.g. the senior citizen fall down) regarding to their location information. The SMF of the parented person therefor subscribes at the mobile SMF of the assistants. The assistants can notify and confirm the request, can directly communicate with the parented person (e.g. for call backs) or can contact the other assistants using the communication services of the SMF (IM, audio/video call).

Community services for senior citizens: Senior citizens, relatives, friends and other service provider (e.g. shopping service) act as peers. They exchange information according to the current needs of the senior citizen (availability of food, wishes for walks, support for public authorities, talks etc.) and current service offers (e.g. shopping, read outs, walks etc.). The senior citizen subscribes for service and the peer providing the service notifies if the service can be performed currently or reschedules the time/date.

Community service residential building security/keeping: households, relatives, friends, neighbours, police, fire fighters, security service, facility manager act as peers. They send their current position (location information) continuously to the observed peer and will react on exceptional events in the household (e.g. temperature, water level, door/window contacts etc.). They provide the service to react in case of an alarm. As service composition, while alarm situation, the platform (home surveillance system, connected to SMF) generates an optimised subscription schedule with respect to the current location information of the observing peers (or triggers pre-configured alarm chains, consisting of own mobile SMF and other peers in the same community). The observing peers have subscribed for the behaviour of all other observing peers, thus they stay informed how the other peers will react. This offers the possibility to implement a decentralised monitoring and alarm system. The communication solution of the SMF (sending IMs, establish audio/video calls) can also be used to inform the closed user group (e.g. neighbourhood) about irregular activities during absence of house owners.

\section{CONCLUSION}

This publication presents a novel solution for service provisioning and service usage in different application fields. The presented platform forms the main part of the solution in provision and use of M2M services by connecting service providers and service users, using an overall solution for information exchange. The concept of a P2P-connected Energy-Community became more generalised and the advantages of the solution are no more limited to the energy sector, but can also be applied in various sectors of M2M. The P2P networked community concept offers advantages in all application fields where decentralised challenges exist and central solutions for service provisioning or optimisation are not realisable. The novel concept provides a great possibility to form Smart Villages and the community-based approach is a new comfortable way to join a network of numerous participants, whereby the connection of peers is done by joining the users to a social network. Existing infrastructure can be used to provide different kind of services in different application field with same and cost efficient hardware (e.g. mobile Smartphone). The joint optimisation is based on information exchange between the peers which creates maximal benefit for all participants.

\section{REFERENCES}

[1] M. Steinheimer, U. Trick, and P. Ruhrig, "Energy communities in Smart Markets for optimisation of peer-to-peer interconnected Smart Homes", Proc. CSNDSP 2012, 2012.

[2] U. Trick, M. Steinheimer, P. Ruhrig, R. Tōnjes, and D. Hölker, "Smart Grid - Integration von Haushalten mittels Vernetzung und EnergieCommunity" ${ }^{\prime \prime}$ Proc. VDE-Kongress 2012, 2012.

[3] M. Steinheimer, U. Trick, P. Ruhrig, P. Wacht, R. Tönjes, M. Fischer, and D. Hölker, "SIP-basierte P2P-Vermetzung in einer EnergieCommunity", Proc. VDE/ITG Fachtagung Mobilkommunikation 2013, 2013.

[4] M. Steinheimer, U. Trick, P. Ruhrig, W. Fuhrmann, and B. Ghita, "Load reduction in distribution networks through P2P networked energycommunity", Proc. ITA 13, 2013

[5] M. Steinheimer, U. Trick, P. Wacht, and P. Ruhrig, "Decentralised optimisation solution for Smart Grids using Smart Market aspects and P2P intemetworked Energy-Community", Proc. VDE World Smart Grid Forum 2013, 2013.

[6] N. Joo Teh, G. Goujon, G. Bortuzzo, and A. Rhodes, "UK Smart Grid Capabilities Development Programme", Study Energy Generation \& Supply KTN, 2011.

[7] A. Weidlich et al., "Smart Houses Interacting with Smart Grids to achieve next-generation efficiency and sustainability", Deliverable D 5.5 EU project Smart House/Smart Grid, 2011

[8] P. Decker: "Facebook of Things - The challenge of Endusers within M2M Systems", Symphony Teleca Corporation, M2M Summit 2012, 2012.

[9] I. Stoica et al., "Chord: A Scalable Peer-to-peer Lookup Service for Intermet Applications", IEEE/ACM Transactions on Networking, Vol. 11,2003 , pp. 17-32.

[10] M. Castro, A. J. Jara, and A. F. Skarmeta, "An analysis of M2M platforms: challenges and opportunities for the Intemet of Things", Proc. 6. Int. Conf. on Innovative Mobile and Intemet services in Ubiquitous Computing, 2012 .

[11] A. B. Roach, "Session Initiation Protocol (SIP)-Specific Event Notification", IETF RFC 3265, June 2002.

[12] J. Rosenberg et al., "SIP: Session Initiation Protocol", IETF RFC 3261, June 2002.

[13] B. Campbell et al., "Session Initiation Protocol SIP Extension for Instant Messaging", IETF RFC 3428, December 2002.

[14] J. Rosenberg, "Interactive Connectivity Establishment (ICE): A Protocol for Network Address Translator (NAT) Traversal for Offer/Answer Protocols", IETF RFC 5245, April 2010.

[15] I. Fette, Google Inc., A. Melnikov, and Isode Ltd., "The WebSocket [1] 


\title{
$\mathrm{P} 2 \mathrm{P}$ based service provisioning in M2M networks
}

\author{
Michael Steinheimer, Ulrich Trick \\ Research Group for Telecommunication Networks \\ Frankfurt University of Applied Sciences \\ Frankfurt/M., Germany \\ steinheimer@e-technik.org
}

\author{
Michael Steinheimer, Bogdan Ghita \\ Centre for Security, Communications and Network \\ Research \\ University of Plymouth \\ Plymouth, UK
}

\author{
Woldemar Fuhrmann \\ Department for Computer Science \\ University of Applied Sciences Darmstadt \\ Darmstadt, Germany
}

\begin{abstract}
This publication presents a novel concept to provide and use services in a Peer-to-Peer (P2P) connected Machine-To-Machine (M2M) network. Based on the successful implementation in Smart Grid application field, the concept became more generalised and now it is part of M2M application fields of 2nd and 3rd generation. The concept provides an overall solution for information exchange, extended by community mechanism, for convenient networking of peers to use and provide optimised services. Inside an M2M community, the peers can operate as a single peer or operate as a cluster of peers to provide and use the services.
\end{abstract}

Keywords-Service Provisioning; Peer-to-Peer; Machine-toMachine

\section{INTRODUCTION}

The existing concepts for provisioning services in IT (Information Technology) infrastructures are based on a centralised approach. ITIL (IT Infrastructure Library), which is "the most widely adopted framework for IT service management" [1] specifies that a service provider is "An Organisation supplying Services to one or more Internal Customers or External Customers." [2]

\section{A. Conventional approach for service provisioning}

Service providers are central instances which offer a service at a central location (see Fig. 1). The service consumers are usually end-users. The service provider has to provide more or less resources for service offering to cover the demand for the service. Services are provided in this type of service provisioning according to a top-down approach.

If multiple instances are needed to provide a service, the same approach is followed. Several key service providers offer individual services, which are in turn combined to form a complex service that is used by customers. For this purpose, a central service management (for the complex service) is again required. "Service Management is a set of specialized organizational capabilities for providing value to customers in the form of services. "[2] Thus, it is again a central approach (top-down). Also, there is usually a $1 \mathrm{n}$ relationship of service provider to service user. The service provider may also offer $n$

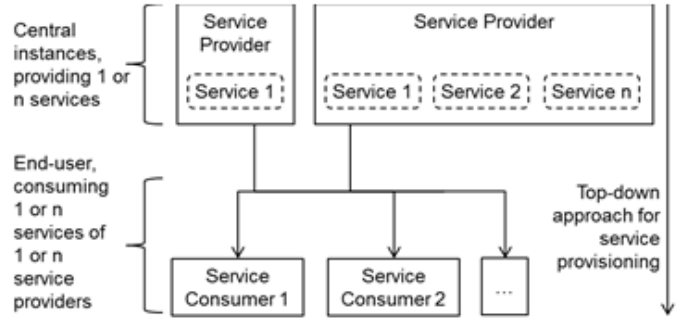

Fig. 1. Conventional approach for service provisioning

services, then it is an n:n relationship from the service to service users, but still a 1 in relationship of service provider to service user. If end-users consume different services from different service providers an $n: n$ relationship from service providers to service users exists (still following the top-down approach). In general, the relationship of service provider to service user can be identified as a vertical service relationship (in one direction, i.e. from provider to user).

\section{B. Service Provisioning by end-users}

The aim of the concept described in this publication is to enable end-users (previous service consumer) to take the role of a service provider. The end-user should be able to provide services as well as consume services. The approach aims that consumers are able to offer services for other end-users and higher authorities (e.g. companies). These services should be aggregated and can be used by central authorities (bottom-up) and other customers (horizontal service usage). Fig. 2 shows the concept for horizontal service provisioning and Fig. 3 shows the concept for bottom-up service provisioning.

Until now, service provisioning follows the centralised approach (central service provider which provides services to the end-user). In the described concept services can be provided by the end-user and can be used by central institutions or other end-users. Thus the resulting relationship from service provider to service consumer can be described as bottom-up service relationship (end-user jointly offers a service that can 


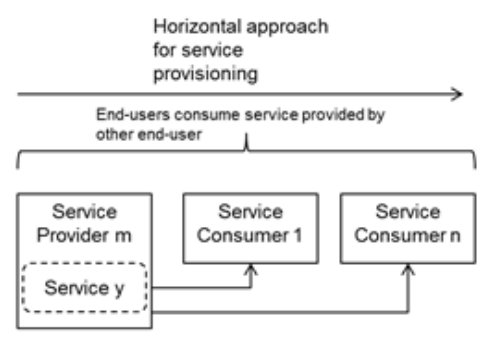

Fig. 2. Horizontal approach for service provisioning

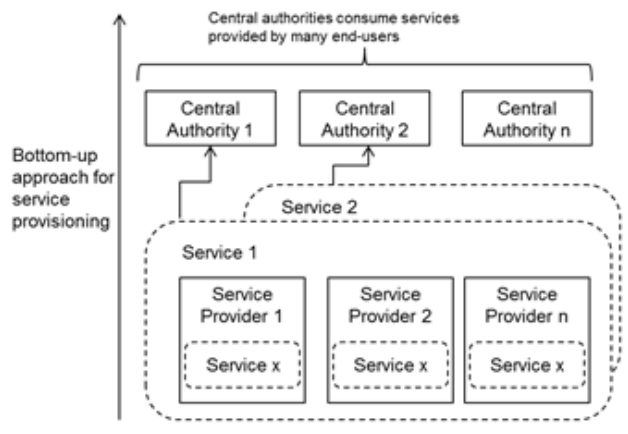

Fig. 3. Bottom-up approach for service provisioning

be used by central authorities) or as a horizontal service relationship (end-user offer services to other end-users).

\section{Challenges and requirements of service provisioning by end-user}

Service provisioning by end-user results in new challenges. The infrastructure that is used has to offer a possibility that services can be provided in a simple and comfortable way. Services need to be generated easily by the end-user. A service description has to be available to identify and use services according to the service functionality. The services need to be described in a unique way to offer the possibility for unified service requests. There shall be a mechanism describing how the services can be used. There must be a possibility to communicate between service providers and service user (to arrange the service usage and the joint service provisioning). It needs to be defined who manages the services. For joint service provisioning a network of the end-users (that provide the service) is required. Service provisioning, especially joint service provisioning (requires information exchange between the end-users) may affect the network (be sending many and large messages). Special challenge for this type of service provisioning is that the service creation/ provision can be offered with the available resources at the service provider (end-user) side as well as that the services can be published and used easily. A possibility to provide services without great technical effort is the use of existing resources. Both, for communication between service providers and service consumer as well as for the service execution (especially for technical services, e.g. energy management or surveillance services), the existing hardware at the end-user side should be used. This requirement is also in non-technical services in focus. Social services (non-technological, driven by human interests, e.g. assistance services for senior citizens) also require certain resources to be able to provide the service (e.g. available time to perform the service or existing skills to solve the service tasks). This resource is not tied to technical prerequisites. As the availability and the type of resources for a generalisation of the service provisioning are difficult to look at, the resources should generally be considered and included as a "resource" to abstract the consideration of the prerequisites for service provisioning by end-users.

For service provisioning by end-users several preconditions can be identified. Services have to be described (categorisation of services, unified description format, service description has to be published; service description has to be understood by service consumers). Services have to be combinable. No central instance has to be integrated whereof service delivery depends on. Facts against a central instance inside the infrastructure for service provisioning are e.g. large effort for management and maintenance, mostly high performance hardware required (costly), reduced reliability etc. No central instances means e.g. avoidance of central service repositories, as utilisation is only possible if this service repository is available. Another difficulty would be to define who should operate the repository. There should be an instance present which ensures operation, free of charge and permanently. This is hardly possible with a centralised approach. Decentralised, however, the end-users are responsible for hosting the service repositories to increase availability, redundancy and allow permanent offering guarantees. Communication options for service providers and service consumer must be available. Communication is an essential part for organisation of service usage or organisation of joint service provisioning. Especially for social services often personal communication between service provider and service consumer is required to arrange an appointment (via Email, IM or call). Multimedia communication is required to build up services such as surveillance services (e.g. monitoring buildings via video streams etc.). Constraints for the services (e.g. temporal and local availability) have to be defined. Existing hardware has to be used to fulfil minimum hardware requirements (e.g. installation of service platform in Integrated Access Devices).

The concept that is outlined in this paper offers the possibility to connect devices, service platforms, environment and people using IP (Internet Protocol)-based communication networks and exchange information between them to make each of them more intelligent. For decentralised networking of service provider and service consumer P2P mechanisms will be used. The possibility to create and provide services in a comfortable way can be defined for several application fields. The concept that is presented proposes a generalised approach for M2M applications, based on the solution for decentralised energy management with $\mathrm{P} 2 \mathrm{P}$ connected communities. The concept is part of M2M application fields, that [3] classifies as 2nd generation (M2M end user/ devices/ environment interaction) and 3rd generation (Social M2M, integration of people and networking of people). This paper proposes the concept that uses the advantages of providing the infrastructure 
for different kind of services in different M2M application fields with same and cost efficient hardware, software, elementary services (e.g. communication service) and the independence of central service provider.

The goal of the presented concept is to address the issues in a way that enables service providers to publish new services and also to use these services. Service provider needn't be a single instance, but services can also be provided by an aggregation of single service providers. The goal of the presented concept is to optimise the provisioning and usage of distributed services for several application fields, especially M2M application field. The objective is a framework that provides autonomous service provisioning and usage based on distributed atomic services using a peer-to-peer infrastructure and community mechanisms.

\section{FRAMEWORK FOR DECENTRALISED SERVICE PROVISIONING IN SMART GRIDS AND SMART HOMES}

Previous publications, e.g. [13], [14] and [15] describes that existing research and development activities for optimisation of Smart Homes and Smart Grids do not consider service provisioning, networking of instances and decentralised optimisation via information exchange. Therefore, a continuous solution is presented in [13], [14] and [15], oriented at the personal needs of users, to offer cost-efficient energy management, according to user personal needs, and integration within their house automation as well as combined with multimedia communication. Also the interaction between households is described to exchange information regarding the local energy production and consumption. This information is used for joint, independent and decentralised energy optimisation. The principles and architectures of the proposed framework for provisioning of services and optimisation possibilities in Smart Grids are presented in [13] and [14]. The Concept is mainly based on a Service Management Framework (SMF), consisting of a Service Delivery Platform (SDP) and a Service Creation Environment (SCE), see [15], which is installed in the local households. The SCE brings the functionality to design and configure value-added services graphically, according to the personal needs of the users, to monitor and control actuators, sensors and devices in user households. The SDP is used to provide a solution for service provisioning, service controlling and service management and serves as a runtime environment for the generated services. SCE and SDP in combination allow easy service design by the customer, as well as local controlling/ management of energy producers and devices (energy consumer) in Smart Homes. With that solution the optimisation of energy consumption by optimal load distribution is possible and can reduce the load in users' households (scheduling, eliminating temporal peak loads by using batteries for power supply). The presented concepts include the clustering of households for more effective optimisation because of independent optimisation of individual households does not automatically lead to optimisation of the local or distribution grid. The concept is totally based on decentralised approaches to avoid central instances in the infrastructure. In order to provide the aggregation of households, the SMFs of individual households are connected using a SIP-based peer-to-peer (P2P) network as introduced in
[16]. To solve issues for networking, avoid legal restrictions, optimisation, missing mechanisms to form the P2P network or the fact that user aim different goals for optimisation (e.g. cost reduction, obtain only "green energy", most benefit for energy supply) the P2P networking approach was extended by community mechanism (see [13], [14], [15]). The communitybased approach forms a new comfortable way to join a network of peers, whereby the connection of peers is done by joining the users to a social network. Using IP-based Information exchange guarantees the applicability by everyone that has access to the Internet. Therefore the realisation is possible with minimal costs. Inside that Energy-Community not only the single customer goals are considered, the goal is to reach a benefit for every participant in the community. In addition the participants can form sub-communities to address common shared interests (e.g. energy saving, cost reduction, obtain only "green energy").

References [13], [14] and [15] present the principles of the proposed framework for creation, provisioning and optimisation of services in Smart Homes and Smart Grids. Also the architecture of the implementation of the proposed solution is described. As exemplary service the optimisation of the energy consumption in single and clustered Smart Homes is outlined that forms a novel solution for optimisation in Smart Grids. This described framework demonstrates that service provisioning and joint optimisation by end-users can be implemented in an application field that hadn't addressed those topics yet.

III

\section{P2P BASED SERVICE PROVISIONING IN M2M NETWORKS}

The framework mentioned in section II serves as a work basis for more generalised consideration of the principles in M2M application field. The solution focuses on the consideration of $\mathrm{M} 2 \mathrm{M}$ aspects, because $\mathrm{M} 2 \mathrm{M}$ is a special field of networked devices with special requirements and limitation for available data storage, processing power, bandwidth etc. The platform forms the main part of the solution in provision and use of M2M services by connecting service providers and service users, using an overall solution for information exchange. The concept of a P2P-connected Community offers many advantages and can be applied in various sectors of M2M. The P2P networked community concept offers advantages in all application fields where decentralised challenges exist and central solutions for service provisioning or optimisation are not realisable. The novel concept provides a great possibility to form intelligent environments and the community-based approach is a new comfortable way to join a network of numerous participants, whereby the connection of peers is done by joining the users to a social network. Existing infrastructure can be used to provide different kind of services in different application field with same and cost efficient hardware (e.g. mobile Smartphone). The joint optimisation is based on information exchange between the peers which creates maximal benefit for all participants.

\section{A. Requirements for M2M application field}

In the following section the requirements will be described to achieve service provisioning in $\mathrm{M} 2 \mathrm{M}$ application fields. 
The overall goal is to provide a concept for service provisioning in $\mathrm{M} 2 \mathrm{M}$ application field and to provide this services using P2P mechanisms. The networking enables the participating peers to provide a service that can be consumed by others. Especially the jointly service provisioning is considered in the concept (e.g. to provide services consumed by central authorities). The communication between the peers for service provisioning and service consuming is realised without any central authority. Therefore the overall goal can be separated in the goals "joint service provisioning" and "autonomous service provisioning". Joint service provisioning is separated to service aggregation (provide a joint service by simultaneously offering the same service) and service composition (provide a joint service by offering different services). This leads to the following requirements.

Service provisioning have to be autonomous. To avoid centralised management and controlling, service provisioning and service consuming have to be realised without any central authority. This enables flexibility because of no limitations for specialised application fields (central service provisioning will limit services to specific domain). To enhance the flexibility of the services and to provide the possibility for detailed coordination of service offering and usage (e.g. by human communication), multimedia communication and other IT services (e.g. email, calendar, IM etc.) have to be integrated into services and service platform. Services have to be provided and executed locally (using existing hardware). This requires a minimum of hardware requirements. To provide a service in Smart Grids or to optimise a Smart Grid, a Smart Home is required that is able to exchange information with other Smart Homes. The Smart Home must be as flexible as possible. That means many opportunities for controlling have to exist (connection of different technologies for home automation, provide mechanisms for energy management and integration into services). Devices and people which provide services have to describe the service (functionality, conditions for usage, description of required parameters etc.) and make the services available to others. Therefore a service description is required. Service provider and service consumer have to be networked to exchange information regarding service provisioning and service usage. Therefore communication between them is required. The network of peers (devices and people) has to be scalable and self-organised. The networking and data storage (if required) have to be completely decentralised. Assuming that electronic media are used to communicate between the peers, technical "communication endpoints" exist. Therefore especially communication between devices has to be considered (M2M). The Quality of Service (QoS) must be estimable in dependence of the service. That means evaluation of requirements for service provisioning/ service usage have to be provided, such as high availability, robustness, low packet loss, low latency, real-time capability, prioritisation, guaranteed bit rates, throughput, dependencies from other services, diagnostic facilities, self-detection of problems and failures, self-healing. A peer-to-peer network should be created (or many P2P networks) in which the peers offer their services and use services (jointly offered services). The granularity of the P2P network has to be flexible. I.e. peers can represent companies, households, people or devices. Services can be available local to an area, e.g. a building etc.
The joint service provisioning should be completely decentralised. That means, no central instances have to be involved. Possibly required concentrators don't have to be equipped with additional (costly) infrastructure. To avoid centralised management tasks, the service provisioning and registration has to be completely decentralised. Since the granularity of the P2P network, and therefore the number of service providers is scalable (and thus, any number of service providers and service users in any combination may exist) a central service management cannot be implemented, because effort and costs for administration and maintenance of the infrastructure cannot be estimated/ realised. Service providers must register their services in the P2P network. The service consumer should request for the service usage.

\section{B. Scope of concept for P2P based Service Provisioning in M2M}

The concept for P2P based Service Provisioning in M2M is limited to the following restrictions. Only IP networks are considered. The restriction is made because it may be assumed that many devices (or communication endpoints, as M2M) will hold its own IP address. "The global market for machine-tomachine (M2M) device connections will grow from 62 million devices in 2010 to 2.1 billion devices in 2020." [4]. Reference [5] reports that the "Global M2M connections will increase from two billion at the end of 2011 to 18 billion at the end of 2022." They also estimate that "Short range technology will dominate: over $70 \%$ of $\mathrm{M} 2 \mathrm{M}$ devices will be connected by shortrange technologies, mostly WiFi." Reference [6] also describe that IP will be the basis for future communication. The responsible group for M2M at ETSI "aims to provide an end-to-end view of Machine to Machine standardization, and will co-operate closely with ETSI's activities on Next Generation Networks (NGN), and also with the work of the 3GPP standards initiative for mobile communication technologies." [7]. This suggests also that IP-based communication will play a decisive role and therefore IP will be set as basis for communication in the presented concept.

\section{SIP as protocol to connect peers}

The information exchange between the peers and the connection of the peers should be based on the Session Initiation Protocol (SIP) [8]. The communication protocol SIP is the standard protocol for signalling in NGN and already provides a mechanism to exchange information based on the subscribe/ notify principle. Therefore, it makes sense to use SIP to exchange messages between the peers, but also for further communication e.g. via audio/video call, IM. This allows the communication of service consumers and service providers for detailed coordination of service usage (e.g. personal consultations, etc.). The SMF is connected via IP to external networks such as the Internet or fixed and mobile networks. Therefore communication between the peers should occur based on IP-technology, because of existing mechanisms for security, availability and quality of service, preferable via Next Generation Networks. Therefore it is again advisable to use SIP as application layer protocol for signalling and e.g. RTP (Realtime Transport Protocol) for transport of multimedia data (e.g. audio, video). Hence the SMF is a combination of a Smart Home control and a SIP Application Server. The SIP protocol 
is used because it is an IETF standard which is widely adopted and it offers interfaces for integration of services. SIP is the standard protocol in multimedia communication (for controlling communication sessions), which was defined as a requirement for the platform to offer total communication possibility. The integration of telecommunications services to the presented platform already requires the usage of SIP. SIP is similar to HTTP which allows the easy integration of multimedia communication and e.g. Web services and other IPbased applications. Also SIP has standardised functionality for transport of XML-data and mechanisms for reliable communication, realised in Application Layer that offers the functionality to act in heterogeneous networks. SIP has already integrated control functionalities for point-to-point/ multicast connections and provides many features that can be advantageously used for the connection of peers and the exchange of information. Advantageous functionalities of SIP are: The globally defined SIP address (SIP URI) for users that allows location-independent availability via unique identifiers; Location Service functionality for worldwide localisation of users over the Internet (including mobile users); Presence functionality to request information about the availability of users; Event Notification Framework [9] that "enables SIP entities to subscribe to specific events occurring on other SIP entities." [12] and Instant Messaging [10] that can be used "to carry short text messages in a simple way from one SIP entity to another." [12] In addition SIP provides existing security functionalities, described in [8]. Furthermore Interactive Connectivity Establishment (ICE) [11] can be used to traverse a Network Address and Port Translation (NAPT) gateway or a Firewall.

\section{Application fields and exemplary services}

Several services can be implemented by a generalised approach in M2M environments, especially in next generation $\mathrm{M} 2 \mathrm{M}$. The platform, described in [13], [14] and [15], forms the main part in provision and use of M2M services by connecting service providers and service consumers, using an overall solution for information exchange. The focus is the P2P connection of all participants and the community mechanism. Based on that concept, different kind of services are realisable in different application fields (e.g. energy management, electro mobility, traffic, Ambient Assisted Living, Industry 4.0). Fig. 4 describes the generalised structure for service provision and usage in P2P networked communities.

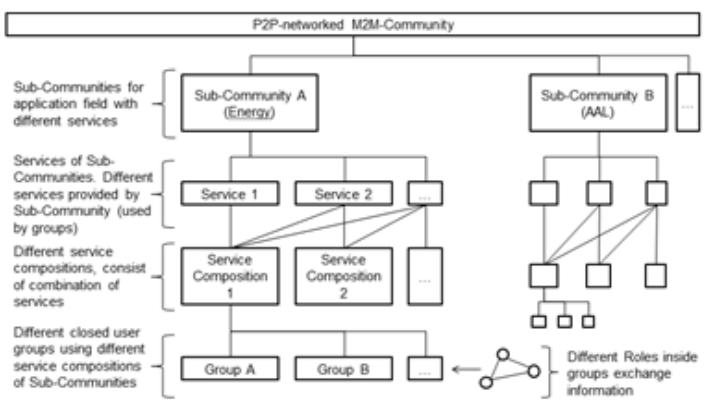

Fig. 4. Generalised structure of $\mathrm{P} 2 \mathrm{P}$ networked M2M communitiy services
The concept identifies several elements in the structure: The P2P networked M2M-Community itself, sub-communities for different application fields, different services provided by the sub-communities, different service compositions consisting of several services and different closed user groups that use the service compositions. Within the groups different roles exist that are central parts for the service realisation (e.g. peers which supply energy or peers which shift energy consumer as service provider for load reduction; distribution grid provider as service consumer which aims to reduce the load in the grid to avoid grid expansion). This structure is used to apply the concept for several M2M applications/ services like energy management, traffic management, electro mobility (e.g. electric vehicle charging service), logistic (parcel, relocation/ cold transport services), public/ private transport, Ambient Assisted Living (or senior citizens services), residential building security/ keeping.

\section{CATEGORISATION OF SERVICES AND \\ OPTIMISATION TOPICS FOR M2M APPLICATION FIELD}

For application and evaluation of the presented concept and the required architecture, categories have been defined to divide the several variants of relationships regarding the kind of service provisioning/ usage (see Fig. 5). The different kind of relationships can be categorised relating to the user who consumes the service and the number of users which provide and consume the service. The constellation can be identified as shown in Table 1. For determination/ evaluation of different types of services that can be provided under different preconditions such as different network infrastructures (e.g. preconditions of access networks), categorisation has been done regarding network requirements: availability, bandwidth, delay, jitter, packet loss, data volume. This categorisation is also used for evaluation of the scalability of services regarding provisioning and usage. To narrow the several optimisation problems of the services that are related to optimisation tasks and to look at that optimisation tasks from a generalised perspective, categories of optimisation problems have been identified: chronological order tasks focus on sorting algorithm, Route planning or distance detection tasks focus on algorithm to detect shortest paths. Tasks for being at the right time at the right place focus on matching algorithm and availability of resources (ability, time etc.) focuses on scheduling algorithm.

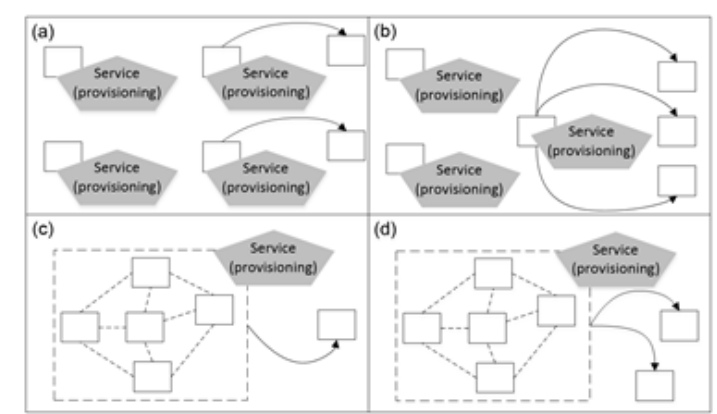

Fig. 5. Categories regarding service usage/ provisioning 


\begin{tabular}{|c|c|}
\hline TABLE I. & $\begin{array}{l}\text { CATEGORISATION REGARDING RELATIONSHIP SERVICE } \\
\text { PROVISIONING' USAGGE }\end{array}$ \\
\hline $\begin{array}{c}\text { Relationship } \\
\text { category }\end{array}$ & Category Description \\
\hline $1: 1$ & $\begin{array}{l}1 \text { service provider (1 platform) provides one service } \\
\text { used by } 1 \text { service consumer (Fig. } 5 \text { section (a)) }\end{array}$ \\
\hline 1:n & $\begin{array}{l}1 \text { service provider (1 platform) provides one service } \\
\text { used by n service consumer (Fig. } 5 \text { section (b)) }\end{array}$ \\
\hline $\mathrm{n}: 1$ & $\begin{array}{l}\text { n service provider ( } \mathrm{n} \text { platforms) provide one (same) } \\
\text { service used by } 1 \text { service consumer (Fig. } 5 \text { section } \\
\text { (a)) }\end{array}$ \\
\hline $\mathrm{n} \cdot \mathrm{m}$ & $\begin{array}{l}\text { n service provider (n platforms) provide one (same) } \\
\text { service used by } \mathrm{m} \text { (different) service consumer (Fig. } \\
5 \text { section (b)) }\end{array}$ \\
\hline 1-n:1 & $\begin{array}{l}1 \text { to } \mathrm{n} \text { (different) peers provide } 1 \text { service in } \\
\text { cooperation that is used by } 1 \text { service consumer (Fig. } 5 \\
\text { section (c)) }\end{array}$ \\
\hline 1-n:m & $\begin{array}{l}1 \text { to } \mathrm{n} \text { (different) peers provide } 1 \text { service in } \\
\text { cooperation that is used by } \mathrm{m} \text { (different) service } \\
\text { consumer (Fig. } 5 \text { section (d)) }\end{array}$ \\
\hline
\end{tabular}

\section{CONCLUSION}

The categorisation of services and optimisation strategies allows evaluation of the requirements that are placed on the infrastructure. Particularly, the requirements for the access network change, because this is faced with new challenges regarding the service provisioning. A large number of service providers now are located in the mobile access network, instead of the fixed network or the core network. Also the increasing communication between the clients inside the access network changes the requirements for the access network.

In the presented approach service users are not necessarily the end-user, but can also be higher authorities such as grid operators. Such higher authorities can honour the service provision, respectively achievement of a given goal, through e.g. lower network charges and thus create a financial benefit for the customer (end-user)

The P2P networked community concept offers advantages in all application fields where decentralised challenges exist and central solutions for service provisioning or optimisation are not realisable (e.g. because of costs, infrastructural precondition or real-time requirements). The joint optimisation is realisable, based on information exchange between the peers. On the one side information are exchanged from machine to machine (SMF to SMF, device to device, device to SMF, SMF to device) and from machine to process (optimisation algorithm and other services), on the other side information become available for customers (machine to human and human to human information exchange). The challenges to M2M platforms or IoT (Internet of Things) mentioned in [17] (scalability, robustness, openness, flexibility etc.) are addressed/ solved by the SMF and the P2P networked community concept. The SMF forms a platform for automated information exchange (using communication networks) between devices/ humans. The SMF is the basis for exchange of information with limited human interaction. The integration of different house automation systems and communication technologies into SMF brings the possibility to build many services based on $\mathrm{M} 2 \mathrm{M}$. These services can be provided by one or many peers and used by one or many peers (service user and provider are linked in a n:n constellation).
Using a mobile implementation of the SMF, the mobile phone (with e.g. Android as operating system) is the runtime environment and at the same times the interface to the customer. The implementation on mobile platforms offers possibilities for many applications and services, independent of the location, using location data and environment information.

\section{REFERENCES}

[1] V. Arraj, Compliance Process Partners LLC, "ITIL: the basics", White Paper, The APM Group and The Stationery Office 2013 AXELOS.com, July 2013

[2] ITIL V3 Glossary V3.1.24, "Glossary of Terms, Definitions and Acronyms", AXELOS Limited, May 2007

[3] P. Decker, "Facebook of Things - The challenge of Endusers within M2M Systems", Symphony Teleca Corporation, M2M Summit 2012, Düsseldorf, Germany, September 2012

[4] Analysys Mason, "Machine-to-machine device connections: worldwide forecast 2010-2020", http://www.analysysmason.com/Research Content/Reports/RRE02_M2M_devices_forecast' (last visited 2015-02 17), December 2010

[5] Machina Research, "Report - M2M Global Forecast and Analysis 2011 $22^{11}, \quad$ https: $/ /$ machinaresearch.com $/ \mathrm{m} 2 \mathrm{~m}$-global-forecast-analysis-2011 22/ (last visited 2013-12-27), November 2012

[6] GSMA, "Future Communications", http:/iwww.gsma.com/future communications/ (last visited 2013-12-27), December 2013

[7] ETSI (European Telecommunications Standards Institute), "Machine to Machine Communications -Introduction", http://www.etsi.org/ technologies-clusters technologies $/ \mathrm{m} 2 \mathrm{~m}$ (last visited 2013-12-20), technologies-clust

[8] IETF (The Internet Engineering Task Force), Request For Comments (RFC) 3261, "SIP: Session Initiation Protocol", June 2002

[9] IETF (The Internet Engineering Task Force), Request For Comments (RFC) 3265, "Session Initiation Protocol (SIP) Specific Event Notification", June 2002

[10] IETF (The Internet Engineering Task Force), Request For Comments (RFC) 3428, "Session Initiation Protocol (SIP) Extension for Instant Messaging", December 2002

[11] IETF (The Internet Engineering Task Force), Request For Comment (RFC) 5245, "Interactive Connectivity Establishment (ICE): A Protocol for Network Address Translator (NAT) Traversal for Offer/Answer Protocols", April 2010

[12] A. Lehmann, "Service composition based on SIP peer-to-peer network", $\mathrm{PhD}$ Thesis, School of Computing and Mathematics, University Plymouth, United Kingdom, March 2014

[13] M. Steinheimer, U. Trick, W. Fuhrmann, B. Ghita, "Load reduction in distribution networks through P2P networked energy-community", Proc of Fifth International Conference on Internet Technologies \& Applications (ITA 13), Wrexham, Wales, UK, September 2013

[14] M. Steinheimer, U. Trick, W. Fuhrmann, B. Ghita, "P2P-based community concept for M2M Applications", Proc. of Second International Conference on Future Generation Communication Technologies (FGCT 2013), London, UK, December 2013

[15] M. Steinheimer, U. Trick, P. Ruhrig, R. Tönjes, M. Fischer, D. Hōlker "SIP-basierte P2P-Vernetzung in einer Energie-Community", ITGFachbericht 242: Mobilkommunikation, VDE Verlag $\mathrm{GmbH}$, Osnabrück, Germany, ISBN: 978-3-8007-3516-7, ISSN: 0932-6022, pp. 64, Mai 2013

[16] A. Lehmann, W. Fuhrmann, U. Trick, B. Ghita, "New possibilities for the provision of value-added services in SIP-based peer-to-peer networks", Proc. of SEIN (Symposium on Security, E-learning, Internet and Networking), University of Wrexham, UK, November 2008

[17] M. Castro, A. J. Jara, F. Antonio, "An analysis of M2M platforms: challenges and opportunities for the Internet of Things", Proc. of Sixth Intemational Conference on Innovative Mobile and Intemet services in Ubiquitous Computing, pp. 757-762, Palermo, Sicily, July2012 
Published in Proceedings of the Eleventh International Network Conference (INC 2016), pp. 115-120, July 2016, Frankfurt, Germany

\title{
P2P-based M2M Community Applications
}

\author{
Michael Steinheimer ${ }^{1,3}$, Ulrich Trick ${ }^{1}$, Woldemar Fuhrmann ${ }^{2}$, and Bogdan Ghita \\ ${ }^{1}$ Research Group for Telecommunication Networks, Frankfurt University of Applied Sciences, Frankfurt/M., Germany \\ ${ }^{2}$ Department for Computer Science, University of Applied Sciences Darmstadt, Darmstadt, Germany \\ ${ }^{3}$ Centre for Security, Communications and Network Research, University of Plymouth, Plymouth, UK \\ steinheimer@e-technik.org, trick@e-technik.org,w.fuhrmann@fbi.h-da.de, bogdan.ghita@plymouth.ac.uk
}

\begin{abstract}
This publication presents a novel concept for decentralised service and application provision in a Peer-to-Peer (P2P) connected Machine-To-Machine (M2M) network based on community mechanisms. Several execution environments are introduced and evaluated. Requirements are defined to achieve application/ service provision in M2M application fields. This publication also introduces an application, consisting of several services, for building surveillance, realised via the presented concept of P2P based application provision in M2M networks.
\end{abstract}

Keywords-Service/Application Provision; P2P; M2M

\section{INTRODUCTION}

In previous publication a concept for decentralised service provision by end-users has been presented.[4] The aim of the concept described in that publication is to enable end-users (previous service consumer) to take the role of a service provider and offer services for other end-users and corporations. The end-users can be networked to provide cooperative services, consisting of several identical or different services, realised by individual end-users. These services can be aggregated/ composed and can be consumed by central corporations or other customers. The approach of provision services by end-users and consummation of these services by end-users is defined as "horizontal service provision" (illustrated in Fig. 1). The approaches of service provision by end-users (individual or cooperative) which are consumed by central corporations or other end-users are defined as "bottomup service provision" (illustrated in Fig. 2).

Horizontal approach for service provision

\begin{tabular}{|l|l|l|}
\hline Service Provider $\mathrm{m}$ & Service Consumer 1 \\
\hline Service y & \multicolumn{1}{|c|}{ Service Consumer $\mathrm{n}$} \\
\hline
\end{tabular}

Fig. 1. Horizontal approach for service provisioning

Until now, service provision followed the centralised approach (central service provider which provides services to the end-user). The presented concept changes the relationship from service provider to service consumer which can be described as bottom-up service relationship (end-user jointly offers a service that can be used by central corporation) or as a horizontal service relationship (end-user offer services to other end-users). The described concept is realised by M2M. For this a framework has been defined, that realises service/ application provision using SIP-based P2P networking, so that no central appliances for realisation are required. Furthermore the concept includes social networking approaches, by forming communities and sub-communities for establishing the P2P network and networking the participants corresponding to their interests, geographical locations as well as different application field etc. Through the described concept, in addition of service application provision in end-user domain, also service, application provision in M2M application field becomes possible, whereby M2M devices also are considered as peers.

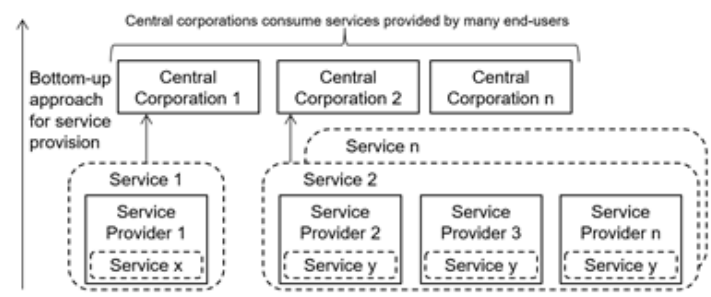

Fig. 2. Bottom-up approach for service provisioning

This publication specifies the concept more detailed to provide community applications which are not covered (and not focused) by the ETSI M2M standard. The presented concept defines a novel framework for application provision, based on M2M Systems.

The service/ application provision in M2M, according to the presented concept, can be separated in two main categories: 1. Autonomous service provision (compare Fig. 1). Here peers provide a service which is consumed by other peers on the same hierarchical level. 2. Cooperative service provision (illustrated in Fig. 3). Here many peers provide a service which is consumed by other peers on same or higher hierarchical level. In case of service aggregation peers e.g. provide a joint service by simultaneously offering the same service. In case of service composition, peers provide a joint service by offering different services.

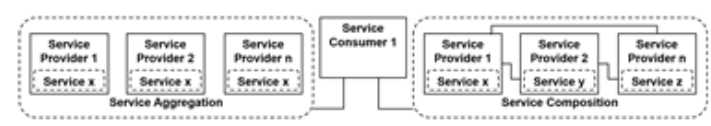

Fig. 3. Cooperative service Provisioning 


\section{TERMS AND DEFINITIONS OF SERVICE PROVISION IN M2M}

In order to clarify how an application in M2M context is structured, definitions of $[5,6,7]$ are used to classify and separate service and application in context of application service provision in M2M (illustrated in Fig. 4). It illustrates that an application consists of one or more underlying services that are combined (i.e. aggregated or composed) and, if required, exchange information. The services are realised by one or more service components, which form the building blocks of services. The service components itself are realised via several software applications executed on several execution environments

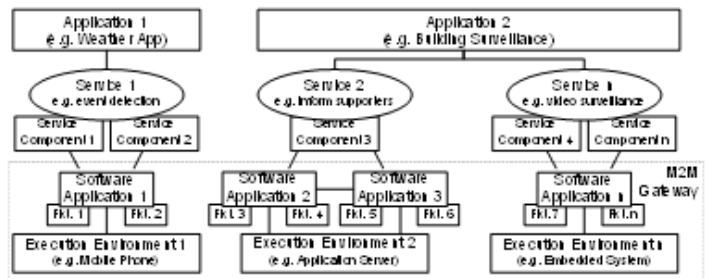

Fig. 4. Classification of Service and Application

A service as well as an application can be realised on technical or non-technical principles (i.e. it can be provided using technical devices, e.g. computers, or by a human, e.g. personal assistance services). To distinguish the kind of services and applications, if necessary they are indicated as technical service/ application or non-technical service/ application. General indication of both is service/ application.

In the presented concept of $\mathrm{P} 2 \mathrm{P}$ based application provision in $\mathrm{M} 2 \mathrm{M}$, definition of service and application follows the definition illustrated in Fig. 4. Same as for service and application, peers can be technical devices or humans (if applicable supported by technical devices). To distinguish the kind of peer, if necessary, it is indicated as technical peer or non-technical peer. General indication of both is peer. Peers are able to provide services. The service is executed on the execution environment of the specific device or, in case of a non-technical service, provided by the personal execution of the human service provider (if applicable supported by a device providing a technical service, e.g. a Smartphone).

Based on references $[8,9,10,11] \mathrm{M} 2 \mathrm{M}$ is defined as follows: Machine-to-Machine communications (M2M) "refer to physical telecommunication based interconnection for data exchange between two ETSI M2M compliant entities, like: device, gateways and network infrastructure"[8] M2M area network provides "physically and MAC layer connectivity between different $\mathrm{M} 2 \mathrm{M}$ devices connected to the same M2M area network or allowing an M2M device to gain access to a public network via [...] a gateway."[10] "M2M Applications: are respectively Device Application (DA), Gateway Application (GA) and Network Application (NA)."[9] "M2M Applications [...] run the service logic [...]."[8] In general [8] defines an application as an "entity (typically in software) designed to perform specific tasks on behalf of /in order to help a user to operate for a specific goal." "M2M Application Service Provider: is an entity (e.g. a company) that provides M2M Application Services in the M2M System to the end user."[8] ,M2M value-added services are not services at the $\mathrm{M} 2 \mathrm{M}$ application level. The M2M value-added services relate to the data communication services themselves, not to the application in the M2M device and M2M server. Examples of M2M value-added services are: QoS and priority differentiation; charging and subscription management; device management; connection monitoring; fraud control; secure connections." [10] This statement is substantiated by [11] who summarises that despite all networking and communication abilities all M2M applications focus on optimisation of collaboration of $\mathrm{M} 2 \mathrm{M}$ devices based on M2M applications within a defined, separated overall process, often using a M2M service platform.[11] According to ETSI the end user operates M2M Application Services (provided by M2M Application Service Provider, i.e. companies). This especially means that the end user (according to the ETSI standard of M2M) does not create and provide services as well as is a different role separated from the M2M Application Service Provider. In summary this means that up to this point only a framework is defined for provision of M2M Network Applications and no framework for application provision on end users domain has been defined yet.

III.

EXECUTION ENVIRONMENTS FOR SERVICES/ APPLICATIONS

Currently several concepts for hosting services/ applications exists. Well-known approaches of service/ application provision are central server approach (using servers, hosting the applications on central locations in the network) and Cloud Computing approach. Next to this approaches two new architectural approaches for hosting services and applications came up: Edge and Fog Computing.

"Edge computing is all about pushing processing for certain data intensive, remotely isolated applications away from the core of the data center to the outer edges of the network where all the interactions are happening and the actual processing needs to take place."[12] Mobile-edge Computing is a part of Edge Computing which "provides IT and cloud-computing capabilities within the Radio Access Network (RAN) in close proximity to mobile subscribers."[13] "Mobile-edge Computing can be seen as a cloud server running at the edge of a mobile network and performing specific tasks that could not be achieved with traditional network infrastructure. Machineto-Machine gateway and control functions are one example [...]."[13] The second new approach of hosting services and applications is Fog Computing, which "is a highly virtualized platform that provides compute, storage, and networking services between end devices [...], typically, but not exclusively located at the edge of network."[14] Also [15] describes, that virtualisation plays a big role in Fog Computing and ,the Fog enables user devices to become the virtualisation platform themselves". "In the fog, both the network and the services running on top of it can be deployed on demand in a fog of [..] devices."[15] In [15] Fog Computing is defined as „a scenario where a huge number of heterogeneous (wireless and sometimes autonomous) ubiquitous and decentralised devices 
communicate and potentially cooperate among them and with the network to perform storage and processing tasks without the intervention of third parties. These tasks can be for supporting basic network functions or new services and applications that run in a sandboxed environment. Users leasing part of their devices to host these services get incentives for doing so". Fog Computing is defined according to [14] as "a platform to deliver [...] services and applications at the edge of the network".

The main intention of Edge and Fog Computing is to support network services, reduce the load in the core network, enlarge the Quality of Service for the customers, provide storage as well as perform distributed computing tasks. Considering the definition of Cloud Computing and application provision using centralised server approach as well as the definitions of Edge and Fog Computing acc. to $[12,13,14,15]$, Fig. 5 illustrates the execution environments of services/ applications according to the several approaches.

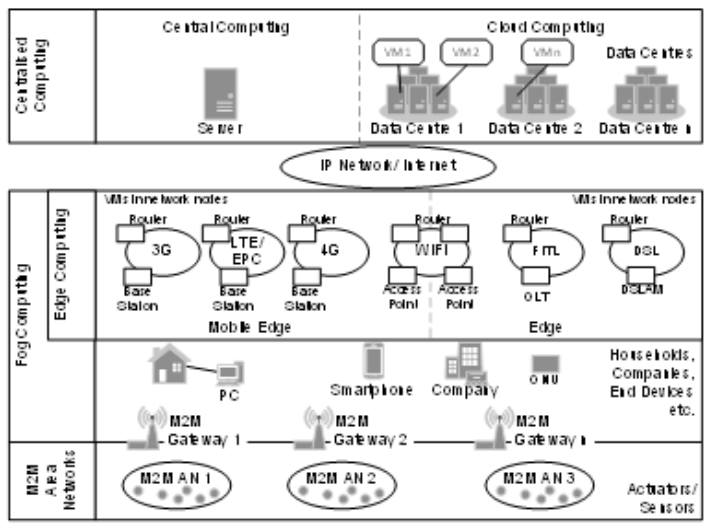

Fig. 5. Execution Environments of Services and Applications

Edge Computing is realised in the infrastructure provided by the network operator (i.e. access networks or devices located close to the customers, e.g. services executed in base stations). This eliminates service provision by end-users in the edge. Fog computing also is executed in same infrastructure and, in addition, more close to the customer (e.g. in IADs) and extended by virtualisation concepts. Edge computing can only be operated by the network operators because no one else has access to the network equipment located in the access network. Fog computing also requires the existence of central corporations for coordination of the distributed computing tasks as well as the management and arrangement of the virtualised services executed in devices next to the customers. Despite Fog Computing offers the possibility for service execution next to the customer, end-users service provision is still not integrated due to missing access to the infrastructure of the Fog Computing provider.

Combining the concept of P2P based service/ application provision by end-users with Fog Computing aspects of virtualisation and service execution next to end-users, by enabling the platforms next to the end-users for execution of services provided by the end-users, with possibility of information exchange between end-users devices, forms a novel concept for provision of $\mathrm{M} 2 \mathrm{M}$ services and enables endusers to participate in application provision on end-users domain.

IV.

P2P BASED SERVICE/ APPLICATION PROVISION IN M2M

The following chapter describes a solution for $\mathrm{P} 2 \mathrm{P}$ based service provision in M2M. First a framework is introduced to provide a solution for service provision in M2M networks. Afterwards the requirements are defined to achieve application service provisioning in M2M application fields. This chapter closes with a description of the communication between the involved parties and an evaluation of the presented approach regarding existing execution environments (described in chapter III)

Previous publications, e.g. $[1,2,3]$ describe that existing research and development activities for optimisation of Smart Homes and Smart Grids do not consider service provisioning networking and decentralised optimisation via information exchange. Therefore, a solution is presented in $[1,2,3]$, oriented at the personal needs of users, to offer cost-efficient energy management, according to users personal needs, and integration within their house automation as well as combined with multimedia communication. Also the interaction between households is included to exchange information regarding the local energy production and consumption. This information is used for joint, independent and decentralised energy optimisation. The principles and architectures of the proposed framework for provision of services and optimisation possibilities in Smart Grids are presented in $[1,2]$. The Concept is mainly based on a Service Management Framework (SMF), consisting of a Service Delivery Platform (SDP) and a Service Creation Environment (SCE), see [3], which is installed in the local households. The SCE brings the functionality to design and configure value-added services graphically, according to the personal needs of the users, to monitor and control actuators, sensors and devices in user households. The SDP is used to provide a solution for service provision, service control and service management and serves as a runtime environment for the generated services. SCE and SDP in combination allow easy service design by the customer, as well as local controlling/management of energy producers and devices (energy consumer) in Smart Homes. With that solution the optimisation of energy consumption e.g. by optimal load distribution is possible.

The presented concept include the clustering of households for more effective optimisation because of independent optimisation of individual households does not automatically lead to optimisation of the local or distribution grid. The concept is totally based on decentralised approaches to avoid central instances in the infrastructure. In order to provide the aggregation of households, the SMFs of individual households are connected using a SIP-based peer-to-peer $(\mathrm{P} 2 \mathrm{P})$ network as introduced in [16]. To solve issues for networking, avoid legal restrictions, optimisation, missing mechanisms to form the $\mathrm{P} 2 \mathrm{P}$ network or the fact that user aim different goals for 
optimisation (e.g. cost reduction, obtain only "green energy", most benefit for energy supply) the P2P networking approach was extended by community mechanism (see $[1,2,3]$ ). The community-based approach forms a new comfortable way to join a network of peers, whereby the connection of peers is done by joining the users to a social network. Using IP-based information exchange guarantees the applicability by everyone that has access to the Internet. Therefore the realisation is possible with minimal costs. Inside that Energy-Community not only the single customer goals are considered, the goal is to reach a benefit for every participant in the community. In addition the participants can form sub-communities to address common shared interests (e.g. energy saving, cost reduction, obtain only "green energy").

References $[1,2,3]$ present the principles of the proposed framework for creation, provisioning and optimisation of services in Smart Homes and Smart Grids. Also the architecture of the implementation of the proposed solution is described. As exemplary service the optimisation of the energy consumption in single and clustered Smart Homes is outlined that forms a novel solution for optimisation in Smart Grids. This described framework demonstrates that service provisioning and joint optimisation by end-users can be implemented in an application field that hadn't addressed those topics yet.

The novel concept of a P2P-connected community offers many advantages and can be applied in various sectors of M2M to form intelligent environments. The SMF is the main component of the solution in provision and consummation of M2M services by connecting service providers and service consumers, using an overall solution for information exchange. All peers, providing services and applications, are associated in a M2M community. The M2M community is the mechanism to form the P2P network and avoid legal restrictions. The M2M community forms a social network of peers. Various subcommunities are used to address different application fields, interests, geographical locations etc. Fig. 6 illustrates the collocation of M2M Community, sub-communities and applications based on the classification of service and application illustrated in Fig. 4. Existing infrastructure (IP network and devices, e.g. IAD or smartphone) can be used to provide different kind of services in different application field with same and cost efficient hardware (e.g. Smartphone). Fig. 6 illustrates the overall architecture of execution environments for applications/ services, where the community and the subcommunities are integrated. The overall goal is to provide a concept for application/ service provisioning in M2M application field and to provide this applications/ services using P2P mechanisms without central instances.

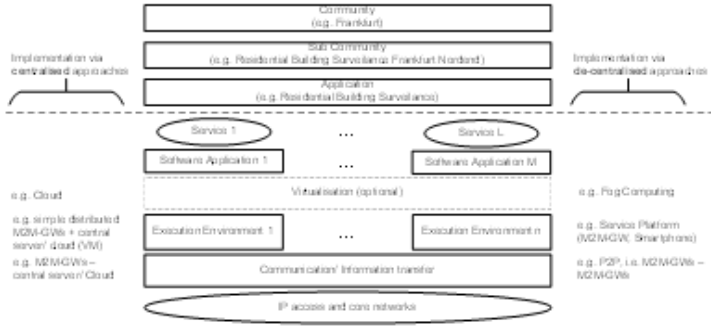

Fig. 6. Collocation of M2M Community, Sub-Communities, Applications

The networking enables the participating peers to provide a service that can be consumed by others. Especially the cooperative service provisioning is considered in the concept (e.g. to provide services consumed by central corporations).

The overall goal can be separated in the goals "joint service provisioning" and "autonomous service provisioning". Joint service provision is separated into service aggregation (provide a joint service by simultaneously offering the same service) and service composition (provide a joint service by offering different services).

M2M is a special field of networked devices with special requirements and limitation for available data storage, processing power, bandwidth etc. For application/ service provision and consummation in $\mathrm{M} 2 \mathrm{M}$, according to the presented concept, following requirements have been defined: Possibility for service provision (incl. cooperative service provision) by end-users using existing resources (e.g. IAD or mobile phone); autonomous service provision; decentralised mechanism for service description/ registration by devices and people; unified communication of service provider and consumer; management of constraints for services (e.g. temporal and local availability). Requirements according networking and resources: Integrated multimedia communication (and other IT services) to services and service platform; minimal hardware requirements for decentralised service execution; networking of service provider and service consumer for decentralised communication and cooperative service provision; estimable Quality of Service (QoS) in dependence of service; avoidance of central instances for (joint) service provision; efficient usage of limited resources (available data storage, processing power, network connectivity); determinability and predictability of network capabilities (bandwidth, availability, latency); flexible, scalable, and robust self-organised network; solutions must address scalability, robustness, openness, and flexibility.

For satisfying the requirement of integrated multimedia communication, the communication unit of the SMF provides an interface for Next Generation Network conform multimedia communication which also offers total communication possibility between the peers. The communication between the peers is realised based on IP-technology (via Internet/ NGNs) which is todays and future standard in network communication. Also IPv6 offers a large scalability for addressing devices. The information exchange between the peers and the connection of the peers is based on the Session Initiation Protocol (SIP)[17]. The communication protocol SIP is a standardised, widely 
adopted communication protocol for signalling in Internet/ NGNs. SIP provides standardised functionality for information exchanged based on the subscribe/ notify principle. In addition for messages exchange between the peers, SIP is also used for further communication e.g. via audio/ video call, IM. This allows the communication of service consumers and service providers for detailed coordination of service usage (e.g. personal consultations, etc.). Also SIP has standardised functionality for transport of XML-data and mechanisms for reliable communication, realised in OSI Application Layer that offers the functionality to act in heterogeneous networks. SIP has already integrated control functionalities for point-to-point/ multicast connections and provides many features that can be advantageously used for the connection of peers and the exchange of information. Advantageous functionalities of SIP are: The globally defined SIP address (SIP URI) for users that allows location-independent availability via unique identifiers; Location Service functionality for worldwide localisation of users over the Internet (including mobile users); Presence functionality to request information regarding the availability of users; Event Notification Framework[19] that "enables SIP entities to subscribe to specific events occurring on other SIP entities." [11] and Instant Messaging[20] that can be used "to carry short text messages in a simple way from one SIP entity to another."[11] In addition SIP provides existing security functionalities, described in [17]. Furthermore Interactive Connectivity Establishment (ICE)[18] can be used to traverse a Network Address and Port Translation (NAPT) gateway or a Firewall.

TABLE I. EVALUATION OF EXECUTION ENVIRONMENTS

\begin{tabular}{|c|c|c|c|c|c|}
\hline $\begin{array}{l}\text { Advantages/Dis- } \\
\text { advantages reg. single } \\
\text { M2M Application }\end{array}$ & Centr. & Cloud & \multicolumn{2}{|c|}{$\begin{array}{c}\text { Fog } \\
\text { Computing }\end{array}$} & P2P \\
\hline Costs & - & 0 & + & + & + \\
\hline Short time to market & - & + & + & + & + \\
\hline Demand for energy & - & t & + & + & + \\
\hline Large flexibility & - & + & - & + & + \\
\hline $\begin{array}{l}\text { High availability reg. } \\
\text { platform breakdown }\end{array}$ & 0 & + & o & 0 & + \\
\hline Privacy & - & - & - & + & + \\
\hline $\begin{array}{l}\text { Ability of end-users for } \\
\text { service provision }\end{array}$ & 0 & 0 & - & + & + \\
\hline Scalability & - & + & 0 & + & + \\
\hline $\begin{array}{l}\text { M2M Gateway } \\
\text { integrated }\end{array}$ & - & - & - & + & + \\
\hline $\begin{array}{l}\text { Support of sensor } \\
\text { networks with high } \\
\text { amount of nodes }\end{array}$ & - & - & 0 & + & + \\
\hline Virtualisation possible & - & + & + & + & - \\
\hline Distributed data storage & - & 0 & - & + & + \\
\hline $\begin{array}{l}\text { Independence of } \\
\text { network/ platform } \\
\text { operator }\end{array}$ & - & - & - & - & + \\
\hline $\begin{array}{l}\text { Avoidance of central } \\
\text { instances }\end{array}$ & - & - & - & + & + \\
\hline $\begin{array}{l}\text { Automated (optimised) } \\
\text { networking }\end{array}$ & - & o & - & o & + \\
\hline $\begin{array}{l}\text { Corporate application } \\
\text { provision by cooperation } \\
\text { of end-users }\end{array}$ & + & + & - & - & + \\
\hline $\begin{array}{l}\text { Community approach is } \\
\text { realisable }\end{array}$ & + & + & - & - & + \\
\hline
\end{tabular}

\section{V.EVALUATION OF APPROACHES FOR APPLICATION PROVISION}

TABLE I illustrates an extract of an evaluation for M2M application provision regarding the approaches introduced in chapter III (central server approach, Cloud Computing, Edge Fog Computing and application provision according to the proposed P2P approach). Especially the topics "Independence of network/ platform operator", "Automated (optimised) networking", "Corporate application provision by cooperation of end-users" and "Community approach is realisable" shows the advantages of P2P based application provision as. The positive evaluation of the $\mathrm{P} 2 \mathrm{P}$ approach is illustrated once more when evaluations are not carried out respect a single $\mathrm{M} 2 \mathrm{M}$ application, but considers multiple applications.

\section{VI.}

\section{APPLICATION EXAMPLE}

In the following the application " $\mathrm{P} 2 \mathrm{P}$ Building Surveillance" is described with the basic functionality of building surveillance. The application observes certain buildings if defined events occur in the building (e.g. fire or intrusion detection) and informs the corresponding supporter (peers) about this event. The application is realised by a subcommunity for building surveillance with P2P interconnected participants. The aim of that sub-community is to assist each other for surveillance of certain buildings. The specialty of this application, as same as for the complete approach, is that no central corporation for application provision are required.The application consists of in total seven different services which are composed to realise the application and provided by the different peers (e.g. executed inside of a Smart Home platform or a mobile phone). All services can be executed independently and in particular provided directly by different peers, located at different locations in the network (i.e. that it is possible that all peers are connected via separated access networks to the core network). For information of the supporter (offering the nontechnical service of building monitoring) two different variants exist. Variant 1: Supporting peers register at service 1 with indication of kind of event, specific building $\mathrm{ID}$, and their corresponding contact information. Service 1 generates a list of supporters which are responsible for a specific event occurred in a specific building (or many buildings, e.g. fire brigade). The supporters also report their current availability status and geographical locations continuously to service 2 . Service 2 manages the availability information as well as the geographical locations of supporters and responses location and availability information of supporter, if requested. If a supporting peer should be informed about an event, then a SIP NOTIFY message is sent to it (Fig. 7, step 10). Variant 2 : Supporters don't report their current availability and location information. If the support is required they receive a SIP SUBSCRIBE message and response with a SIP NOTIFY message about acceptance (Fig. 7, step 10). Service 3 records sensor data (e.g. smoke detector or intrusion detection sensor) and forwards the sensor value for requested sensor ID to service 4 (incl. destination address contained in request. Sensors transmit sensor values periodically or at state changes to service 3 . Service 4 transmits requested sensor values to the contact destination contained in the request. Service 5 receives the current sensor values of a specific building and evaluates if an event has occurred. This service can be configured for self- 
contained request of sensor values at service 3 or advices same service to transmit the sensor value to a specific destination address. If this service detects an event, it generates a notification event. Service 6 specifies which supporter should be notified. This service receives an alarm event as well as building $\mathrm{ID}$ and determines (using service 1) the supporter which should be notified, depending on its availability and location information (using service 2). If supporter is available, the service returns the contact information as well as event occurred. Service 7 contacts the supporter about occurred events in monitored buildings, including information regarding event and location of building, either by sending a SIP SUBSCRIBE or by sending a SIP NOTIFY. Fig. 7 shows the combination of the services described above for realisation of the application for building surveillance.

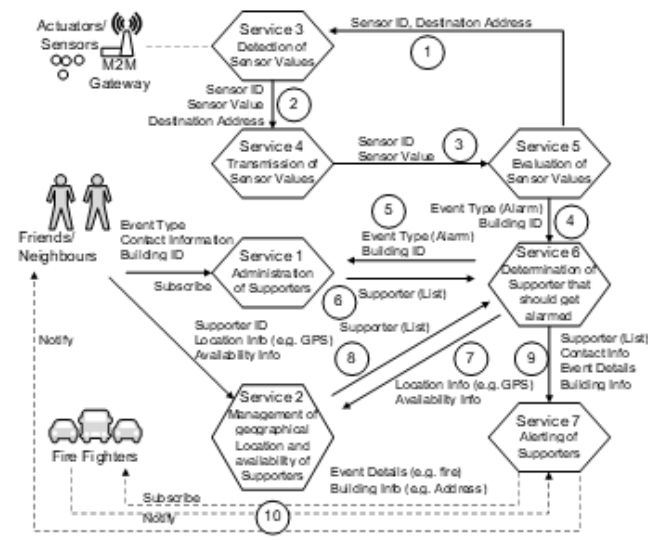

Fig. 7. Service Interaction of Application „P2P Building Surveillance

VII.

CONCLUSION

This publication presented a novel concept of service/ application provision in $\mathrm{M} 2 \mathrm{M}$ and defines a so far missing framework using SIP-based P2P networks with communities. This concept offers new possibilities for applications, realised by several peers, independent of central appliances or corporations. Especially the concept of cooperative service provision enables new application fields. Requirements have been defined to realise service/ application provision in M2M. Definition and classification of software application/ service and applications in M2M as well as classification of execution environments of services and applications offers detailed indexing and evaluation of several approaches for service application provision. Based on this the evaluation of approaches for service/ application provision has indicated that the concept of P2P-based service/ application provision has explicit advantages compared with the other approaches, especially when combining the presented concept with elements of Fog Computing. The presented application "P2P Building Surveillance" clarifies the concept and illustrates exemplarily which new application fields can be addressed by the concept, without utilisation of central appliances or corporations. Using the existing IP network infrastructure (Internet or NGNs) and devices (e.g. IADs or Smartphones) enables the realisation of $\mathrm{M} 2 \mathrm{M}$ applications without additional hardware.

\section{REFERENCES}

[1] M. Steinheimer, U. Trick, W. Fuhrmann, B. Ghita, "Load reduction in distribution networks through P2P networked energy-community", Proc. of Fifth International Conference on Internet Technologies \& Applications (ITA 13), Wrexham, Wales, UK, September 2013

[2] M. Steinheimer, U. Trick, W. Fuhmann, B. Ghita, "P2P-based community concept for M2M Applications", Proc. of Second Intermational Conference on Future Generation Communication Technologies (FGCT 2013), London, UK, December 2013

[3] M. Steinheimer, U. Trick, P. Ruhrig, R. Tönjes, M. Fischer, D. Hölker, "SIP-basierte P2P-Vernetzung in einer Energie-Community", ITGFachbericht 242: Mobilkommunikation, VDE Verlag $\mathrm{GmbH}$, Osnabrück, Germany, ISBN: 978-3-8007-3516-7, ISSN: 0932-6022, pp. 64, Mai 2013

[4] M. Steinheimer, U. Trick, W. Fuhrmann, B. Ghita, "P2P based service provisioning in M2M networks", Proc. of Sixth International Conference provisioning in on Intemet Technologies \& Applications (ITA 15), Wrexham, Wales, on Intermet Technolo
UK, September 2015

[5] ITU-T Y.101 (2000), Recommendation, "Global Information Infrastructure terminology: Terms and definitions", ITU

[6] ISO IEC 20000-1:2011 (2011) "Part 1: Service management system requirements", IEEE

[7] ITIL V3.1.24 (2007) "Glossary of Terms, Definitions and Acronyms", ITIL

[8] ETSI TR 102 725, V1.1.1, 2013-06: Technical Report, "Machine-toMachine communications (M2M); Definitions", ETSI TISPAN

[9] TS 102 690: Machine-to-Machine communications (M2M); Functional architecture, V.2.1.1. ETSI, October 2013

[10] Boswarthick, David; Elloumi, Omar; Hersent, Olivier: M2M Communications - A Systems Approach. Wiles; 2012

[11] Trick, Ulrich and Weber, Frank (2015), "SIP und Telekommunikationsnetze", De Greuvter, Berlin, Germany, ISBN: 9783-486-77853-3

[12] LeClair 2014, http://insights.wired.com/profiles/blogs/the-edge-ofcomputing-it-s-not-all-about-the-cloud\# axzz3rC9 $\mathrm{fm} J 23$, The Edge of Computing_It is Not All About the Cloud.pdf, posted by Dave LeClair on July 22,2014 , Last visited $2015-11-11$

[13] ETSI 2014, Mobile-Edge Computing-Introductory Technical White Paper, https://portal etsiorg/Portals/0/TBpages/MEC/Docs/Mobileedge Computing - Introductory Technical White Paper V1\%201809-14.pdf, Mobile-edge Computing - Introductory Technical White Paper_V1 18-09-14.pdf

[14] Bonomi 2012, "Fog Computing and Its Role in the Internet of Things", ACM, p13.pdf, http://conferences.sigcomm.org/sigcomm/2012/paper/ mcc/p13.pdf

[15] Vaquero 2014, "Finding your Way in the Fog: Towards a Comprehensive Definition of Fog Computing", ACM SIGCOMM, 0000000-0000003.pdf

[16] A. Lehmann, W. Fuhrmann. U. Trick, B. Ghita "New possibilities for the provision of value-added services in SIP-based peer-to-peer networks", Proc. of SEIN (Symposium on Security, E-leaming, Intemet networks", Proc. of SEIN (Symposium on Security, E-leaming,
and Networking), University of Wrexham, UK, November 2008

[17] IETF (The Intemet Engineering Task Force), Request For Comments (RFC) 3261, "SIP: Session Initiation Protocol", June 2002

[18] IETF (The Intermet Engineering Task Force), Request For Comments (RFC) 5245, "Interactive Connectivity Establishment (ICE): A Protocol for Network Address Translator (NAT) Traversal for Offer/Answer Protocols", April 2010

[19] IETF (The Internet Engineering Task Force), Request For Comments (RFC) 3265, "Session Initiation Protocol (SIP) Specific Event Notification", June 2002

[20] IETF (The Intemet Engineering Task Force), Request For Comments (RFC) 3428, "Session Initiation Protocol (SIP) Extension for Instant Messaging", December 2002 


\title{
M2M Application Service Provision: An autonomous and decentralised Approach
}

\author{
Michael Steinheimer ${ }^{1,3}$, Ulrich Trick ${ }^{1}$, Woldemar Fuhrmann ${ }^{2}$, Bogdan Ghita $^{3}$, and Gregor Frick ${ }^{1}$ \\ ${ }^{1}$ Research Group for Telecommunication Networks, Frankfurt University of Applied Sciences, Frankfurt/M., Germany \\ ${ }^{2}$ Department for Computer Science, University of Applied Sciences Darmstadt, Darmstadt, Germany \\ ${ }^{3}$ Centre for Security, Communications and Network Research, University of Plymouth, Plymouth, UK \\ Email: \{steinheimer, trick, frick\}@e-technik.org; woldemar.fuhrmann@h-da.de; bogdan.ghita@plymouth.ac.uk
}

\begin{abstract}
This publication presents a novel concept for autonomous and decentralised M2M application service provision. The functional architecture of the approach is introduced as well as a detailed description of the system structure and process for application creation. Furthermore, this publication describes details about the proposed process for decentralised M2M application service management and formal description of M2M application services as well as independent validation of M2M application service configurations. Finally, the prototype $\mathrm{M} 2 \mathrm{M}$ system realising the proposed concept is introduced and several scenarios are evaluated.
\end{abstract}

Index Terms - M2M application service, P2P, service provision

\section{INTRODUCTION}

Devices are becoming more and more intelligent (i.e. they include complex functionality for monitoring and control). Additionally the devices receive the functionality for communication (this enables remote monitoring and controlling of devices). Because of the increasing number of intelligent devices, which support forming of intelligent environments, many new application fields can be established (e.g. as presented in [1] smart building, electro mobility, smart city, energy optimisation etc.). Especially the end user domain is an application field with powerful potential, however not addressable by external service providers.

Machine-to-Machine Communications (M2M) systems realise the integration of such intelligent devices by provision of specific $\mathrm{M} 2 \mathrm{M}$ applications. Traditional $\mathrm{M} 2 \mathrm{M}$ systems and the corresponding M2M application services have the focus to support specific business processes. Traditional M2M applications provide their functionality as a service for users (end users or companies). Currently existing concepts from standardisation and research to implement M2M application services provision focus on central approaches. Projects from research area are e.g. INOX Managed Service Platform [2], M2M Platform Project based on SOA [3], BOSP Business Operation Support

Manuscript received Month Day, 2016; revised Month Day, 2016 This work was supported by the Foundation Name under Grant No. Xxxxxx.

Corresponding author email: author@hostname.org. doi: 10.12720 jcm.v.n.p-p
Platform [4], IMS enabled M2M Service Platform [5], eDSON [6]. Commercial M2M platforms implement acc. [7] mostly the oneM2M standard for M2M [8]. Disadvantages of the centralised approaches are e.g. that the user of the application is dependent of the platform provider as a central instance. Often M2M platforms provide their functionality only for specific application fields and are therefore less flexible. Centralised provision of platforms requires many resources (costs for operation, maintenance, availability, application development). End users often have no possibility to define applications themselves (since the creation of applications requires expert knowledge or the end user does not have access to the platform) or the devices present in user's personal environment cannot be connected to the M2M platforms.

The personal environment of the end user described above is difficult to address by external service providers, since the activities (controlling and monitoring of $\mathrm{M} 2 \mathrm{M}$ devices) carried out in this area would severely affect the end user or challenge data security. To antagonise this issue, it was proposed in [9] to integrate the end user. For this purpose, an approach was presented in [9-12], which allows end user to define services and automation tasks for their personal environment. The Service Management Framework (SMF) presented in [9-11, 13] consists of a local Service Creation Environment (SCE) and a Service Delivery Platform (SDP). The presented SMF integrates the devices present in the personal area of the end users regardless of their technology and provides basic services, e.g. for multimedia communication. The end user can combine intelligent and communicable devices as well as the basic services using the SCE and thus define personalised services for his personal area (e.g. automation tasks, sensor-dependent signalling or monitoring). An elementary requirement for the platform described above was that it provides an opportunity to develop services intuitively. An intuitive development can be realised by a graphical development process and by modelling the behaviour of a system independently from underlying technology. Both were implemented in the above-mentioned approach. The graphical development process and the underlying methodology are discussed in section III. 
By the approach proposed in $[1,9-13]$, the end user has thus been given the opportunity to create graphically and intuitively services for his personal environment. An approach has been defined in $[1,10$, and 12] as to how these services can be made available to other end users or organisations in order to gain added value through the use of the services. In $[1,10$, and 12] it was proposed that end users combine the individually offered services to provide a more complex, intelligent and more powerful service. As shown in Fig. 1, a complex service consists of the combination of several distributed services that are networked together. Two ways exist to combine services into an application. Multiple end users can offer the same service with the same local functionality running parallel/ synchronously (service aggregation). Alternatively, end users can offer services that have a different functionality and are composed to an application by linking the services together (service composition).

As described above, the central approaches for defining $\mathrm{M} 2 \mathrm{M}$ applications have various disadvantages and are not very flexible. Therefore, as a prerequisite for the approach introduced above, it was defined that creation and provision of individual services and the cooperative provision of an application (consisting of distributed services) is exclusively decentralised, i.e. without the integration of logical as well as physical central entities into the overall system. Under these circumstances, the realisation of the approach cannot be implemented with the existing architectures for M2M platforms. Therefore, an approach has been presented in $[1,10$, and 12$]$, which allows implementation following a purely decentralised approach.

This publication dedicates to present a more flexible methodology for realisation of M2M systems with the focus on dissolving the bindings to centralised entities, integration of the end user for realisation of $\mathrm{M} 2 \mathrm{M}$ applications satisfying the individual requirements of end users, and realisation without specialised and dedicated M2M infrastructures. Furthermore, this publication aims to introduce details about the functional architecture of the proposed concept as well as methodologies forming the basis for the presented concept of autonomous decentralised M2M application service provision

\section{FUNCTIONAL ARCHTTECTURE}

In order to clarify how an application in M2M context is structured, definitions of $[14,15$, and 16] are used to classify and separate service and application in context of application/ service provision in M2M (illustrated in Fig. 2 ). It illustrates that an application consists of one or more underlying services that are combined (i.e. aggregated or composed) and, if required, exchange information. An application service contains the application and an application interface to make the application consumable for other entities. The services are realised by one or more service components, which form the building blocks of services. The service

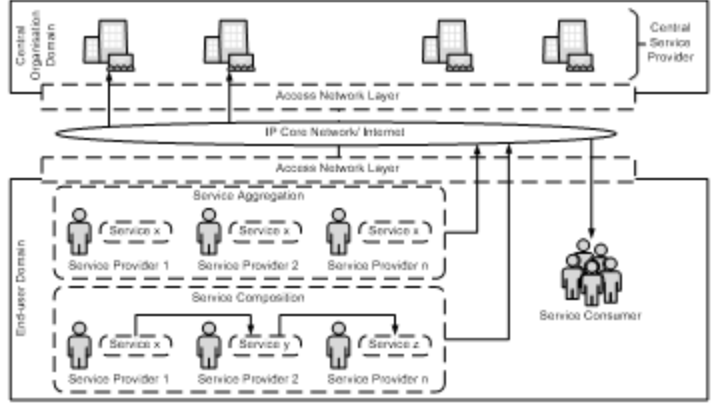

Fig. 1. Cooperative M2M Application Service Provision.

components itself are blocks of services. The service components itself are realised via several software applications executed on several execution environments.A service as well as an application can be realised on technical or non-technical principles (i.e. it can be provided using technical devices, e.g. computers, or by a human, e.g. personal assistance services). To distinguish the kinds of services and applications, if required they are indicated as technical service/ application or non-technical service/ application. General indication of both is service/ application.

Fig. 3 shows the functional architecture of the concept for the autonomous P2P-based provision of $\mathrm{M} 2 \mathrm{M}$ application services. The architecture is structured into four layers. The following describes the respective layers in their functionality. Network Layer: End user's M2M gateways are connected with each another via an IP network, respectively the Internet. End users can be located at different geographic locations as well as in different access networks (wireless mobile networks or fixed access networks), which are interconnected via a core network. P2P Network Layer: End user nodes represent equivalent instances and are connected to each other via a P2P network. The P2P Network Layer includes the sub-layer P2P Overlay Layer and P2P Communication Layer. Any data storage in the presented system is decentralised via the end user clients within the P2P Overlay layer. For this purpose, the clients, respectively the peers form a $\mathrm{P} 2 \mathrm{P}$ Overlay network. The Overlay network is either a pure structured Overlay network or a pure unstructured Overlay network (due to the general restriction to avoid also partly centralised system architectures). The Overlay network is used as explained in $[10,11,13]$ to store the addressing

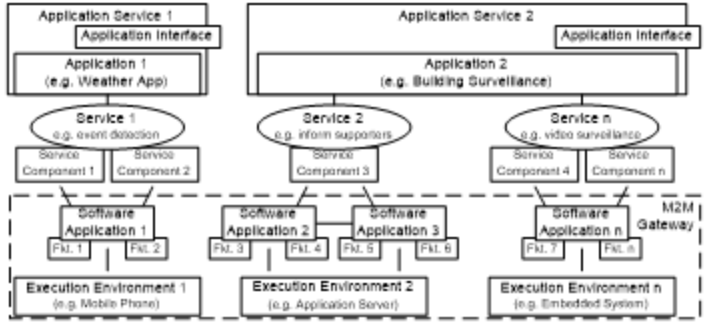

Fig. 2. Classification of Service and Application. 


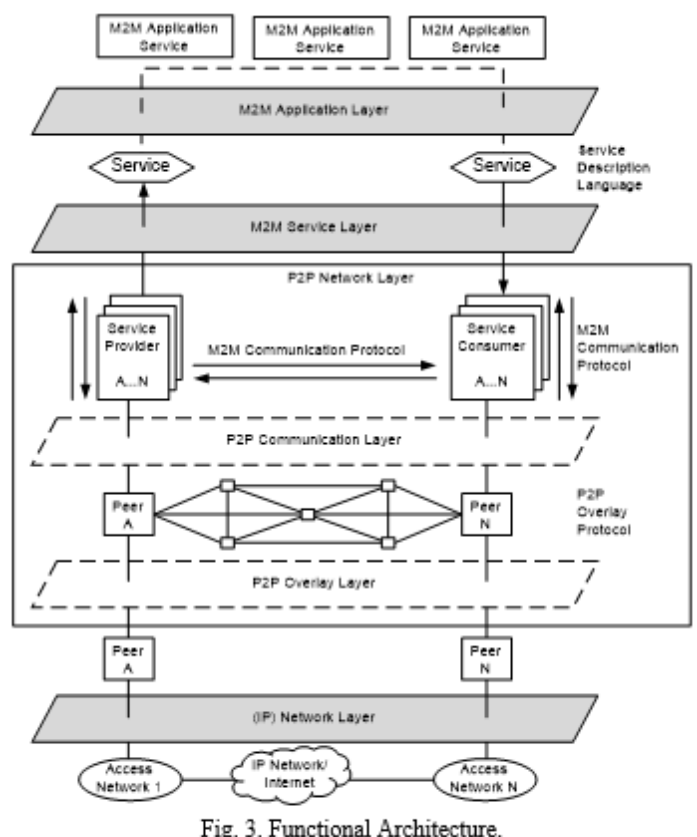

information of the peers (assignment temporary to static contact address). In addition, the P2P Overlay network is used to manage and configure services and applications (described in section IV). The information exchange between the peers for the service utilisation (service requests, confirmations) as well as the necessary signalling to generate the application automatically is also done directly between the peers. For this purpose, various M2M communication protocols (e.g., CoAP, HTTP, SIP, MQTT) can be used. M2M Service Layer: The services defined and provided by the end users are available via the M2M service layer and can be used via the corresponding interface. As described in section III and [9], the services are formally described by a service description language. The service interface is described by an interface specification and is available in the M2M Service Layer (explained in section III). M2M Application Layer: The distributed M2M application services are implemented within the M2M application layer. For this purpose, the services available via the M2M Service Layer are combined with each other as described in section III. The exchange of information between the services, respectively applications takes place via the $\mathrm{P} 2 \mathrm{P}$ network layer by $\mathrm{P} 2 \mathrm{P}$ communication protocols. [17]

\section{SYSTEM STRUCTURE AND APPLICATION CREATION}

In existing platforms for providing $\mathrm{M} 2 \mathrm{M}$ applications, the configuration of automation tasks or the creation of application logic is highly dependent on the implementing systems. To prevent this, the concept defined in this research work was designed with a modelbased system structure, derived from [18]. Models, expressed by modelling languages, describe model-based infrastructures and systems. The behaviour of an application or a system is described by means of a platform-independent model, separated from the technology-specific realisation of the system [18]. The behaviour of a system can be modelled with a behavioural model in an abstract manner with much lesser complexity than the platform that implements the behaviour of the system or the application [19]. By stepwise abstraction, respectively transformation, an abstract modelled system is transformed acc. [20] into a concrete system by means of multi-step transformation. Fig. 4 shows the transformation level defined for the approach described in this publication. Derived from [20], transformation and mapping rules have been defined to automatically process the transformation from the abstract model of the system created by the user to the platform-specific models shown in Fig. 4 of the novel architecture for provision of distributed M2M applications described in this publication. As described above, the intuitive development of services and applications is done by graphically modelling the behaviour of a service or creating a behaviour model of service/ application. This allows end users to describe the system without having to have any specific knowledge about the execution platform. The behaviour of a system can be modelled intuitively with a state machine. For this purpose, states represent the devices/ services that should be combined, and transitions represent the connections (information flow) between the devices/ services. To enable end user to model a system using state machine concept, a domain independent modelling language is required which can be used to describe the behaviour of a system abstracted in form of a state machine. [17] The following requirements were initially defined for the selection of an optimal modelling language for this purpose: Machine readability: Since no further entities are to be involved in the application generation or its implementation, i.e. the application, respectively the service should be generated automatically after modelling, the modelling language must be machinereadable. It must therefore be a formal language. Standardised language: It must be a standardised

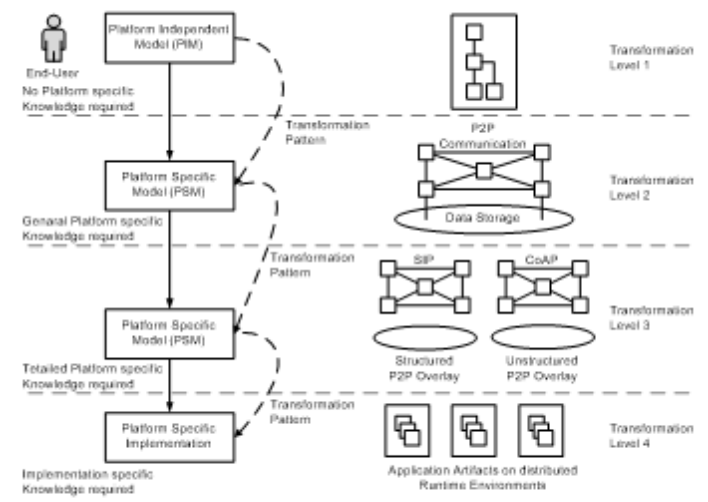

Fig. 4. Transformation Level. 
modelling language in order to ensure the easiest portability. Immediate mapping of graphical and machine-readable notation: Since graphical modelling by the end user was defined as a central aspect of the concept, a direct mapping of the graphical notation (elements of the state machine) to the machine- readable notation must exist. Intuitive usability: Since the system is to be used by an average technically experienced end user after a short training, the complexity of the graphical notation has to be low. Complex and non-intuitive modelling forms reduce or eliminate end user usability. Existing parser/ interpreter implementation: To enable further processing the formal language by a machine, an implementation of a corresponding parser or interpreter must exist. Nested states: Combination of application services. A system (M2M application service) should be combined with other systems. A previously modelled system is to be reused by being embedded in another application. Parallel flows: Within an application, it should be possible to define parallel sequences in order to implement concurrent tasks. State parametrisation: The services that are to be combined must have the possibility to be parameterised (definition of configuration/ input and output parameters). Therefore, the states in the state machine must also be parameterisable. Conditional state transitions: The transition of a state or transmission of information to a service or the activation of a service should be provided with a condition. Synchronisation of states: To synchronise parallel flows, there must be a possibility for synchronisation. According to these requirements, the following state machine-based modelling languages and modelling concepts have been examined: Business Process Modeling Notation (BPMN), Business Process Modeling Language (BPEL), UML State Machines, Petri Nets, and Finite State Machines. The results of the evaluation are shown in TABLE I. Requirements the modelling languages/ concepts satisfy are marked with "+"; requirements not satisfied are marked with "-" ; requirements partially satisfied are marked with " 0 ". The evaluation of the above-mentioned modelling languages showed that UML State Machines fully meet the requirements. UML State Machines are based on Harel Statecharts, which, due to their structure, meet the structural requirements for the state machine. The standardised description language StateChart XML (SCXML) makes it possible to express Harel statecharts in a formal notation. An SCXML parser, which is also available, enables the machine readability and thus the automatic processing of the formally described state machine. [17]

For modelling of a system, the SMF, as described in $[9-11,13]$, provides a GUI to the end user. Using this GUI, the end user graphically creates a state machine that represents the behaviour of the system. The GUI provides an overview of all available devices and services. The services and smart devices that are in the personal environment of the end users, e.g. in its Smart Home, are
Table I: Evaluation of State Machine Modeling Languages

\begin{tabular}{|l|c|c|c|c|c|}
\hline \multirow{2}{*}{} & \multicolumn{5}{|c|}{ Modelling Language } \\
\cline { 2 - 7 } & BPMN & BPEL & UML & $\begin{array}{c}\text { Petri } \\
\text { Nets }\end{array}$ & FSM \\
\hline Machine read. & - & + & + & + & - \\
\hline Standardised & + & + & + & + & - \\
\hline Immediate map. & - & + & + & + & - \\
\hline Intuitive usability & + & $\circ$ & + & - & + \\
\hline $\begin{array}{l}\text { Parser/ } \\
\text { Interpreter }\end{array}$ & - & + & + & + & - \\
\hline Nested states & $\circ$ & $\circ$ & + & + & - \\
\hline Parallel Flows & + & + & + & + & - \\
\hline State param. & - & + & + & - & - \\
\hline $\begin{array}{l}\text { Conditional } \\
\text { trans. }\end{array}$ & + & + & + & $\circ$ & - \\
\hline Sync. states & $\circ$ & + & + & + & - \\
\hline
\end{tabular}

stored within the SMF in a local repository. The services offered by other peers are stored in the P2P Overlay as described in following section IV. An overview of the services stored in the P2P Overlay is also loaded by the SCE as described in section IV and displayed in the GUI. The end user now graphically combines the available services and devices by first selecting the desired devices or services and then connecting the input and output interfaces defined for the services and devices (transition of the state machine). As described above, the user models the behaviour of service/ application by defining a state machine. The following is a description of the elementary elements of the state machine, as well as the semantic defined by mapping the elements of the state machine to the service/ application components. In addition to these elements, further elements of a state machine can be used, which are not further explained at this point. State element: Devices, services, and applications are represented by states. State parameter: The state parameters represent the data model of the devices, services and applications. Input, output and configuration parameters can be defined. Global state machine parameter: This defines global attributes of a service/ application. Global attributes are available for all services and applications and are queried directly from the source. Transition: The transition from one state to another state (transition target) represents the connection of devices, services and applications and the associated information exchange. Transition condition: Transitions can be provided with conditions. This defines the precondition when device, service or application sends an information. Conditions can be optionally defined. Transition assign element: The transitions of a state machine have assign elements. These assign elements define how the output parameters are assigned to the input parameters of a device, service or application. Thus, the contents of the messages that are exchanged are specified via this element. Nested state element: Using the nested state element enables embedding an already 
defined state machine, i.e. a service or an application, into another service or application. For this purpose, the corresponding state machine is embedded into a nested state element. The nested state element then is integrated as a simple state into the sequence of the state machine. Parallel state element: The parallel state element is used to model parallel sequences that can be synchronised as required at the end. [17]

The interface description of a device, service or application represents the template for a state. It is embedded in a service or application description or managed separately. The interface description specifies the input/ output and configuration parameters as well as the value ranges of a service/ application. The interface description is used as a template to represent the devices, services or applications graphically and functionally in the GUI. In addition to the above parameters, the interface description contains further parameters for classifying and describing a device, service or application by e.g. keywords or prose. The interface description can be stored in a local repository or stored in the Overlay network. In addition, it is possible to request the interface description directly from a peer that offers a service. [17]

The SCE automatically generates the formal description of the end user-modelled system using SCXML. Service/ application are completely described by the generated SCXML document. If it is a local service, the application description is stored locally, parsed, interpreted and executed according to the defined conditions. If it is a distributed application, i.e. consisting of distributed services offered by different peers, the application description is distributed to the peers that offer a service in the application context. In this case, each peer receives only the part of the application description that is required to configure and execute its individual service. Peers therefore do not have an overview of the overall system, which performs as a basis for securing the system. [17]

\section{APPLICATION REGISTRY, CONFIGURATION, EXECUTION}

As described in section II, data storage in the described approach is performed via structured or unstructured P2P Overlay networks. The following is a description of the functions for registering and searching, which are implemented using the Overlay network for the management of services and applications. Services can be offered by different peers. The peers register their services via the Overlay network. To do so, they store the combination of service ID and their associated personal temporary contact information in the Overlay. If different peers offer the same services, several associated peers are stored under the same entry in the Overlay network. Each peer represents a specific instance of a service. Peers can request the Overlay network to get an overview of all available services or a certain subset of all services. This overview is required to generate an application from the various services as described in section III. Sub-sets of services may include e.g. that services are only available for a particular region, have further meta information that can specify a service more detailed, can only be consumed by a particular group, or implement a particular interface description. Depending on the type of request, the Overlay networks offer various ways to provide the overview of the services. For example, the meta-informations stored for a service can be searched by keywords. In order to obtain a specific instance of a service during the application configuration (see below), a peer makes a request (possibly restricted by search criteria) to the Overlay network. This returns a list of all found instances of a service. [17]

An M2M application service consists of several services that are interconnected and exchange information. An M2M application is thus a concatenation of distributed services. Each service has input and output interfaces. To realise an application, the output interfaces of a service are connected to the input interfaces of another service, and the information is passed on from one service to the other. The description of the application, i.e., which service is associated with which other service, is defined by a formal application description (described in section III). The approach described in this publication has two phases to realise an application (illustrated in Fig. 5). A configuration phase in which the application is generated automatically and an execution phase in which the application is running. First, the following section describes the configuration phase. Each peer providing a service involved in an application receives the formal description of the application. As described in section III, each peer only receives the segment of the application description, which contains the necessary information to configure its individual service and insert it into the overall context of the application according to the application description. Inserting into the overall context of the application means that the peer connects the interfaces (input, output) of his service to the other associated services in order to exchange information. By automatically parsing the application description, the peer determines the following information. Service connections: To which other

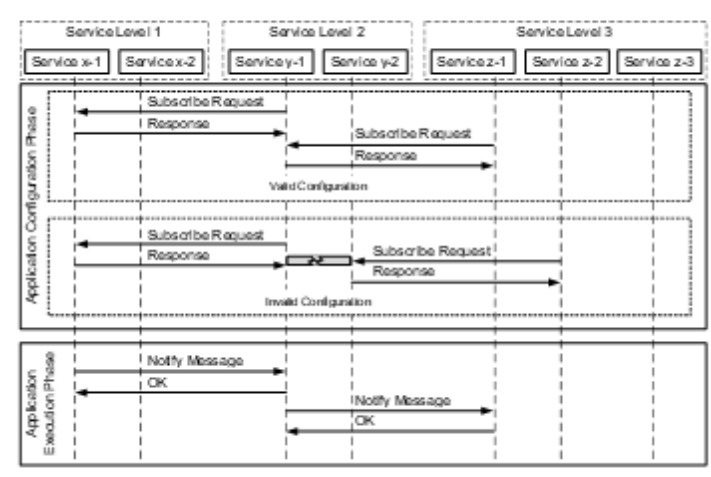

Fig. 5. Application Configuration and Execution Phase. 
services his offered service should be connected. A distinction is made here between input interfaces served by another service and output interfaces which are served by the service itself. Input interface information content: Which information is received via the input interface. Output interface information content: What information via the output interface should be transferred to another service. Output interface condition: Under which prerequisites an information is to be sent to a service via an output interface (directly on request or time-delayed, if a defined condition is met). Service configuration parameter: If configuration parameters have been defined for the offered service, the service must be configured accordingly. Global attributes: Information about the attributes provided by services that are not directly connected to their own service via an interface. [17]

Each peer now uses the configuration information, determined by the automated parsing of the formal application description, to integrate its service autonomously into the overall context of the application. First, each peer determines the specific instances of the services to be connected to it, i.e. determines a list of peers that offer the service based on the service ID. This is done as described above. The exchange of information takes place in the approach described in this publication according to the Subscribe/ Notify principle. The connection of an input interface of a service with the output interface of another service is performed by sending the peer with its output interface a subscribe request. If the requested service is available, the requested peer confirms service utilisation request with a positive response. If the service is not available or is available later, the requested peer responds with a negative response and rejects to consume the service or defines a later usage time. Once the connections between the instances of the services are established, the local configuration of the service is performed. This consists of the assignment of the defined input and output information content to the interfaces (transition assign). Furthermore, the transfer of any existing service configuration parameters to the local service is performed. In addition, the conditions are configured locally that define the preconditions to transmit an information to a connected service via the output interface (transition condition). [17]

As described above and shown in Fig. 5, the described approach also includes the execution phase additionally to the configuration phase of an application. In the execution phase, the exchange of information takes place between the peers, which represents the M2M application service. Once the connections between the service instances have been established, the information is transmitted between the services using Notify messages. Sending a notification message either is done directly on request (global attribute or condition less transition) or as soon as a defined condition (transition condition) applies. [17]
It should be noted that the same service can be provided by several instances (peers). This may result in incomplete configurations of an application during the configuration phase. In addition to a complete configuration of an application, Fig. 5 also shows an incomplete configuration of an application). For this purpose section $\mathrm{V}$ presents an algorithm allowing all peers to determine independently if they have embedded themselves in a valid application configuration. The algorithm developed for this purpose is based on the computation of the transitive closure derived from [21] of all connections established in the P2P network in the application context. If a peer detects that it has embedded itself in an invalid application configuration, the established connections are automatically disconnected and, if necessary, a new local configuration process is performed. Thus, it is possible to determine independently a valid application configuration without having to have a complete view of the system. [17]

\section{APPLICATION VALIDATION}

Fig. 5 shows in the Invalid Configuration section that no path exists between the first services (service at most left service level) and the last services (service at most right service level). This means, although the peers have correctly configured their individual service connections according the application description, the application was not configured correctly in total. A correctly configured application contains at least one closed path through all service levels, starting at an instance at first service level and ending at an instance of a service at the most right service level. Because the invalid configurations are unusable for the application configuration, they first must be identified as described in the following and afterwards removed again.

The peers require an overview of the overall configuration of the application in order to identify independently whether they have established an invalid connection to another peer. I.e. they need an overview of peers included in a specific instance of the application service connected to other peers. In order to provide such an overview of the overall configuration, the peers must document to which other peers they have established a connection. This documentation have to be available to all other peers, which in turn use the documentation to identify the invalid connections. As the P2P Overlay inside the P2P network layer is a shared database, it is used to store the connection information.

The connections of the services are mapped to a directed graph by representing the peers as nodes and the connections between the peers as edges between the nodes. Arithmetic operations applied to that graph enable to determine whether a closed connection exists from an initial node to an end node. All nodes whose connections are not in the path between the start and end nodes have invalid connections and must remove them again. The structure of the graph, represented as adjacency matrix, is 
stored as a data structure inside the Overlay. The structure of the adjacency matrix is defined as follows. The nodes of a graph are specified as row and column names in the adjacency matrix, and the connections between the nodes as entries in the associated fields in the adjacency matrix. Because it is a directed graph, the nodes from which a connection originates are specified in the row names, and the destination nodes of these links in the column entries. A uniform name for the column and row entries is defined as starting with the service ID as prefix and appending the specific peer ID as a suffix.

If a peer has established a connection according to the application description with another peer, respectively has associated a service offered by him with another service, it executes the algorithm shown in Fig. 6 in order to document the established connection in the adjacency matrix. The following describes how peers can use the connection overview stored in the Overlay in order to identify and remove the invalid connections.

The arithmetic operations and algorithms applicable on graphs allow the evaluation of characteristics of a graph The Transitive Closure of a binary relation in a graph (link between nodes), calculated e.g. by the algorithm of Warshall [21], indicates which node pairs are mutually accessible. This information determines whether a destination node is reachable from a start node, possibly with the inclusion of other nodes. All nodes contained in the transitive closure of a node can be accessed from the associated node. Since the connection graph is stored in the Overlay, all peers request and use it to compute the transitive closure of the connection graph independently. Thus, each peer gets an overview of which peers are reachable among each other. In order to verify the complete configuration of an application, each peer, for its individual node in the connection graph autonomously executes the algorithm described in Fig. 7. First, the peer queries the complete adjacency matrix of the connection

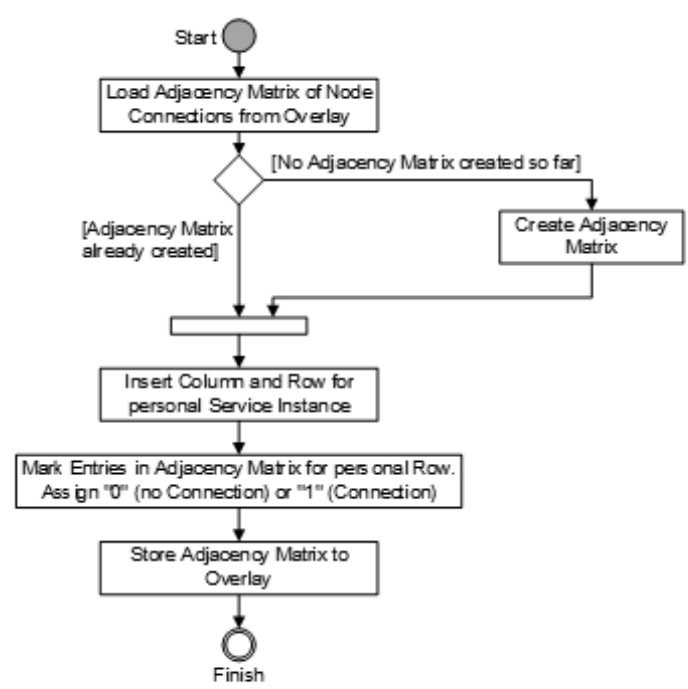

Fig. 6. Algorithm for Documentation of Connection Establishments.

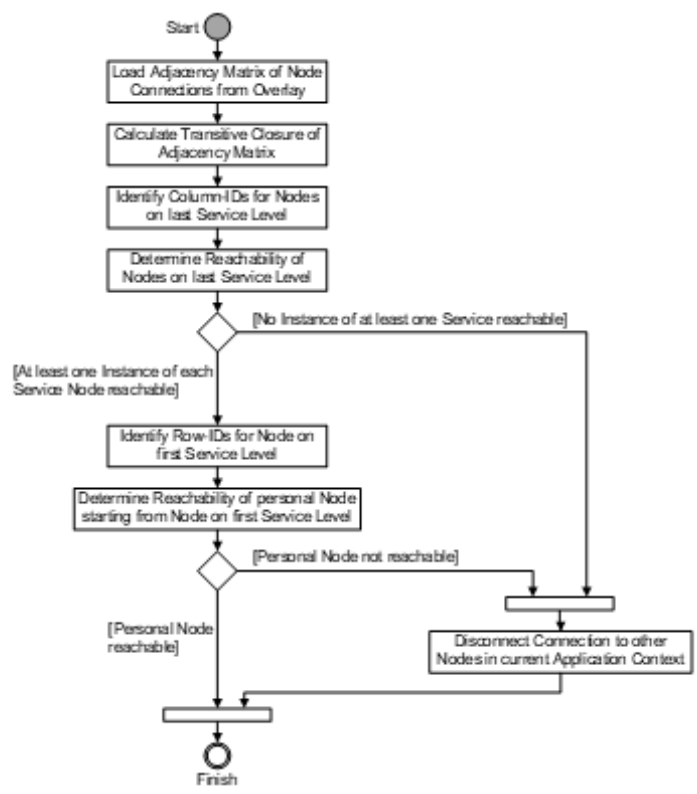

Fig. 7. Algorithm for Determination of invalid Application Service Configuration.

graph from the Overlay. For the resulting connection graph, the peer then calculates the transitive closure. Thus, the peer has an overview of which nodes are mutually accessible. The column entries for the nodes on the last service level are determined afterwards. The determination of the services at the last service level is made possible since the last services in the formal application description are marked as "final state". This determines the prefix of the column description. The calculating peer does not know the suffix, but by the uniformly defined format of the column names enables the determination of the column name without knowledge about the suffix. After identifying the nodes at the last service level, it is checked whether these nodes, starting from the own node, are reachable. If there is not at least one instance of each service at the last service level reachable, an invalid configuration of the application exists and the connection to the peers must be removed again. If a connection to the instances at the last service level exists, the next step checks whether the own node is accessible by an instance of a service on the first service level. For this purpose, the row $\mathrm{ID}$ of the nodes on the first service level is determined first. The determination of the service on the first service level is made possible by marking the first service in the formal application description as "initial state". This determines the prefix of the line description. Similar to the determination of the column ID, this makes it possible to identify the row IDs of nodes on the first service level. After identifying the nodes on the first service level, it is checked whether the own node is accessible from one of these nodes. If this is the case, a continuous connection from the first and the last service level exists.

Incomplete configuration of the application exists 
when: first, at least one instance of each service on the last service level of the connection graph cannot be reached from its own node and/ or second, the own node cannot be reached by at least one instance of the service at the first service level of the connection graph. If a peer detects that it is within an invalid configuration of the application, it removes the connections to other peers. Removing a connection between the peers is done by sending a request to the connected peer with the request to disconnect.

\section{PROTOTYPE AND EVALUATION RESULTS}

In order to verify the above introduced concepts for autonomous decentralised M2M Application Service Provision as well as to evaluate the behaviour of the P2P Network Layer a prototype has been realised implementing the described M2M service platform. This section presents the testbed setup and the performance results. As prototype hardware a high performance server system is used equipped with $2 \times 24$ CPUs und $2 \times 64$ GB RAM. This server system contains the hypervisor OS Proxmox [22] that provides virtual host systems and virtual networks. This makes it possible to implement the prototype system without affecting the measurements by activities that can occur in a physical network. In order to implement the scaling of the M2M system, the container virtualisation system Docker [23] is used within the virtual hosts. This makes it possible to emulate a large number of standalone containers, i.e. peers, without being restricted by the hardware requirements of virtual machines, as would be the case when using a hypervisor virtualisation solution. Fig. 8 shows the prototype setup. It consists of two VMs each implementing a Docker host. Docker hosts are connected to each other via two separate virtual networks. The Docker hosts contain several Docker containers whose number is scalable as well as internal networks. Through the internal networks, different access networks are emulated via which the peers, represented by the Docker containers, are connected. This makes it possible to evaluate not only the P2P system itself, but also the behaviour of distributed M2M applications in different network topologies. The prototype includes two separate networks to which the Docker hosts as well as the peers themselves are connected: the P2P/ M2M Network (illustrated with solid lines) and a separated Management Network (illustrated

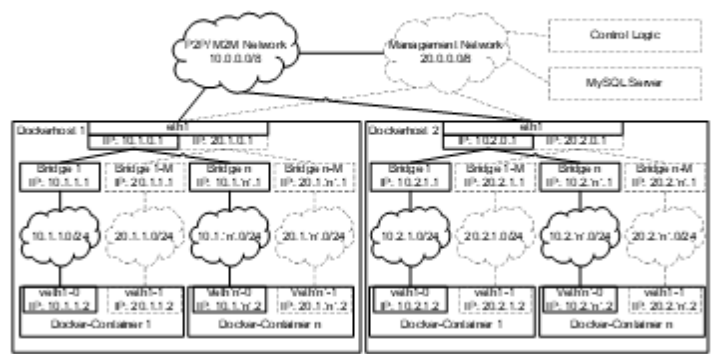

Fig. 8. Prototype Setup.
Table II: Evaluation of M2M Protocol Behaviour

\begin{tabular}{|l|c|c|c|c|}
\hline \multirow{2}{*}{ Use Case } & \multicolumn{4}{|c|}{ M2M Protocol } \\
\cline { 2 - 5 } & \multicolumn{2}{|c|}{ CoAP } & \multicolumn{2}{c|}{ SIP } \\
\cline { 2 - 5 } & Packets & Bytes & Packets & Bytes \\
\hline $\begin{array}{l}\text { Successful subscription } \\
\text { establishment }\end{array}$ & 2 & 138 & 6 & 2593 \\
\hline $\begin{array}{l}\text { Unsuccessful subscription } \\
\text { establishment }\end{array}$ & 2 & 119 & 2 & 837 \\
\hline Sending notification & 2 & 126 & 2 & 913 \\
\hline Subscription refreshment & 2 & 139 & 4 & 1743 \\
\hline $\begin{array}{l}\text { Subscription termination by } \\
\text { a subscriber }\end{array}$ & 2 & 126 & 4 & 1718 \\
\hline $\begin{array}{l}\text { Subscription termination by } \\
\text { a notifier }\end{array}$ & 2 & 120 & 2 & 860 \\
\hline
\end{tabular}

with dashed lines). The management network is used to control the individual peers, perform and control performance measurements as well as to log them without affecting the behaviour of the networks over which the peers are connected. Two additional VMs are also connected to the management network: the Control Logic Server, which contains the control logic of the prototype, and a MySQL server that is used to log measurement results. The Docker containers contain the distributed application logic to provide the M2M services and to perform the autonomous configuration of the $\mathrm{M} 2 \mathrm{M}$ application. Based on this prototype setup the behaviour of the P2P network layer was analysed. The M2M communication protocols SIP and CoAP has been analysed as well as unstructured P2P Overlay networks represented by the Gnutella architecture [24] and structured P2P Overlay networks represented by the Chord architecture [25]. The analysis results are explained below. Table II shows the comparison of the M2M communication protocols SIP and CoAP for various communication scenarios for $\mathrm{M} 2 \mathrm{M}$ application configuration and execution. It can be seen here that the number of packets sent and the required data volumes when using CoAP is significantly lower than with SIP. The use of CoAP offers advantages with regard to the required number of packages as well as the data volume. If an existing telecommunication infrastructure is to be used for peer communication, SIP offers the advantage that no additional protocol stack is required. The network resources when using SIP are significantly reduced if the alternative TinySIP messaging library [26] is used for message exchange. It enables sending SIP messages with a maximum message size of 29 bytes. Fig. 9-12 shows the analysis of the significant characteristics of $\mathrm{P} 2 \mathrm{P}$ Overlays with scaling number of peers. Fig. 9 shows the effort for inserting/ searching/ removing operation of a data record in a structured P2P Overlay, as well as the initial entry of a peer to the Overlay. The effort in scaling the peers does not increase significantly. In comparison, Fig. 10 shows the effort for searching a data record in an unstructured P2P Overlay on the basis of the transmitted messages. The effort increases linearly to the number of peers and is dependent on the number of connections between nodes (ABN). This is caused by the Query 
Flooding process for message forwarding, which is typical for unstructured P2P systems. In Fig. 10 the queries (Query) and the corresponding responses (QueryHit) are shown. The queries are significantly higher than the answers. Compared to structured P2P Overlays, this is a crucial disadvantage. Both types of P2P Overlays require a stabilisation process of the network, which is executed continuously parallel to the data record requests. The effort for both approaches scales linearly. However, this is dependent on the respective interval of the stabilisation process of the algorithm. The analysis shows that a structured P2P Overlay requires significantly more effort for the stabilisation process than an unstructured Overlay. This is justified by the fact that the algorithm for validating the topology of the Overlay with a structured P2P Overlay is much more complex. In the case of an unstructured P2P Overlay, the simple request of accessibility of neighbouring nodes is done. The analysis of the P2P Overlay approaches has shown that the effort for finding a data record in an unstructured P2P Overlay is significantly higher than with a structured P2P Overlay. An unstructured P2P Overlay, offers the advantage that the effort to re-organise the Overlay when a peer leaves is significantly less. This is particularly advantageous when a frequent fluctuation of peers exist. Furthermore, in structured P2P Overlays the finding of an existing data record is guaranteed; on the other hand, this aspect cannot be ensured in unstructured P2P Overlays. In unstructured Overlays, however, a keyword-based search is possible.

\section{VII.CONCLUSIONS}

This publication presented principles and details of a novel concept for autonomous and decentralised M2M application service provision. This concept offers new possibilities for applications, realised by several peers, independent of central appliances or corporations. Especially the independent structure of presented system architecture and the simplicity of M2M service/ application creation combined with the autonomous management of $\mathrm{M} 2 \mathrm{M}$ application services form the strength of the proposed framework and offer a promising approach for supporting M2M networks with end user environment integration. The analysis of the M2M communication protocols and the P2P Overlay architectures has shown that all of them have their strengths and weaknesses. Optimal selection of the M2M protocols, respectively P2P Overlay architecture depends on the application scenario and network topology.

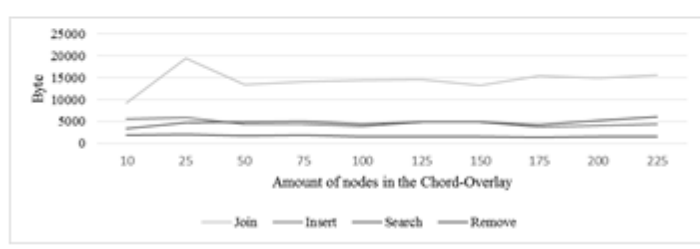

Fig. 9. Operations in structured P2P Overlay.

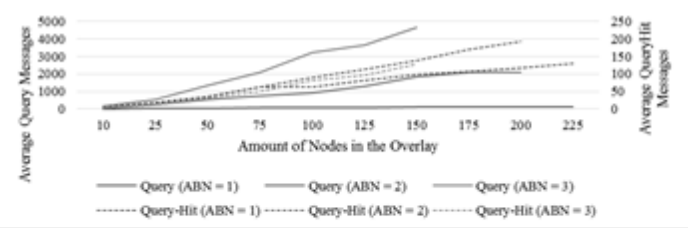

Fig. 10. Dataset Request Operation in unstructured P2P Overlay.

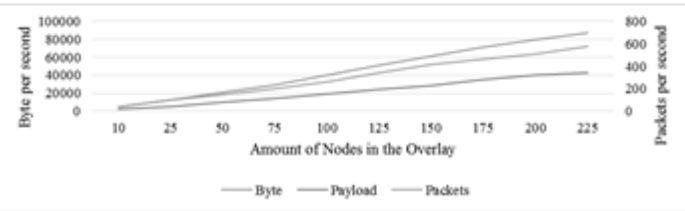

Fig. 11. Stabilisation Process of a structured P2P Overlay

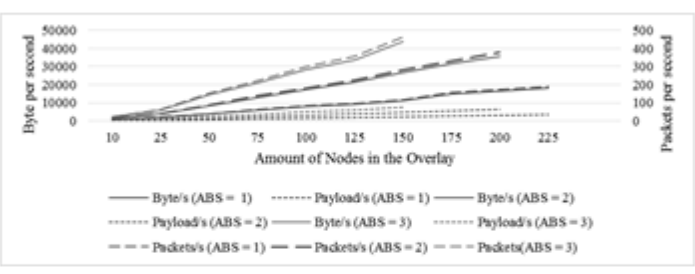

Fig. 12. Stabilisation Process of an unstructured P2P Overlay.

\section{ACKNOWLEDGMENT}

The research project $\mathrm{P} 2 \mathrm{P} 4 \mathrm{M} 2 \mathrm{M}$ providing the basis for this publication is partially funded by the Federal Ministry of Education and Research (BMBF) of the Federal Republic of Germany under grant number 03FH022IX5. The authors of this publication are in charge of its content.

\section{REFERENCES}

[1] M. Steinheimer, U. Trick, W. Fuhrmann, B. Ghita, "P2P based service provisioning in M2M networks", Proc. of Sixth International Conference on Internet Technologies \& Applications (ITA 15), Wrexham, Wales, UK, September 2015.

[2] S. Clayman, A. Galis, "INOX: a managed service platform for interconnected smart objects", Proc. of the workshop on Internet of Things and Service Platforms 2011 (loTSP'11), pp. 1-8, December 2011.

[3] S. Zhang, J. Zhang, W. Li, "Design of M2M Platform Based on J2EE and SOA", 2010 International Conference on E-Business and E-Government, pp. 2029-2032, September 2010.

[4] Q. Xiaocong, Z. Jidong, "Study on the structure of "Internet of Things(IOT)" business operation support platform", 2010 12th IEEE Int. Conf. Commun. Technology (ICCT), pp. 1068-1071, November 2010.

[5] L. Foschini, T. Taleb, A. Corradi, D. Bottazzi, "M2Mbased metropolitan platform for IMS-enabled road traffic management in IoT", IEEE Commun. Mag., vol. 49, no. 11, pp. 50-57, November 2011.

[6] Y. J. Kim, E. K. Kim, B. W. Nam, I. Chong, "Service composition using new DSON platform architecture for M2M service", Proceedings of the International 
Conference on Information Network 2012, pp. 114-119, February 2012.

[7] J. Kim, J. Lee, J. Kim, J. Yun, "M2M Service Platforms: Survey Issues and Enabling Technologies", IEEE Communications Surveys \& Tutorials, vol. 16, no. 1, pp. 61-76, First Quarter 2014.

[8] oneM2M Standardisation Committee, "oneM2M Release 2 specifications", http://www.onem $2 \mathrm{~m}$.org/technical/publishe d-documents, December 2016.

[9] M. Steinheimer, U. Trick, W. Fuhrmann, B. Ghita, "Load reduction in distribution networks through $\mathrm{P} 2 \mathrm{P}$ networked energy-community", Proc. of Fifth International Conference on Internet Technologies \& Applications (ITA 13), Wrexham, Wales, UK, September 2013.

[10] M. Steinheimer, U. Trick, W. Fuhrmann, B. Ghita, "P2Pbased community concept for M2M Applications", Proc. of Second International Conference on Future Generation Communication Technologies (FGCT 2013), London, UK, December 2013.

[11] M. Steinheimer, U. Trick, P. Ruhrig, R. Tönjes, M. Fischer, D. Hölker, "SIP-basierte P2P-Vernetzung in einer Energie-Community", ITG-Fachbericht 242: Mobilkommunikation, VDE Verlag $\mathrm{GmbH}$, Osnabrück, Germany, ISBN: 978-3-8007-3516-7, ISSN: 0932-6022, pp. 64, Mai 2013.

[12] M. Steinheimer, U. Trick, W. Fuhrmann, B. Ghita, "P2Pbased M2M Community Applications", Proc. of Eleventh International Network Conference (INC 2016), Frankfurt, Germany, July 2016.

[13] M. Steinheimer, U. Trick, P. Wacht, P. Ruhrig, "Decentralised optimisation solution for Smart Grids using Smart Market aspects and P2P internetworked EnergyCommunity", IEC/ SGCC/VDE World Smart Grid Forum 2013, Berlin, Germany, September 2013.

[14] ITU-T Y.101 (2000), Recommendation, "Global Information Infrastructure terminology: Terms and definitions", ITU, 2000.

[15] ISO IEC 20000-1:2011 (2011) "Part 1: Service management system requirements", IEEE, 2011.

[16] ITIL V3.1.24 (2007) "Glossary of Terms, Definitions and Acronyms", ITIL, 2007.

[17] M. Steinheimer, "Optimierte P2P-Dienstearchitektur für hochverfugbare M2M-Applikationen (P2P4M2M)", TR004, Frankfurt, Germany, October 2016.

[18] Object Management Group, "OMG Model Driven Architecture", http://www.omg.org/mda/, January 2017.

[19] Object Management Group, "Model Driven Architecture (MDA) MDA Guide rev. 2.0", Boston, USA, June 2014

[20] R. Petrasch, O. Meimberg, "Model Driven Architecture", dpunkt.verlag, Heidelberg, Germany, ISBN: 978-3-89864$343-6,2006$

[21] V. Turau, "Algorithmische Graphentheorie", De Gruyter Oldenbourg, Munich, Germany, ISBN: 978-3-486-59057$9,2009$.

[22] Proxmox Server Solutions, https://www.proxmox.com/en/, April 2017.

[23] Docker, https://www.docker.com/, April 2017.

[24] The Gnutella Protocol Specification v0.4, http://web.stanfo rd.edu/class/cs244b/gnutella_protocol_0.4.pdf, April 2017.

[25] I. Stoica, R Morris, D. Karger, M. Kaashoek, H. Balakrishnan, "Chord: A Scalable Peer-to-peer Lookup
Service for Internet Applications", IEEE/ACM Transactions on Networking, Vol. 11, 2013.

[26] TinySIP, EUROPEAN PATENT SPECIFICATION EP 1 $968275 \mathrm{BI}$, October 2016

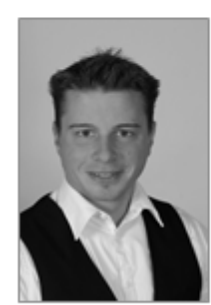

Michael Steinheimer received the B.Sc degree from the University of Applied Sciences Darmstadt, Germany, in 2008 and the M.Sc. degree from the University of Applied Sciences Darmstadt, Germany, in 2011 , both in computer science. He is currently pursuing the $\mathrm{Ph} . \mathrm{D}$. degree with the Centre for Security, Communications and Network Research, University of Plymouth UK. His research interests include decentralised service provision, Machine-to-Machine Communications, and Peer-to-Peer Networks.

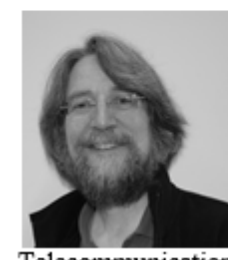

Ulrich Trick received the Dipl. Ing. degree from the University of Kaiserslautern, Germany, in 1983 in Electrical Engineering telecommunications. He received his Doctoral degree from the University of Kaiserslautern, Germany, in 1987. Since 2001 he is Professor for Telecommunication Networks with the Department for Computer Science and Engineering at Frankfurt University of Applied Sciences, Germany. His research interests include NGN, M2M, IoT, P2P and virtualisation.

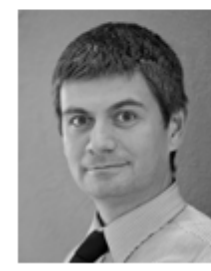

Bogdan Ghita received the Dipl. Eng. from Politehnica University of Bucharest, Romania, in 1998 and his $\mathrm{PhD}$ from Plymouth University, UK, in 2005. He is Associate Professor at Plymouth University and leads the networking area within the Centre for Security, Communications, and Network research. His research interests include computer networking and security, focusing on the areas of network performance modelling and optimisation, wireless and mobile networking, and network security. He has been principal investigator in several industryled, national, and $\mathrm{EU}$ research projects. He is the chair of the International Networking Conference series.

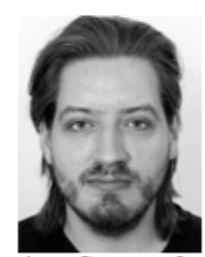

Gregor Frick received the B. Eng. degree in Electrical Engineering and Information Technology from the Frankfurt University of Applied Sciences, Germany, in 2014 and the M. Eng. degree in Information Technology from the Frankfurt University of Applied Sciences, Germany, in 2017. He is currently preparing his $\mathrm{Ph} . \mathrm{D}$. course with the Centre for Security, Communications and Network Research, University of Plymouth, UK. His research interests include Wireless Mesh Networks, Network Functions Virtualisation, and Disaster Networks.

Woldemar Fuhrmann is Professor at Department for Computer Science at the University of Applied Sciences Darmstadt, Germany. His research interests include Next Generation Mobile Networks and Telecommunications. 
Published in Proceedings of the 2017 IEEE International Conference on Smart Grid and Smart Cities (ICSGSC 2017), pp. 312-317, July 2017, Singapore, IEEE

\title{
Decentralised System Architecture for autonomous and cooperative M2M Application Service Provision
}

\author{
Michael Steinheimer, Ulrich Trick \\ Research Group for Telecommunication Networks \\ Frankfurt University of Applied Sciences \\ Frankfurt/M., Germany \\ steinheimer@e-technik.org
}

\author{
Michael Steinheimer, Bogdan Ghita \\ Centre for Security, Communications and Network \\ Research \\ University of Plymouth \\ Plymouth, UK
}

\author{
Woldemar Fuhrmann \\ Department for Computer Science \\ University of Applied Sciences Darmstadt \\ Darmstadt, Germany
}

\begin{abstract}
This publication presents a novel concept for autonomous and decentralised M2M application service provision. The functional architecture of the approach is introduced as well as a detailed description of the system structure and process for application creation. Furthermore, this publication describes details about the proposed process for decentralised M2M application service management and formal description of M2M application services.
\end{abstract}

Keywords-M2M application service; P2P; service provision

\section{INTRODUCTION}

Devices are becoming more and more intelligent (i.e they include complex functionality for monitoring and control). Additionally the devices receive the functionality for communication (this enables remote monitoring and controlling of devices). Because of the increasing number of intelligent devices, which support forming of intelligent environments, many new application fields can be established (e.g. as presented in [1] smart building, electro mobility, smart city, energy optimisation etc.). Especially the end user domain is an application field with powerful potential, however not addressable by external service providers.

Machine-to-Machine Communications (M2M) systems realise the integration of such intelligent devices by provision of specific M2M applications. Traditional M2M systems and the corresponding M2M application services have the focus to support specific business processes. Traditional M2M applications provide their functionality as a service for users (end users or companies). Currently existing concepts from standardisation and research to implement M2M application services provision focus on central approaches. Projects from research area are e.g. INOX Managed Service Platform [2], M2M Platform Project based on SOA [3], BOSP Business Operation Support Platform [4] IMS enabled M2M Service Platform [5], e-DSON [6] Commercial M2M platforms implement acc. [7] mostly the
oneM2M standard for M2M [8]. Disadvantages of the centralised approaches are e.g. that the user of the application is dependent of the platform provider as a central instance. Often M $2 \mathrm{M}$ platforms provide their functionality only for specific application fields and are therefore less flexible. Centralised provision of platforms requires many resources (costs for operation, maintenance, availability, application development). End users often have no possibility to define applications themselves (since the creation of applications requires expert knowledge or the end user does not have access to the platform) or the devices present in user's personal environment cannot be connected to the M2M platforms.

The personal environment of the end user described above is difficult to address by external service providers, since the activities (controlling and monitoring of M2M devices) carried out in this area would severely affect the end user or challenge data security. To antagonise this issue, it was proposed in [9] to integrate the end user. For this purpose, an approach was presented in [9-12], which allows end user to define services and automation tasks for their personal environment. The Service Management Framework (SMF) presented in [9-11, 13] consists of a local Service Creation Environment (SCE) and a Service Delivery Platform (SDP). The presented SMF integrates the devices present in the personal area of the end users regardless of their technology and provides basic services, e.g. for multimedia communication. The end user can combine intelligent and communicable devices as well as the basic services using the SCE and thus define personalised services for his personal area (e.g. automation tasks, sensordependent signalling or monitoring). An elementary requirement for the platform described above was that it provides an opportunity to develop services intuitively. An intuitive development can be realised by a graphical development process and by modelling the behaviour of a system independently from underlying technology. Both were implemented in the above-mentioned approach. The 
graphical development process and the underlying methodology are discussed in section III.

By the approach proposed in $[1,9-13]$, the end user has thus been given the opportunity to create graphically and intuitively services for his personal environment. An approach has been defined in $[1,10$, and 12] as to how these services can be made available to other end users or organisations in order to gain added value through the use of the services. In $[1,10$, and 12$]$ it was proposed that end users combine the individually offered services to provide a more complex, intelligent and more powerful service. As shown in Fig. 1, a complex service consists of the combination of several distributed services that are networked together. Two ways exist to combine services into an application. Multiple end users can offer the same service with the same local functionality running parallel/ synchronously (service aggregation). Alternatively, end users can offer services that have a different functionality and are composed to an application by linking the services together (service composition)

As described above, the central approaches for defining M2M applications have various disadvantages and are not very flexible. Therefore, as a prerequisite for the approach introduced above, it was defined that creation and provision of individual services and the cooperative provision of an application (consisting of distributed services) is exclusively decentralised, i.e. without the integration of logical as well as physical central entities into the overall system. Under these circumstances, the realisation of the approach cannot be implemented with the existing architectures for M2M platforms. Therefore, an approach has been presented in $[1$, 10 , and 12], which allows implementation following a purely decentralised approach.

This publication dedicates to present a more flexible methodology for realisation of M2M systems with the focus on dissolving the bindings to centralised entities, integration of the end user for realisation of M2M applications satisfying the individual requirements of end users, and realisation without specialised and dedicated M2M infrastructures. Furthermore, this publication aims to introduce details about the functional architecture of the proposed concept as well as methodologies forming the basis for the presented concept of autonomous decentralised M2M application service provision.

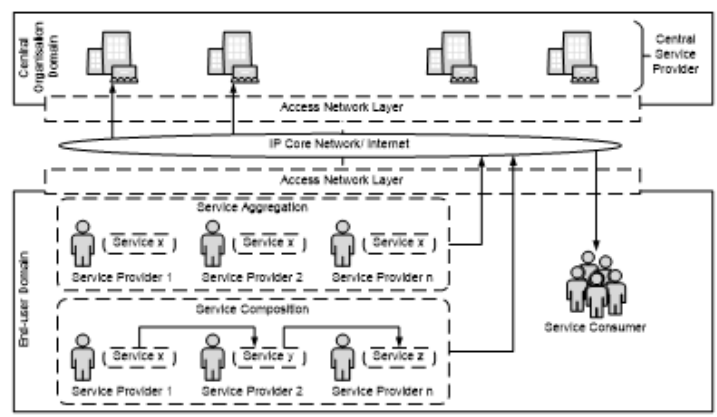

Figure 1. Cooperative M2M Application Service Provision.

\section{FUNCTIONAL ARCHITECTURE}

In order to clarify how an application in M2M context is structured, definitions of $[14,15$, and 16] are used to classify and separate service and application in context of application/ service provision in M2M (illustrated in Fig. 2). It illustrates that an application consists of one or more underlying services that are combined (i.e. aggregated or composed) and, if required, exchange information. An application service contains the application and an application interface to make the application consumable for other entities. The services are realised by one or more service components, which form the building blocks of services. The service components itself are blocks of services. The service components itself are realised via several software applications executed on several execution environments.A service as well as an application can be realised on technical or non-technical principles (i.e. it can be provided using technical devices, e.g. computers, or by a human, e.g. personal assistance services). To distinguish the kinds of services and applications, if required they are indicated as technical service/ application or non-technical service/ application. General indication of both is service/ application.

Fig. 3 shows the functional architecture of the concept for the autonomous P2P-based provision of M2M application services. The architecture is structured into four layers. The following describes the respective layers in their functionality. Network Layer: End user's M2M gateways are connected with each another via an IP network, respectively the Internet. End users can be located at different geographic locations as well as in different access networks (wireless mobile networks or fixed access networks), which are interconnected via a core network. P2P Network Layer: End user nodes represent equivalent instances and are connected to each other via a P2P network. The P2P Network Layer includes the sub-layer P2P Overlay Layer and P2P Communication Layer. Any data storage in the presented system is decentralised via the end user clients within the P2P overlay layer. For this purpose, the clients, respectively the peers form a P2P overlay network. The overlay network is either a pure structured overlay network or a pure unstructured overlay network (due to the general restriction to avoid also partly centralised system architectures). The overlay network is among other things used as explained in $[10,11,13]$ to store the addressing information of the peers (assignment temporary to static

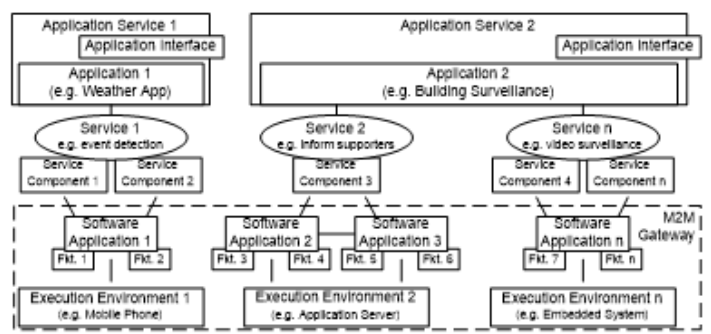

Figure 2. Classification of Service and Application. 


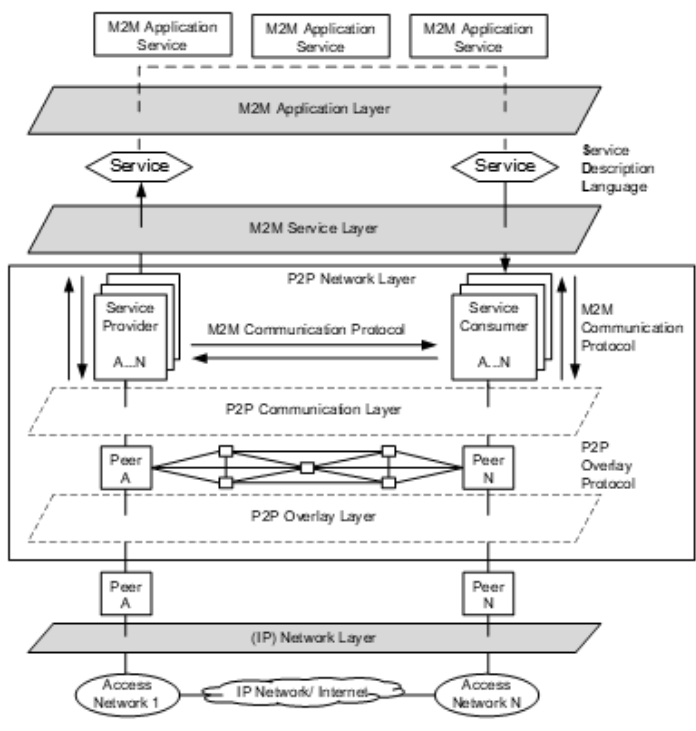

Figure 3. Functional Architecture.

contact address). In addition, the P2P overlay network is used to manage and configure services and applications (described in section IV). The information exchange between the peers for the service utilisation (service requests, confirmations) as well as the necessary signalling to generate the application automatically is also done directly between the peers. For this purpose, various M2M communication protocols (e.g., CoAP, HTTP, SIP, MQTT) can be used M2M Service Layer: The services defined and provided by the end users are available via the M2M service layer and can be used via the corresponding interface. As described in section III and [9], the services are formally described by a service description language. The service interface is described by an interface specification and is available in the M2M Service Layer (explained in section III). M2M Application Layer: The distributed M2M application services are implemented within the M2M application layer. For this purpose, the services available via the M2M Service Layer are combined with each other as described in section III. The exchange of information between the services, respectively applications takes place via the P2P network layer by P2P communication protocols. [17]

\section{SYSTEM STRUCTURE AND APPLICATION CREATION}

In existing platforms for providing $\mathrm{M} 2 \mathrm{M}$ applications, the configuration of automation tasks or the creation of application logic is highly dependent on the implementing systems. To prevent this, the concept defined in this research work was designed with a model-based system structure, derived from [18]. Models, expressed by modelling languages, describe model-based infrastructures and systems. The behaviour of an application or a system is described by means of a platform-independent model, separated from the technology-specific realisation of the system [18]. The behaviour of a system can be modelled with a behavioural model in an abstract manner with much lesser complexity than the platform that implements the behaviour of the system or the application [19]. By stepwise abstraction, respectively transformation, an abstract modelled system is transformed acc. [20] into a concrete system by means of multi-step transformation. Fig. 4 shows the transformation level defined for the approach described in this publication. Derived from [20], transformation and mapping rules have been defined to automatically process the transformation from the abstract model of the system created by the user to the platform-specific models shown in Fig. 4 of the novel architecture for provision of distributed M2M applications described in this publication. As described above, the intuitive development of services and applications is done by graphically modelling the behaviour of a service or creating a behaviour model of service/ application. This allows end users to describe the system without having to have any specific knowledge about the execution platform. The behaviour of a system can be modelled intuitively with a state machine. For this purpose, states represent the devices/ services that should be combined, and transitions represent the connections (information flow) between the devices/ services. To enable end user to model a system using state machine concept, a domain independent modelling language is required which can be used to describe the behaviour of a system abstracted in form of a state machine. [17] The following requirements were initially defined for the selection of an optimal modelling language for this purpose: Machine readability: Since no further entities are to be involved in the application generation or its implementation, i.e. the application, respectively the service should be generated automatically after modelling, the modelling language must be machine-readable. It must therefore be a formal language. Standardised language: It must be a standardised modelling language in order to ensure the easiest portability. Immediate mapping of graphical and machine-readable notation: Since graphical modelling by the end user was defined as a central aspect of the concept, a direct mapping of the graphical notation (elements of the state machine) to the machine- readable notation must exist. Intuitive usability: Since the system is to be used by an average technically experienced end user after a short training, the complexity of the graphical notation has to be low. Complex and non-intuitive modelling forms reduce or

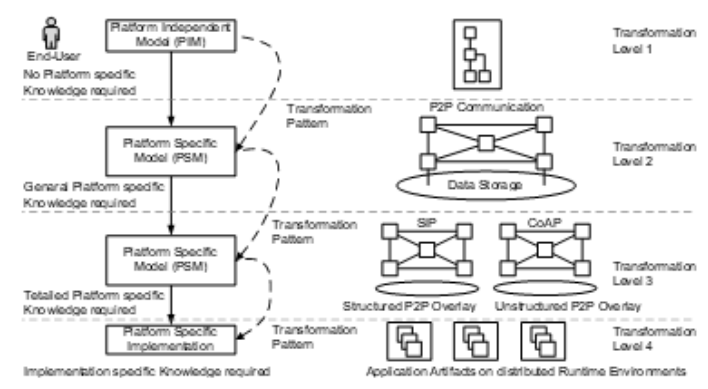

Figure 4. Transformation Level. 
eliminate end user usability. Existing parser/ interpreter implementation: To enable further processing the formal language by a machine, an implementation of a corresponding parser or interpreter must exist. Nested states: Combination of application services. A system (M2M application service) should be combined with other systems. A previously modelled system is to be reused by being embedded in another application. State parametrisation: The services that are to be combined must have the possibility to be parameterised (definition of configuration input and output parameters). Therefore, the states in the state machine must also be parameterisable. Conditional state transitions: The transition of a state or transmission of information to a service or the activation of a service should be provided with a condition. Synchronisation of states: To synchronise parallel flows, there must be a possibility for synchronisation. According to these requirements, the following state machine-based modelling languages and modelling concepts have been examined: Business Process Modeling Notation (BPMN), Business Process Modeling Language (BPEL), UML State Machines, Petri Nets, and Finite State Machines. The results of the evaluation are shown in TABLE I. Requirements the modelling languages/ concepts satisfy are marked with " + "; requirements not satisfied are marked with "-"; requirements partially satisfied are marked with "o". The evaluation of the above-mentioned modelling languages showed that UML State Machines fully meet the requirements. UML State Machines are based on Harel Statecharts, which, due to their structure, meet the structural requirements for the state machine. The standardised description language StateChart XML (SCXML) makes it possible to express Harel statecharts in a formal notation. An SCXML parser, which is also available, enables the machine readability and thus the automatic processing of the formally described state machine. [17] For modelling of a system, the SMF, as described in $[9-11,13]$, provides a GUI to the end user. Using this GUI, the end user graphically creates a state machine that represents the behaviour of the system. The GUI provides an overview of all available devices and services. The services and smart devices that are in the personal environment of the end users, e.g. in its Smart Home, are stored within the SMF in a local repository. The services offered by other peers are stored in the P2P overlay as described in following section IV. An overview of the services stored in the P2P overlay is also loaded by the SCE as described in section IV and displayed in the GUI. The end user now graphically combines the available services and devices by first selecting the desired devices or services and then connecting the input and output interfaces defined for the services and devices (transition of the state machine). As described above, the user models the behaviour of service/application by defining a state machine. The following is a description of the elementary elements of the state machine, as well as the semantic defined by mapping the elements of the state machine to the service/ application components. In addition to these elements, further elements of a state machine can be used, which are not further explained at this point. State element: Devices, services, and applications are represented by states. State
TABLE I. Evaluation of State Machine Modelling LANGUAGES

\begin{tabular}{|l|c|c|c|c|c|}
\hline & \multicolumn{5}{|c|}{ Modelling Languages } \\
\cline { 2 - 6 } & BPMN & BPEL & UML & Petri Nets & FSM \\
\hline Machine read. & - & + & + & + & - \\
\hline Standardised & + & + & + & + & - \\
\hline Immediate map. & - & + & + & + & - \\
\hline Intuitive usability & + & $\circ$ & + & - & + \\
\hline Parser/ Interpreter & - & + & + & + & - \\
\hline Nested states & $\circ$ & $\circ$ & + & + & - \\
\hline State param. & - & + & + & - & - \\
\hline Conditional trans. & + & + & + & $\circ$ & - \\
\hline Sync. states & $\circ$ & + & + & + & - \\
\hline
\end{tabular}

parameter: The state parameters represent the data model of the devices, services and applications. Input, output and configuration parameters can be defined. Global state machine parameter: This defines global attributes of a service/ application. Global attributes are available for all services and applications and are queried directly from the source. Transition: The transition from one state to another state (transition target) represents the connection of devices, services and applications and the associated information exchange. Transition condition: Transitions can be provided with conditions. This defines the precondition when device, service or application sends an information. Conditions can be optionally defined. Transition assign element: The transitions of a state machine have assign elements. These assign elements define how the output parameters are assigned to the input parameters of a device, service or application. Thus, the contents of the messages that are exchanged are specified via this element. Nested state element: Using the nested state element enables embedding an already defined state machine, i.e. a service or an application, into another service or application. For this purpose, the corresponding state machine is embedded into a nested state element. The nested state element then is integrated as a simple state into the sequence of the state machine. Parallel state element: The parallel state element is used to model parallel sequences that can be synchronised as required at the end. [17] The interface description of a device, service or application represents the template for a state. It is embedded in a service or application description or managed separately. The interface description specifies the input/ output and configuration parameters as well as the value ranges of a service/ application. The interface description is used as a template to represent the devices, services or applications graphically and functionally in the GUI. In addition to the above parameters, the interface description contains further parameters for classifying and describing a device, service or application by e.g. keywords or prose. The interface description can be stored in a local repository or stored in the overlay network. In addition, it is 
possible to request the interface description directly from a peer that offers a service. [17] The SCE automatically generates the formal description of the end user-modelled system using SCXML. Service/ application are completely described by the generated SCXML document. If it is a local service, the application description is stored locally, parsed, interpreted and executed according to the defined conditions. If it is a distributed application, i.e. consisting of distributed services offered by different peers, the application description is distributed to the peers that offer a service in the application context. In this case, each peer receives only the part of the application description that is required to configure and execute its individual service. Peers therefore do not have an overview of the overall system, which performs as a basis for securing the system. [17]

\section{APPLICATION REGISTRY, CONFIGURATION, EXECUTION}

As described in section II, data storage in the described approach is performed via structured or unstructured P2P overlay networks. The following is a description of the functions for registering and searching, which are implemented using the overlay network for the management of services and applications. Services can be offered by different peers. The peers register their services via the overlay network. To do so, they store the combination of service ID and their associated personal temporary contact information in the overlay. If different peers offer the same services, several associated peers are stored under the same entry in the overlay network. Each peer represents a specific instance of a service. Peers can request the overlay network to get an overview of all available services or a certain subset of all services. This overview is required to generate an application from the various services as described in section III. Sub-sets of services may include e.g. that services are only available for a particular region, have further meta information that can specify a service more detailed, can only be consumed by a particular group, or implement a particular interface description. Depending on the type of request, the overlay networks offer various ways to provide the overview of the services. For example, the metainformations stored for a service can be searched by keywords. In order to obtain a specific instance of a service during the application configuration (see below), a peer makes a request (possibly restricted by search criteria) to the overlay network. This returns a list of all found instances of a service. [17] An M2M application service consists of several services that are interconnected and exchange information. An M2M application is thus a concatenation of distributed services. Each service has input and output interfaces. To realise an application, the output interfaces of a service are connected to the input interfaces of another service, and the information is passed from one service to the other. The description of the application, i.e., which service is associated with which other service, is defined by a formal application description (described in section III). The approach described in this publication has two phases to realise an application (illustrated in Fig. 5). A configuration phase in which the application is generated automatically and

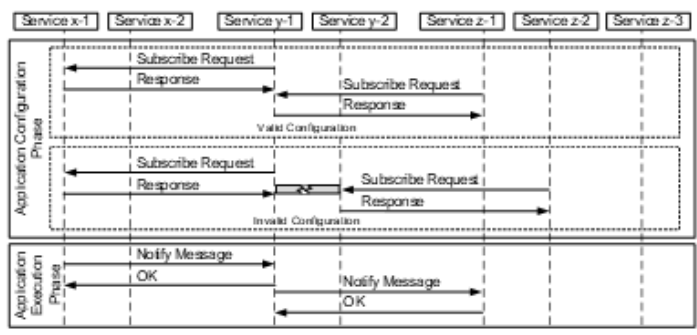

Figure 5. Application Configuration and Execution Phase.

an execution phase in which the application is running. First, the following section describes the configuration phase. Each peer providing a service involved in an application receives the formal description of the application. As described in section III, each peer only receives the segment of the application description, which contains the necessary information to configure its individual service and insert it into the overall context of the application according to the application description. Inserting into the overall context of the application means that the peer connects the interfaces (input, output) of his service to the other associated services in order to exchange information. By automatically parsing the application description, the peer determines the following information. Service connections: To which other services his offered service should be connected. A distinction is made here between input interfaces served by another service and output interfaces which are served by the service itself. Input interface information content: Which information is received via the input interface. Output interface information content: What information via the output interface should be transferred to another service. Output interface condition: Under which prerequisites an information is to be sent to a service via an output interface (directly on request or time-delayed, if a defined condition is met). Service configuration parameter: If configuration parameters have been defined for the offered service, the service must be configured accordingly. Global attributes: Information about attributes provided by services that are not directly connected to their own service via an interface. [17]

Each peer now uses the configuration information, determined by the automated parsing of the formal application description, to integrate its service autonomously into the overall context of the application. First, each peer determines the specific instances of the services to be connected to it, i.e. determines a list of peers that offer the service based on the service $\mathrm{ID}$. This is done as described above. The exchange of information takes place in the approach described in this publication according to the Subscribe/ Notify principle. The connection of an input interface of a service with the output interface of another service is performed by sending the peer with its output interface a subscribe request. If the requested service is available, the requested peer confirms service utilisation request with a positive response. If the service is not available or is available later, the requested peer responds with a negative response and rejects to consume the service or defines a later usage time. Once the connections between 
the instances of the services are established, the local configuration of the service is performed. This consists of the assignment of the defined input and output information content to the interfaces (transition assign). Furthermore, the transfer of any existing service configuration parameters to the local service is performed. In addition, the conditions are configured locally that define the preconditions to transmit an information to a connected service via the output interface (transition condition). [17] As described above and shown in Fig. 5, the described approach also includes the execution phase additionally to configuration phase of an application. In execution phase, the exchange of information takes place between the peers, which represents the M2M application service. Once the connections between the service instances have been established, the information is transmitted between the services using Notify messages. Sending a notification message either is done directly on request (global attribute or condition less transition) or as soon as a defined condition (transition condition) applies. [17]

It should be noted that the same service can be provided by several instances (peers). This may result in incomplete configurations of an application during the configuration phase. In addition to a complete configuration of an application, Fig. 5 also shows an incomplete configuration of an application). For this purpose, an algorithm has been developed which allows all peers to determine independently whether they have embedded themselves in a valid application configuration. The algorithm developed for this purpose is based on the computation of the transitive closure derived from [21] of all connections established in the P2P network in the application context. If a peer detects that it has embedded itself in an invalid application configuration, the established connections are automatically disconnected and, if necessary, a new local configuration process is performed. Thus, it is possible to determine independently a valid application configuration without having to have a complete view of the system. [17]

\section{$\mathrm{V}$ CONCLUSTON}

This publication presented principles and details of a novel concept for autonomous and decentralised M2M application service provision. This concept offers new possibilities for applications, realised by several peers, independent of central appliances or corporations. Especially the independent structure of presented system architecture and the simplicity of $\mathrm{M} 2 \mathrm{M}$ service/ application creation combined with the autonomous management of M2M application services form the strength of the proposed framework and offer a promising approach for supporting M2M networks with end user environment integration.

\section{ACKNOWLEDGMENT}

The research project $\mathrm{P} 2 \mathrm{P} 4 \mathrm{M} 2 \mathrm{M}$ providing the basis for this publication is partially funded by the Federal Ministry of Education and Research (BMBF) of the Federal Republic of Germany under grant number 03FH022IX5. The authors of this publication are in charge of its content.

\section{REFERENCES}

[1] M. Steinheimer, U. Trick, W. Fuhrmann, B. Ghita, "P2P based service provisioning in M2M networks", Proc. of Sixth International Conference on Internet Technologies \& Applications (ITA 15), Wrexham, Wales, UK, September 2015.

[2] S. Clayman, A. Galis, "INOX: a managed service platform for interconnected smart objects", Proc. of the workshop on Internet of Things and Service Platforms 2011 (1oTSP'11), pp. 1-8, 2011

[3] S. Zhang, J. Zhang, W. Li, "Design of M2M Platform Based on J2EE and SOA", 2010 International Conference on E-Business and EGovernment, pp. 2029-2032, September 2010.

[4] Q Xiaocong Z Jidong "Study on the structure of "Internet of Things(IOT)" business operation support platform", 2010 12th IEEE Int. Conf. Commun. Technology (ICCT), pp. 1068-1071, 2010.

[5] L. Foschini, T. Taleb, A. Corradi, D. Bottazzi, "M2M-based metropolitan platform for IMS-enabled road traffic management in IoT", IEEE Commun. Mag., vol. 49, no. 11, pp. 50-57, 2011.

[6] Y. J. Kim, E. K. Kim, B. W. Nam, I. Chong, "Service composition using new DSON platform architecture for M2M service", Proc. of the International Conference on Information Network 2012, pp. 114119, February 2012

[71 J. Kim, J. Lee, J. Kim, J. Yun, "M2M Service Platforms: Survey Issues and Enabling Technologies", IEEE Communications Surveys \& Tutorials, vol. 16, no. 1, pp. 61-76, First Quarter 2014.

[8] oneM2M Standardisation Committee, "oneM2M Release 2 specifications", http://www.onem2m.org/technical/publisheddocuments, December 2016.

[9] M. Steinheimer, U. Trick, W. Fuhrmann, B. Ghita, "Load reduction in distribution networks through P2P networked energy-community", Proc. of Fifth International Conference on Internet Technologies \& Applications (ITA 13), Wrexham, Wales, UK, September 2013.

[10] M. Steinheimer, U. Trick, W. Fuhrmann, B. Ghita, "P2P-based community concept for M2M Applications", Proc. of Second International Conference on Future Generation Communication Technologies (FGCT 2013), London, UK, December 2013.

[11] M. Steinheimer, U. Trick, P. Ruhrig, R. Tönjes, M. Fischer, D. Hölker, "SIP-basierte P2P-Vernetzung in einer Energie-Community", ITG-Fachbericht 242: Mobilkommunikation, VDE Verlag $\mathrm{GmbH}$, Osnabrück, Germany, ISBN: 978-3-8007-3516-7, ISSN: 0932-6022, pp. 64, Mai 2013.

[12] M. Steinheimer, U. Trick, W. Fuhrmann, B. Ghita, "P2P-based M2M Community Applications", Proc. of Eleventh International Network Conference (INC 2016), Frankfurt, Germany, July 2016.

[13] M. Steinheimer, U. Trick, P. Wacht, P. Ruhrig, "Decentralised optimisation solution for Smart Grids using Smart Market aspects and P2P internetworked Energy-Community", IEC/ SGCC/ VDE World Smart Grid Forum 2013, Berlin, Germany, September 2013.

[14] ITU-T Y.101 (2000), Recommendation, "Global Information Infrastructure terminology: Terms and definitions", ITU, 2000.

[15] ISO IEC 20000-1:2011 (2011) "Part 1: Service management system requirements", IEEE, 2011

[16] ITIL V3.1.24 (2007) "Glossary of Terms, Definitions and Acronyms", ITIL, 2007.

[171 M. Steinheimer, "Optimierte P2P-Dienstearchitektur für hochverfugbare M2M-Applikationen (P2P4M2M)", TR-004, Frankfurt, Germany, October 2016.

「18] Object Management Group, "OMG Model Driven Architecture", http://www.omg.org/mda/, January 2017

[19] Object Management Group, "Model Driven Architecture (MDA) MDA Guide rev. 2.0", Boston, USA, June 2014

[20] R. Petrasch, O. Meimberg, "Model Driven Architecture", dpunkt.verlag, Heidelberg, Germany, ISBN: 978-3-89864-343-6, 2006

[21] V. Turau, "Algorithmische Graphentheorie", De Gruyter Oldenbourg, Munich, Germany, ISBN: 978-3-486-59057-9, 2009. 


\title{
Autonomous decentralised M2M Application Service Provision
}

\author{
Michael Steinheimer, Ulrich Trick \\ Research Group for Telecommunication Networks \\ Frankfurt University of Applied Sciences \\ Frankfurt/M., Germany \\ steinheimer@e-technik.org
}

\author{
Michael Steinheimer, Bogdan Ghita \\ Centre for Security, Communications and Network \\ Research \\ University of Plymouth \\ Plymouth, UK
}

\author{
Woldemar Fuhrmann \\ Department for Computer Science \\ University of Applied Sciences Darmstadt \\ Darmstadt, Germany
}

\begin{abstract}
In this publication a novel concept for M2M service platform architecture is presented enabling decentralised and autonomous M2M application service provision. This publication introduces the functional architecture of the approach and describes the system structure and application creation process. Additionally several M2M service platforms are evaluated regarding requirements necessary for decentralised M2M application service provision with end user integration. This publication describes also a decentralised M2M application service management process and the corresponding formal notation of $\mathrm{M} 2 \mathrm{M}$ application services.
\end{abstract}

Keywords-M2M application service, P2P, service provision

\section{INTRODUCTION}

Devices are becoming more and more intelligent by including complex functionality for monitoring and control. Additionally the devices receive the functionality for communication enabling remote monitoring and controlling of devices. Due to the increasing number of intelligent devices, which support forming of intelligent environments, many new application fields can be established (e.g. as presented in [11] smart building, electro mobility, smart city, energy optimisation etc.). Especially the end user domain is an application field with powerful potential, however not or only complicated addressable by external service providers due to e.g. legal restrictions.

Machine-to-Machine Communications (M2M) systems realise the integration of such intelligent devices by provision of specific M2M applications. Traditional M2M systems and the corresponding $\mathrm{M} 2 \mathrm{M}$ application services have the focus to support specific business processes. Traditional M2M applications provide their functionality as a service for users (end users or companies). Currently existing concepts from standardisation and research to implement M2M application services provision (as illustrated in section II) mostly focus on central approaches.

This publication dedicates to present a more flexible methodology for realisation of M2M systems with the focus on dissolving the bindings to centralised entities, integration of the end user for realisation of M2M applications satisfying the individual requirements of end users, and realisation without specialised and dedicated M2M infrastructures. Furthermore this publication aims to introduce details about the functional architecture of the proposed concept as well as methodologies forming the basis for the presented concept of autonomous decentralised $\mathrm{M} 2 \mathrm{M}$ application service provision.

\section{M2M SERVICE PLATFORMS}

As introduced in section I of this publication the realisation of $\mathrm{M} 2 \mathrm{M}$ applications and provision their functionality as a service is typically done using M2M Service Platforms. This section presents requirements for $\mathrm{M} 2 \mathrm{M}$ service provision, defined by analysis of existing M2M Service Platform projects. Projects from research area are e.g. INOX Managed Service Platform [4], M2M Platform Project based on SOA [5], BOSP Business Operation Support Platform [6], IMS enabled M2M Service Platform [7], e-DSON [8]. Commercial M2M platforms implement acc. [9] mostly the oneM2M standard for $\mathrm{M} 2 \mathrm{M}[10]$. In order to determine the requirements for $\mathrm{M} 2 \mathrm{M}$ service provision with end user environment integration the oneM2M standard as well as projects from research were examined. Table I specifies the analysed projects and an extract of the requirements defined resulting of the strengths and weaknesses of the projects. Disadvantages of the centralised approaches are e.g. that the user of the application is dependent of the platform provider as a central entity. Often M2M platforms provide their functionality only for specific application fields and are therefore less flexible. Centralised 
provision of platforms requires many resources (costs for operation, maintenance, availability, application development) End users often have no possibility to define applications themselves (since the creation of applications requires expert knowledge or the end user does not have access to the platform) or the devices present in user's personal environment cannot be connected to the M2M platforms.

The personal environment of the end user described above is difficult to address by external service providers, since the activities (controlling and monitoring of M2M devices) carried out in this area would severely affect the end user or challenge data security. To antagonise this issue, it was proposed in [12] to integrate the end user. For this purpose, an approach was presented in $[12-14,16]$, which allows end user to define services and automation tasks for their personal environment. The Service Management Framework (SMF) presented in [1215] consists of a local Service Creation Environment (SCE) and a Service Delivery Platform (SDP). The presented SMF integrates the devices present in the personal area of the end users regardless of their technology and provides basic services, e.g. for multimedia communication. The end user can combine intelligent and communicable devices as well as the basic services using the SCE and thus define personalised services for his personal area (e.g. automation tasks, sensordependent signalling or monitoring). An elementary requirement for the platform described above was that it provides an opportunity to develop services intuitively. An intuitive development can be realised by a graphical development process and by modelling the behaviour of a system independently from underlying technology. Both were implemented in the above-mentioned approach. The graphical development process and the underlying methodology are discussed in section III.

table i. Evaluation Related M2M Service Platform Projects

\begin{tabular}{|c|c|c|c|c|c|c|c|c|c|c|c|c|c|}
\hline \multirow[t]{2}{*}{ Requirements } & \multicolumn{13}{|c|}{ M2M Service Platform Projects } \\
\hline & 흘 & 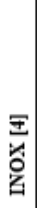 & 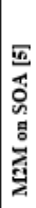 & 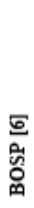 & 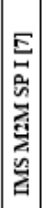 & 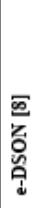 & 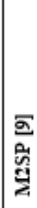 & 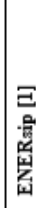 & & & $\begin{array}{l}0 \\
0 \\
0\end{array}$ & & 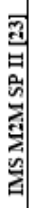 \\
\hline $\begin{array}{l}\text { End user environment } \\
\text { integration }\end{array}$ & - & 1 & - & + & - & + & + & + & & + & - & & + \\
\hline End user integration & - & - & - & - & - & $\circ$ & - & + & + & - & - & & - \\
\hline $\begin{array}{l}\text { End user service } \\
\text { provision and } \\
\text { utilisation }\end{array}$ & - & - & - & - & - & + & - & - & - & - & - & & - \\
\hline $\begin{array}{l}\text { Corporate end user } \\
\text { service provision }\end{array}$ & - & - & - & - & - & $\circ$ & - & - & - & - & - & & - \\
\hline $\begin{array}{l}\text { Decentralised system } \\
\text { architecture }\end{array}$ & $\circ$ & - & - & - & - & $\circ$ & - & - & + & - & $\circ$ & & - \\
\hline $\begin{array}{l}\text { Multimedia } \\
\text { communication }\end{array}$ & l & l & & + & + & - & - & - & - & - & - & & + \\
\hline $\begin{array}{l}\text { Device/ service lookup } \\
\text { mechanism }\end{array}$ & + & + & + & + & + & + & + & l & + & + & - & & 1 \\
\hline Simplicity & - & 7 & - & - & - & - & - & $\circ$ & $\circ$ & 0 & - & & 1 \\
\hline
\end{tabular}

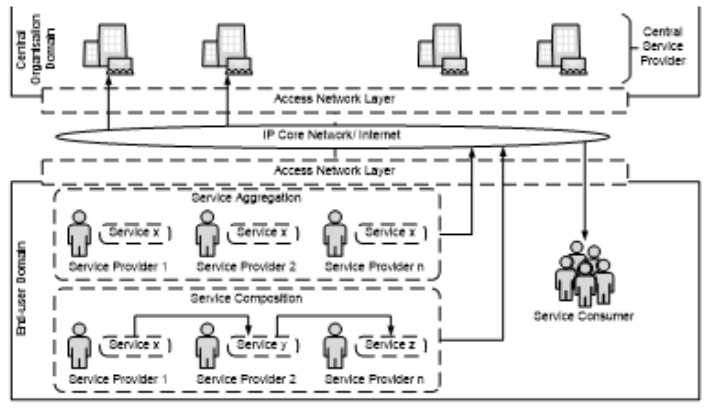

Fig. 1. Cooperative M2M Application Service Provision

By the approach proposed in [11-16], the end user has thus been given the opportunity to create graphically and intuitively services for his personal environment. An approach has been defined in $[11,13$, and 16] as to how these services can be made available to other end users or organisations in order to gain added value through the use of the services. In $[11,13$, and 16] it was proposed that end users combine the individually offered services to provide a more complex, intelligent and more powerful service. As shown in Fig. 1, a complex service consists of the combination of several distributed services that are networked together. Two ways exist to combine services into an application. Multiple end users can offer the same service with the same local functionality running parallel/ synchronously (service aggregation). Alternatively, end users can offer services that have a different functionality and are composed to an application by linking the services together (service composition).

As described above, the central approaches for defining M2M applications have various disadvantages and are not very flexible. Therefore, as a prerequisite for the approach introduced above, it was defined that creation and provision of individual services and the cooperative provision of an application (consisting of distributed services) is exclusively decentralised, i.e. without the integration of logical as well as physical central entities into the overall system. Under these circumstances, the realisation of the approach cannot be implemented with the existing architectures for M2M platforms. Therefore, an approach has been presented in [11, 13 , and 16], which allows implementation following a purely decentralised approach.

\section{FUNCTIONAL ARCHITECTURE}

Fig. 2 shows the functional architecture of the concept for the autonomous P2P-based provision of M2M application services. The architecture is structured into four layers. The following describes the respective layers in their functionality. Network Layer: End user's M2M gateways are connected with each another via an IP network, respectively the Internet. End users can be located at different geographic locations as well as in different access networks (wireless mobile networks or fixed access networks), which are interconnected via a core network. P2P Network Layer: End user nodes represent 


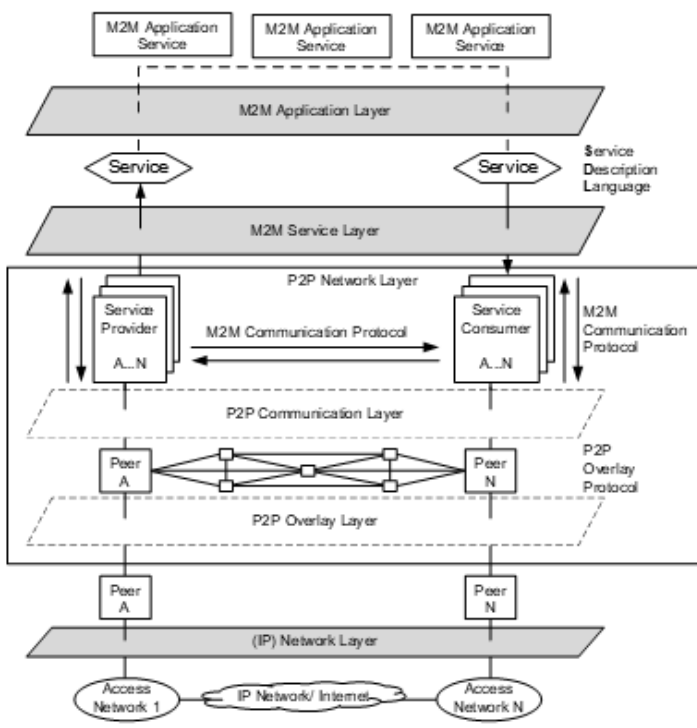

Fig. 2. Functional Architecture

equivalent instances and are connected to each other via a P2P network. The P2P Network Layer includes the sub-layer P2P Overlay Layer and P2P Communication Layer. Any data storage in the presented system is decentralised via the end user clients within the P2P overlay layer. For this purpose, the clients, respectively the peers form a $\mathrm{P} 2 \mathrm{P}$ overlay network. The overlay network is either a pure structured overlay network or a pure unstructured overlay network (due to the general restriction to avoid also partly centralised system architectures). The overlay network is among other things used as explained in [13-15] to store the addressing information of the peers (assignment temporary to static contact address). In addition, the P2P overlay network is used to manage and configure services and applications (described in section $\mathrm{V}$ ). The information exchange between the peers for the service utilisation (service requests, confirmations) as well as the necessary signalling to generate the application automatically is also done directly between the peers. For this purpose, various M2M communication protocols (e.g., CoAP, HTTP, SIP, MQTT) can be used. M2M Service Layer: The services defined and provided by the end users are available via the $\mathrm{M} 2 \mathrm{M}$ service layer and can be used via the corresponding interface. As described in section IV and [12], the services are formally described by a service description language. The service interface is described by an interface specification and is available in the M2M Service Layer (explained in section IV). M2M Application Layer: The distributed M2M application services are implemented within the M2M application layer. For this purpose, the services available via the M2M Service Layer are combined with each other as described in section IV. The exchange of information between the services, respectively applications takes place via the P2P network layer by P2P communication protocols. [17]

\section{SYSTEM STRUCTURE AND APPLICATION CREATION}

In existing platforms for providing M2M applications, the configuration of automation tasks or the creation of application logic is highly dependent on the implementing systems. To prevent this, the concept defined in this research work was designed with a model-based system structure, derived from [19]. Models, expressed by modelling languages, describe model-based infrastructures and systems. The behaviour of an application or a system is described by means of a platformindependent model, separated from the technology-specific realisation of the system [19]. The behaviour of a system can be modelled with a behavioural model in an abstract manner with much lesser complexity than the platform that implements the behaviour of the system or the application [20]. By stepwise abstraction, respectively transformation, an abstract modelled system is transformed acc. [18] into a concrete system by means of multi-step transformation. Fig. 3 shows the transformation level defined for the approach described in this publication. Derived from [18], transformation and mapping rules have been defined to automatically process the transformation from the abstract model of the system created by the user to the platform-specific models shown in Fig. 3 of the novel architecture for provision of distributed $\mathrm{M} 2 \mathrm{M}$ applications described in this publication. As described above, the intuitive development of services and applications is done by graphically modelling the behaviour of a service or creating a behaviour model of service/ application. This allows end users to describe the system without having to have any specific knowledge about the execution platform. The behaviour of a system can be modelled intuitively with a state machine. For this purpose, states represent the devices/ services that should be combined, and transitions represent the connections (information flow) between the devices/ services. To enable end user to model a system using state machine concept, a domain independent modelling language is required which can be used to describe the behaviour of a system abstracted in form of a state machine. [17] The following requirements were initially defined for the selection of an optimal modelling language for this purpose: Machine readability: Since no further entities are to be involved in the application generation or its implementation, i.e. the application, respectively the service should be generated automatically after modelling the modelling language must be machine-readable. It must therefore be a formal language Standardised language: It must be a standardised modelling language in order to ensure the easiest portability. Immediate mapping of graphical and machine-readable notation: Since graphical modelling by the end user was defined as a central aspect of the concept, a direct mapping of the graphical notation (elements of the state machine) to the machinereadable notation must exist. Intuitive usability: Since the system is to be used by an average technically experienced end user after a short training, the complexity of the graphical notation has to be low. Complex and non-intuitive modelling forms reduce or eliminate end user usability. Existing parser/ interpreter implementation: To enable further processing the formal language by a machine, an implementation of a corresponding parser or interpreter must exist. Nested states: Combination of application services. A system (M2M application service) should be combined with other systems. A 


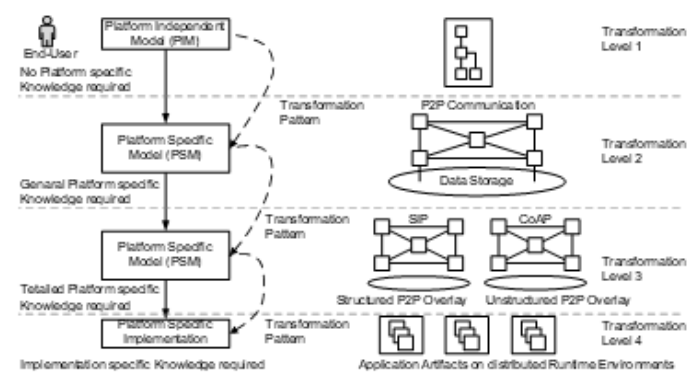

Fig. 3. Transformation Level

previously modelled system is to be reused by being embedded in another application. Parallel flows: Within an application, it should be possible to define parallel sequences in order to implement concurrent tasks. State parametrisation: The services that are to be combined must have the possibility to be parameterised (definition of configuration/ input and output parameters). Therefore, the states in the state machine must also be parameterisable. Conditional state transitions: The transition of a state or transmission of information to a service or the activation of a service should be provided with a condition. Synchronisation of states: To synchronise parallel flows, there must be a possibility for synchronisation. According to these requirements, the following state machinebased modelling languages and modelling concepts have been examined: Business Process Modeling Notation (BPMN), Business Process Modeling Language (BPEL), UML State Machines, Petri Nets, and Finite State Machines. The results of the evaluation are shown in TABLE II. The evaluation of the above-mentioned modelling languages showed that UML State Machines fully meet the requirements. UML State Machines are based on Harel Statecharts, which, due to their structure, meet the structural requirements for the state machine. The standardised description language StateChart XML (SCXML) makes it possible to express Harel statecharts in a formal notation. An SCXML parser, which is also available, enables the machine readability and thus the automatic processing of the formally described state machine. [17]

For modelling of a system, the SMF as described in [1215], provides a GUI to the end user. Using this GUI, the end user graphically creates a state machine that represents the behaviour of the system. The GUI provides an overview of all available devices and services. The services and smart devices that are in the personal environment of the end users, e.g. in its Smart Home, are stored within the SMF in a local repository. The services offered by other peers are stored in the P2P overlay as described in following section V. An overview of the services stored in the P2P overlay is also loaded by the SCE as described in section V and displayed in the GUI. The end user now graphically combines the available services and devices by first selecting the desired devices or services and then connecting the input and output interfaces defined for the services and devices (transition of the state machine). As described above, the user models the behaviour of service/ application by defining a state machine. The following is a description of the elementary elements of the state machine, as
TABlE II. Evaluation of State Machine Modelling Languages

\begin{tabular}{|l|c|c|c|c|c|}
\hline \multirow{2}{*}{} & \multicolumn{5}{|c|}{ Modelling Language } \\
\cline { 2 - 6 } & $B P M N$ & BPEL & UML & Peri Nets & FSM \\
\hline Machine read. & - & + & + & + & - \\
\hline Standardised & + & + & + & + & - \\
\hline Immediate map. & - & + & + & + & - \\
\hline Intuitive usability & + & $\circ$ & + & - & + \\
\hline $\begin{array}{l}\text { Parser/ } \\
\text { Interpreter }\end{array}$ & - & + & + & + & - \\
\hline Nested states & $\circ$ & $\circ$ & + & + & - \\
\hline Parallel Flows & + & + & + & + & - \\
\hline State param. & - & + & + & - & - \\
\hline $\begin{array}{l}\text { Conditional } \\
\text { trans. }\end{array}$ & + & + & + & $\circ$ & - \\
\hline Sync. states & $\circ$ & + & + & + & - \\
\hline
\end{tabular}

well as the semantic defined by mapping the elements of the state machine to the service/ application components. In addition to these elements, further elements of a state machine can be used, which are not further explained at this point. State element: Devices, services, and applications are represented by states. State parameter: The state parameters represent the data model of the devices, services and applications. Input, output and configuration parameters can be defined. Global state machine parameter: This defines global attributes of a service/ application. Global attributes are available for all services and applications and are queried directly from the source. Transition: The transition from one state to another state (transition target) represents the connection of devices, services and applications and the associated information exchange. Transition condition: Transitions can be provided with conditions. This defines the precondition when device, service or application sends an information. Conditions can be optionally defined. Transition assign element: The transitions of a state machine have assign elements. These assign elements define how the output parameters are assigned to the input parameters of a device, service or application. Thus, the contents of the messages that are exchanged are specified via this element. Nested state element: Using the nested state element enables embedding an already defined state machine, i.e. a service or an application, into another service or application. For this purpose, the corresponding state machine is embedded into a nested state element. The nested state element then is integrated as a simple state into the sequence of the state machine. Parallel state element: The parallel state element is used to model parallel sequences that can be synchronised as required at the end. [17]

The interface description of a device, service or application represents the template for a state. It is embedded in a service or application description or managed separately. The interface description specifies the input/ output and configuration parameters as well as the value ranges of a service/ application. The interface description is used as a template to represent the devices, services or applications graphically and functionally in the GUI. In addition to the above parameters, the interface description contains further parameters for classifying and describing a device, service or application by e.g. keywords or 
prose. The interface description can be stored in a local repository or stored in the overlay network. In addition, it is possible to request the interface description directly from a peer that offers a service. [17]

The SCE automatically generates the formal description of the end user-modelled system using SCXML. Service/ application are completely described by the generated SCXML document. If it is a local service, the application description is stored locally, parsed, interpreted and executed according to the defined conditions. If it is a distributed application, i.e. consisting of distributed services offered by different peers, the application description is distributed to the peers that offer a service in the application context. In this case, each peer receives only the part of the application description that is required to configure and execute its individual service. Peers therefore do not have an overview of the overall system, which performs as a basis for securing the system. [17]

\section{V.APPLICATION REGISTRY, CONFIGURATION, EXECUTION}

As described in section III, data storage in the described approach is performed via structured or unstructured P2P overlay networks. The following is a description of the functions for registering and searching, which are implemented using the overlay network for the management of services and applications. Services can be offered by different peers. The peers register their services via the overlay network. To do so they store the combination of service ID and their associated personal temporary contact information in the overlay. If different peers offer the same services, several associated peers are stored under the same entry in the overlay network. Each peer represents a specific instance of a service. Peers can request the overlay network to get an overview of all available services or a certain subset of all services. This overview is required to generate an application from the various services as described in section IV. Sub-sets of services may include e.g. that services are only available for a particular region, have further meta information that can specify a service more detailed, can only be consumed by a particular group, or implement a particular interface description. Depending on the type of request, the overlay networks offer various ways to provide the overview of the services. For example, the metainformations stored for a service can be searched by keywords. In order to obtain a specific instance of a service during the application configuration (see below), a peer makes a request (possibly restricted by search criteria) to the overlay network This returns a list of all found instances of a service. [17]

An M2M application service consists of several services that are interconnected and exchange information. An M2M application is thus a concatenation of distributed services. Each service has input and output interfaces. To realise an application, the output interfaces of a service are connected to the input interfaces of another service, and the information is passed on from one service to the other. The description of the application, i.e., which service is associated with which other service, is defined by a formal application description (described in section IV). The approach described in this publication has two phases to realise an application (illustrated in Fig. 4). A configuration phase in which the application is generated automatically and an execution phase in which the

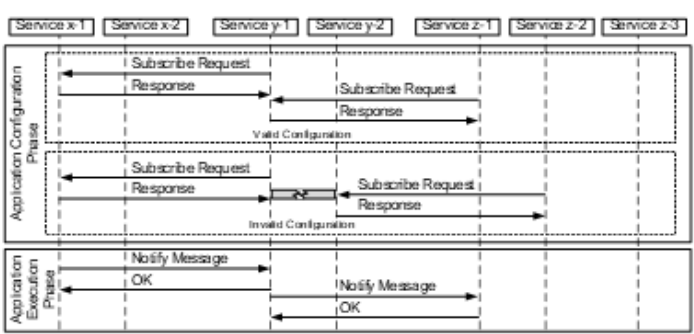

Fig. 4. Application Configuration and Execution Phase

application is running. First, the following section describes the configuration phase. Each peer providing a service involved in an application receives the formal description of the application. As described in section IV, each peer only receives the segment of the application description, which contains the necessary information to configure its individual service and insert it into the overall context of the application according to the application description. Inserting into the overall context of the application means that the peer connects the interfaces (input, output) of his service to the other associated services in order to exchange information. By automatically parsing the application description, the peer determines the following information. Service connections: To which other services his offered service should be connected. A distinction is made here between input interfaces served by another service and output interfaces which are served by the service itself. Input interface information content: Which information is received via the input interface. Output interface information content: What information via the output interface should be transferred to another service. Output interface condition: Under which prerequisites an information is to be sent to a service via an output interface (directly on request or time-delayed, if a defined condition is met). Service configuration parameter: If configuration parameters have been defined for the offered service, the service must be configured accordingly. Global attributes: Information about the attributes provided by services that are not directly connected to their own service via an interface. [17] Each peer now uses the configuration information, determined by the automated parsing of the formal application description, to integrate its service autonomously into the overall context of the application. First, each peer determines specific instances of services to be connected to it, i.e. determines a list of peers that offer the service based on the service ID. This is done as described above. The exchange of information takes place in the approach described in this publication according to the Subscribe/ Notify principle. The connection of an input interface of a service with the output interface of another service is performed by sending the peer with its output interface a subscribe request. If the requested service is available, the requested peer confirms service utilisation request with a positive response. If the service is not available or is available later, the requested peer responds with negative response and rejects to consume the service or defines a later usage time. Once the connections between the of instances the services are established, the local configuration of the service is performed. This consists of the assignment of the defined input and output information content to the interfaces 
(transition assign). Furthermore, the transfer of any existing service configuration parameters to the local service is performed. In addition, the conditions are configured locally that define the preconditions to transmit an information to a connected service via the output interface (transition condition). [17] As described above and shown in Fig. 4, the described approach also includes the execution phase additionally to the configuration phase of an application. In the execution phase, the exchange of information takes place between the peers, which represents the M2M application service. Once the connections between the service instances have been established, the information is transmitted between the services using Notify messages. Sending a notification message either is done directly on request (global attribute or condition less transition) or as soon as a defined condition (transition condition) applies. [17] It should be noted that the same service can be provided by several instances (peers). This may result in incomplete configurations of an application during the configuration phase. In addition to a complete configuration of an application, Fig. 4 also shows an incomplete configuration of an application). For this purpose, an algorithm has been developed which allows all peers to determine independently whether they have embedded themselves in a valid application configuration. The algorithm developed for this purpose is based on the computation of the transitive closure derived from [21] of all connections established in the P2P network in the application context. If a peer detects that it has embedded itself in an invalid application configuration, the established connections are automatically disconnected and, if necessary, a new local configuration process is performed. Thus, it is possible to determine independently a valid application configuration without having to have a complete view of the system. [17]

\section{CONCLUSION}

This publication presented principles and details of a novel concept for autonomous and decentralised M2M application service provision. This concept offers new possibilities for applications, realised by several peers, independent of central appliances or corporations. Especially the independent structure of presented system architecture and the simplicity of M2M service/ application creation combined with the autonomous management of M2M application services form the strength of the proposed framework and offer a promising approach for supporting M2M networks with end user environment integration.

\section{ACKNOWLEDGMENT}

The research project $\mathrm{P} 2 \mathrm{P} 4 \mathrm{M} 2 \mathrm{M}$ providing the basis for this publication is partially funded by the Federal Ministry of Education and Research (BMBF) of the Federal Republic of Germany under grant number 03FH022IX5. The authors of this publication are in charge of its content.

\section{REFERENCES}

[1] G. López, P. Moura, J. I. Moreno, A. Almeida, "ENERsip: M2M-based platform to enable energy efficiency within energy-positive platform to enable energy efficiency within energy-positive
neighbourhoods", Proceedings of the 2011 IEEE Conference on neighbourhoods", Proceedings of the 2011 IEE Conference
Computer Communications Workshops (INFOCOM WKSHPS), 2011
[2] K. J. Lin, N. Reijers, Y. C. Wang, C. S. Shih, J. Y. Hsu, "Building Smart M2M Applications Using the WuKong Profile Framework", Proceedings of the 2013 IEEE International Conference on Green Computing and Communications and IEEE Intemet of Things and IEEE Cyber, Physical and Social Computing, pp. 1175-1180, IEEE, 2013

[3] C. Doukas, F. Antonelli, "COMPOSE: Building smart \& context-aware mobile applications utilizing IoT technologies", Proceedings of the Global Information Infrastructure Symposium (GIIS 2013), 2013

[4] S. Clayman, A. Gali, "INOX: a managed service platform for interconnected smart objects", Proc. of the workshop on Internet of Things and Service Platforms 2011 (loTSP'11), pp. 1-8, December 2011

[5] S. Zhang, J. Zhang, W. Li, "Design of M2M Platform Based on J2EE and $\mathrm{SOA}^{\prime}$, International Conference on E-Business and E-Government pp. 2029-2032 2010 Int. Conf. on E-Business and E-Government, 2010

[6] Q. Xiaocong, Z. Jidong, "Study on the structure of "Intemet of

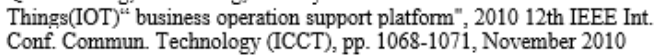

[7] L. Foschini, T. Taleb, A. Corradi, D. Bottazzi, "M2M-based metropolitan platform for IMS-enabled road traffic management in IoT", IEEE Commun. Mag., vol. 49, no. 11, pp. 50-57, November 2011

[8] Y. J. Kim, E. K. Kim, B. W. Nam, I. Chong, "Service composition using new DSON platform architecture for M2M service", Information Networking (ICOIN) 2012 Int. Conf. on, pp. 114-119, February 2012

[9] J. Kim, J. Lee, J. Kim, J. Yun, "M2M Service Platforms: Survey Issues and Enabling Technologies", IEEE Communications Surveys \& Tutorials, vol. 16, no. 1, pp. 61-76, First Quarter 2014

[10] oneM2M Standardisation Committee, "oneM2M Release 2 specifi cations",http://www.onem2m.org/technical/published-documents, 2016

[11] M. Steinheimer, U. Trick, W. Fuhrmann, B. Ghita, "P2P based service provisioning in M2M networks", Proc. of Sixth International Conference on Internet Technologies \& Applications (ITA 15), Wrexham, UK, 2015

[12] M. Steinheimer, U. Trick, W. Fuhrmann, B. Ghita, "Load reduction in distribution networks through P2P networked energy-community", Proc. of Fifh International Conference on Internet Technologies \& Applications (ITA 13), Wrexham, Wales, UK, September 2013

[13] M. Steinheimer, U. Trick, W. Fuhrmann, B. Ghita, "P2P-based community concept for M2M Applications", Proc. of Second community concept for M2M Applications", Proc. of Second International Conference on Future Generation Co
Technologies (FGCT 2013), London, UK, December 2013

[14] M. Steinheimer, U. Trick, P. Ruhrig, R. Tönjes, M. Fischer, D. Hölker, "SIP-basierte P2P-Vernetzung in einer Energie-Community", ITGFachbericht 242: Mobilkommunikation, VDE Verlag $\mathrm{GmbH}$, Osnabrück, Germany, ISBN: 978-3-8007-3516-7, 2013

[15] M. Steinheimer, U. Trick, P. Wacht, P. Ruhrig, "Decentralised optimisation solution for Smart Grids using Smart Market aspects and P2P internetworked Energy-Community", IEC/ SGCC/ VDE World Smart Grid Forum 2013, Berlin, Germany, September 2013

[16] M. Steinheimer, U. Trick, W. Fuhrmann, B. Ghita "P2P-based M2M Community Applications", Proc, of Eleventh Intemational Network Community Applications", Proc. of Eleventh Interna
Conference (INC 2016), Frankfurt, Germany, July 2016

[17] M. Steinheimer, "Optimierte P2P-Dienstearchitektur für hochverfügbare M2M-Applikationen (P2P4M2M)", TR-004, Frankfurt, Germany, 2016

[18] R. Petrasch, O. Meimberg, "Model Driven Architecture", dpunkt.verlag, Heidelberg, Germany, ISBN: 978-3-89864-343-6, 2006

[19] Object Management Group, "OMG Model Driven Architecture", http://www.omg.org/mda/, January 2017

[20] Object Management Group, "Model Driven Architecture (MDA) MDA Guide rev. 2.0", Boston, USA, June 2014

[21] V. Turau, "Algorithmische Graphentheorie", De Gruyter Oldenbourg, Munich, Germany, ISBN: 978-3-486-59057-9, 2009

[22] S. Kitagami, Y. Miyanishi, Y. Urano, N. Shiratori, "Proposal of a Distributed Cooperative M2M System for Flood Disaster Prevention", Distributed Cooperative M2M System for Flood Disaster Prevention",
Proceedings of the 2014 IEEE 11th Intl Conf on Ubiquitous Intelligence and Computing, 2014

[23] J. E. V. Padilla, J. O. Lee, J. H. Kim, "A M2M horizontal services platform implementation over IP multimedia subsystem (IMS)," Proceedings of the 2013 15th Asia-Pacific Network Operations and Management Symposium (APNOMS), 2013 


\section{Appendix C - Low Level Descriptions}

\section{Listings}

\section{Listing C.1: XML Representation of Request Primitive}

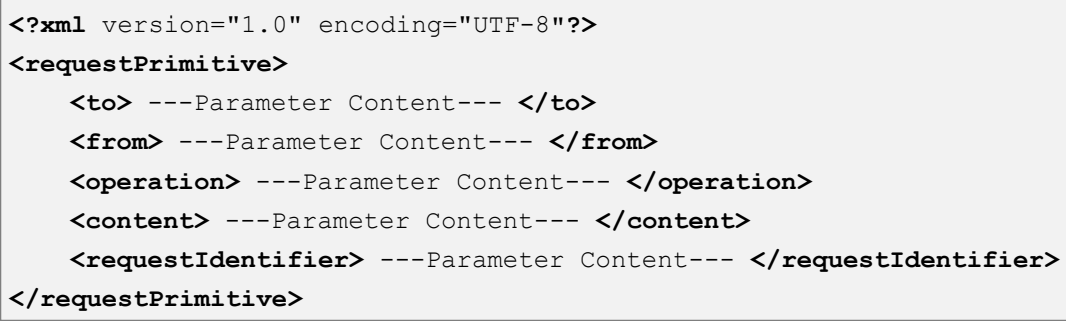

Listing C.2: XML Representation of Response Primitive

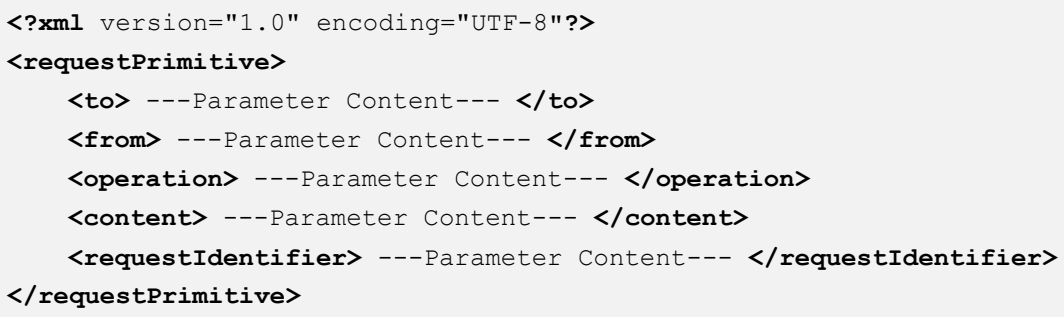

\section{Listing C.3: XML Representation of M2M DCM}

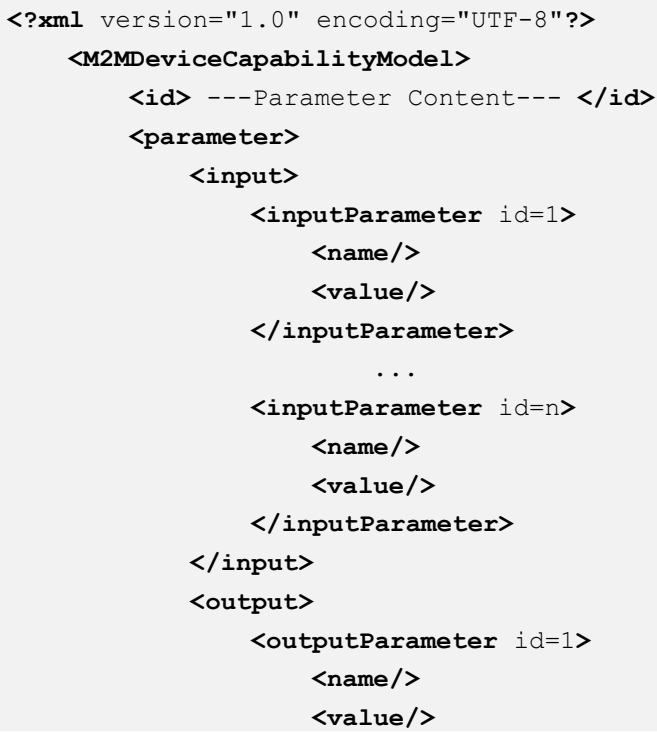




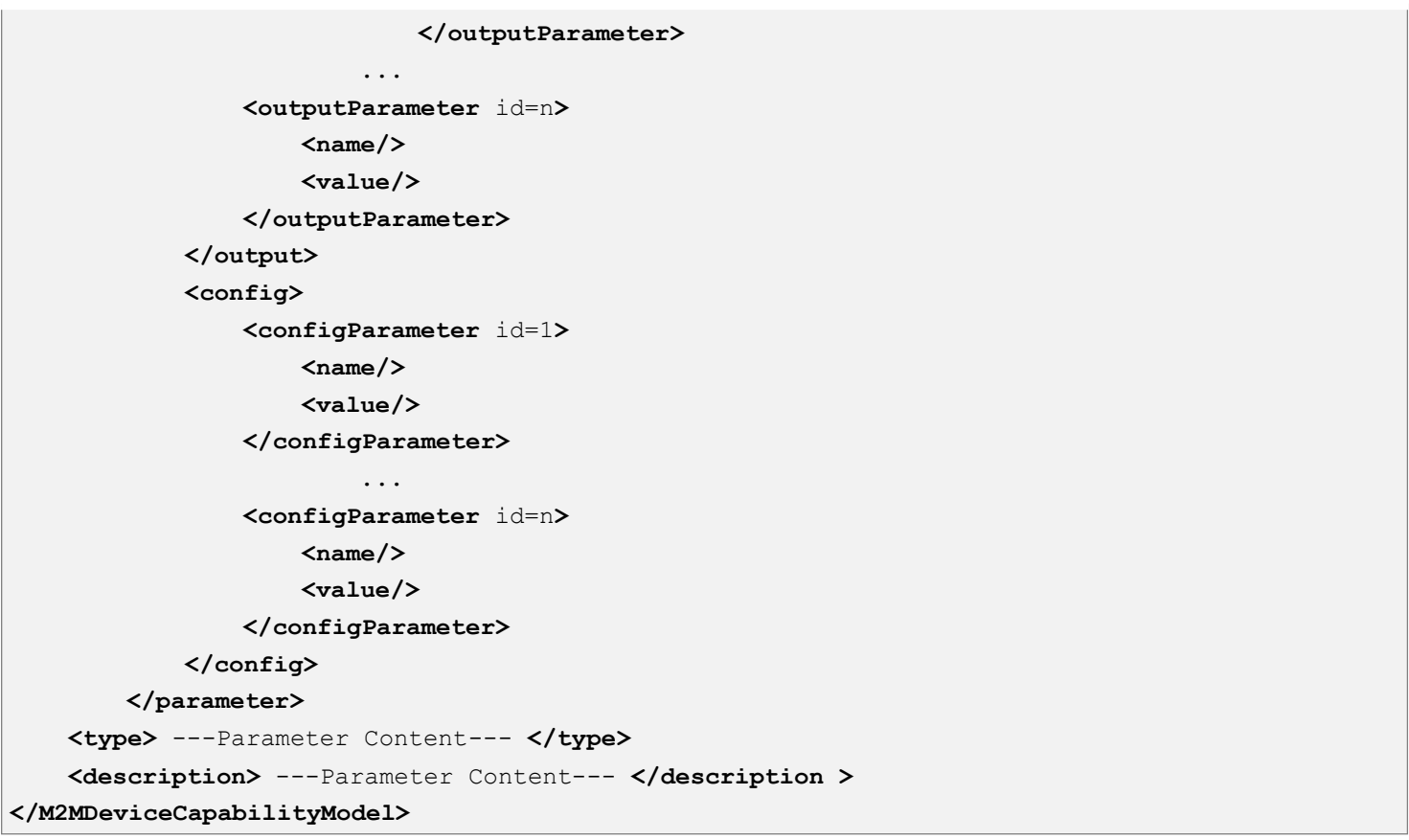

\section{Listing C.4: XML Representation of Request Primitive incl. M2M DCM Parameters}

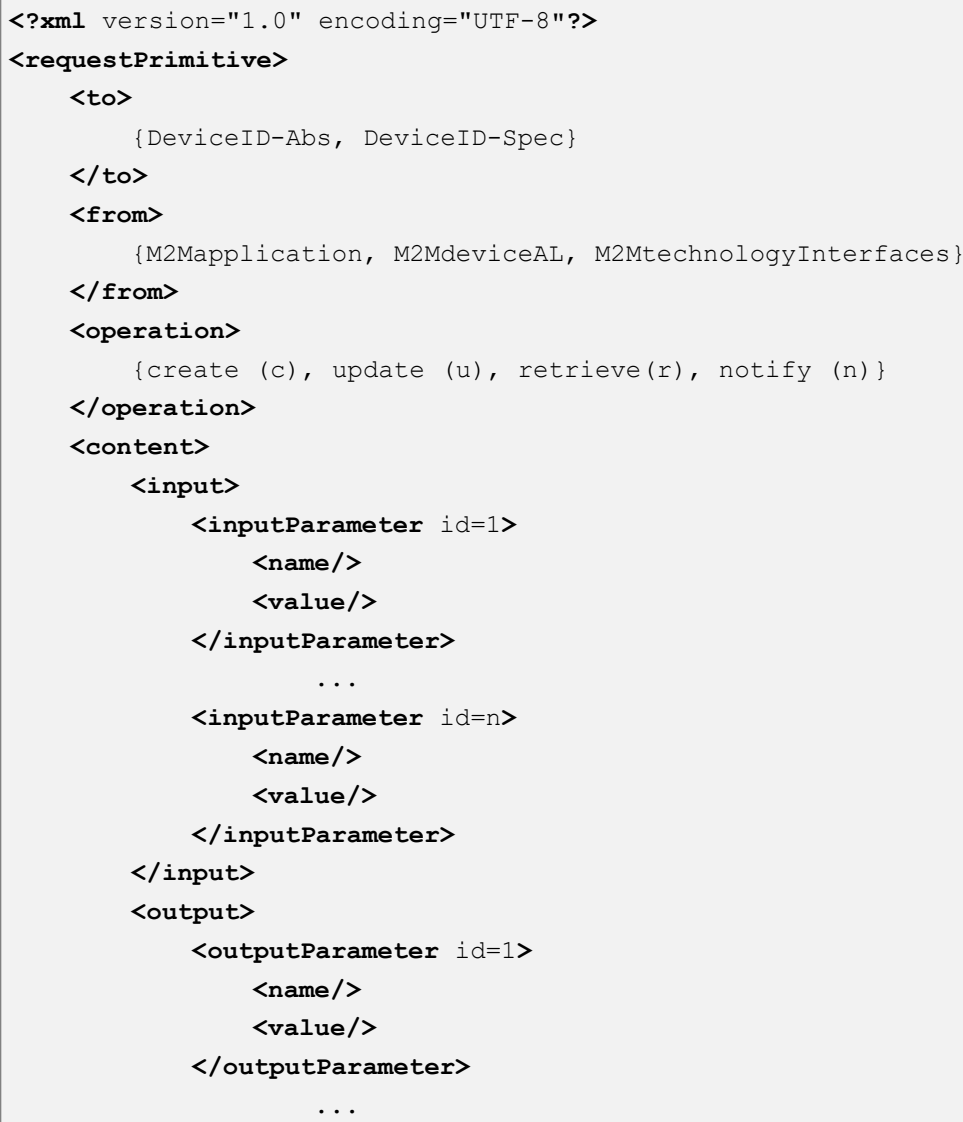




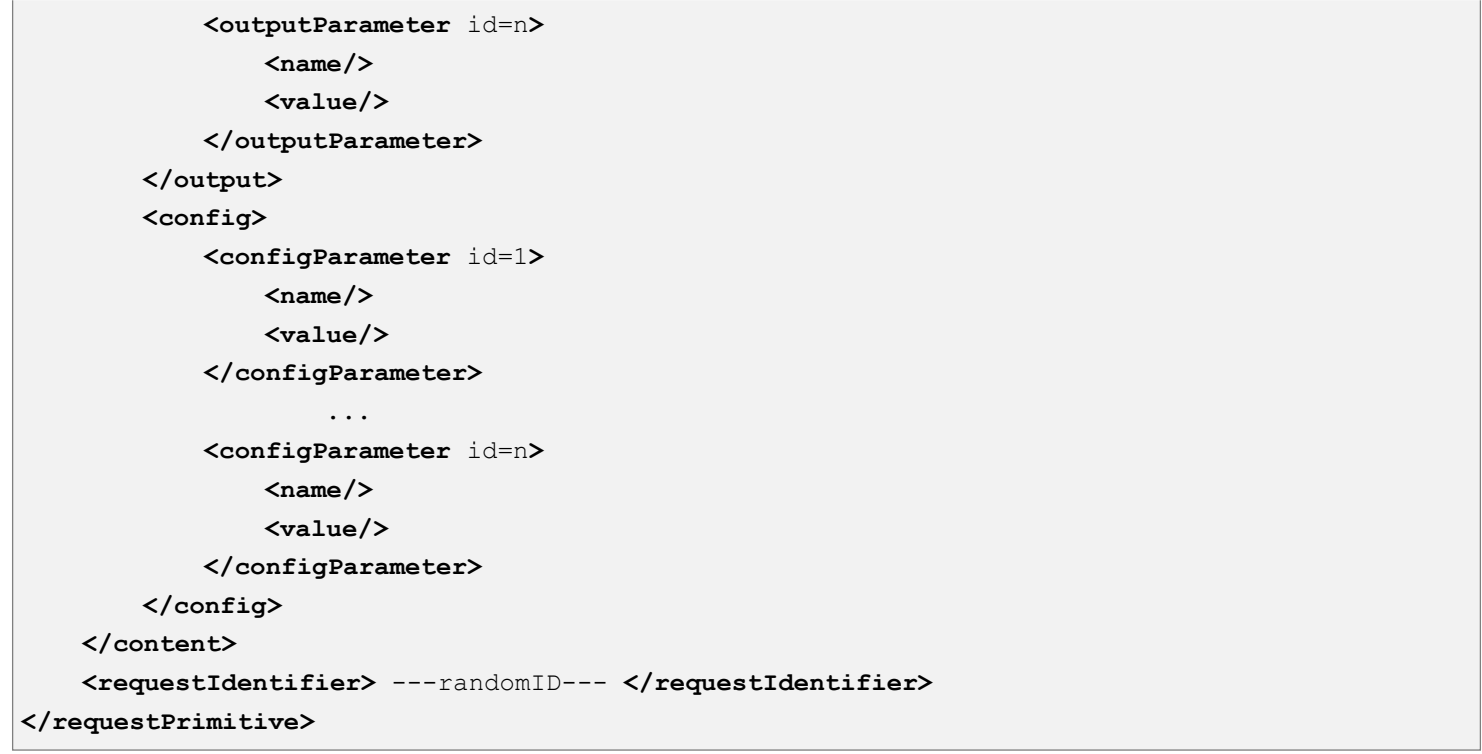

\section{Listing C.5: SCXML Representation of Use Case 1}

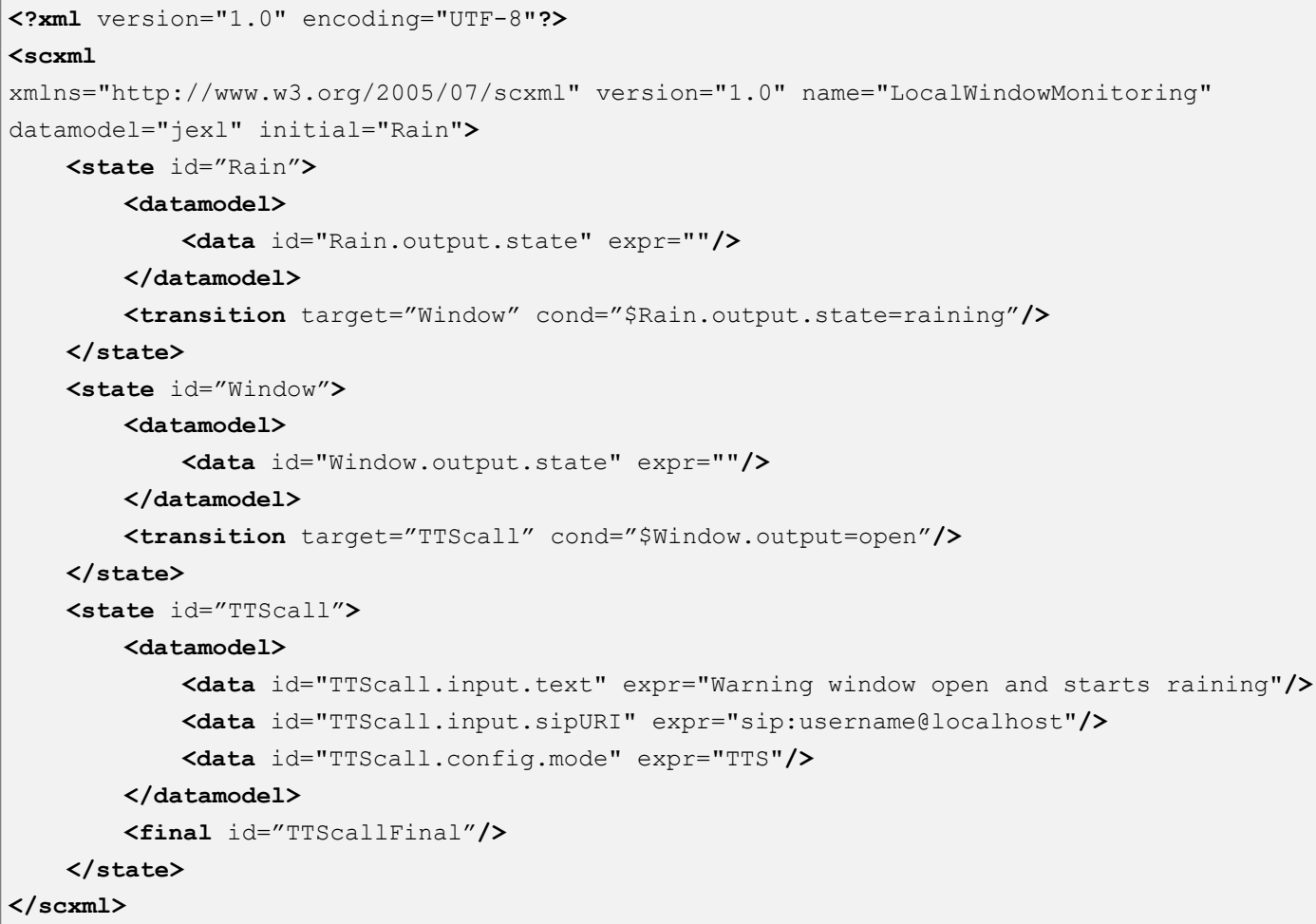




\section{Listing C.6: Principles of SCXML State Parameter Definition and Assign}

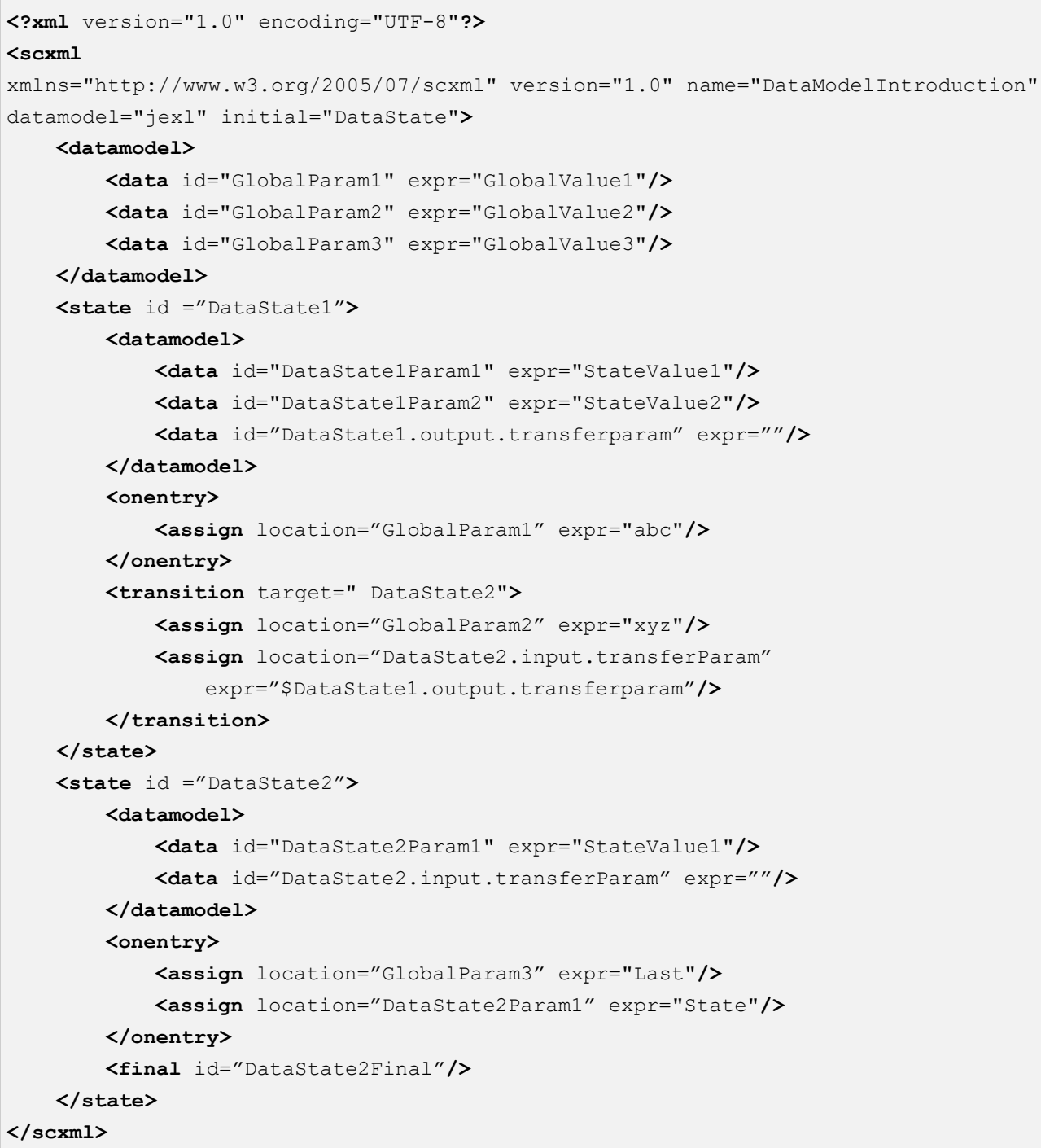

\section{Listing C.7: SCXML Representation of OR-/AND M2M Device Combination}

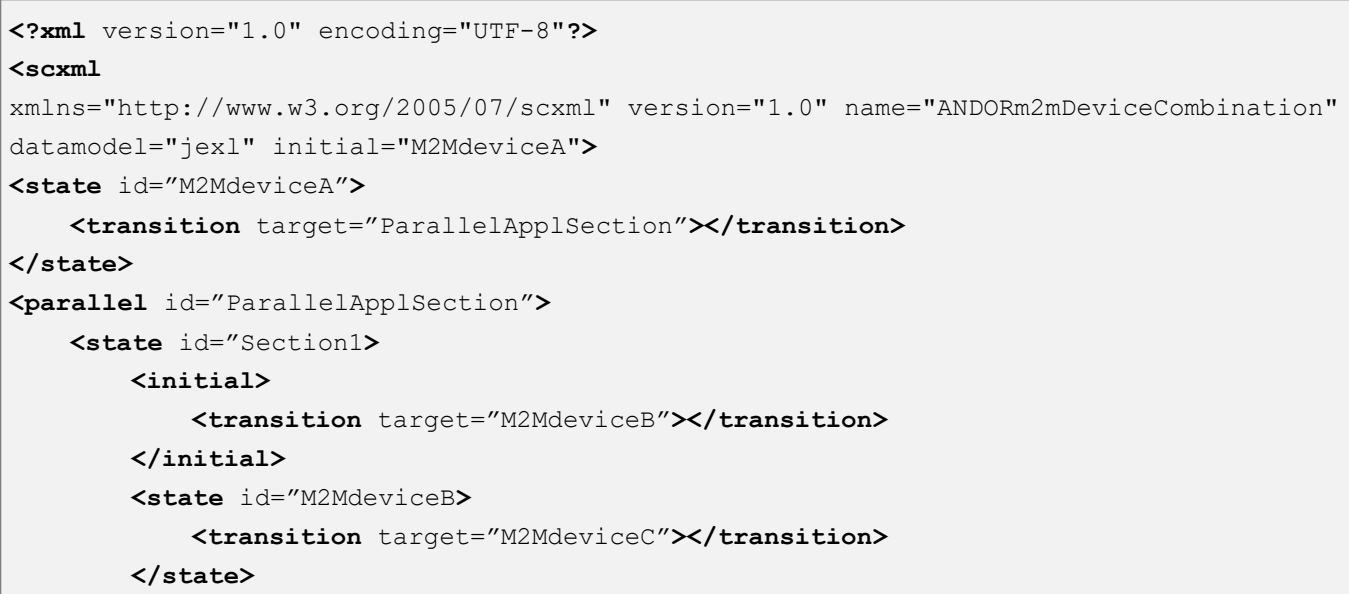




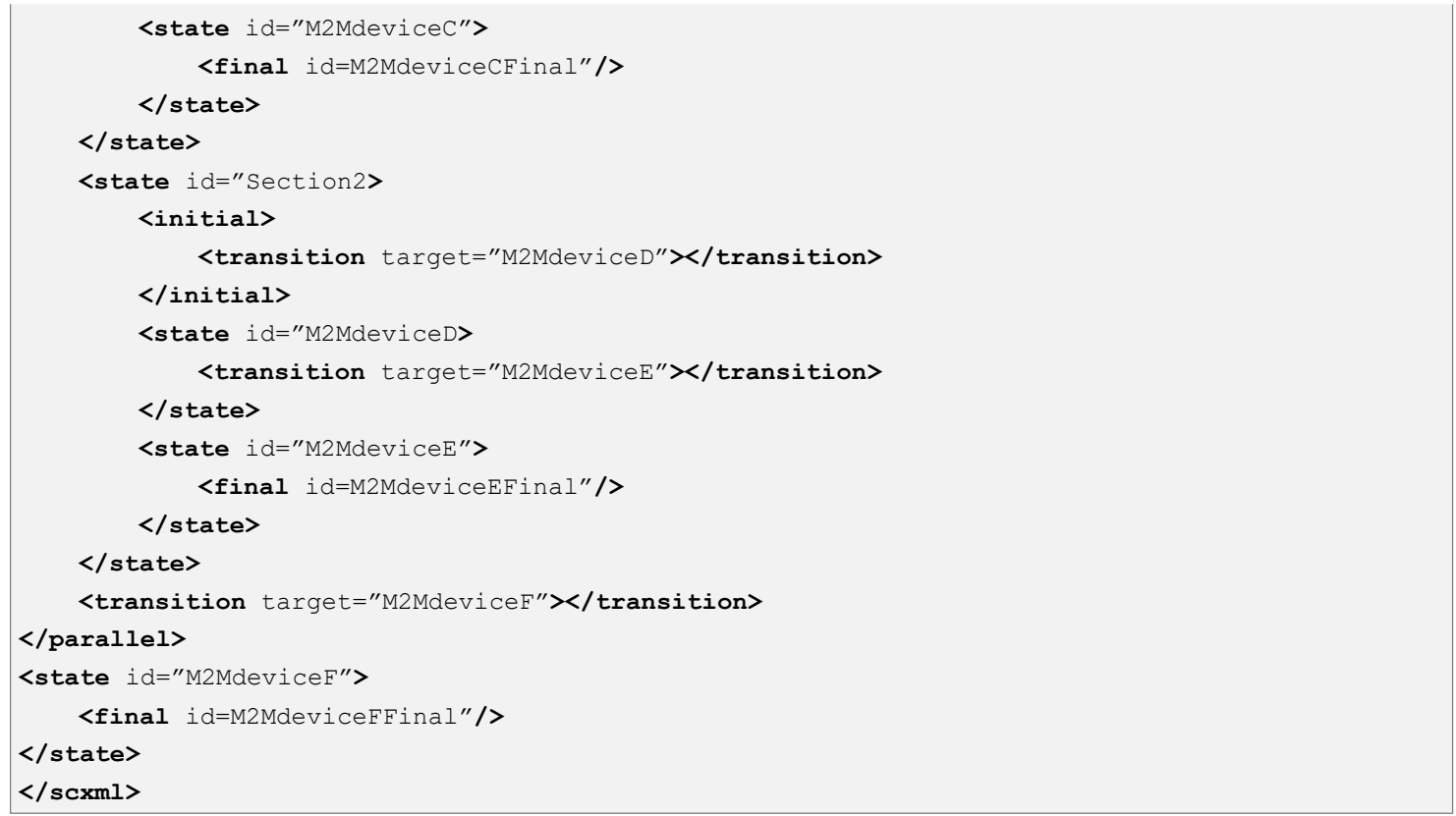

\section{Listing C.8: XML Representation of IFD for remoteRainSensor M2M Application Service}

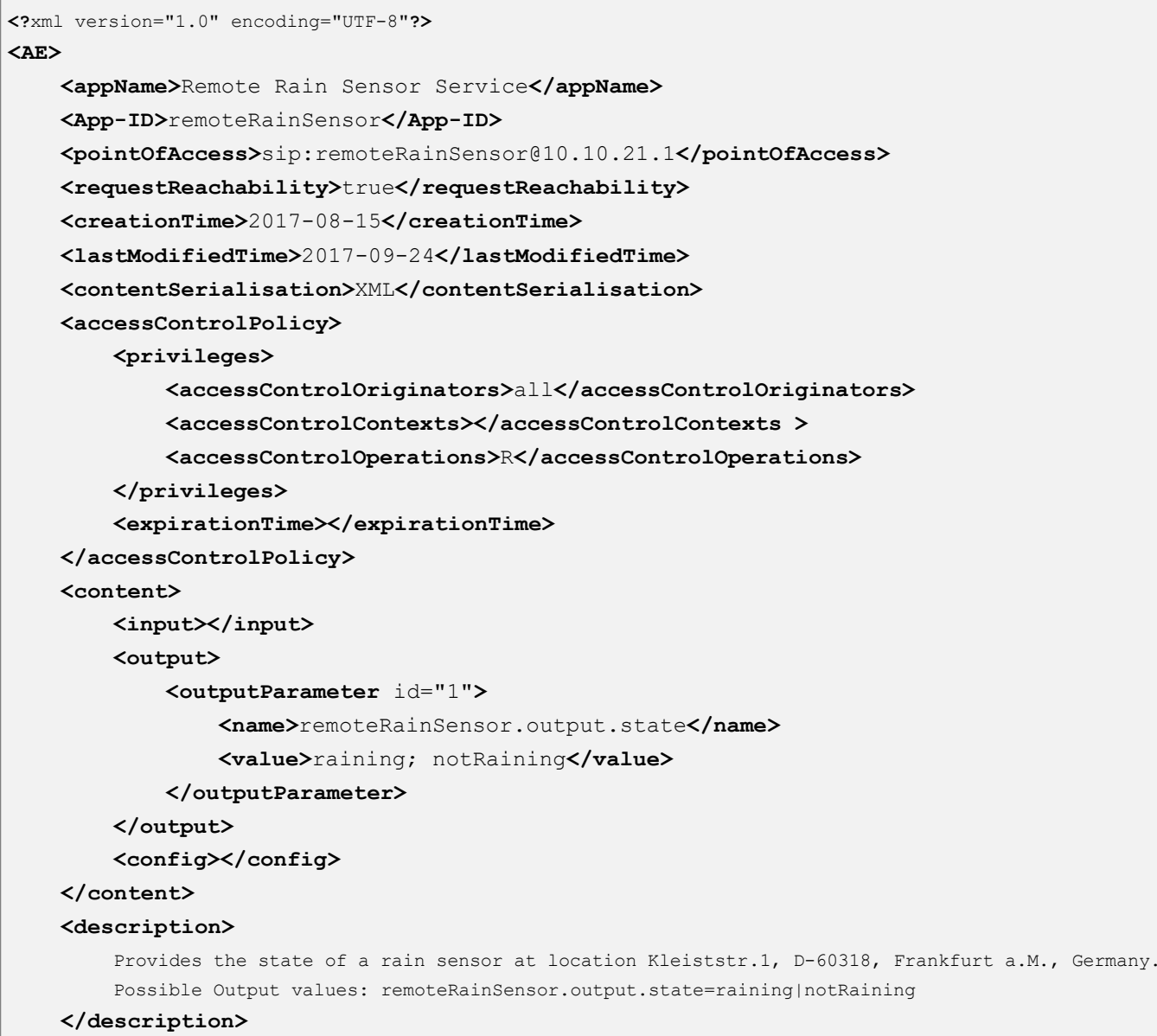




\section{Listing C.9: Extract SCXML Representation Use Case 2}

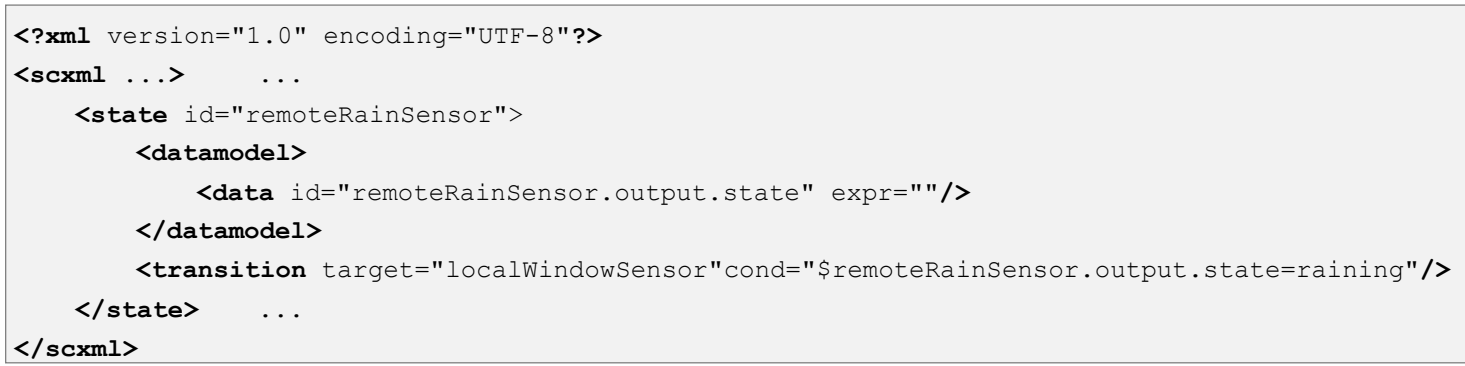

Listing C.10: Formal AD of cooperative M2M application service for Use Case 3 in SCXML format

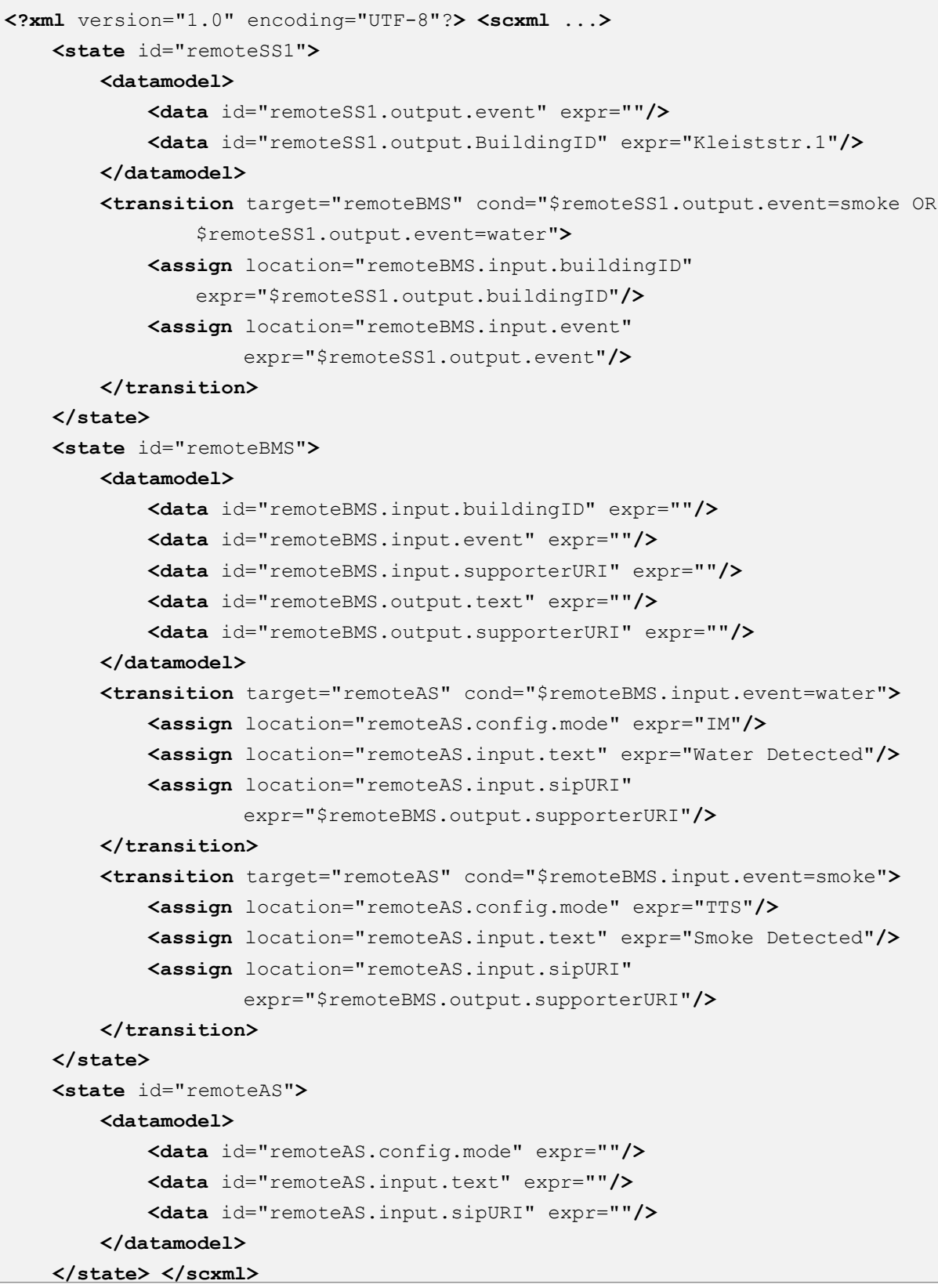


Listing C.11: Formal AD of cooperative M2M Application Service for Use Case 4 in SCXML format

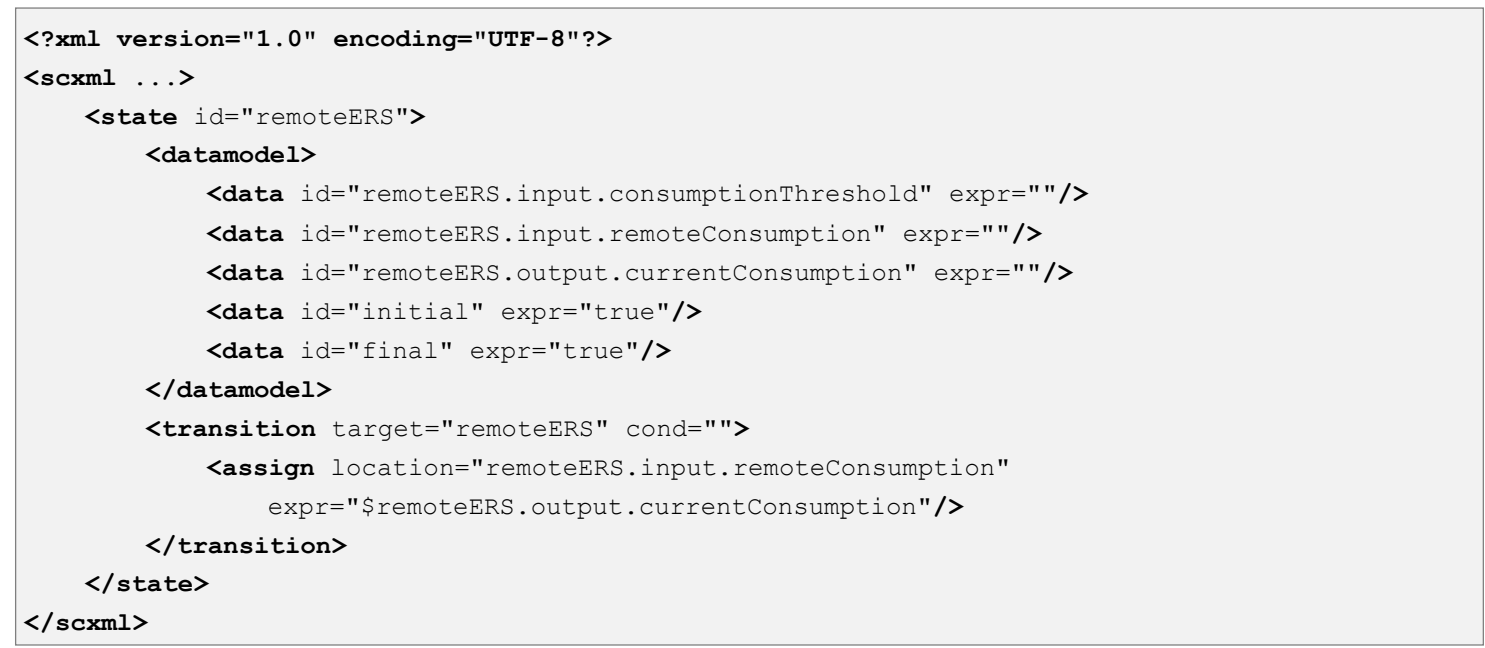

\section{Listing C.12: AccessControlPolicy for Definition of (Sub-) Community Assignment}

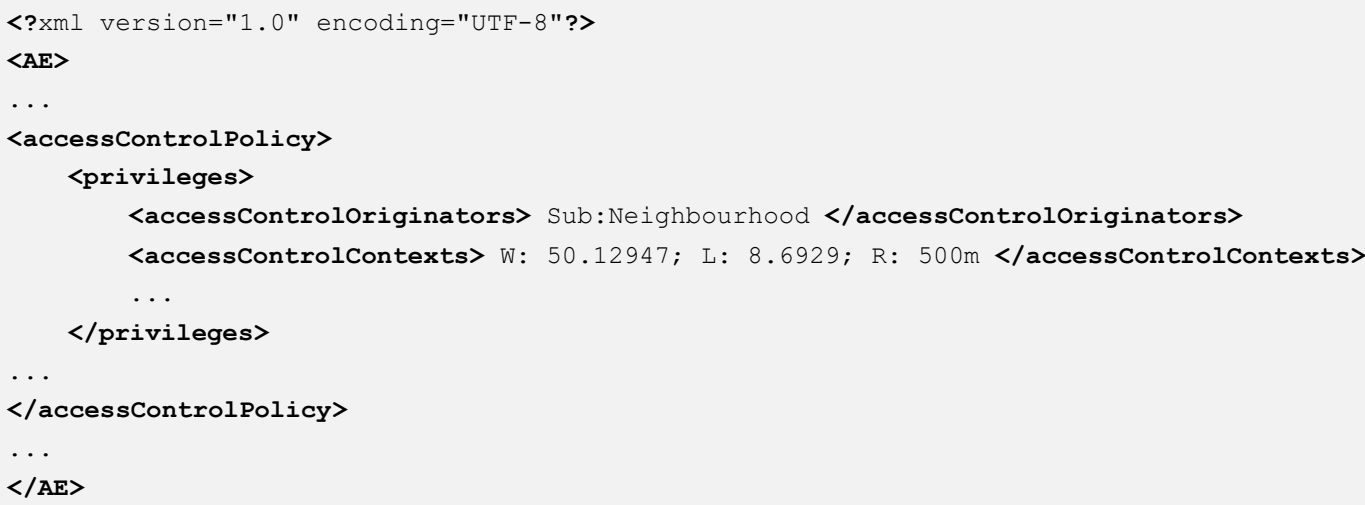

\section{Listing C.13: M2M application service IFD for Social Service}

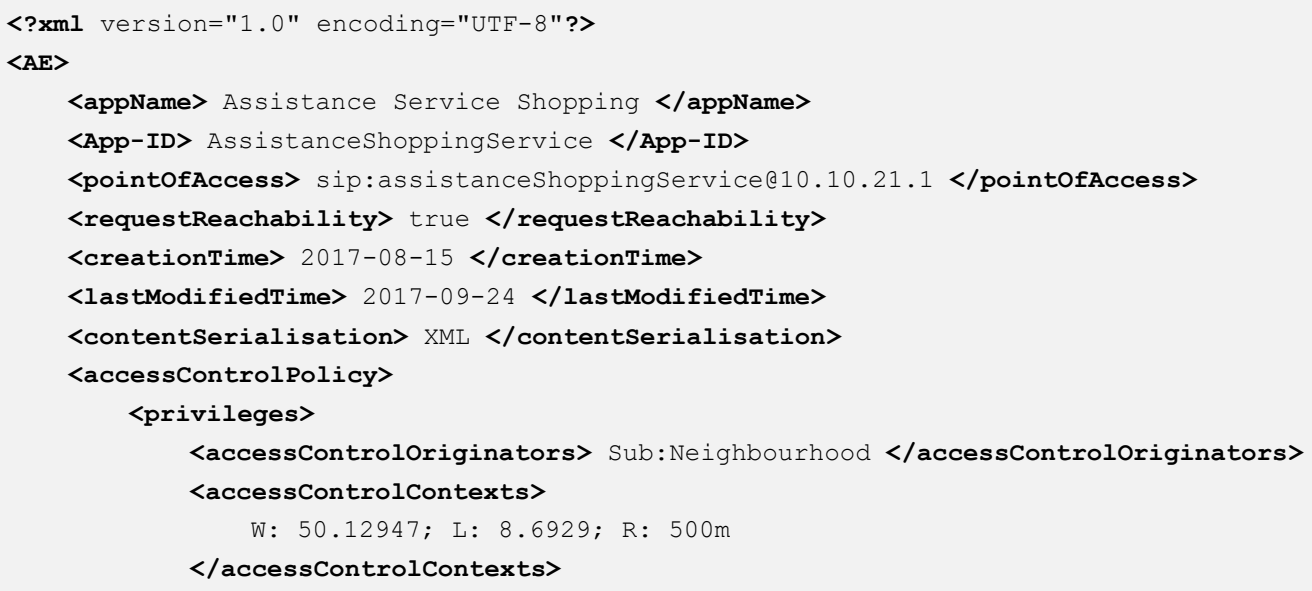




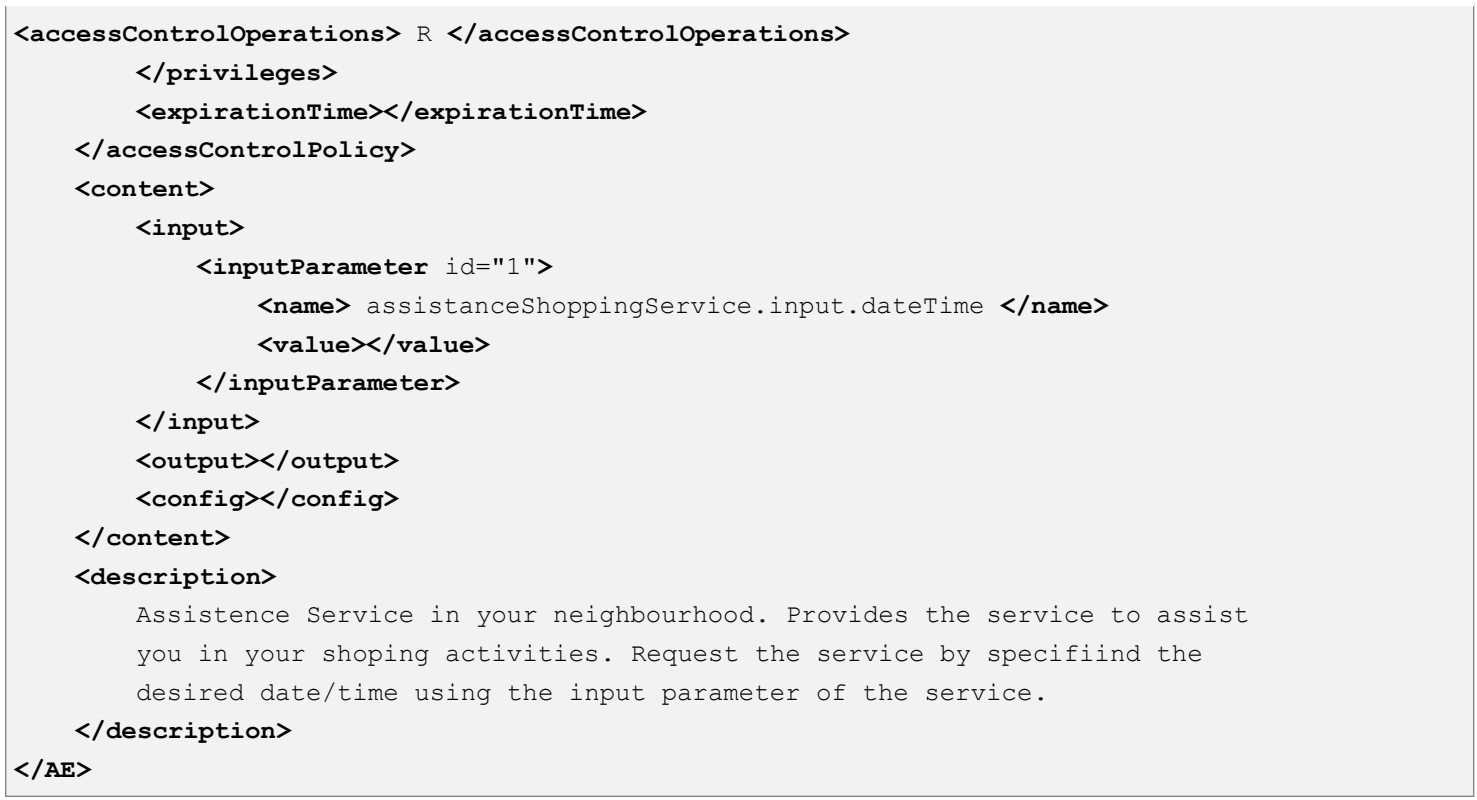




\section{Message Sequence Charts}

\section{Message Sequence Chart C.1: CoAP Messaging for Service/Information Subscription}

\begin{tabular}{|c|c|c|}
\hline $\begin{array}{l}\text { CoAP } \\
\text { Client }\end{array}$ & & $\begin{array}{l}\text { CoAP } \\
\text { Server }\end{array}$ \\
\hline 1 & & 1 \\
\hline 1 & POST coap://10.0.13.20:5683/m2mservice?ty=23 & 1 \\
\hline 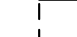 & Version=01 (Version: 1) & \\
\hline 1 & Type $=00$ (Confirmable) & 1 \\
\hline 1 & Token Length $=0100 \quad(4)$ & 1 \\
\hline I & Code $=0.02 \quad(\mathrm{POST})$ & I \\
\hline 1 & Message $I D=3130$ & I \\
\hline 1 & Token= bfb31d74 & 1 \\
\hline 1 & Opt Name \#1: 07 (Uri-Port) : 5683 & 1 \\
\hline I & & i \\
\hline i & Opt Name \#2: 11 (Uri-Path): m2mService & i \\
\hline 1 & Opt Name \#3: 12 (Content-Format): application/xml & 1 \\
\hline 1 & Opt Name \#4: Uri-Query: ty $=23$ & 1 \\
\hline 1 & ... & 1 \\
\hline 1 & Opt Name \#6: 256 (oneM2M-FR) : coap://10.0.4.20:5683/m2mservice & 1 \\
\hline i & Opt Name \#7: 257 (oneM2M-RQI): 333933333834 & i \\
\hline 1 & [Opt Name \#7: 259 (oneM2M-OT): 0] if one-time subscription & i \\
\hline 1 & Payload: & 1 \\
\hline 1 & $<$ Content $>$ & 1 \\
\hline 1 & <output> & 1 \\
\hline 1 & <outputParameter id="1"> & 1 \\
\hline i & $<$ name $>$ & 1 \\
\hline 1 & remoteM2MapplicationService.output.parameter1 & i \\
\hline 1 & $</$ name $\rangle$ & I \\
\hline 1 & </name $>$ & 1 \\
\hline 1 & </outputParameter $>$ & i \\
\hline 1 & $</$ output $>$ & 1 \\
\hline 1 & $</$ Content $>$ & 1 \\
\hline 1 & & i \\
\hline I & & I \\
\hline 1 & $\mathrm{ACK}$ & 1 \\
\hline K & Version=01 (Version: 1) & +1 \\
\hline i & Type $=10$ (Acknowledgement) & 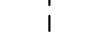 \\
\hline i & Type = 10 (Acknow 1 edgement) & i \\
\hline i & Token Length $=0100$ (4) & i \\
\hline i & Code $=2.05$ (Content) & i \\
\hline 1 & Message $I D=3130$ & i \\
\hline 1 & Token $=\mathrm{bfb} 31 \mathrm{~d} 74$ & 1 \\
\hline 1 & Opt Name \#1: 257 (oneM2M-RQI): 333933333834 & 1 \\
\hline
\end{tabular}




\section{Message Sequence Chart C.2: CoAP Messaging for Service/Information Notification}

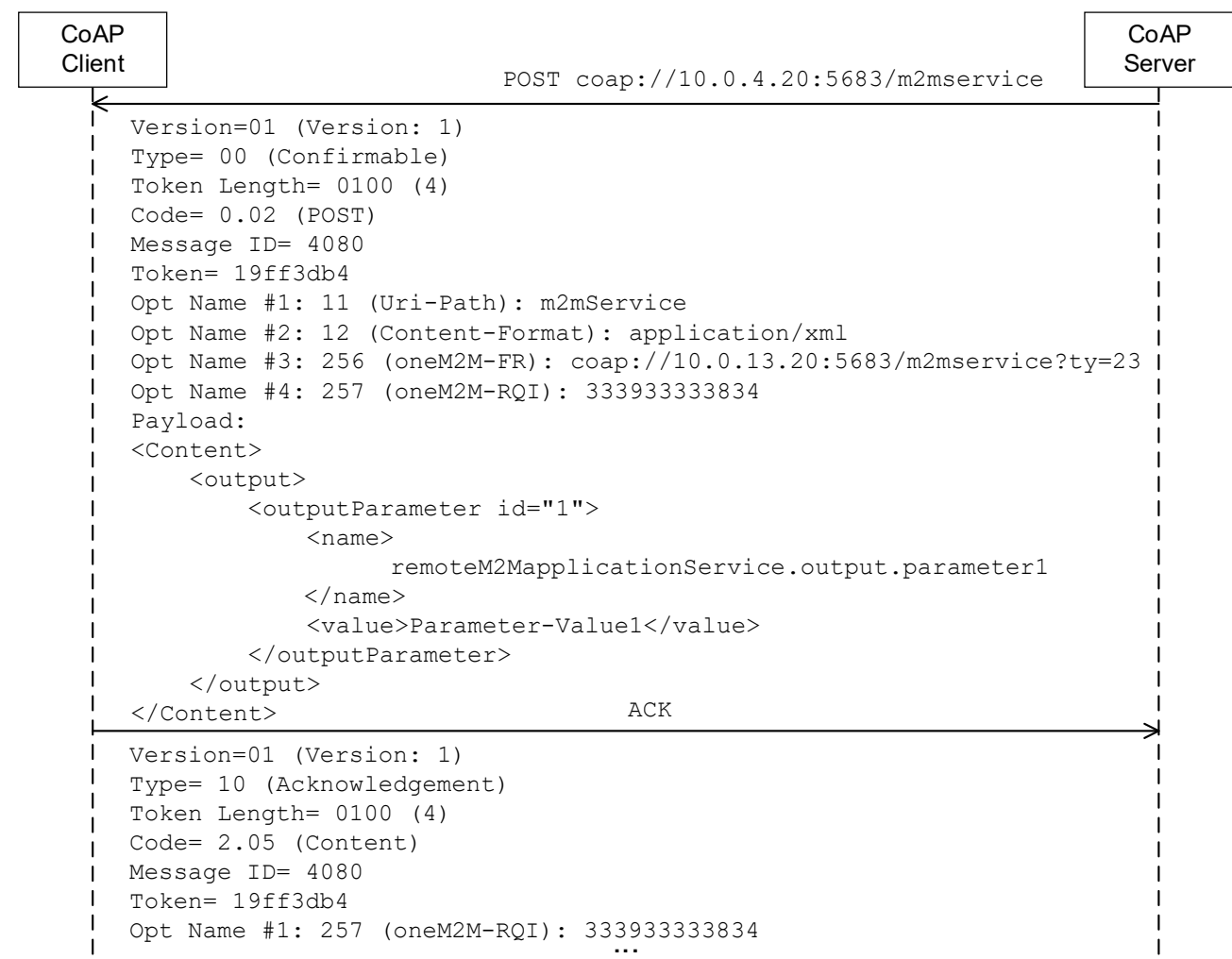

\section{Message Sequence Chart C.3: CoAP Messaging for Service/Information Unsubscription}

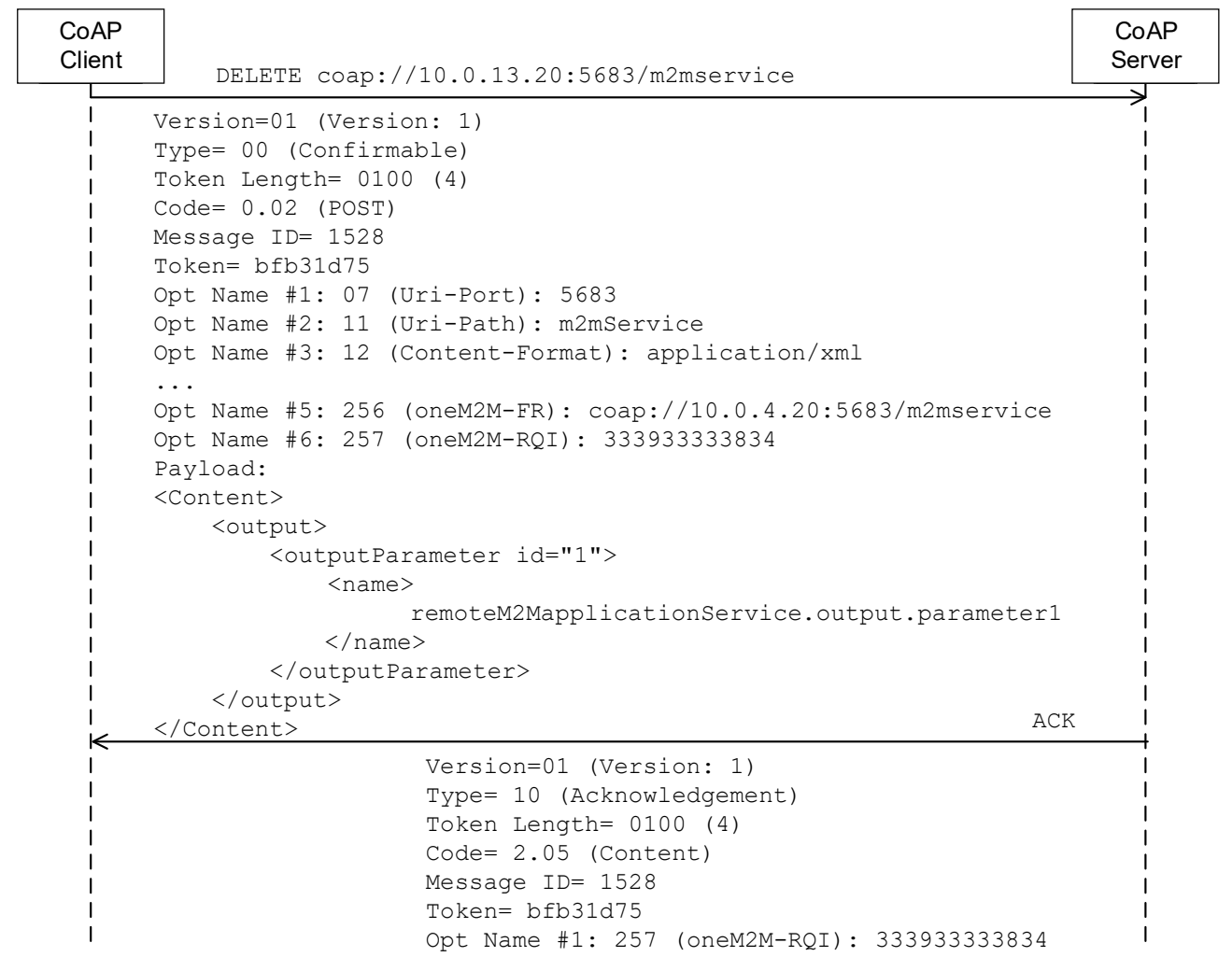




\section{Message Sequence Chart C.4: SIP Messaging for Service/Information Subscription}

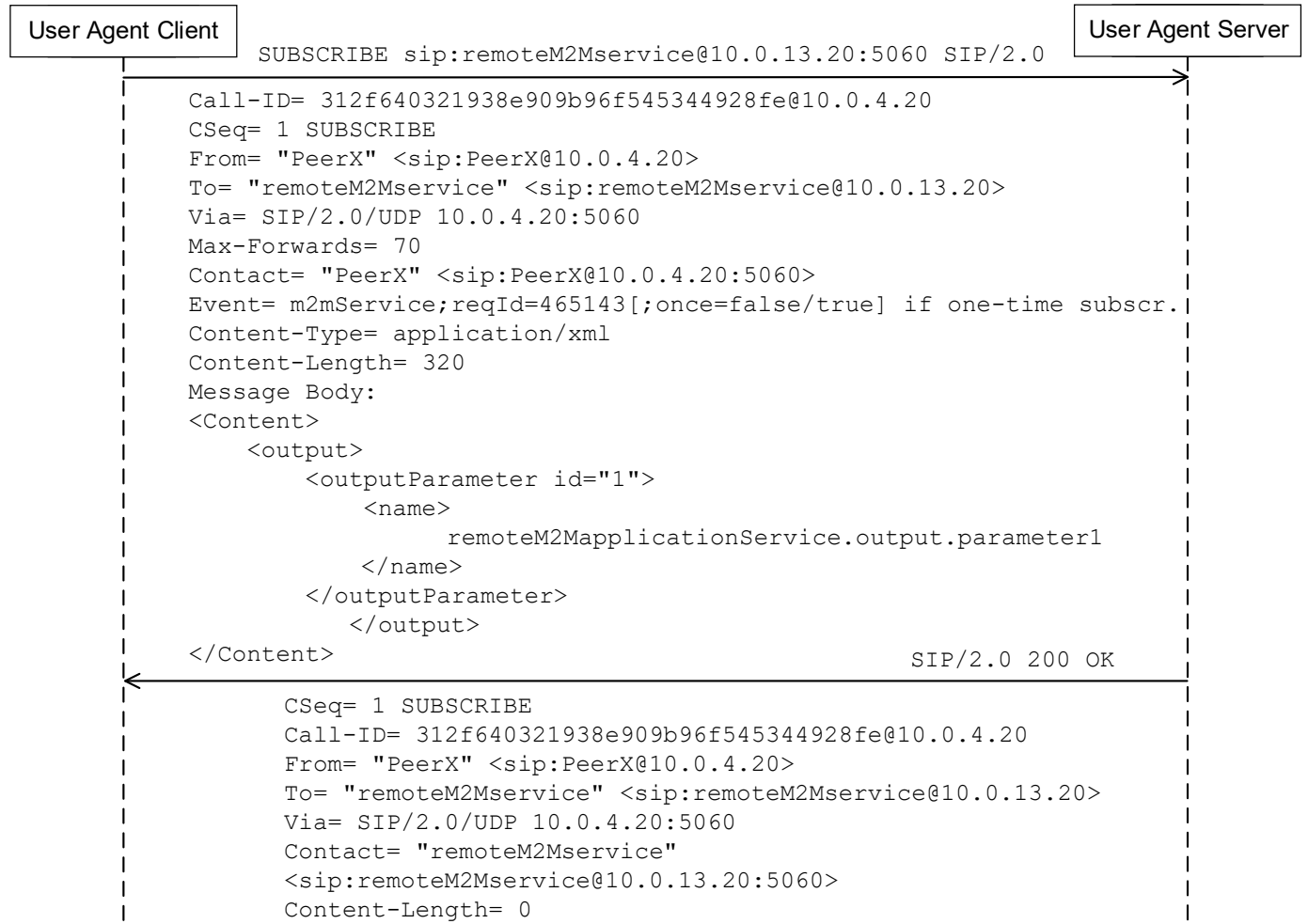

\section{Message Sequence Chart C.5: SIP Messaging for Service/Information Notification}

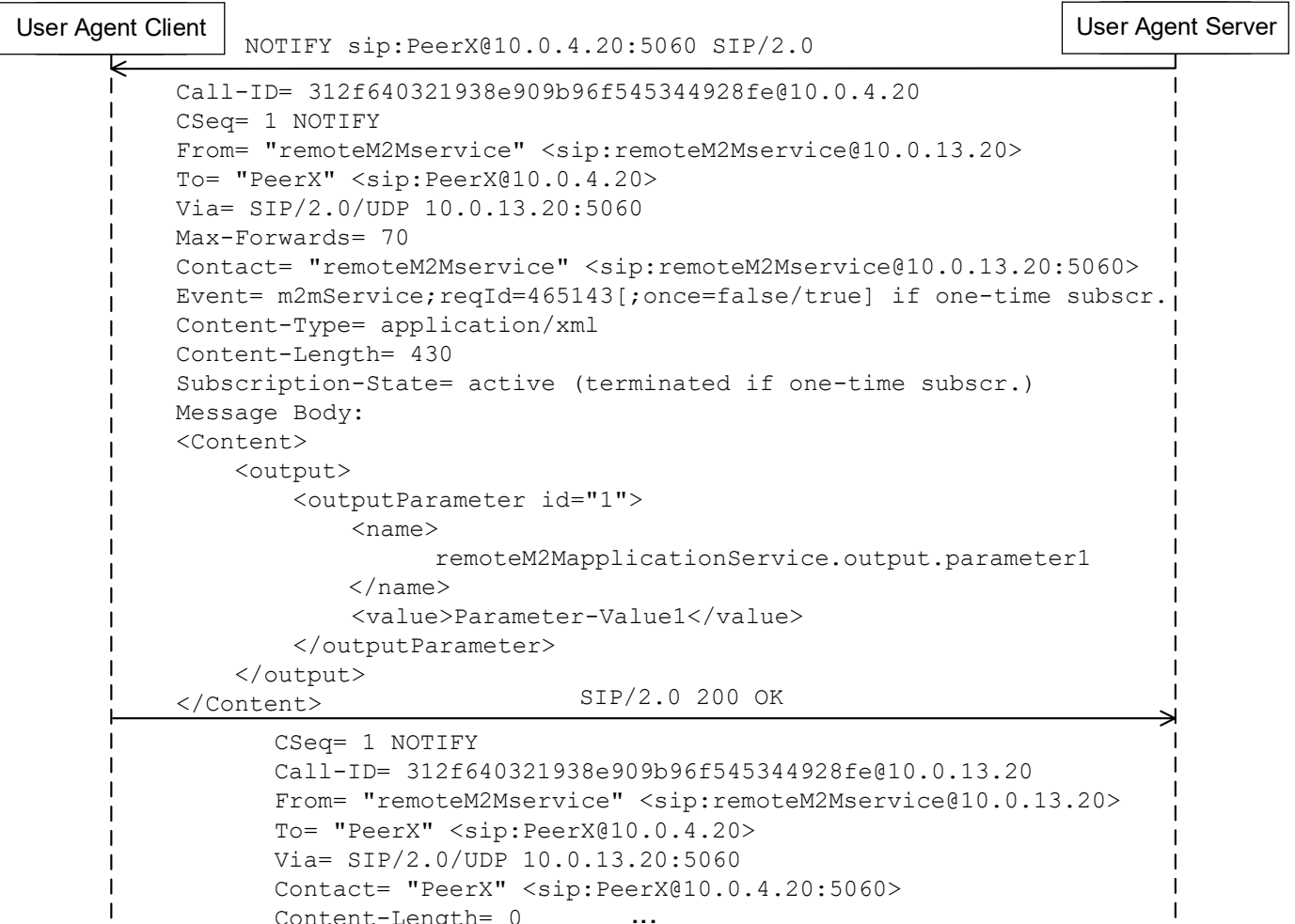




\section{Message Sequence Chart C.6: SIP Messaging for Service/Information Unsubscription}

\begin{tabular}{|c|c|c|c|}
\hline $\begin{array}{l}\text { User Agent } \\
\text { Client }\end{array}$ & SUBSCRIBE sip:remoteM2Mservice@10.0.13.20:5060 & $\mathrm{SIP} / 2.0$ & $\begin{array}{c}\text { User Agent } \\
\text { Server }\end{array}$ \\
\hline I & Call-ID $=312 f 640321938 \mathrm{e} 909 b 96 f 545344928$ fed10.0.4.2 & 20 & $>_{1}$ \\
\hline 1 & $\mathrm{CSeq}=2$ SUBSCRIBE & & i \\
\hline I & & & I \\
\hline I & To= "remoteM2Mservice" <sip:remoteM2Mserviced10.0 & $1320>$ & I \\
\hline 1 & & $.13 .20>$ & I \\
\hline 1 & Via= SIP $/ 2.0 /$ UDP $10.0 .4 .20: 5060$ & & 1 \\
\hline 1 & Max-Eorwards $=70$ & & I \\
\hline 1 & Contact $=$ "PeerX" <sip:PeerX@10.0.4.20:5060> & & 1 \\
\hline 1 & Event $=\mathrm{m} 2 \mathrm{mService} ;$ reqId $=465143[$; once $=$ false $/$ true $]$ & if one-time sub & $\operatorname{scr} \cdot 1$ \\
\hline i & Expires $=0$ & & । \\
\hline i & Content-Type $=$ application $/ x m l$ & & i \\
\hline I & Content-Length $=320$ & & I \\
\hline 1 & Message Body: & & 1 \\
\hline 1 & & & 1 \\
\hline I & <Content> & & i \\
\hline i & <output> & & I \\
\hline i & <outputParameter id="1"> & & i \\
\hline i & $<$ name $>$ & & I \\
\hline 1 & remoteM2MapplicationService.outp & put.parameter 1 & 1 \\
\hline 1 & $<$ /name $>$ (n) & 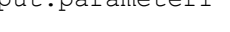 & I \\
\hline i & $</$ name $>$ & & I \\
\hline i & </outputParameter > & & i \\
\hline I & $</$ output $>$ & & I \\
\hline 1 & $</$ Content $>$ & $\mathrm{SIP} / 2.0200 \mathrm{OK}$ & 1 \\
\hline$\hat{\imath}$ & $\mathrm{CSeq}=2 \mathrm{SUBSCRIBE}$ & & i \\
\hline i & Call-ID $=312$ f640321938e909b96f545344928fed 1 & 127.0 .0 .1 & i \\
\hline I & From $=$ "PeerX" <sip:PeerXa10.0.4.20> & & i \\
\hline 1 & To="remeteM2Mservice"<sin.remeteM2Mservic & $\begin{array}{lllll}2 & 0 & 13 & 20 & 20\end{array}$ & I \\
\hline I & IO= remotemZMservice" <sip:remotelMZMservic & $\operatorname{ced} 10.0 .13 .20>$ & I \\
\hline 1 & $\mathrm{Via}=\mathrm{SIP} / 2.0 / \mathrm{UDP} 10.0 .4 .20: 5060$ & & I \\
\hline 1 & Contact= "remoteM2Mservice" & & i \\
\hline 1 & <sip:remoteM2Mserviced10.0.13.20:5060> & & I \\
\hline I & Content-Length $=0$ & & I \\
\hline
\end{tabular}

